Fall 1968

\title{
1968 Miracle Yearbook
}

Cedarville College

Follow this and additional works at: https://digitalcommons.cedarville.edu/yearbooks

Part of the Higher Education Commons, Organizational Communication Commons, and the Public Relations and Advertising Commons

\section{Recommended Citation}

Cedarville College, "1968 Miracle Yearbook" (1968). Yearbooks. 21.

https://digitalcommons.cedarville.edu/yearbooks/21

This Book is brought to you for free and open access by DigitalCommons@Cedarville, a service of the Centennial Library. It has been accepted for inclusion in Yearbooks by an authorized administrator of DigitalCommons@Cedarville. For more information, please contact digitalcommons@cedarville.edu. 


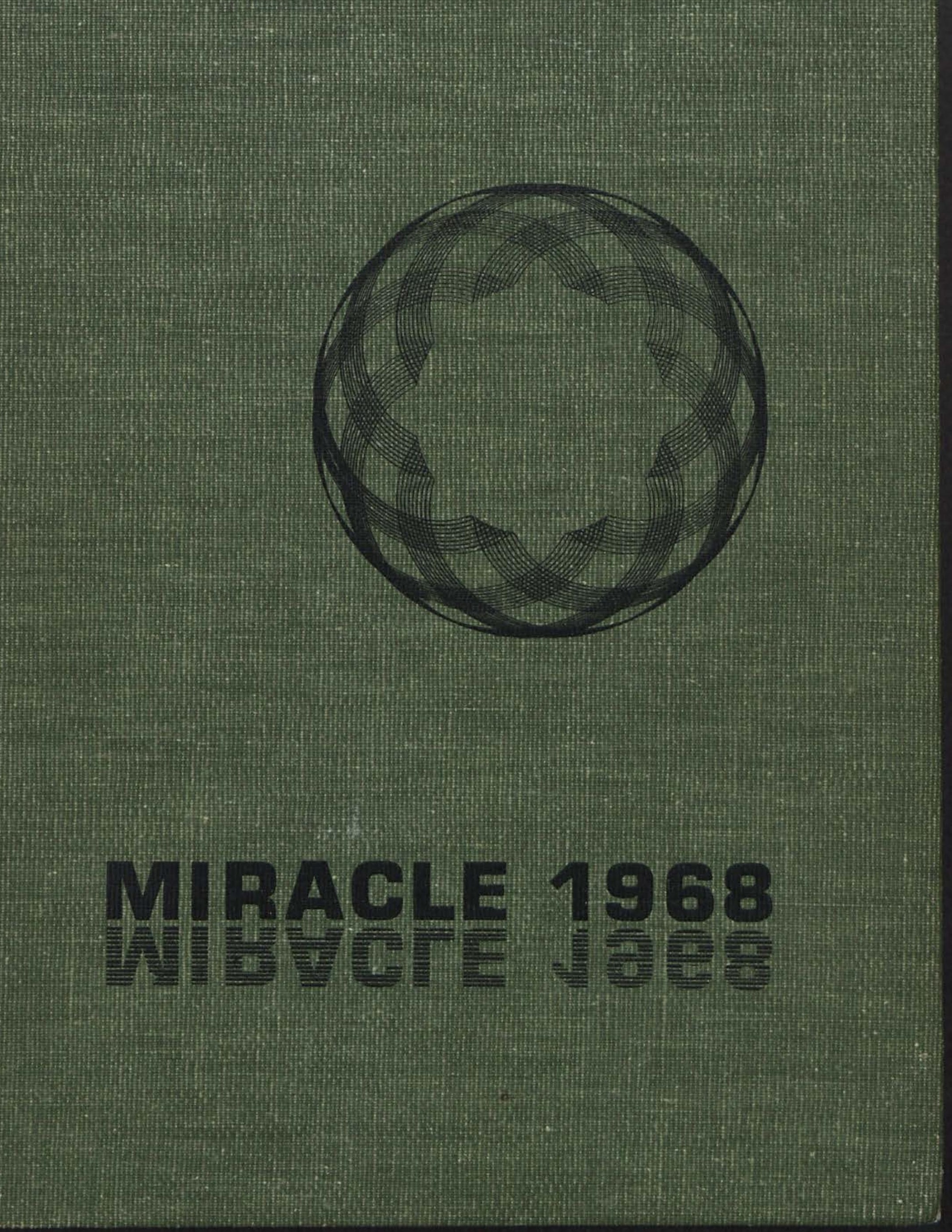







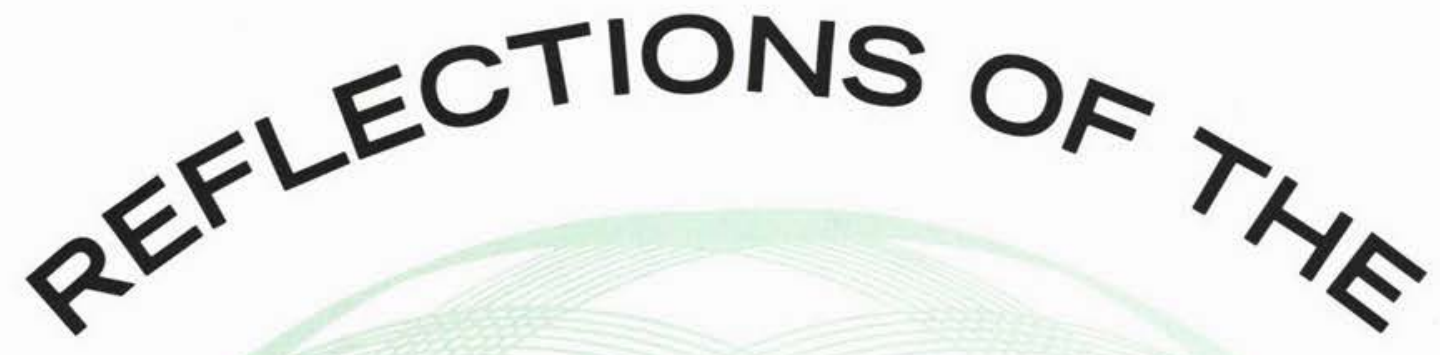

1968

Dianne Chaffe, Editor

Dianna Hutchison, Assistant Editor

David Chumbler, Financial Manager

Dr. Donald Baumann, Advisor

Cedarville College

Cedarville, Ohio

\section{MIRACLE}




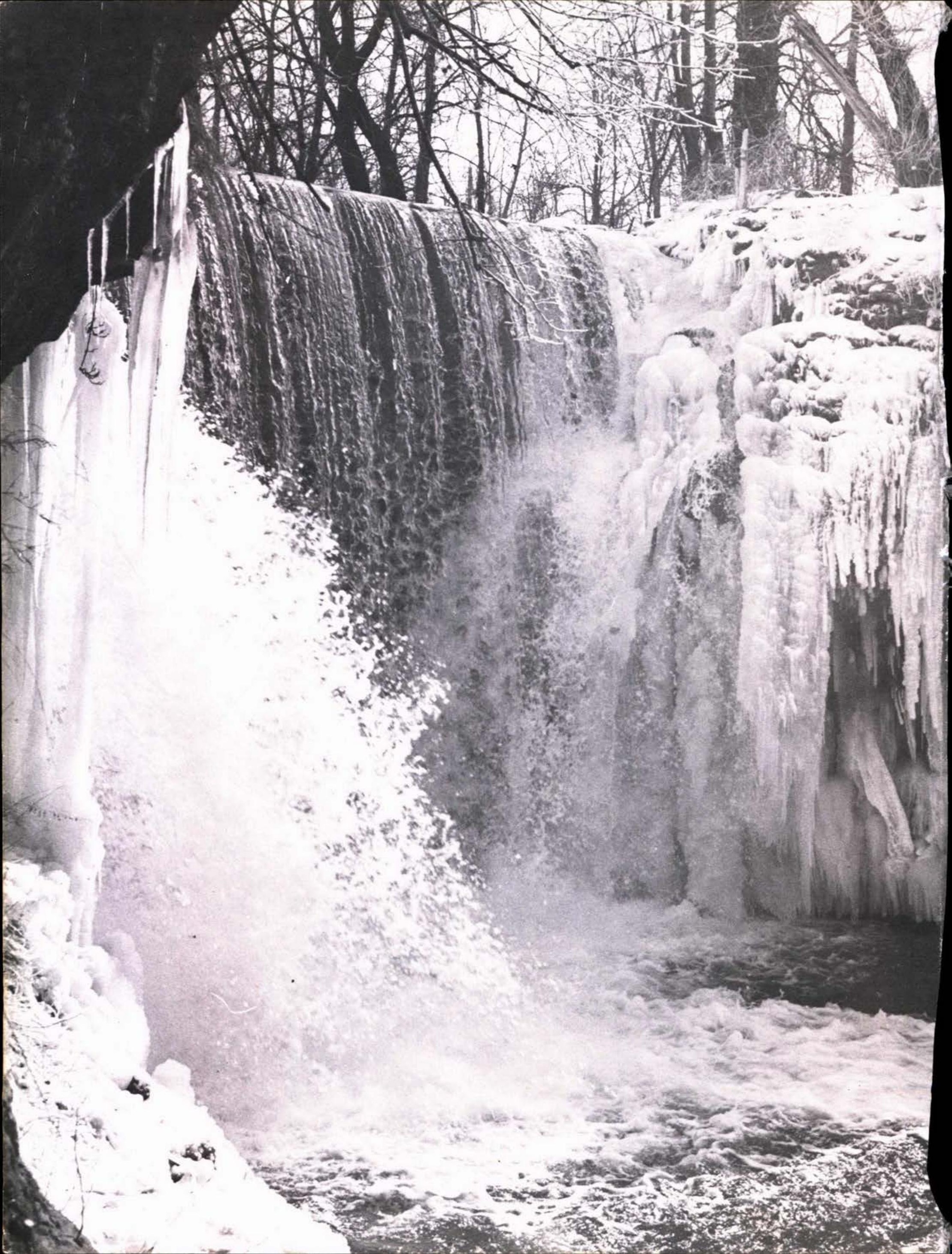




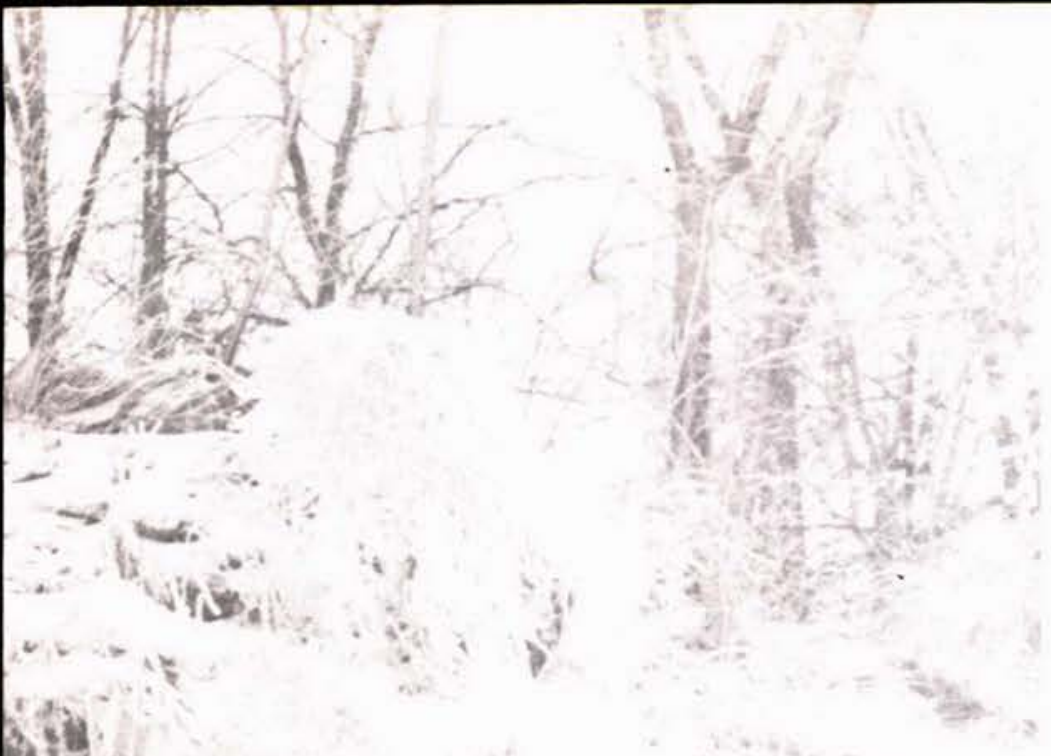

(a)

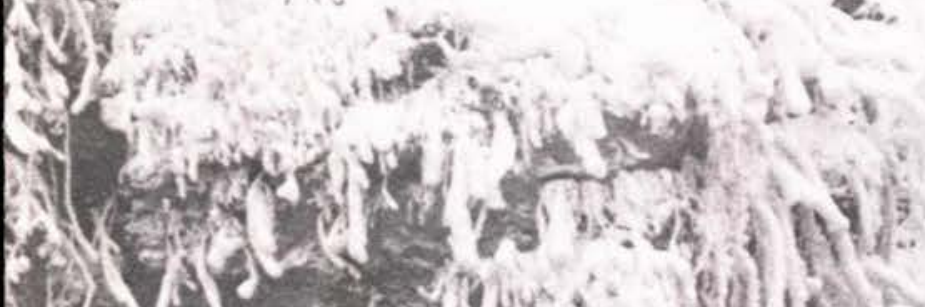

TABLE OF CONTENTS

Dedication .......... 4

Faculty and Staff $\ldots \ldots \ldots 6$

Students ........... 44

Groups and Organizations ... 118

Activities and Special Events 148

In Memoriam . . . . . . . . 180

Advertisements . . . . . . . 190

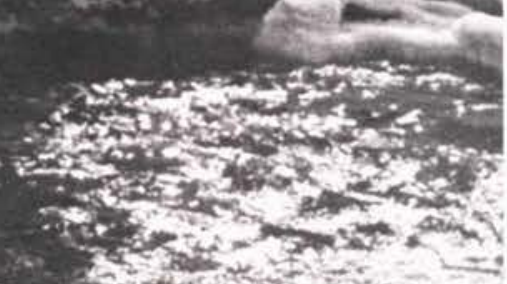

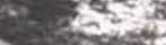




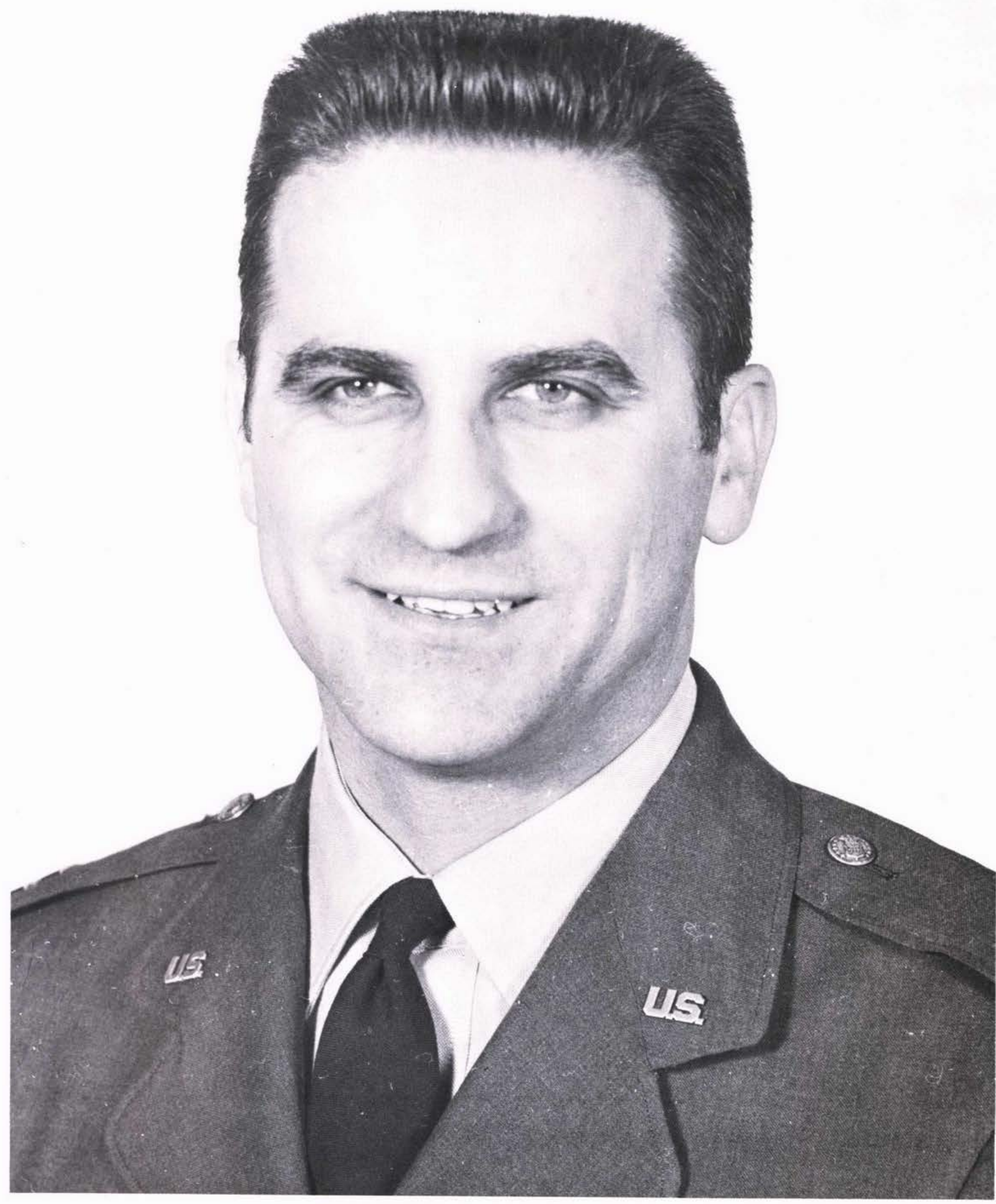

DR. JOHN W. REED 


\section{DEDICATION}

Major, Chaplain, Doctor, Professor ...

A title means so little;

A character and a soul submerged

Mean so much.

While recognizing his outstanding

Degrees of intellectual achievement,

Students respect him more

For his degrees of understanding and concern.

As head of the Speech Department

He communicates very effectively,

But not with words alone,

For his characteristic

Smile, enthusiasm, and warmth

Enable him to express himself

In a way no speech text

Ever comprehended or delineated.

He has been often heard to exclaim,

"It's a great way to live!"

We would affirm.

Having him on our campus,

Having him play

Such a vital part in our lives

Is a great way to live.

Through his energy, help, and interest

He has won the hearts of the student body.

It is with great pride

That we dedicate

The Miracle, 1967-68

To Doctor John W. Reed.
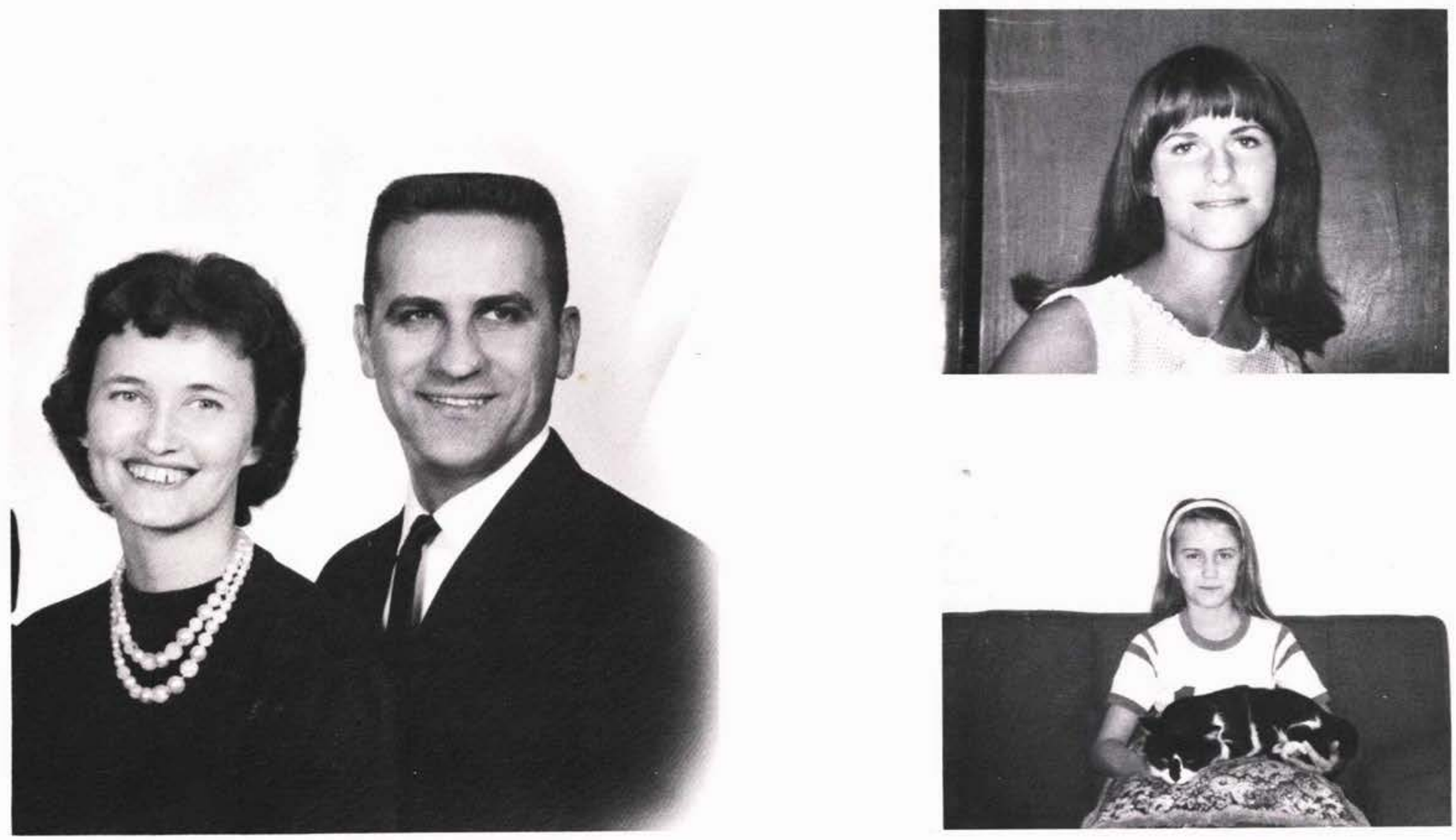


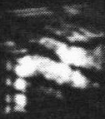

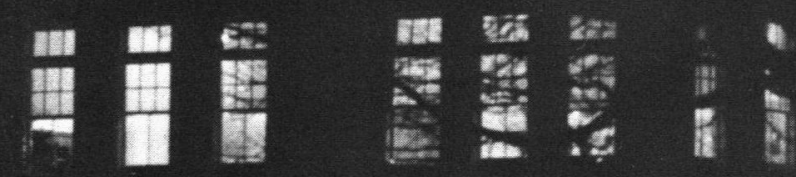
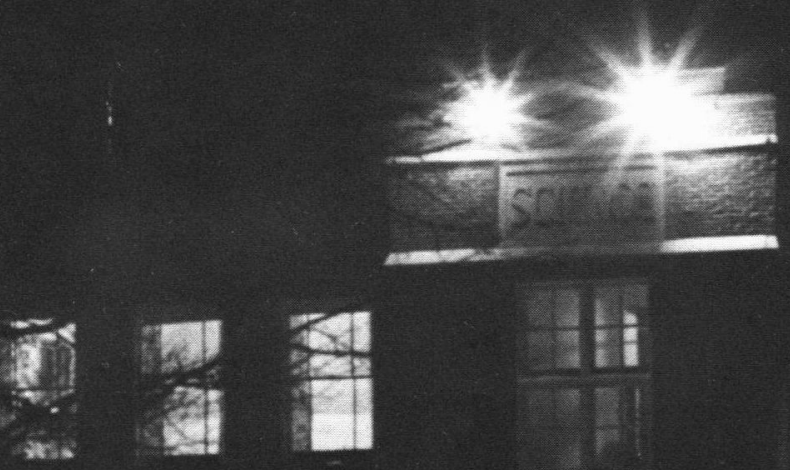

iii

six
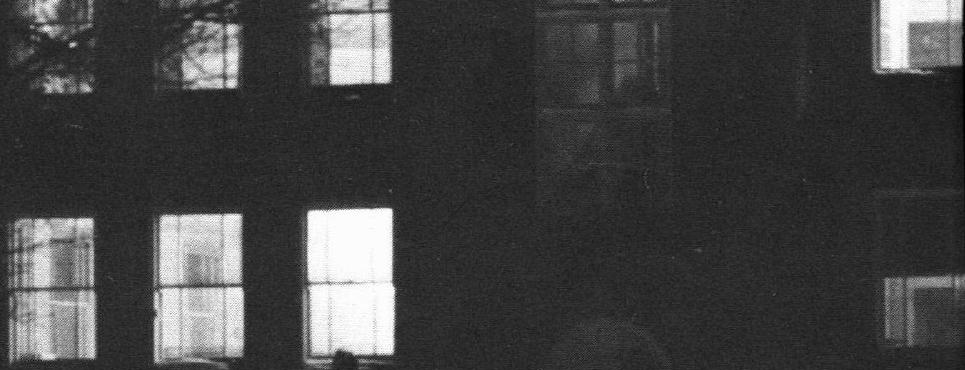

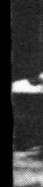

$-$

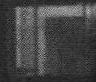

it 1

the

1 


\section{Faculty and Staff}

Cultivate the intellect, and you shall have a mind that produces beautiful thoughts, worthy images, helpful ideas; that will serve as a solace in times of stress, and be to you a refuge 'gainst all the storms that blow. The cultured mind, as compared with the uncultured, is the difference between a beautiful garden which produces vegetables, fruits or flowers, and a tract of land that is overgrown with weeds and brambles.

To be a person of culture is to be at home under all conditions. Your mind is stored with mental images, and memory comes to keep you company, and guide you from nostalgia and the sense of separateness to universality or oneness with the Divine. The country will be beautiful to you in any season, and society and solitude each will be welcomed by you in turn. You are to reject nothing, despise nothing, knowing that everything belongs somewhere, and that it is needed to make up the great mosaic of life. 


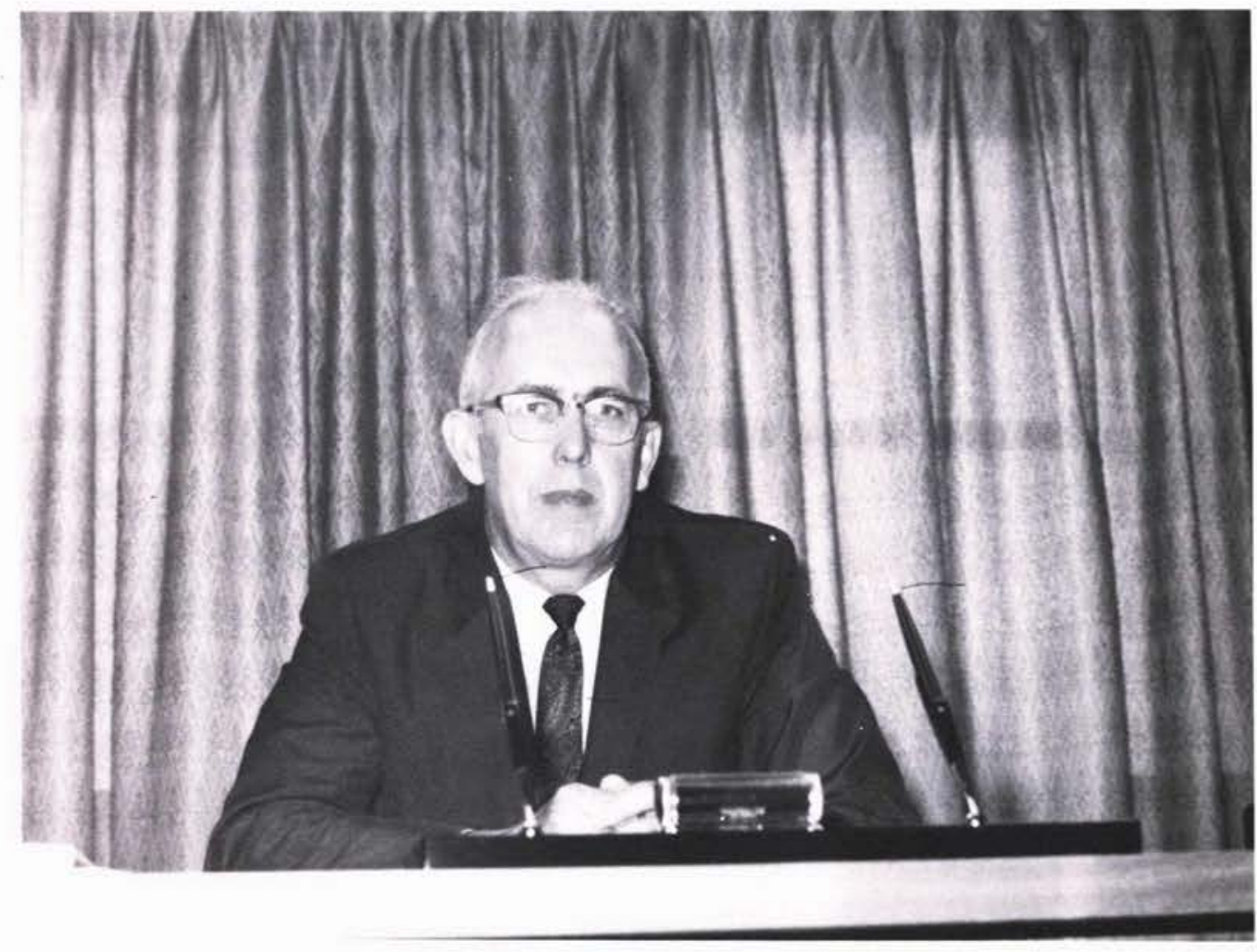

PRESIDENT JAMES T. JEREMIAH

Fifteen years have passed since we became a part of the Cedarville College family. This time has gone by in an unbelievably swift manner. There have been disappointments, but they have vanished from our memory as we have been surrounded with the abundant blessings of our most-gracious God. What a difference there is now as we consider a number of contrasts.

The faculty has grown from eight to fifty; our campus has increased from nineteen to over one hundred acres. In 1953 there were nine buildings on the campus. Since then most of these buildings have undergone some remodeling. Now there are seven additional buildings available for the use of our college family. Then less than one hundred young people made up our student body. Now it numbers more than eight hundred.

Only an all-wise God Who keeps the record knows what the spiritual results of this educational ministry has been through the years which have passed and will become in the future. As we began this experience with the Lord on Cedarville College campus, it was with the assurance that "God is able to make all grace abound toward you, that ye, always having all sufficiency in all things, may abound unto every good work" (II Cor. 9:8). With this same promise we face the future with the confidence that what God has done in the past we can expect Him to do in the years ahead. 


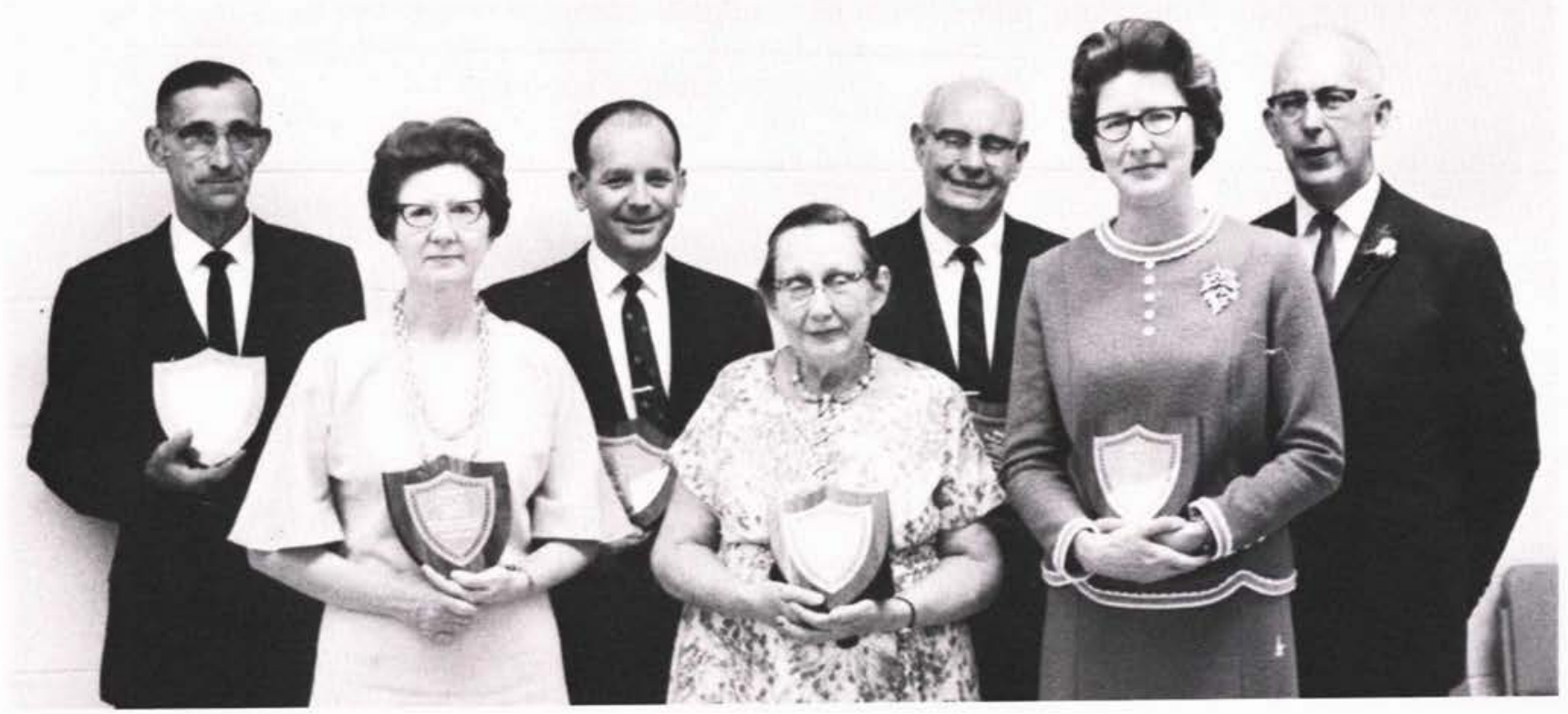

Presidential ten-year service awards.

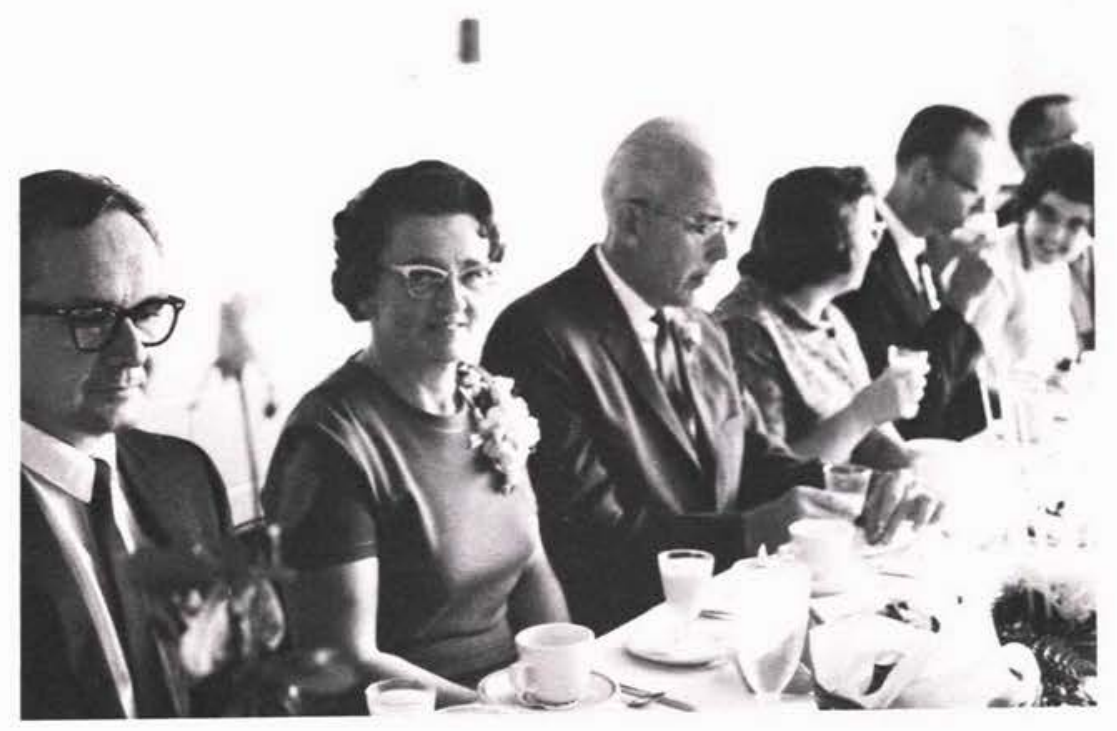

President's Dinner

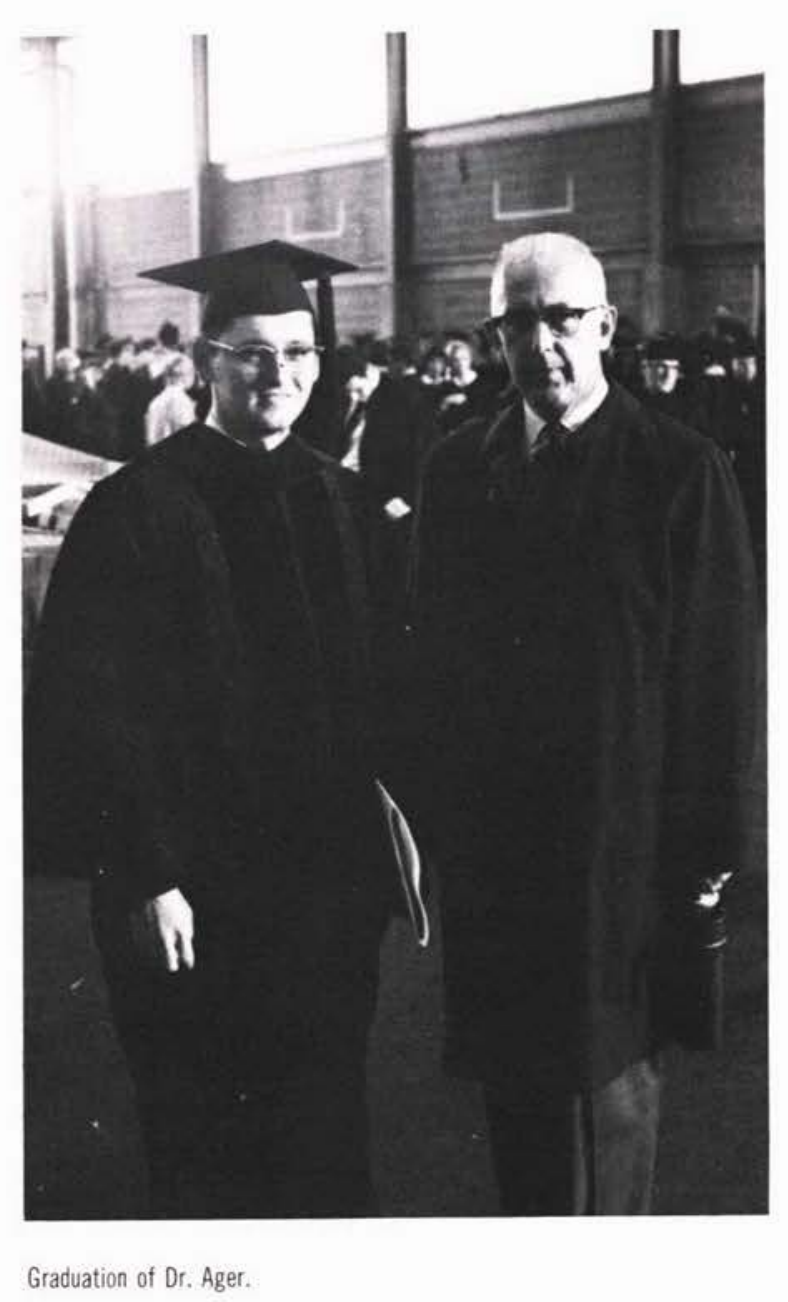

Moving into new library. 


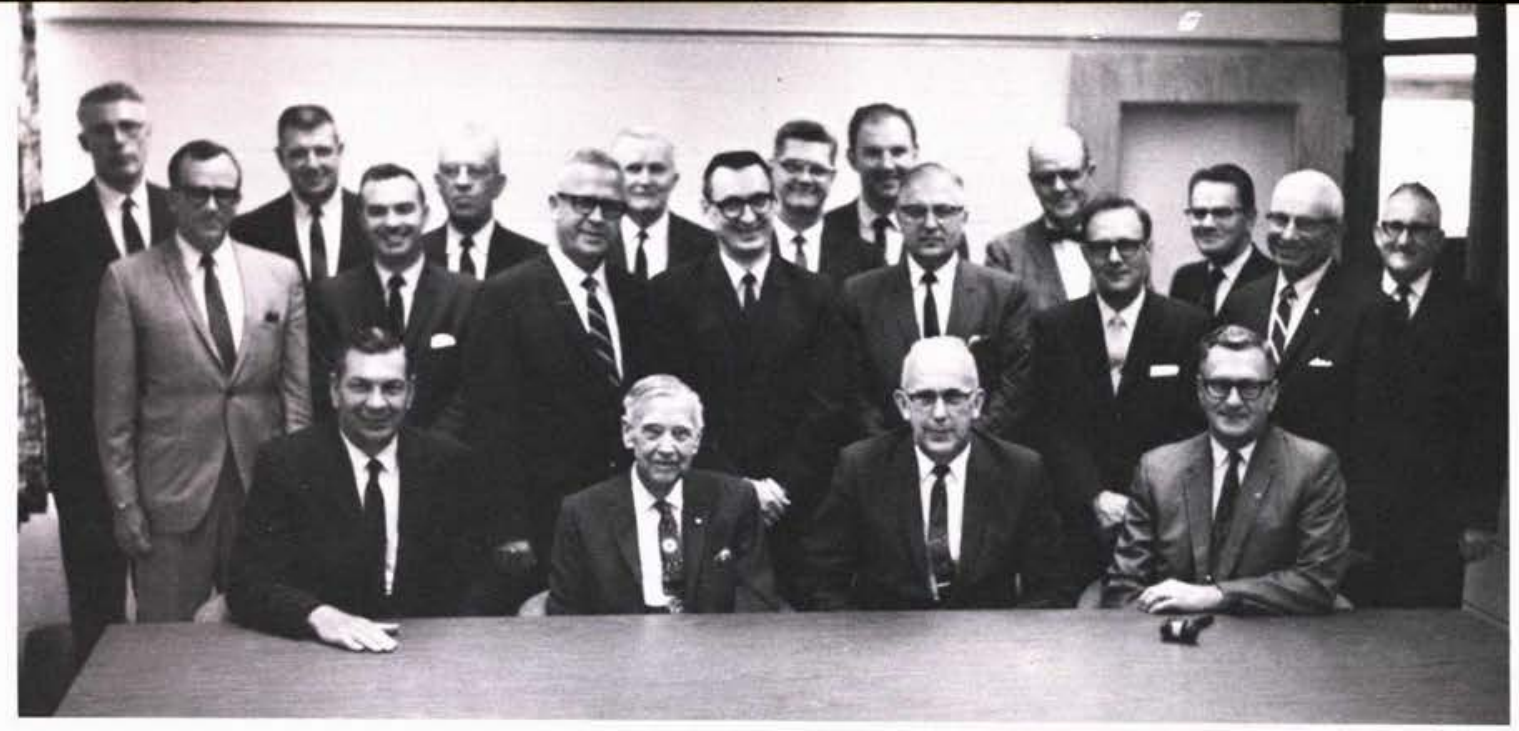

\section{TRUSTEES}

Trustee...

The very name implies a trust,

A grave responsibility,

A holy calling.

Confronted with questions of all natures,

Technical, ethical, and moral,

And with problems of policy and polity

In school administration,

The trustees must be a sharp unit,

Sharp of intellect

And sharp of spiritual stature.

Their direction in the past

Has helped Cedarville emerge and grow.

With the best interests of the institution

At heart,

They govern with discretion and wisdom

And greatly facilitate

The smooth operation

of Cedarville College.

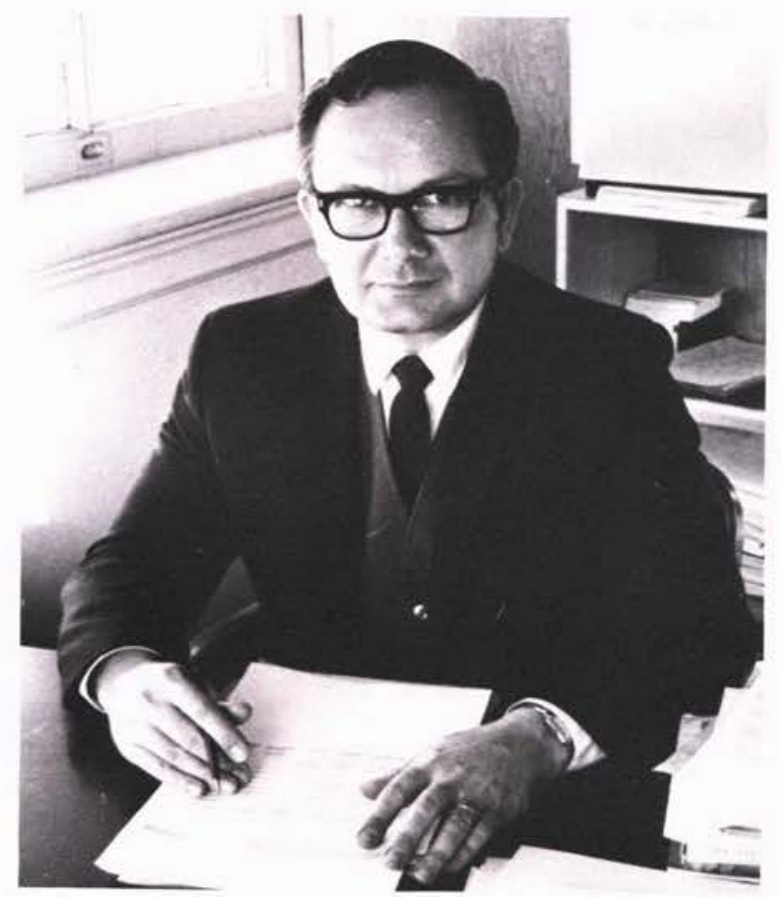

MR. KENNETHH. ST. CLAIR
Dr. George S. Milner, Chairman

James T. Jeremiah, President

Mr. J. Dale Murphy, Chairman

Rev. W. Thomas Younger, Vice-Chairman

Mr. George Boyd, Treasurer

Mr. George Engelmann, Secretary

Rev. William Brock, Rev. Glenn Davis, Mr. John Draxler, Mr. James Richardson, Rev. Wilbur C. Rooke, Rev. Robert Sumner, Rev. Earl Umbaugh, Mr. Rudy Bedford, Dr. Jack Cline, Mr. George O'Bryon, Dr. Paul Tassell, Mr. William Patterson, Mr. Arthur W. Dyke, Rev. Donald Sewell, Rev. Earl Willetts, Mr. Charles Barth, Mr. Vincent Gregg, Mr. Roy Guenin, Rev. Irwin Olson, Rev, Gerald V. Smelser, Rev. Donald Tyler.

\section{BUSINESS MANAGER}

Providing for the future

And the past financially,

The Business Manager

Must utilize discretion and precision

In the performance of his task.

Preparation of the school budget,

Administration of student loan funds,

And supervision of business and maintenance activities

Fall into his capable hands.

While collecting and paying the bills

This essential administrator

Provides for solidarity and efficiency

In the college system. 


\section{INSTRUCTION}

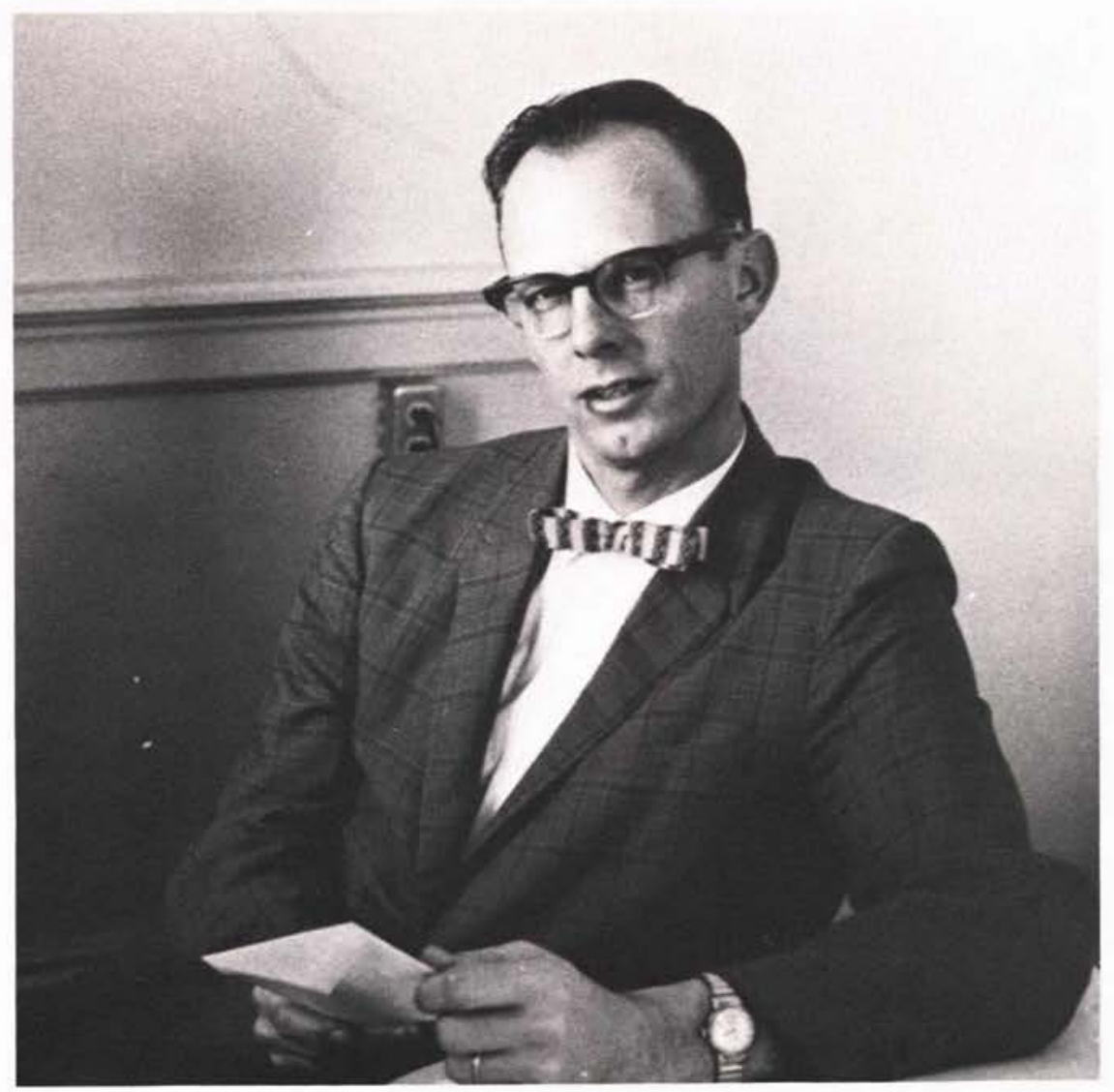

In the role of trouble-shooter

And prophet,

The Academic Dean

Carefully plans the future steps

of a college,

Its faculty and its student body.

In directing academic programs,

He strives to improve the curriculum

By recruiting new faculty members,

Developing up-dated and progressive policies,

And guiding toward accreditation.

Much of the college's well-being

Relies upon

The optimism, perseverance, and toil

of this important administrative position.

DR. CLIFFORD JOHNSON Academic Dean

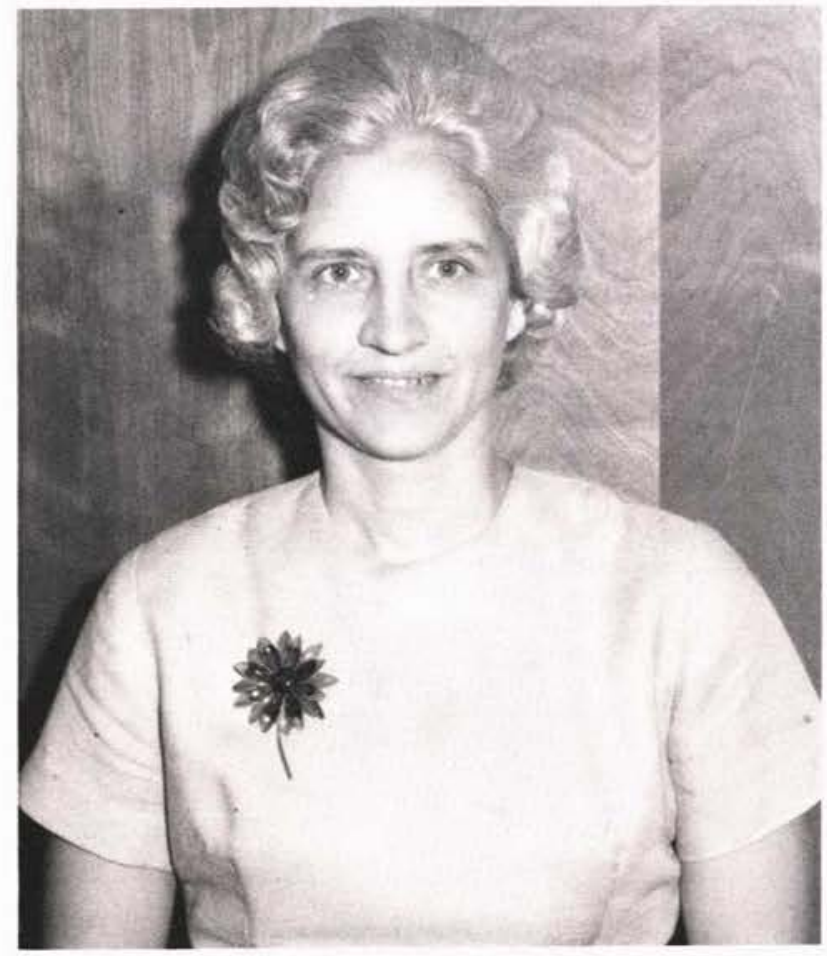

MRS. ALBERTA CHAFFE Librarian

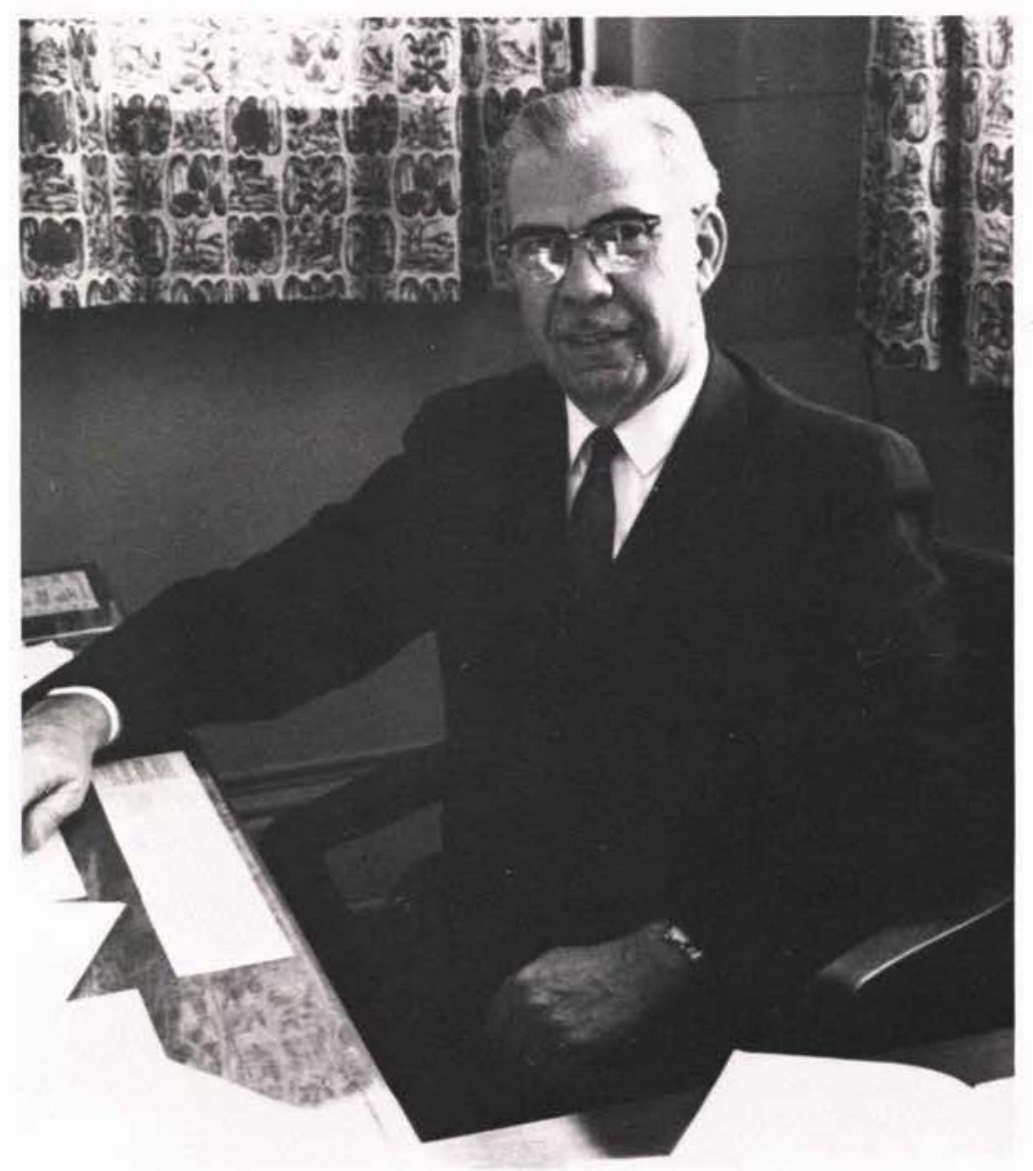

MR. C.B. HURST

Registrar and Director of Admissions 


\section{PUBLIC RELATIONS}

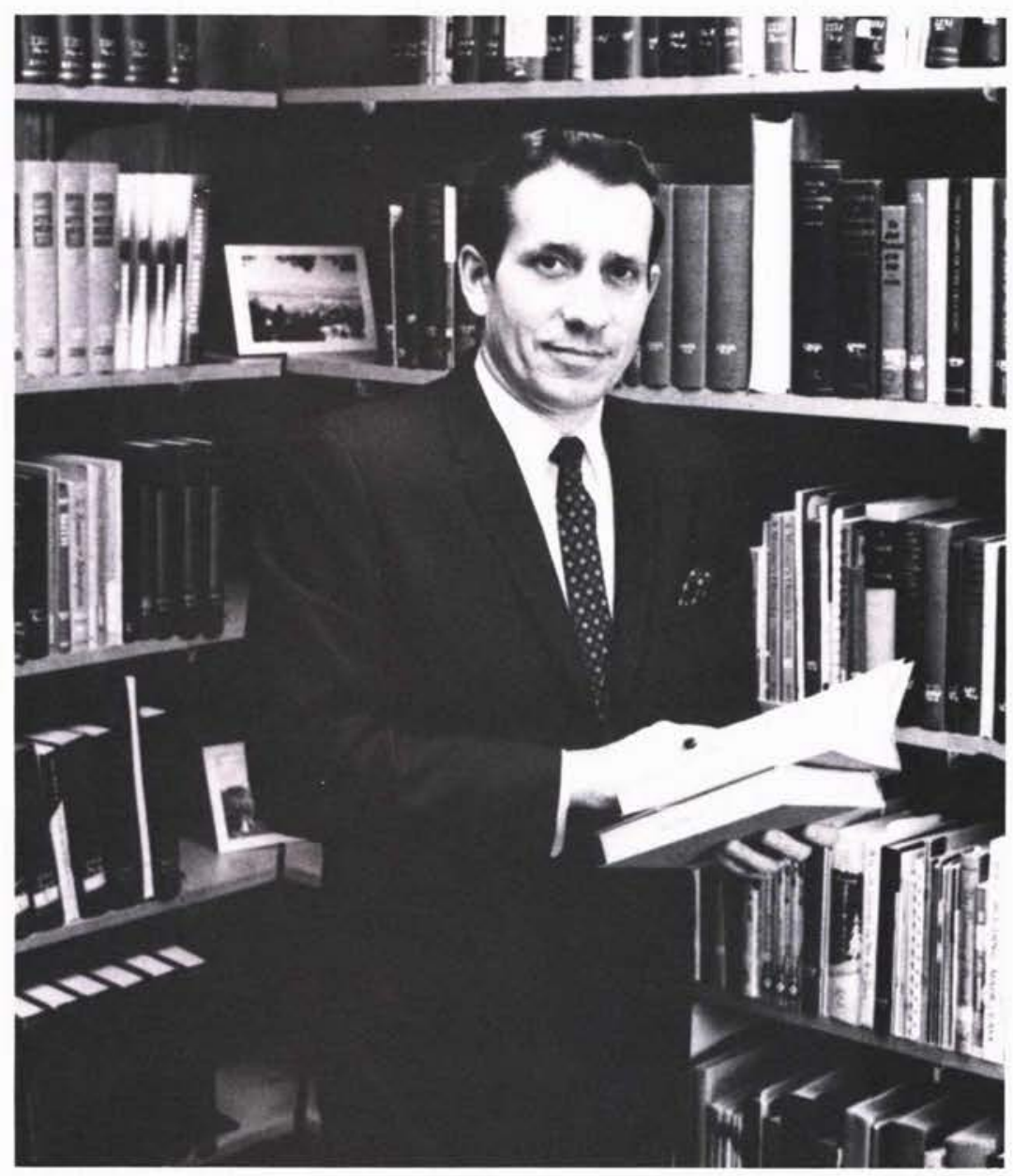

With an eye to the future

And a desire for improvement,

The Director of Development

Designs the long-range master plan

That will fulfill the needs

Of a growing academic institution.

Achievement of these goals

Will be realized

Through public relations programs

With churches, alumni,

Foundations, businesses,

And friends of Cedarville College.

Extensive travel,

Persevering determination,

And undaunted enthusiasm

Envelop this post.

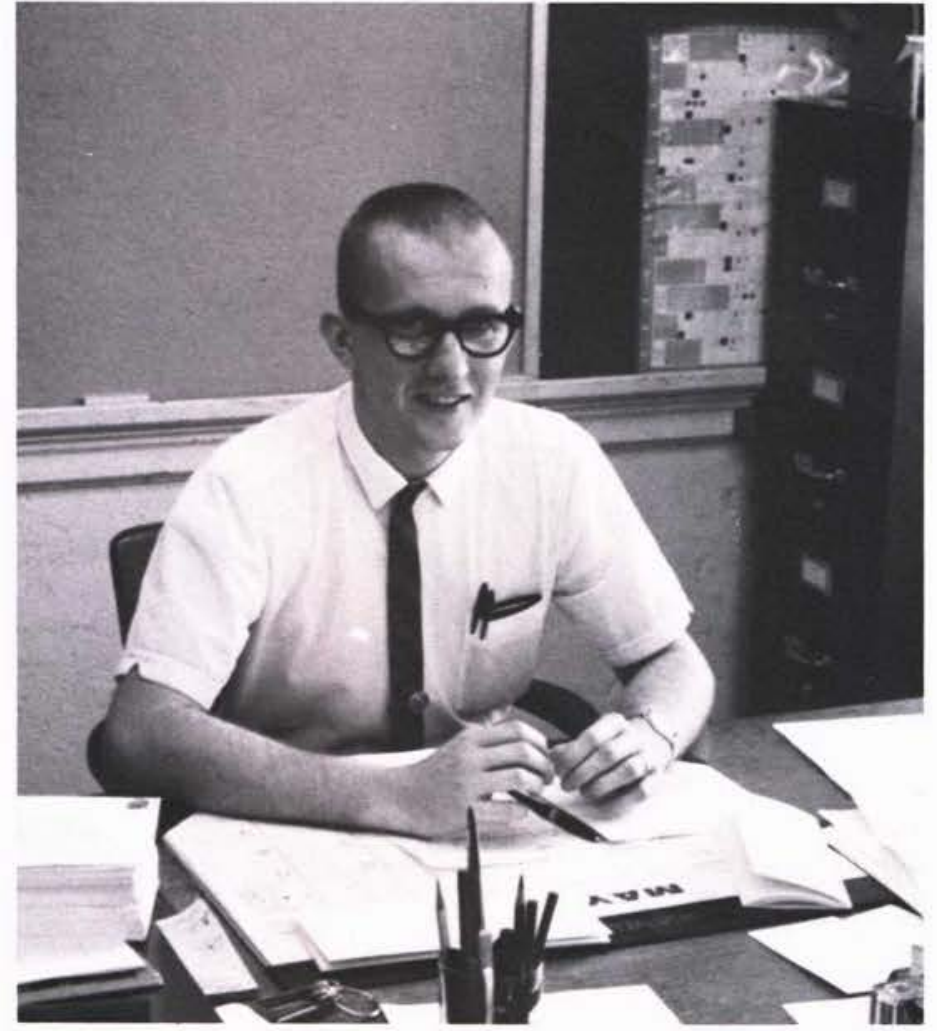

MR. RALPH YARNELL Alumni Relations

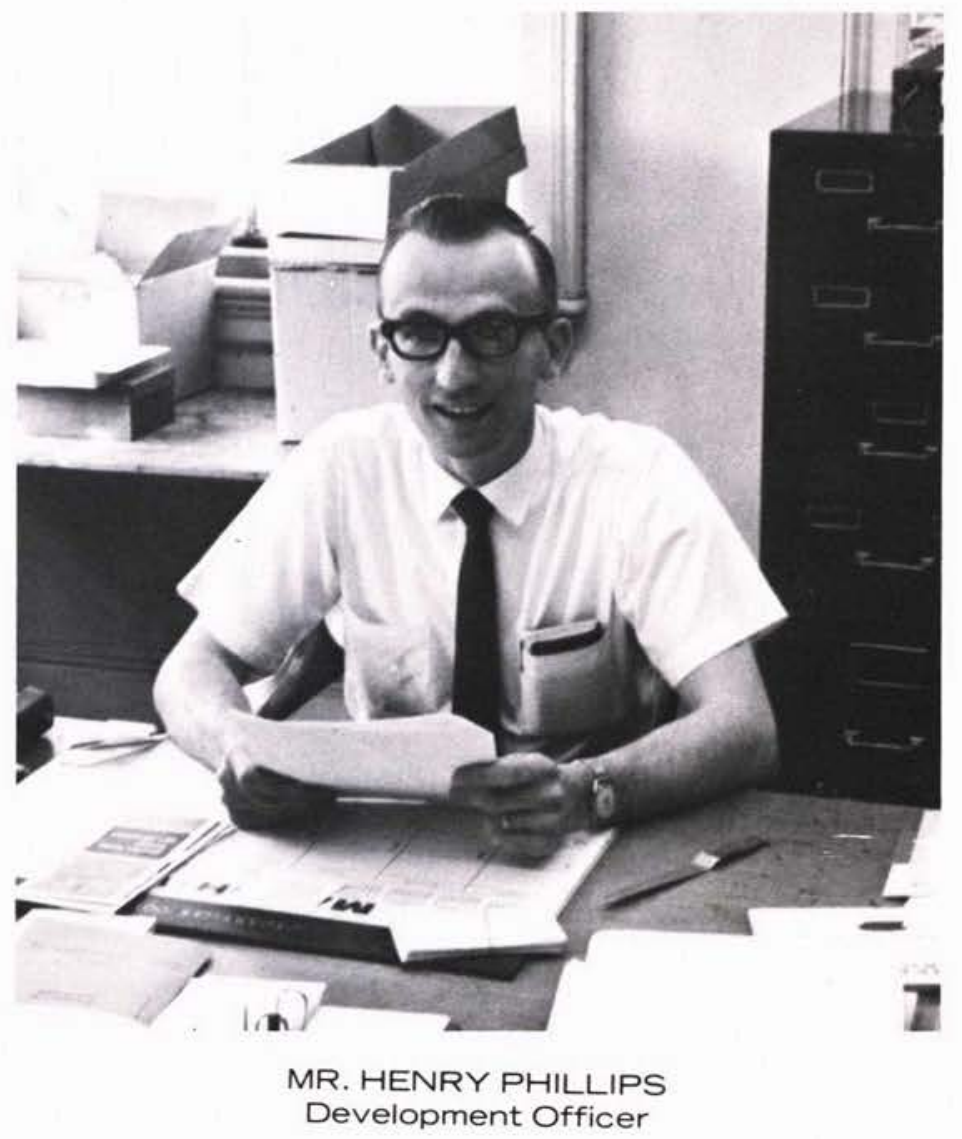




\section{DEANS}

The Dean of Students

The Dean of Men

And the Dean of Women

Carry out responsibilities

Characteristic

Of counselor, clergyman,

Mediator, and parent.

Their variegated tasks

Call for faith and stamina

As they supervise student affairs

By working closely with dorm counselors

And residence hall supervisors,

Proffering academic and vocational guidance,

And helping students with personal problems.

Room assignments,

Close ties with Student Council,

And references for seniors

Are additional duties

Performed by the deans

In this valuable and indispensable

Position.

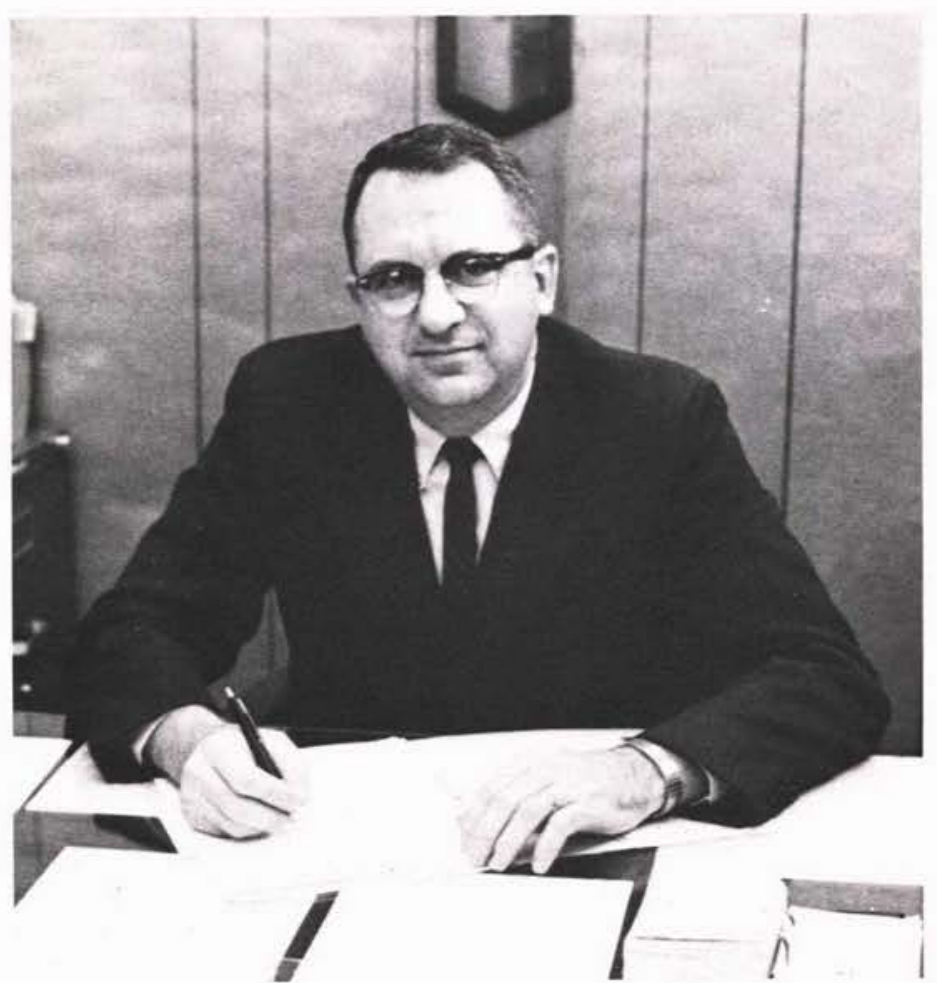

MR. MCINTOSH, Dean of Students

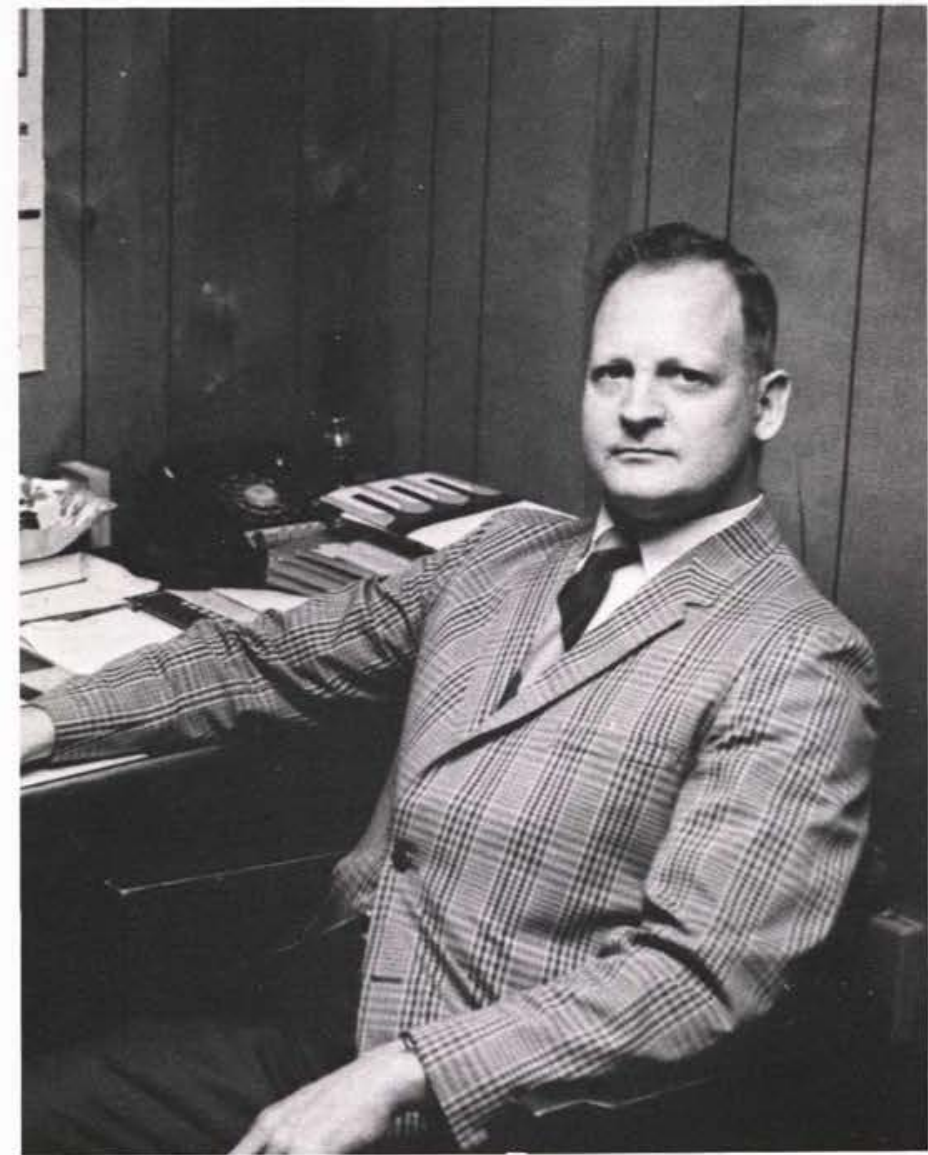

MR. LOPER, Dean of Men 


\section{BIBLICAL EDUCATION}

How do you measure

The value of a life?

A man stands with empty hands,

Searching for the frame of reference.

Words are useless without

The meaning man gives them.

The important words to live by

Are interpreted for us by men

Of God.

Students learn to relate God

To their everyday lives

Through the Bible Education

Department.

The value of a life is seen

Through the wisdom of professors

Who experience God everyday.
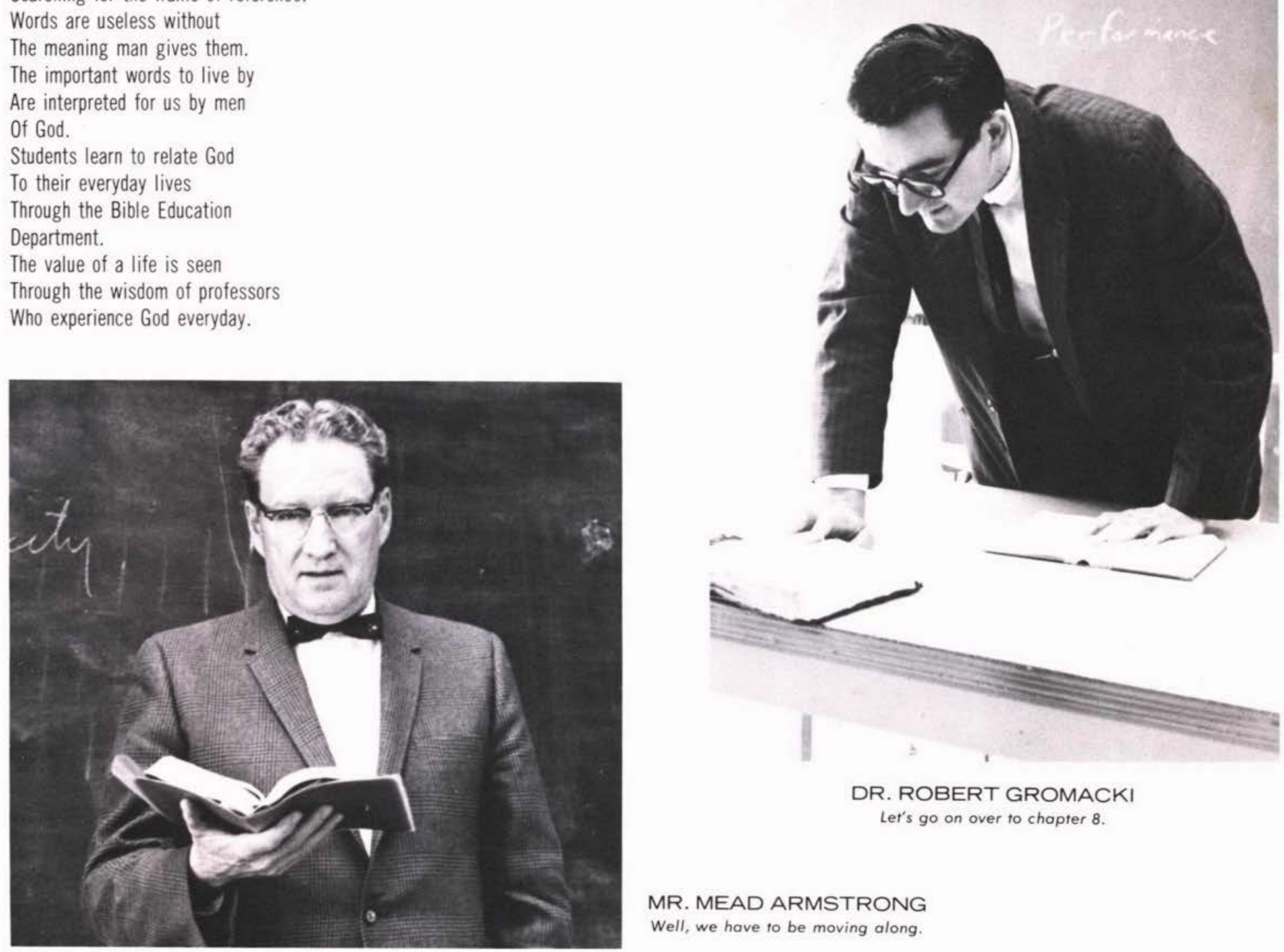

DR. ROBERT GROMACKI

Let's go on over to chapter 8 .

MR. MEAD ARMSTRONG

Well, we have to be moving along.

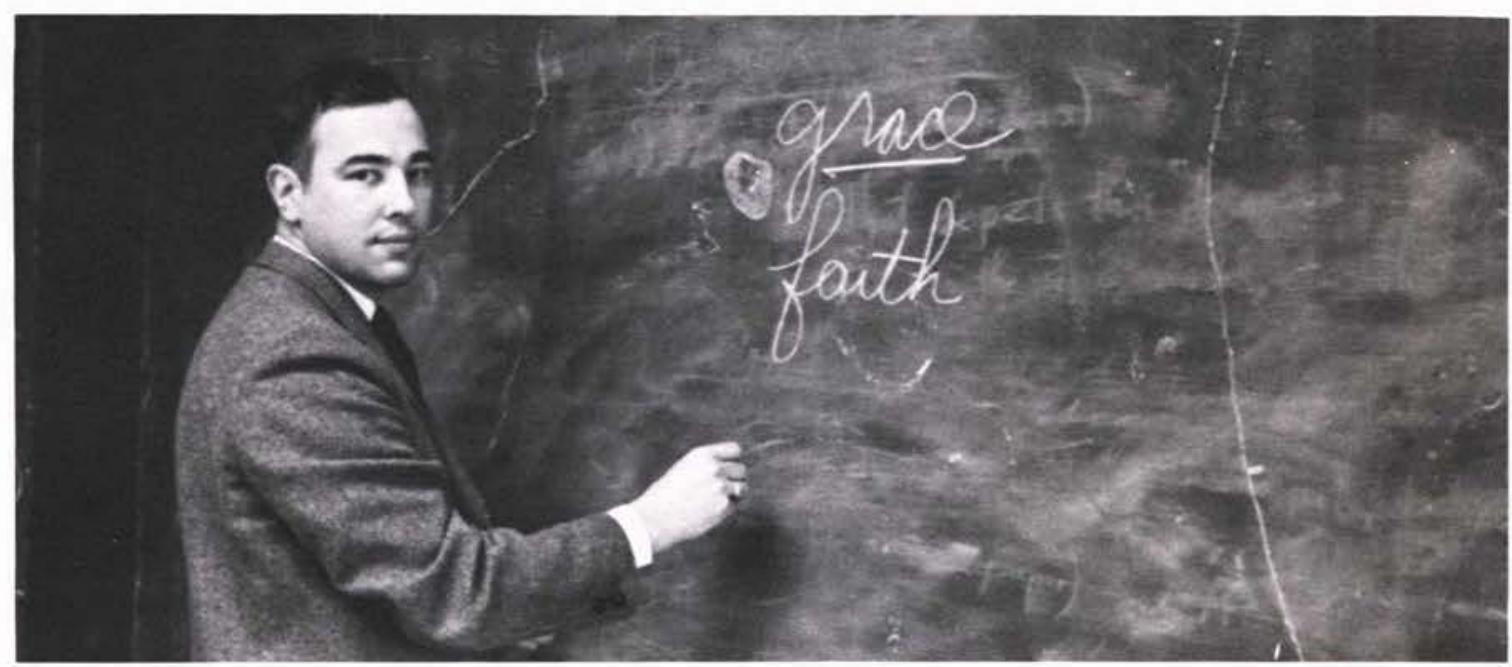

DR. JACK RIGGS

These chapters are important-16, 18, 25, 27, 32, 35, 47, 49, $50 \ldots$ 


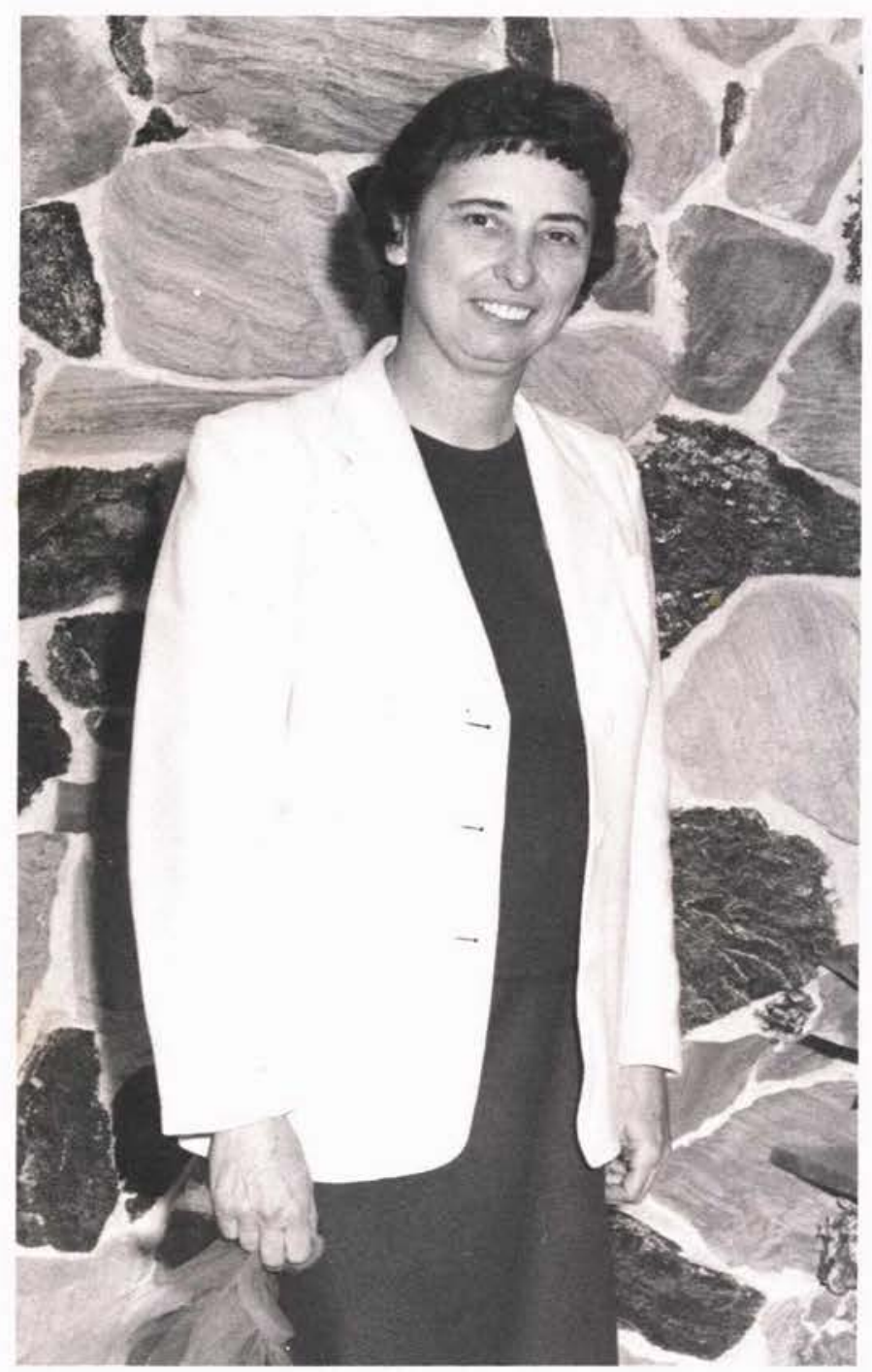

MISS JOY MACKAY

Iruth learned must be truth practised.

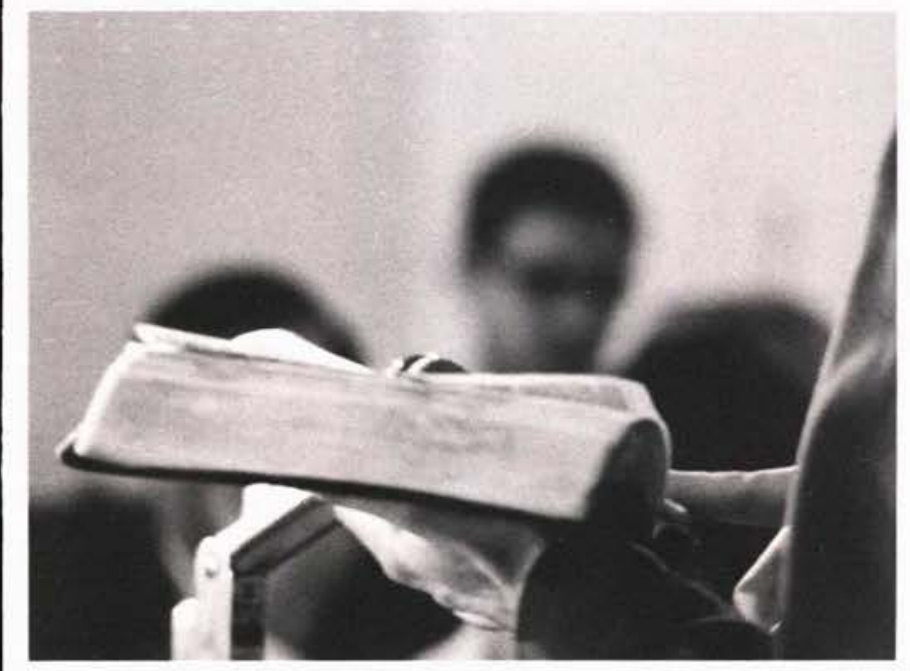

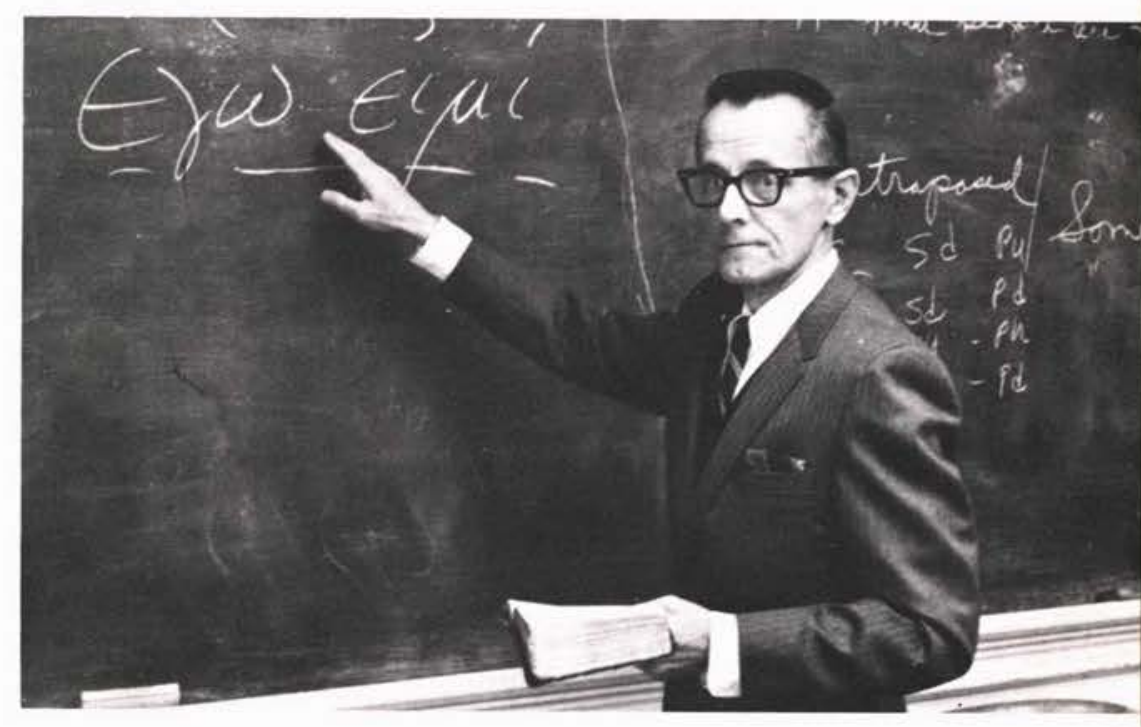

DR. GEORGE LAWLER

Remember your endings, class, remember your endings!

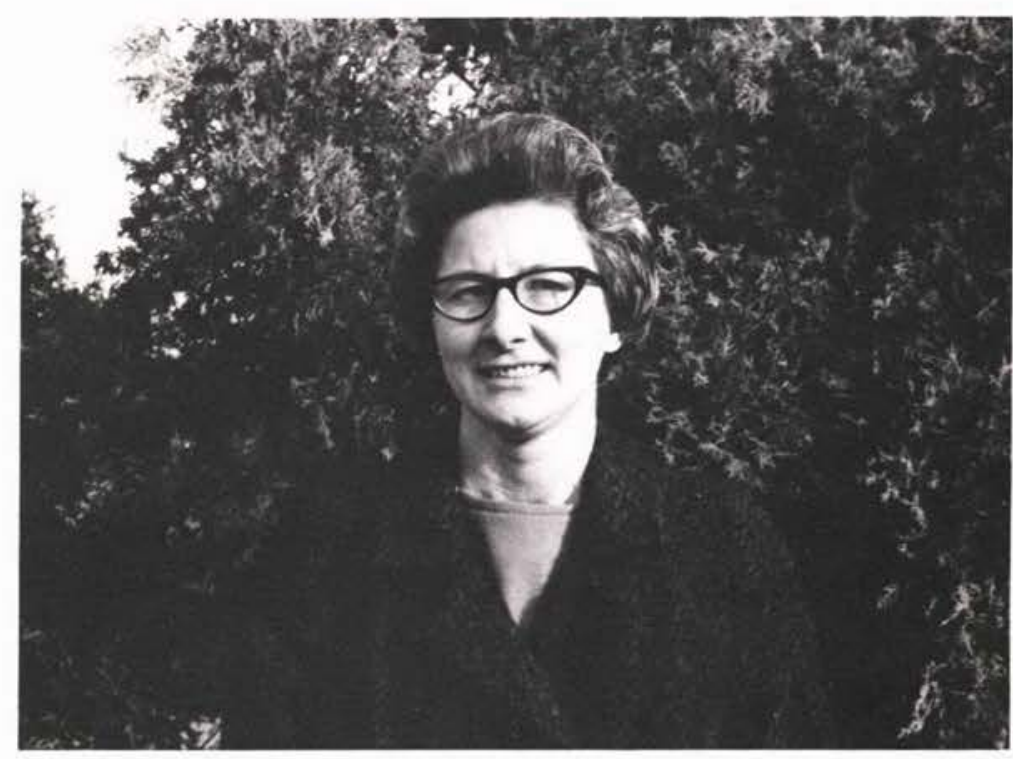

MISS JEAN FISHER

Teach the total man. 


\section{BUSINESS ADMINISTRATION}

The Business Administration Department at Cedarville College Offers the student majoring in this field

More than the essentials:

The rudiments of marketing,

The complexities of accounting,

Or the many facets of business administration;

But incorporates these business fundamentals

With a total college experience.

Under the instruction of competent professors,

Unique in its Christian emphasis,

And unsurpassed among the GARB schools,

The business department is ever striving

To equip its students with the necessary skills

To meet the challenge of our modern business world.
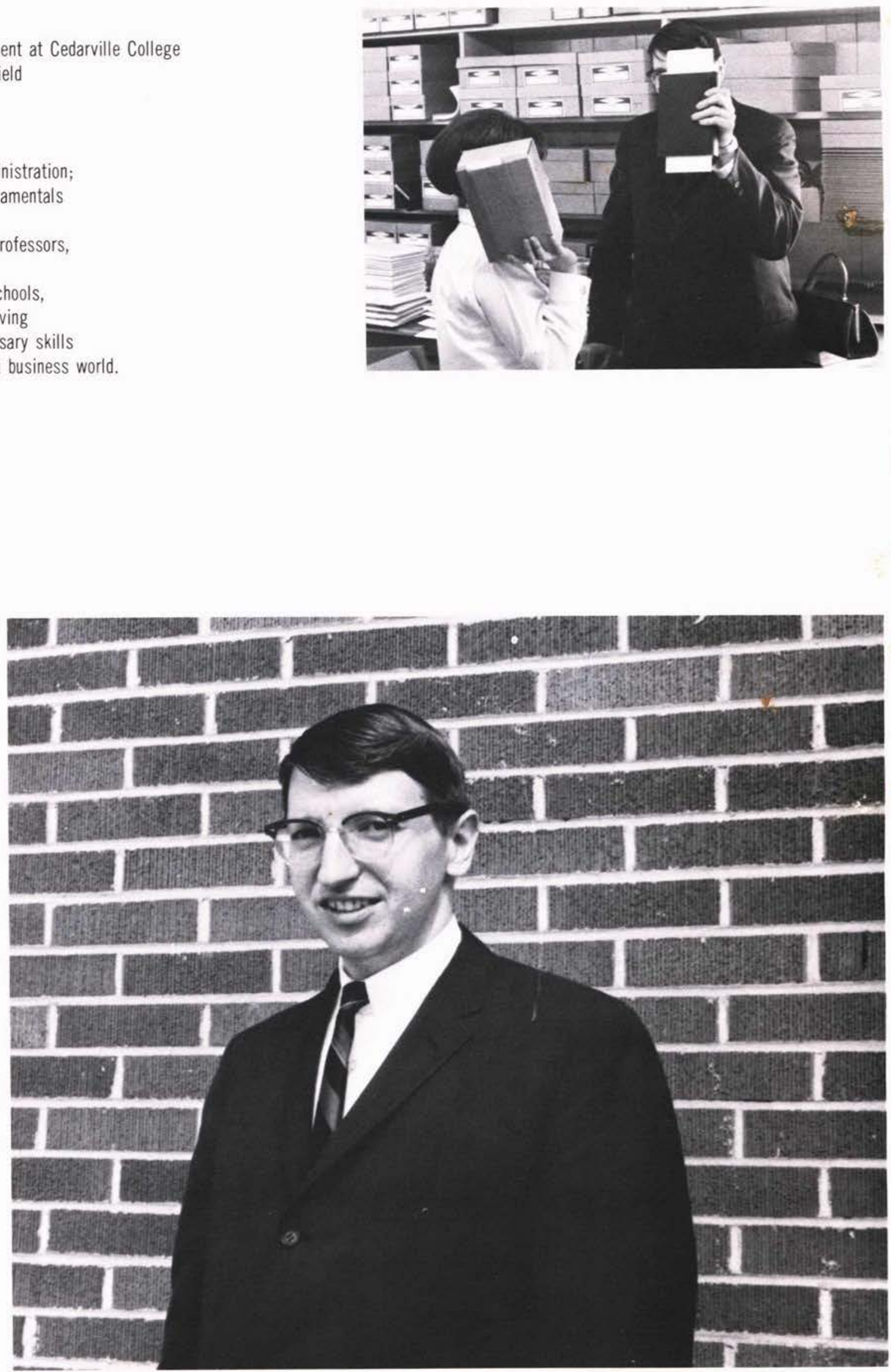

MR. DONALD WILCOXIN

Yes, I think that would be right, in view of the circumstances. 


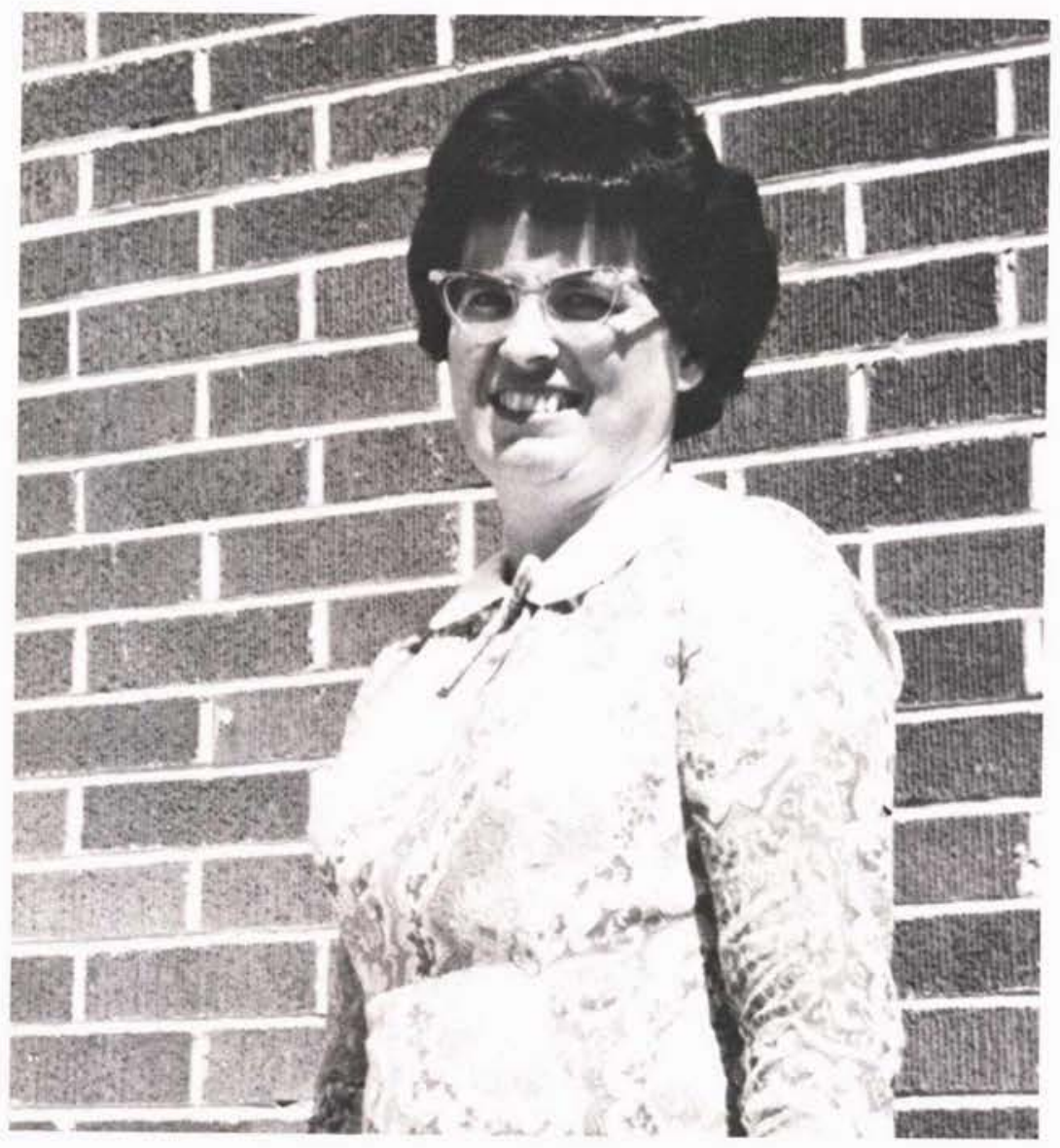

MRS. ARDETH WEBBER

Now, let's take this letter at 140 words per $\mathrm{min}$.

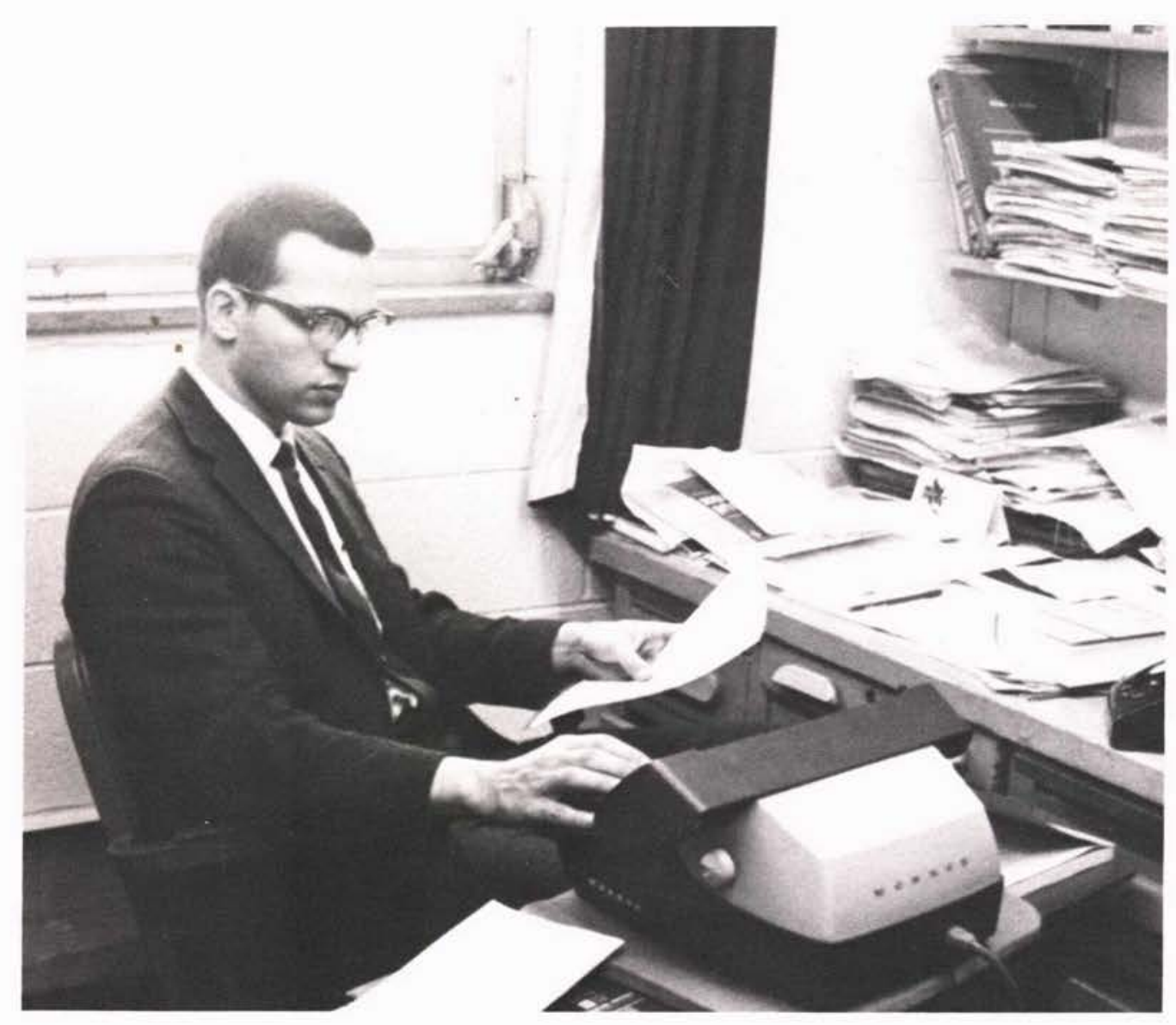

MR. WILLIAM RITER

looks like you guys have been working overtime again. 


\section{EDUCATION AND PSYCHOLOGY}

Learning how society functions,

Imparting insight into behavior,

To understand,

To be understood,

These are goals in Education and Psychology.

Education initiates knowledge

And the starting of ideas.

Psychology enables one to get along with others,

To improve himself.

The interaction between teacher and student,

The challenge of learning,

The fundamentals of knowledge,

Are the beginning of all education.

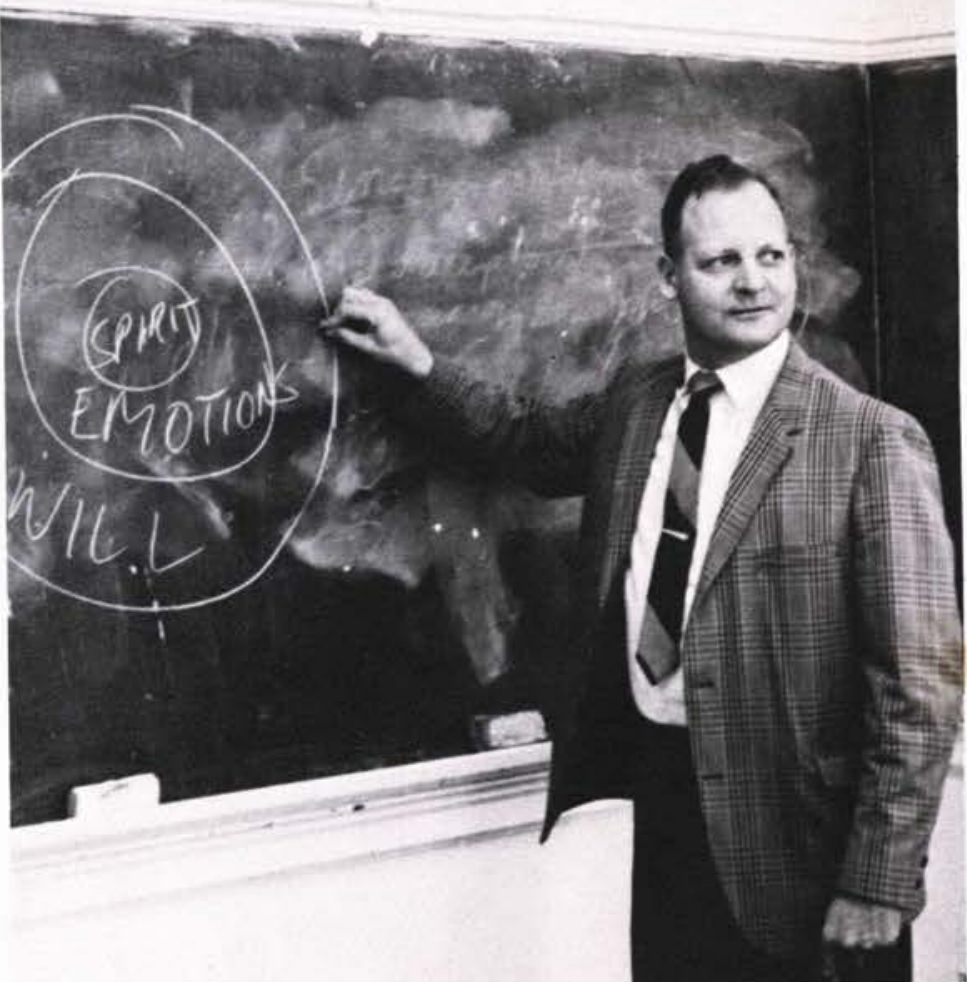

MR. THOMAS LOPER

I want to be an individual
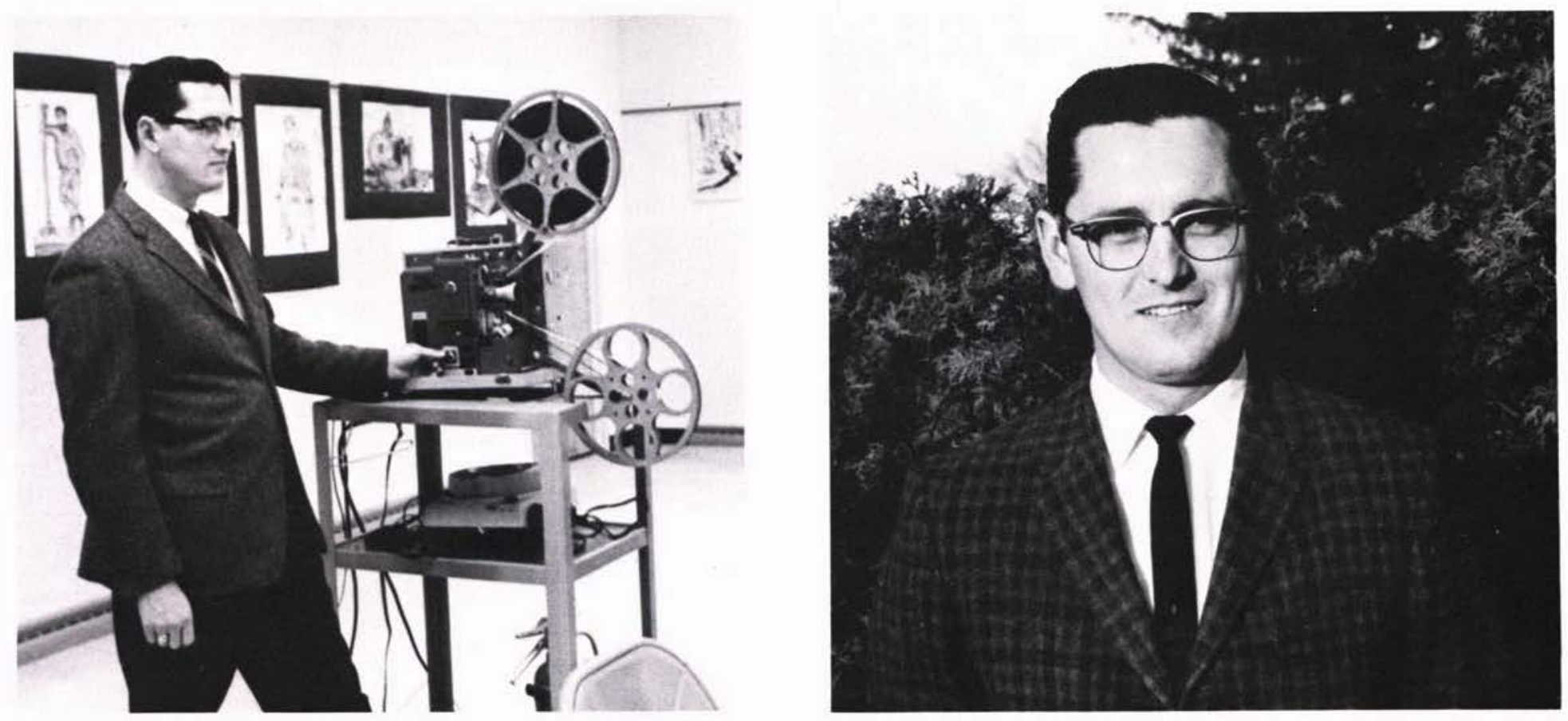

DR. MERLIN AGER

In other words, you're saying that ... 


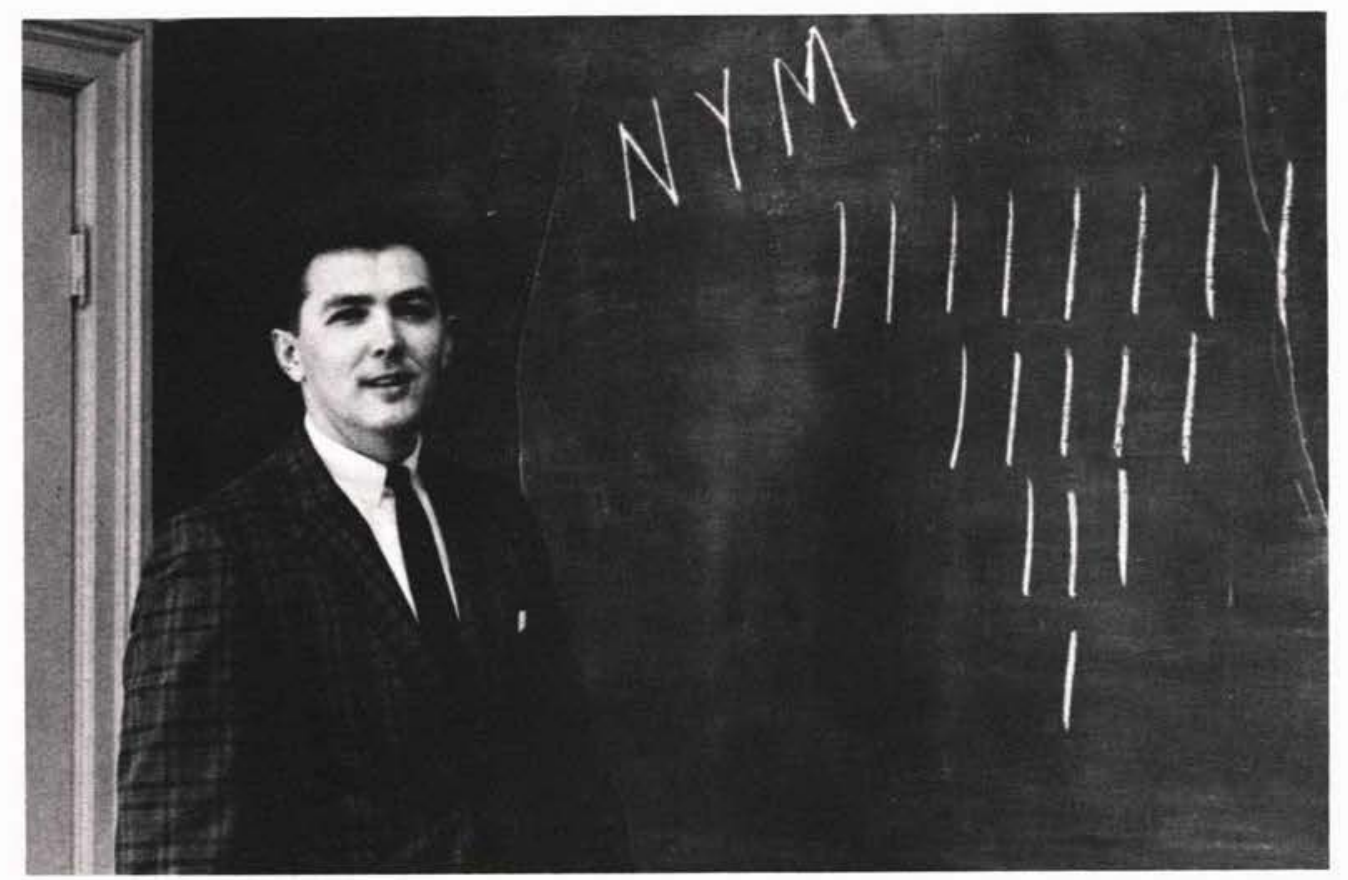

MR. BRAD MOORE

Just for fun, let's do something different today.

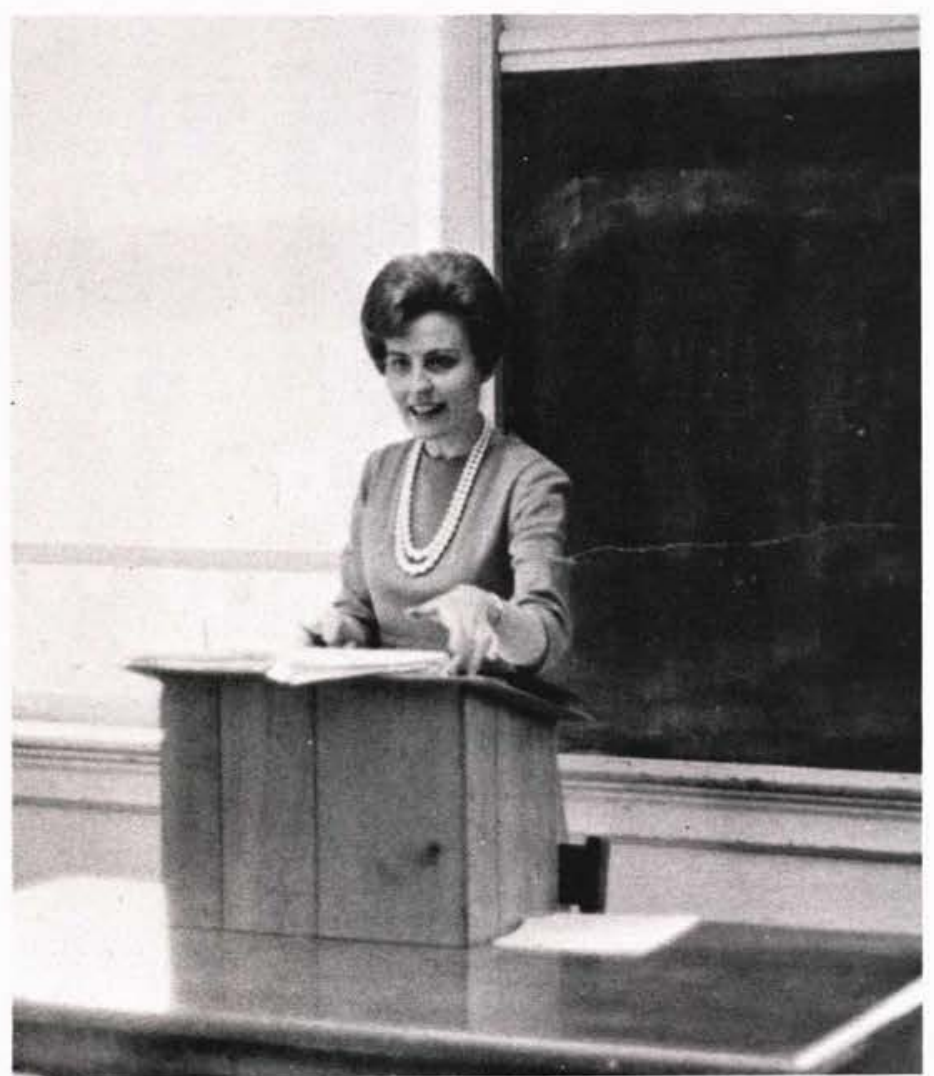

MISS MARTHA DUNN

This is just a small assignment. I'm sure you'll enjoy doing it.

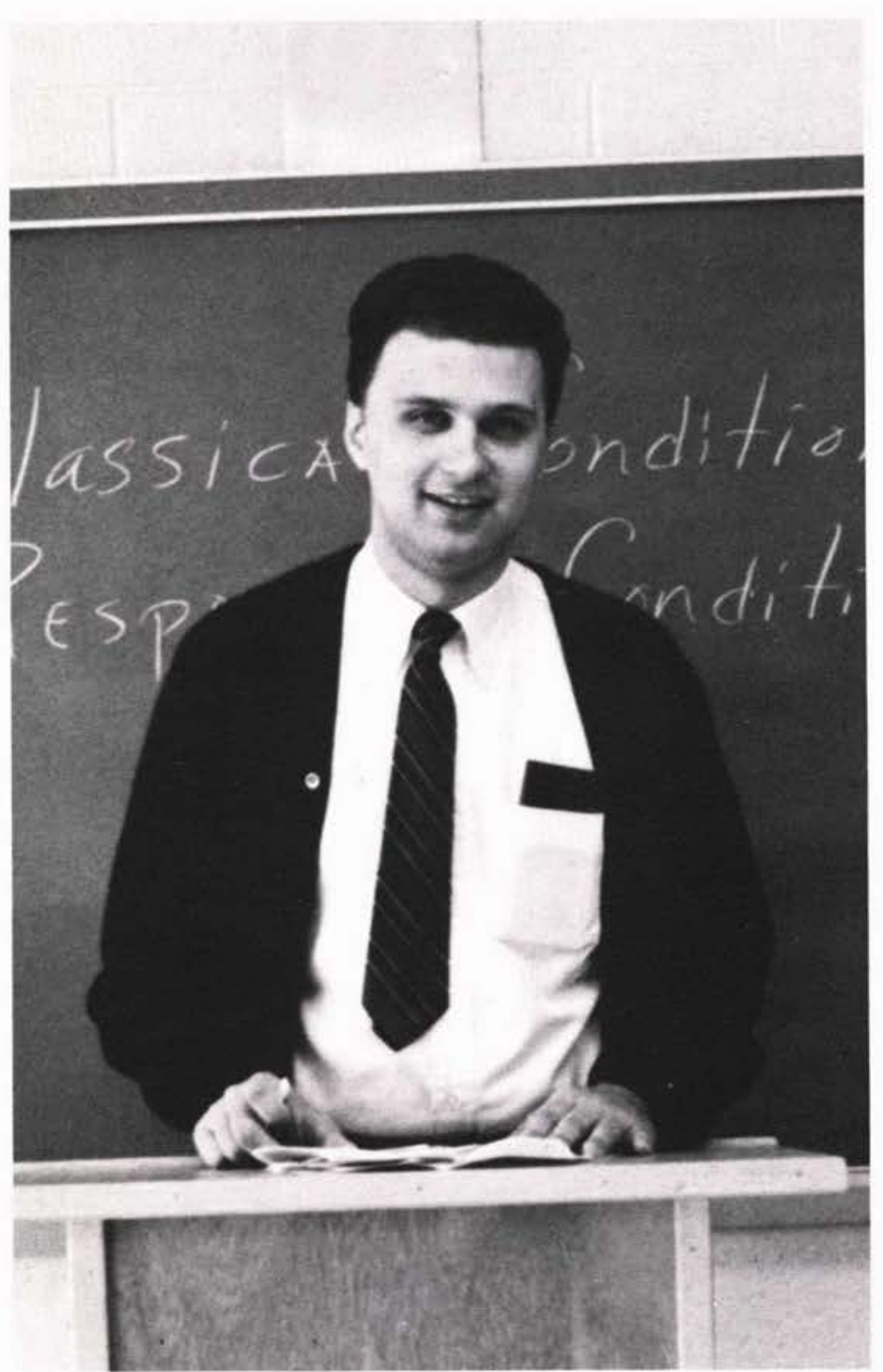

MR. ROLAND FLECK

Well, this test isn't that hard. 


\section{FINE ARTS}

The refinements of art and music

Are diligently surveyed in the Fine Arts Department;

And, the artistic gifts of each student

Are cultivated.

Creativity - the outward expression of ourselves,

Excellence - the refinement of that creation

Are accentuated.

These may take the form of

A multicolored canvas, a resounding sheet of music,

An aesthetically fashioned piece of sculpture

Or an inspiring trio selection.

Yet, whether a spring concert or oil painting,

Each melodious measure and skillful brushstroke

Evinces endless hours of toil.

The consummation of the Fine Arts Department

Is the generation of true artists.

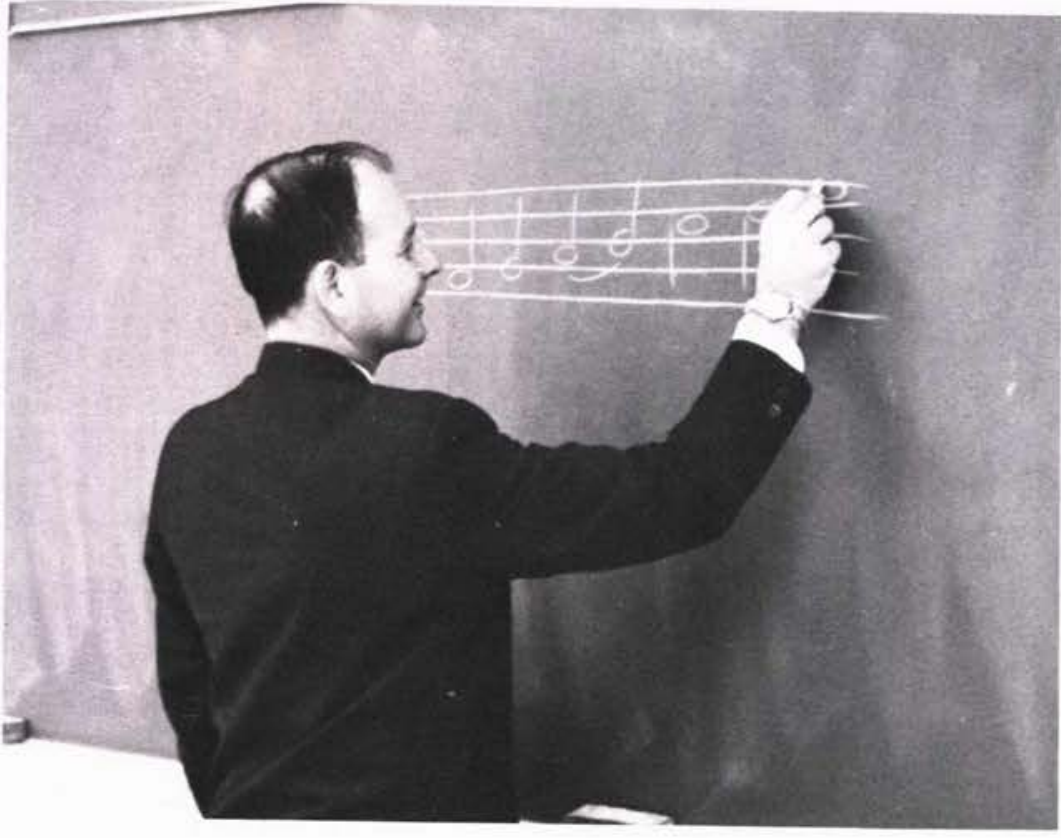

DR. WARREN WEBBER

The question is, where do we go from here?

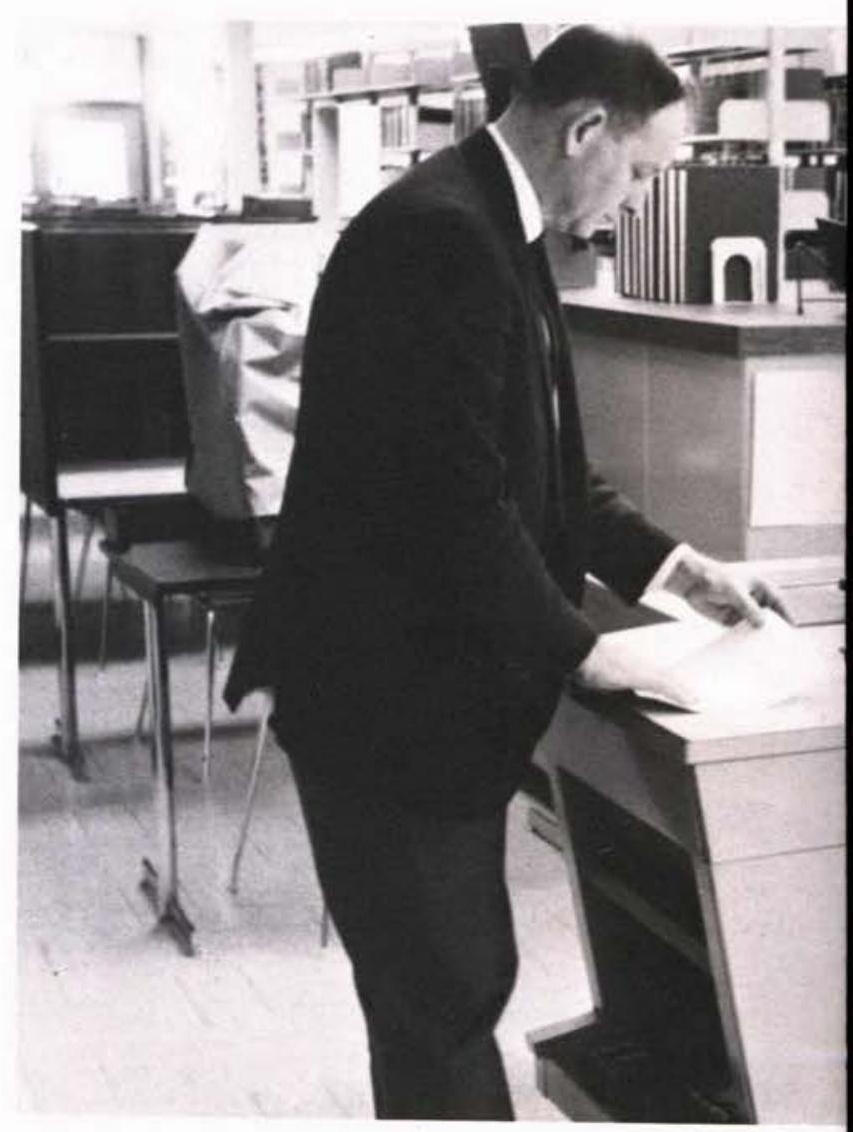




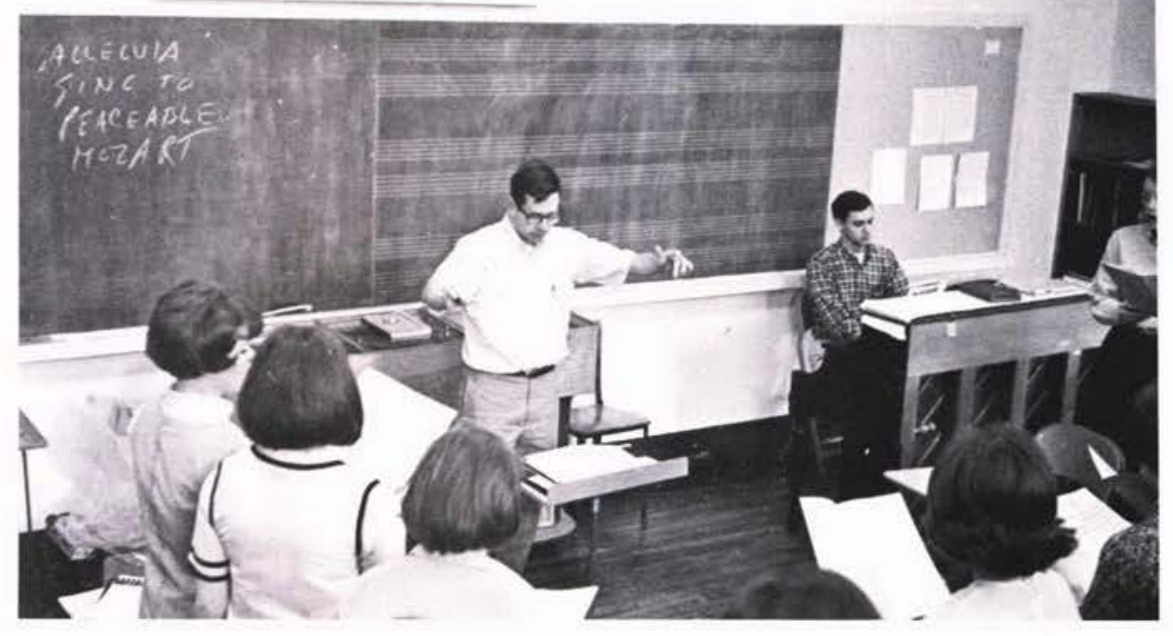

MR. PAUL VANDERKOY

Don't lock your knees or you'll faint.
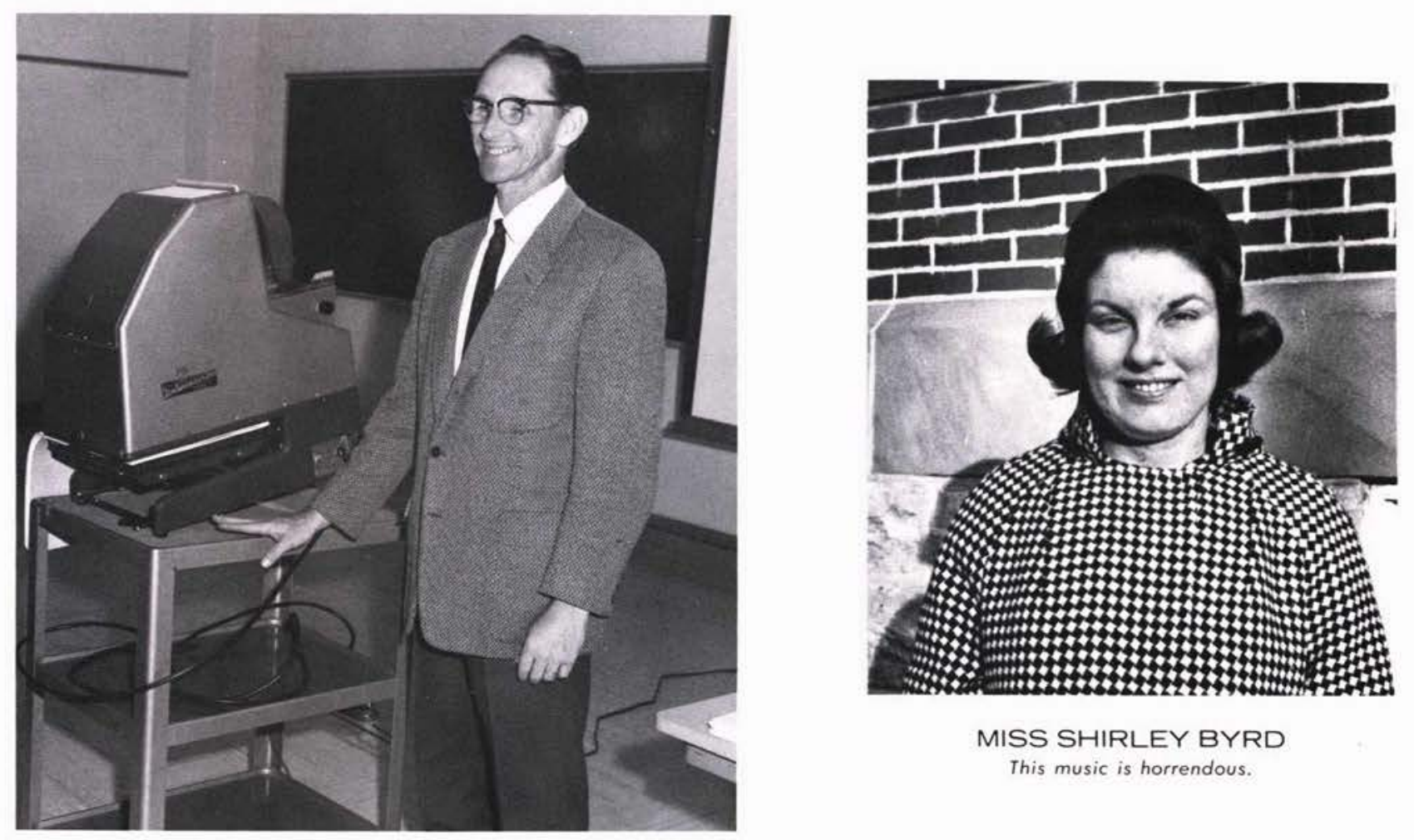

MISS SHIRLEY BYRD

This music is horrendous.

MR. WILLIAM THORNTON

We're going to have a tea party tomorrow - on chapters 1-6.

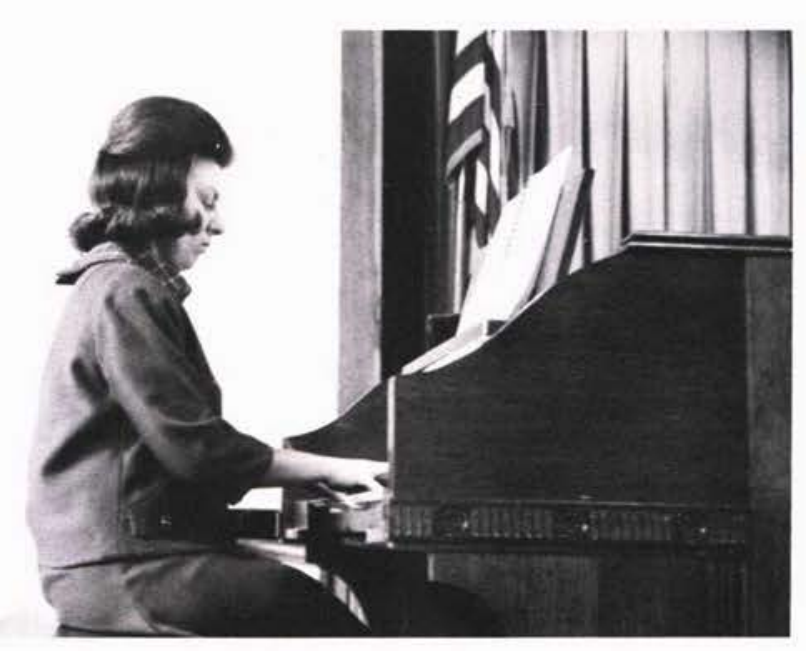



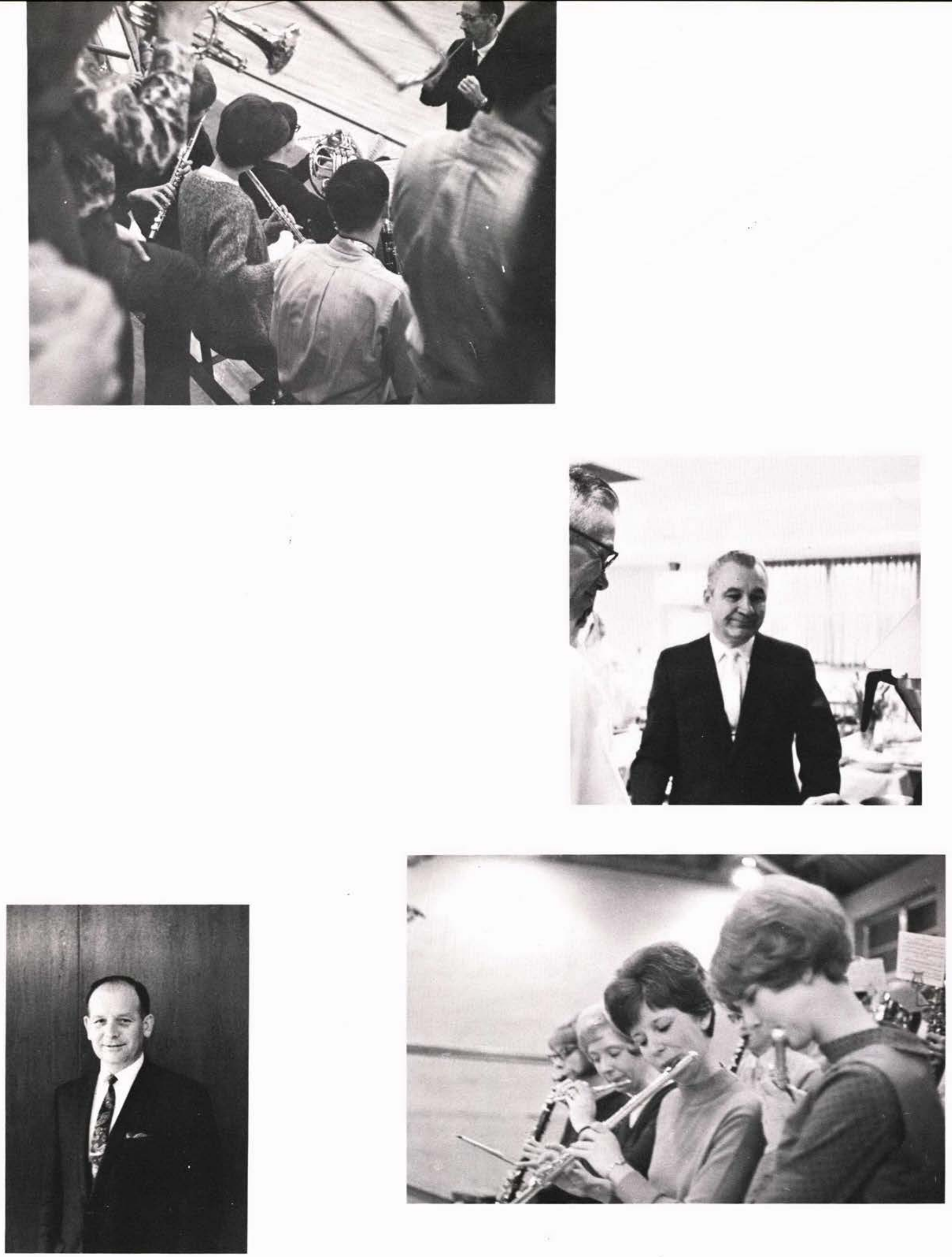


\section{HEALTH AND PHYSICAL EDUCATION}

Disciplining of the body

As well as the mind

Is a part of a liberal arts education.

Flying high on the tramp,

Learning childrens' games

And coaching skills,

The functions of camp life,

Health instruction,

These are part of a total picture

Making up the total man

For a life more abundant.

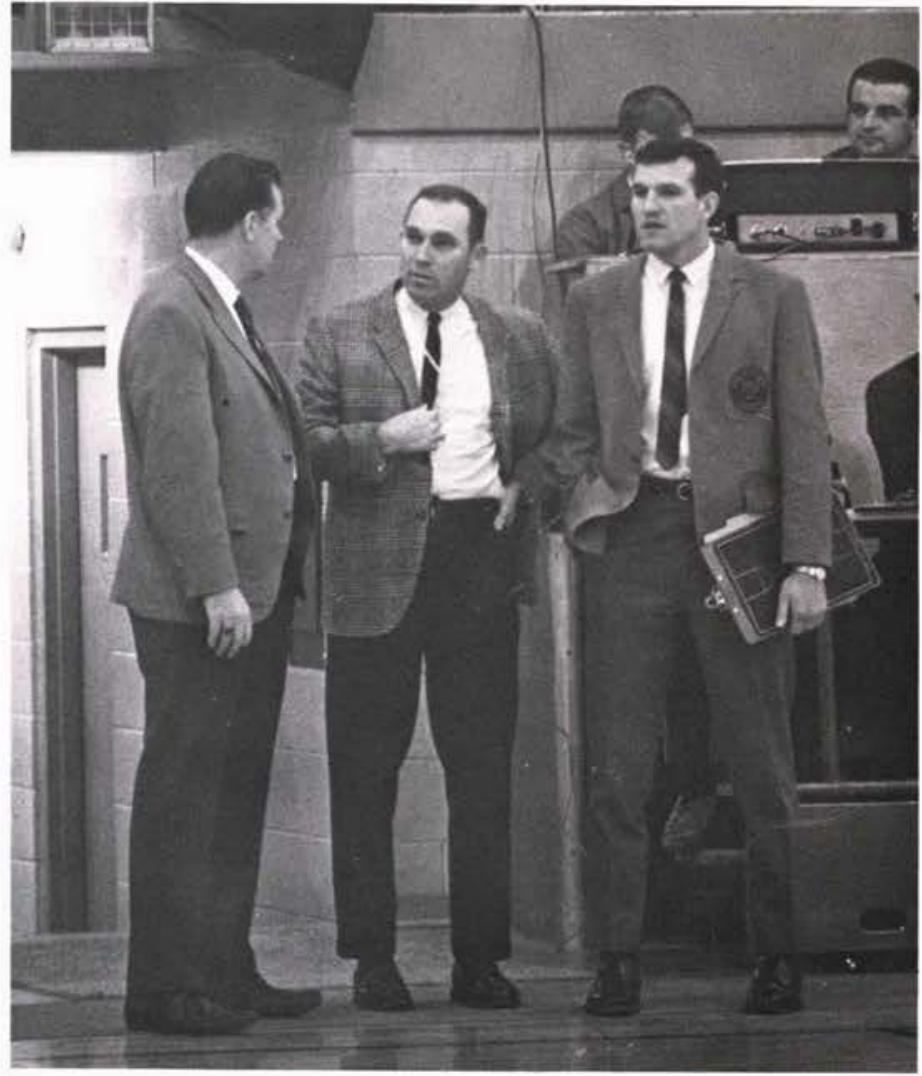

MR. DONALD CALLAN

We're going to have to play "catch-up" now.

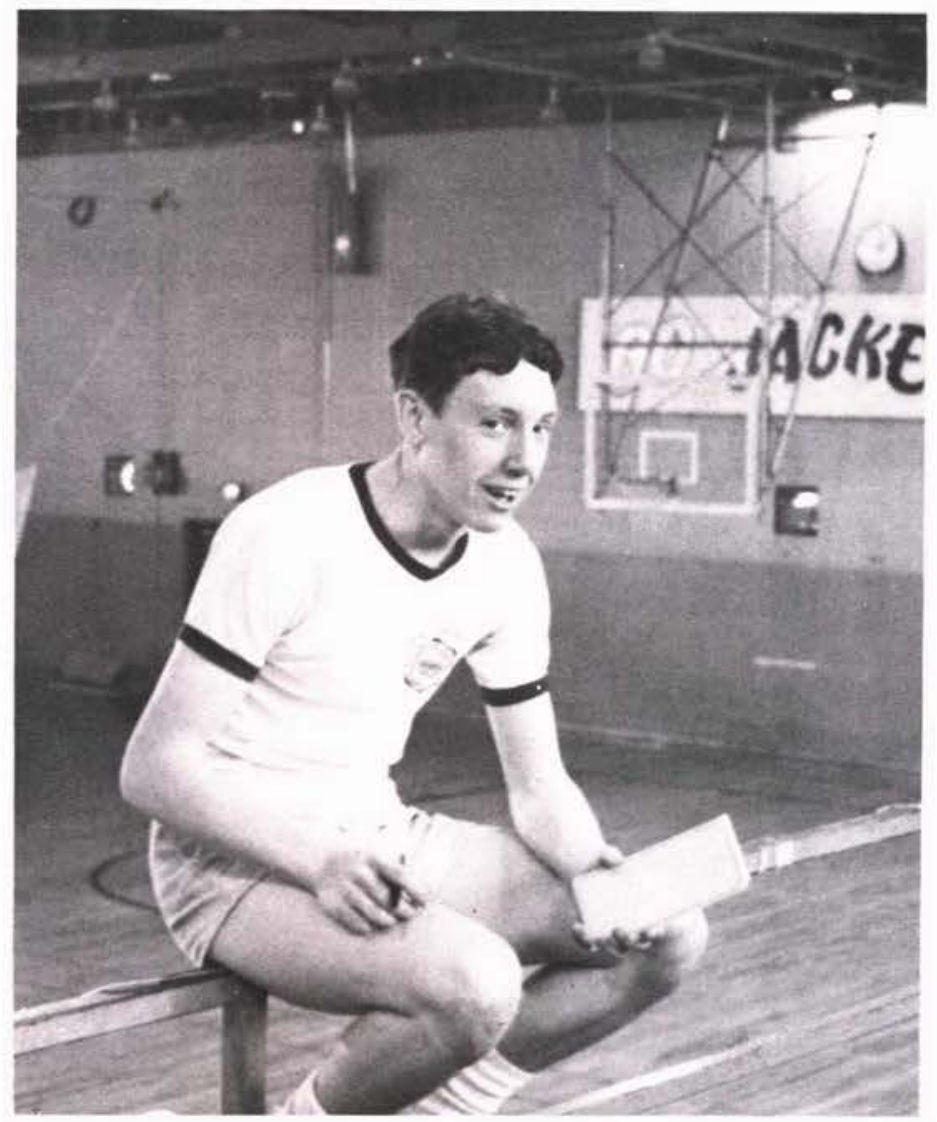

MR. DONALD DUNSTAN

At least I can do a kip-up.

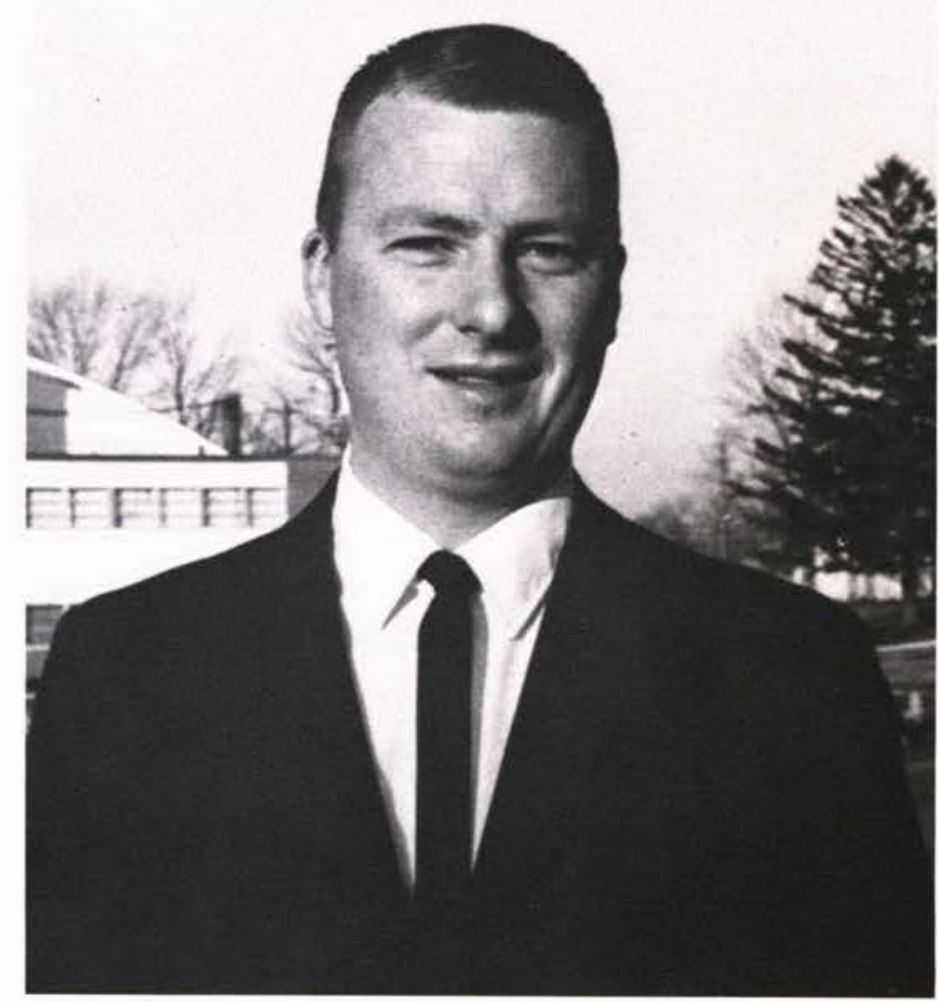

MR. DENNIS OLSON Are you kidding me?! 


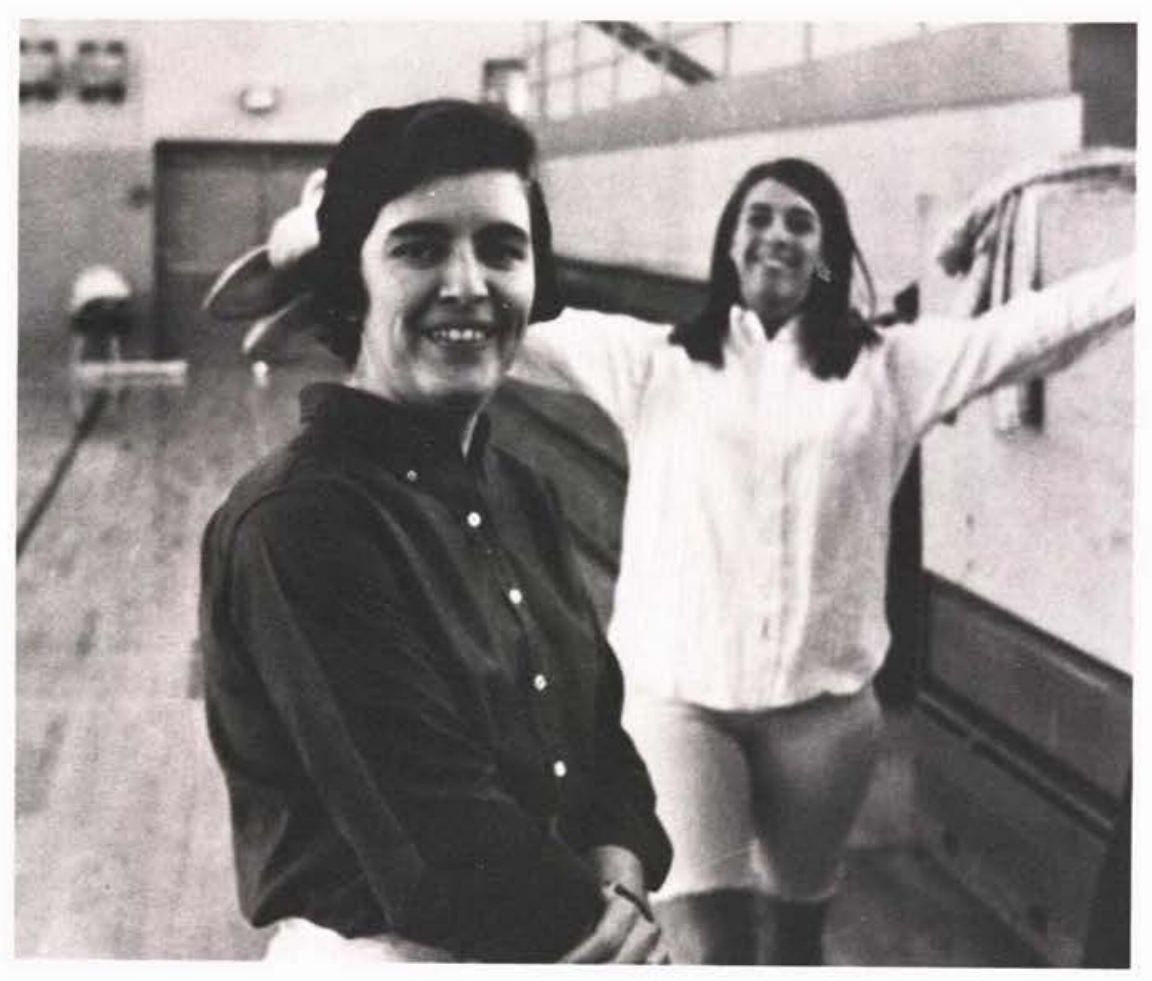

MISS JUNE KEARNEY Just ten more laps, girls.
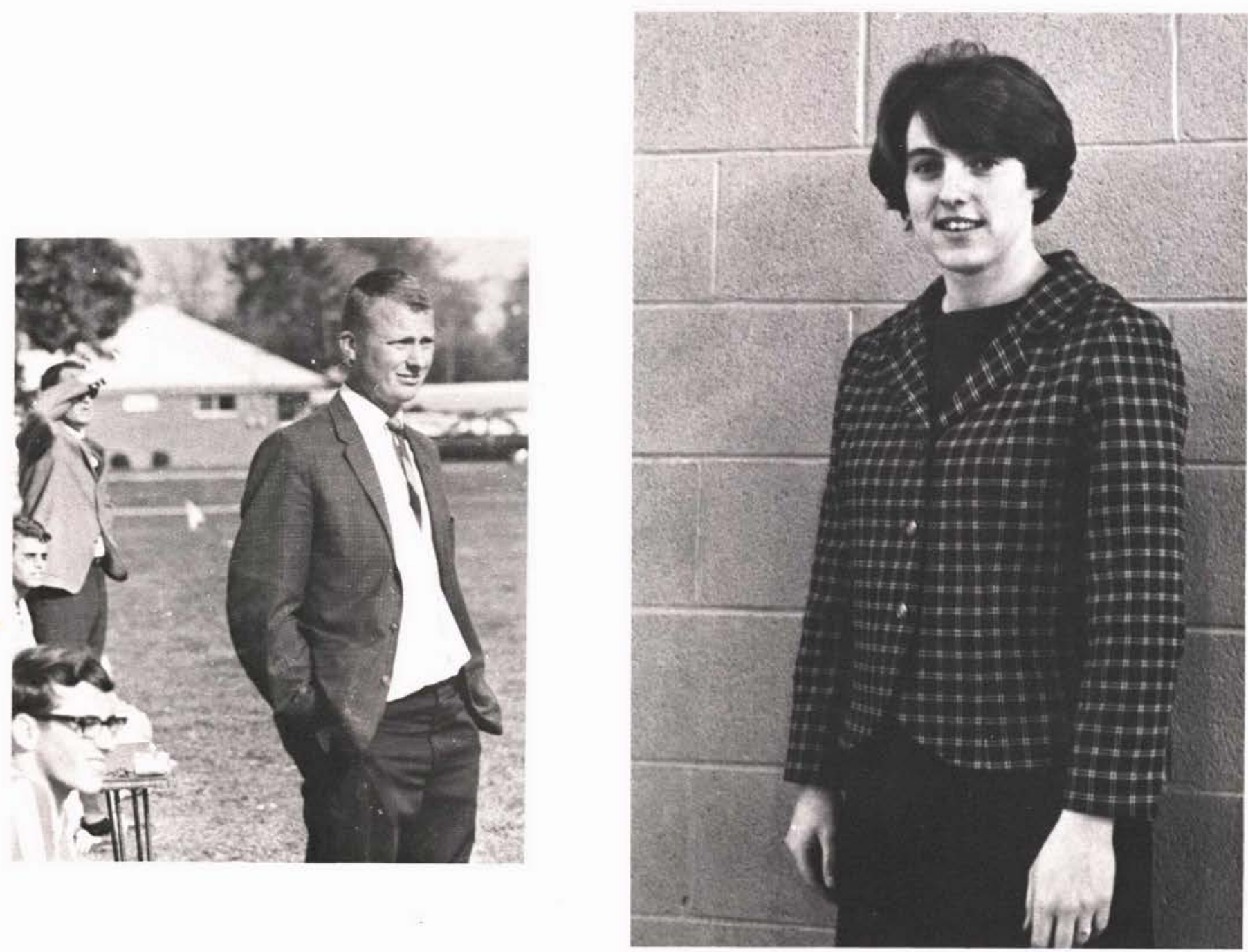

MISS ANN DAMON

We're going to practice our serves today. 


\section{LANGUAGE AND LITERATURE}

Language -

So essential

To a successful career,

Enriching relationships,

And an effective testimony,

Is given close,

Scholarly scrutiny

In German, French, and Spanish courses

As well as

Broad instruction

In English grammar,

Rhetoric, drama, and debate.

Believing that

Aesthetic understanding and literary appreciation Are necessary ingredients

Of the educated, sophisticated man,

The Language and Literature Department

Essays toward that polished goal

With obstruse studies

In British and American literature.

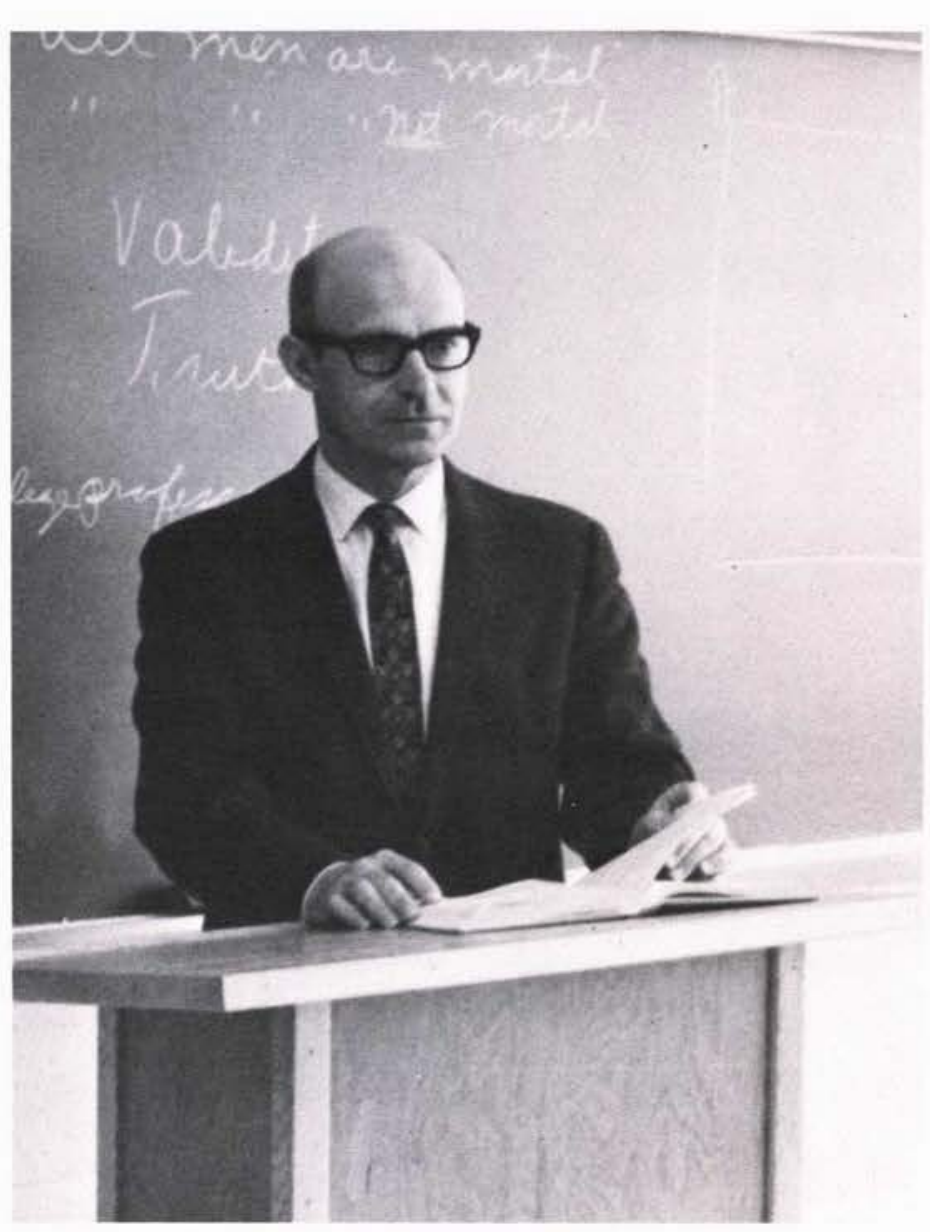

MR. EDWARD SPENCER

Oh, my heart!

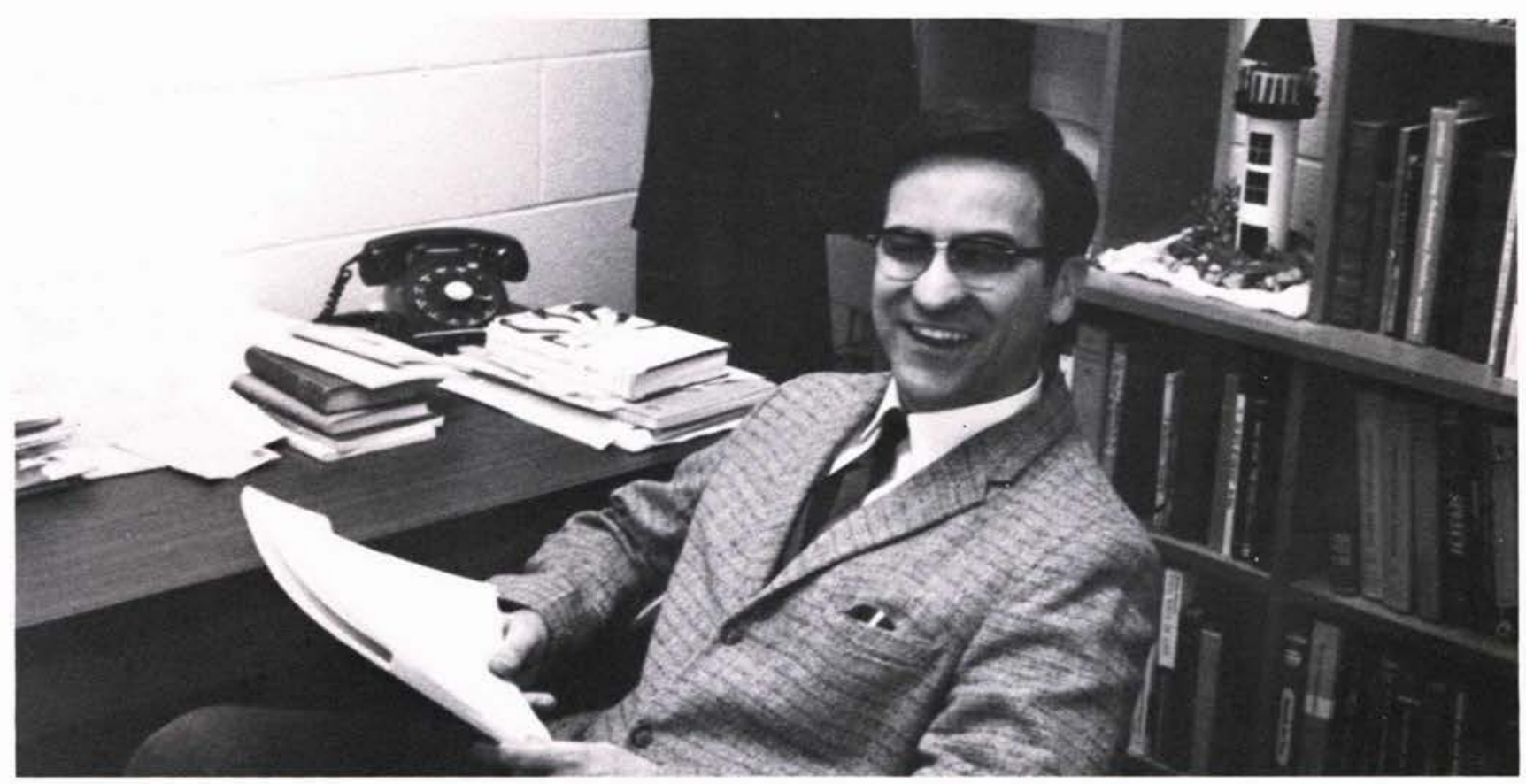

DR. JOHN REED

It's been real. 


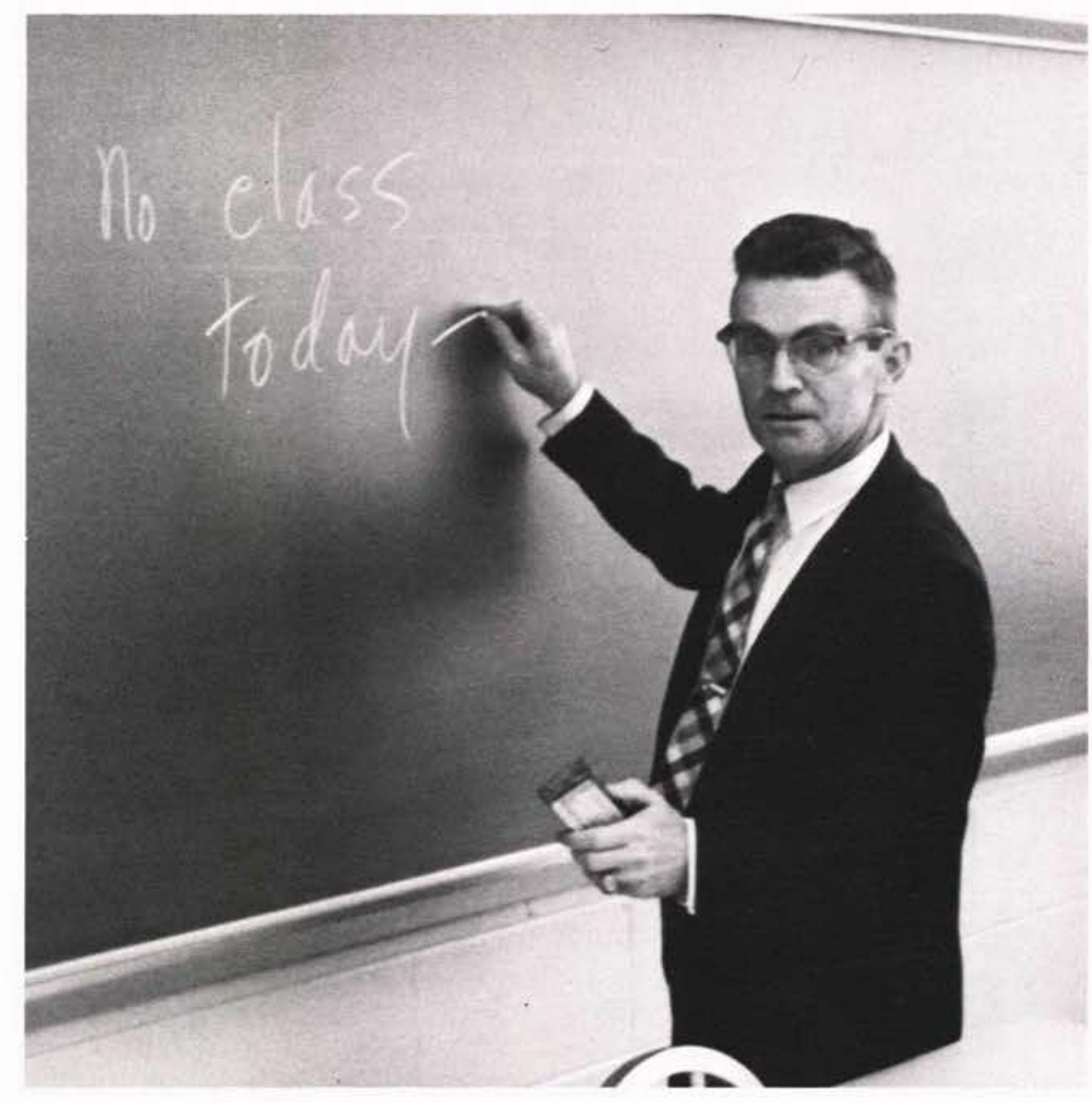

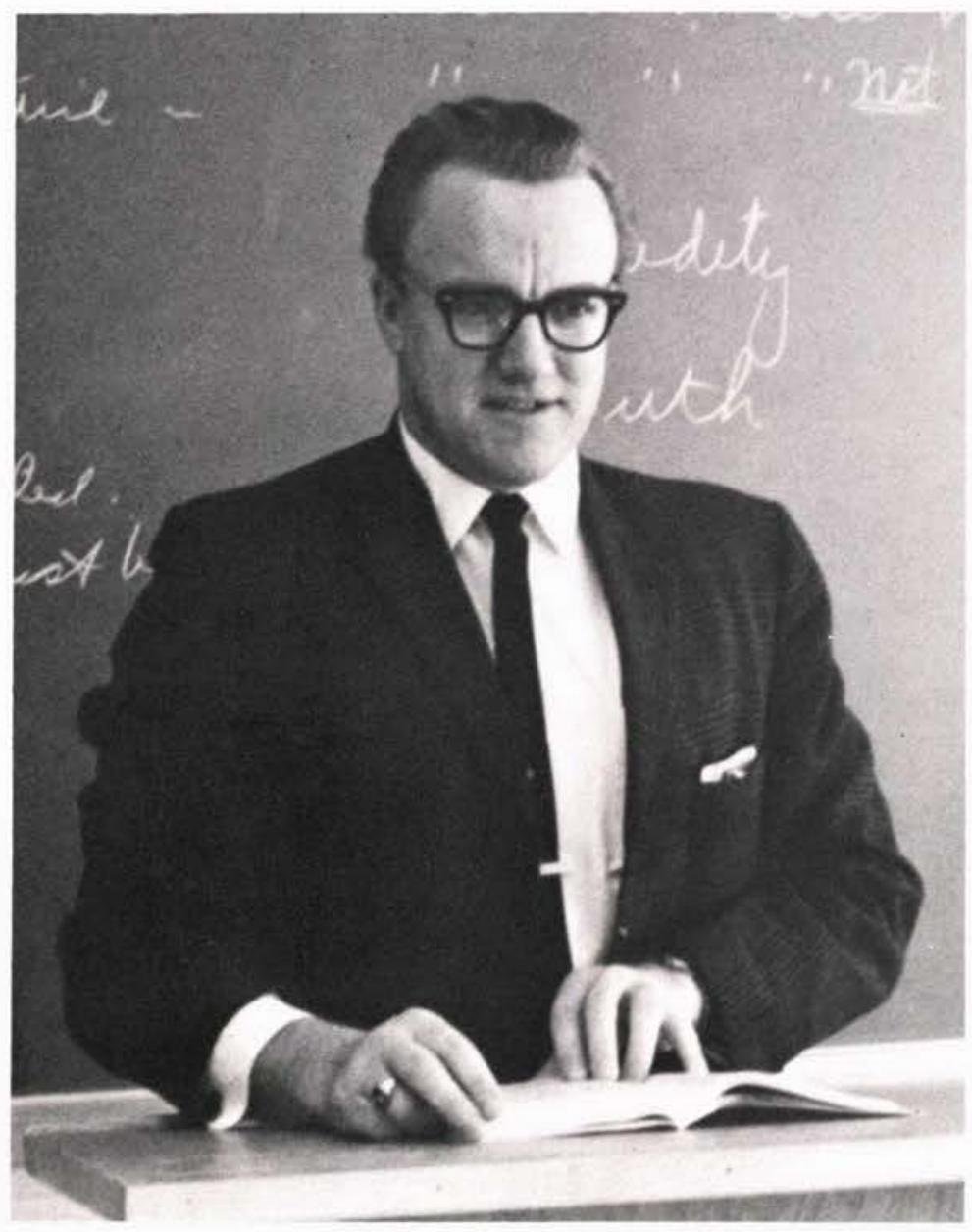

MR. HELMUTH POGGEMILLER Would you believe it?

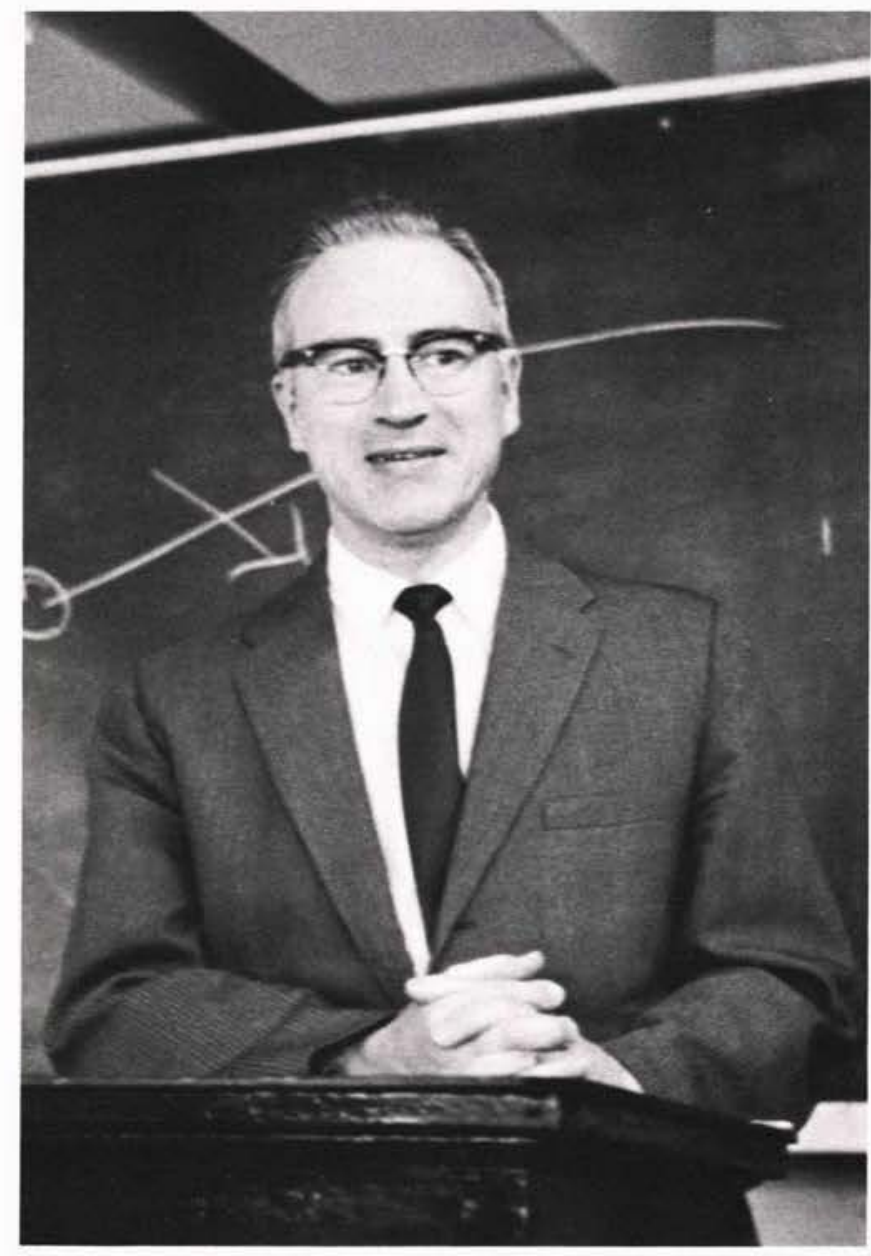

MR. EDWARD GREENWOOD This is getting off the subject but ... 


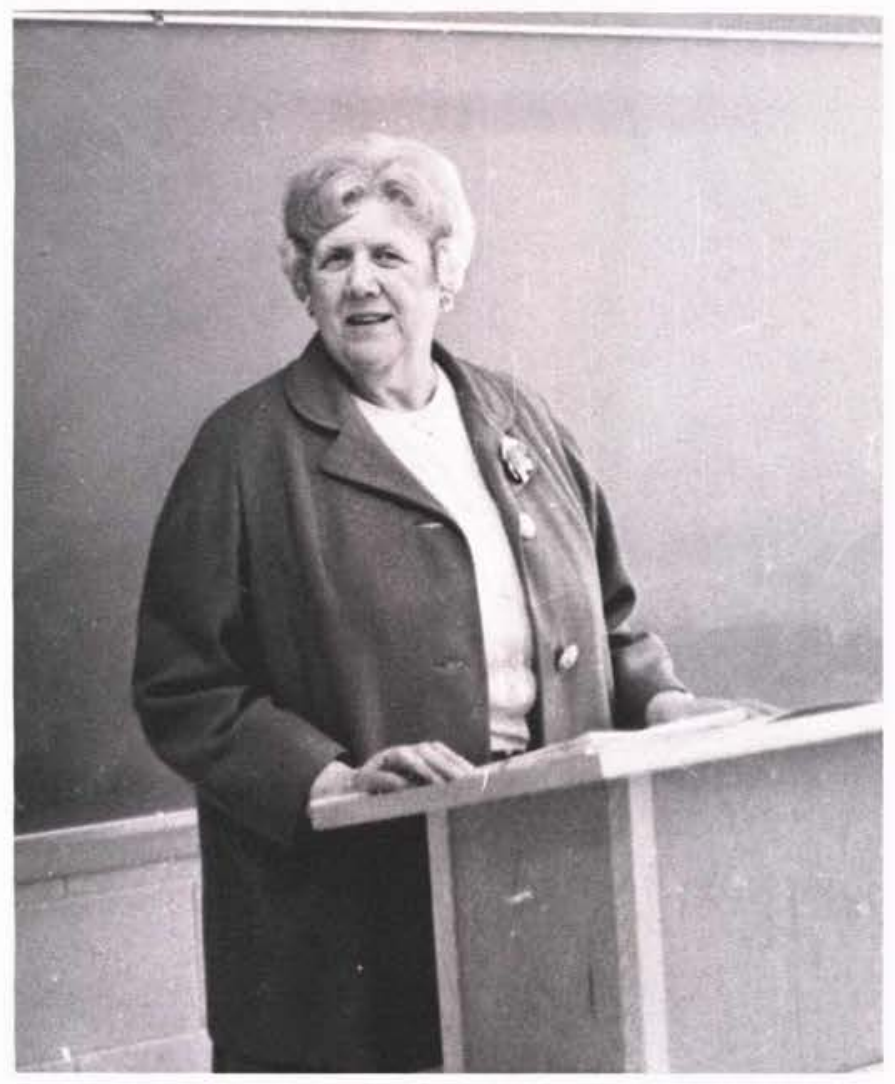

MRS. MIRIAM MADDOX

Are there any comments on that speech?

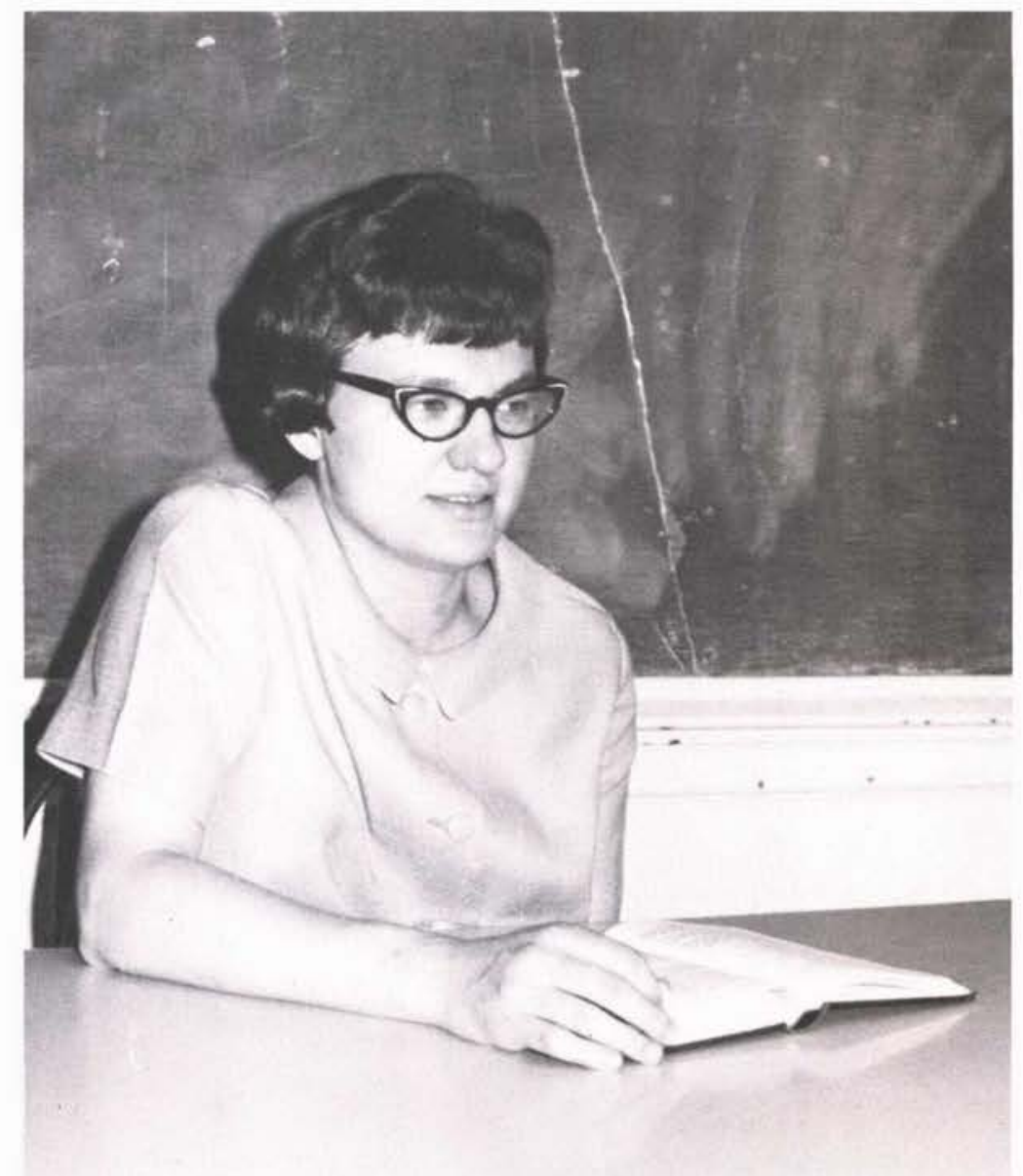

MRS. FAYE RUECK

Vamos a hablar en español hoy.

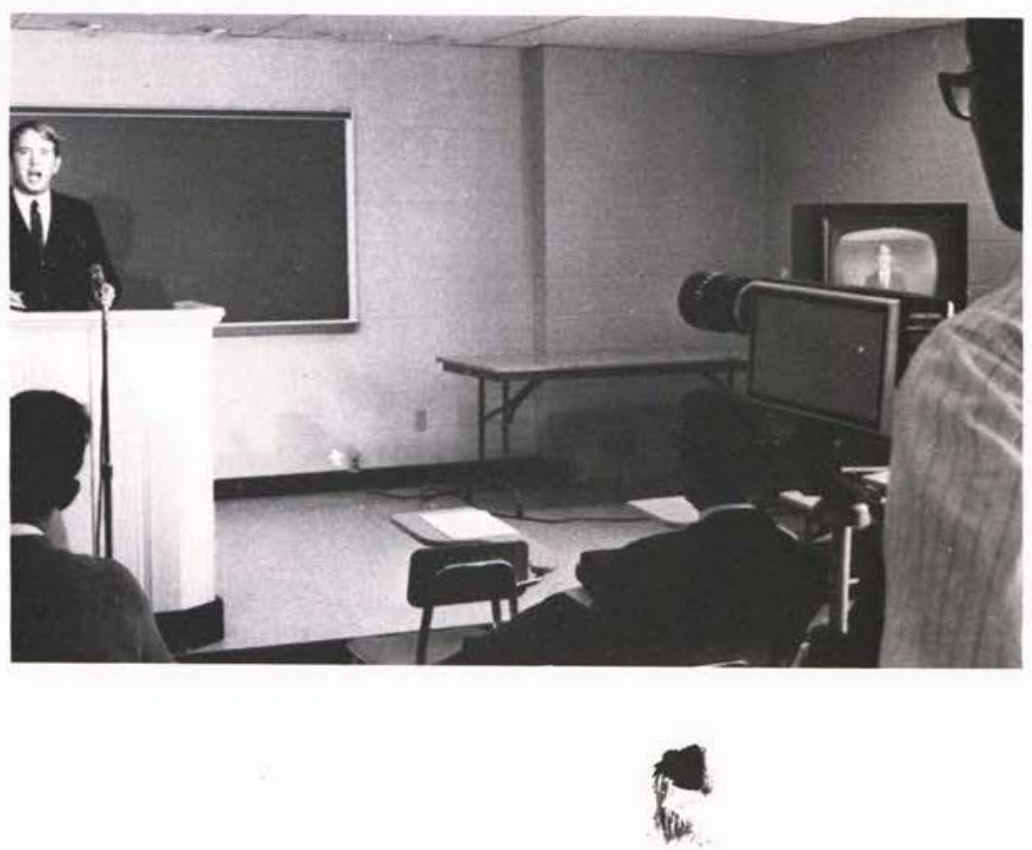




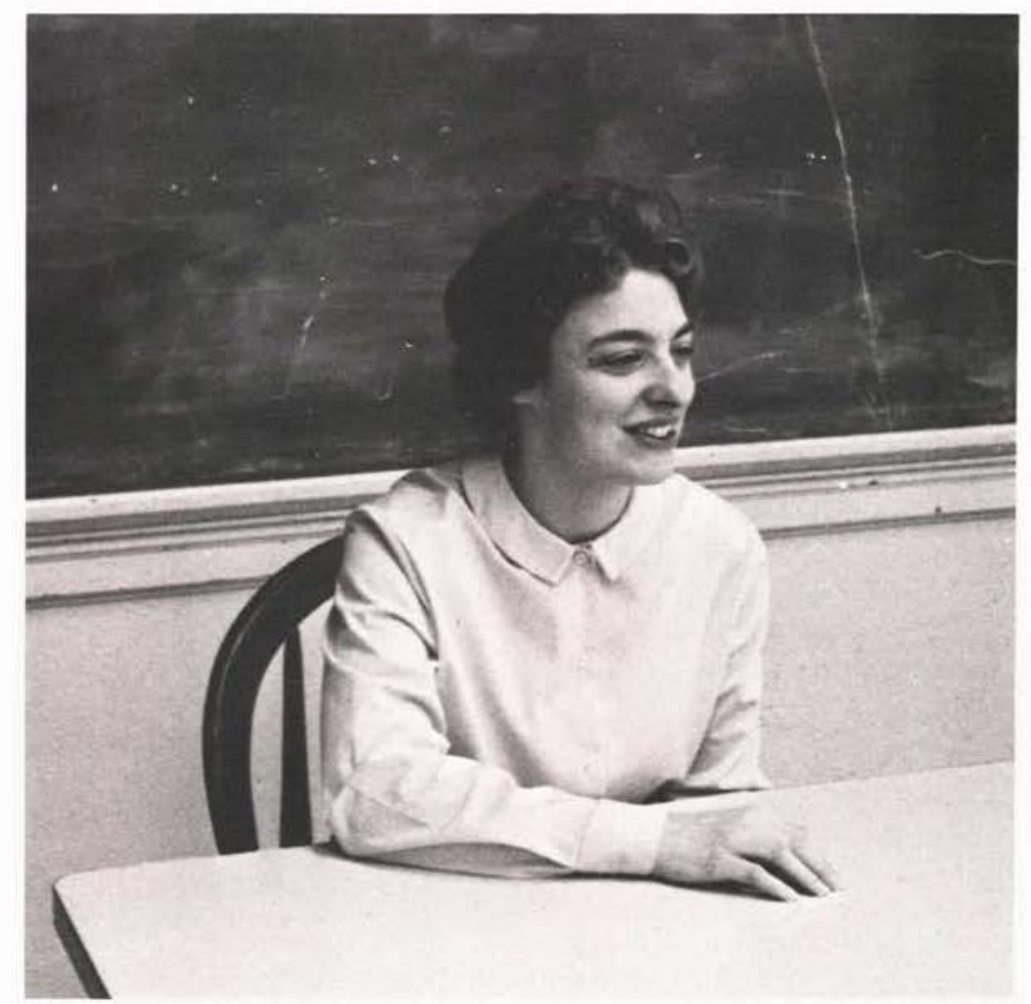

MISS CARLA PACKARD

Bonjour, classe.

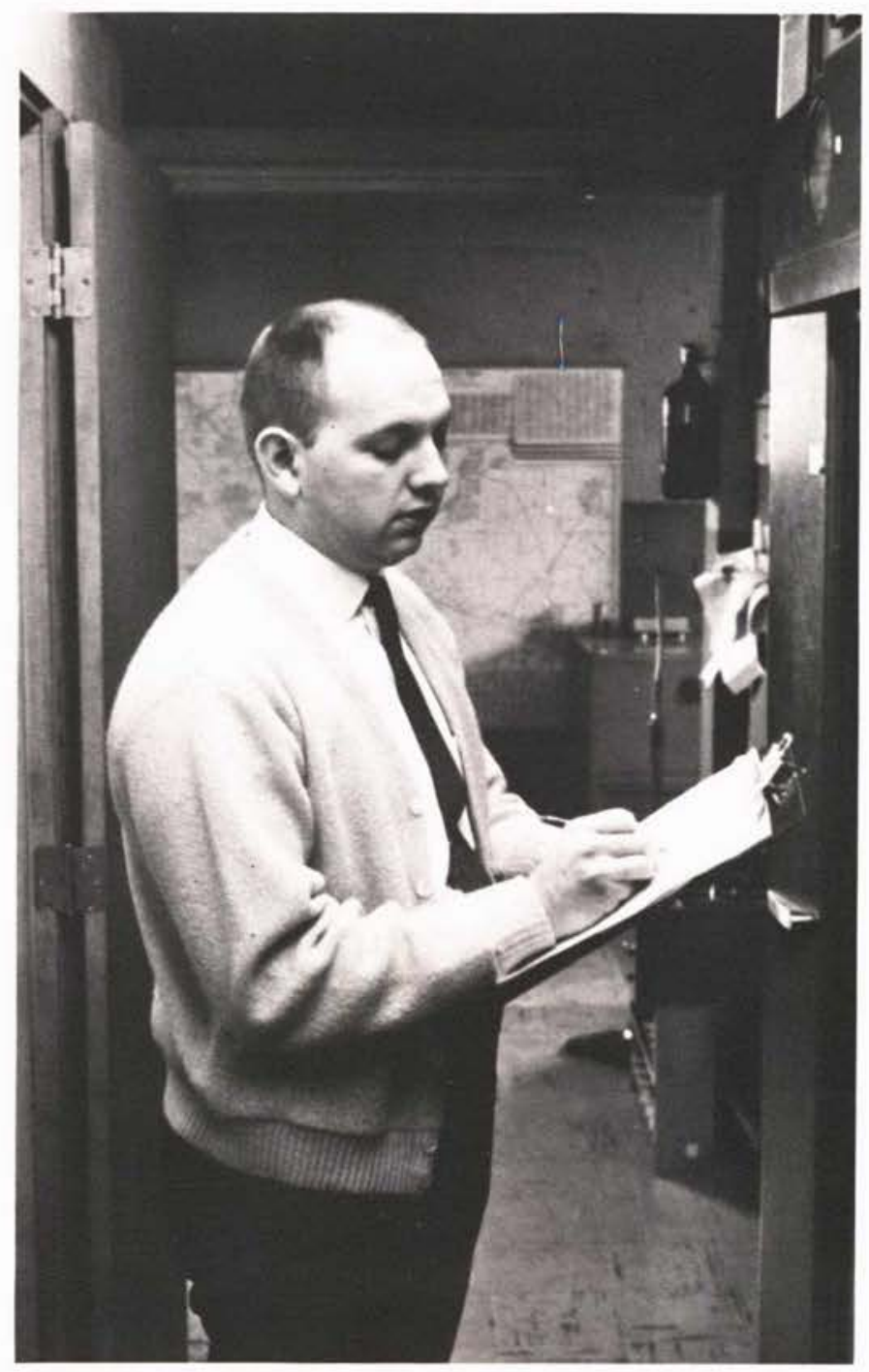

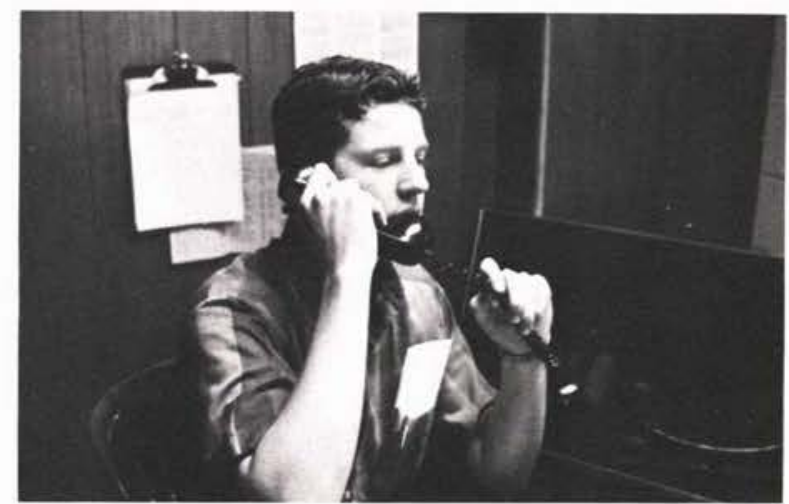

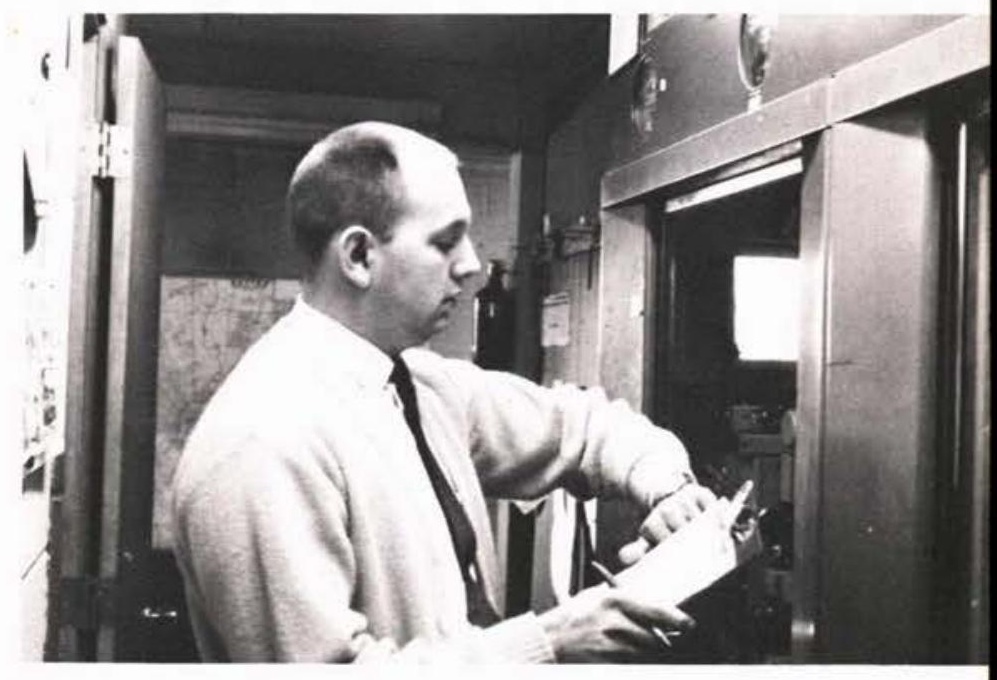

MR. PAUL GATHANY

The time is now 72 degrees and it's 4 o'clock outside our studio. 


\section{SCIENCE \& MATHEMATICS}

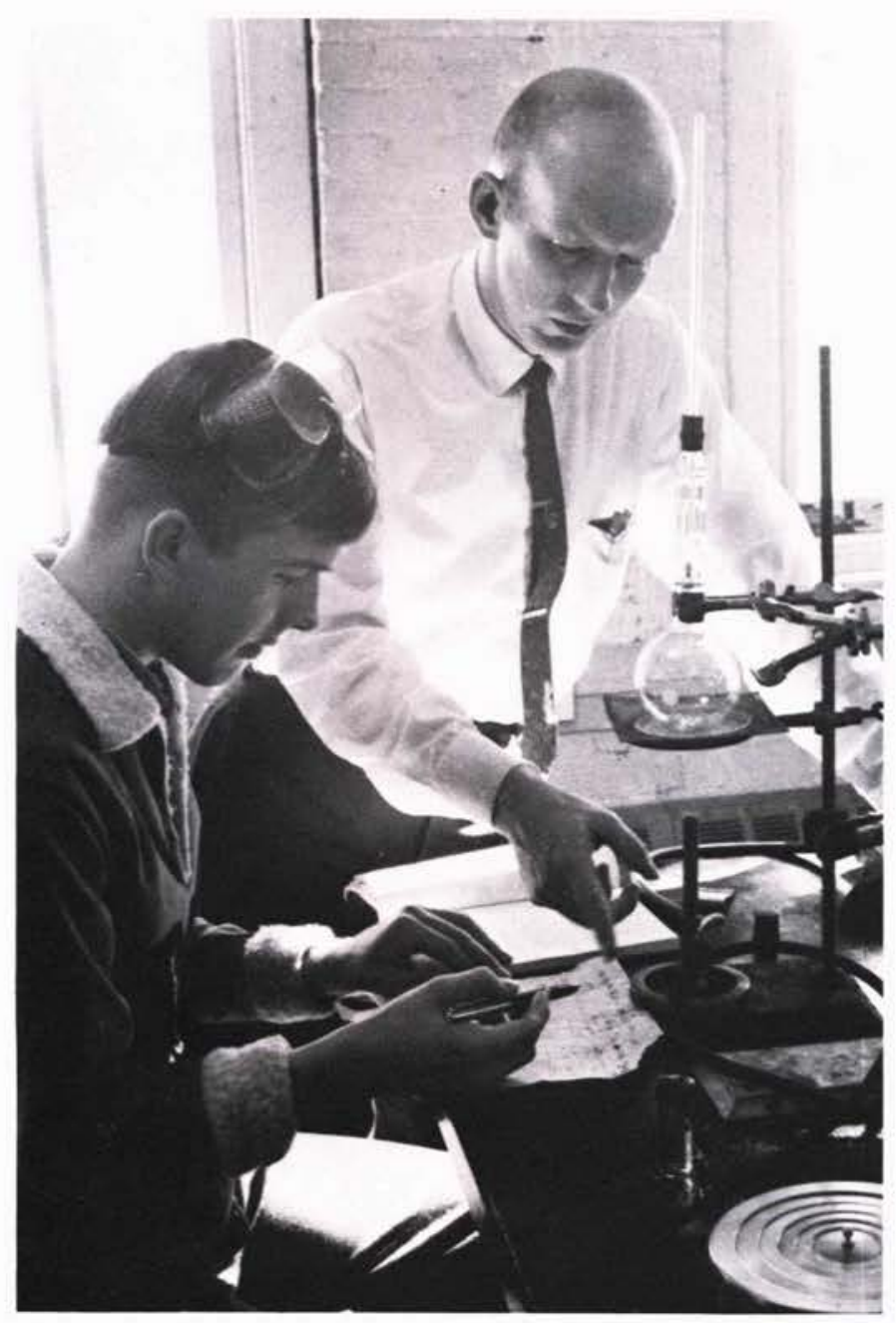

DR. DONALD BAUMANN

1 guess so.

There is a vast world around us,

Challenging our every endeavor

To penetrate its boundless storehouse of knowledge.

The Science Department seeks to satisfy

The Cedarville student's quest for knowledge in this realm.

Whether by gazing at the heavens through a telescope,

Dissecting a felis domestica in the lab,

Or calculating the number of amperes in an electric current;

The quest is made with the instruction of educators.

Trigonometry, digits, logarithms, and the slide rule

Belong to the world of the mathematician.

With these tools, he can do a myriad of things

Beyond our wildest imagination.

The Mathematics Department of Cedarville

Grounds its students in these basic tools.

These two essential areas of study are

Continually striving for excellence.

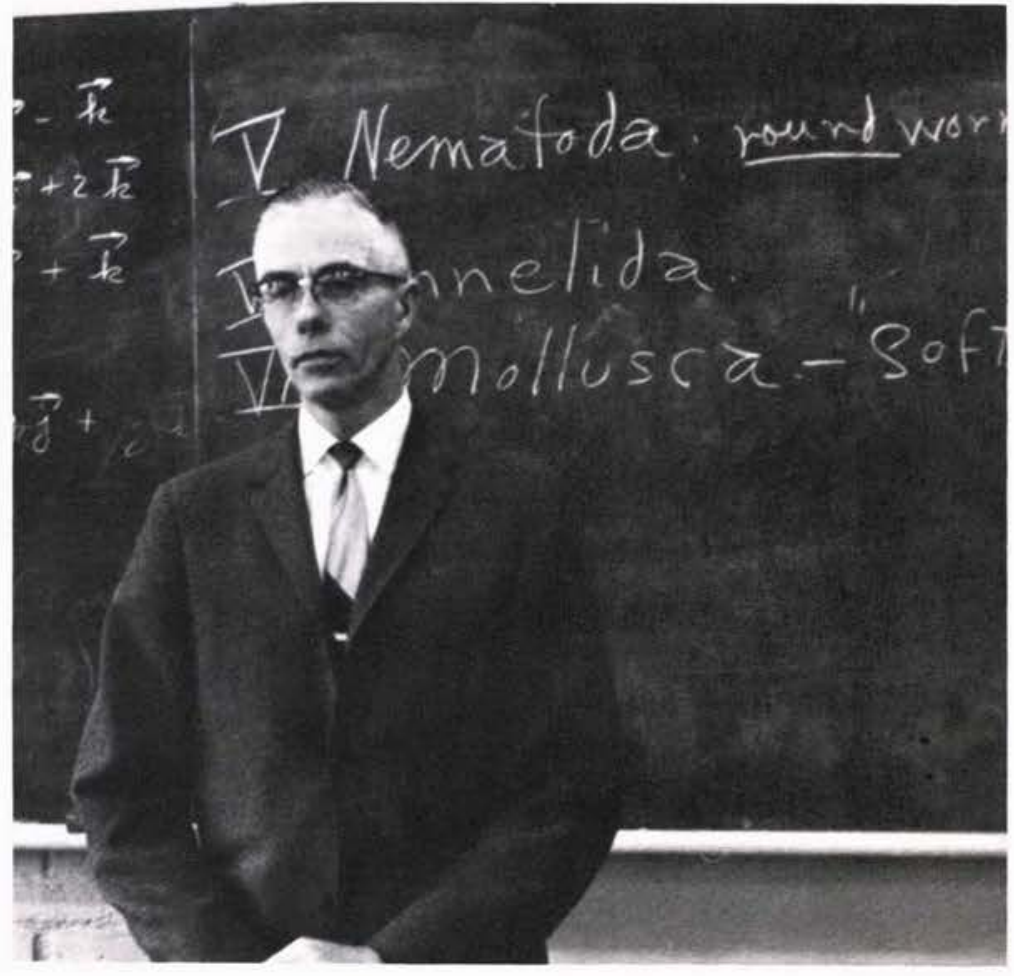

MR. AUSTIN ELMORE

Now that you have completely digested this material ..

4

MR. DANIEL WETZEL

This could get a little messy. 


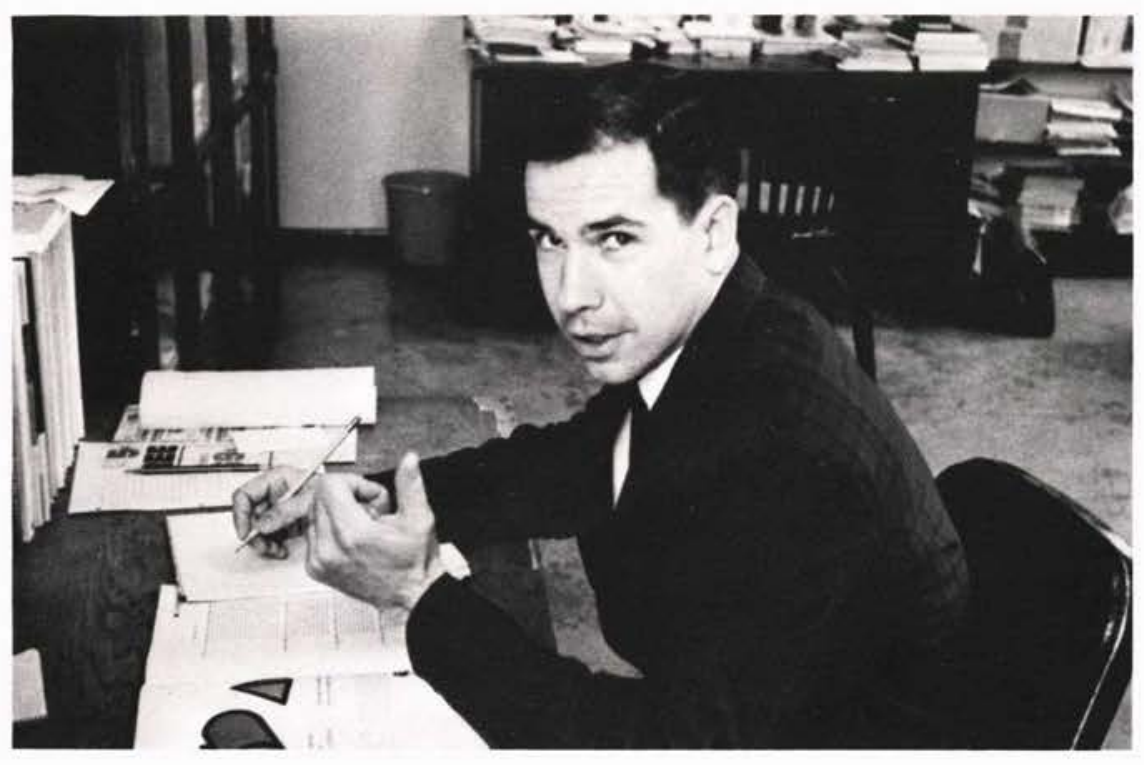

DR. LARRY HELMICK And it furns out.

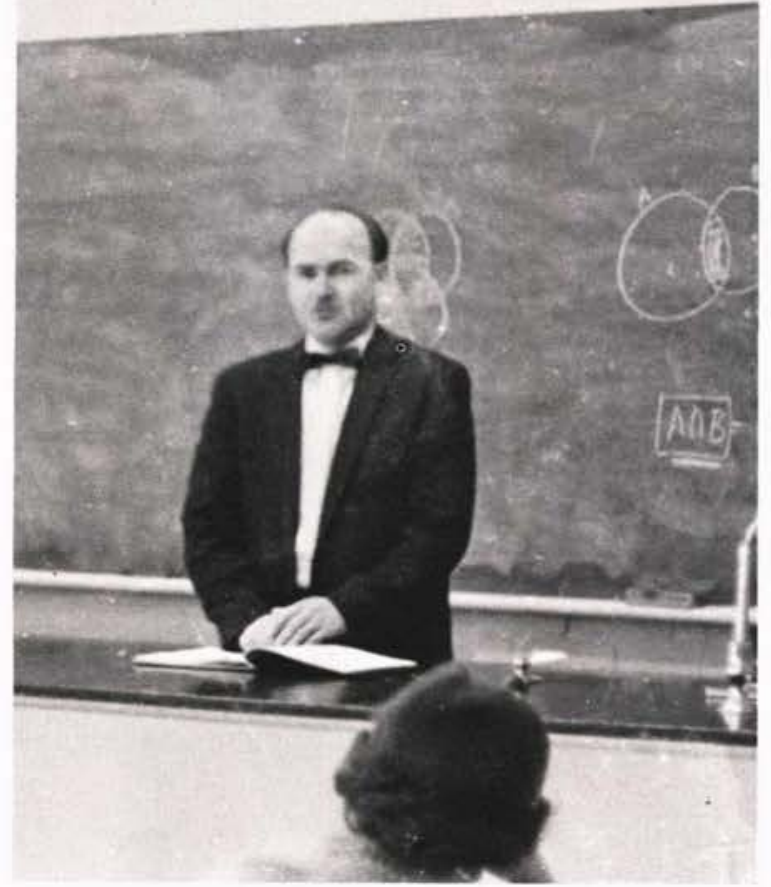

MR. MALCOLM WOODARD

This is simply...
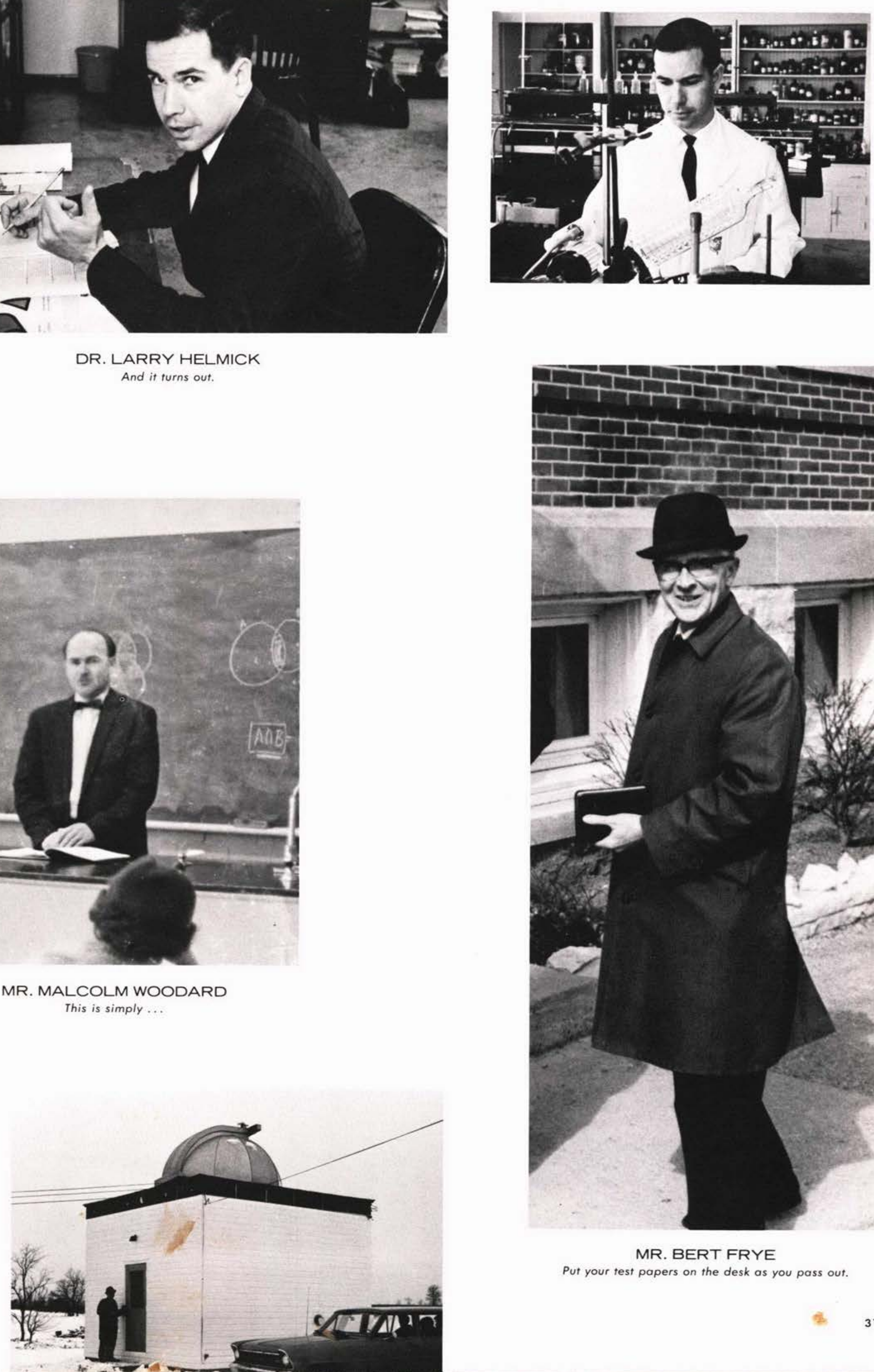

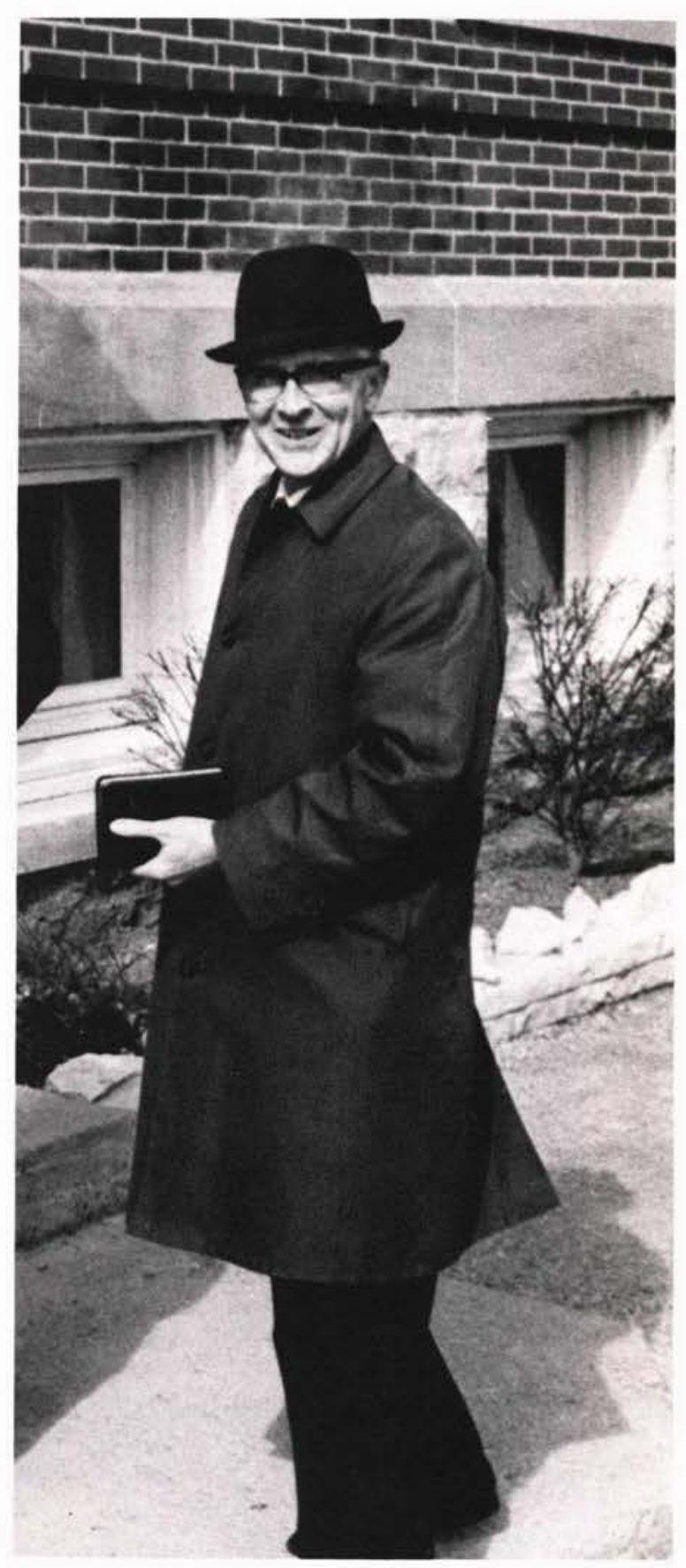

MR. BERT FRYE

Put your test papers on the desk as you pass out. 


\section{SOCIAL SCIENCE}

"It's a man's world"

So they say,

And Viet Nam, the Kremlin, the U.S.A.

Reveal a world

Dependent upon man,

A specimen of man

Whose stature is God-molded

Whose intellect is Spirit-directed

Whose soul is Christ-conscious.

The Social Science Department

Presses toward that mark.

The preparation of responsible citizens

And the investment of patriotism

Are aims which

Color and shape

The curriculum of this field.

Each course is designed

To produce enlightened citizens,

Denizens who can take their place

In this land

While awaiting entrance

Into a more glorious Land.

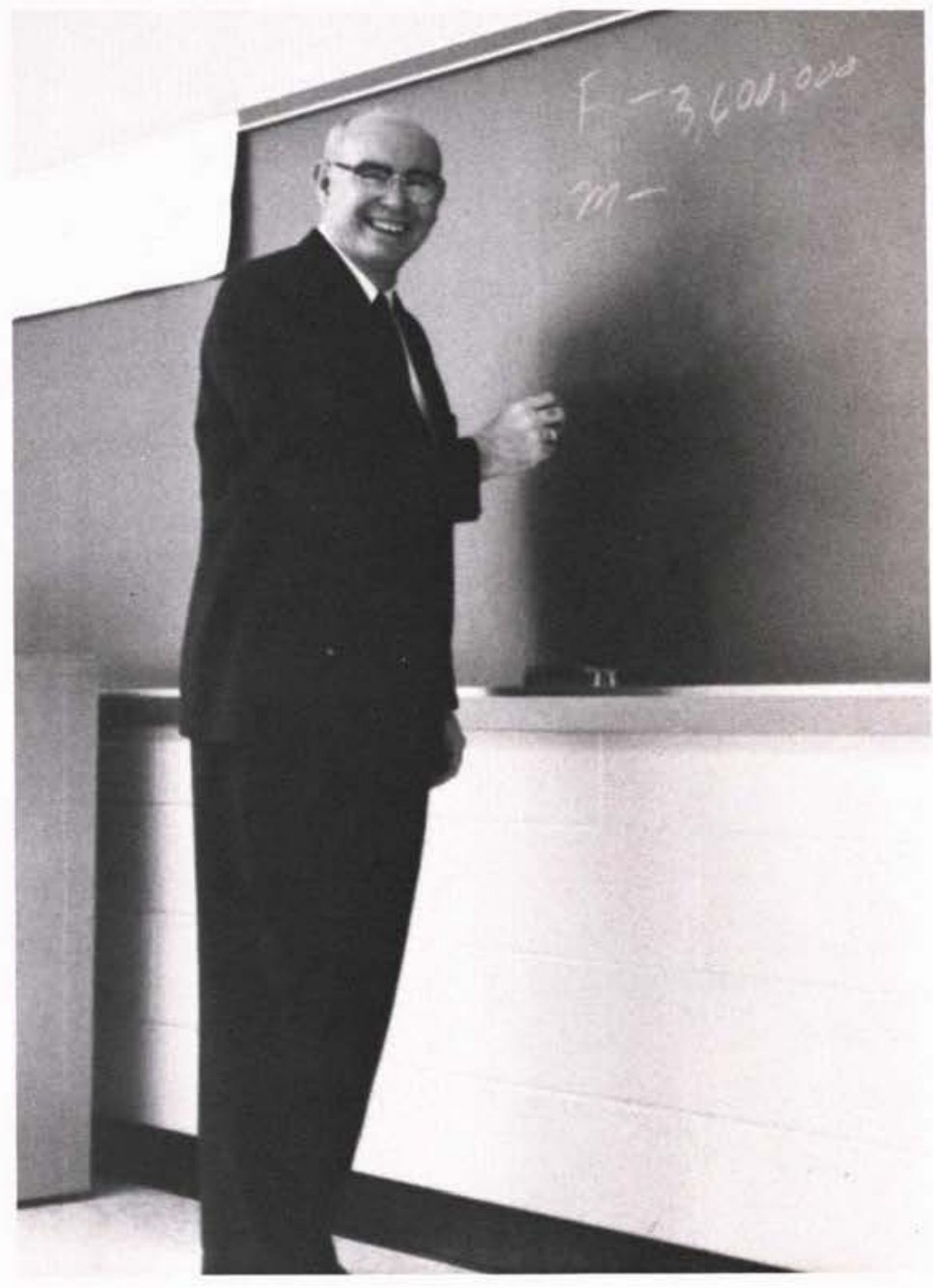

DR. CLEVELAND MCDONALD

1 have a clipping here from 1953.

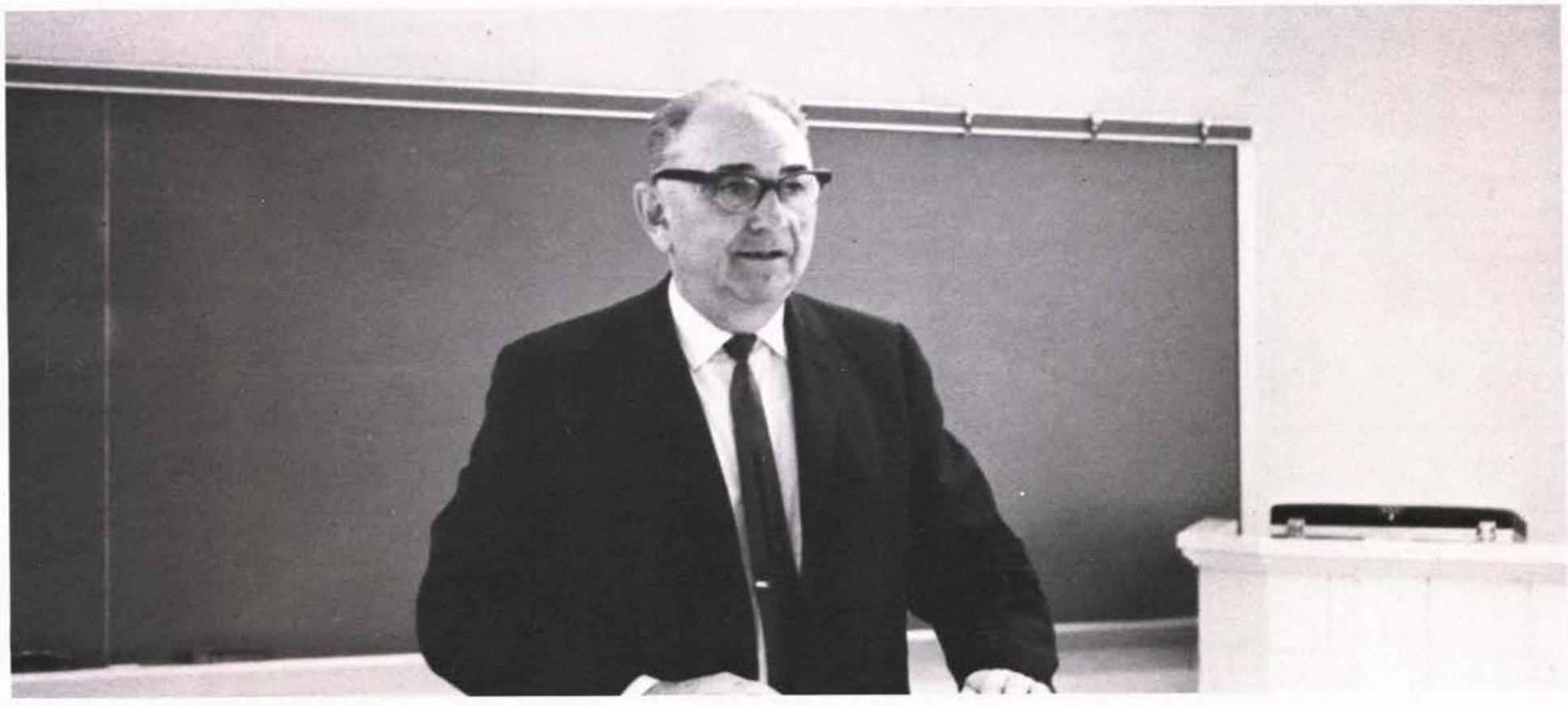

MR. RALPH GALE

Well, it's a little early but it's Friday, so I'll let you go 


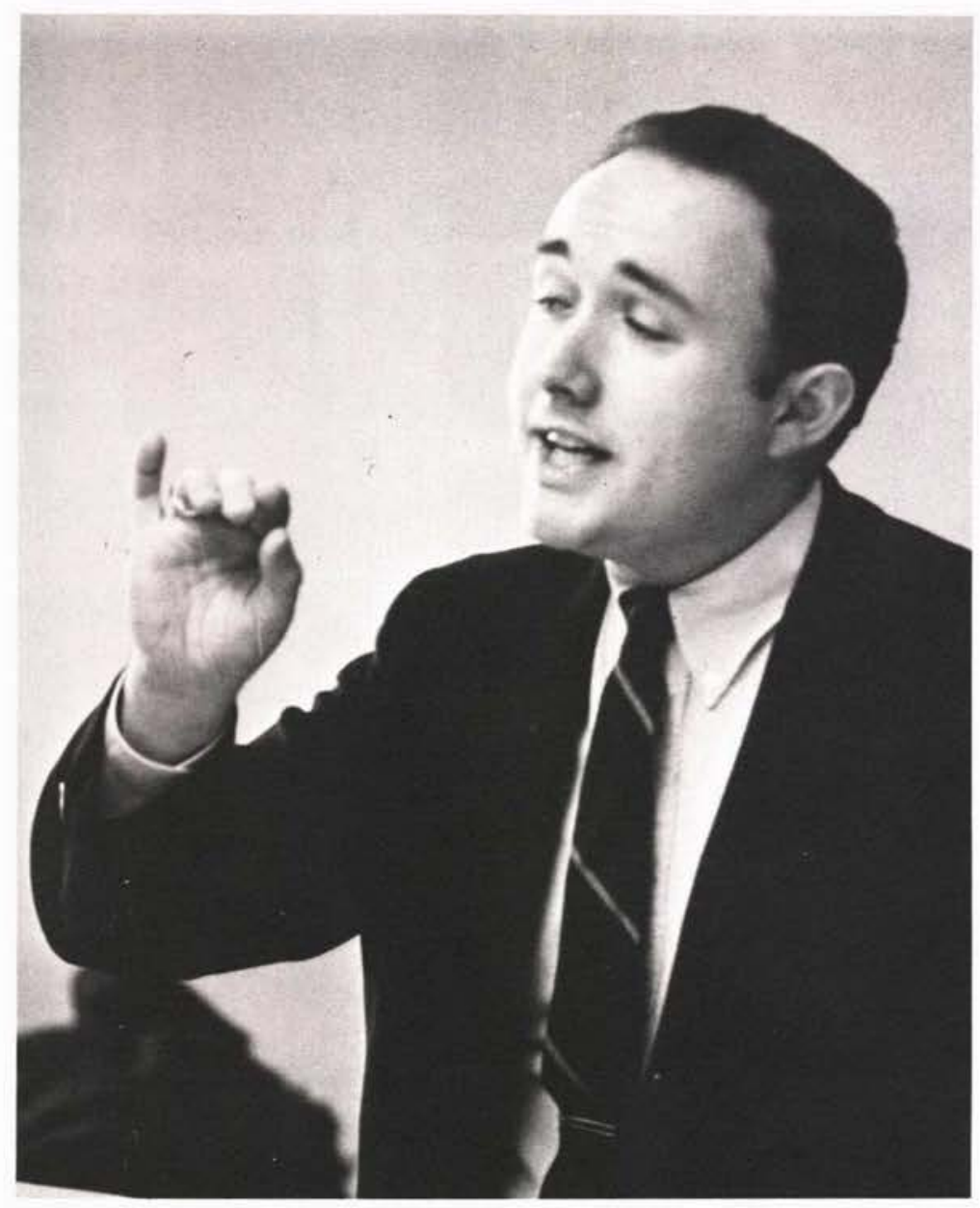

MR. MURRAY MURDOCH

The epitome

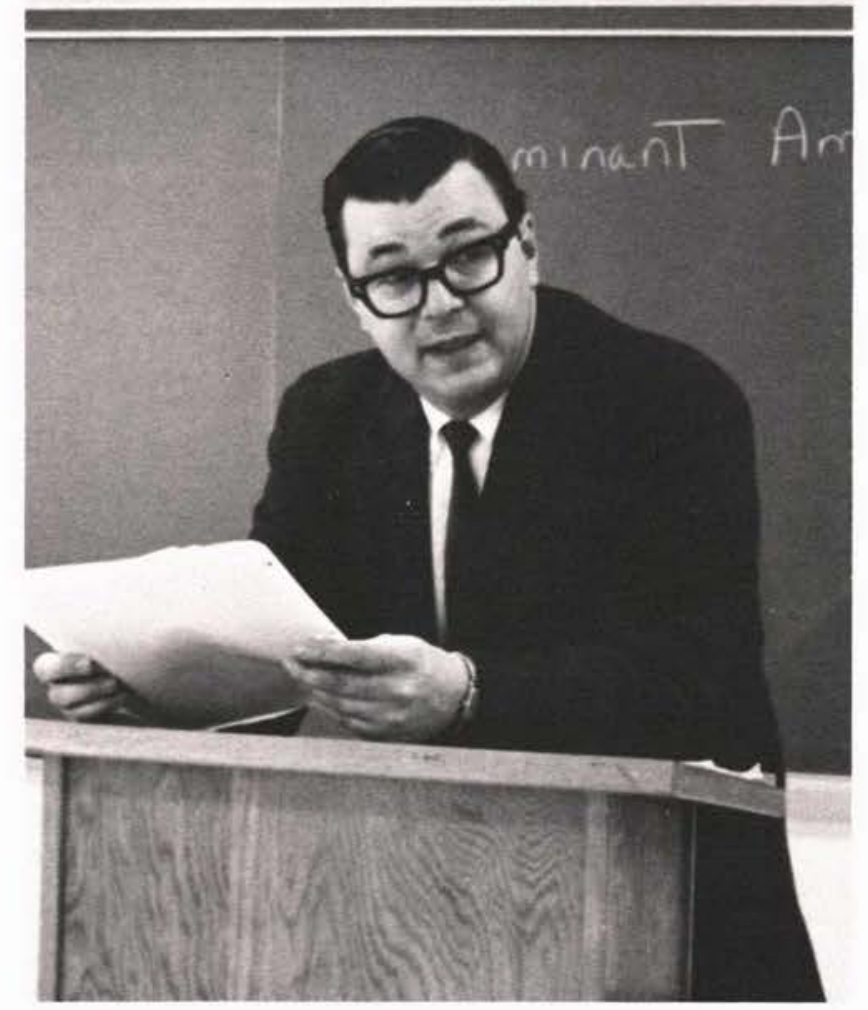

MR. ALLEN MONROE 1 contend that...

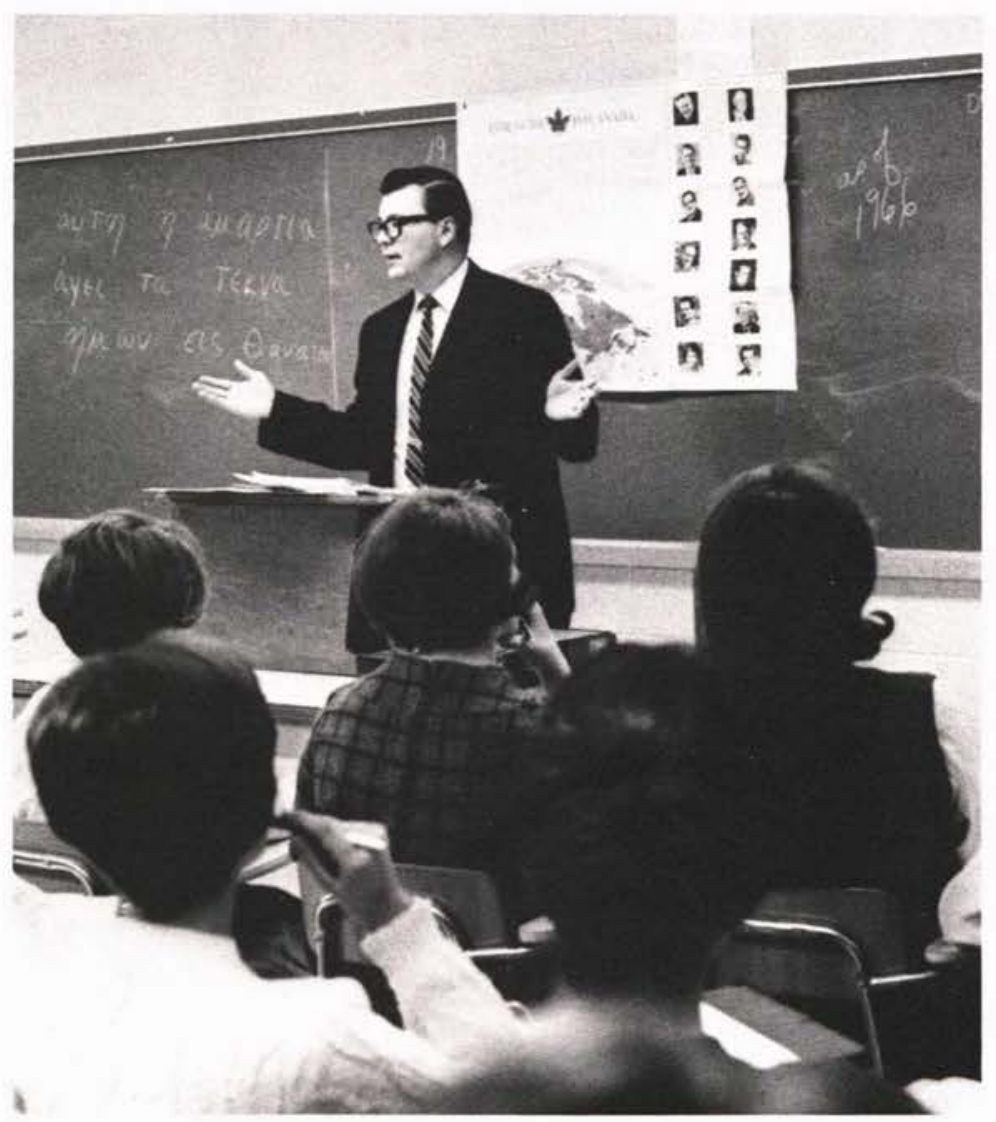




\section{ADMINISTRATIVE SECRETARIES}

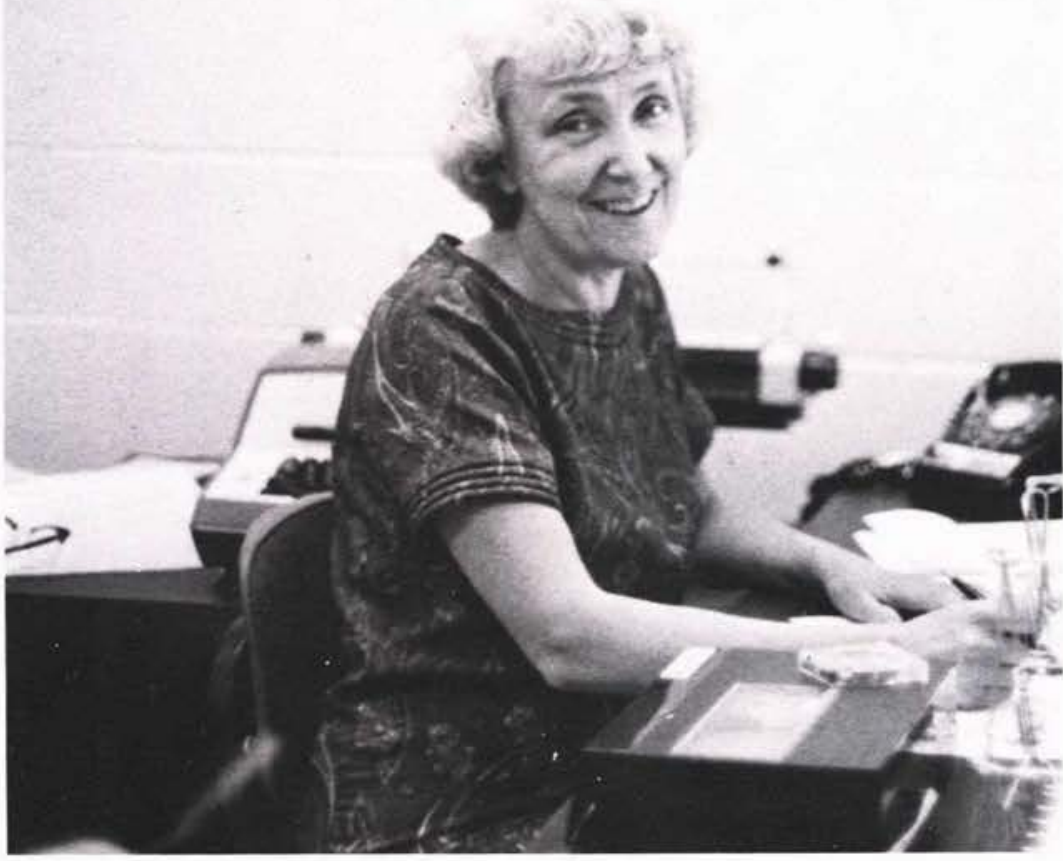

BERYL BROWN

Secretary to the President

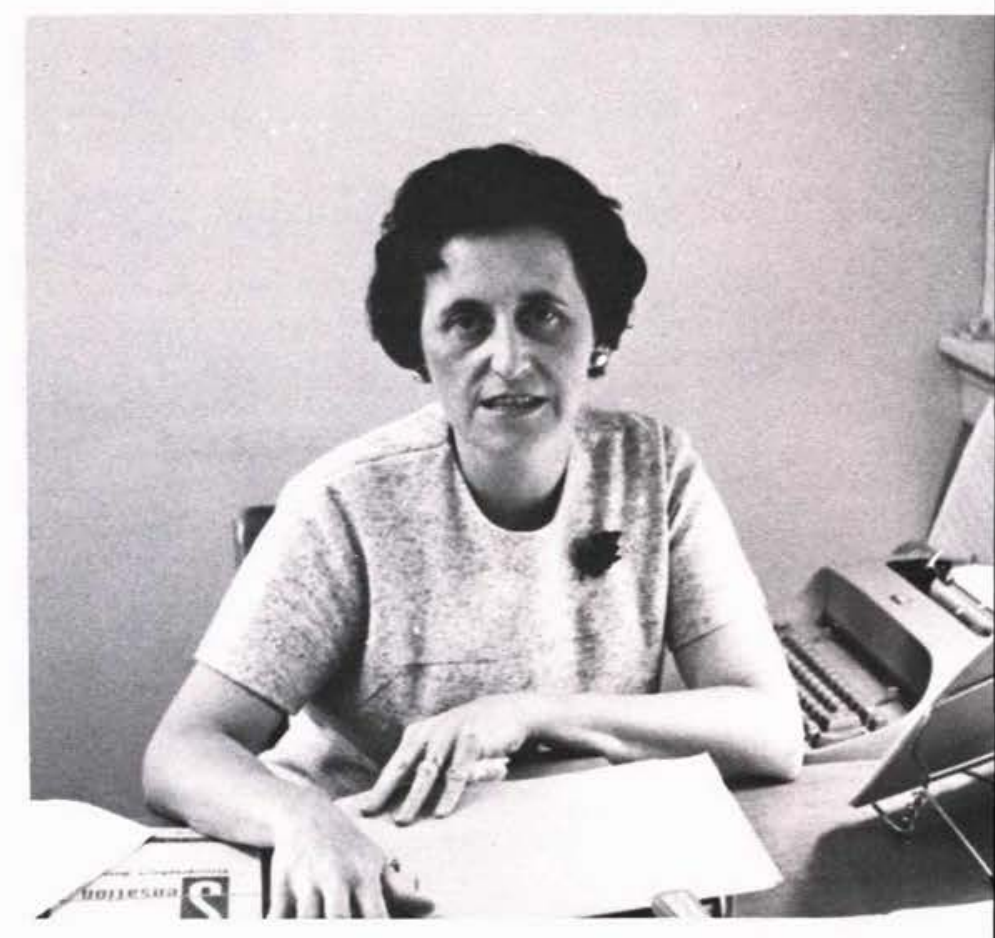

MAY GREENWOOD
Secretary to Academic Dean

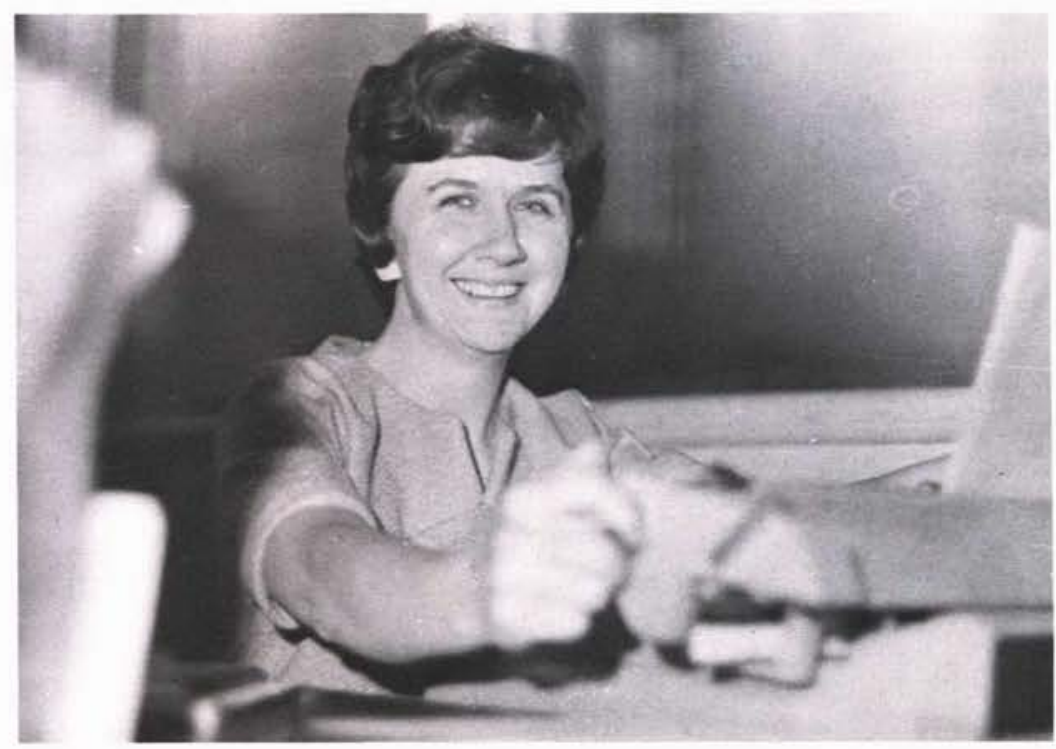

NANCY MCPHEETERS Secretary to Registrar

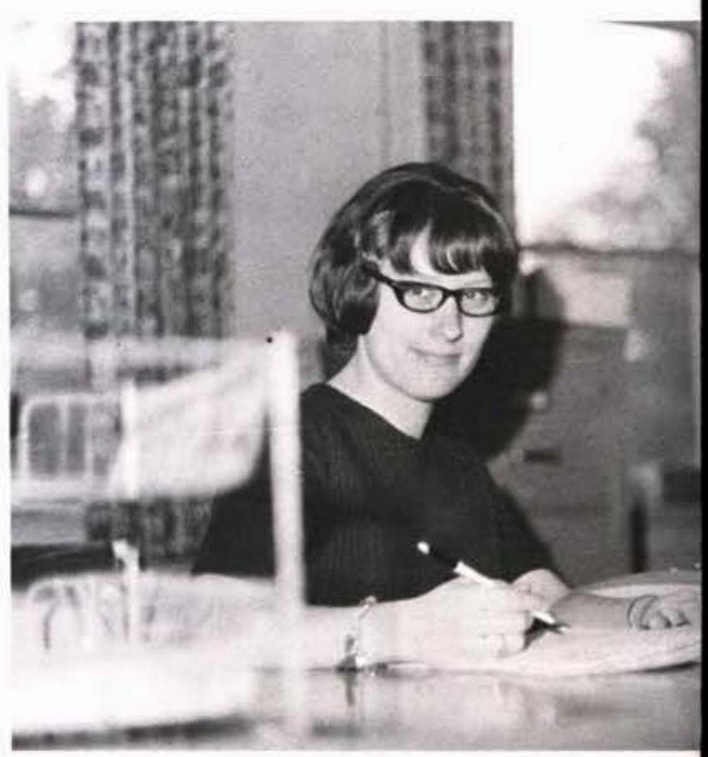

REBECCA WILHITE

Secretary-Admissions and Testing 


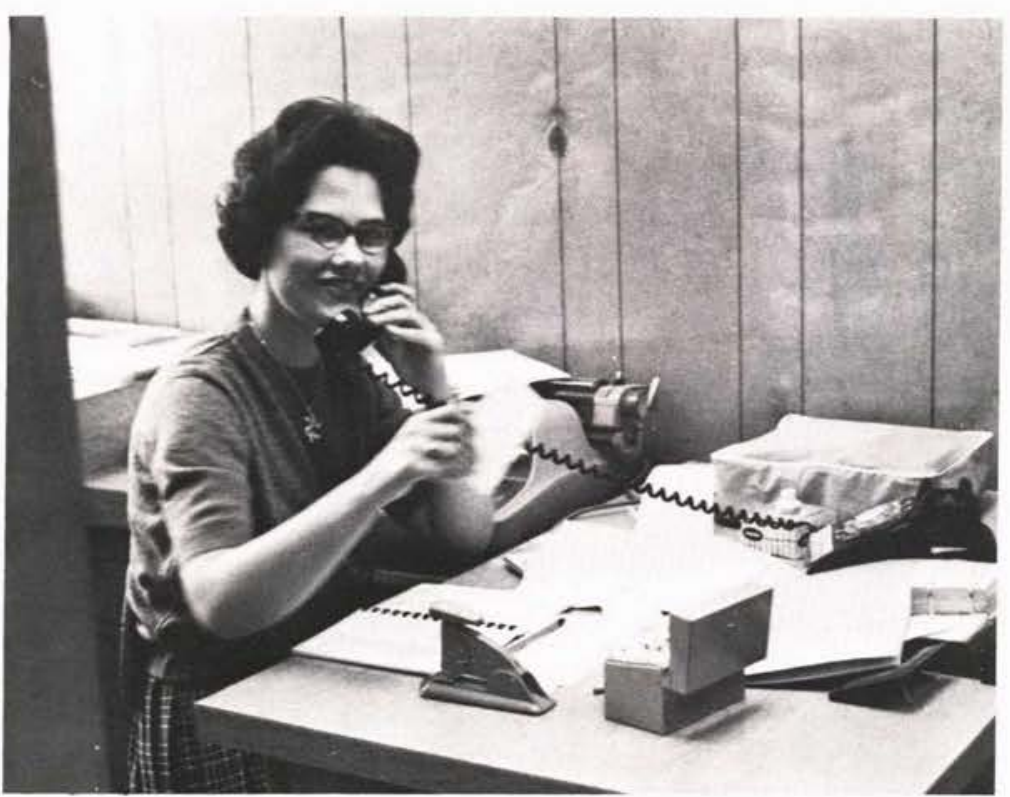

CAROLYN THOMPSON

Secretary to Director of Development

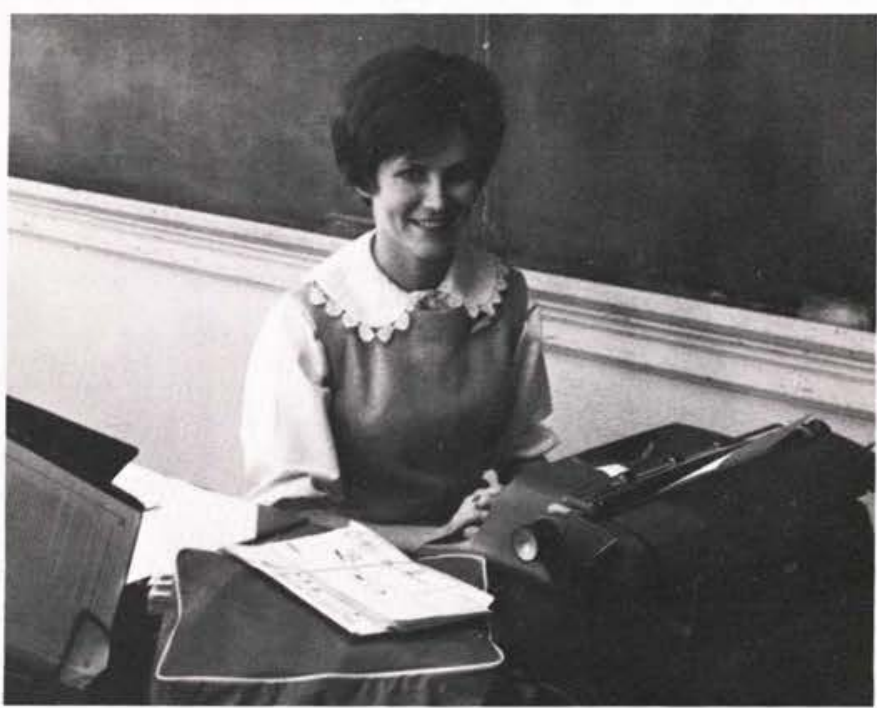

PATTY JOHNSON

Secretary - Alumni and Deferred Giving

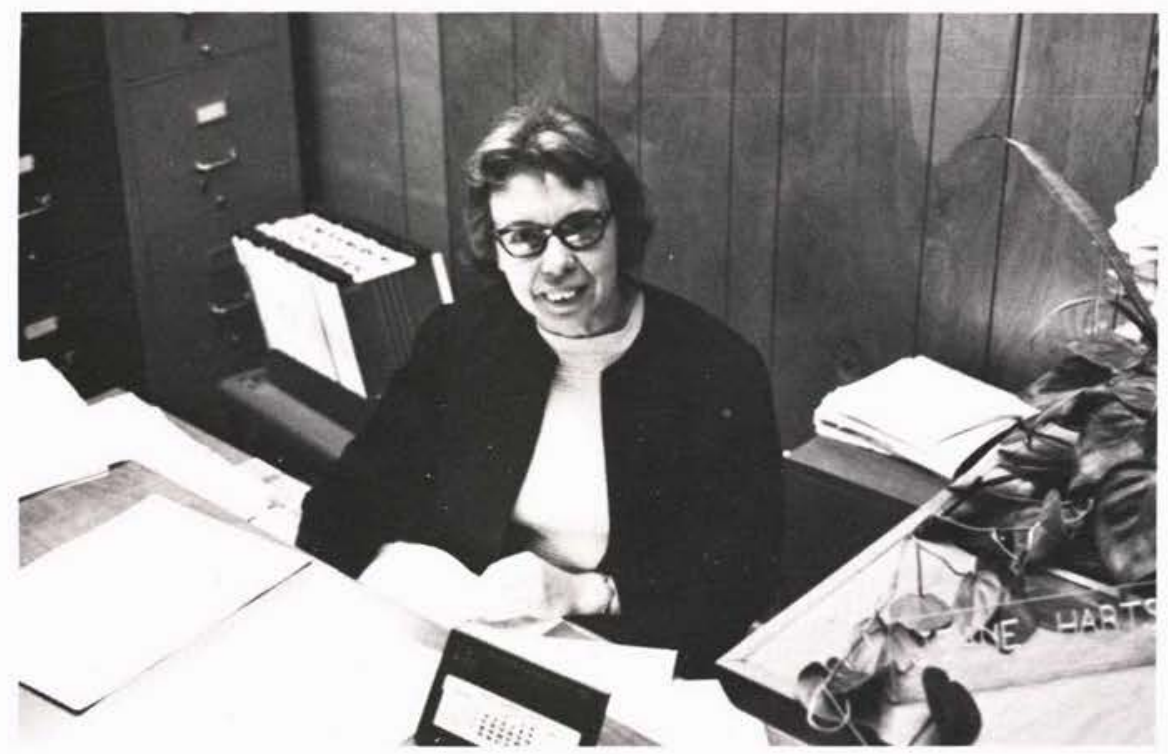

JEANE HARTSELL

Secretary to Dean of Students

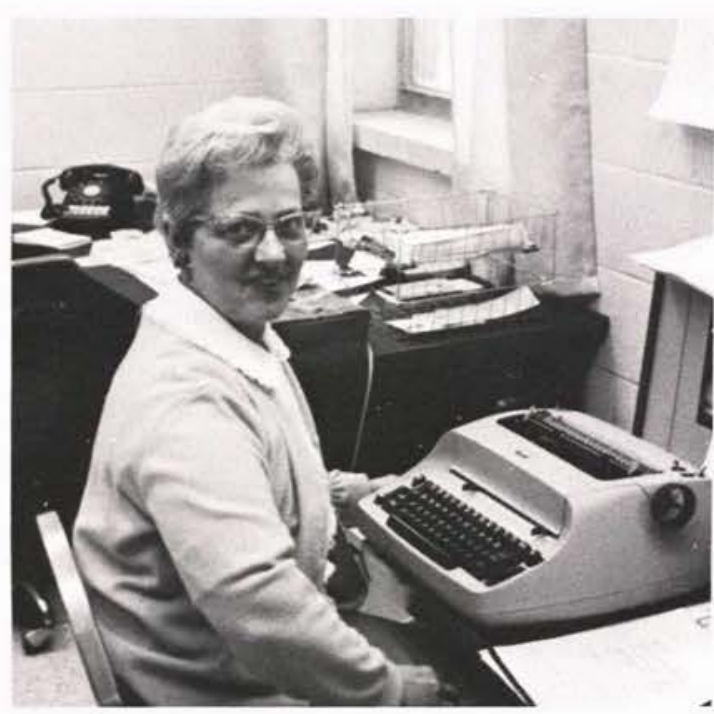

FLORENCE SLABAUGH

Faculty Typist 


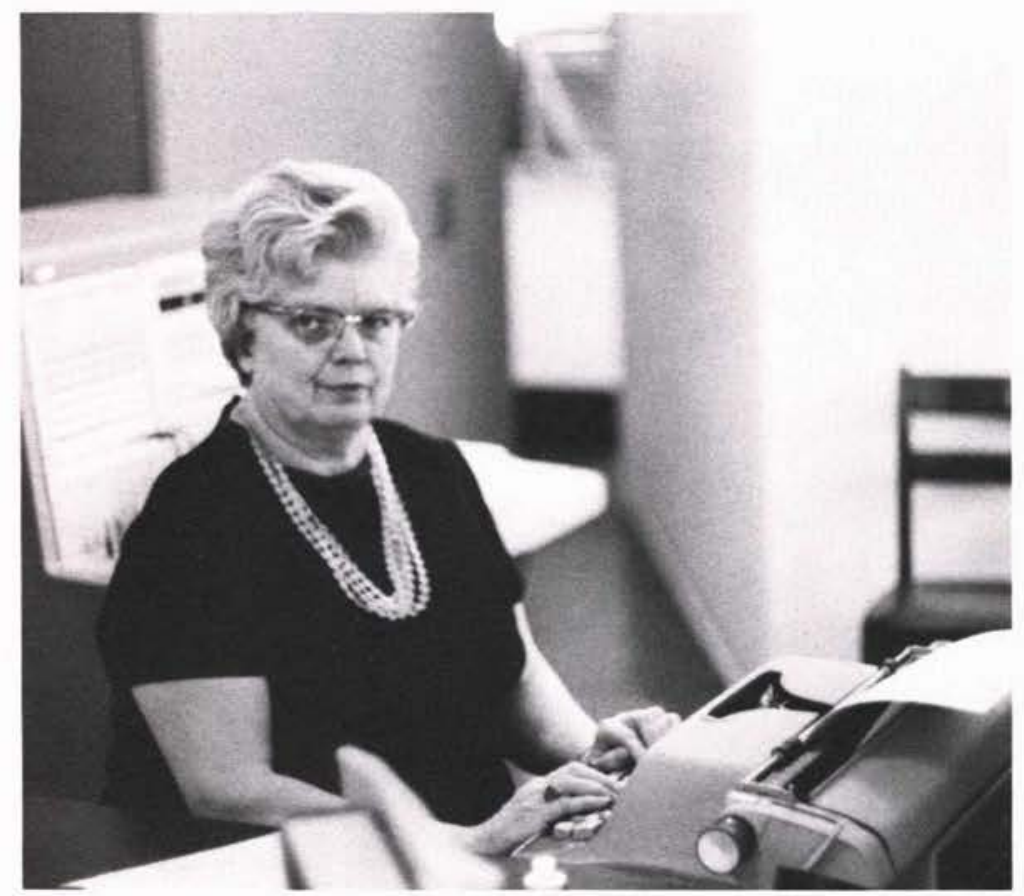

MRS. MARIBETH ELMORE

Receptionist and Secretary to Business Manager
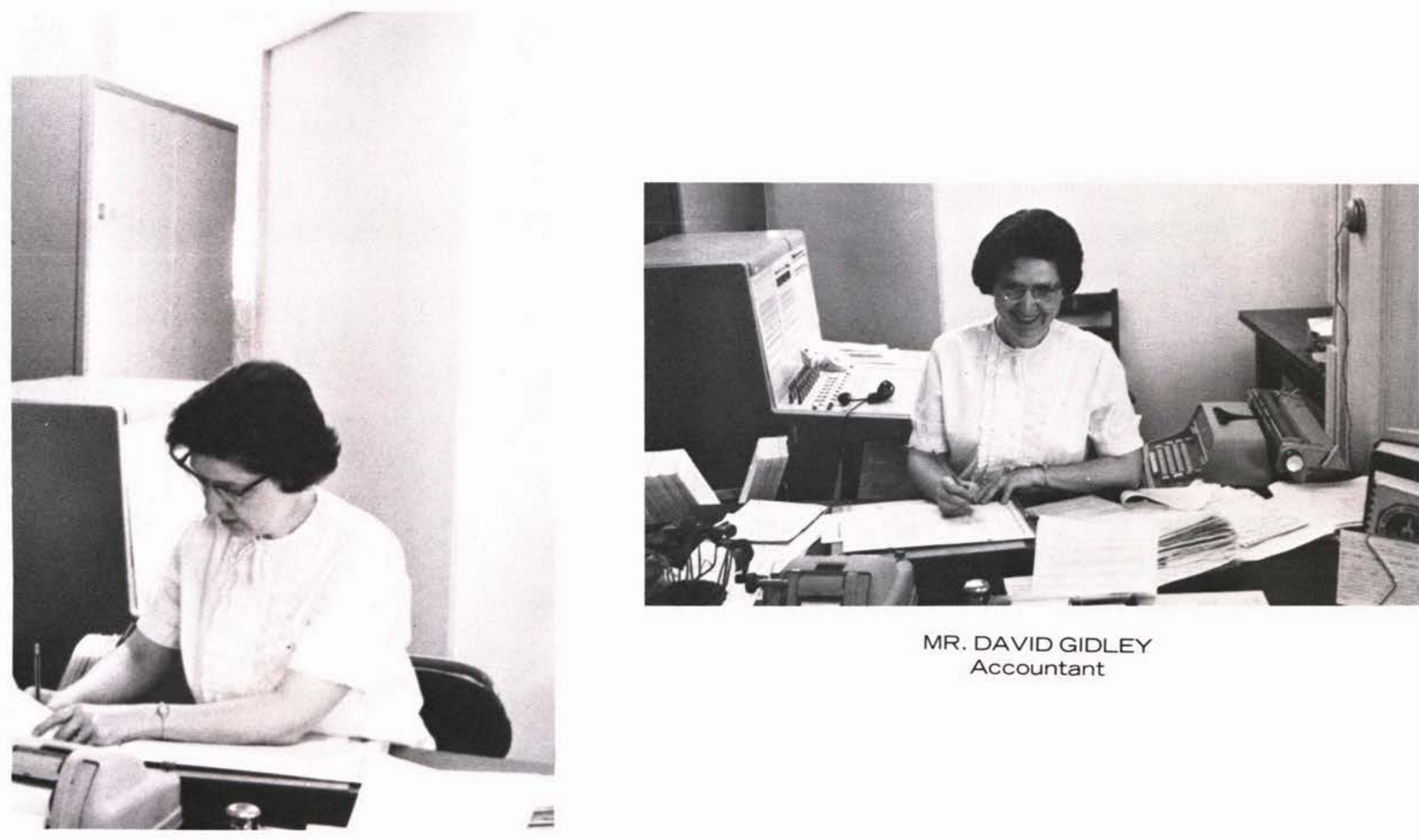

MR. DAVID GIDLEY Accountant

MRS. IDA ST. CLAIR

Accounts Payable 
OFFICE

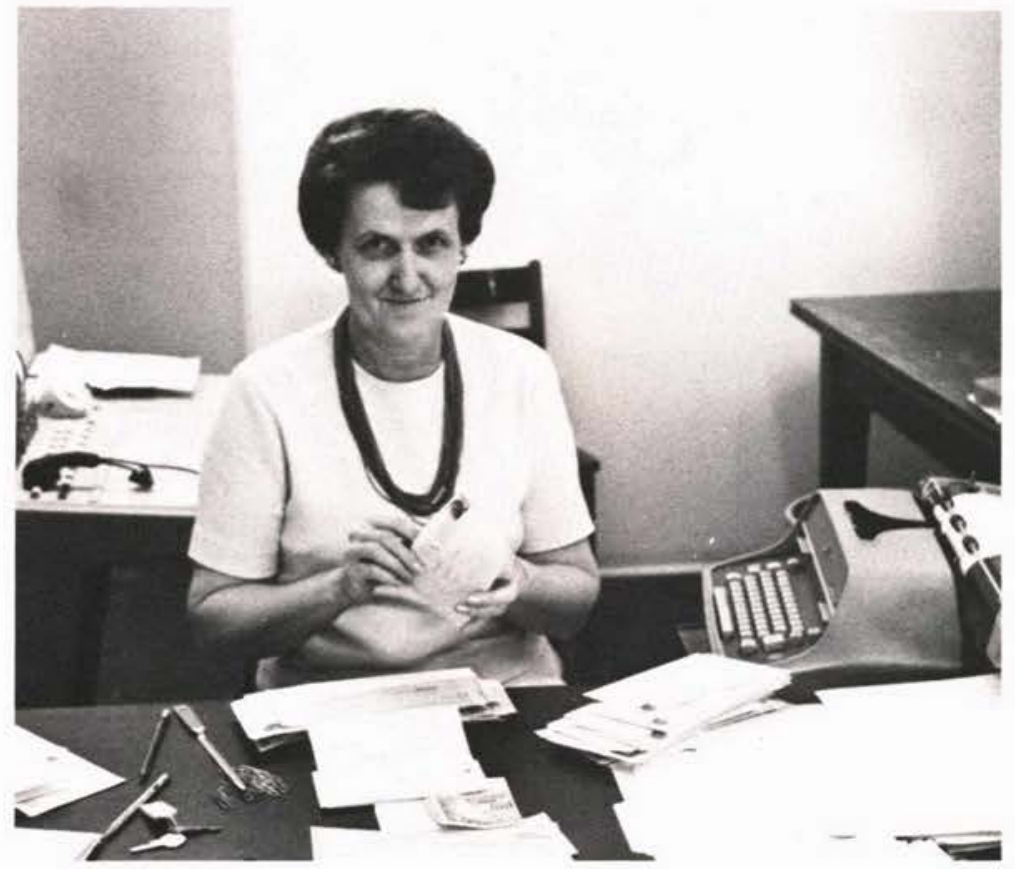

MRS. DOROTHY SPENCER

Cashier

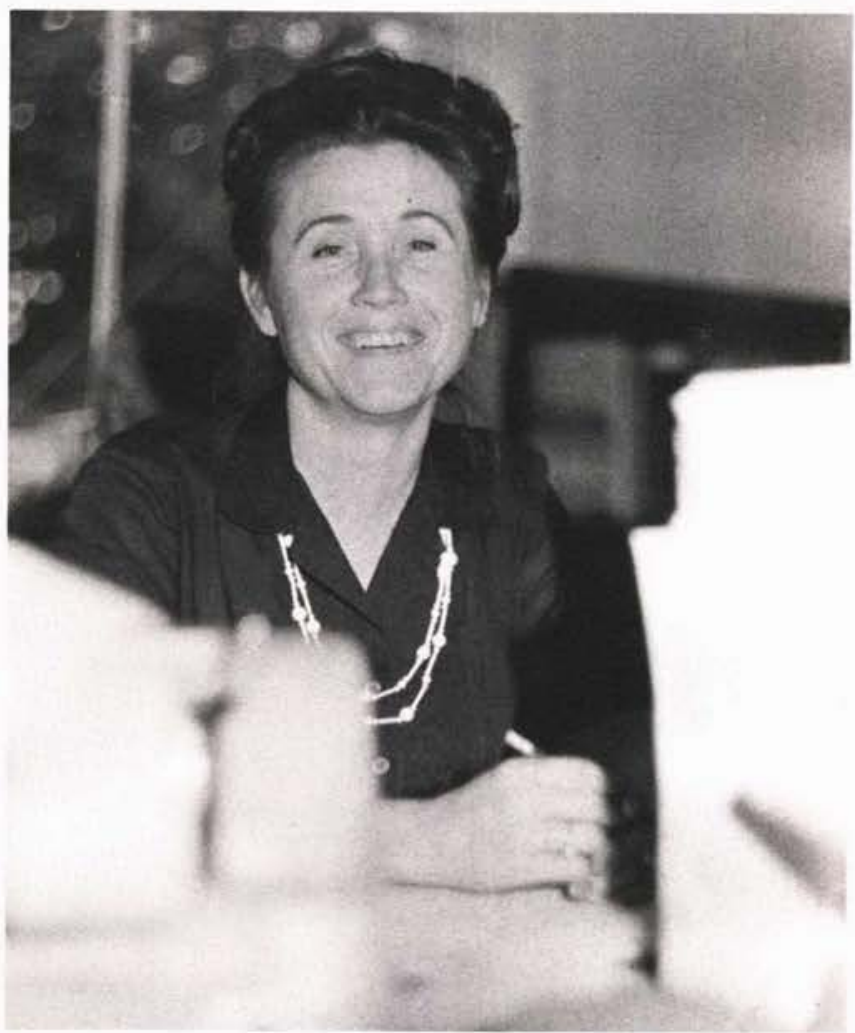

MRS. PATRICIA THORNTON

Secretary to Director of Physical Plant

MRS. BARBARA WILCOXON

Publications 

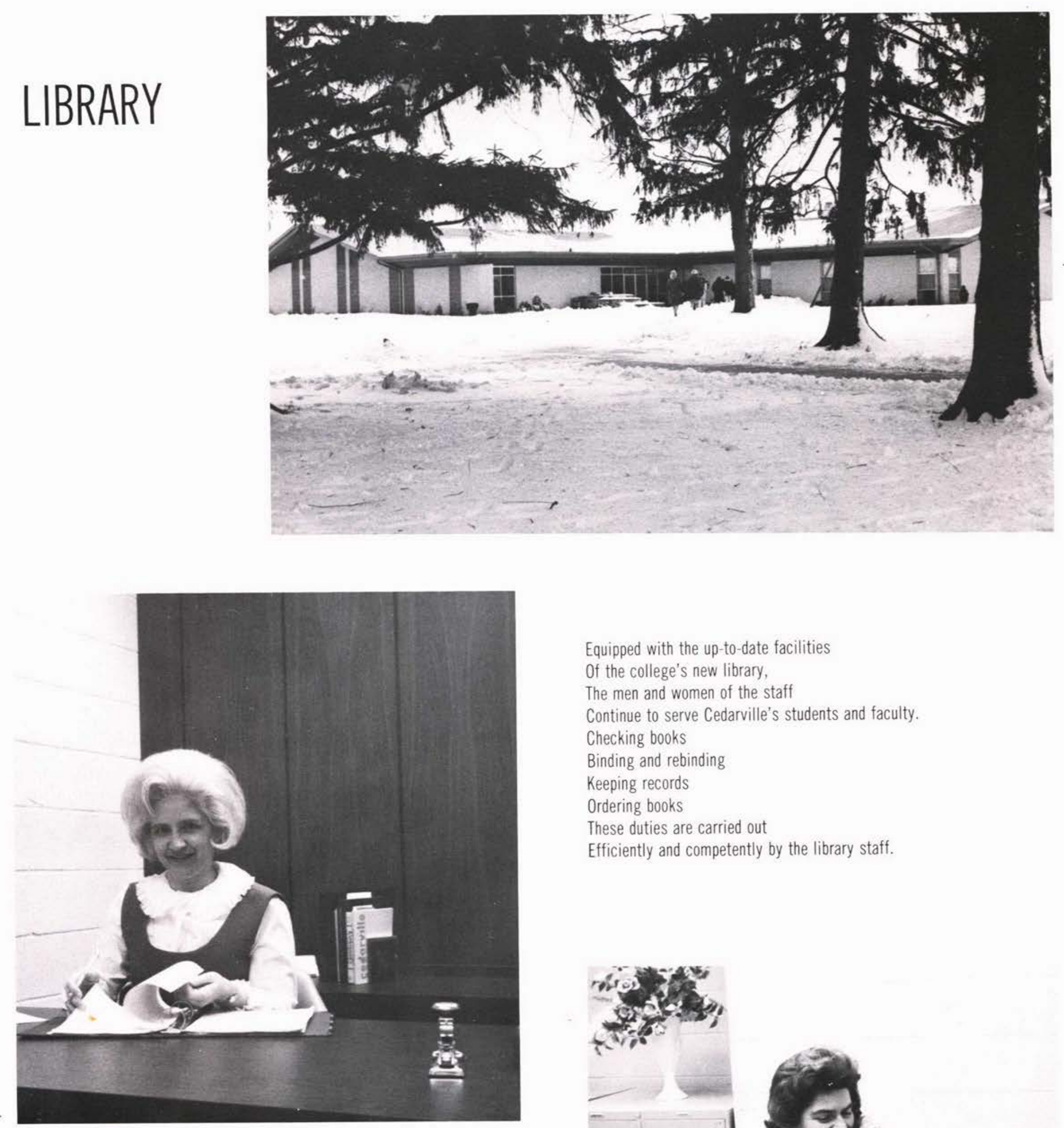

Equipped with the up-to-date facilities

of the college's new library,

The men and women of the staff

Continue to serve Cedarville's students and faculty.

Checking books

Binding and rebinding

Keeping records

Ordering books

These duties are carried out

Efficiently and competently by the library staff.

MRS. CHAFFE, Head Librarian

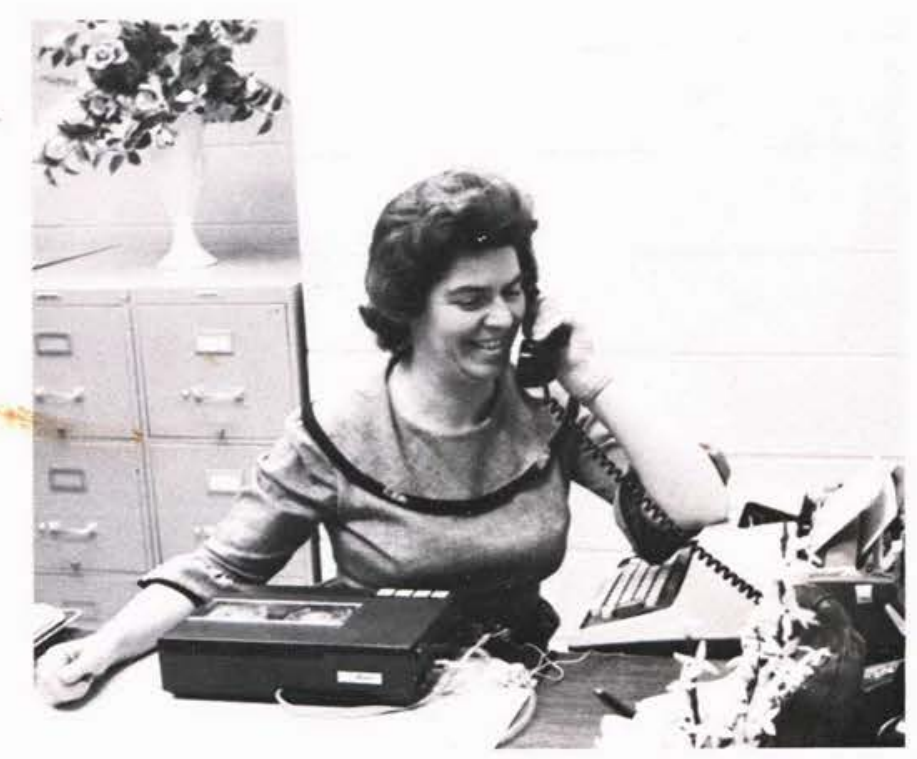

MRS. OSTRANDER, Secretary to Mrs. Chaffe 


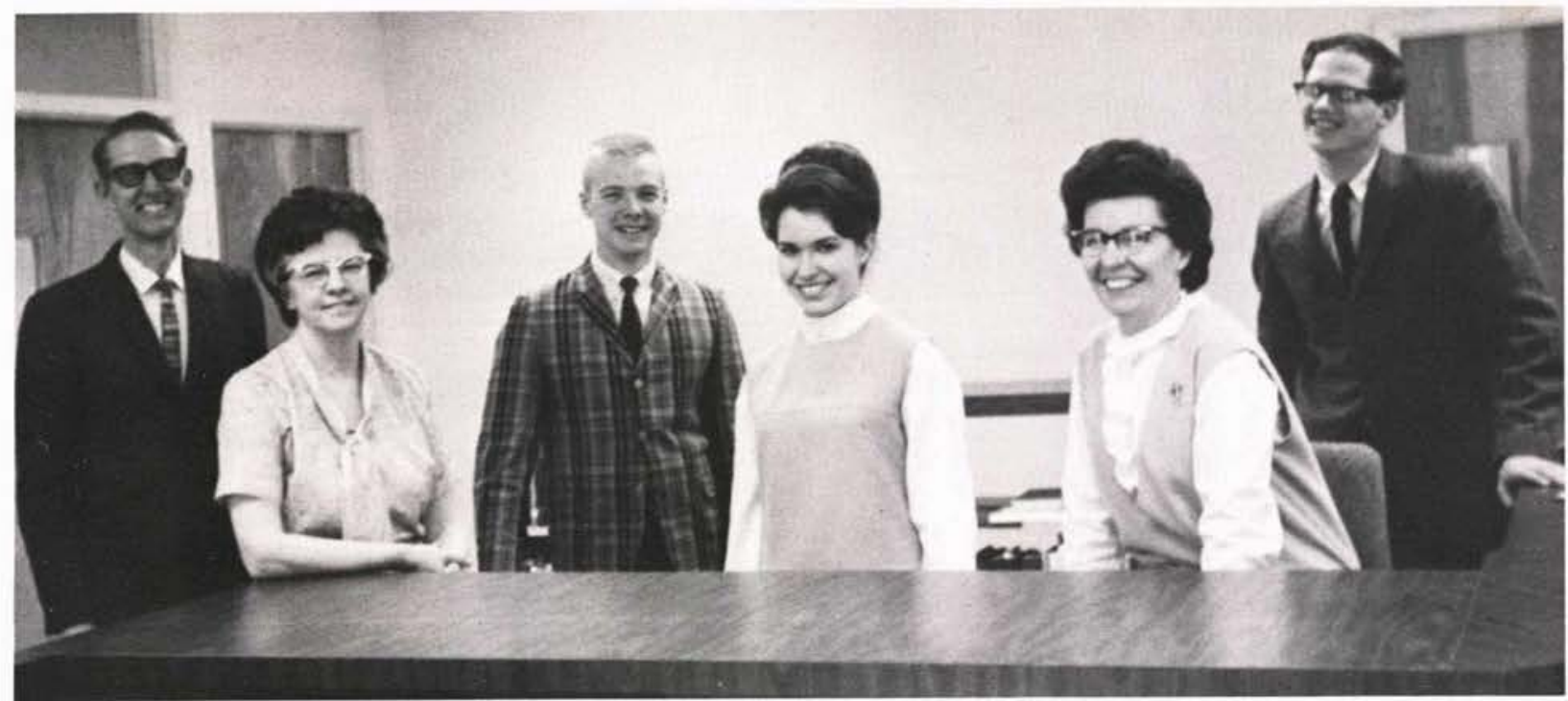

Mr. Eiffert, Mrs. Russell, Lynn Brock, Mrs. Cypher, Mrs. Carter, Stan Brown

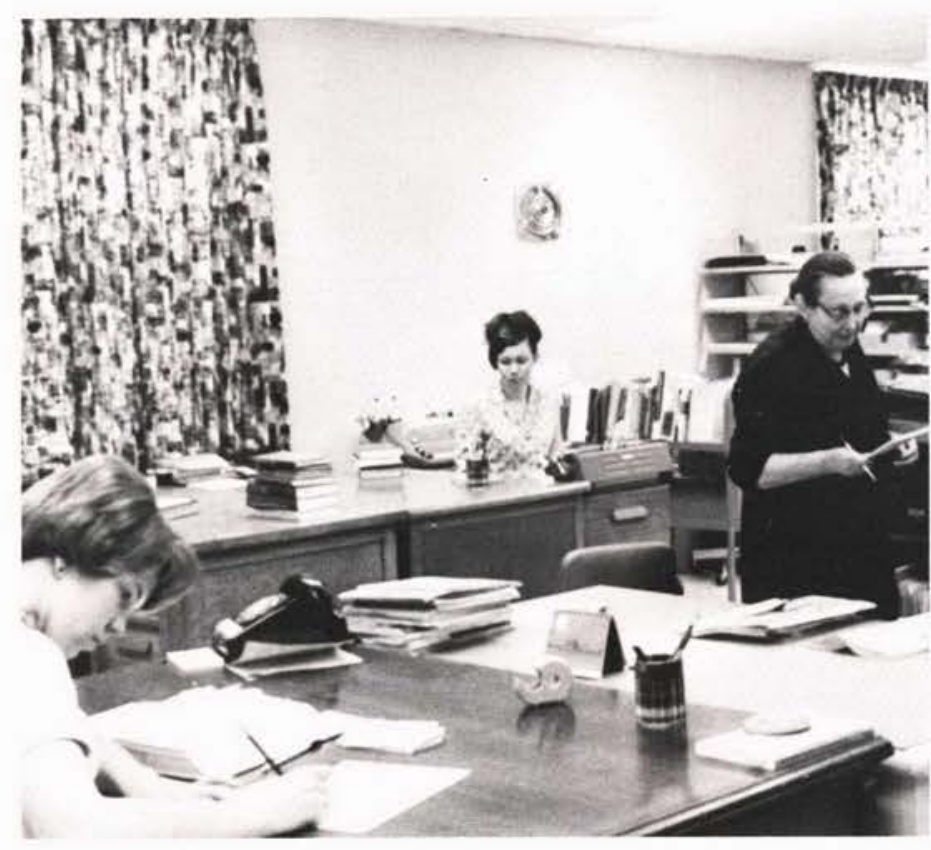

Nadine Queen, Sheryl Zerbe, Mrs. Ruder 


\section{BOOK STORE}

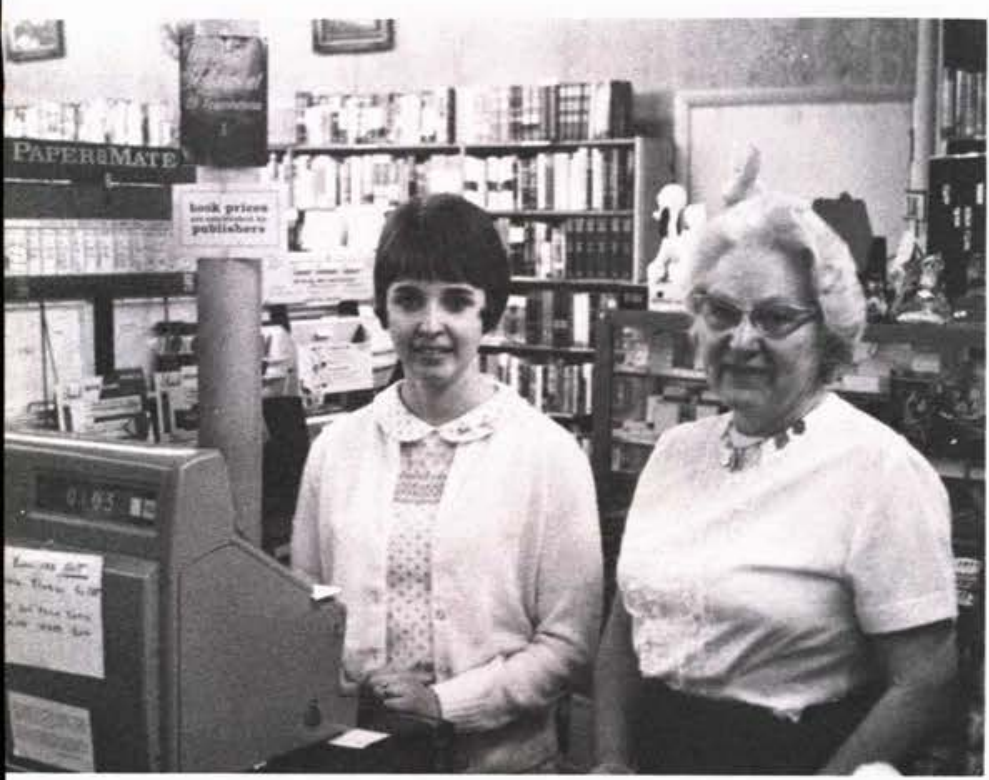

MRS. CHERYL PERRINE MRS. MARY ARMSTRONG

\section{POST OFFICE}

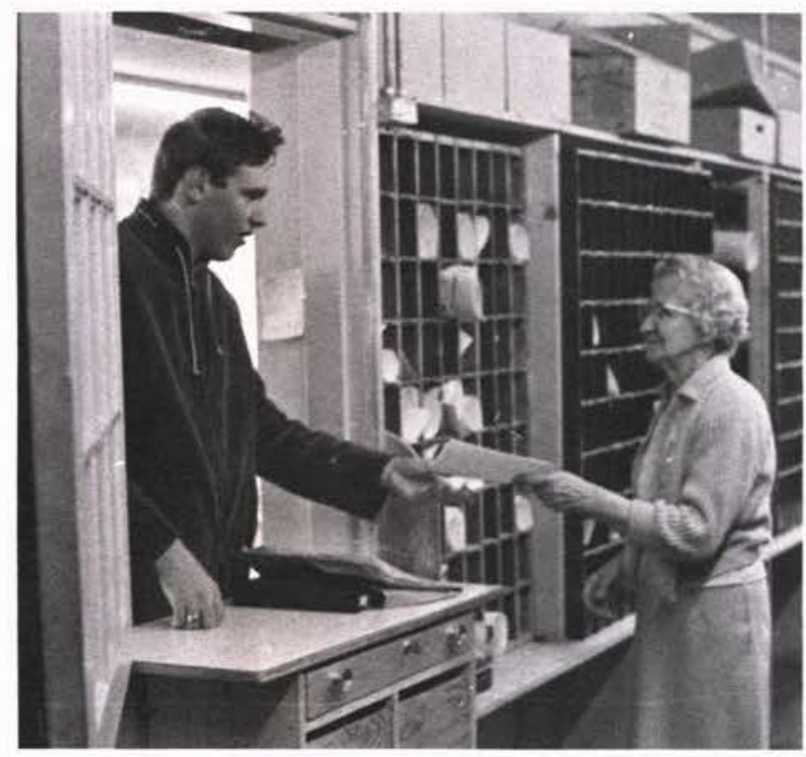

MRS. RUTH UNDERWOOD

MISS KAREN FLEMING

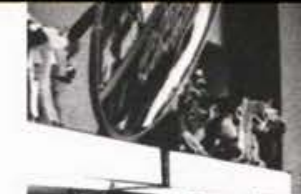

axuI
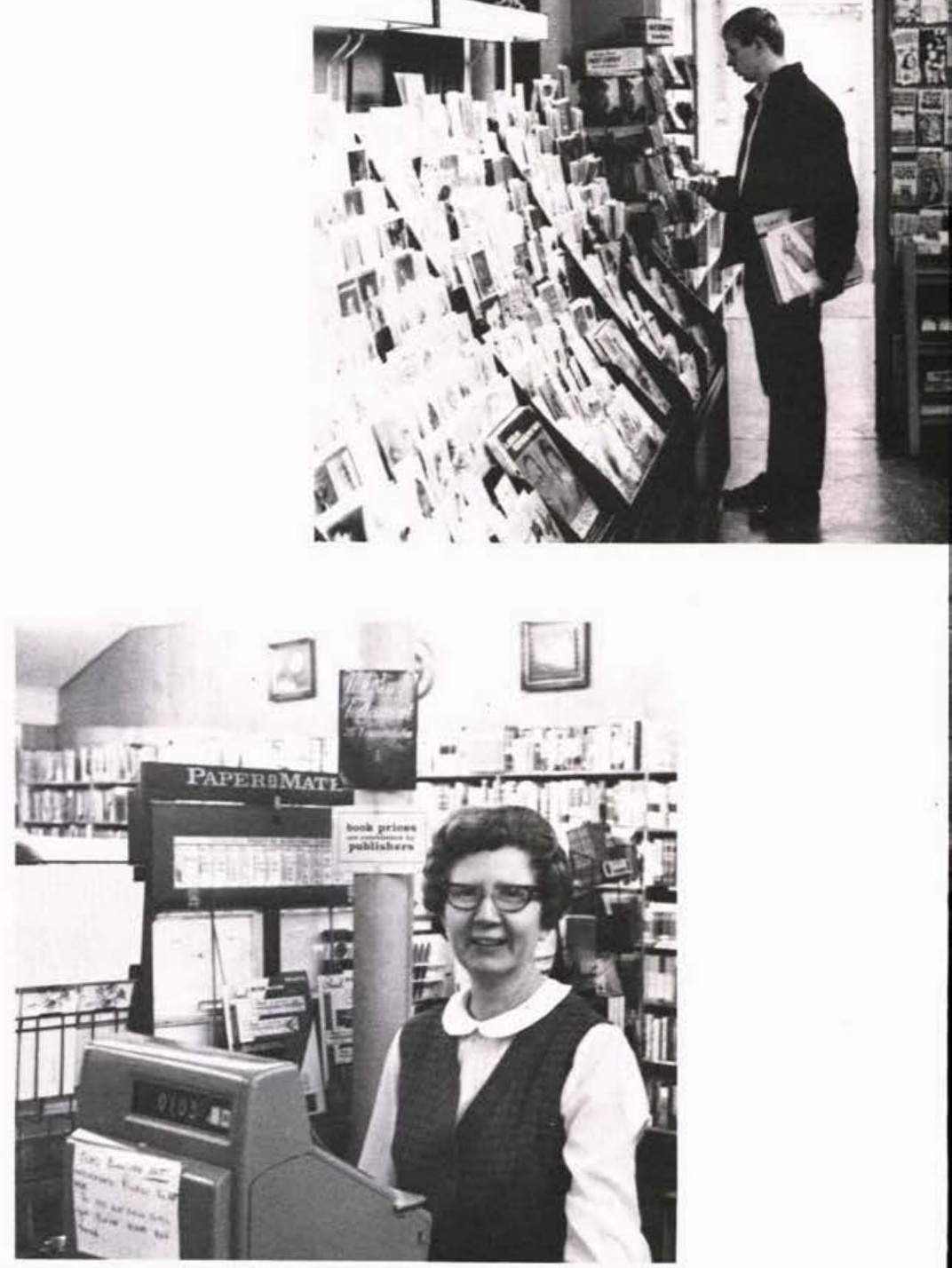

MISS BERNICE MICK, Manager

\section{PRINT SHOP}

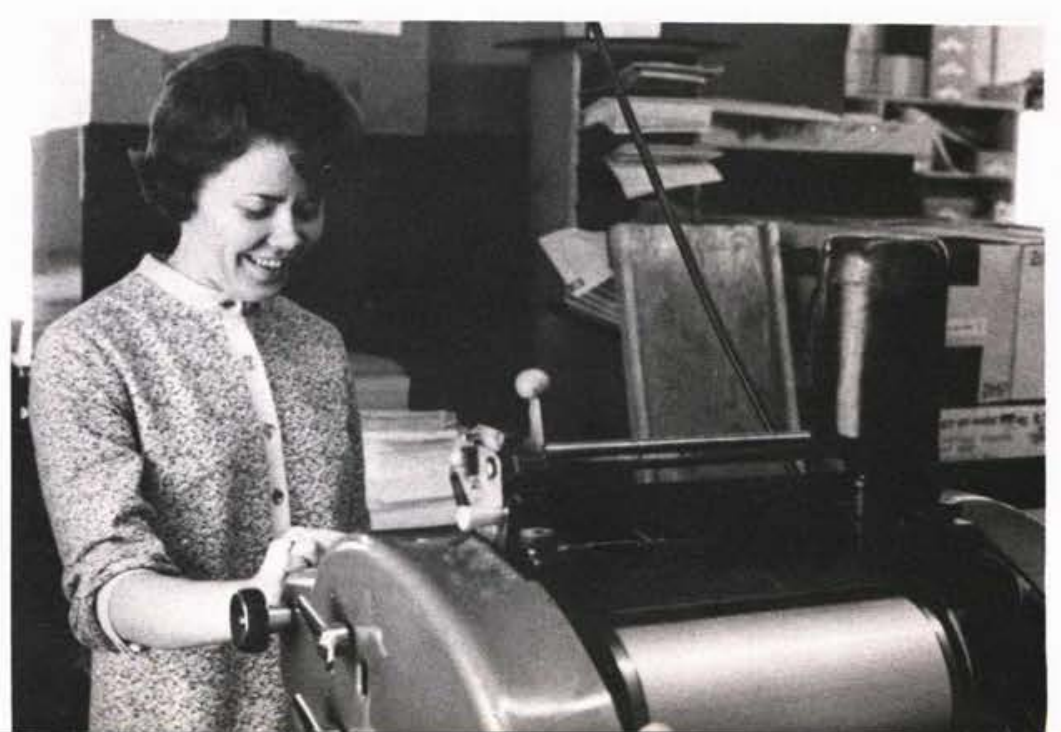




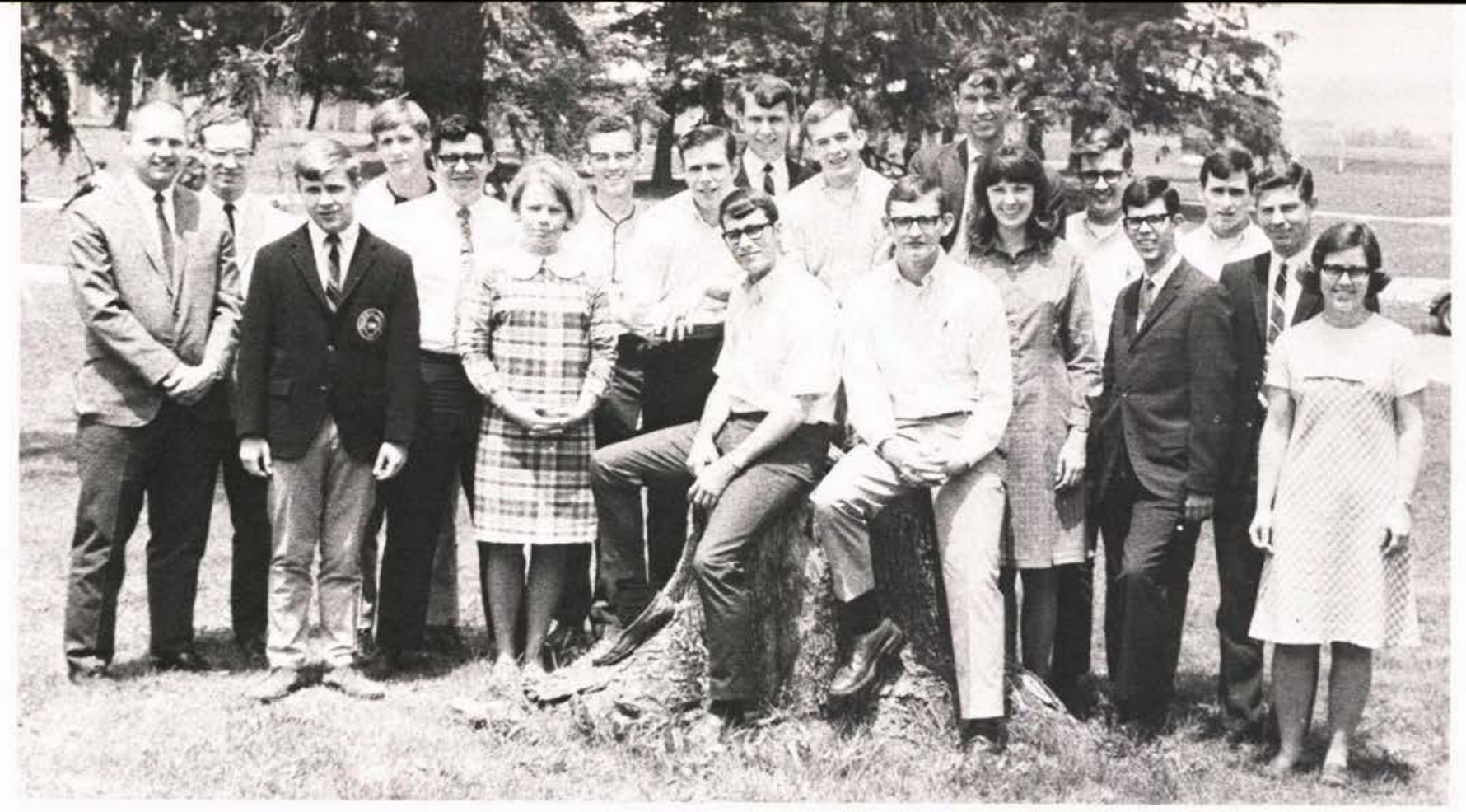

First Row: Mr. P. Gathany, J. Phipps, R. Hamilton, P. Willis, D. Draxler, L. Reno, K. Cole, M. Palmer, D. Fitch, B. Bixel, L. Eichelberger, J. Knapp. Second Row: T. Clater, R. Wita, A. Shuter, R. Patten, M. Crampton, G. Taylor, L. Reno.

\section{RADIO STAFF}

The "sound of the world's beautiful music"

Transmitted near and far

Via the radio waves of WCDR -

Cedarville College's own station

Operated by student workmanship

In connection with its trained station manager,

WCDR offers excellent training,

Invaluable experience for the students involved.

Combining its easy listening theme with

The Gospel message

WCDR is a channel of witnessing for the college.

Into its operation go many man hours of

Diligence, patience

Craftsmanship

Providing the college and community with

Up-to-date news,

Spiritual food, and

The world's beautiful music.

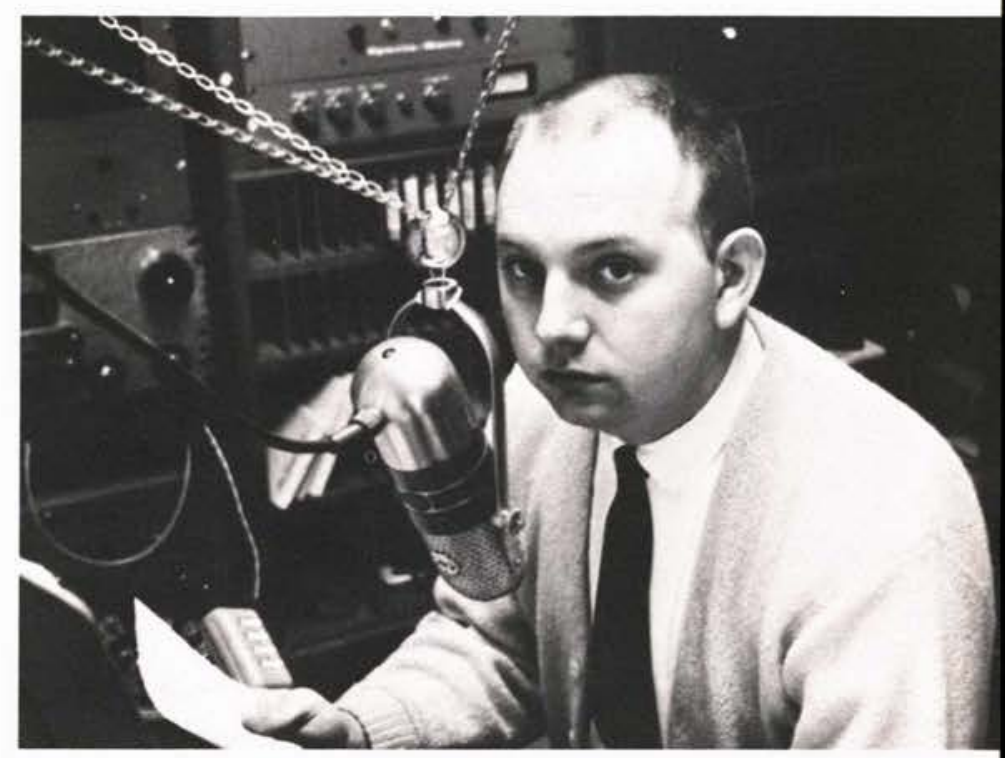

MR. PAUL GATHANY, Radio Instructor.
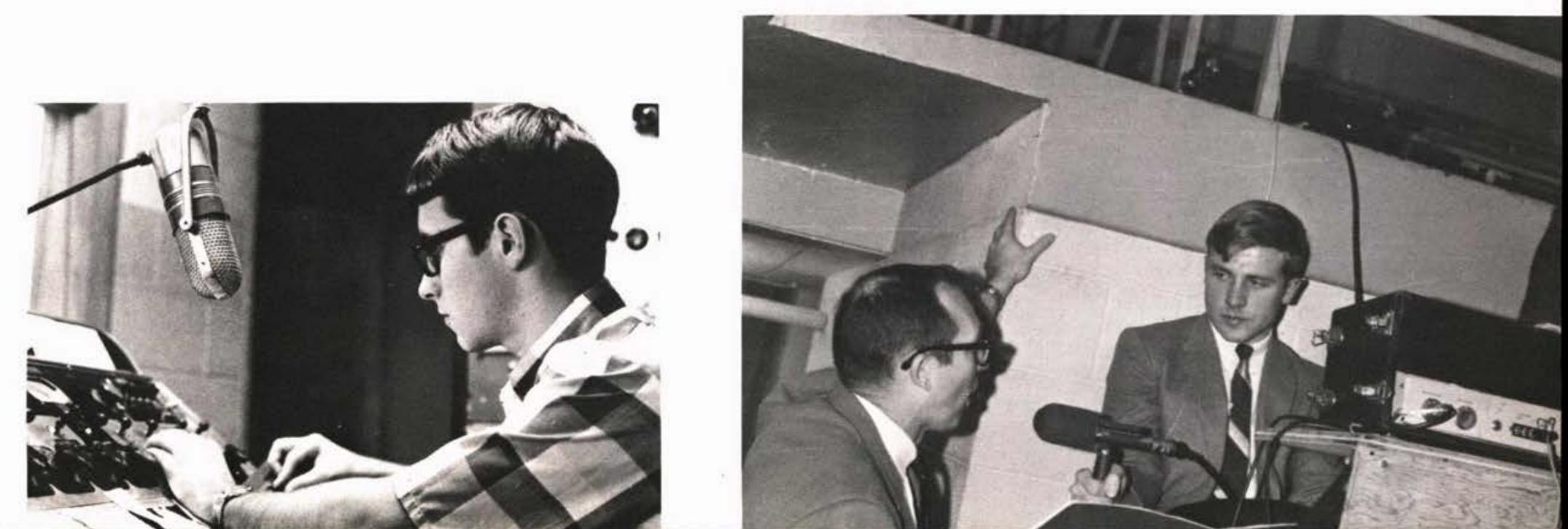


\section{CAFETERIA}

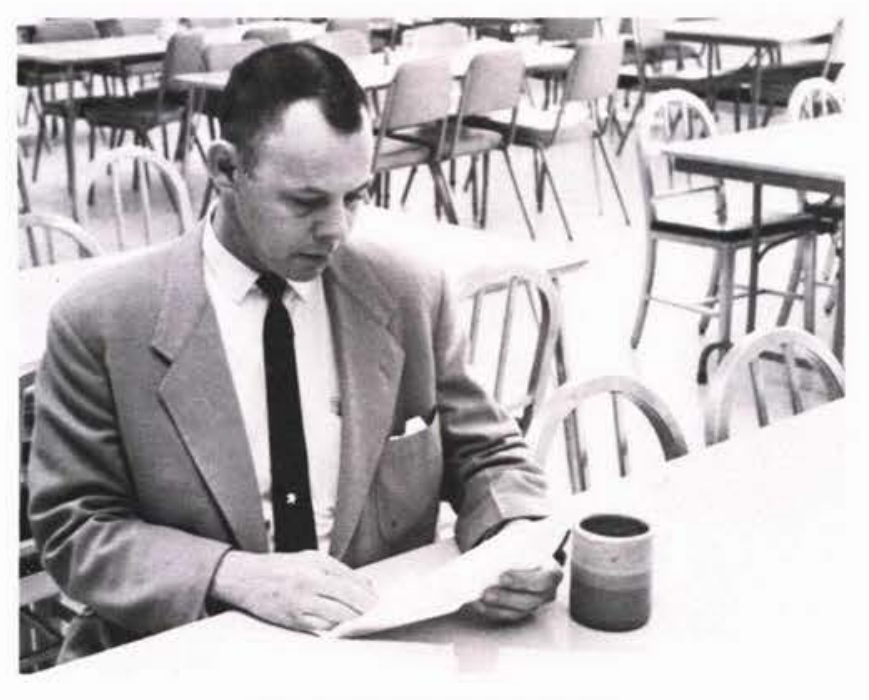

MR. THOMAS SMITH

Food Service Manager

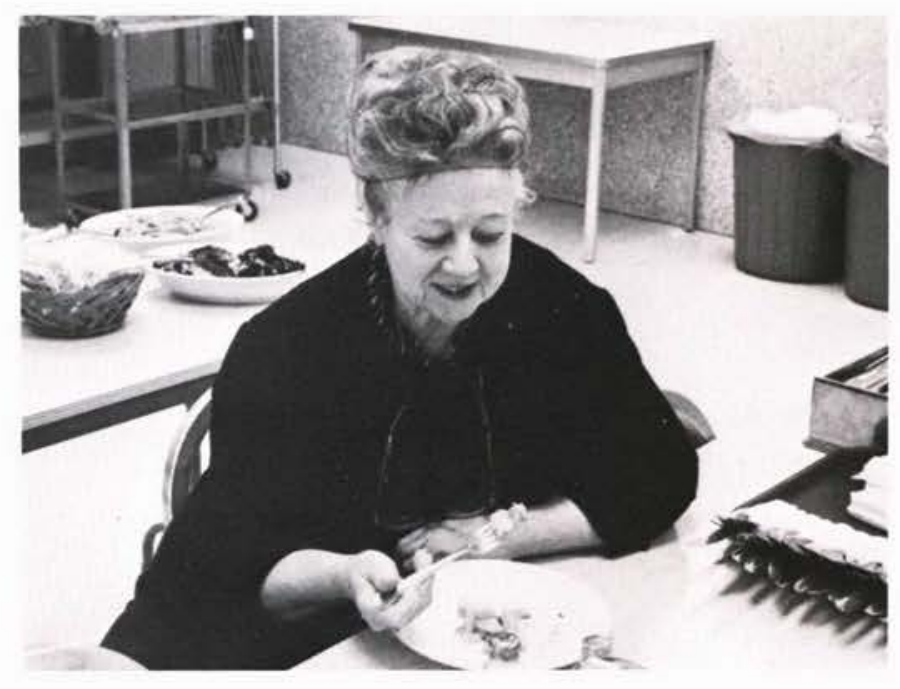

MRS. STELLA SMITH Food Consultant

DORM

PARENTS

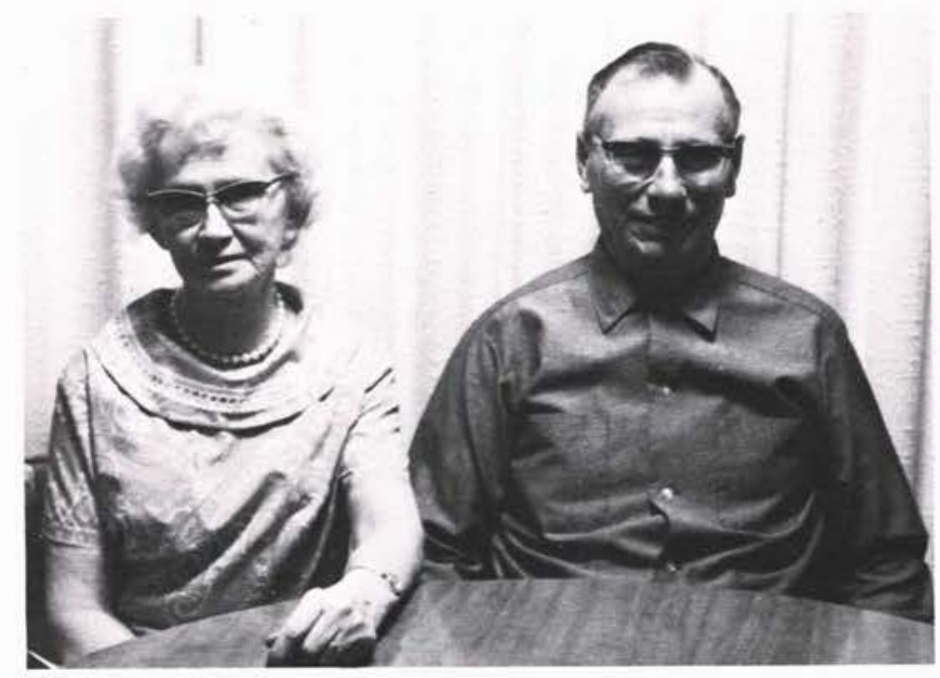

MR. \& MRS. HENRY BRESSON

Faith Hall

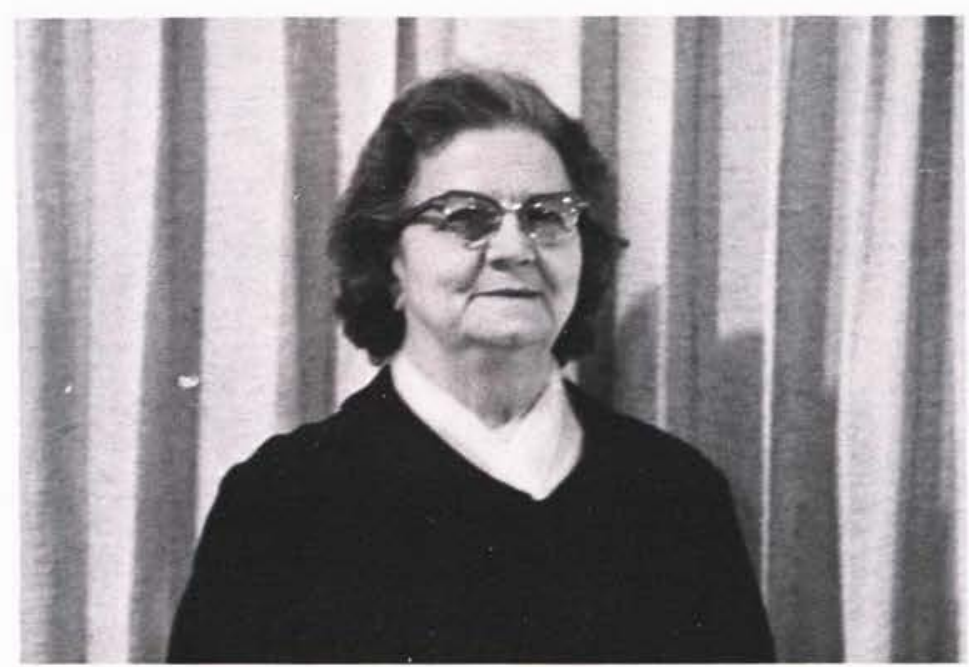

MRS. FRANCIS HOWELL Maddox Hall

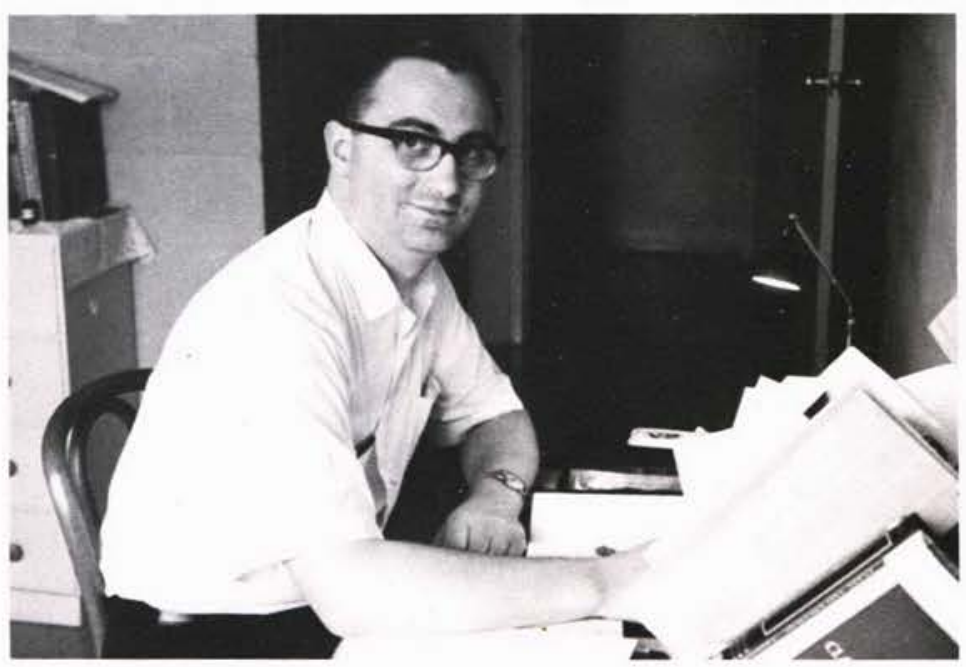

MR. "SKIP" HUGHES Williams Hall 


\section{MAINTENANCE DEPARTMENT}

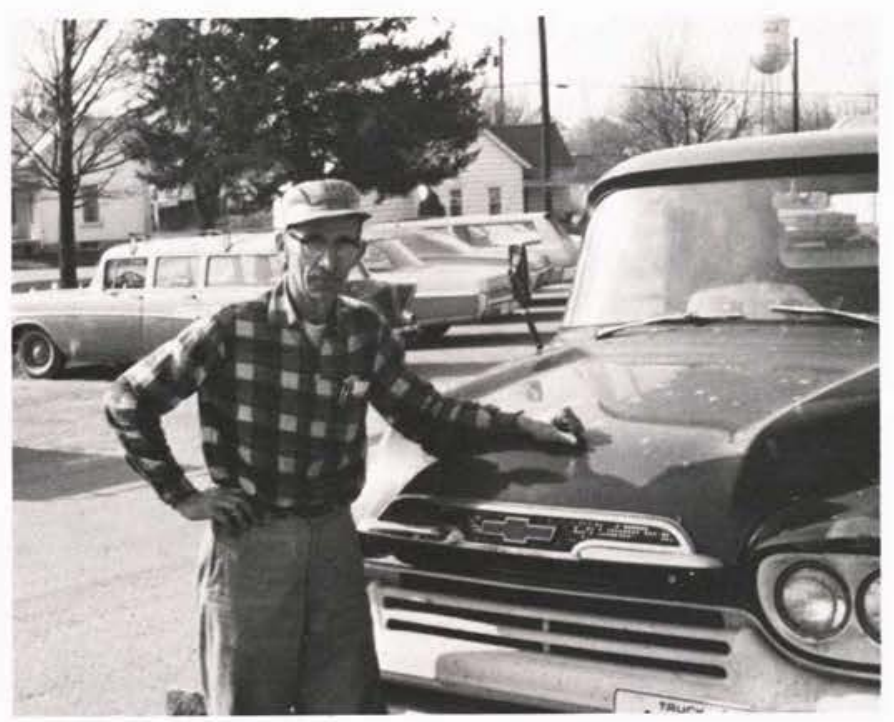

Mr. "Pop" Marshall

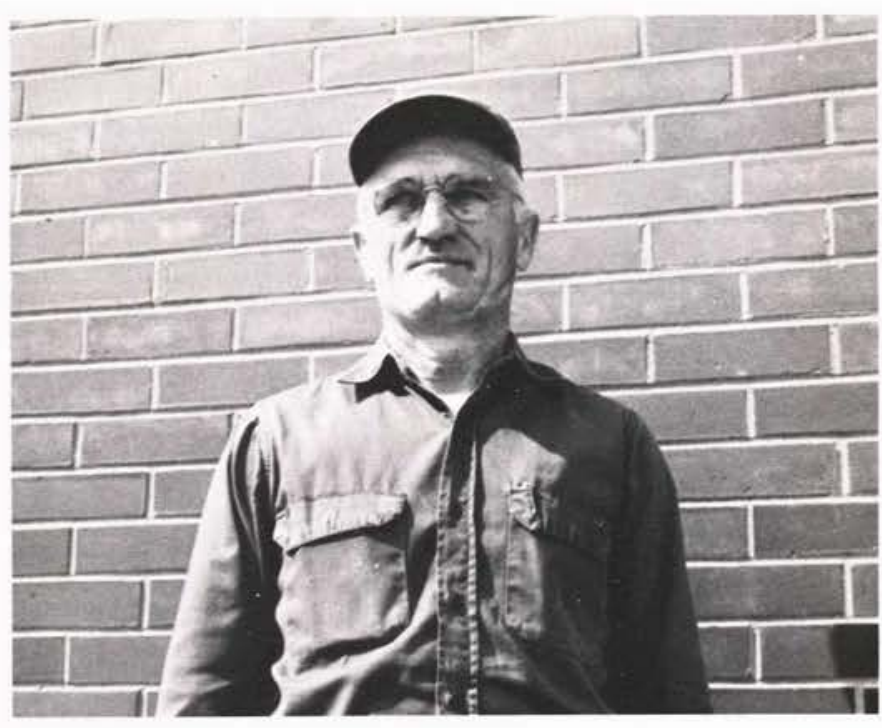

Mr. Robert Dillon

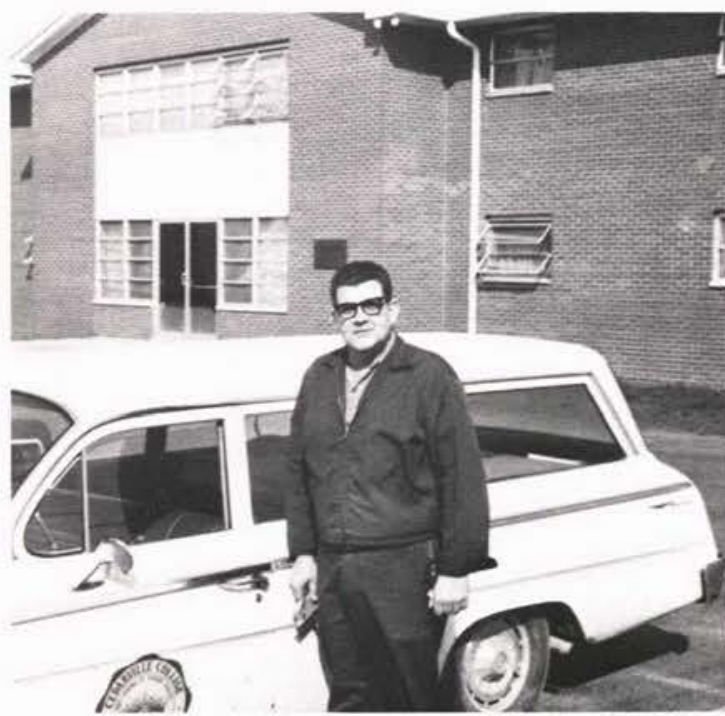

Mr. "Charlie" Tarter
Mr. John Ring

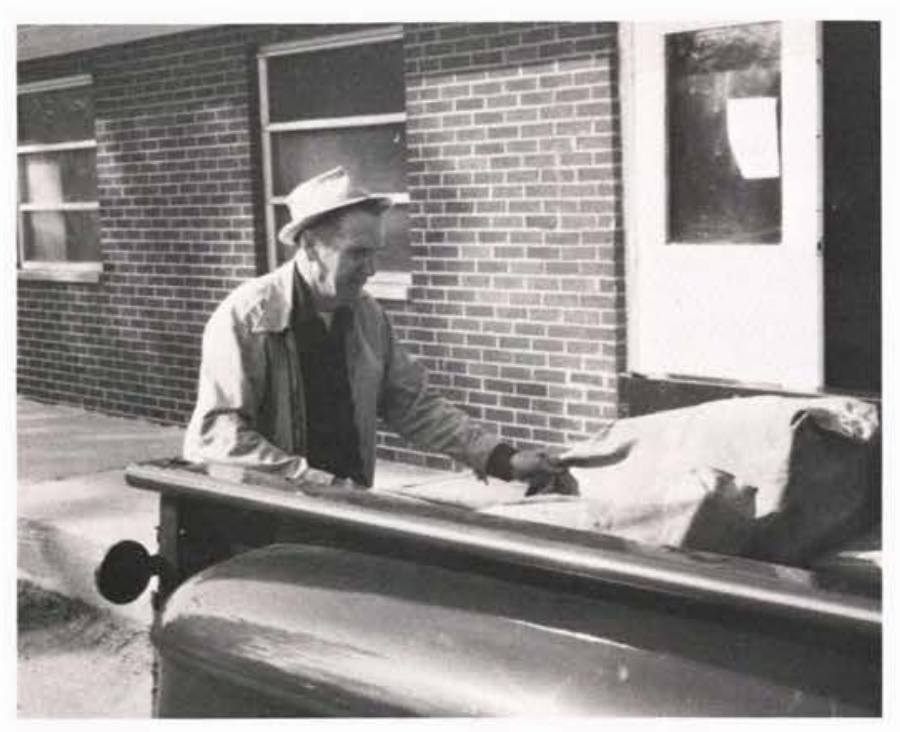

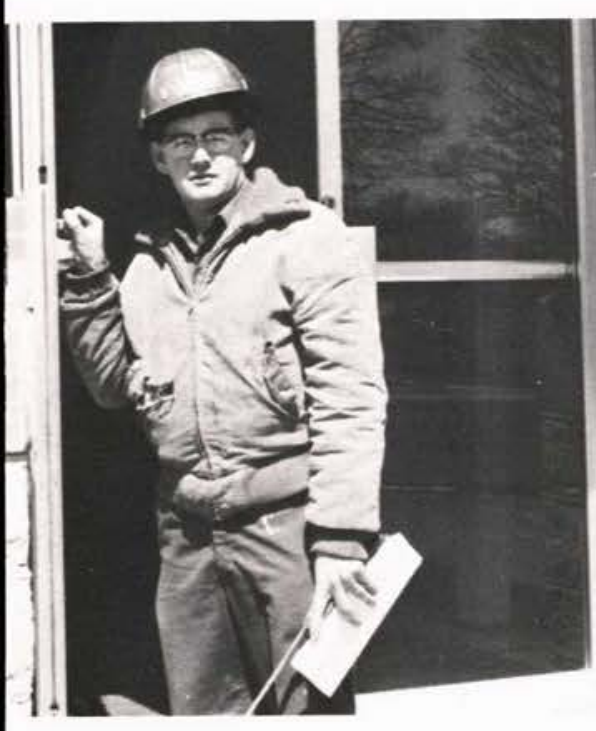

Mr. Tom Foulkrod
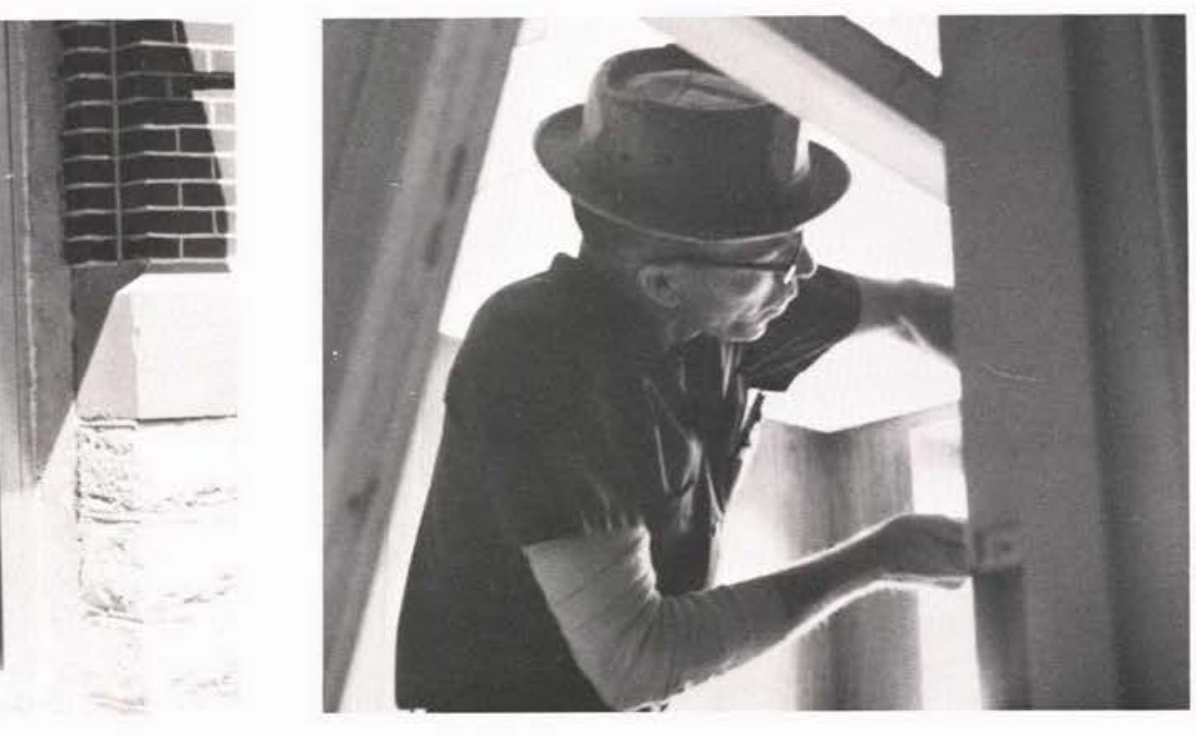

Mr. William Carter 


\section{( T)}

a.

(6) $(7)$

(6)

(2)

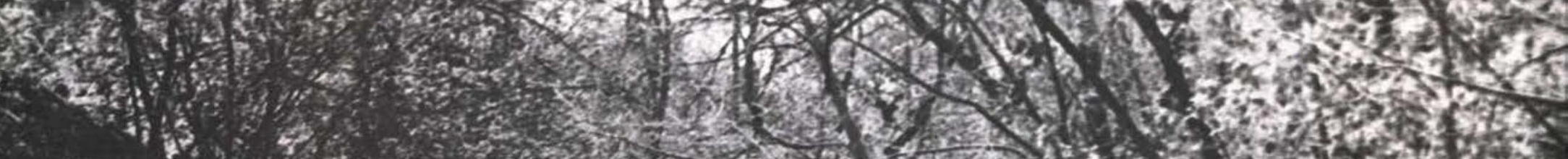

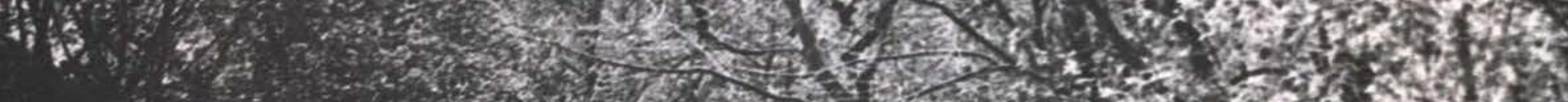

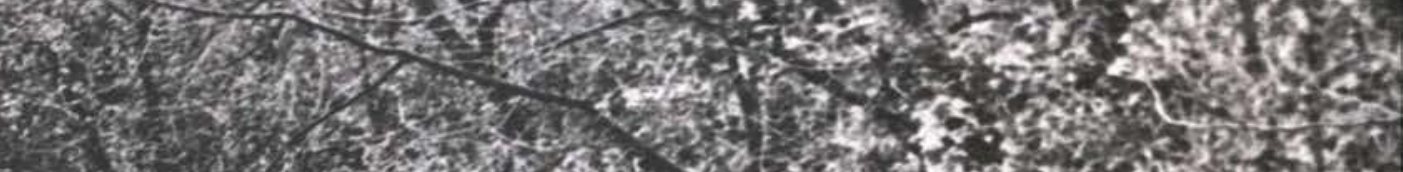

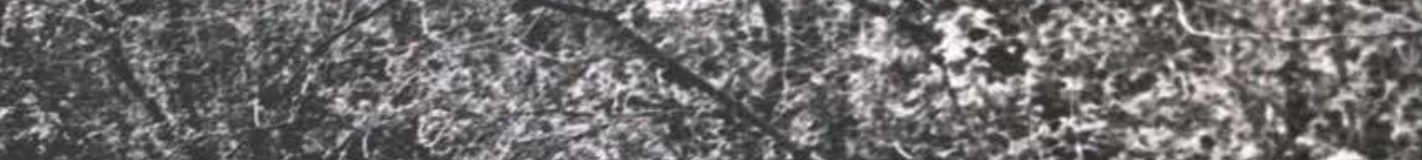

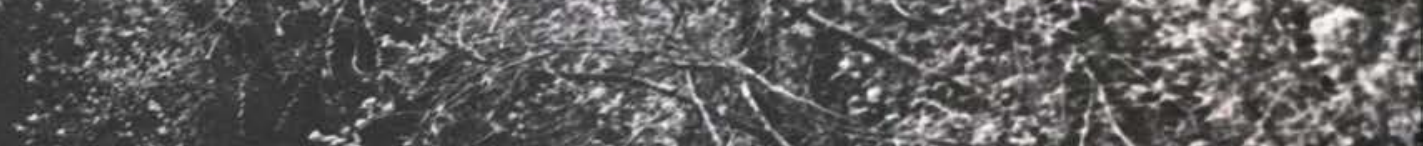

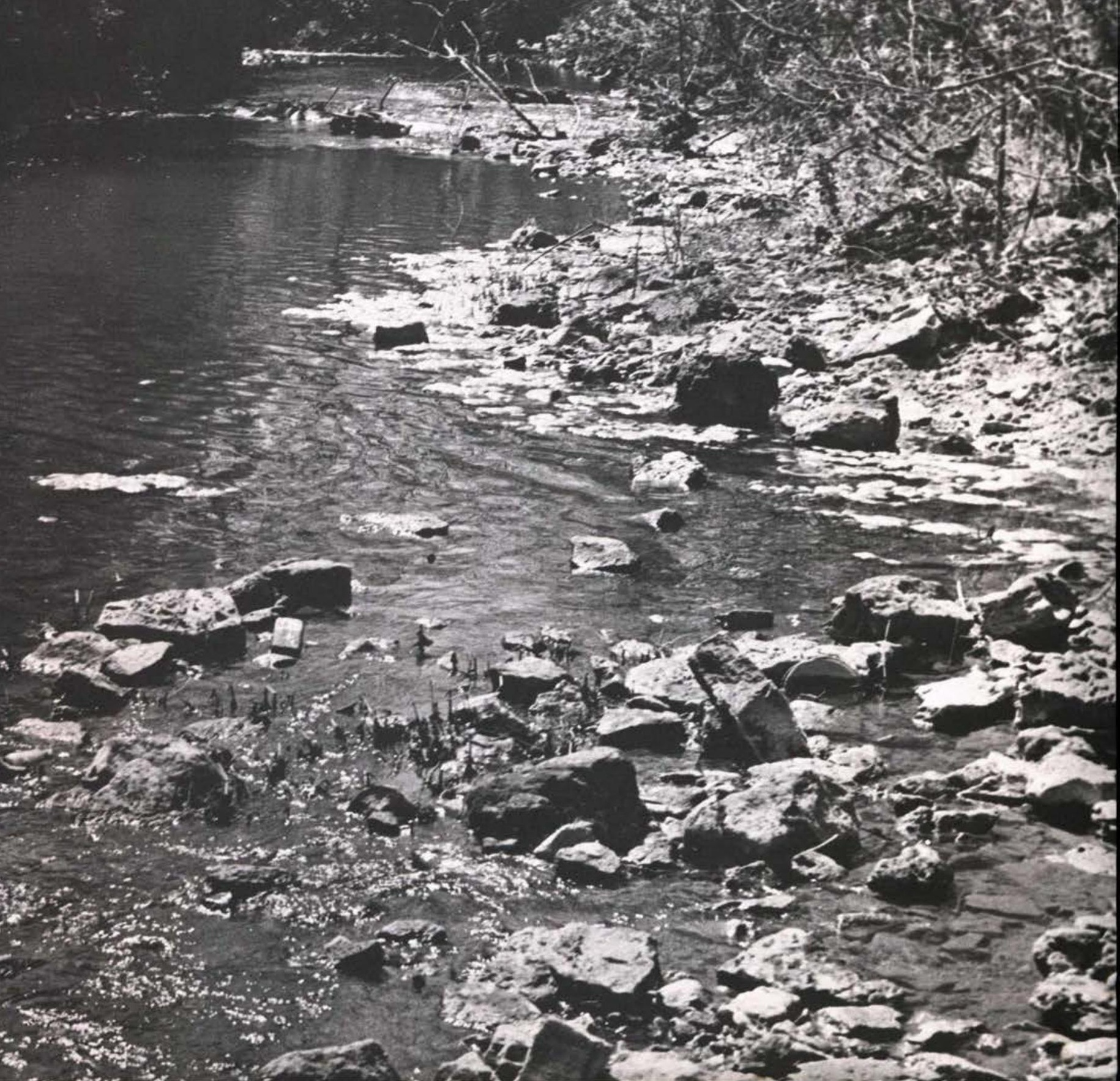




\section{Students}

Friends are like the sturdy oaks that rustle in the breeze when the summer suns are gone... Like the boughs of spicy evergreens pressed against our lives to shelter from the wintry blast ... Friends are like low blooming flowers that break at spring to light our path ... Like the perfumed roses dropping petals of happiness around our door... Friends are like green mosses clinging close to running brooks ... Like the flowing streams spreading their moisture along the fields and asking no reward or pay ... Friends are like the shady nooks giving sweet release at evening's hush .... Like the broad expanse of softest green and copper bronze to delight the eye ... Friends are like the gentle whisperings of a love divine ... Forgiving and forgetting without a tinge of blame.

Bertha Keiningham 


\section{SENIOR OFFICERS}

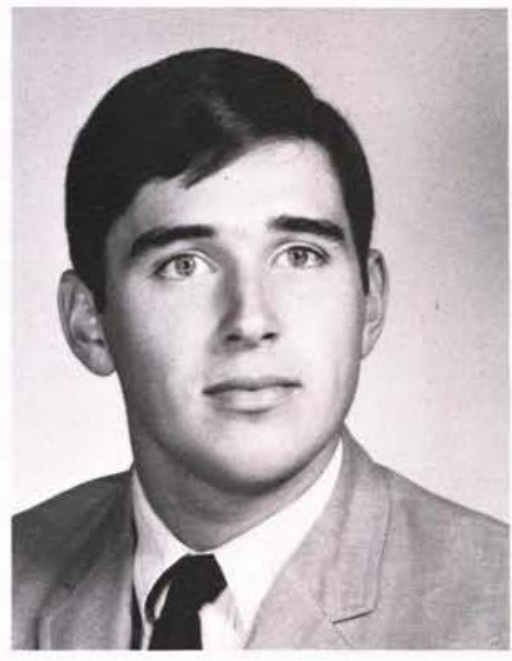

Michael Woodend PRESIDENT Alexandria, Va.

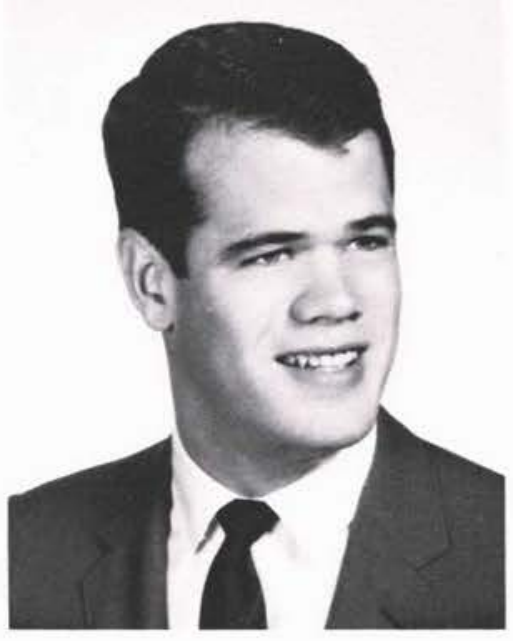

Adrian Sweeney VICE-PRESIDENT Cherry Hill, N.J.

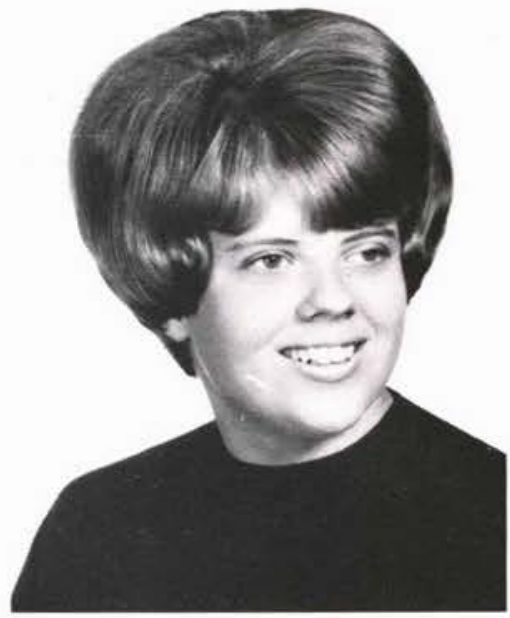

Maxine Stanbery SECRETARY Peoria, III.

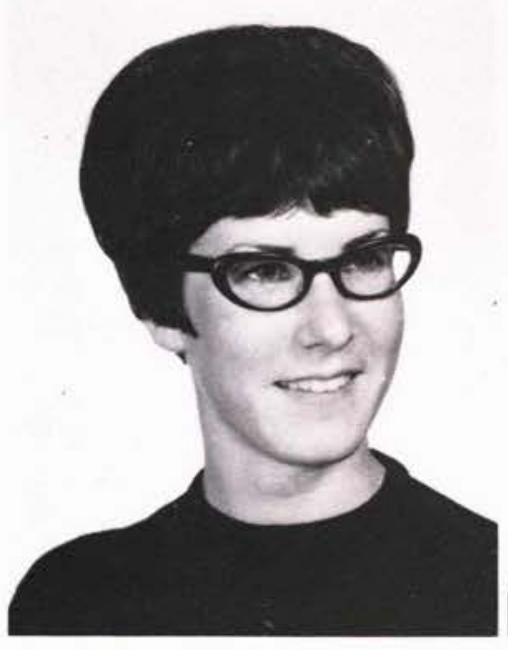

Suzanne 0'Shel TREASURER Olmsted Falls, 0 .

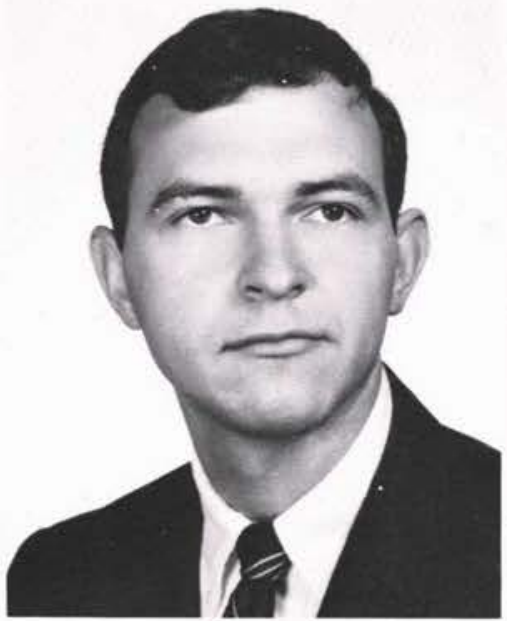

David Wall

STUDENT COUNCIL Decatur, III.

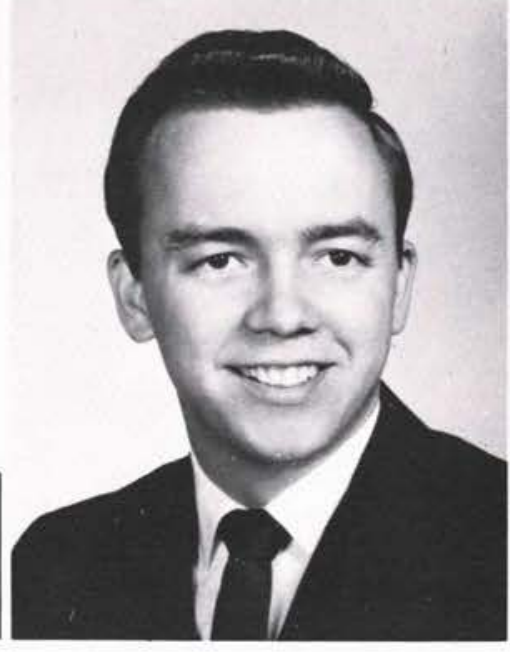

Ron Allerton

STUDENT COUNCIL Prospect, $\mathrm{Pa}$.

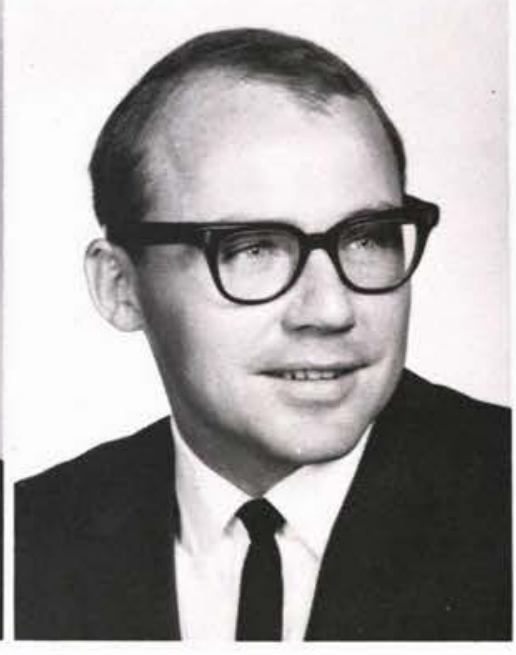

John Rueck CHAPLAIN Alberta, Canada

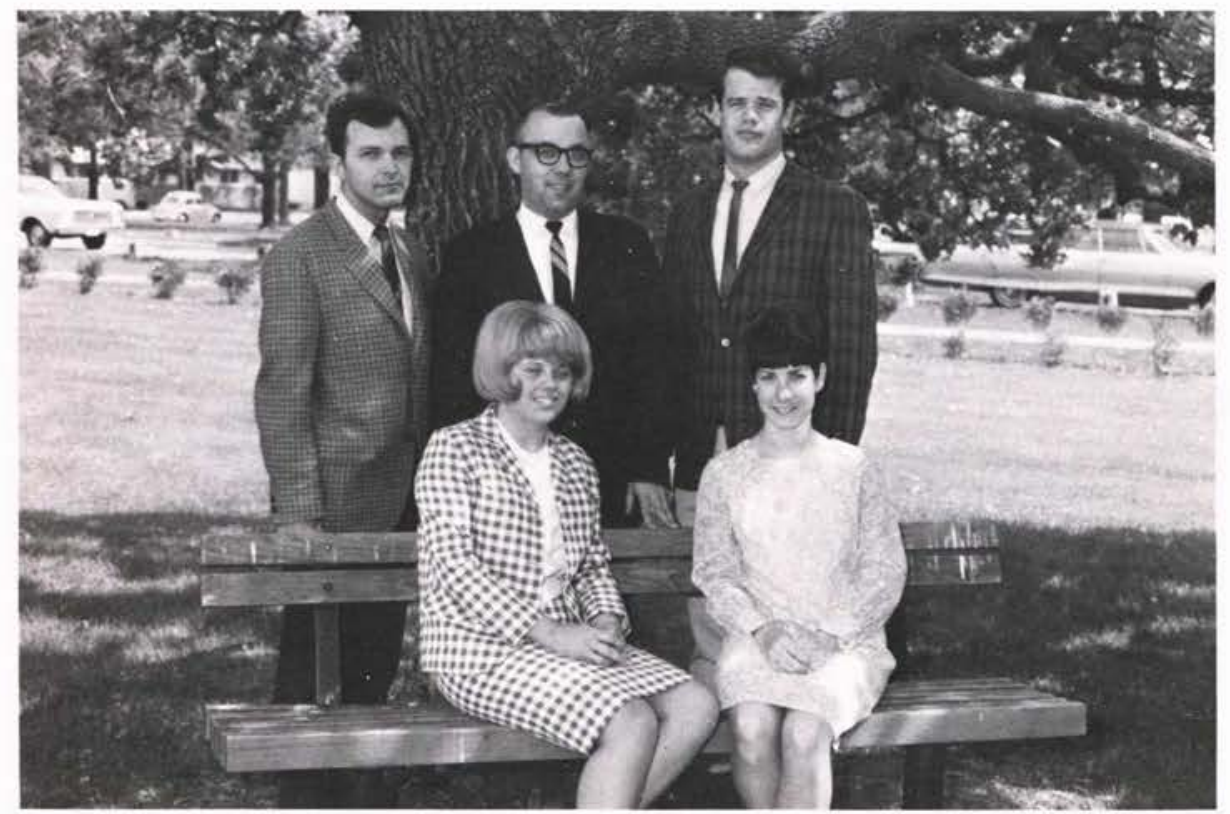


S

$E_{N}$

0

$R$

Ronald Baker

Schenectady, New York

Larry Bellew

Cincinnati, Ohio

Loris Bellintani

Sao Paulo, Brazil

Calvin Biddle

Beloit, Ohio
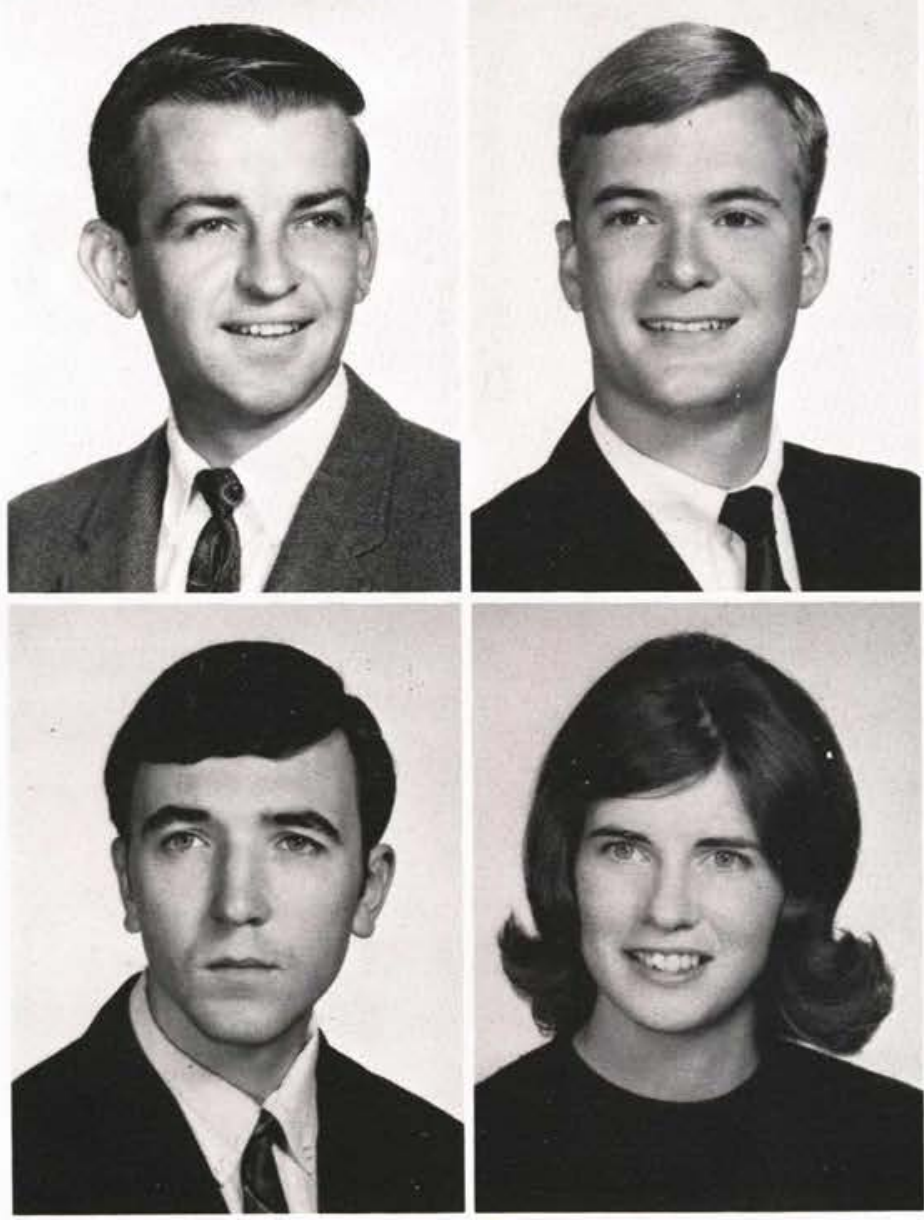

Deane Allen

Hurlock, Maryland

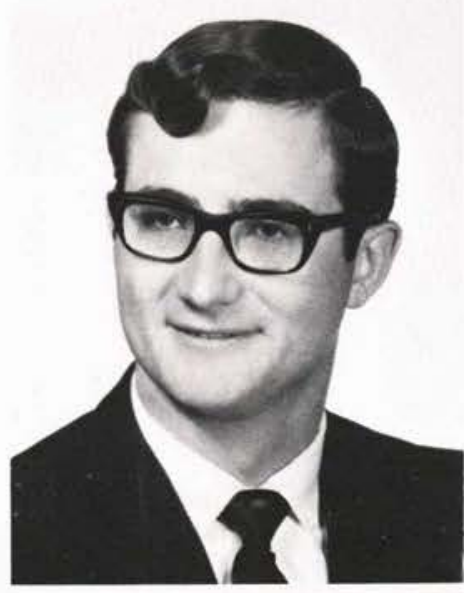

Rebecca Baker

Schenectady, New York

Tom Biller

Valparaiso, Indiana

Bonnie Bird

Broadview Heights, Ohio

Diane Blake

Lexington, Kentucky

James Blaylock

Fairborn, Ohio
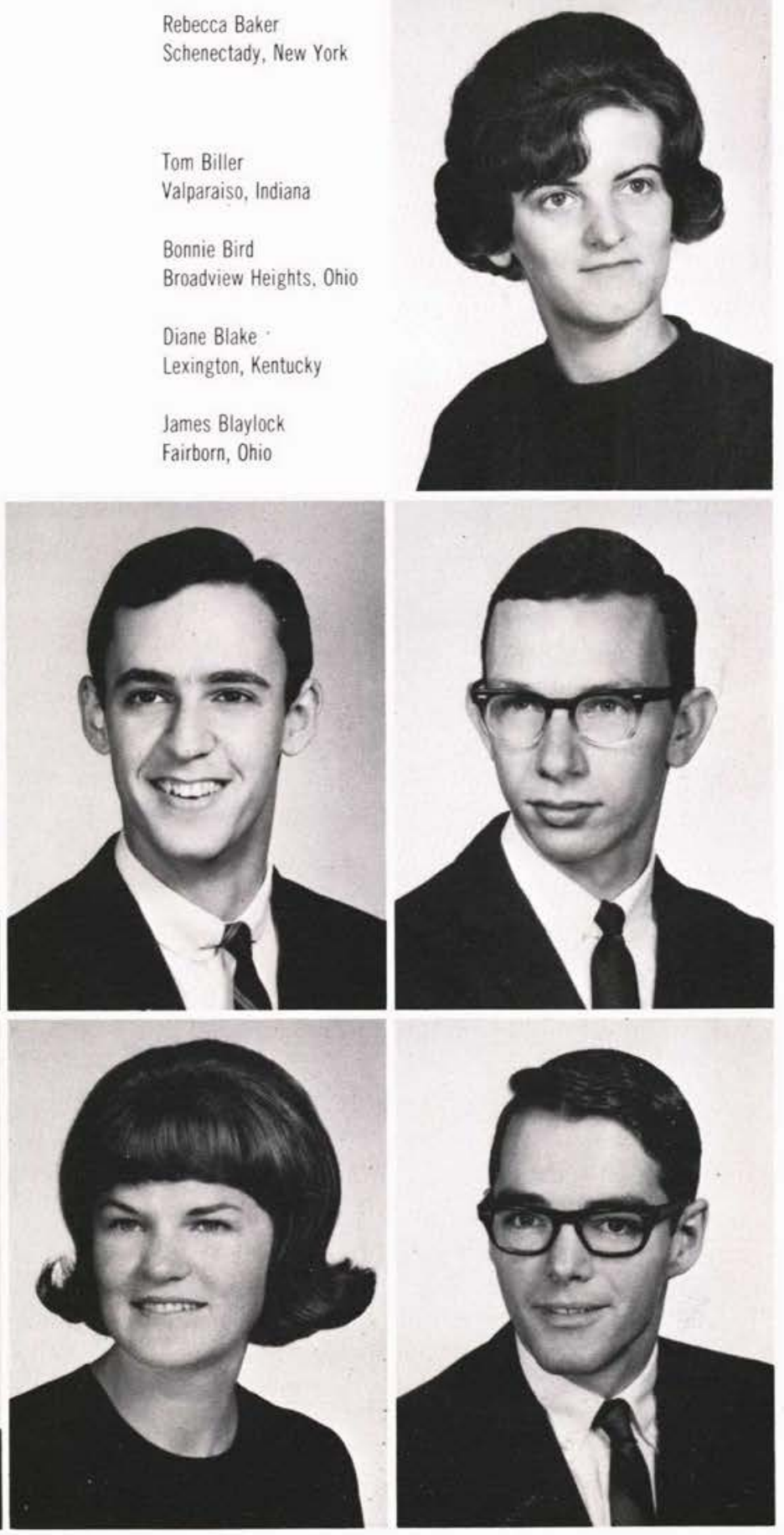


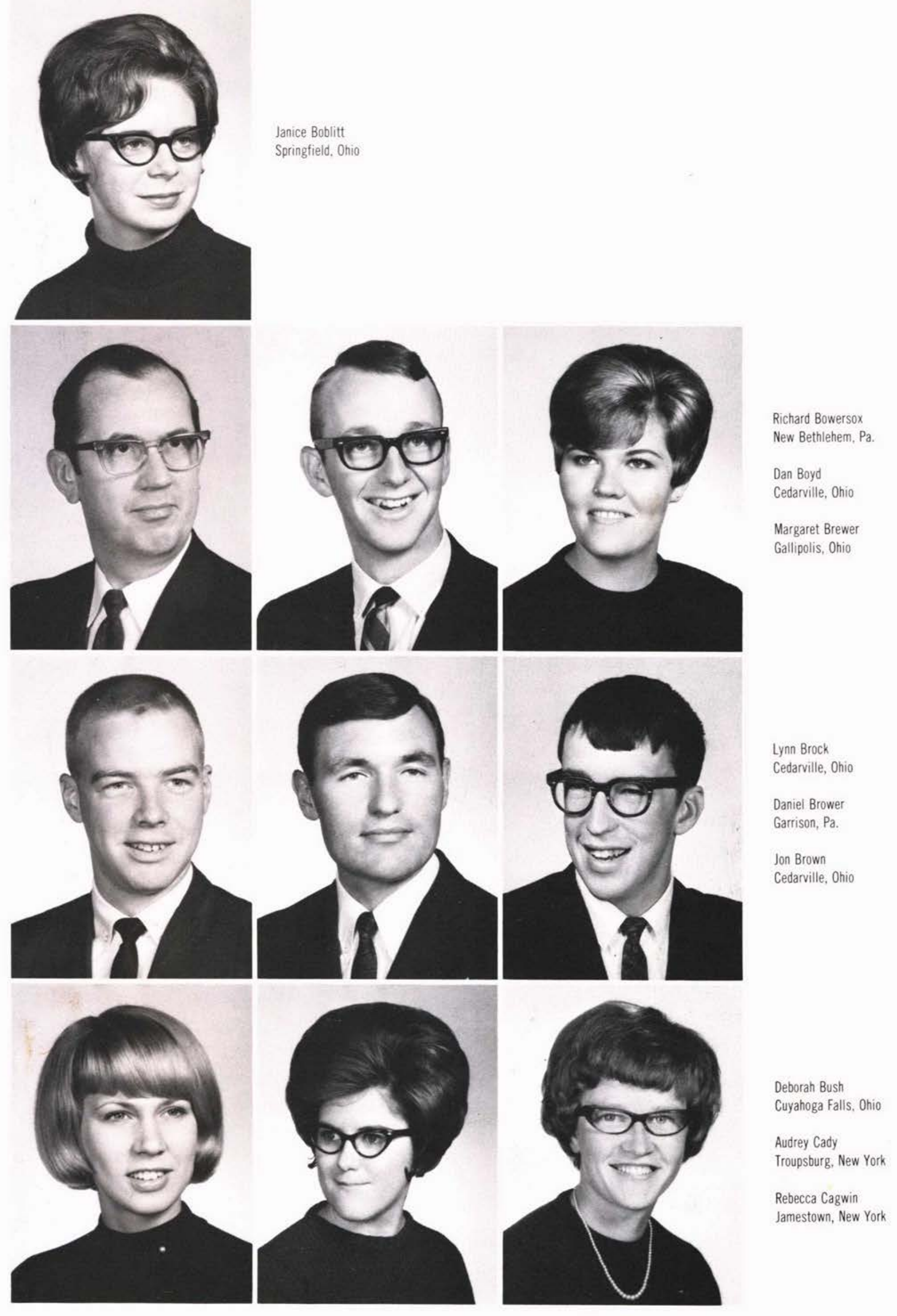


Beverly Carlson

Decatur, Georgia

Theodore Clater

Warsaw, Indiana

Donald Cooper

Belle Center, Ohio
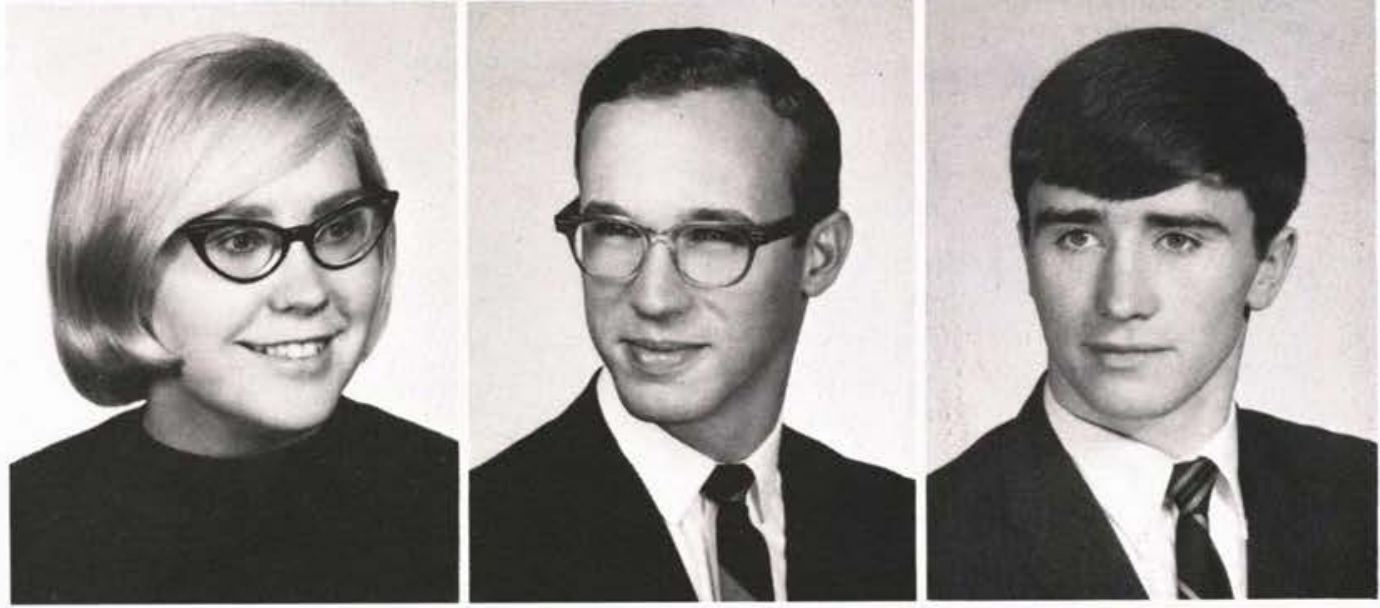

Gwytha Cooper

Ypsilanti, Michigan

Bob Cosgray

Jamestown, Ohio

Michael Crampton

Columbia City, Ind
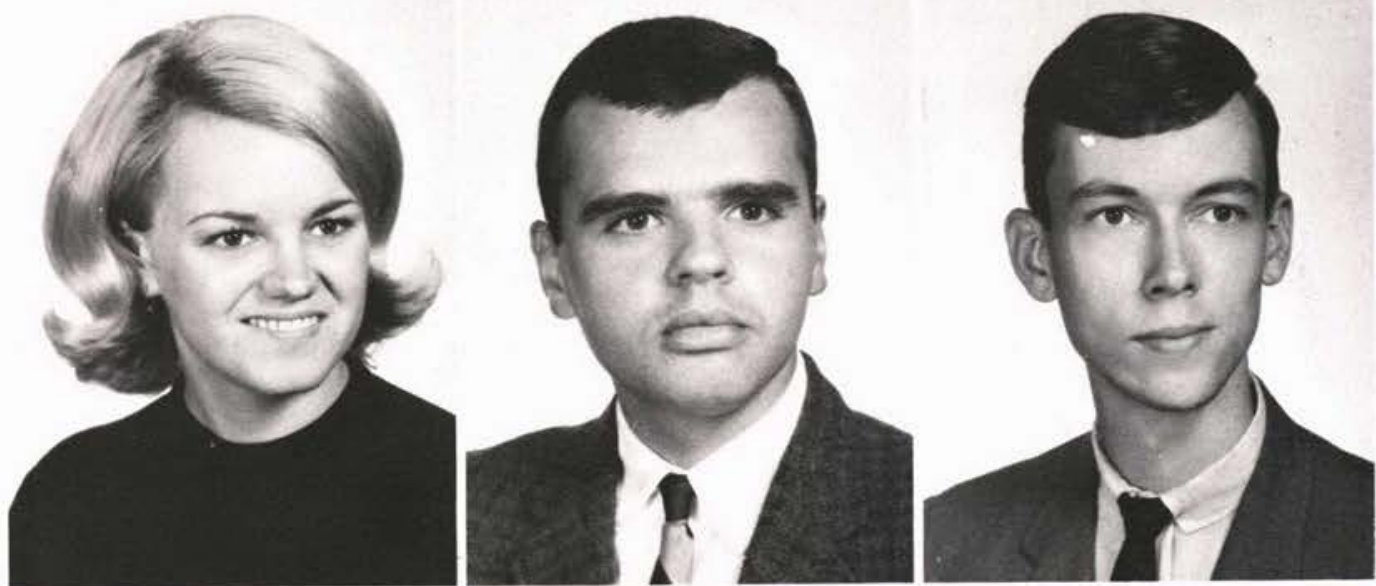

Mary Curtis

Rushville, New York

Russell Daubert

Michigan City, Ind.

James Davis

Homer City, Pa.
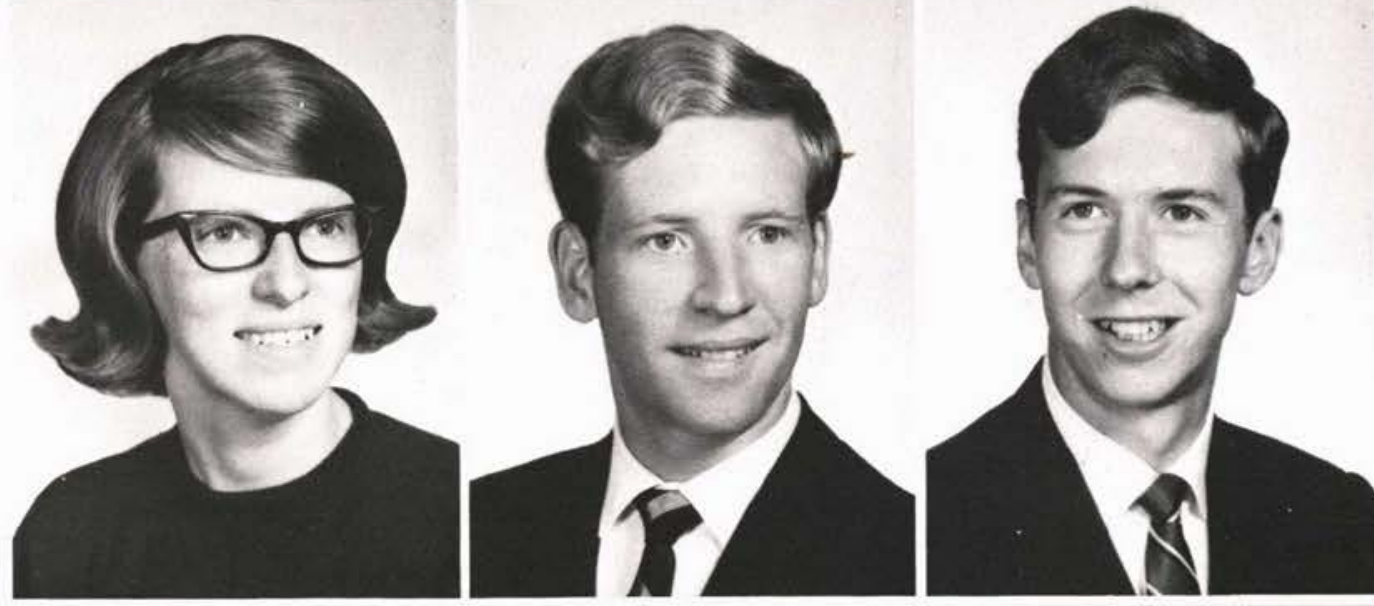

Luiz DeOliveira

Sảo Paulo, Brazil

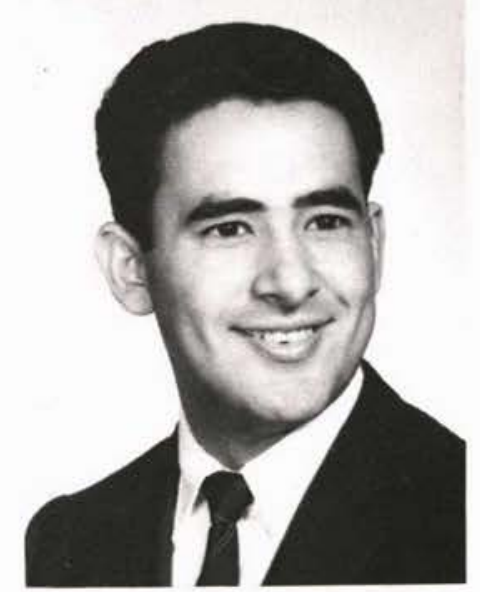



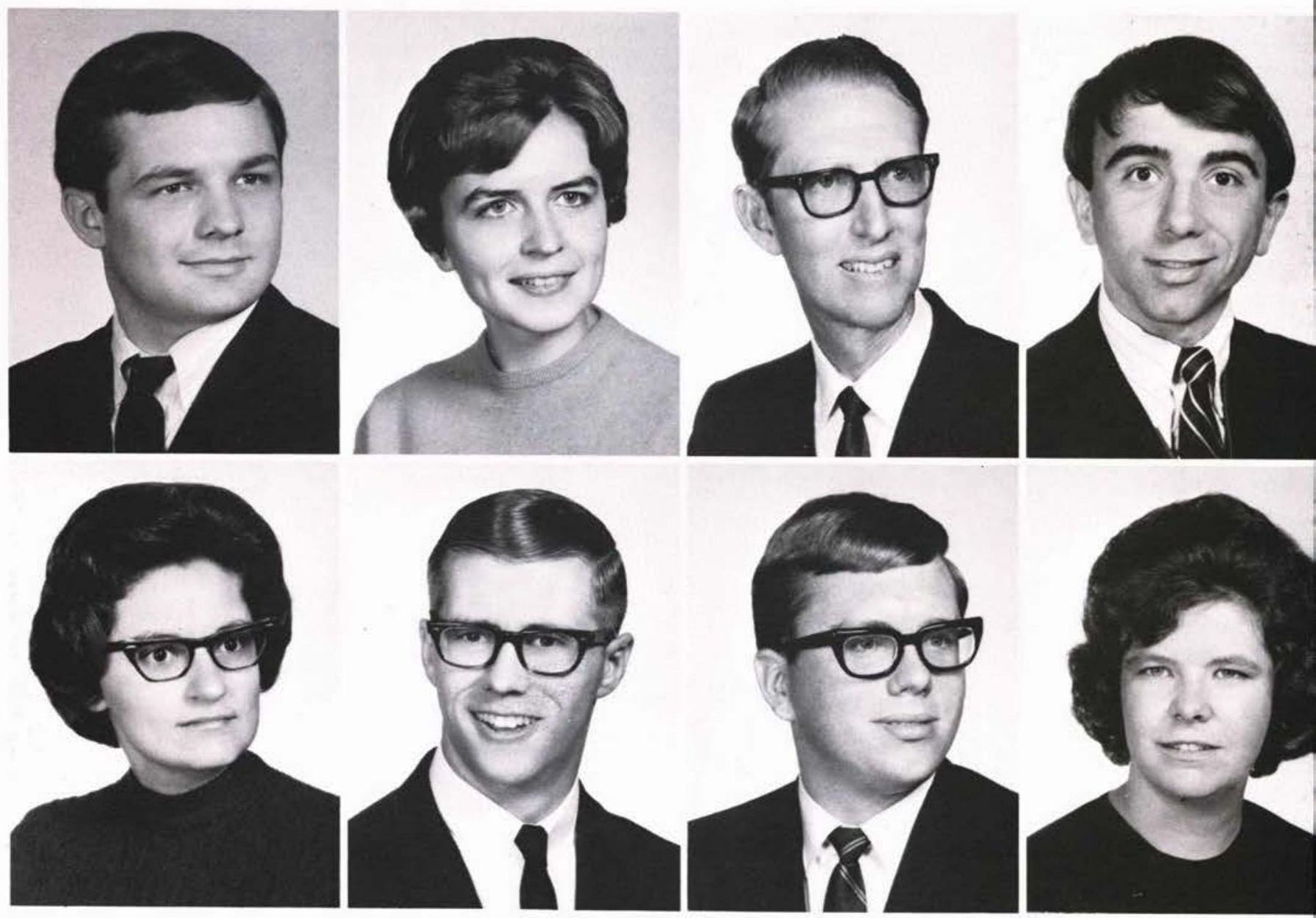

Thomas Duff

Troy, Ohio

Nancy Fourman

Stow, Ohio

Shirley Dufford

Shell Rock, lowa

Gary Fowler

Cedarville, Ohio

Wilbur Eifert

Gerald French

Marathon, New York

John Falci

Hornell. New York

Ann Ganschow

Huntington Woods, Mich.

E. Leroy Gavitt Warren, Ohio

Joyce Goodwin Cedarville, Ohio
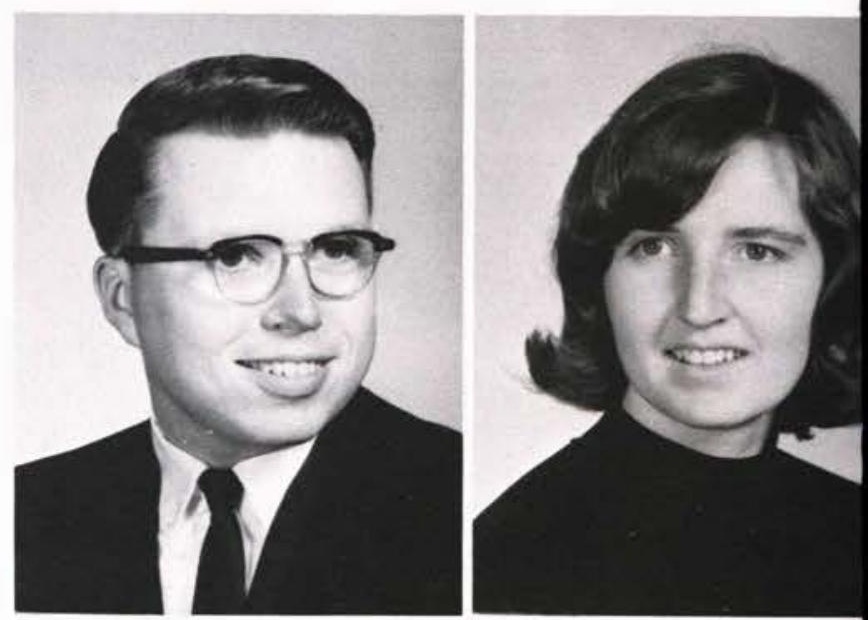
Ruth Hartikainen

Erie, Pennsylvania
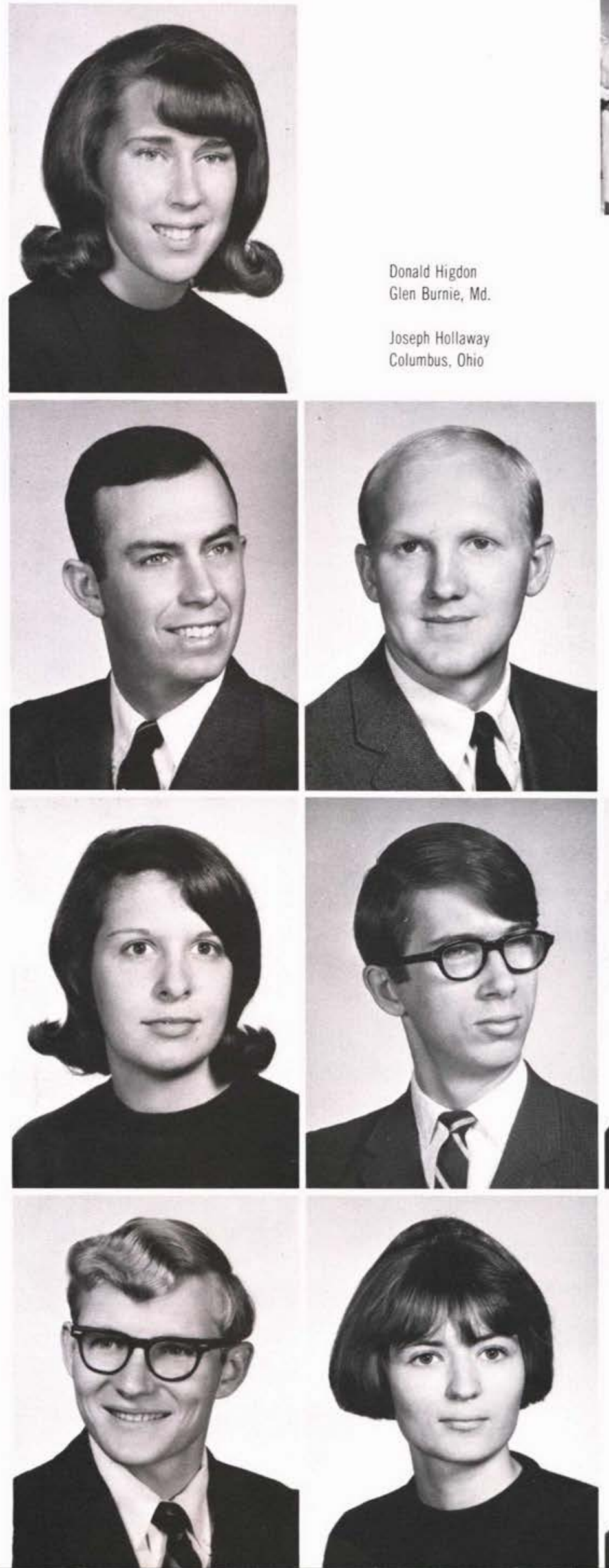

Donald Higdon

Glen Burnie, Md.

Joseph Hollaway

Columbus, Ohio

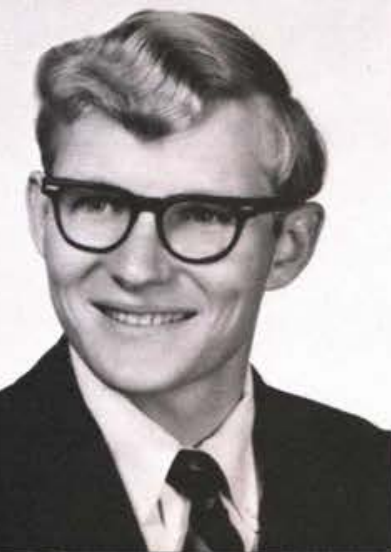

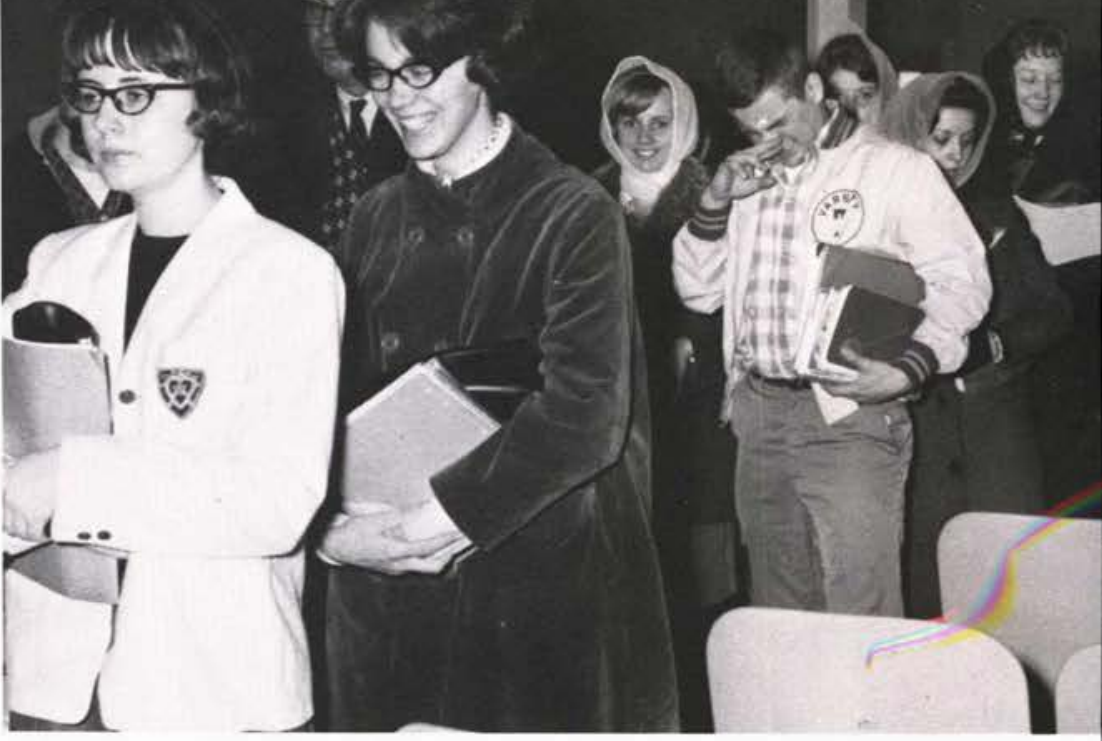
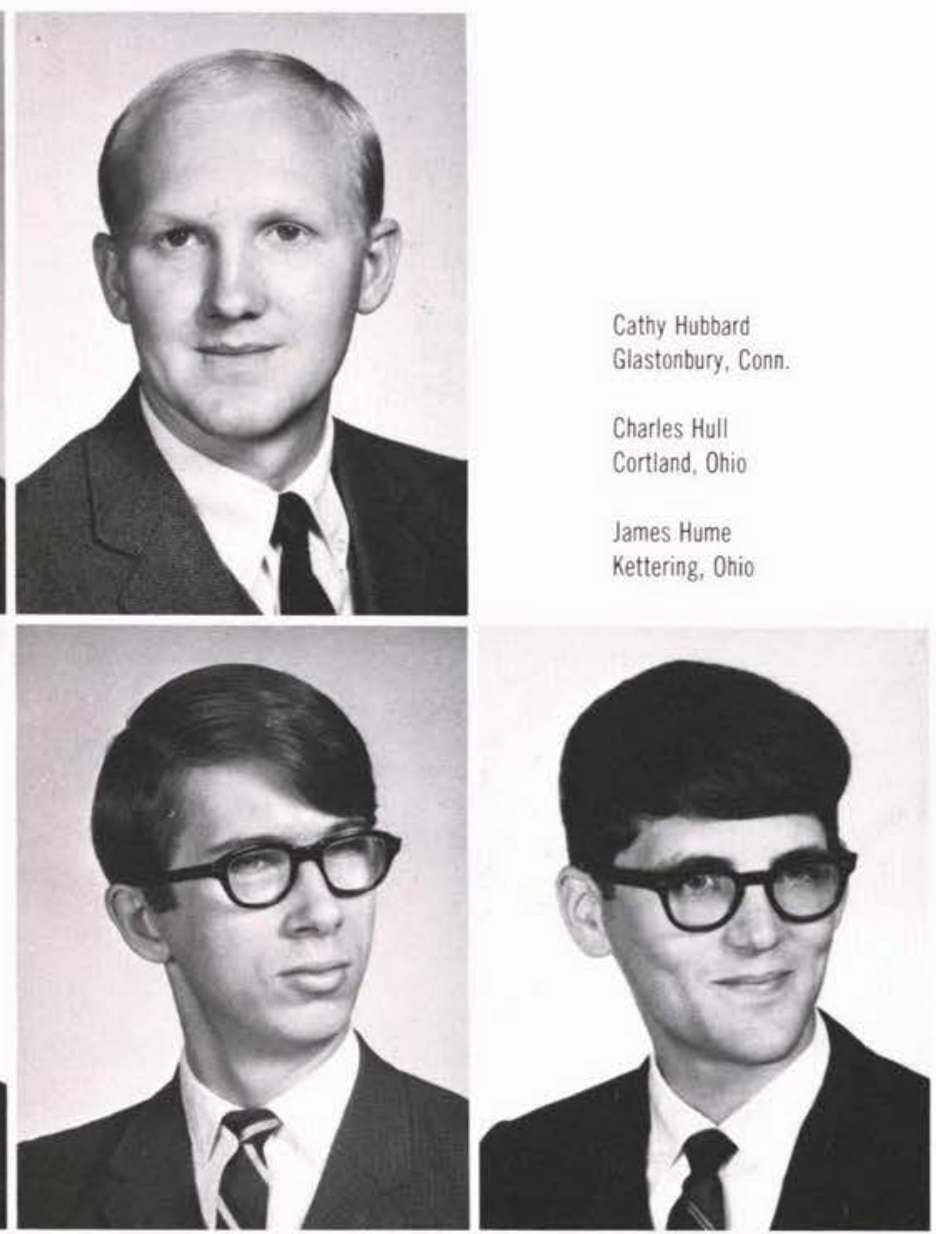

Frank Jenista

lloilo City, Philippines

Ruth Jenista

lloilo City, Philippines

Elaine Johnson

Summerville, S.C.

Kathleen Johnson

Seattle, Washington

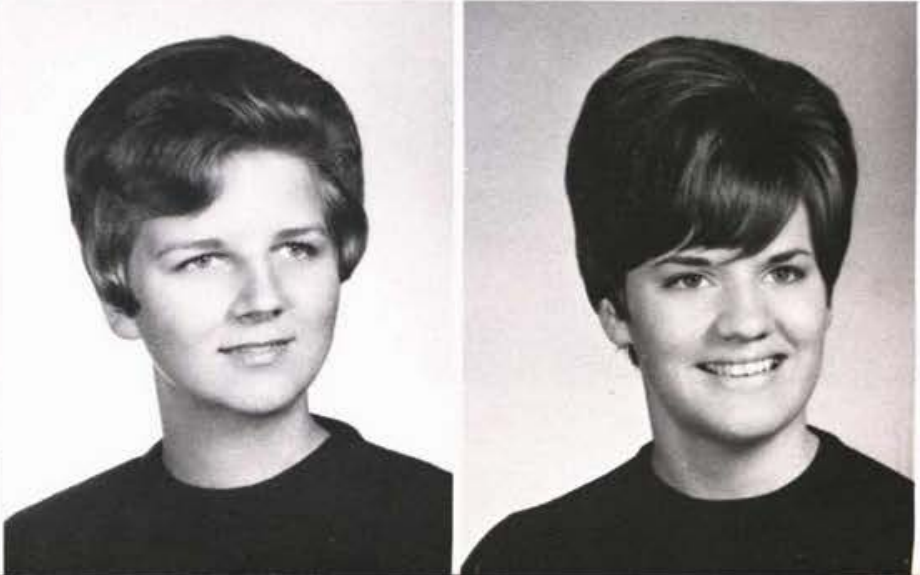




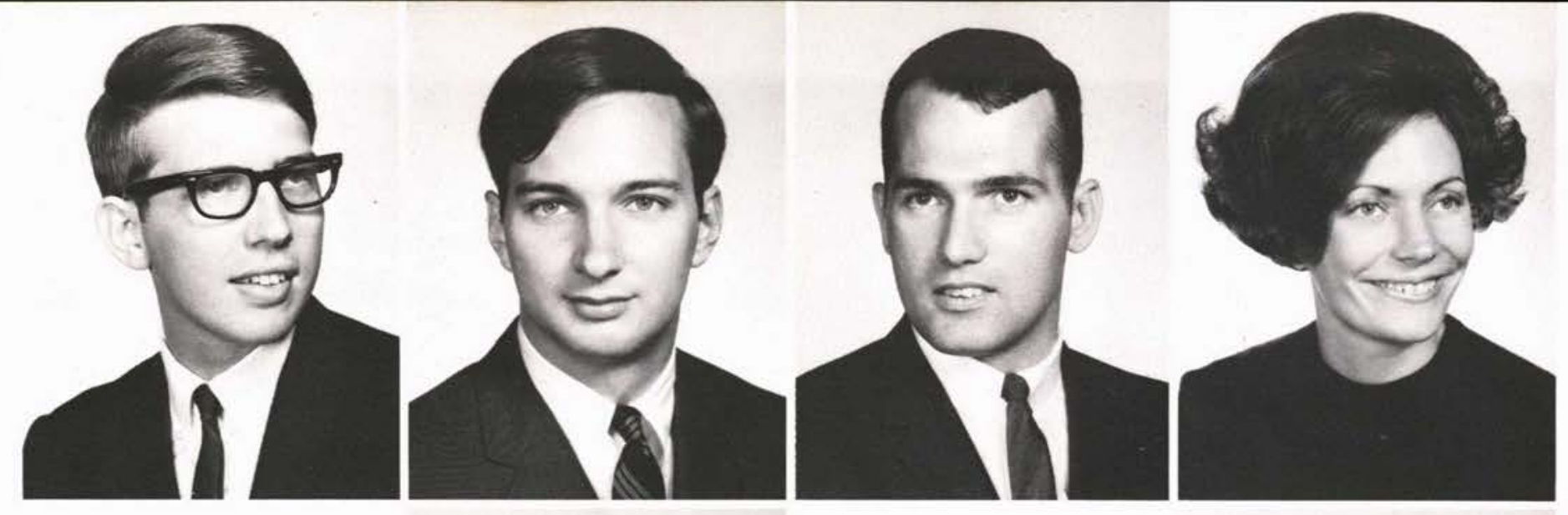

Thomas Johnson

Sciotoville, Ohio

David Jones

Arlington, Virginia

Frances Jones

New Carlisle, Ohio

Marian Kapp

Erie, Pennsylvania
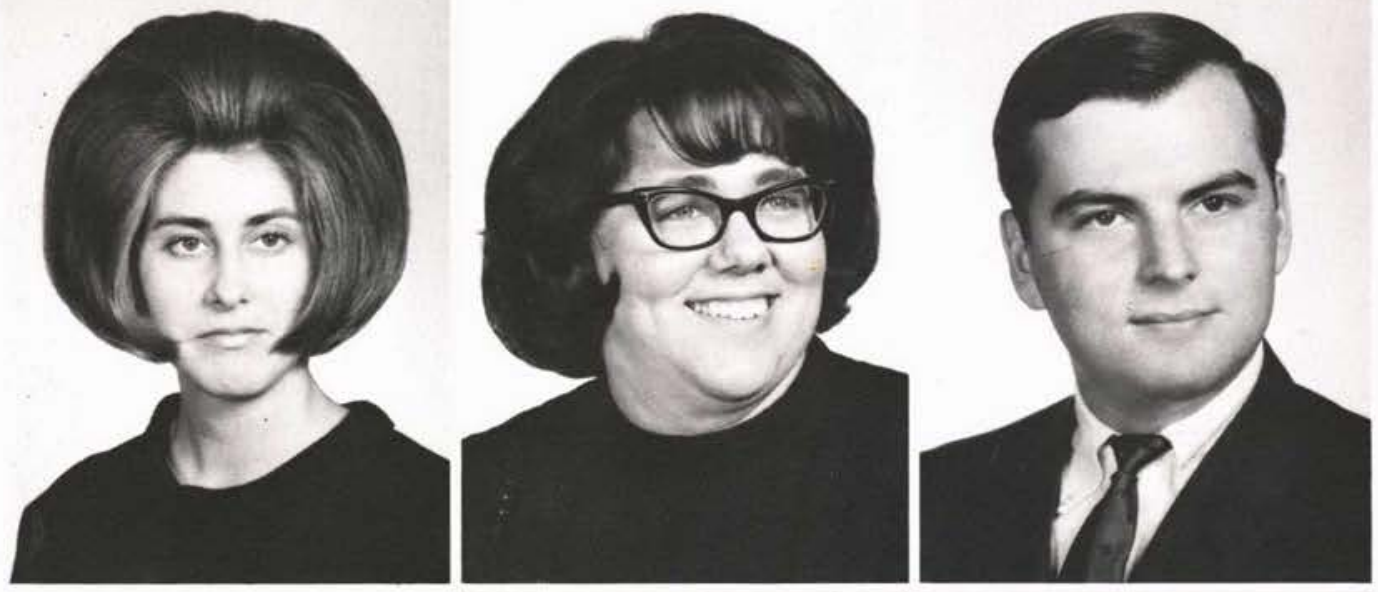

Ruth Kauffold

Dana, Illinois

Donna Keib

Shelby, Ohio

Alan Keim

Holsopple, Pa.
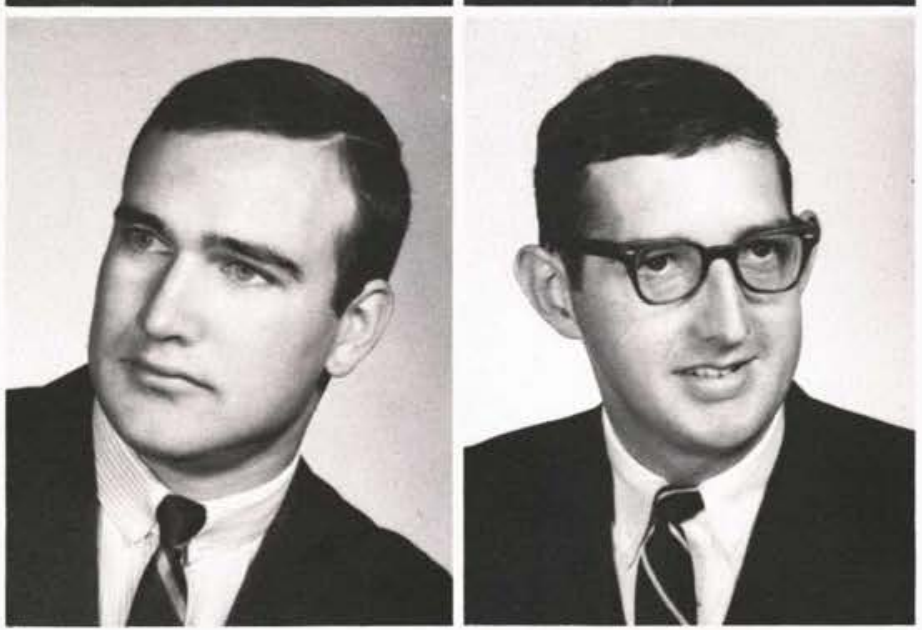

Allen Knott

Cedarville, Ohio

Dale Lauener

Cedarville, Ohio

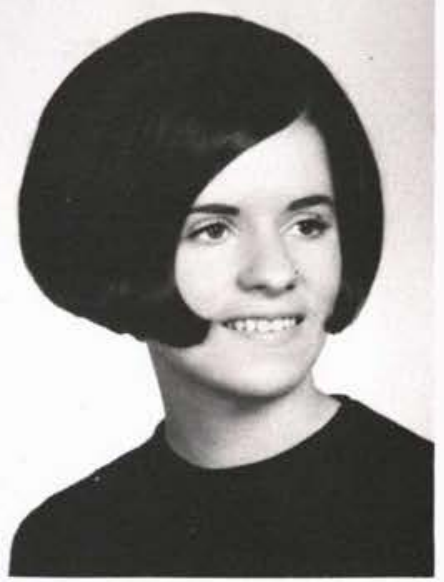

Linda Lauener

Cedarville, Ohio 
Tina Lautt

Michigan City, Ind.

Don Leach

Dayton, Ohio

Lynn Lescure

Hadden Heights. N.J.

Kokomo, Ind

Ron Lewis

Stanton, Michigan

Sandra Lucas

Columbus. Ohio

Bruce Lucius

New Concord, Ohio

John May

Stroudsburg, $\mathrm{Pa}$.

Catherine McGee

Richmond, Virginia

Roberta McInnes

Winona Lake, Ind.
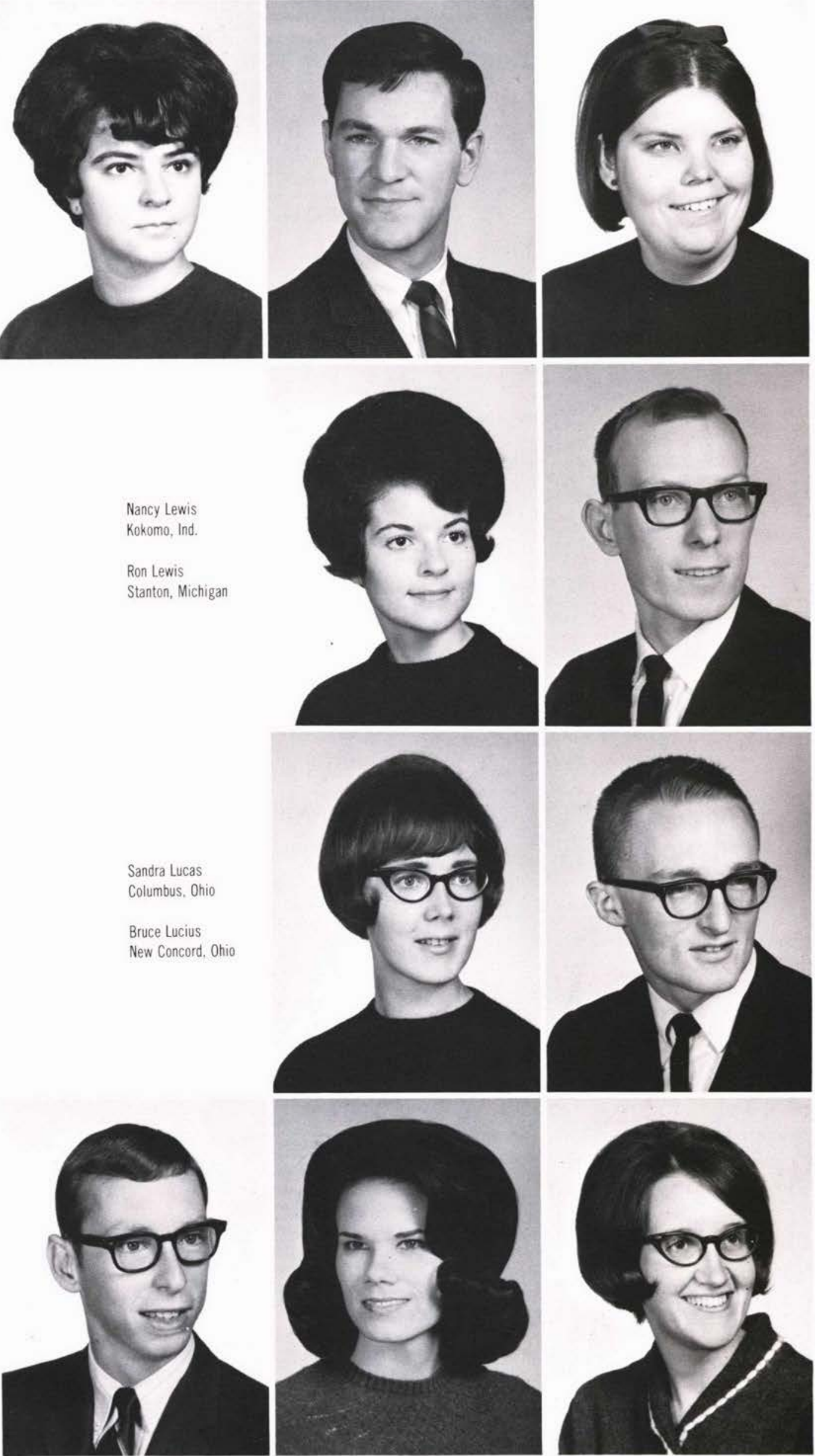

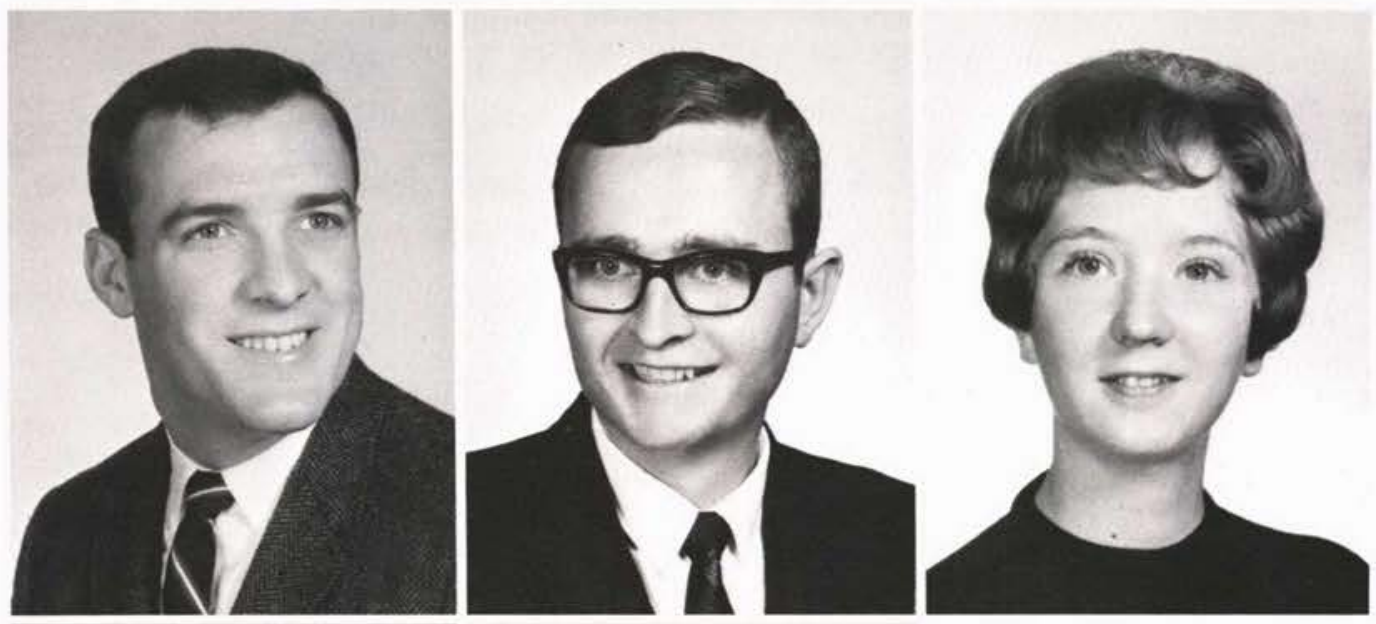

Roger McKenney

Detroit, Michigan

William McVey

Kansas City. Kansas

Betty Meyers

East Moline, Illinois
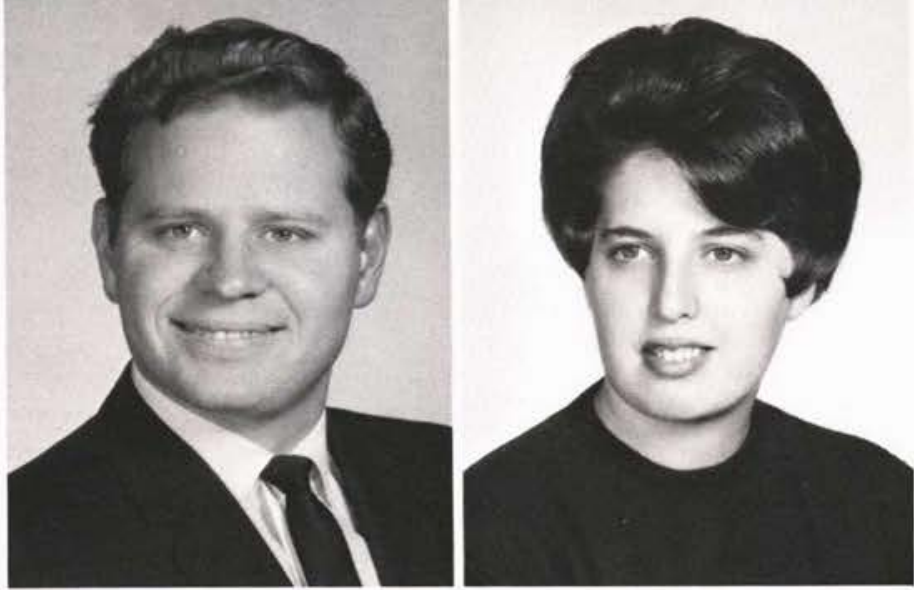

James Miller

Pontiac, Michigan

Roberta Miller

Lowellville, Ohio
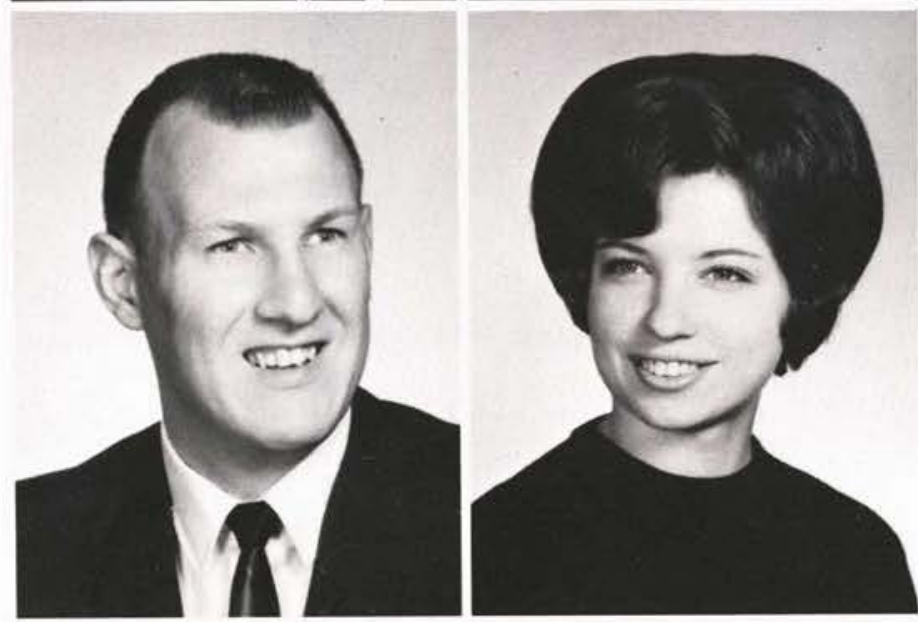

Charles Mitchell

Cedarville, Ohio

Marilyn Mitchell

Cedarville, Ohio
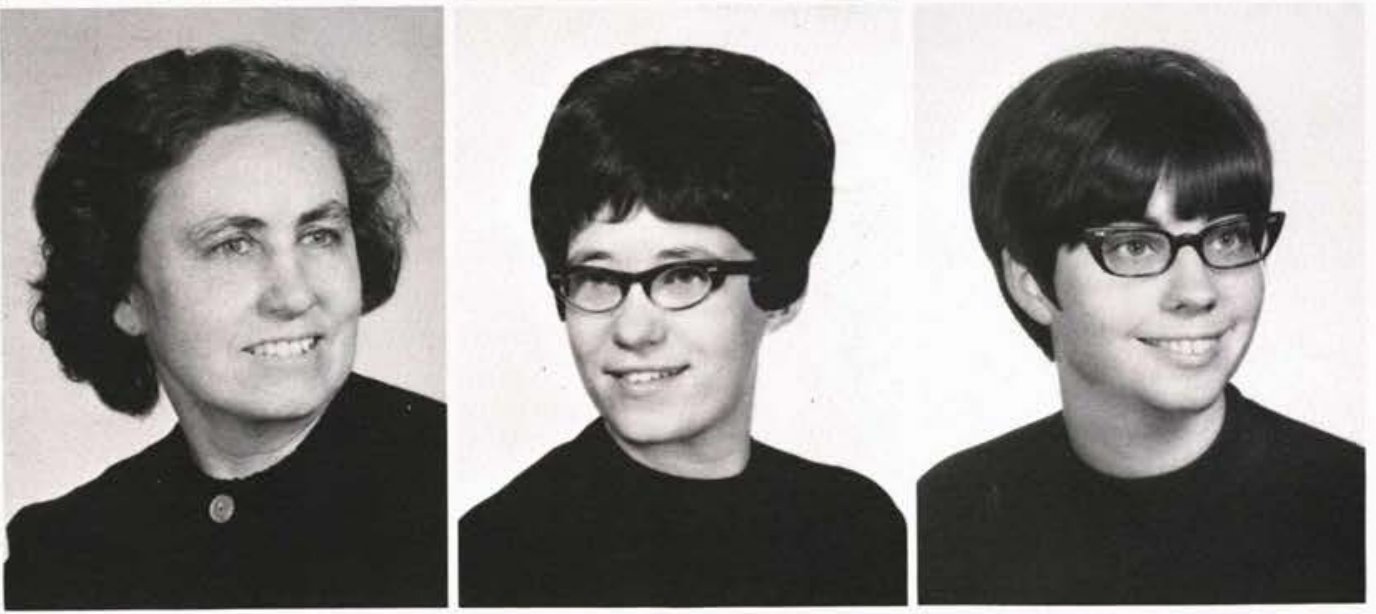

Helen Moose

Crookston, Minnesota

Donna Morgan

Charles City. lowa

Beverly Moseley

Melvindale, Michigan 

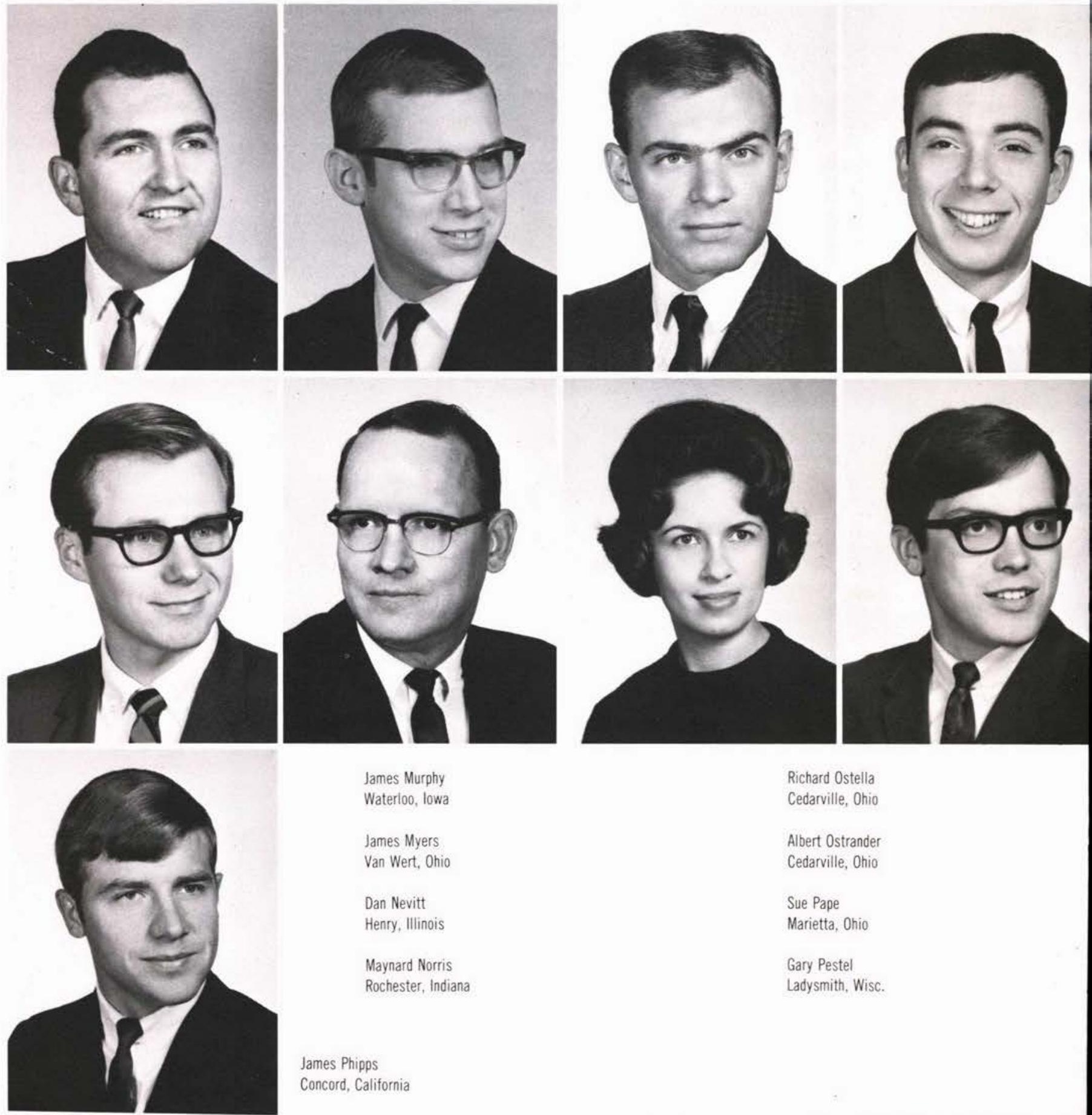

James Murphy

Waterioo, lowa

James Myers

Van Wert, Ohio

Dan Nevitt

Henry, Illinois

Maynard Norris

Rochester, Indiana

Richard Ostella

Cedarville, Ohio

Albert Ostrander

Cedarville, Ohio

Sue Pape

Marietta, Ohio

Gary Pestel

Ladysmith, Wisc.

James Phipps

Concord, California

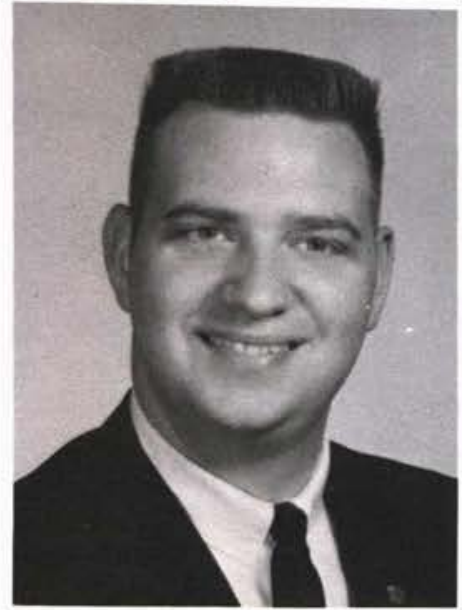

Larry Prentice

Waterloo, New York

Daniel Price

Lebanon, Ohio

Nedra Price

Buffalo, New York
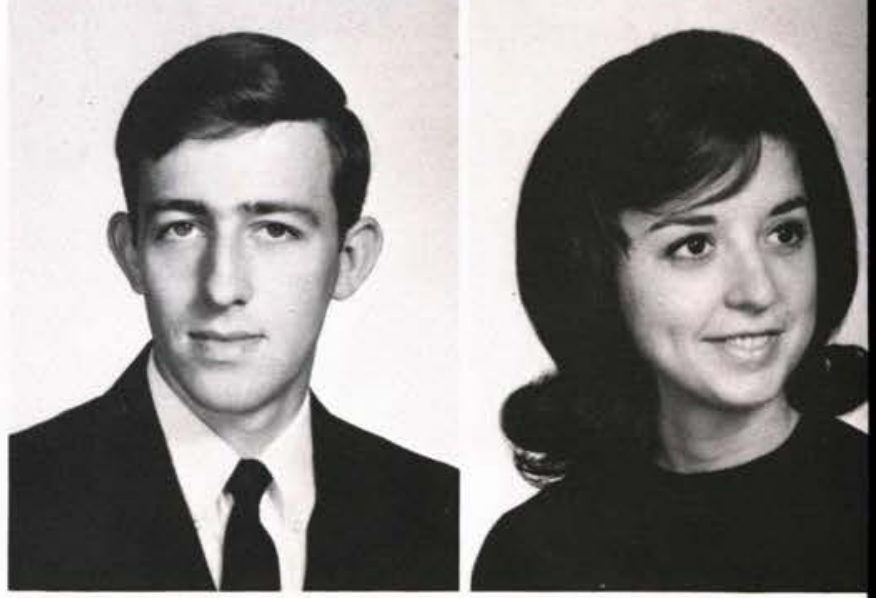
Sandra Schlechty

Lakewood, Ohio

Roy Schisler

Cedarville, Ohio

Marlene Schonscheck
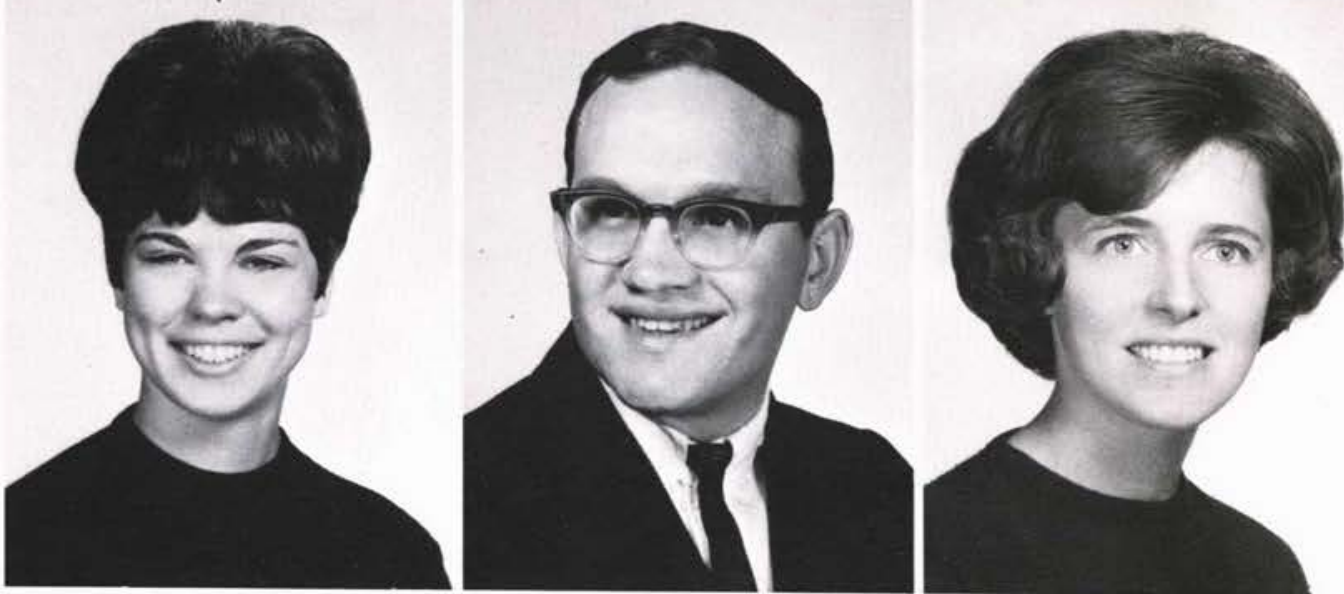

Elaine Schulte

Huntingburg, Indiana

Dean Seignour

Byron Shearer

Pittsburgh, Pa.
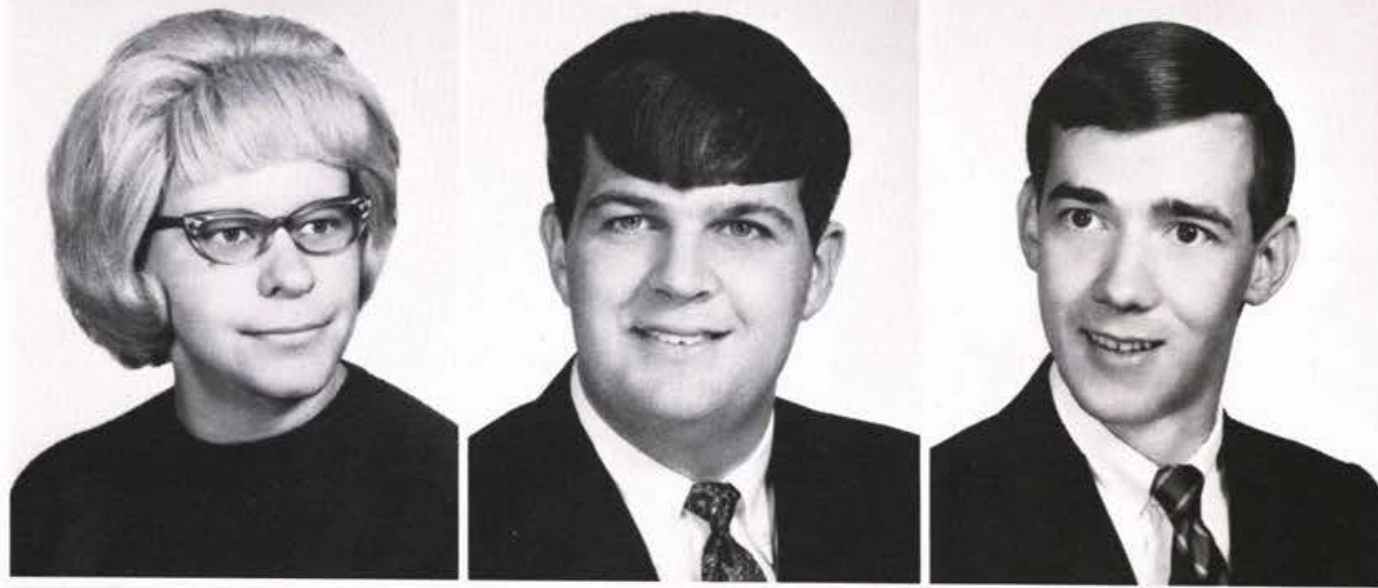

Donna Simpson

Indianapolis, Ind.

Mary Sleichter

Scotland, $\mathrm{Pa}$.

Kathryn Spencer

Cedarville, Ohio

Philip Spencer

Columbus, Ohio

Connie Stevenson

Baker. Montana

Susan Swan

Sabinsville, Pa.
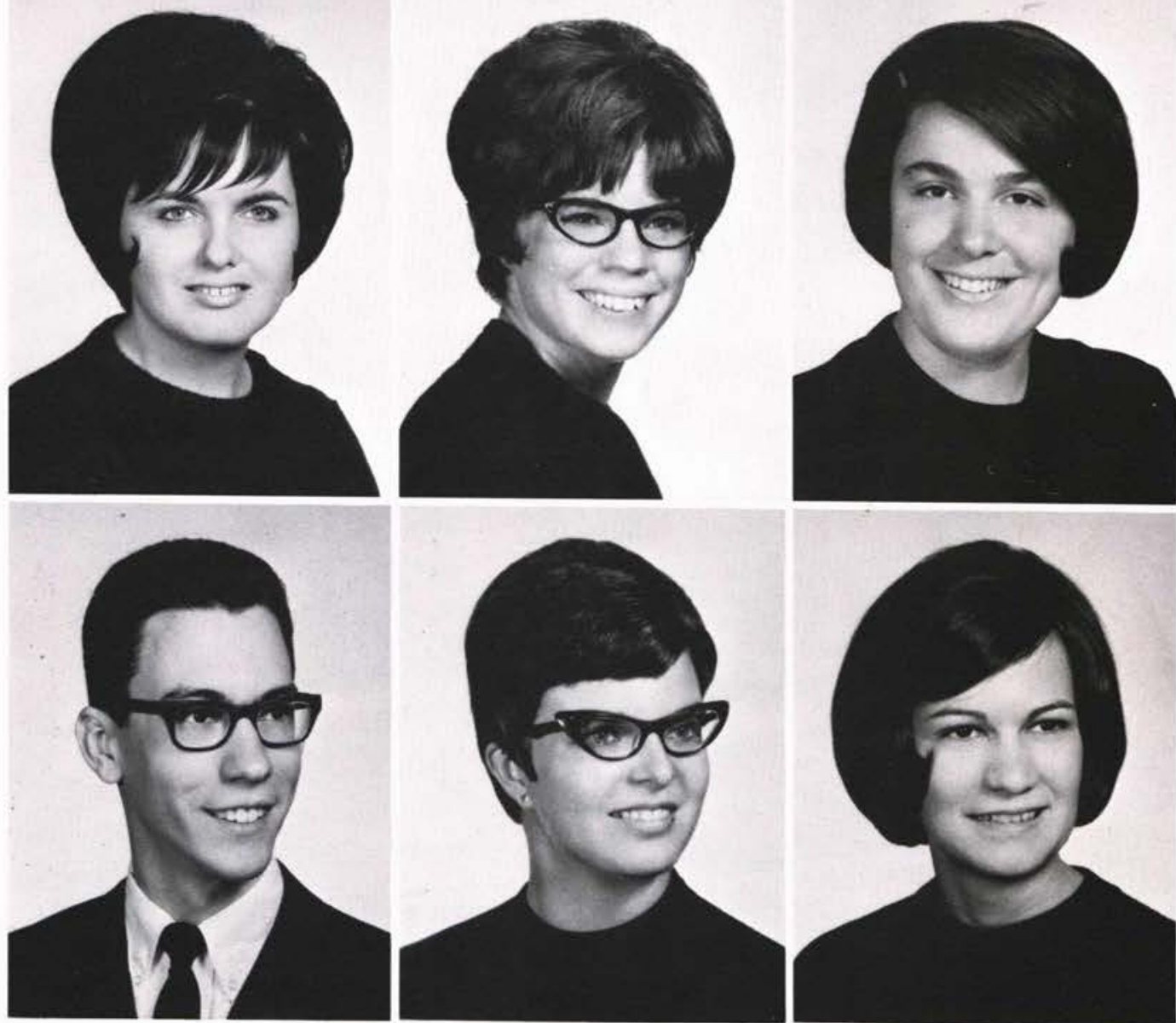


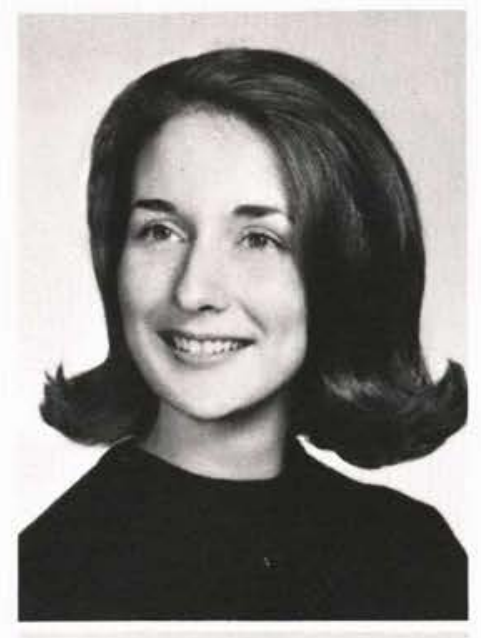

Sharon Tallman

Hallandale, Florida
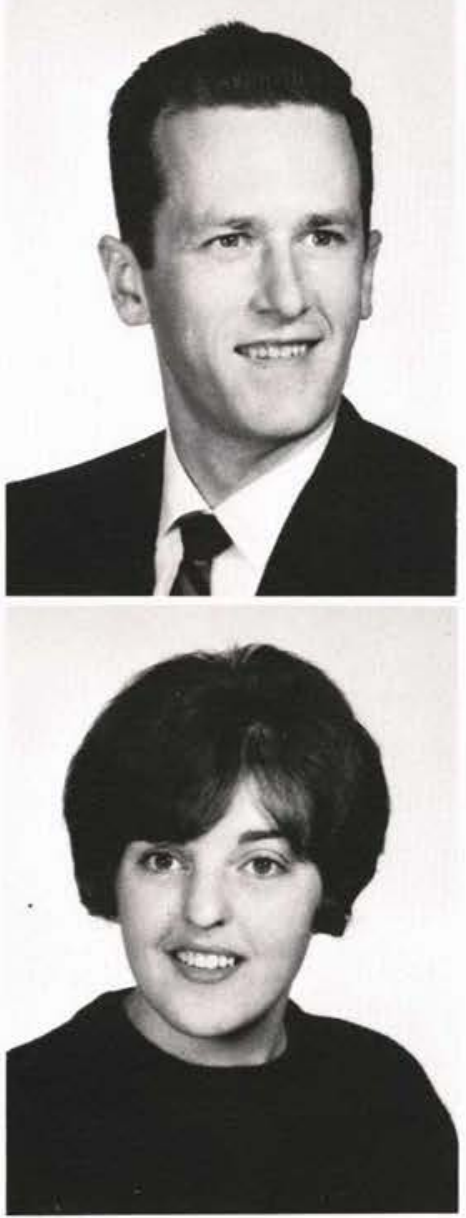

Dale Thompson

Adrian, Michigan

Gail Tompkins

Sherman, N.Y.

Norman Underwood

Dayton, Ohio

Terrance Vlug

Grand Rapids, Mich.

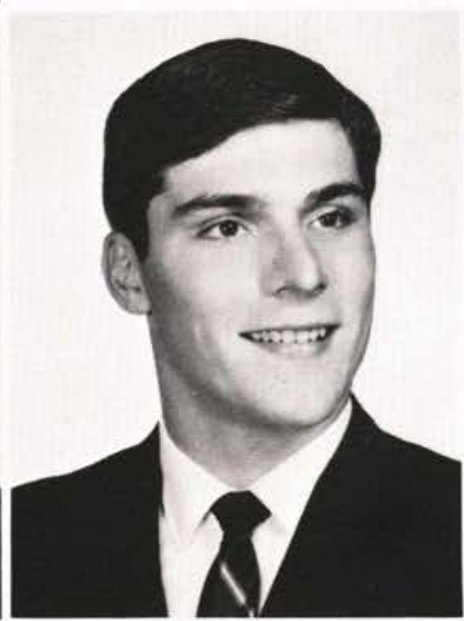

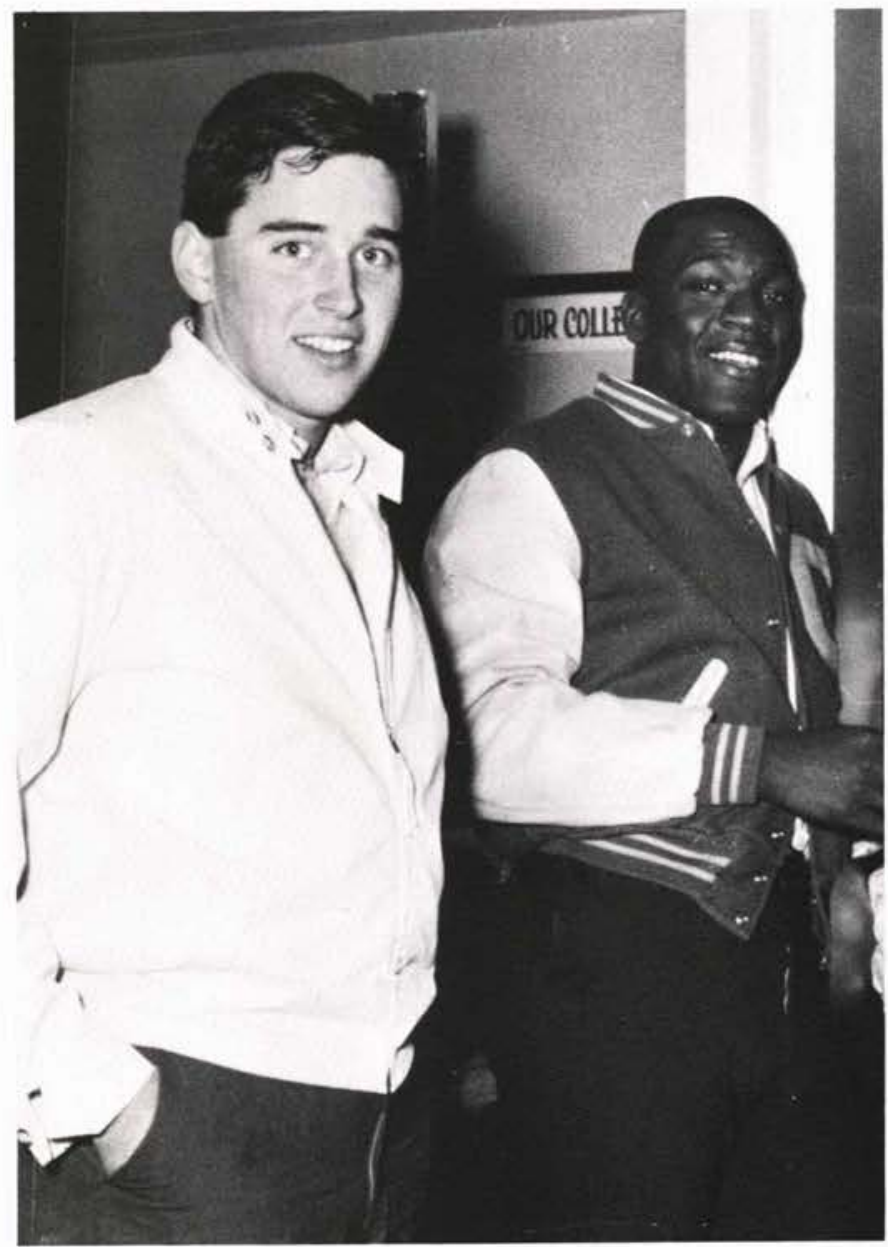

Keith Wagner

Cortland. Ohio

Larry Waite

Hudsonville, Michigan
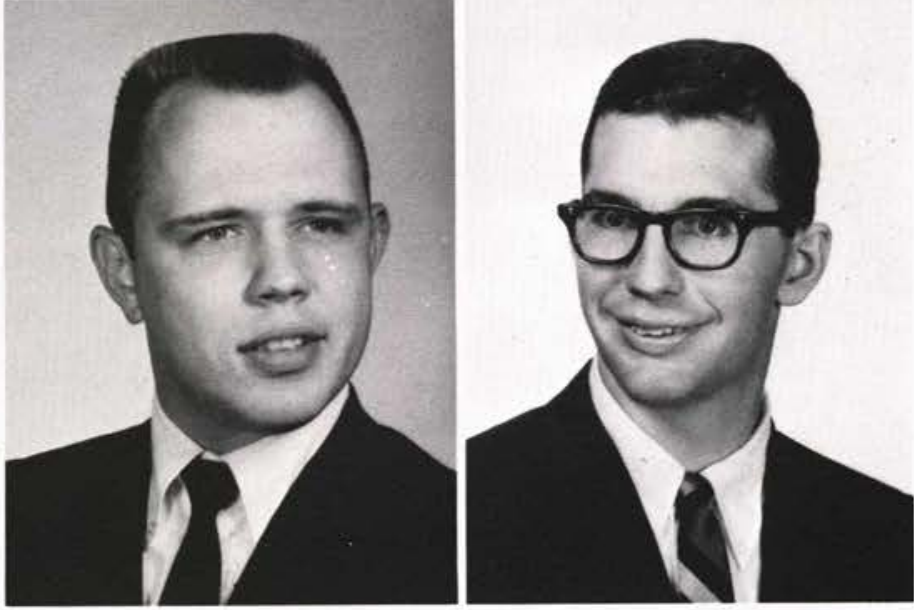

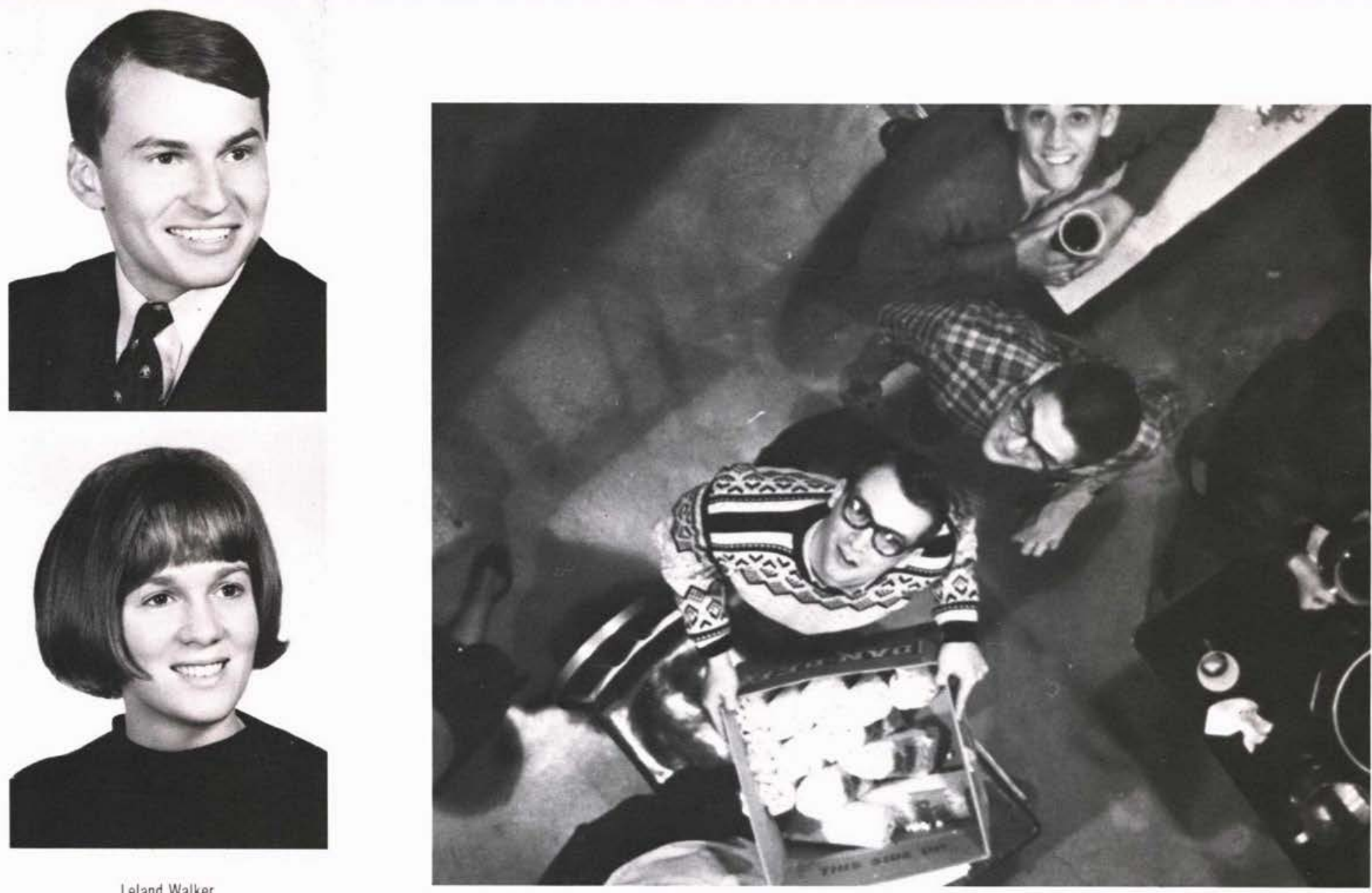

Leland Walker

Carlish, lowa

Lois Walker

Kirtland. Ohio
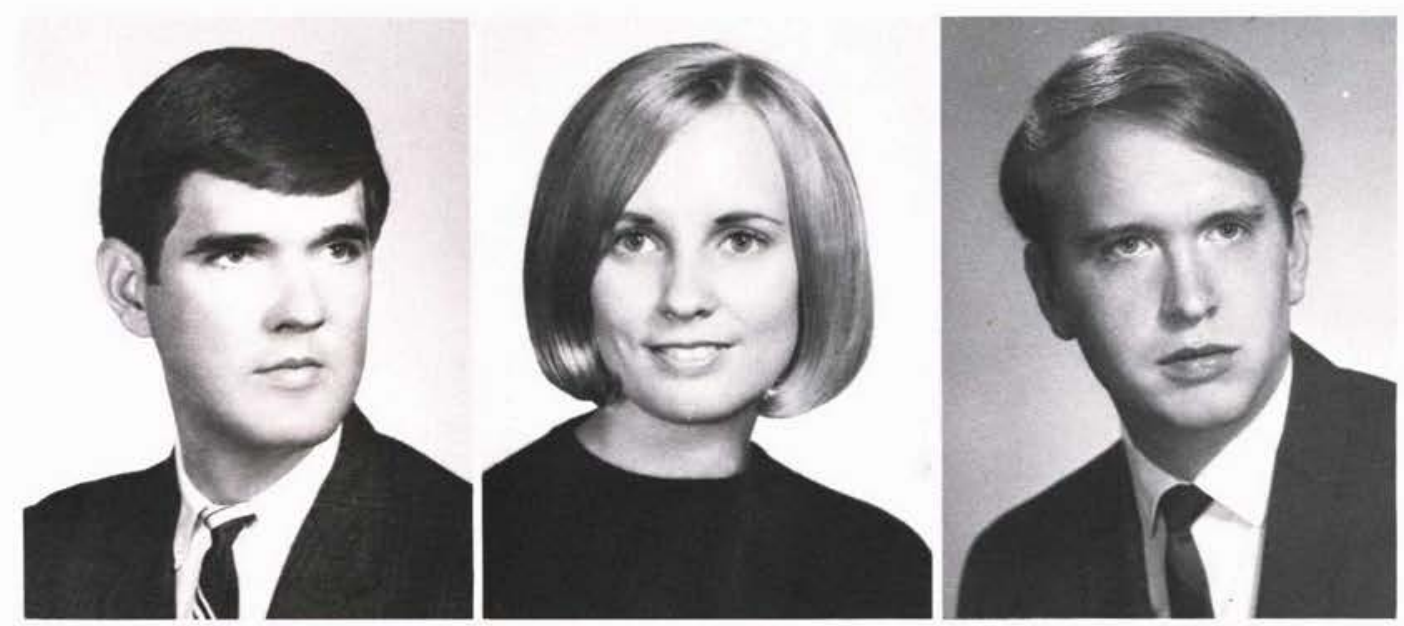

Gary Walthall

Xenia, Ohio

Diana Ward

Cleveland, Ohio

Loren Welch

Shelby, Ohio
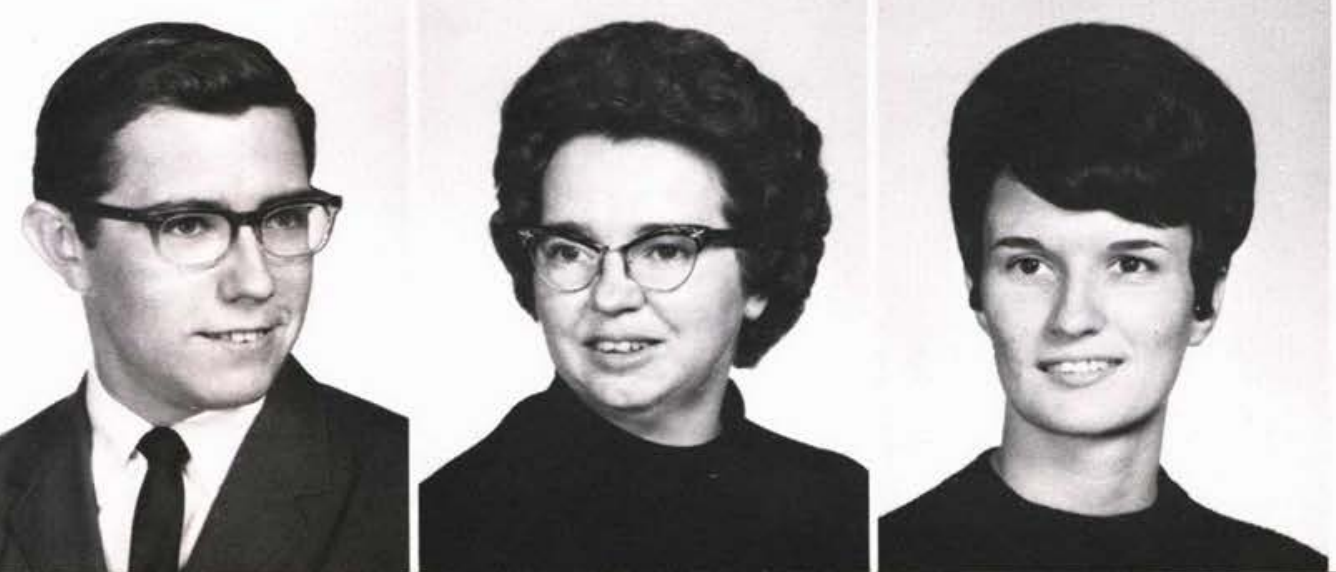

James Whitmire

Butler, Pennsylvania

Leah Whitney

Linda Wintrow

Tipp City. Ohio 

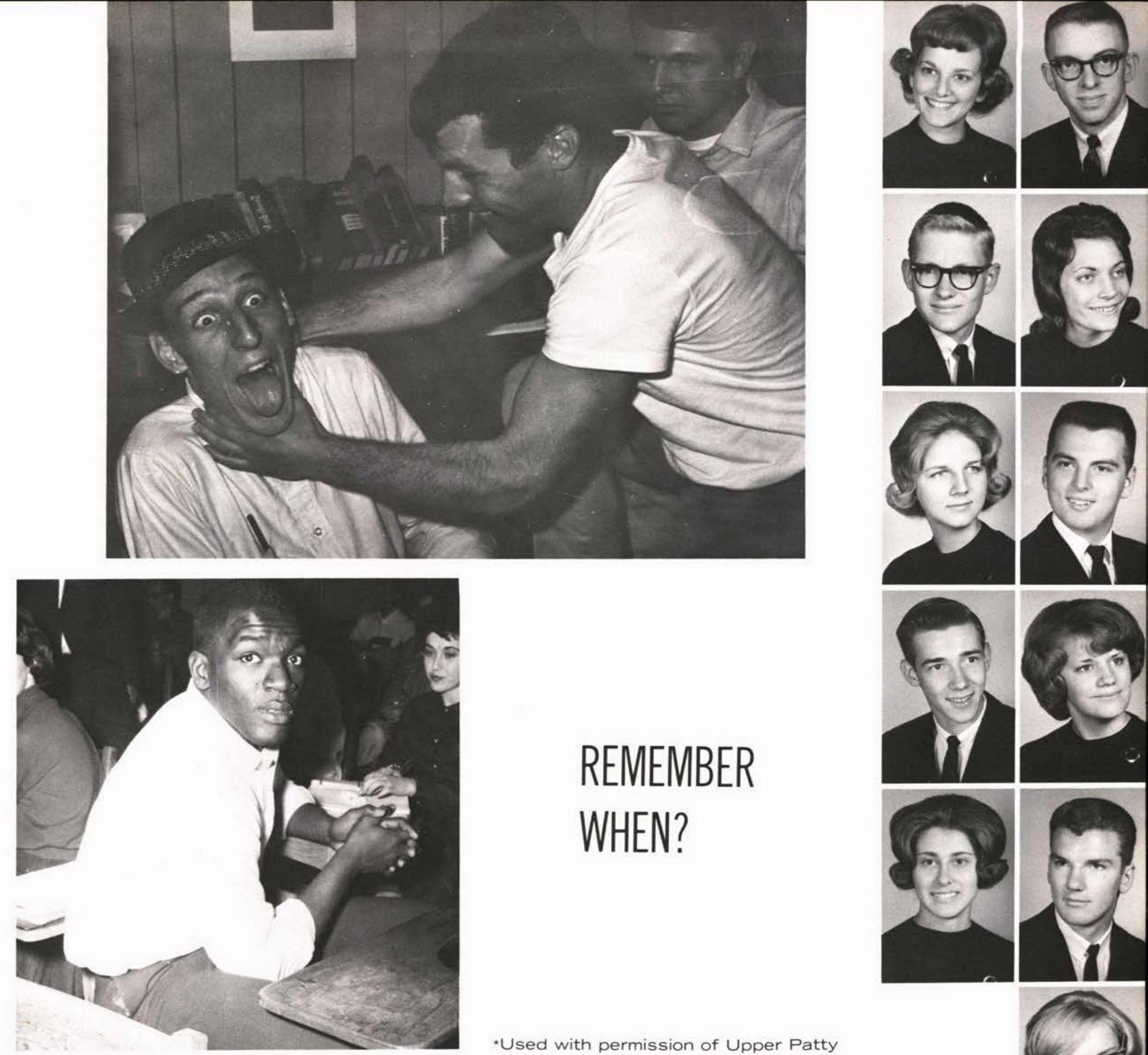

\section{REMEMBER}

WHEN?

*Used with permission of Upper Patty
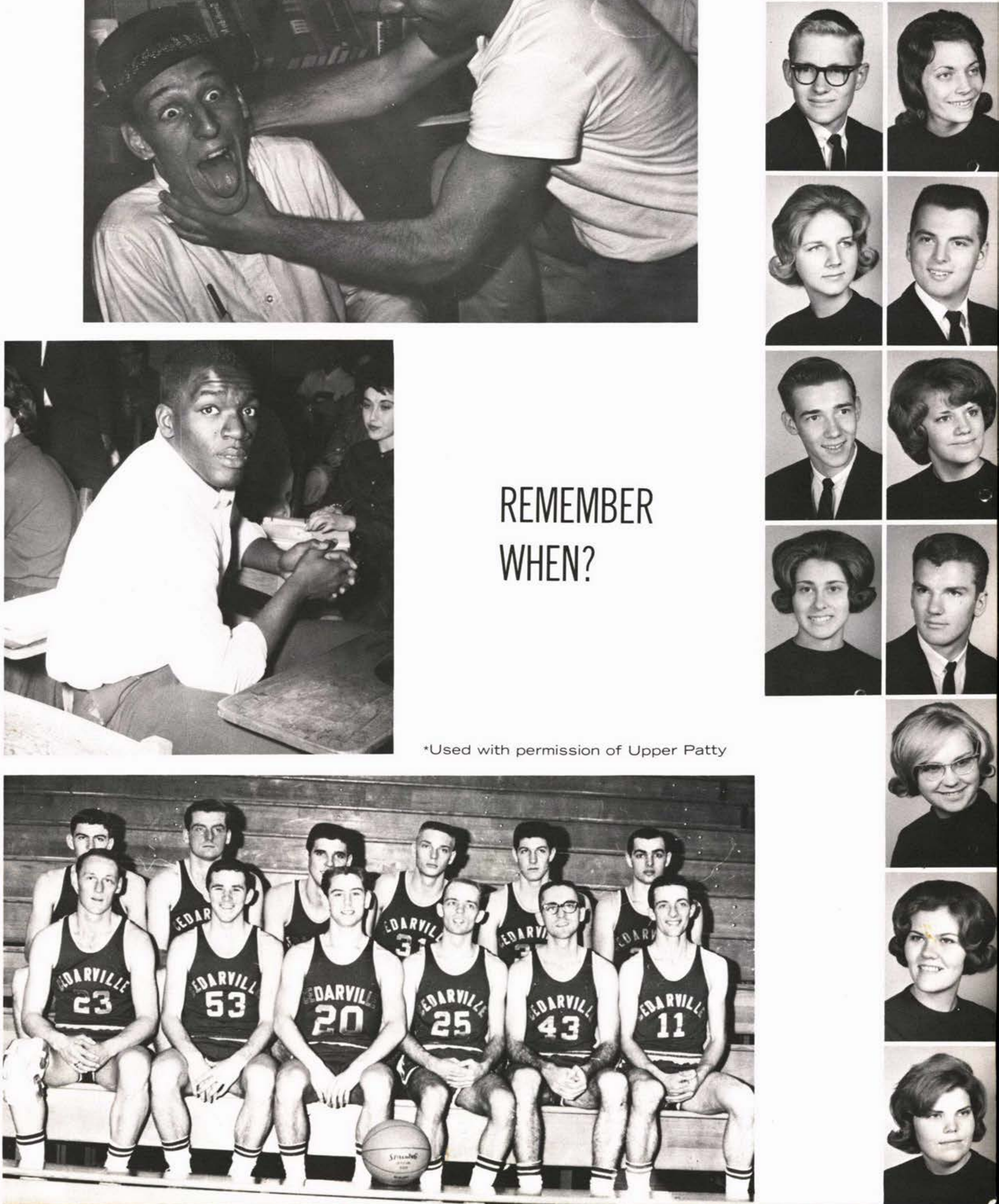

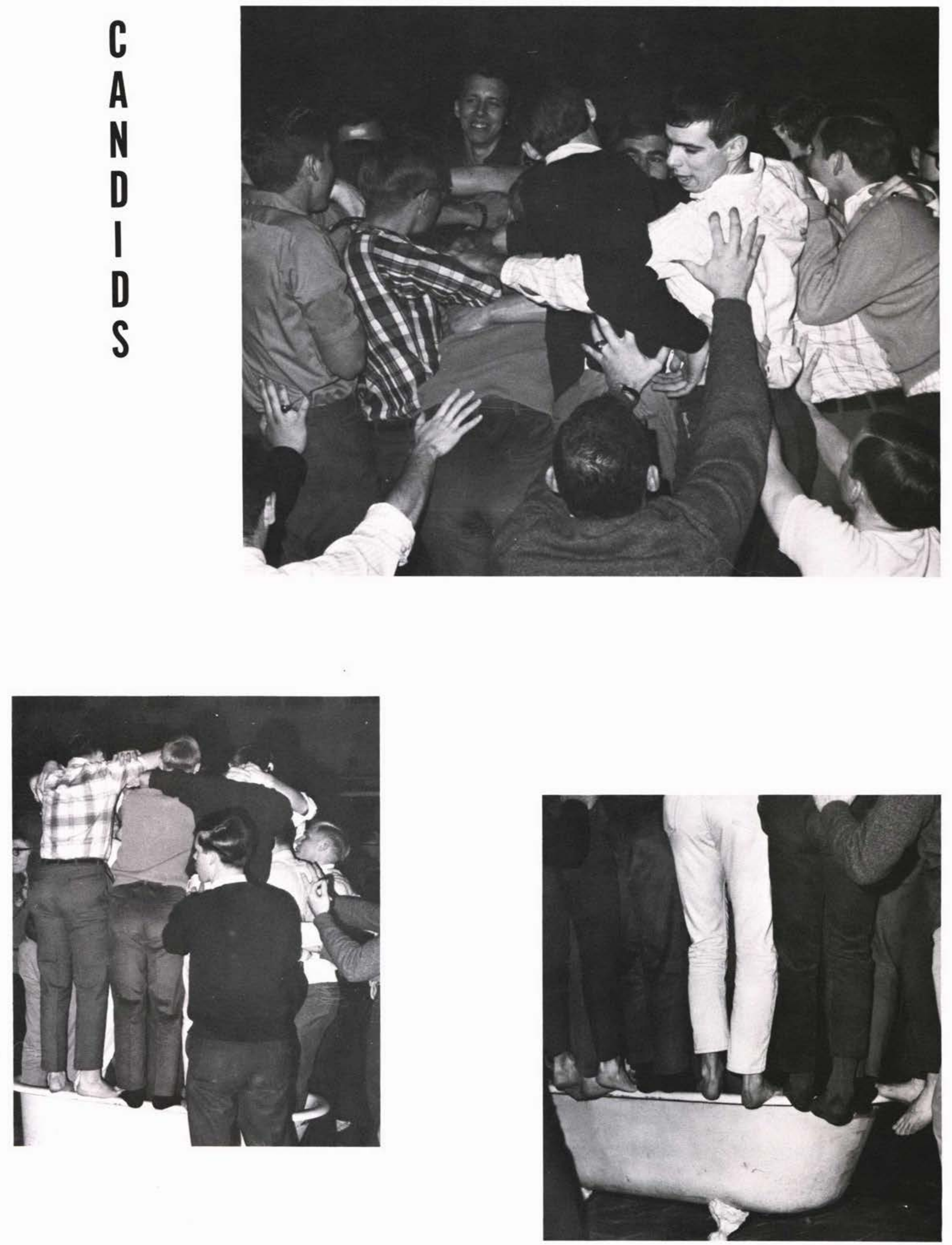

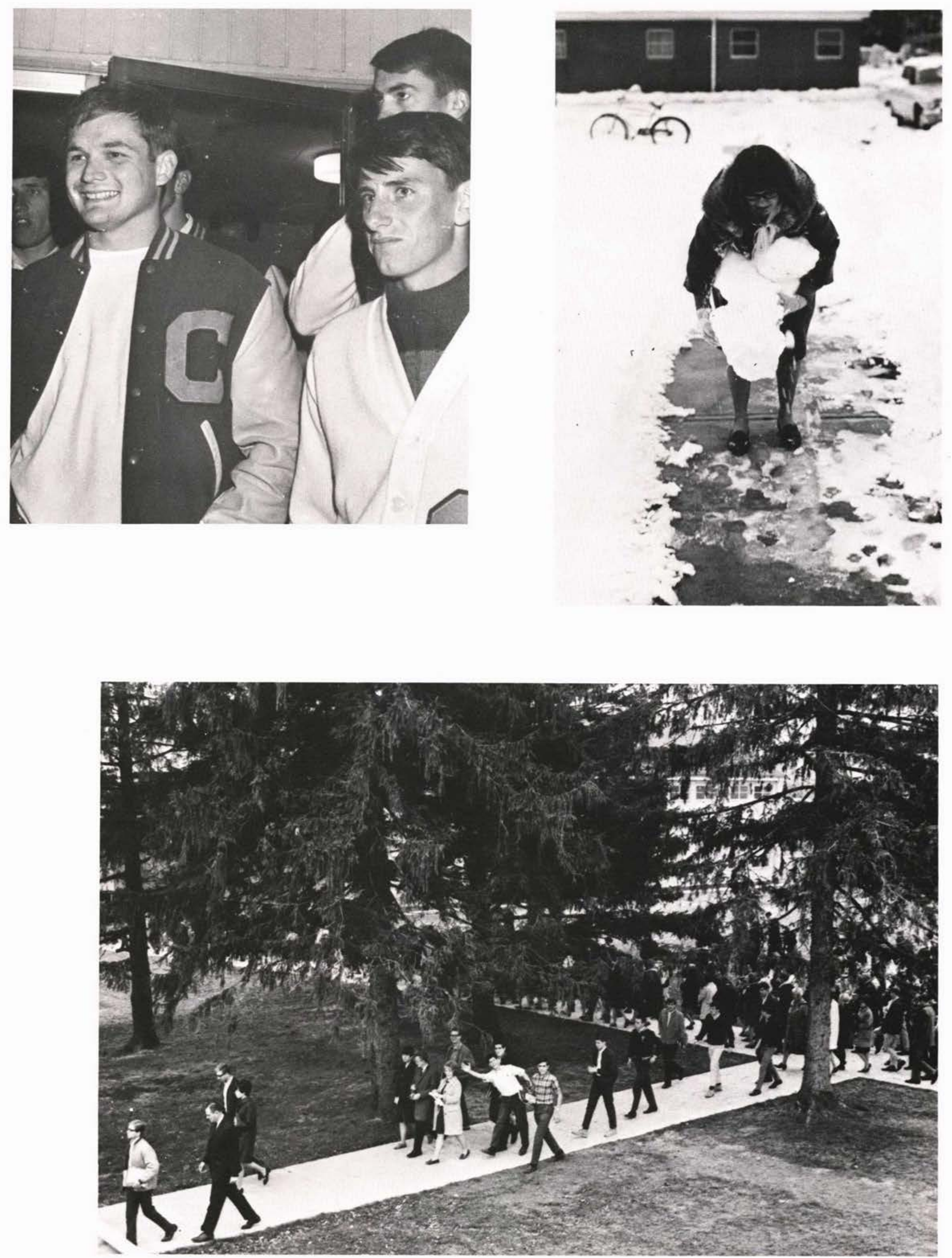

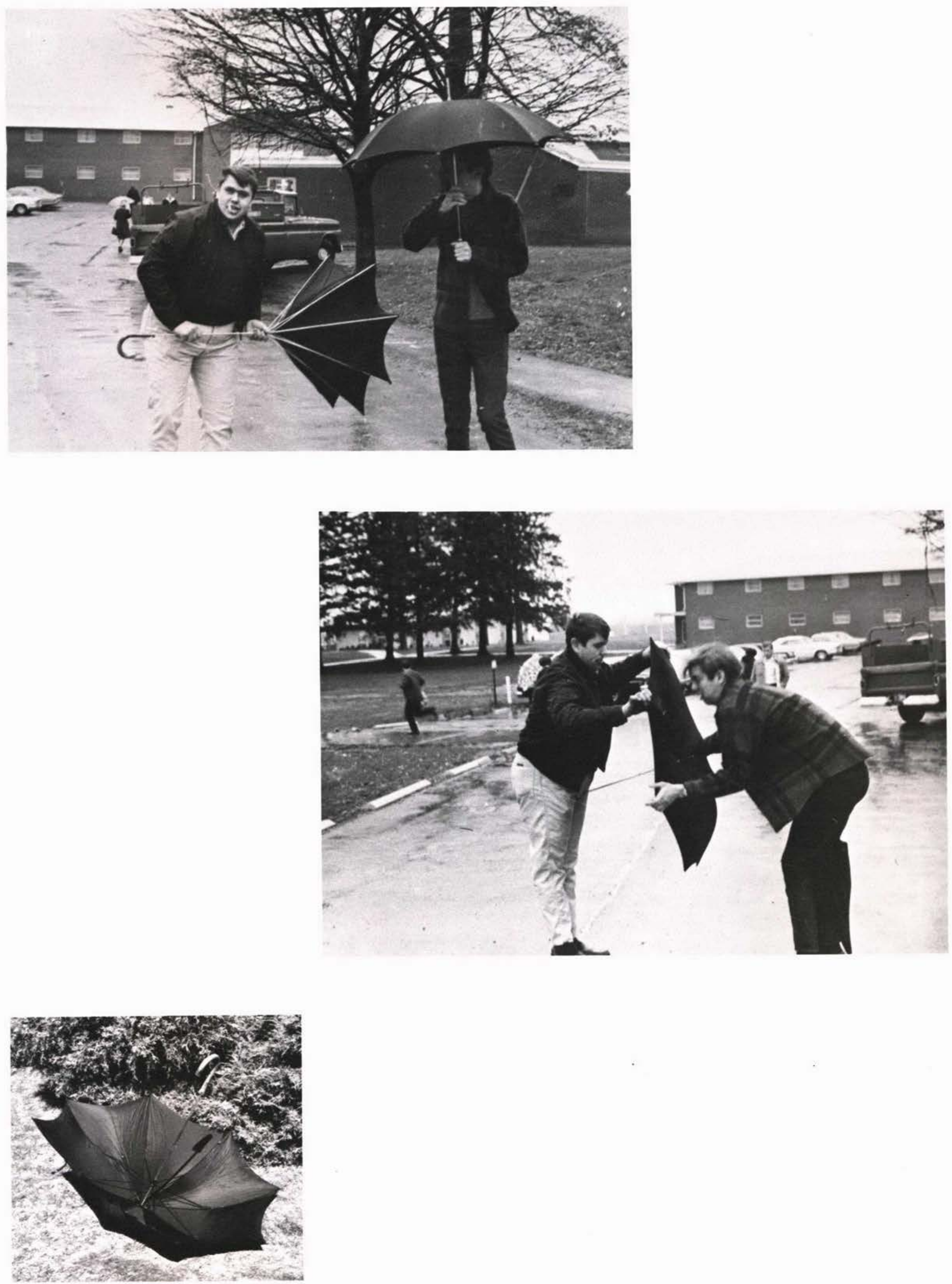

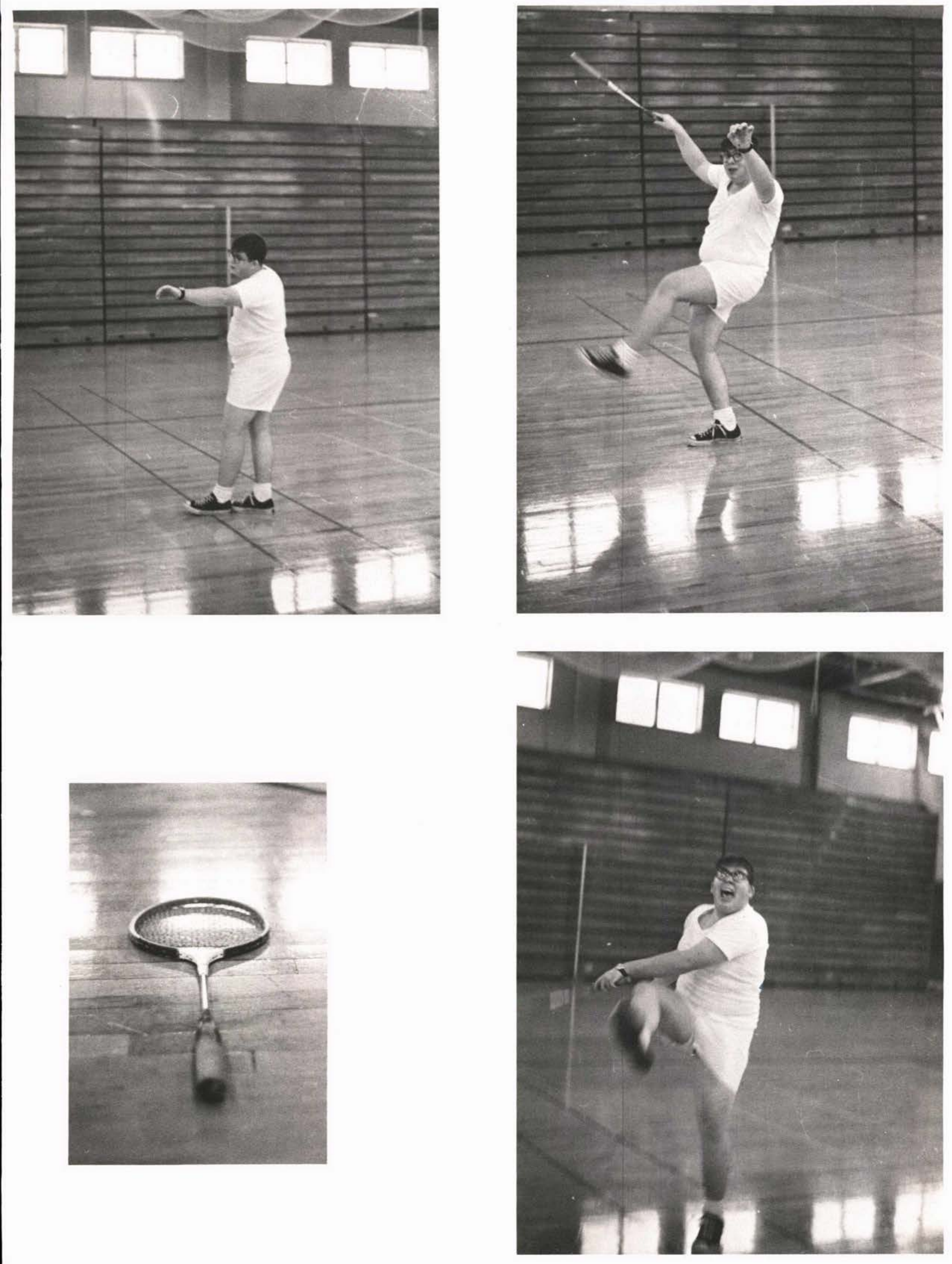

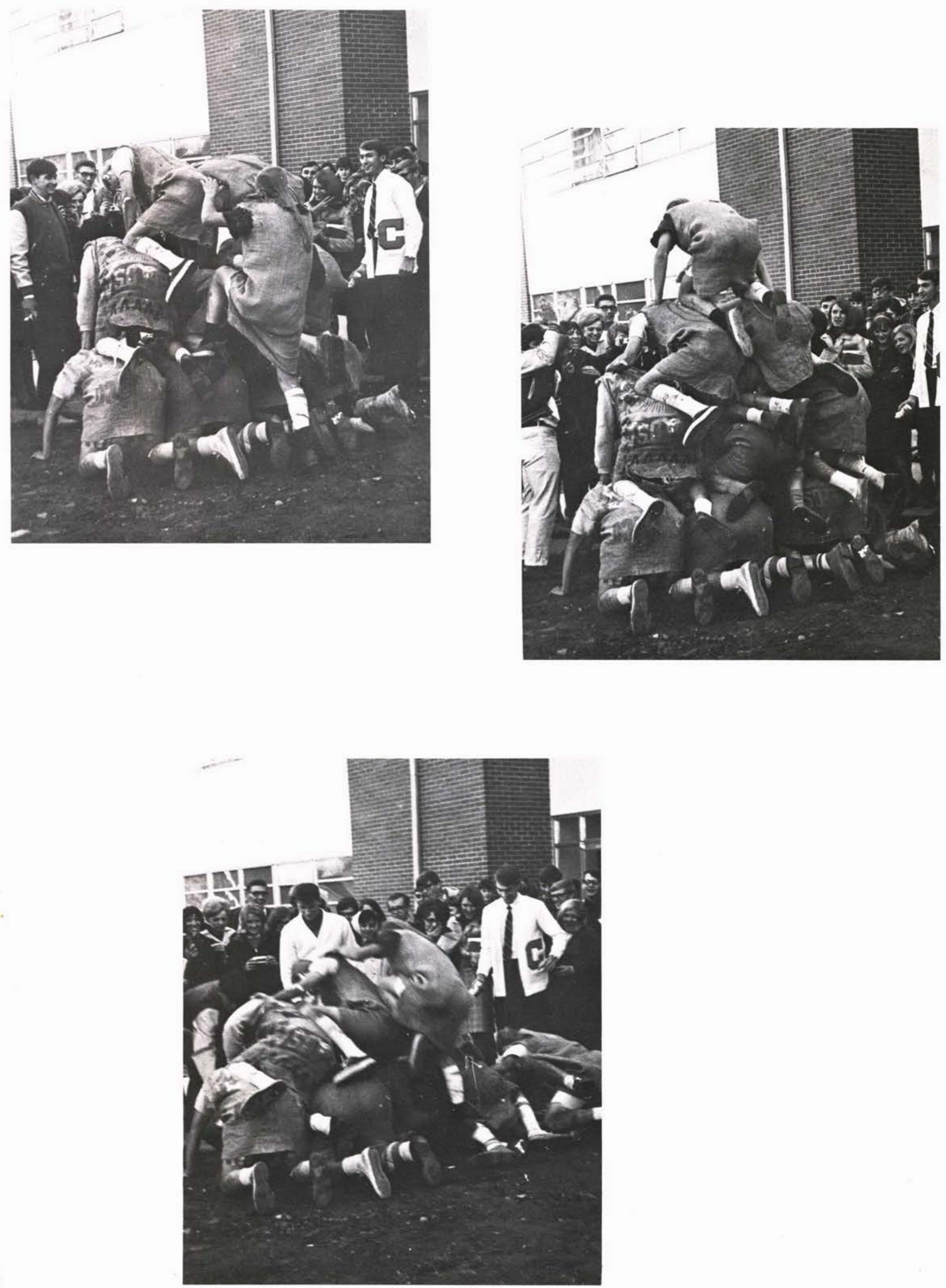

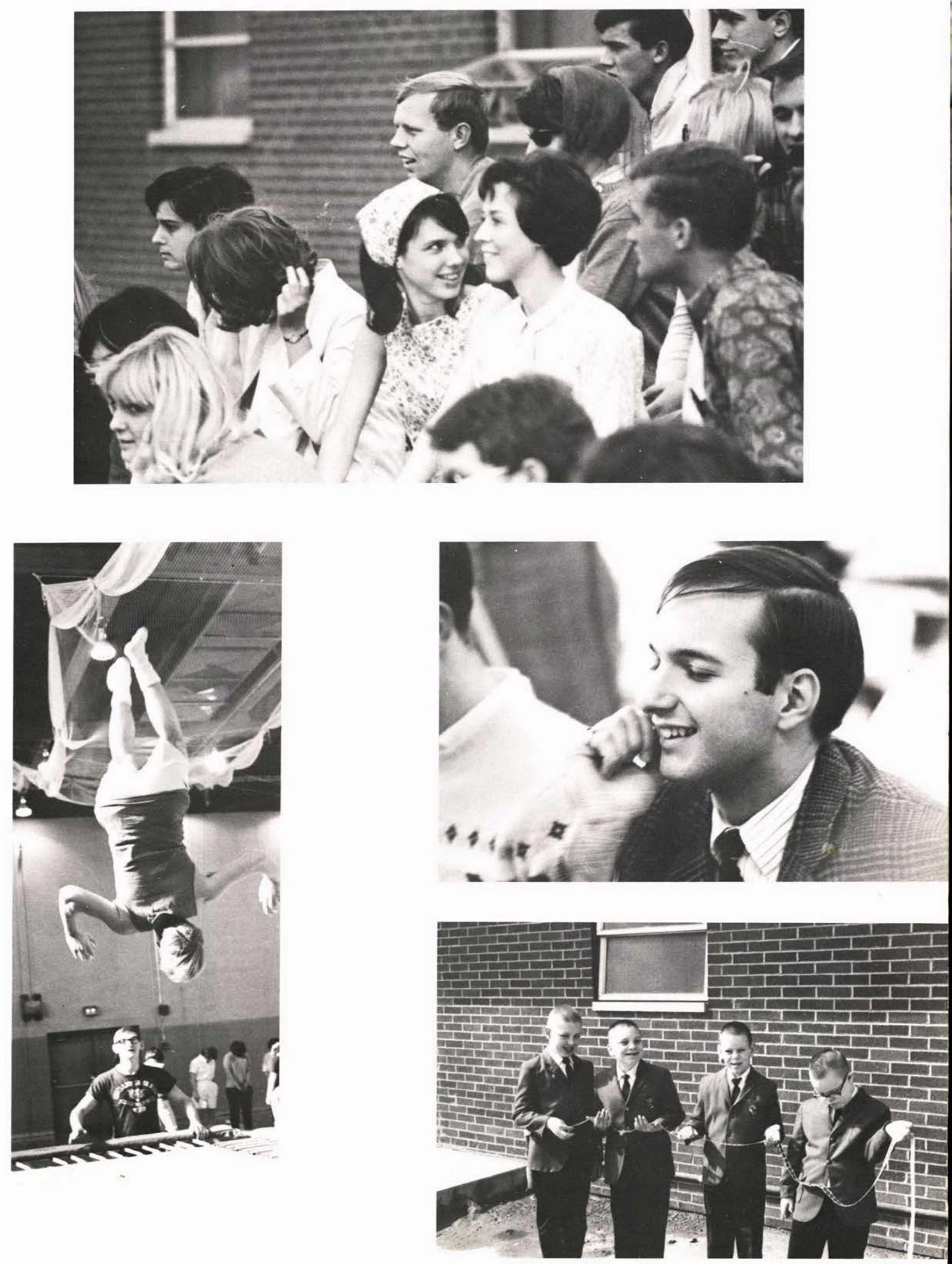

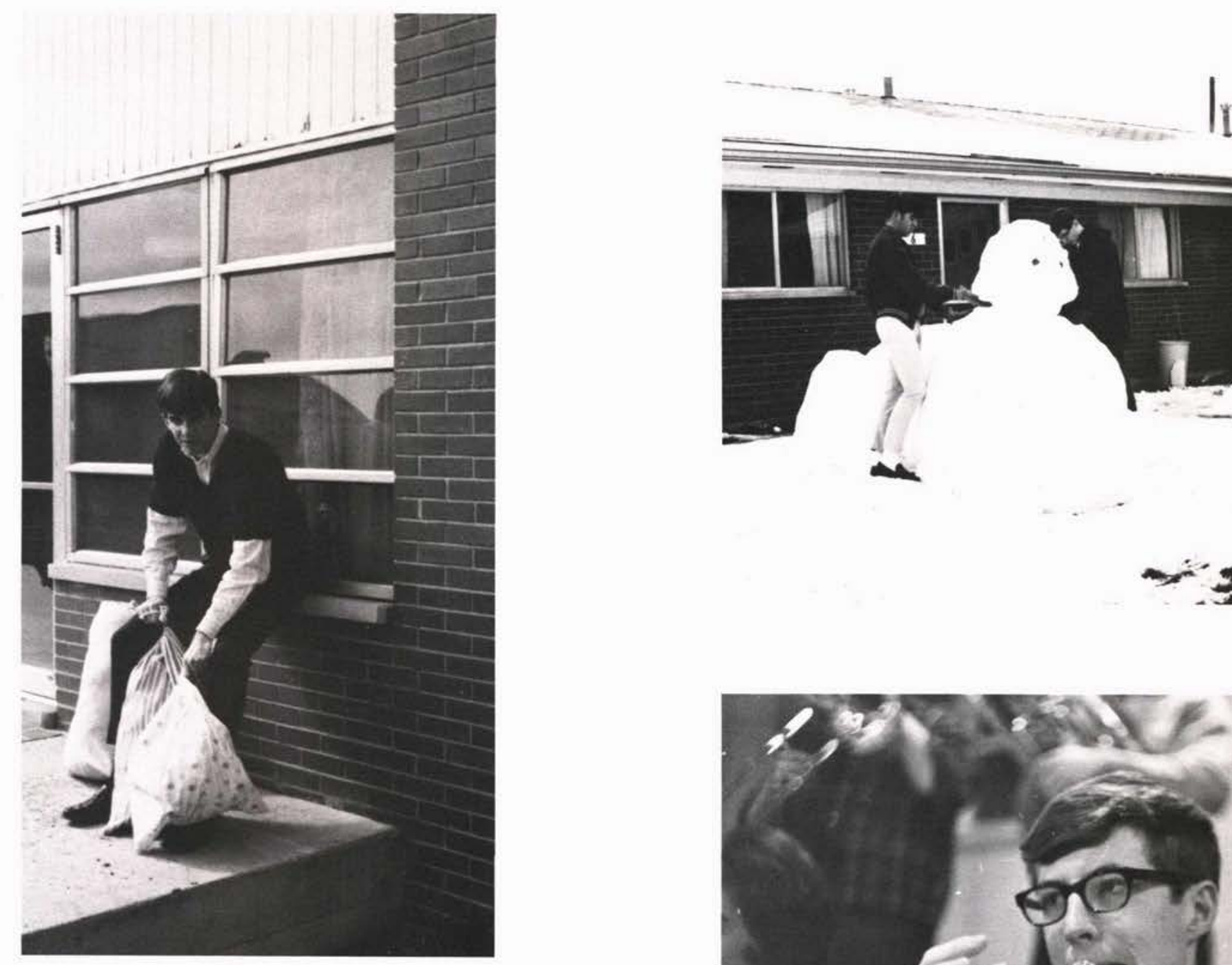

I.

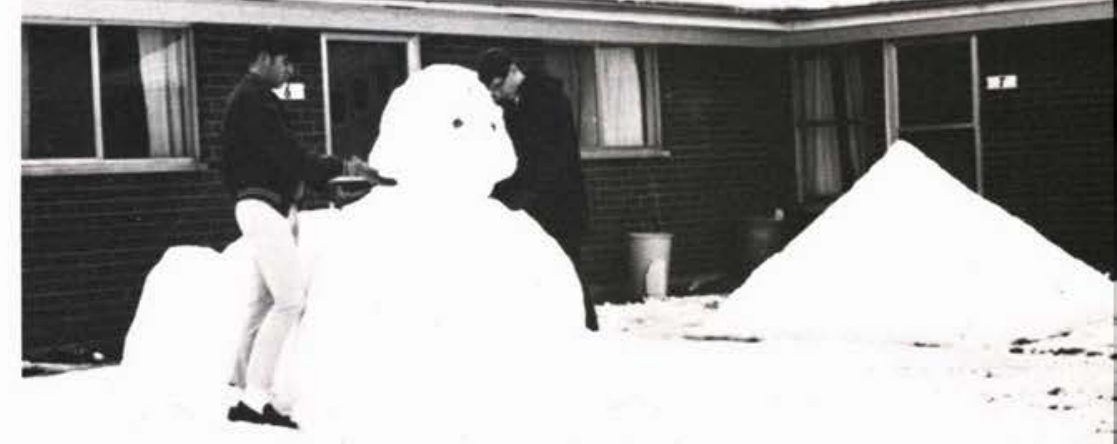

$$
-3 x^{2}
$$
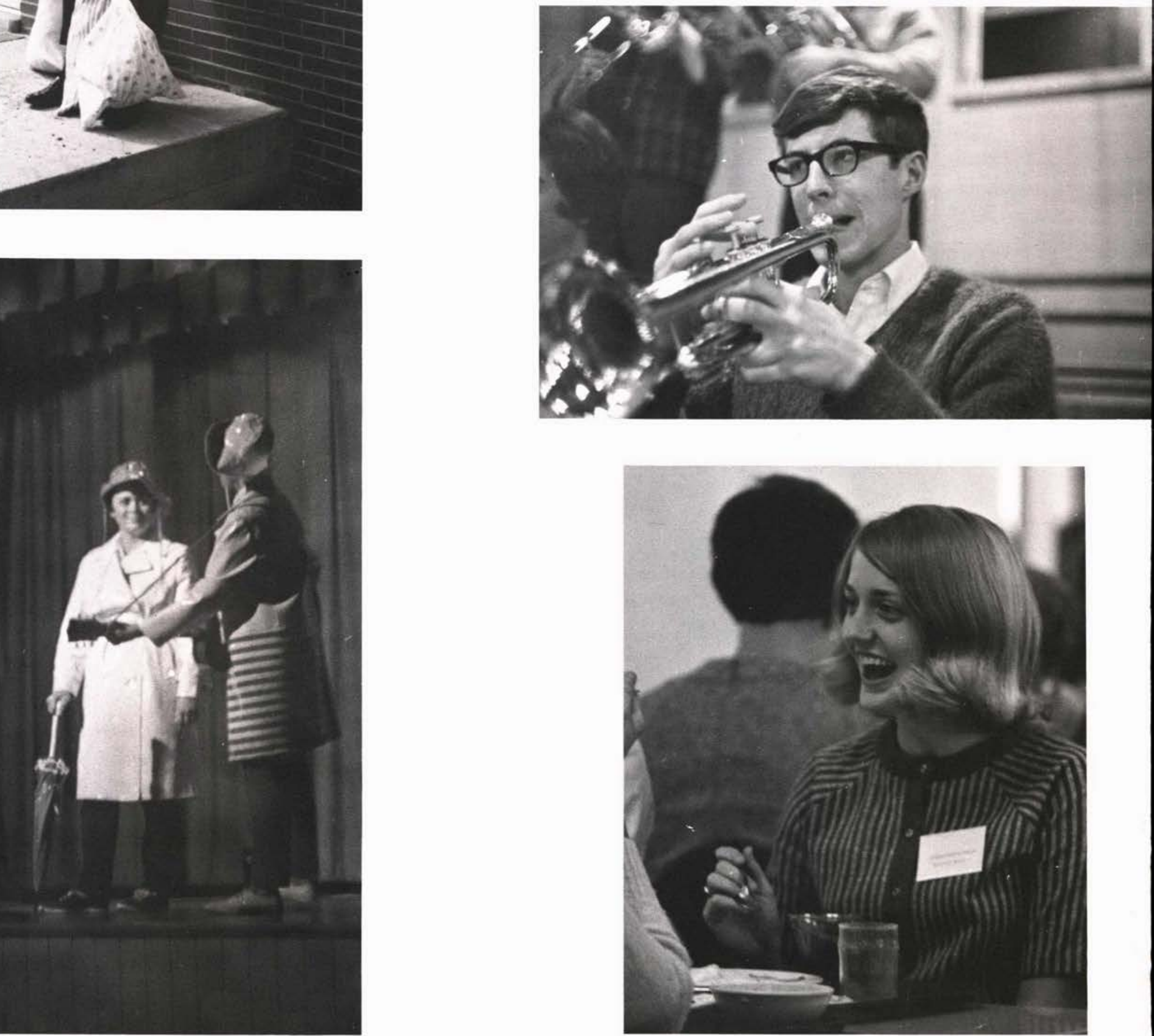

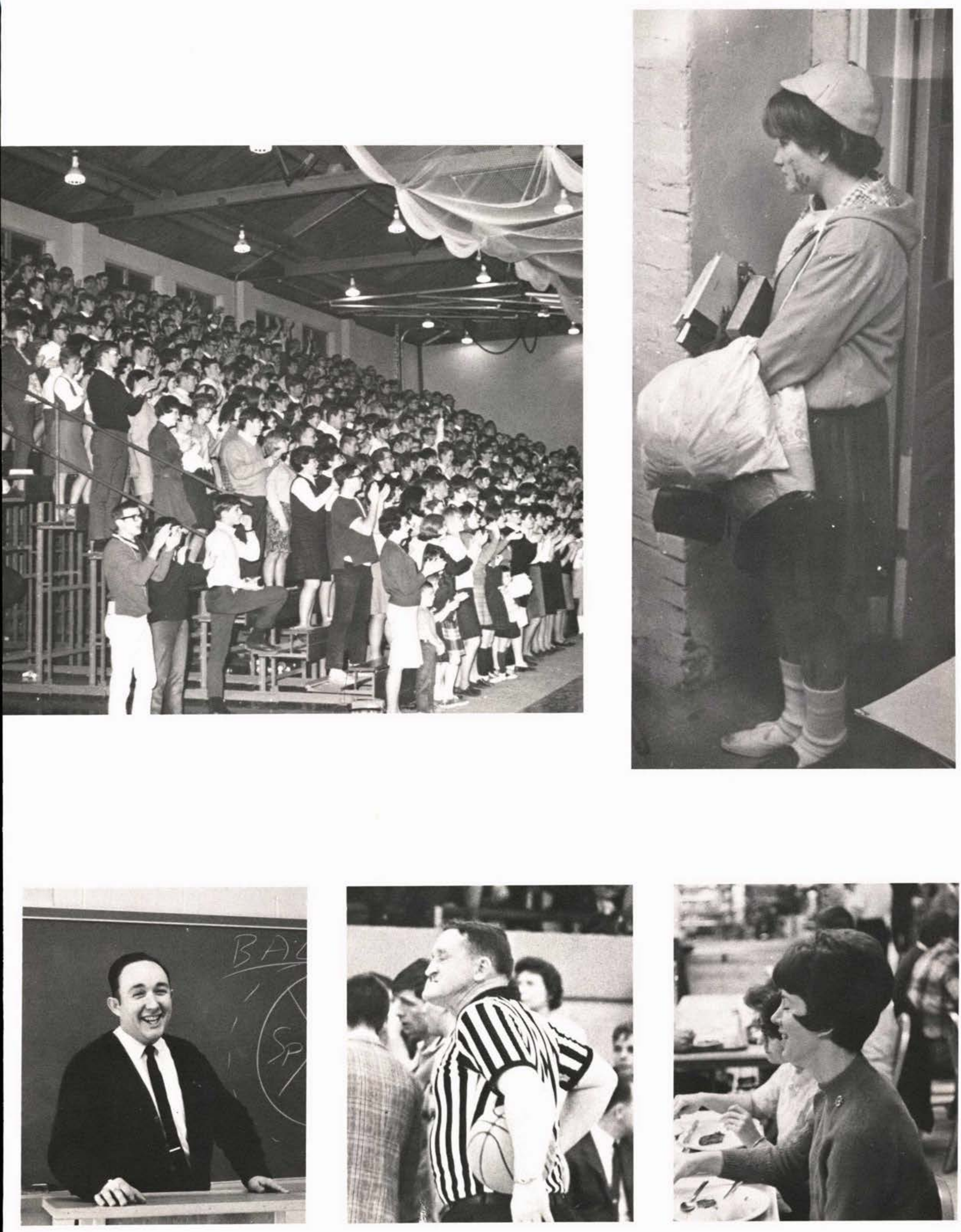


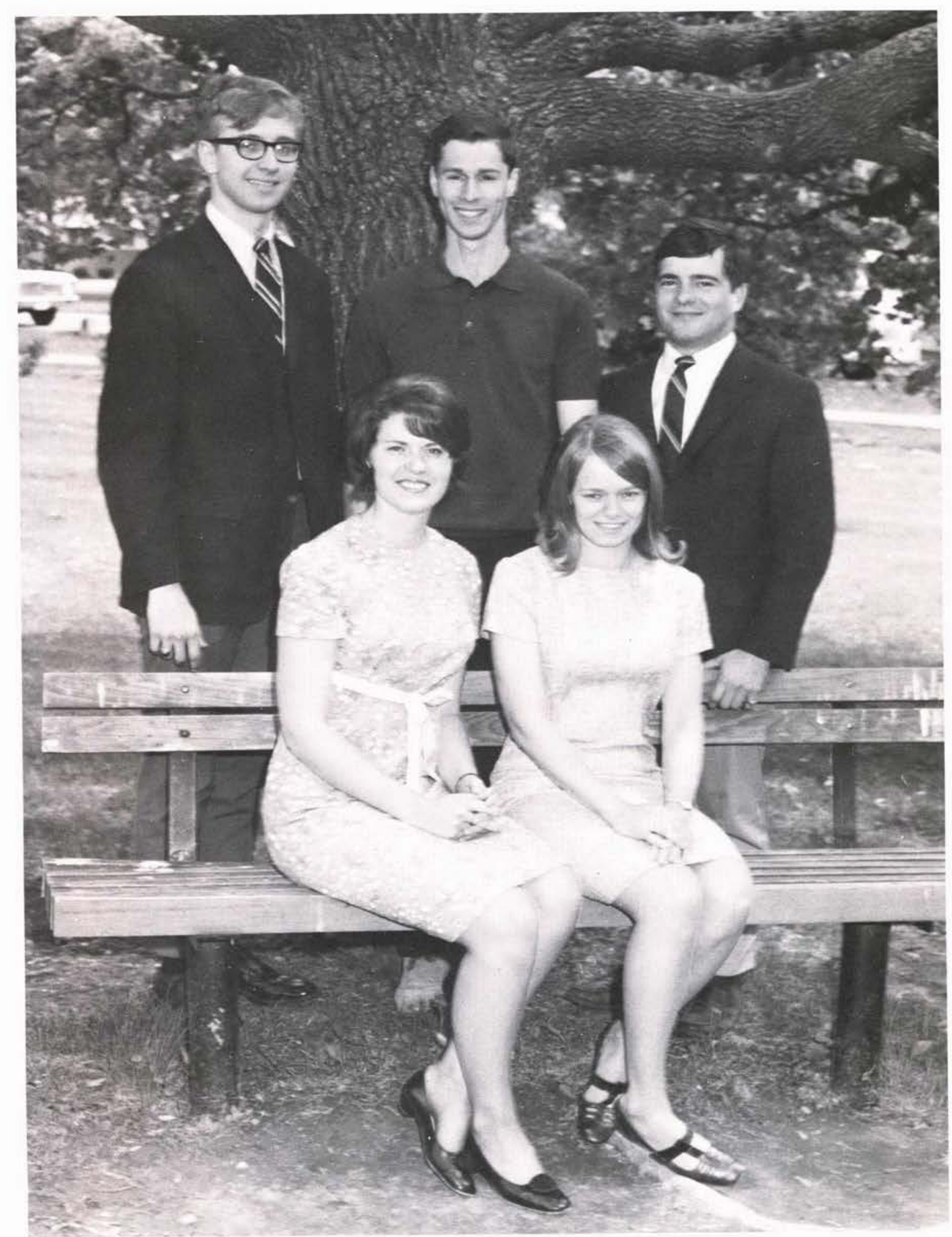

President-Ed Smelser

Vice-President-Martha Brown

Secretary - Sara Empet

Treasurer - Ernest Music

Chaplain-Roy Barnhar

Not Pictured: Advisor - Mr. Riter

Student Council - Paul Entner, Steve Snyder 



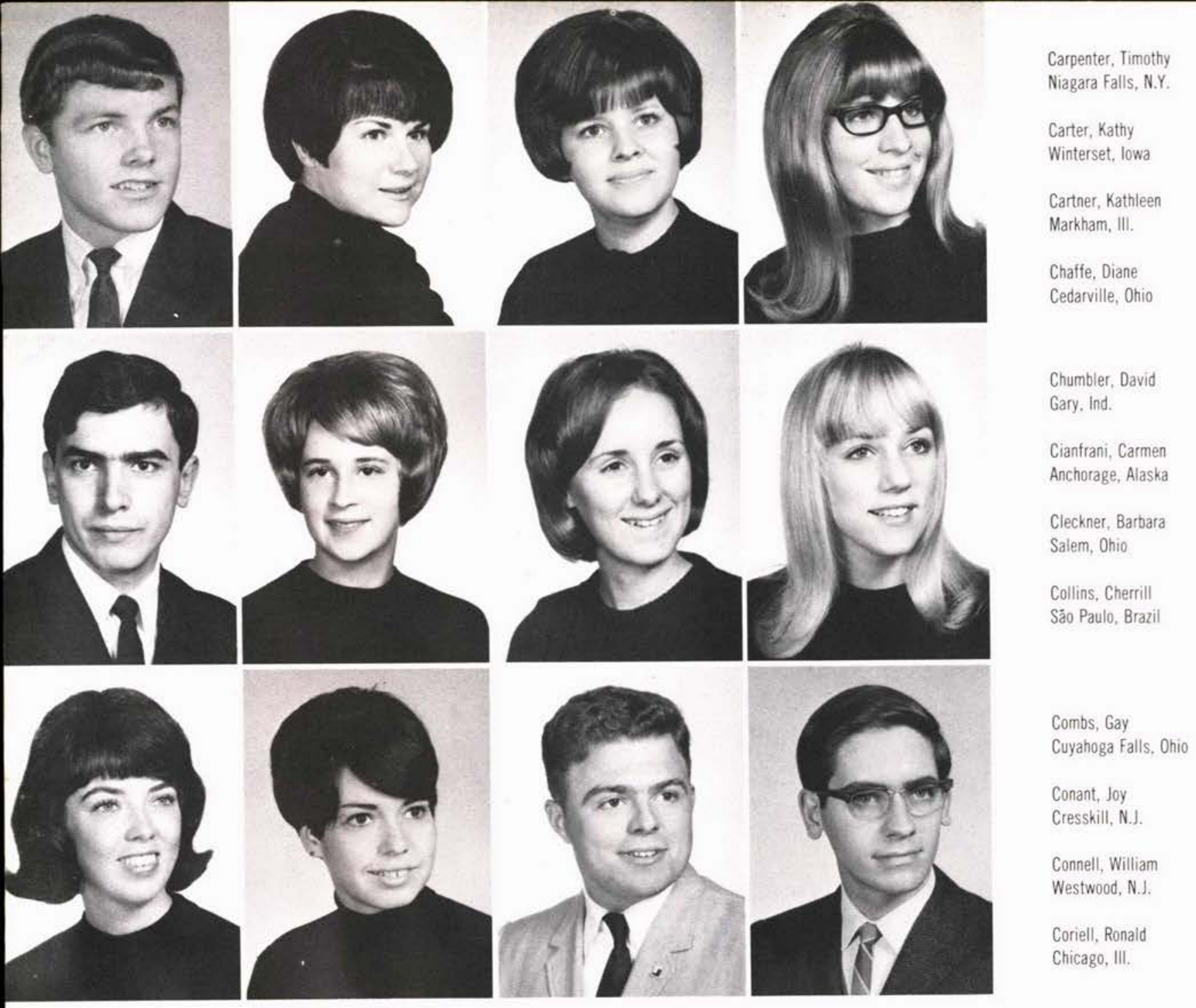



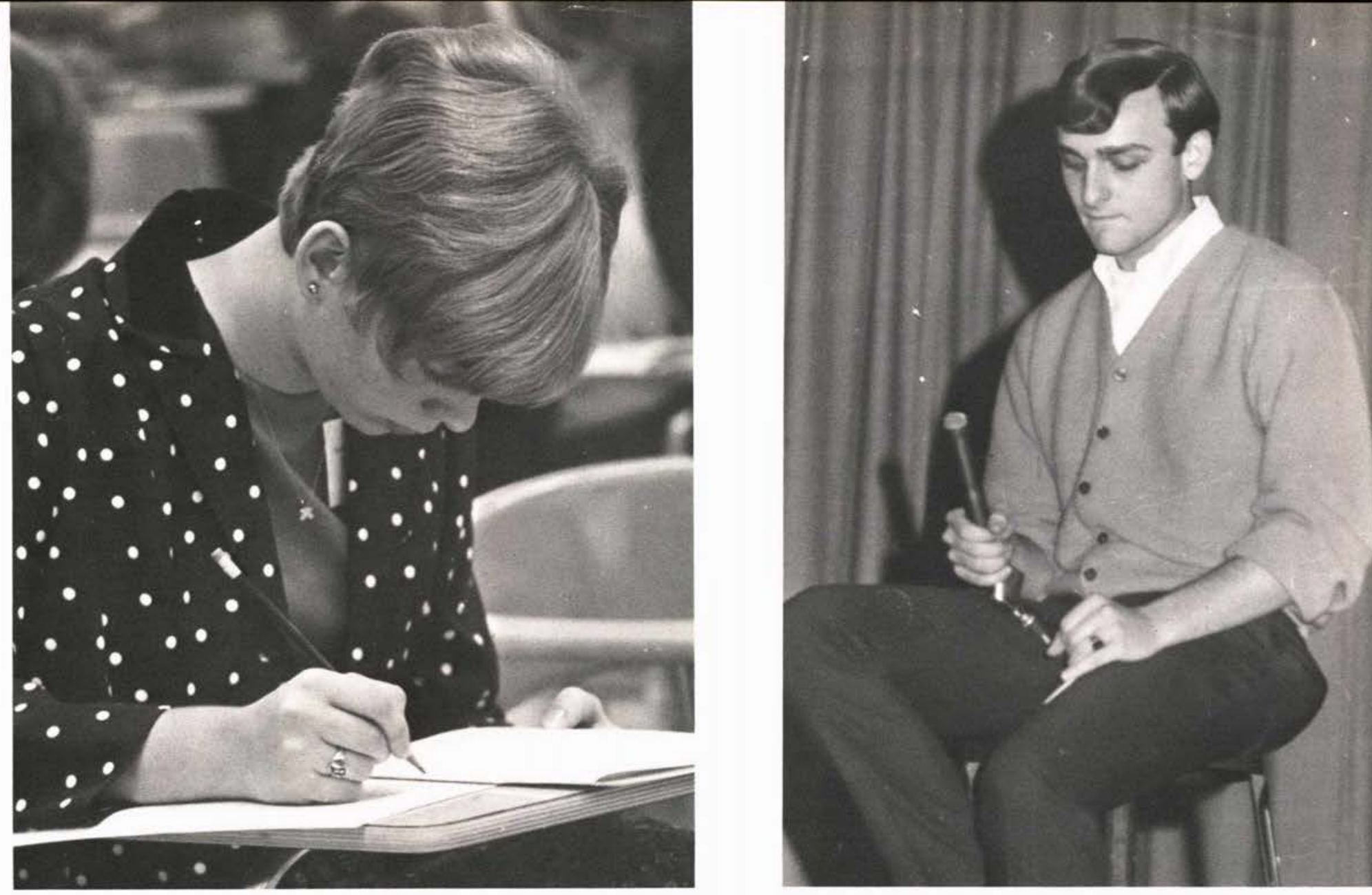

Courtot, Max Cedarville, Ohio

Daab, Donna

East Moline. III.

Darrow, Gary

Westwood, N.J.

Davis, Larry

Homer City, Pa.

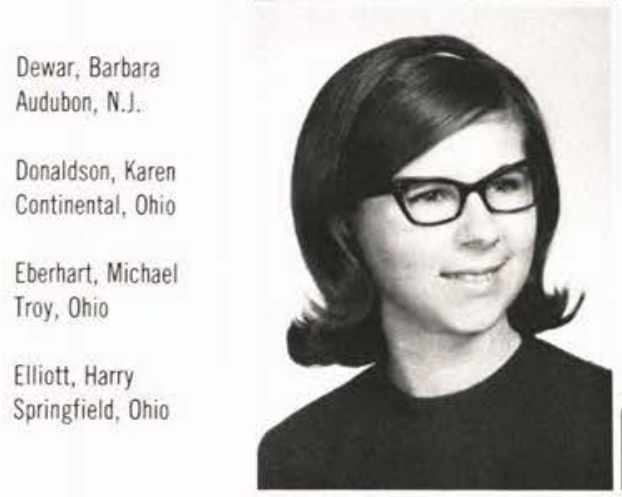

Empet, Sara

Kingsley, $\mathrm{Pa}$

Entner, Paul

Dunkirk, Ind.

Evans, Sylvia

Cedarville, Ohio

Fitch, Darlene

Jamesville, N.Y.

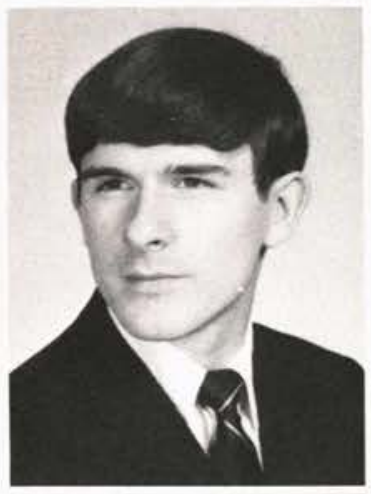

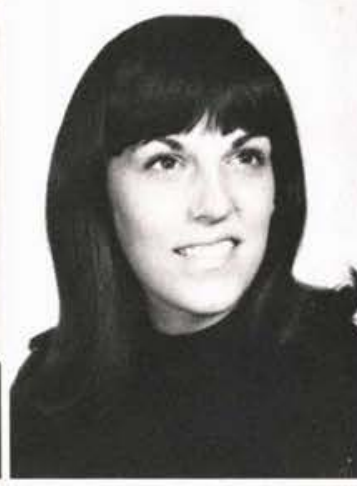
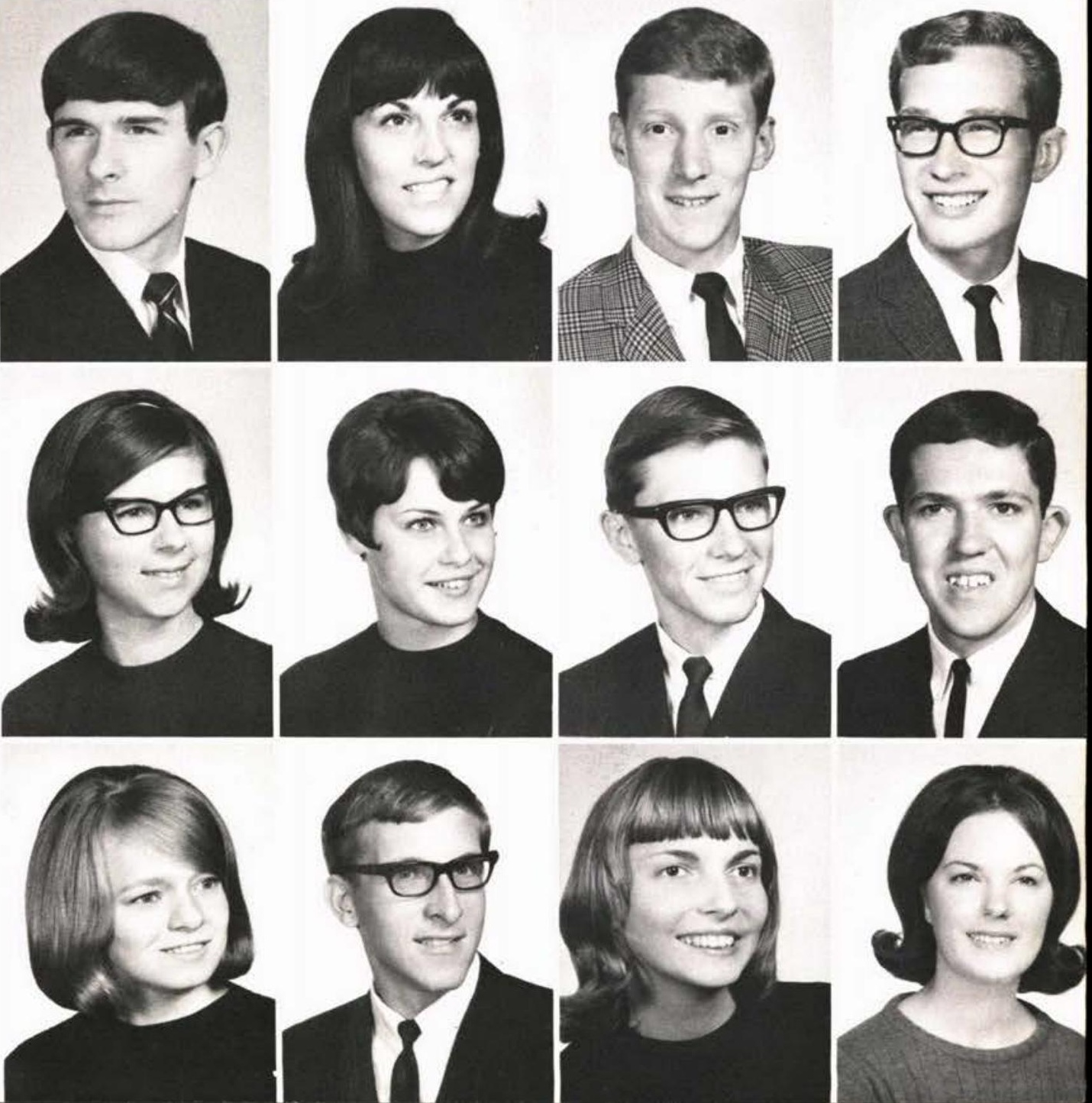



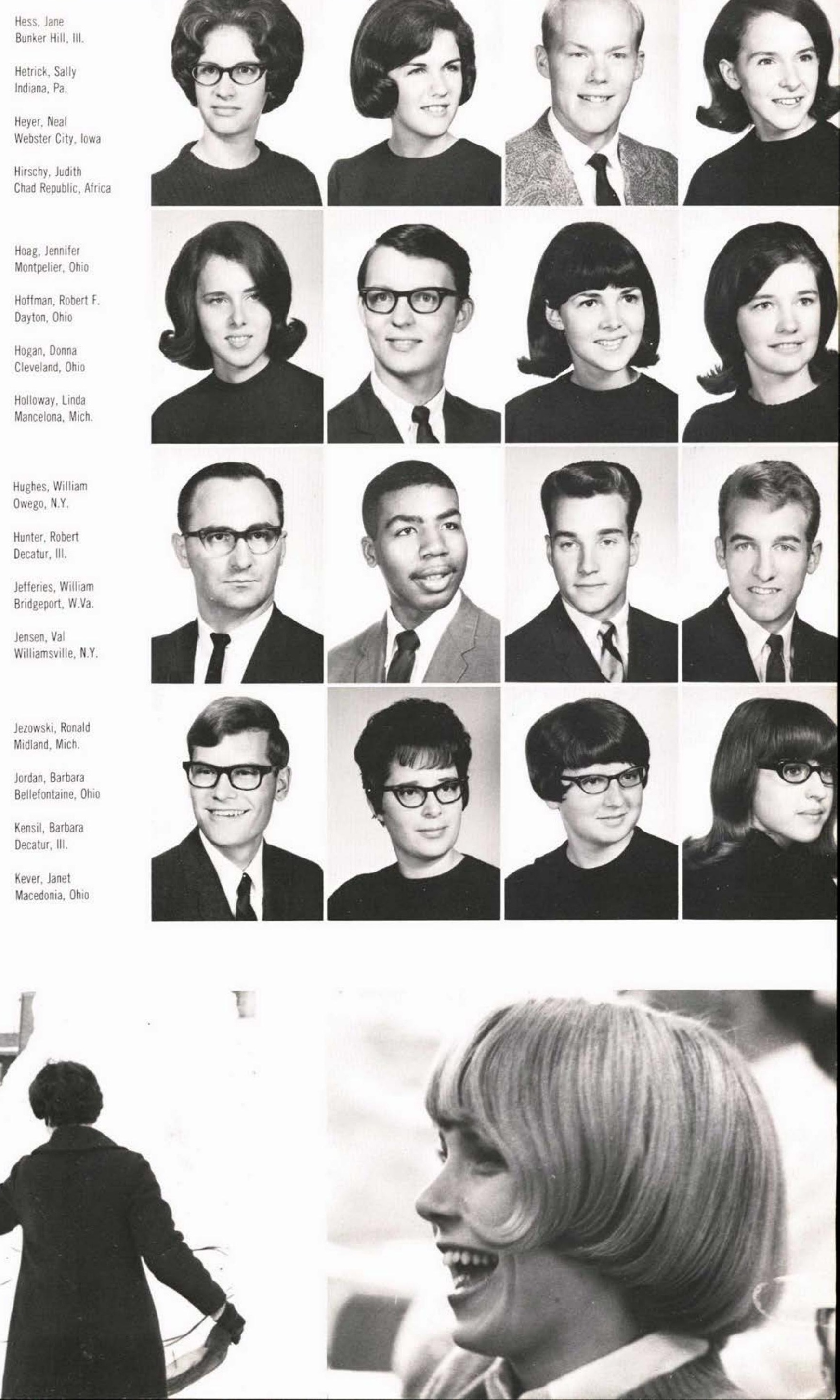




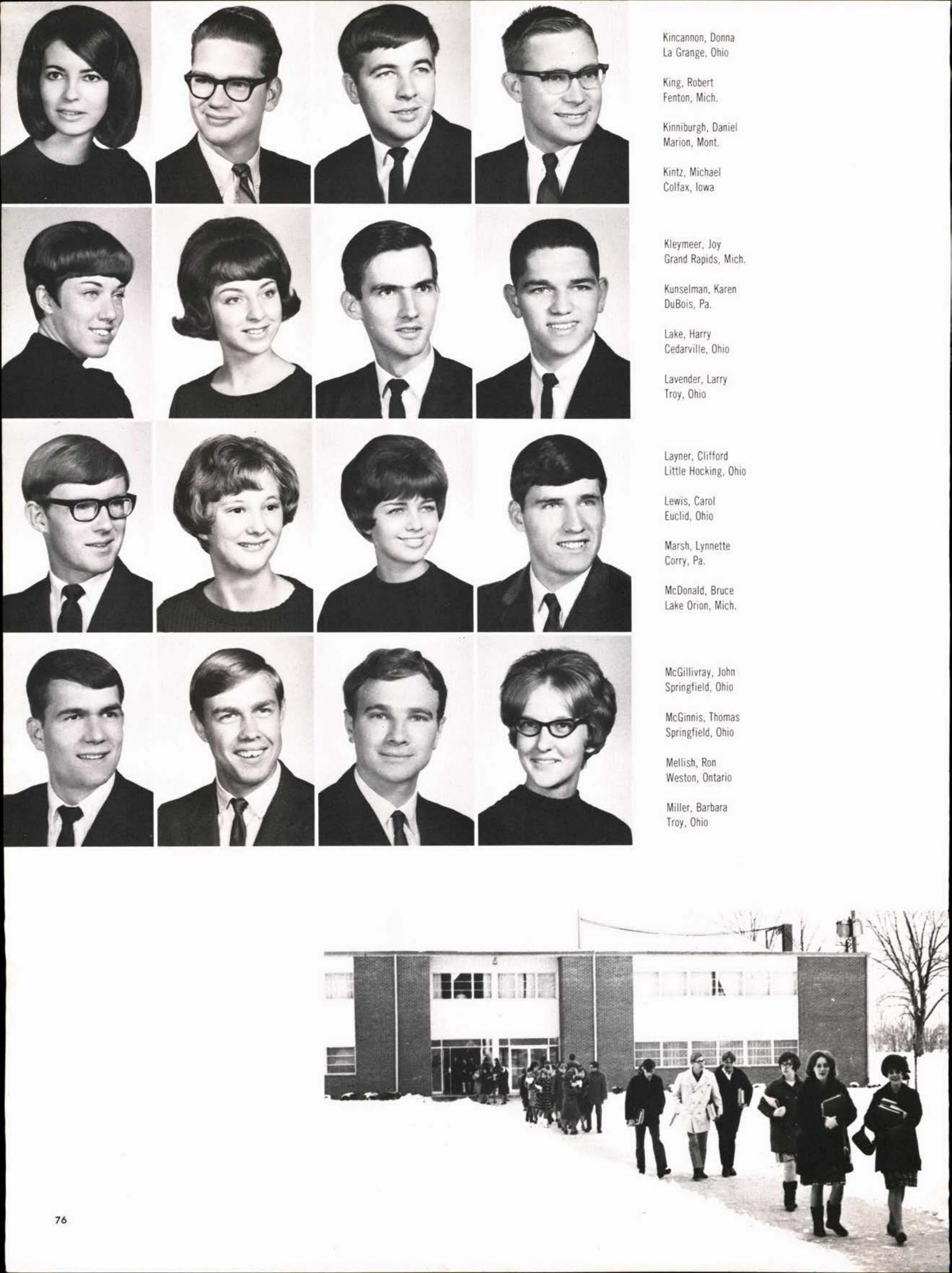






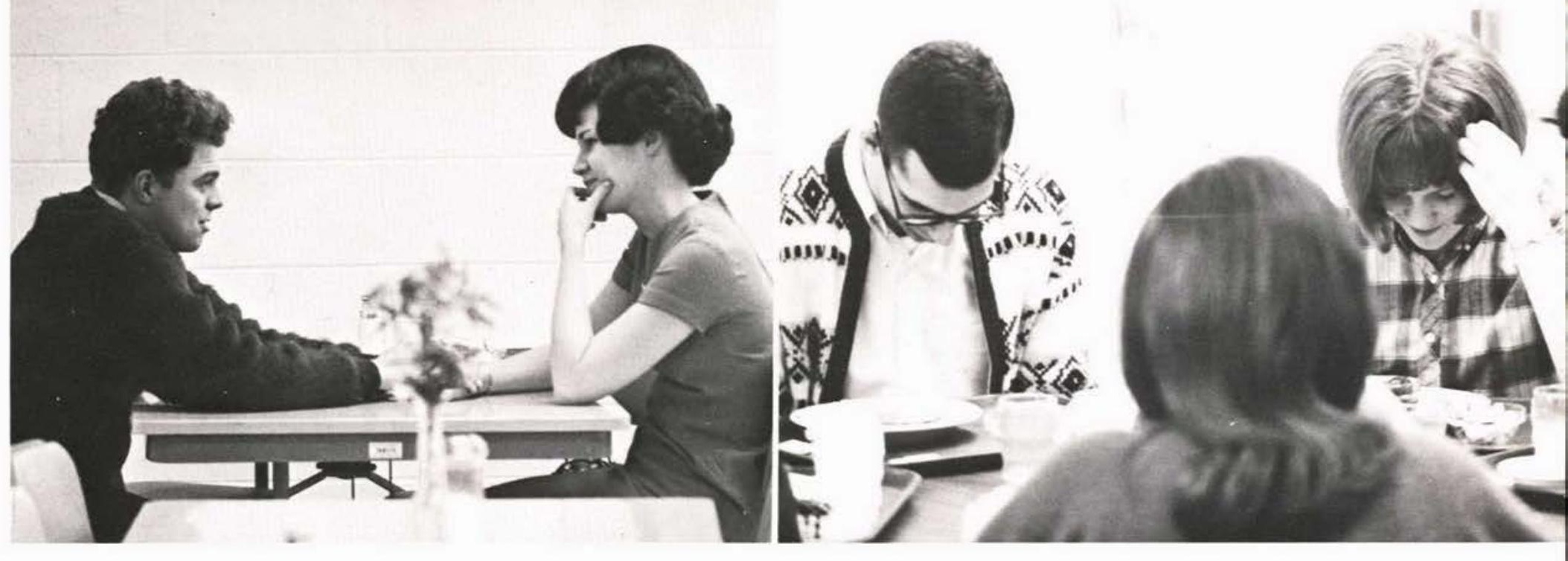

Thorne, Susan

Medford, N.J.

Towle, Nancy

Pataskala, Ohio

Trumbull, Chery

Cedarville, Ohio

Wallis, Nelson

Thurston, Ohio
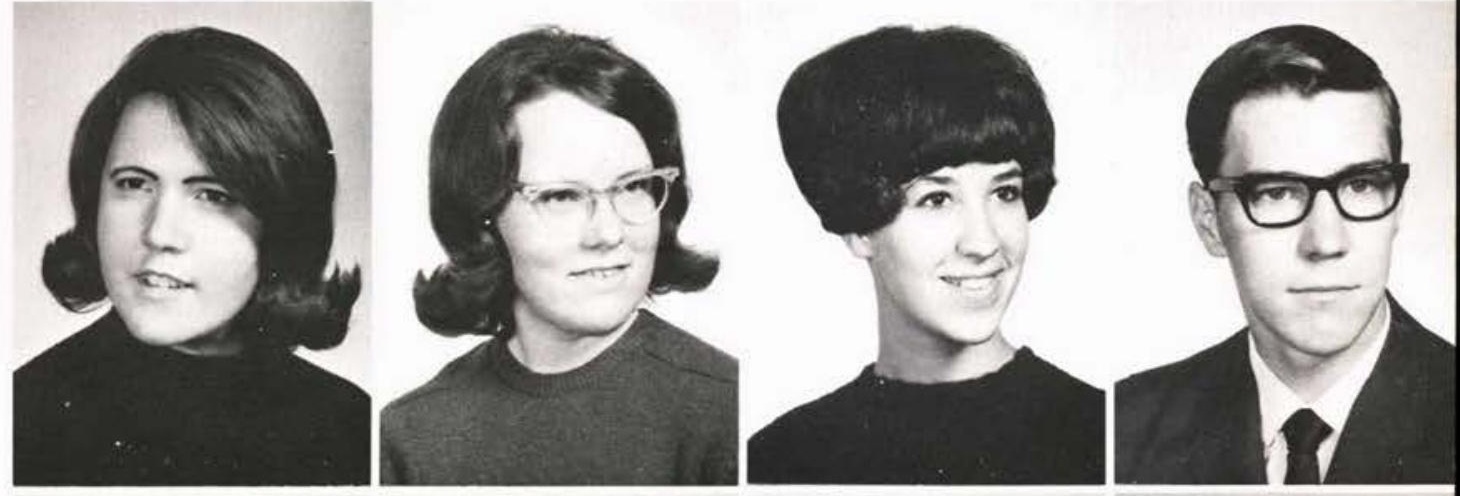

Warren, Carol

Danville, Alabama

Warren, Timothy

Allegan, Mich.

Watson, John

Mishawaka, Ind.

Whattoff, Robert

Ames, lowa
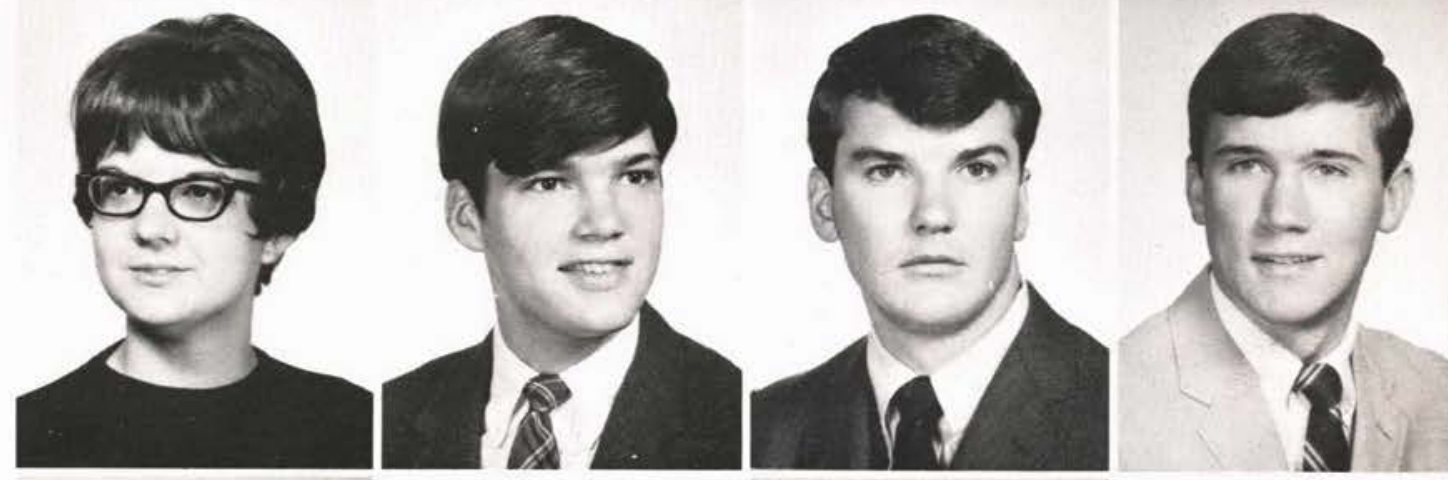

Willis, Pamela

Wenatchee, Wash.

Work, Dale

Coudersport, $\mathrm{Pa}$

Yeater, Sharon

Wampum, $\mathrm{Pa}$.

Yoder, Douglas

West Liberty, Ohio
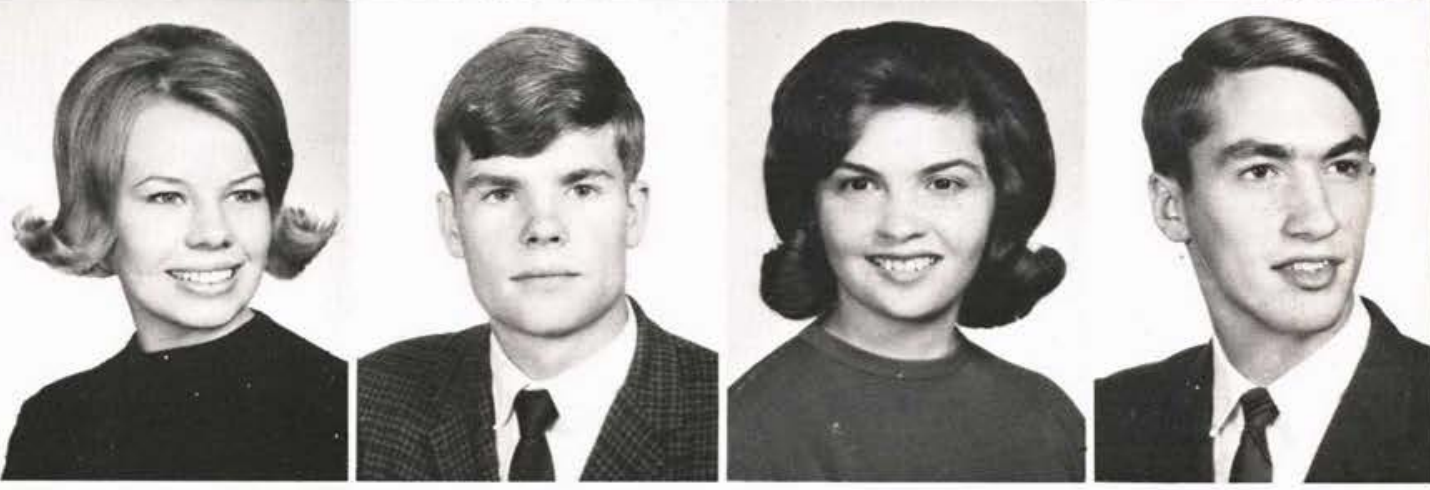

Young, Jeff

Lorain, Ohio

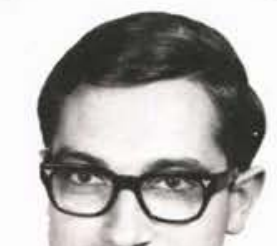

,

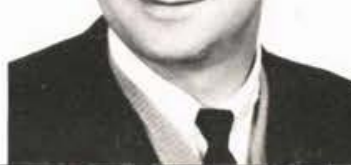




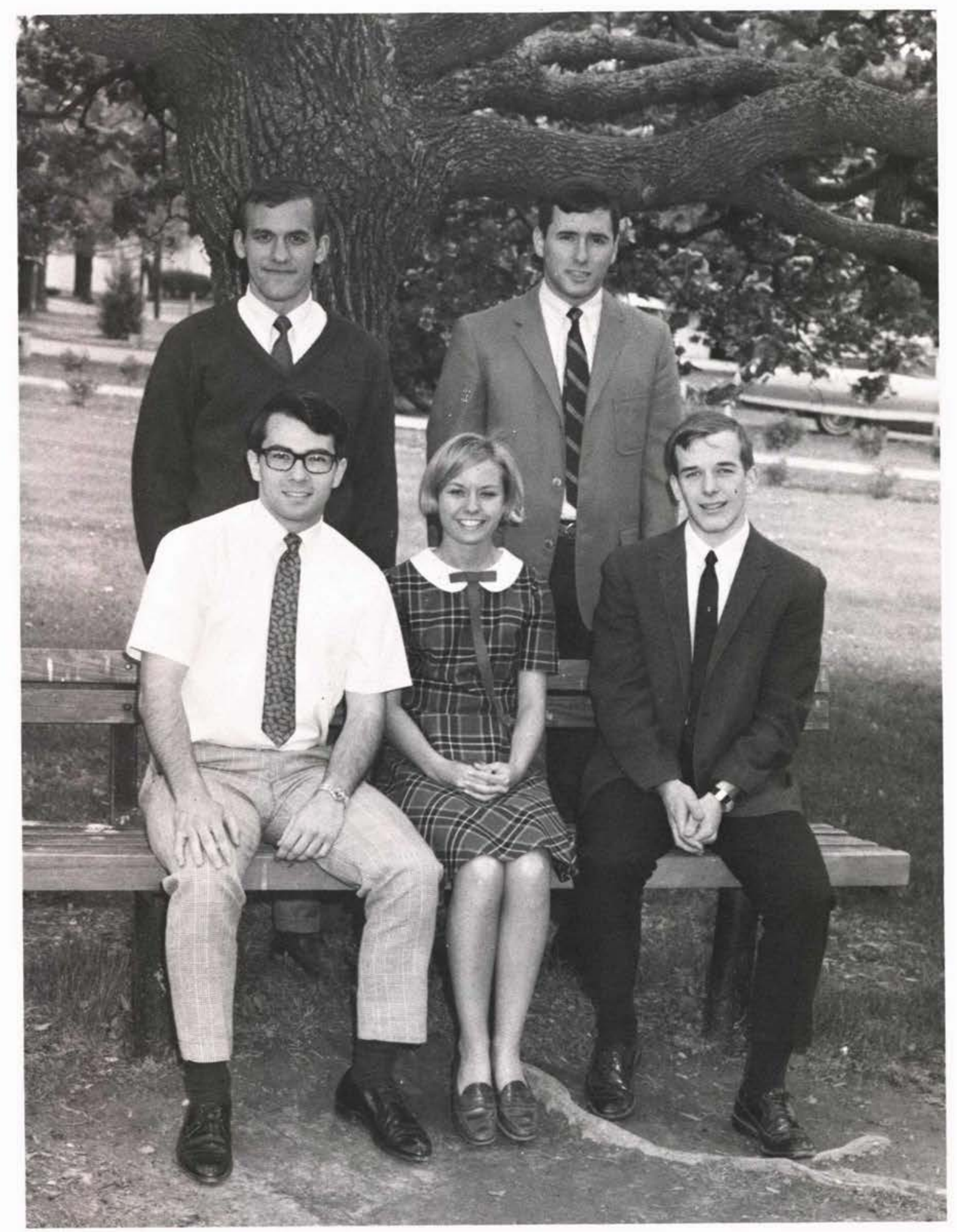

President-Roger Cook

Secretary - Janis Martin

Treasurer - Ken Cole

Student Council-Loren Reno

Chaplain-Robert Carmean

Not Pictured: Advisor - Mr. Spencer

Vice-President-Dan Haseltine

Student Council - Jim Jeremiah 
Abrams, Dorothy

Skaneateles, N.J.

Albright, Kathy

Washington, lowa

Allen, Robert

Hurlock, Md.

Ambroza, Douglas Lima, Ohio

Anderson, Lyle

Des Moines, lowa

Ashley, Janet

Avon Lake, 0.

Austin, Bonfiles Chillicothe, Ohi

Austin, Jane

Bridgetown, N.J.

Bailey, Francis

Cambridge, 0.

Bailey, Nancy

Traverse City, Mich.

Baker, Donita

Cardington, 0 .

Bales, Marilyn

Pana, III.

Beck, Linda

La Porte, Ind.

Beltle, Allen

Manasquan, N.J.

Bennett, Beth

Lyndhurst, 0 .

Bodden, David

Oley, $\mathrm{Pa}$.

Boertje, Darwin

Lima, 0.

Bonzo, Pat

Portsmouth, 0 .

Borger, Karen

New Castle, Pa.

Braswell, Sylvia Sebring, Fla.

Bratka, Donna

West Liberty, 0

Braun, Dale

Wheaton, III.

Brock, Janice

Columbus. 0.
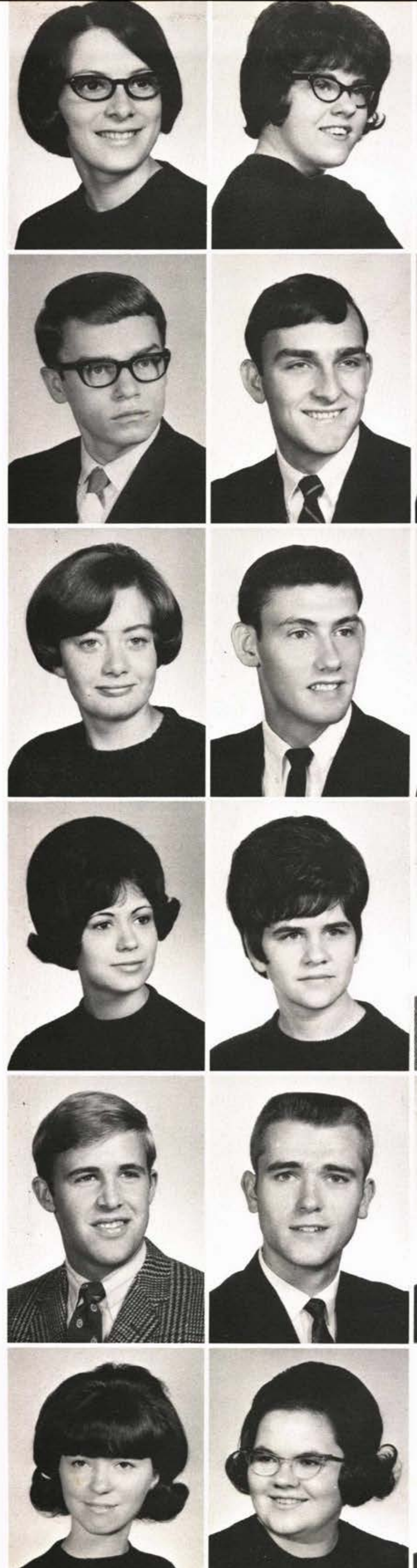
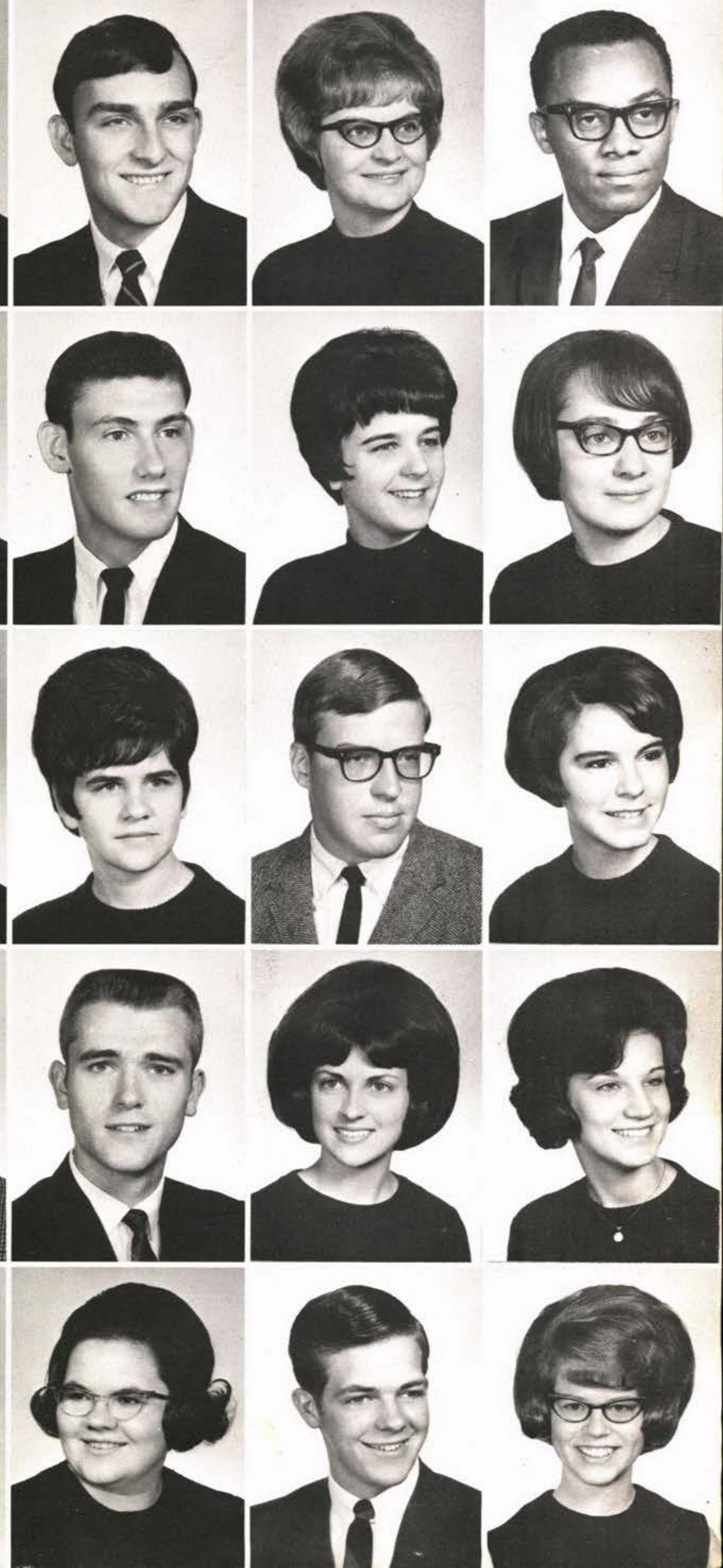



Mansker, Pamela

Connellsville, $\mathrm{Pa}$.

Martin, Janis

Crawfordsville, Ind.

McCann, Judy

Quincy, Mich.

\section{McCullough, Max}

Coshocton, 0.

McDonald, Becky

Cedarville, 0 .

McDowell, Gary

Hobart, Ind.

Meyer, Viann

Waterloo, lowa

\section{Millard, Linda \\ Fairborn, 0. \\ Miller, Alice \\ Columbus, 0 . \\ Miller, Joyce \\ Williamston, Mich. \\ Mills, Roger \\ Columbus, 0}

\section{Mitchell, Richard}

Malaga, N.J.

Moorman, Alice

Xenia, 0 .

Mott, Shirley

Angelica, N.Y.

Motter, Jerry

Genoa, 0.

Muirhead, Margaret

Fulton, III.

Murphy, Coleen

Santa Barbara, Cal.

Nelson, Karen

Quincy, III.

Nelson, Wayne

Shawnee Mission, Kansas 
Lake Orion, Mich.

Price, Wilbur

So. Charleston, 0.

Prindle, Priscilla

Bellevue, Mich.

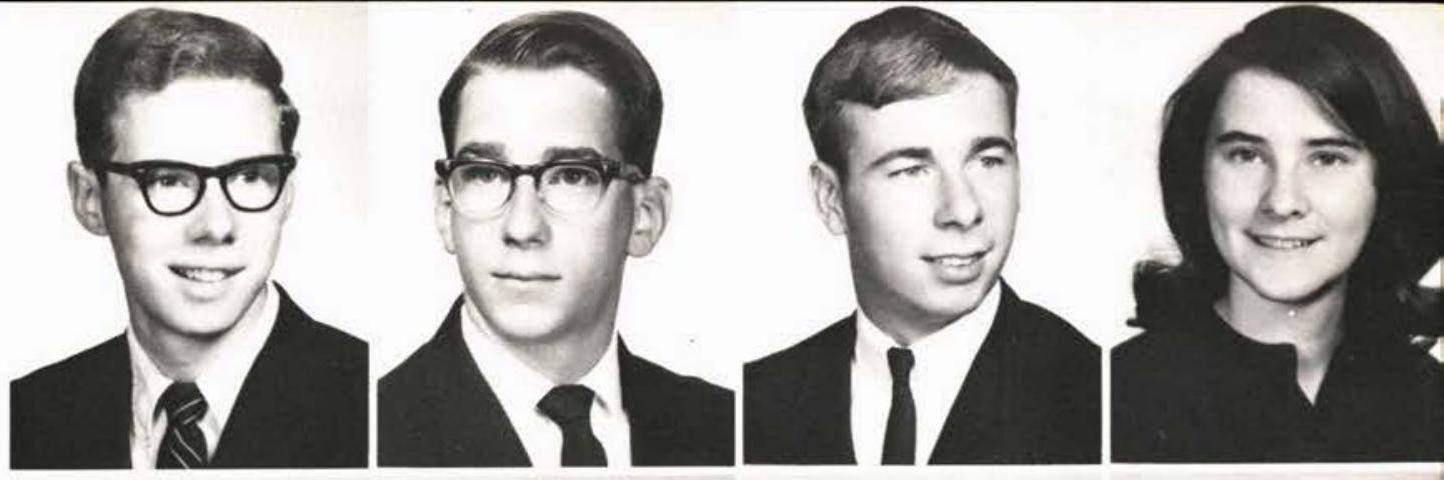

Pugh, Lawrence

Rome, N.Y.

Radcliff, Larry

Caldwell, 0 .

Radcliffe, Diana

Wakenian, 0 .

Ray, Sue

Decatur, III.
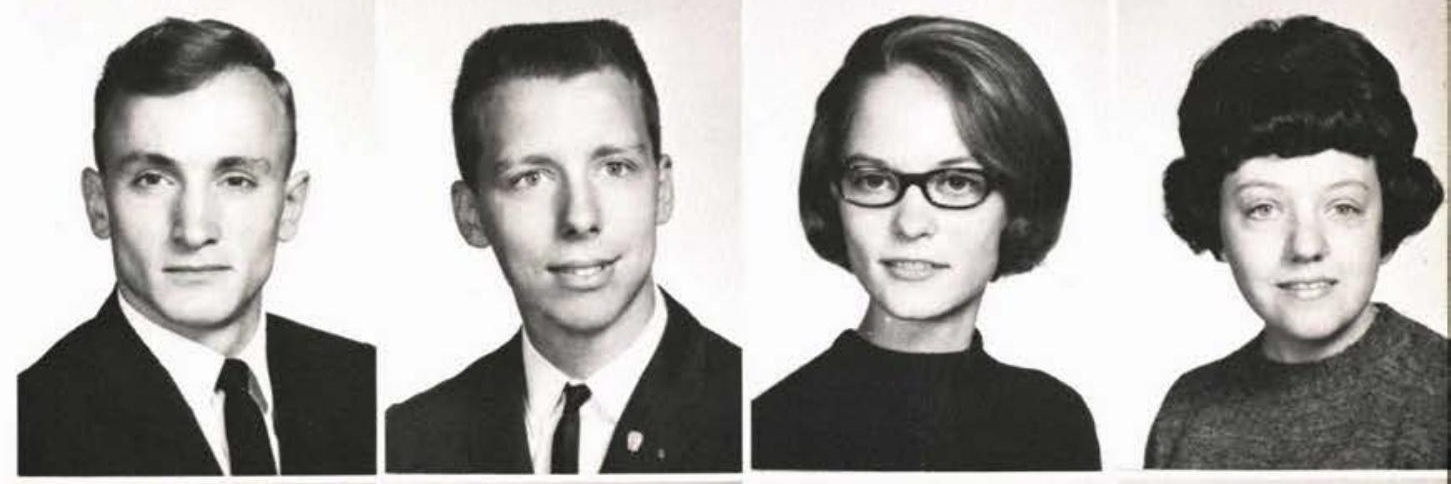

Reed, William

New Kensington, $\mathrm{Pa}$.

Reeder, John

Youngstown, 0.

Reese, Betty

Lock Haven, Pa.

Rehn, Judy

Orangevale, $\mathrm{Cal}$.
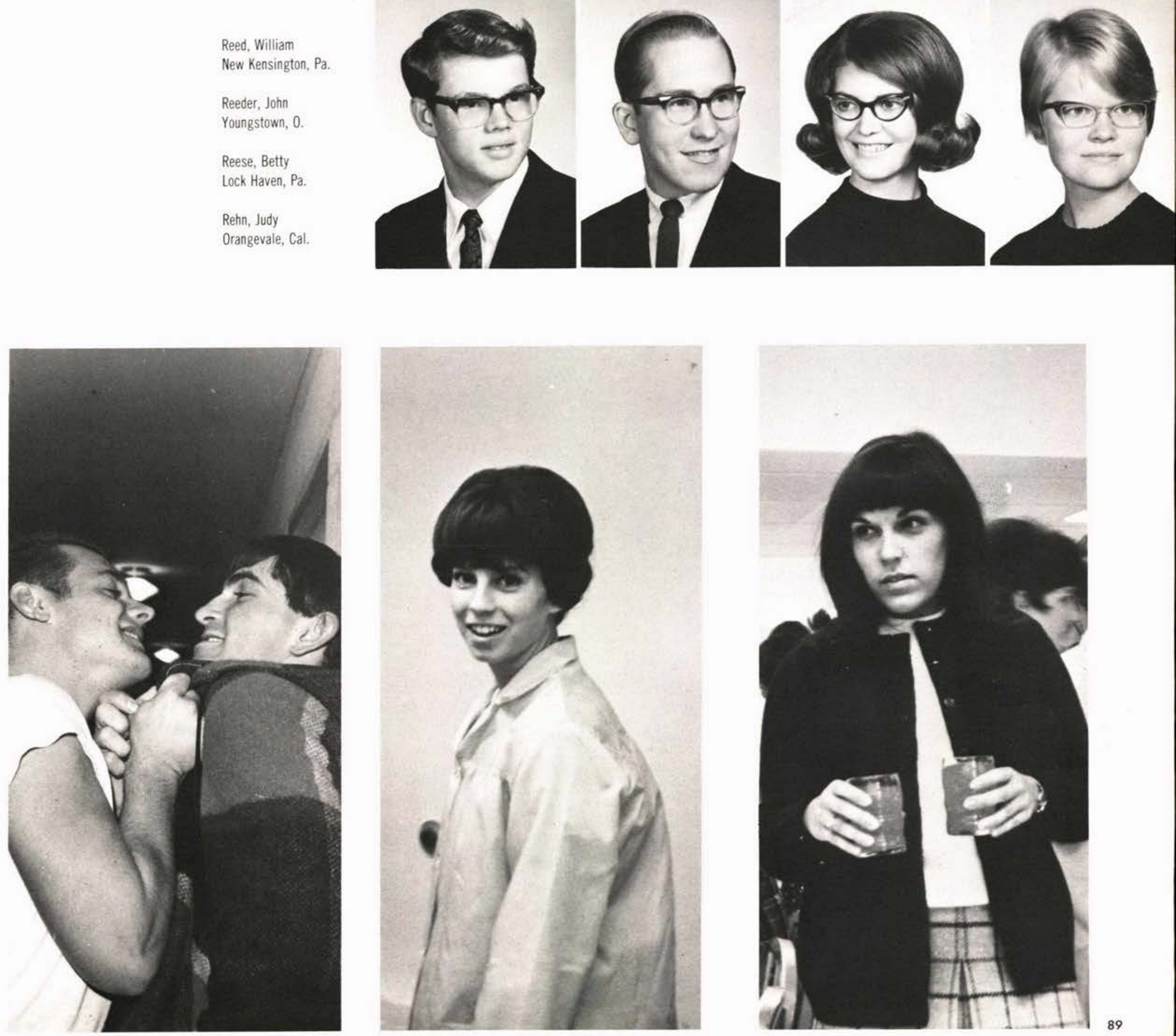



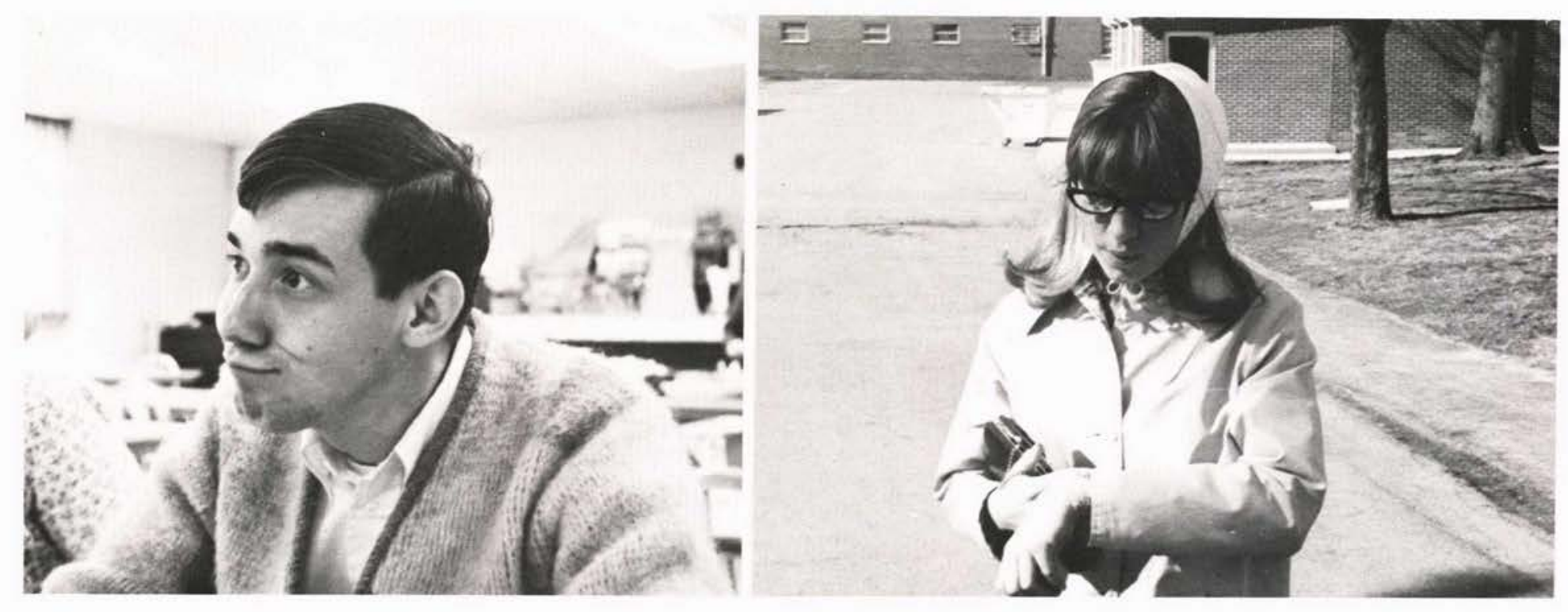

Stewart, Carol Indianapolis, Ind.

Stillwell, Dennis

Huntsburg, 0 .

Stora, Dennis

Allegan, Mich.

Stryker, Tim

Dayton, 0 .
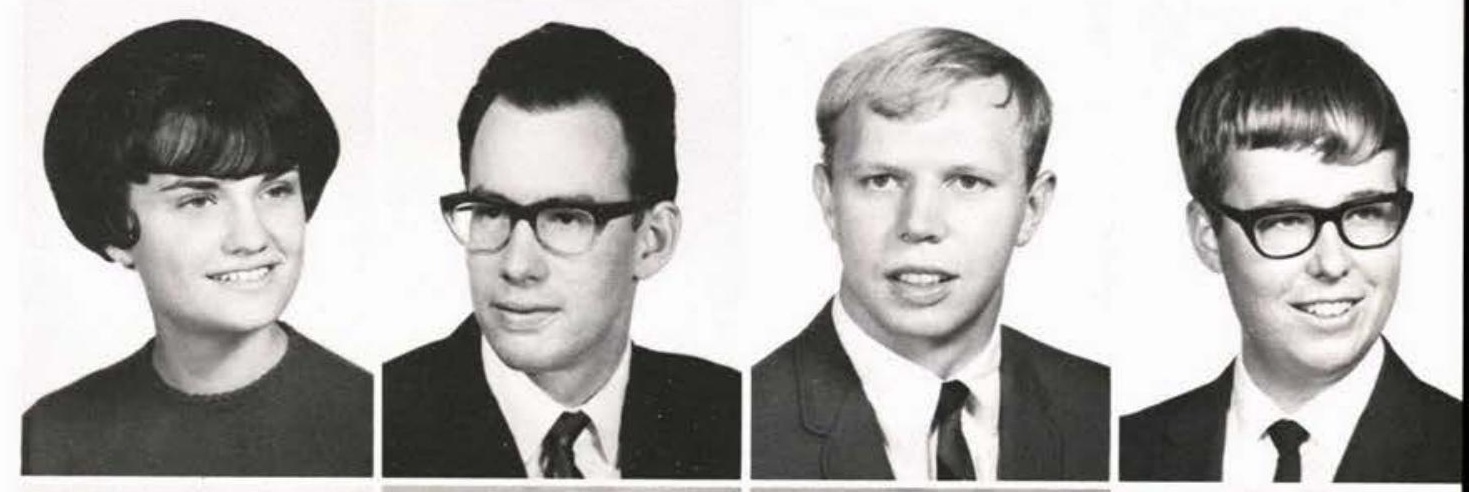

Sturdevant, Patricia Union City, Pa.

Taylor, Richard

Lakewood, 0.

Taylor, Steve

Philadelphia, $\mathrm{Pa}$.

Treat, Ken

Galeton, $\mathrm{Pa}$.
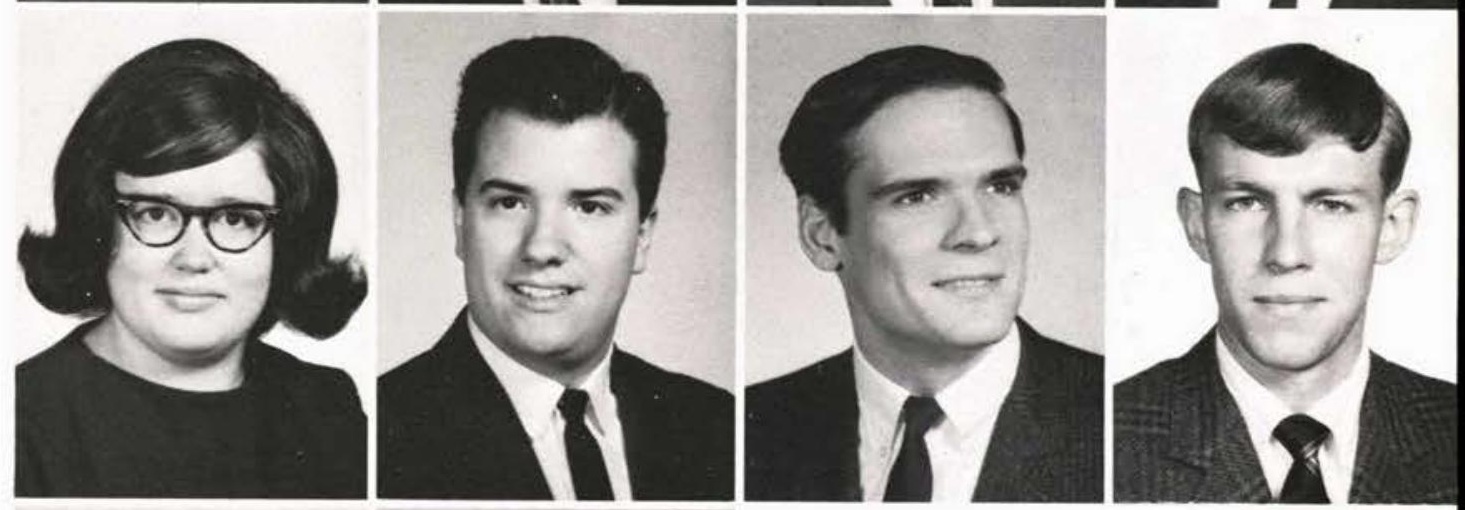

Trotter, Sharon

Liverpool, 0 .

Trumphour, Mary

Warren, 0.

Wagner, Karolyn

Marietta, 0 .

Walstrum, Ann

Jackson, Mich.
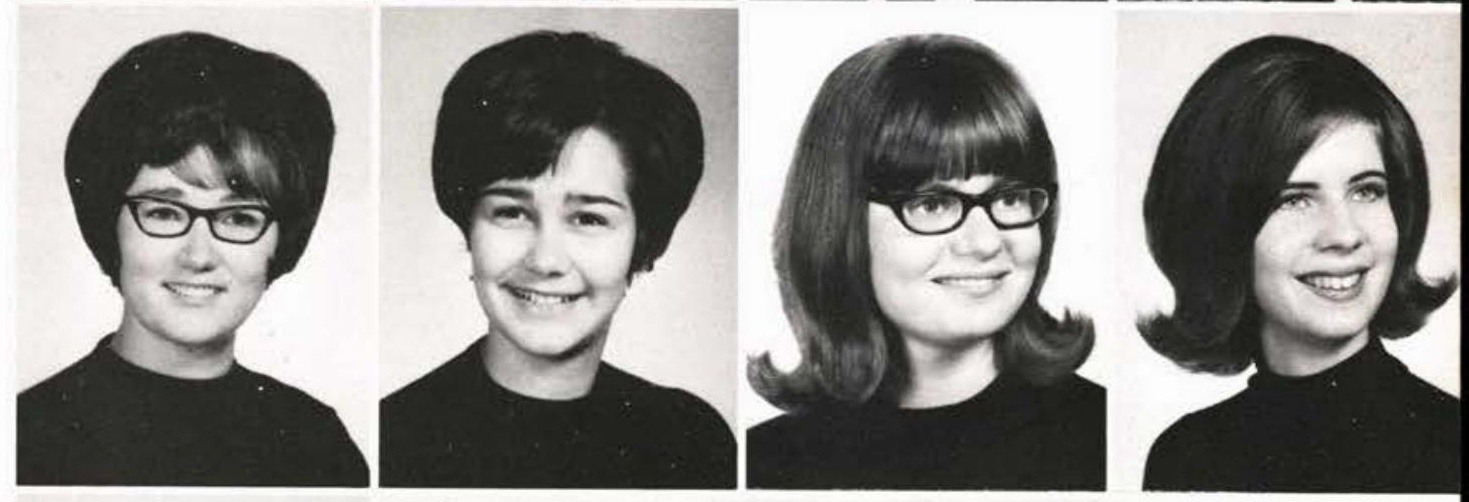

Waltz JoAnn
Warrensville, Pa.

Warnken, Estelle

Wakarusa, Ind.

Watson, Nola

Wakeman, 0 .

Webster, Dan

Hillsboro, 0 .
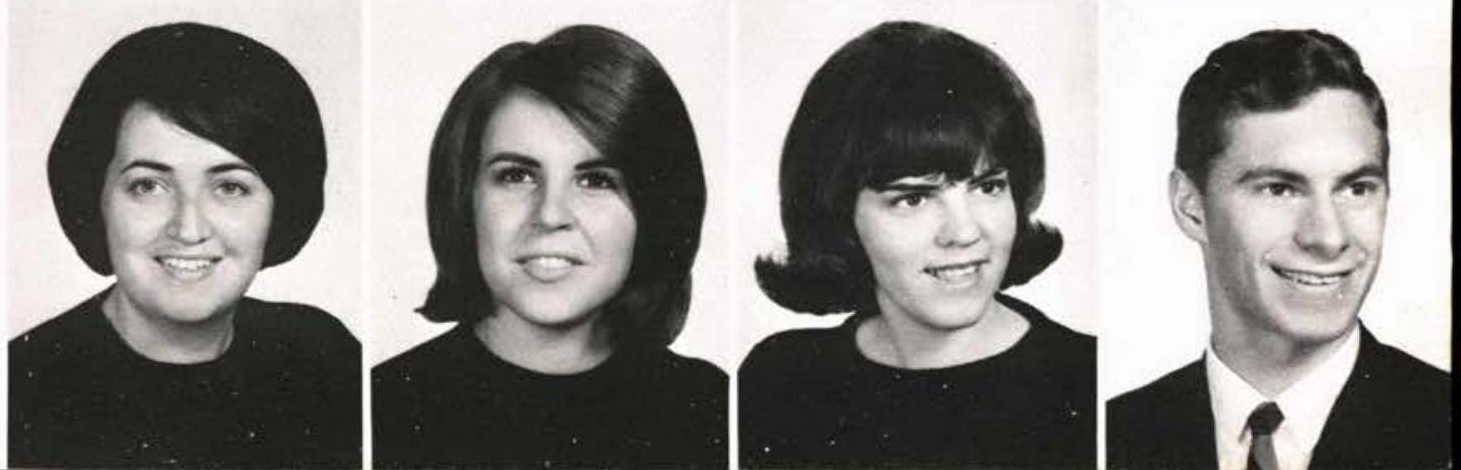

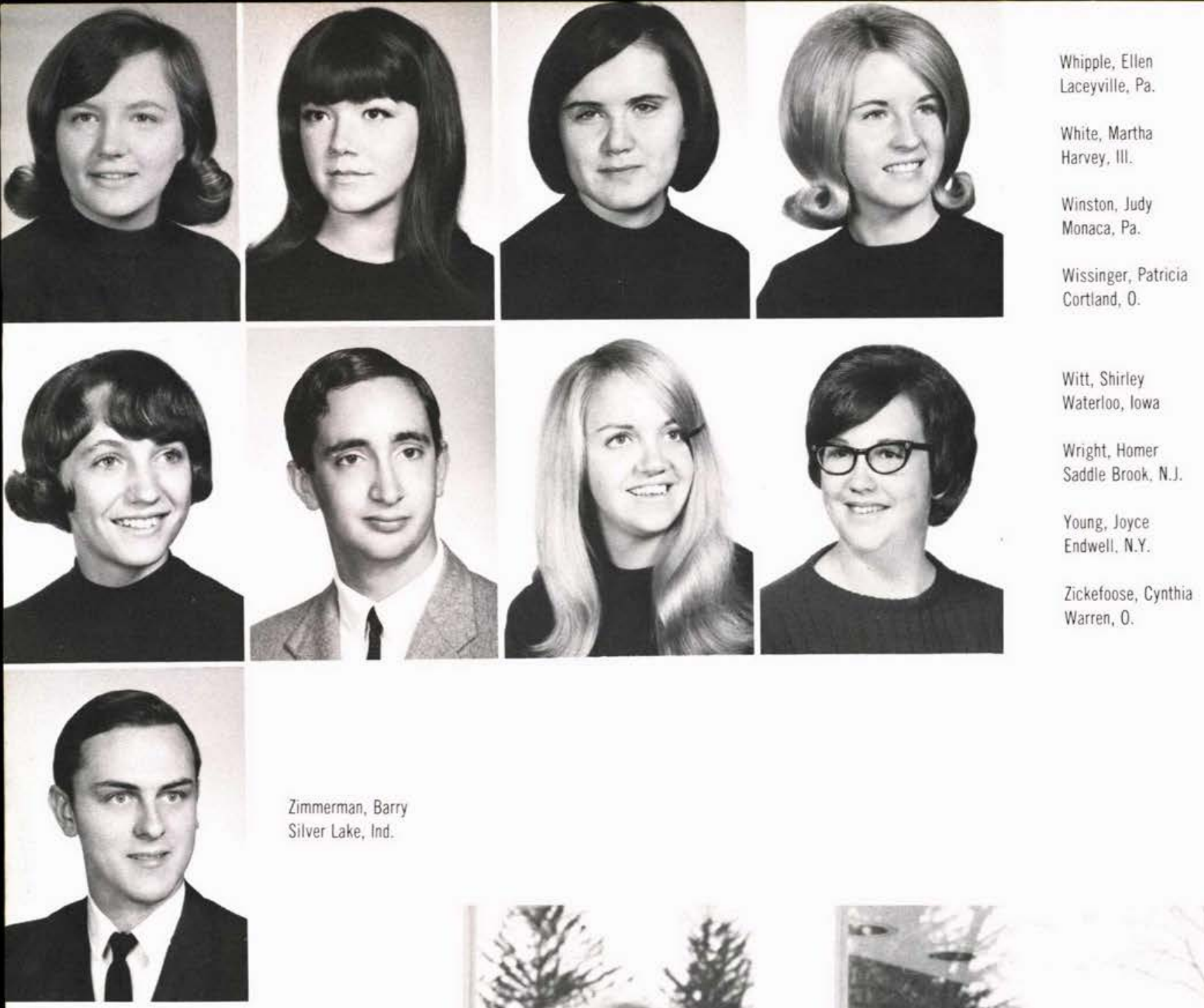

Zimmerman, Barry

Silver Lake, Ind.

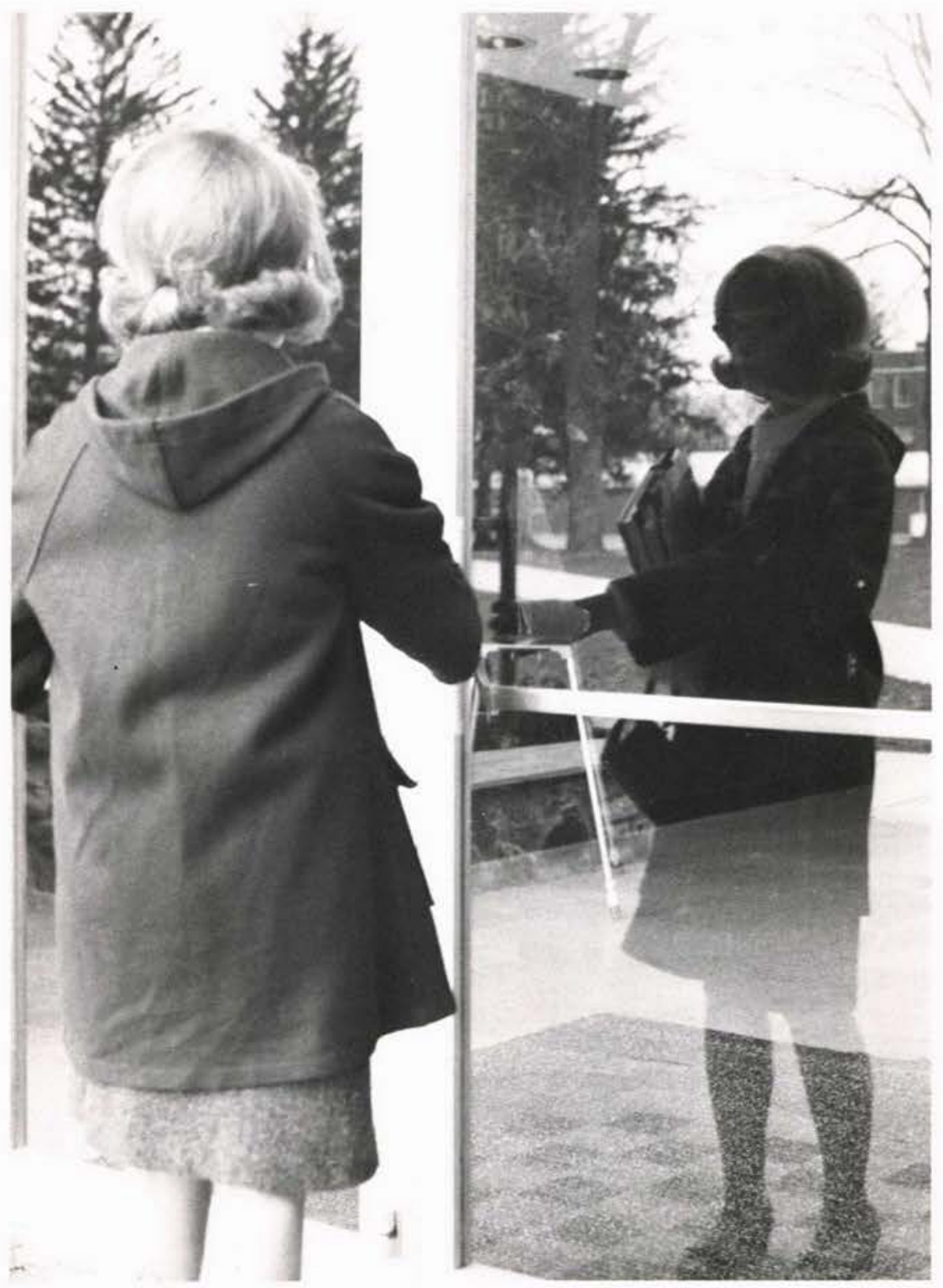



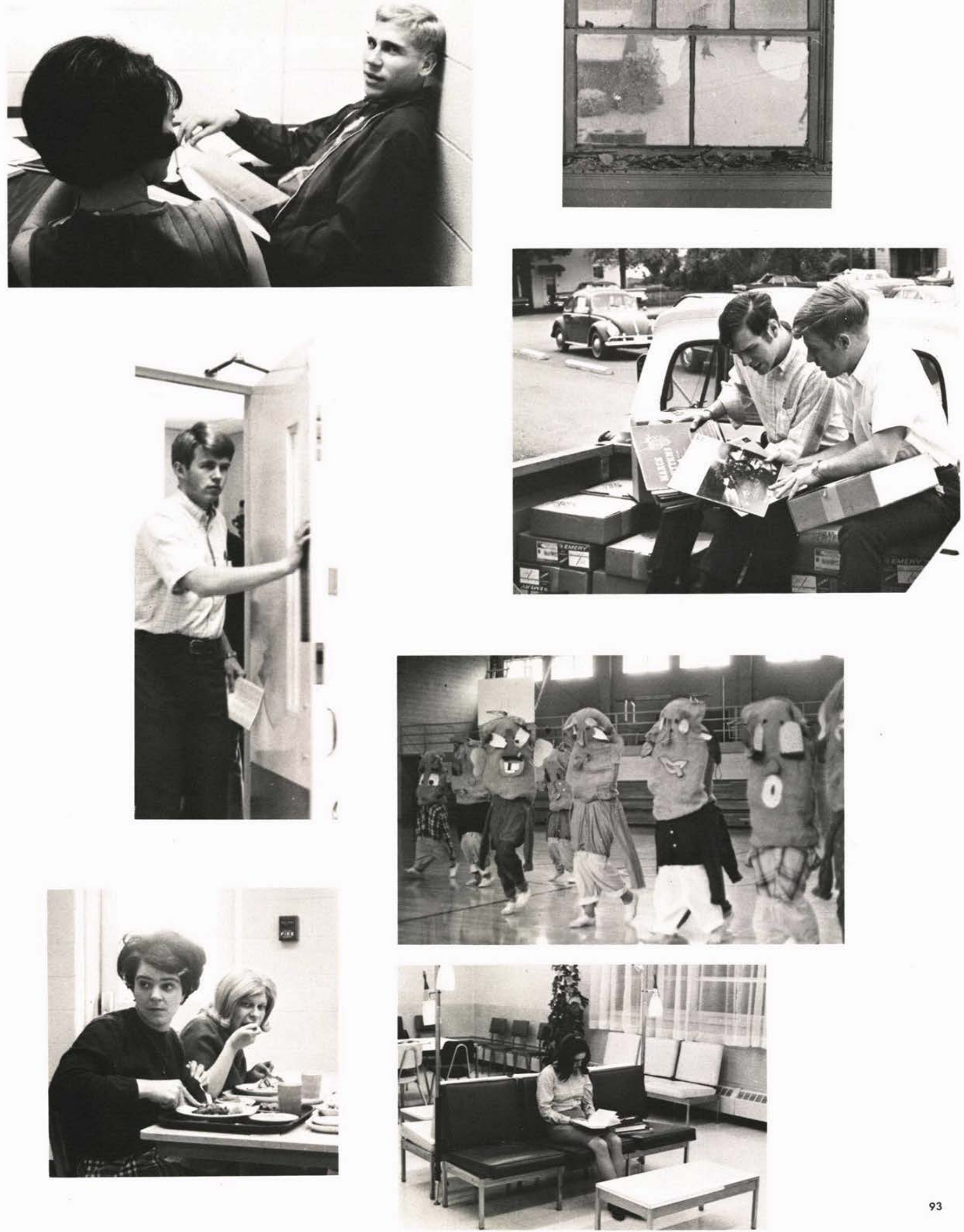


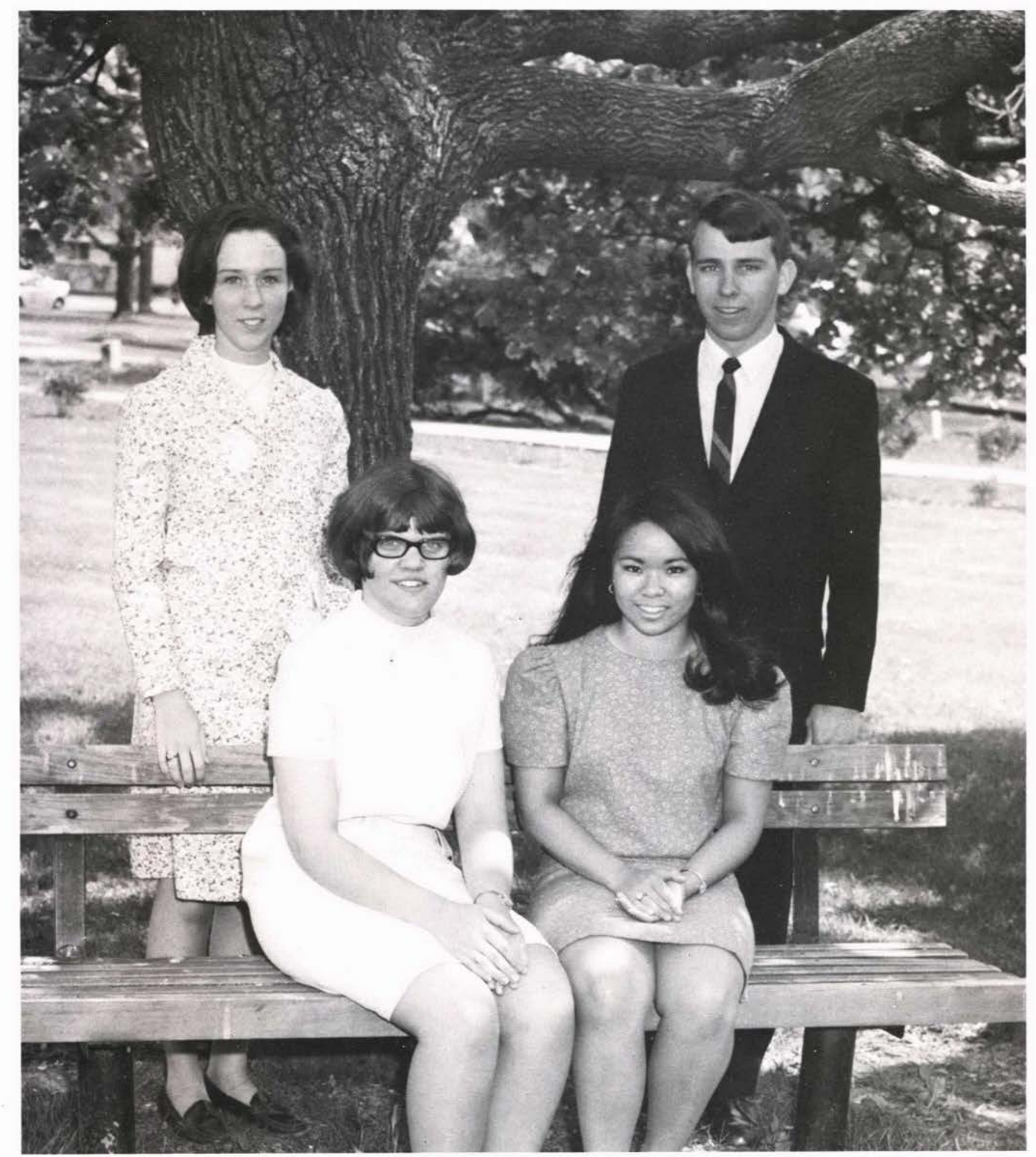

Secretary - Beverly Maidment

Treasurer - Pat Richardson

Student Council - Gayle Furushima Chaplain - Randy Patten

Not Pictured: Advisor - Dr. Ager

President-Stuart Walker

Student Council - Dave Durham 



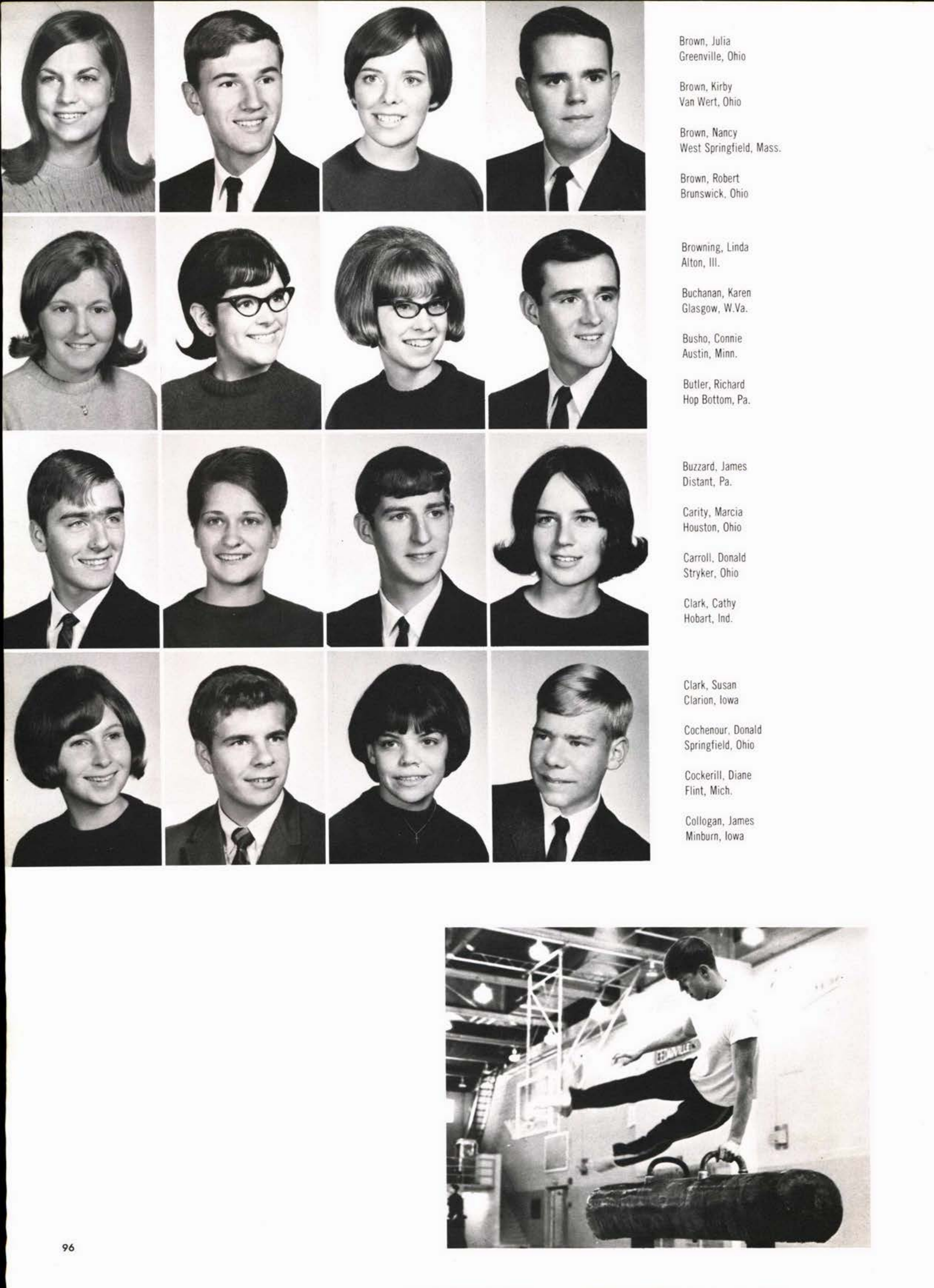




Huddleston, Dennis Dayton, Ohio

Hurst, Harold

Indianapolis, Ind.

Inghram, Richard Morning Sun, lowa
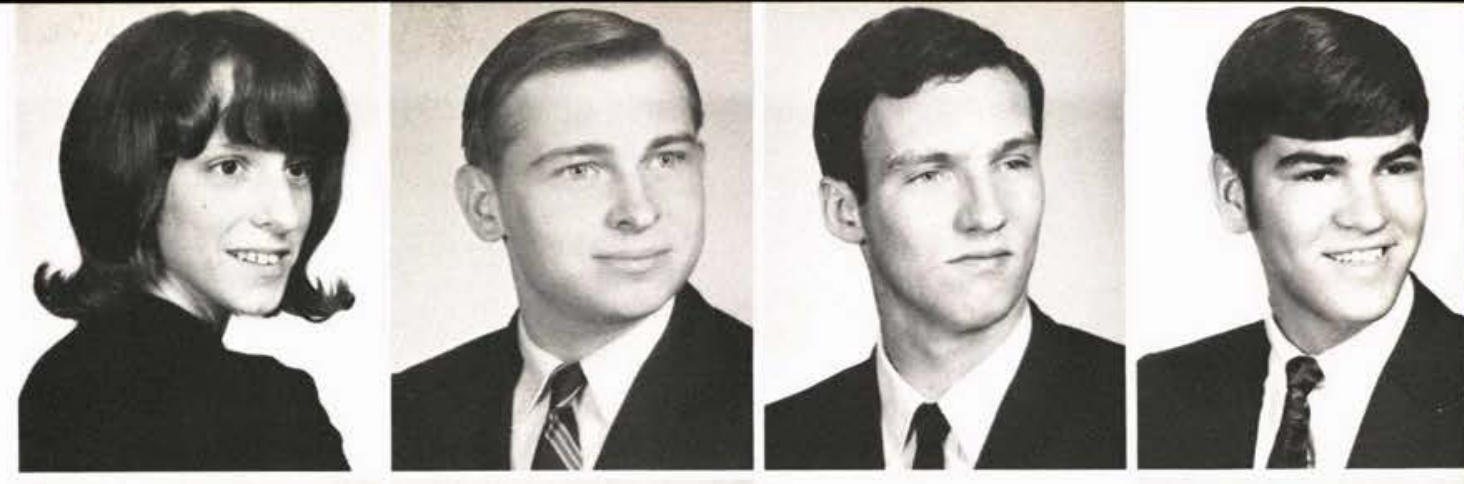

Irwin, Nora

Gidioute, $\mathrm{Pa}$.

Irwin, Patti Jo

Swartz Creek, Mich.

Ishuin, Geraldine

Seabrook, N.J.

Jacoby, Elaine Lima, 0.
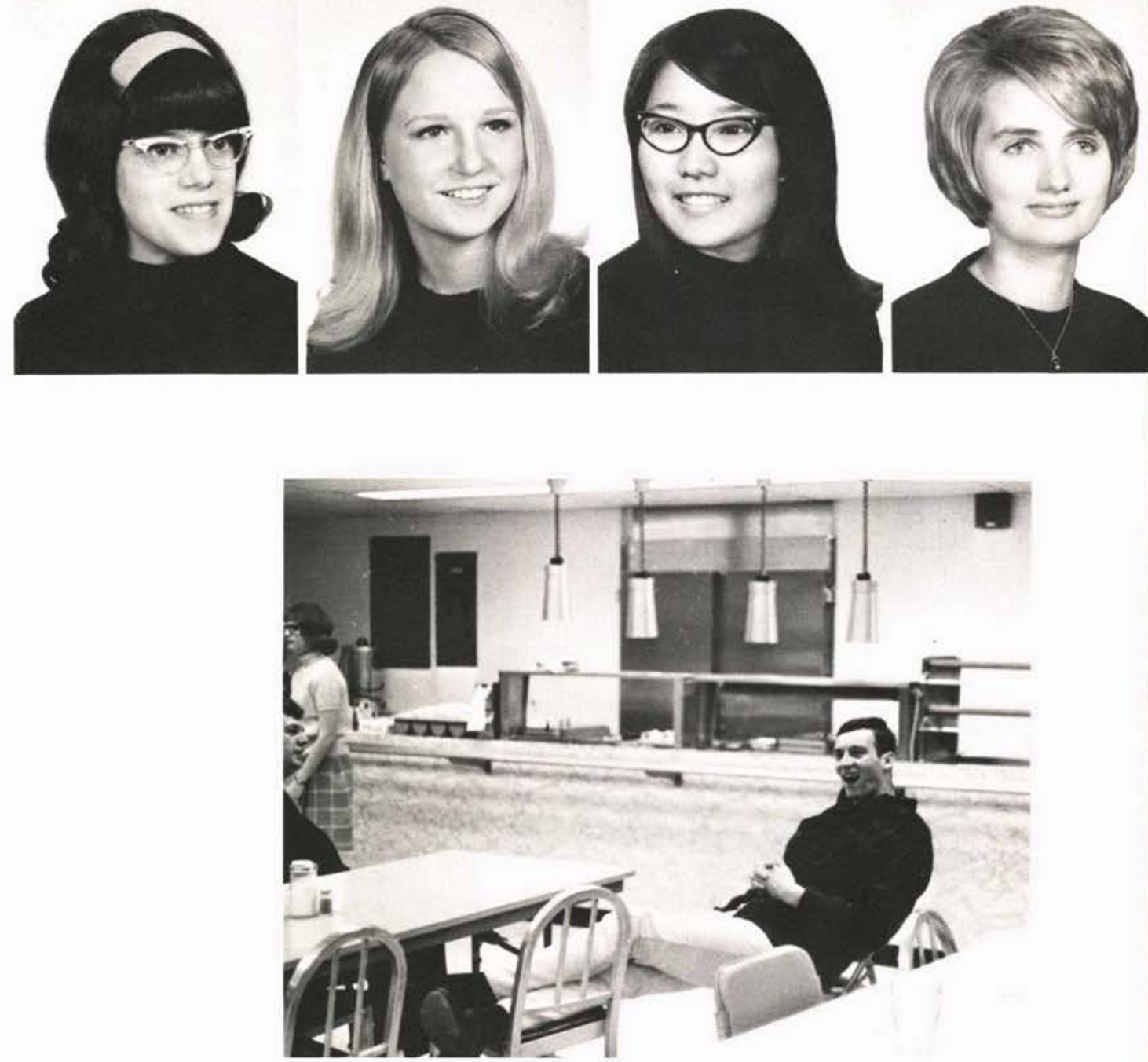

Jenista, Carol

Iloilo City, Philippines

Jenkin, Patricia

Cleveland, 0.

Johnson, Dorena

Paulding, 0 .

Johnson, Faye

Easetake, 0.
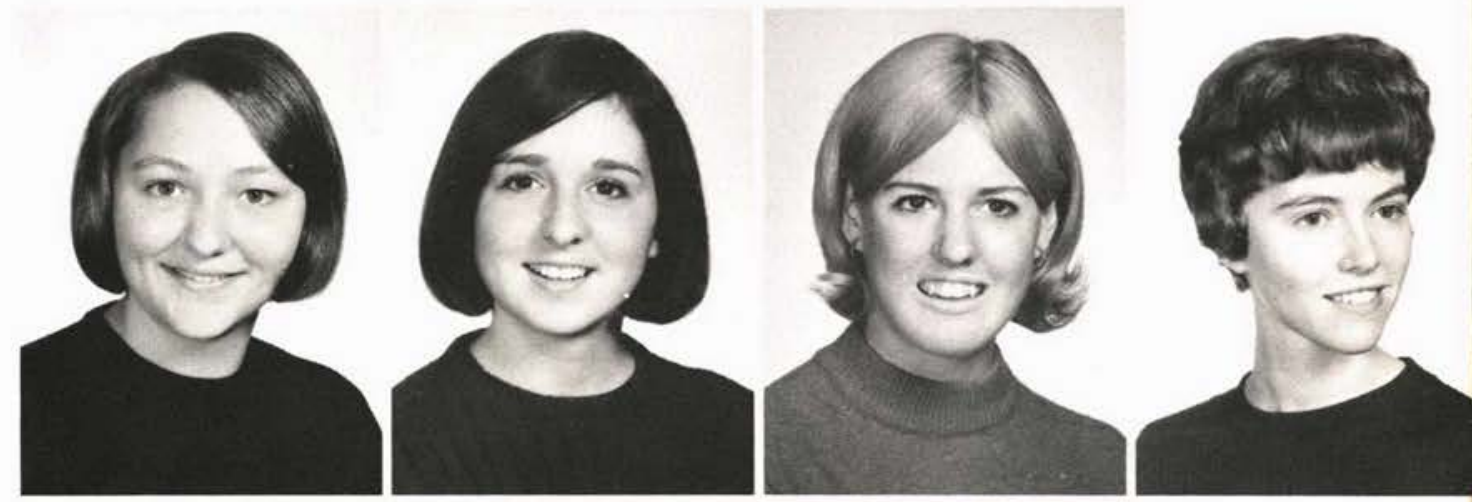

Kaufman, Joan

Roanoke, Ind.

King, Jean

Olmstead Falls, 0 .

King, Maureen

Knapp, Jaris

Prescott, lowa
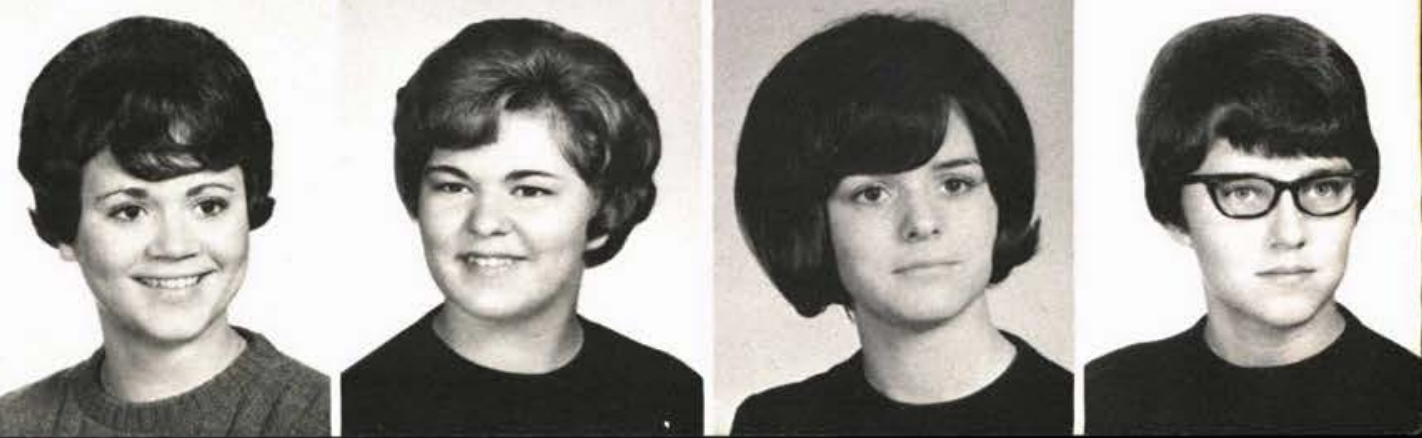



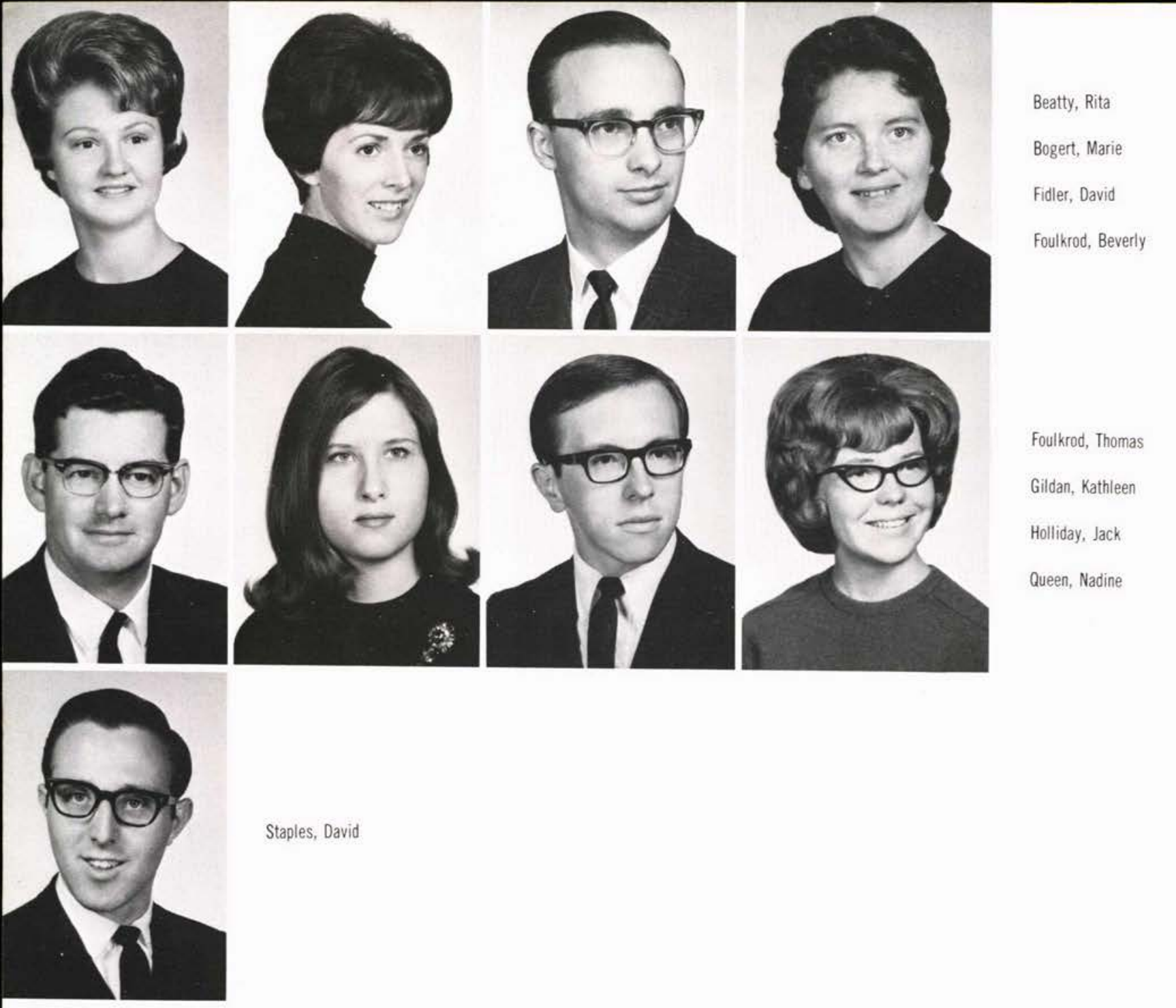

Staples, David

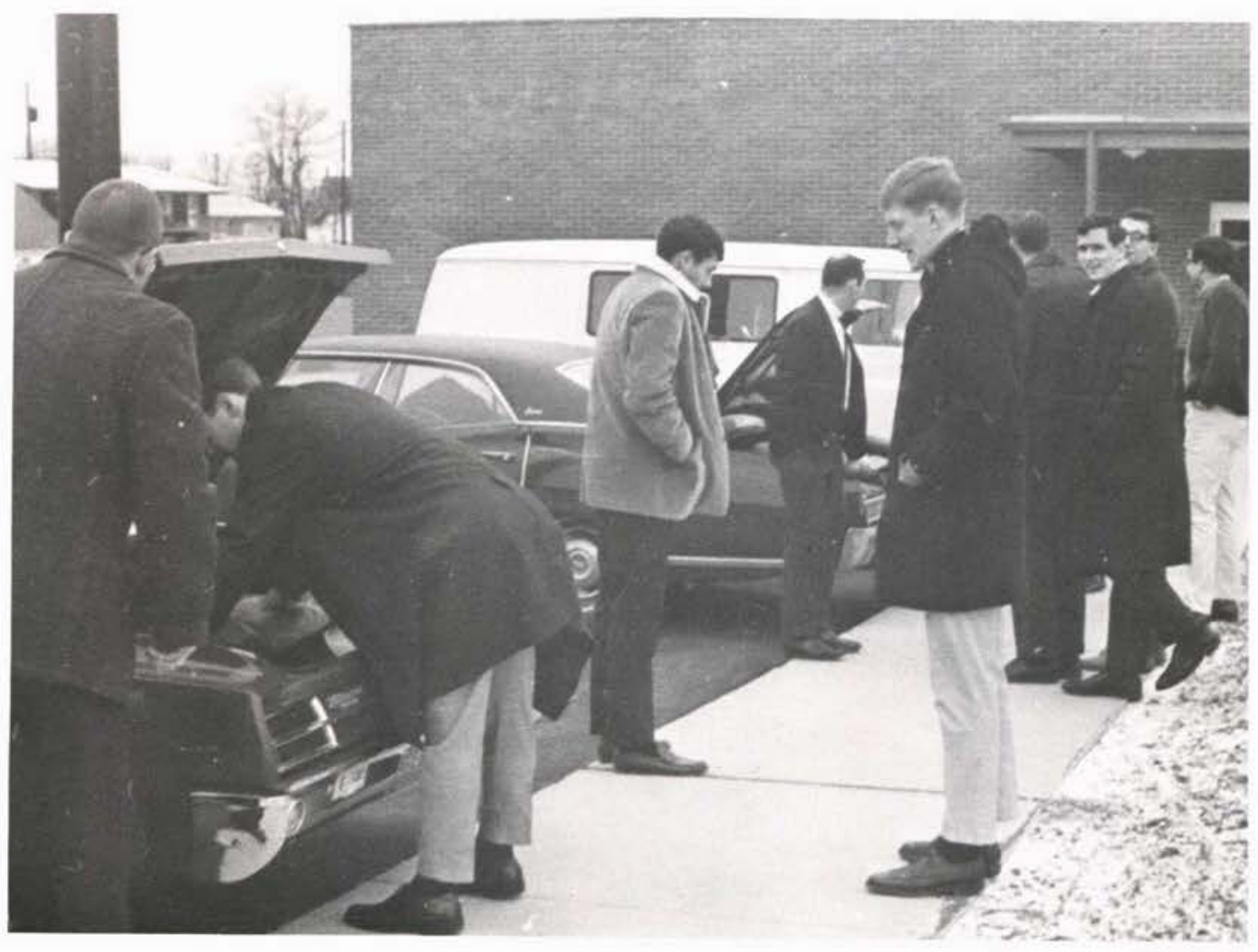




\section{REFLECTING ON}

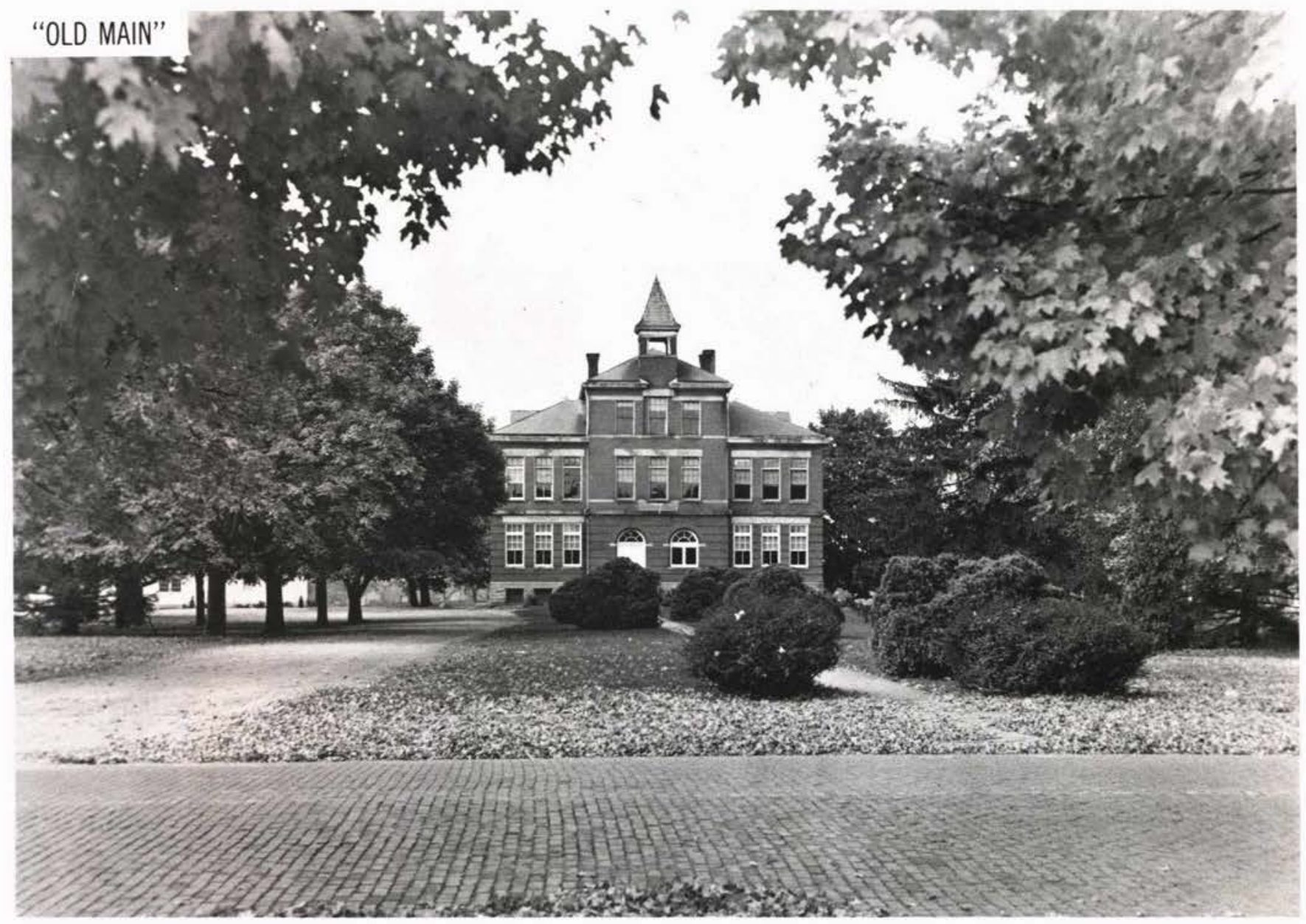

\section{Ehe GAVELYTE}

Published in the interest of CEDARVILLE COLLEGE,

BY

\section{THE GAVEL CLUB}

A MONTHLY PERIODICAI.

Published on the 15th of each month, except

July and August

Subseription price, per year, $\$ 1.00$

Entered as Second Class Mail Matter, in the post office, at Cedarville, Ohio, January 19, 1906.

\section{CONTENTS.}

A Trip To California (Frank Bird)..................Page 100 DiEM IN DeI............................................. " 104

Cedarville in The Oratorical Association...... “ 107

The LAST OF THE RACE (C. G. Ware)............. “ 108

AMERICA - HER OPPORTUNitIES (Wm. Hawthorne).. “ 109

MEN WE HAVE HEard............................... “ 113

Мов SPIRIT (Wilson Hanna)......................... “ " 114

Neath City Roofs (C. G. Ware) .................. “ “ 115

TREASURE IsLANDS (Jeannett• Orr) ................... “ 116

SOCIETIES .............................................. “ “ 120

SAMPLE EXCUSES................................... “ 123

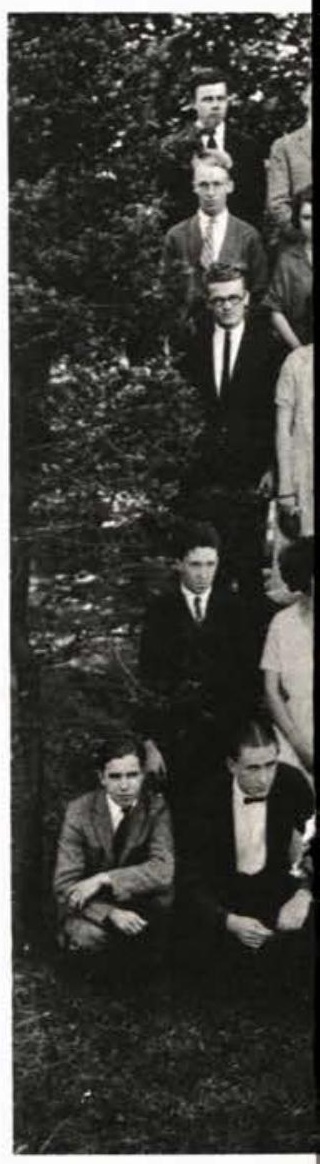




\section{THE PAST . . .}

January $\angle 6,1887$

Cedarville College born,

A dream realized;

Chartered by leaders

of the Reformed Presbyterian Church,

The school has enjoyed

A rich heritage.

The early emphasis

Of a Christ-centered curriculum

Has been perpetuated.

Having been transferred

To Baptist hands

In 1953,

The student enrollment

Has more than tripled

Since 1959.

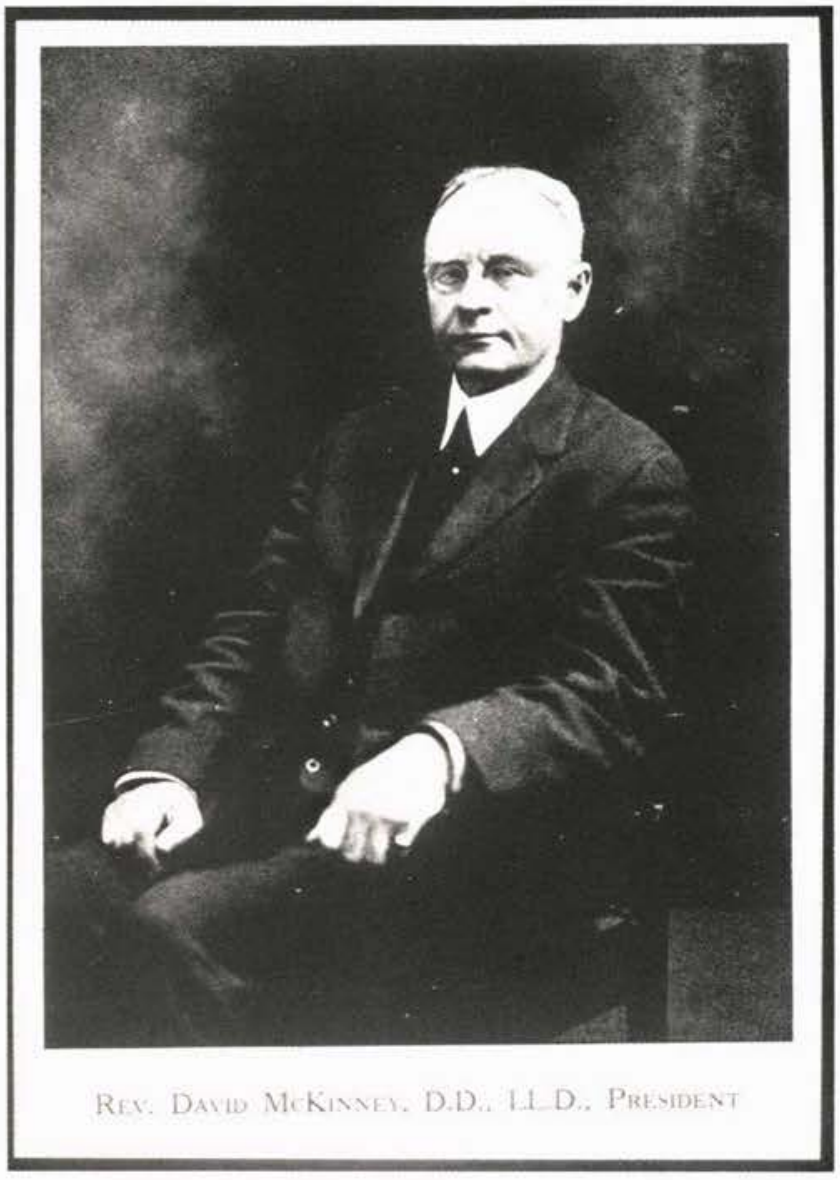




\section{OBSERVING THE}
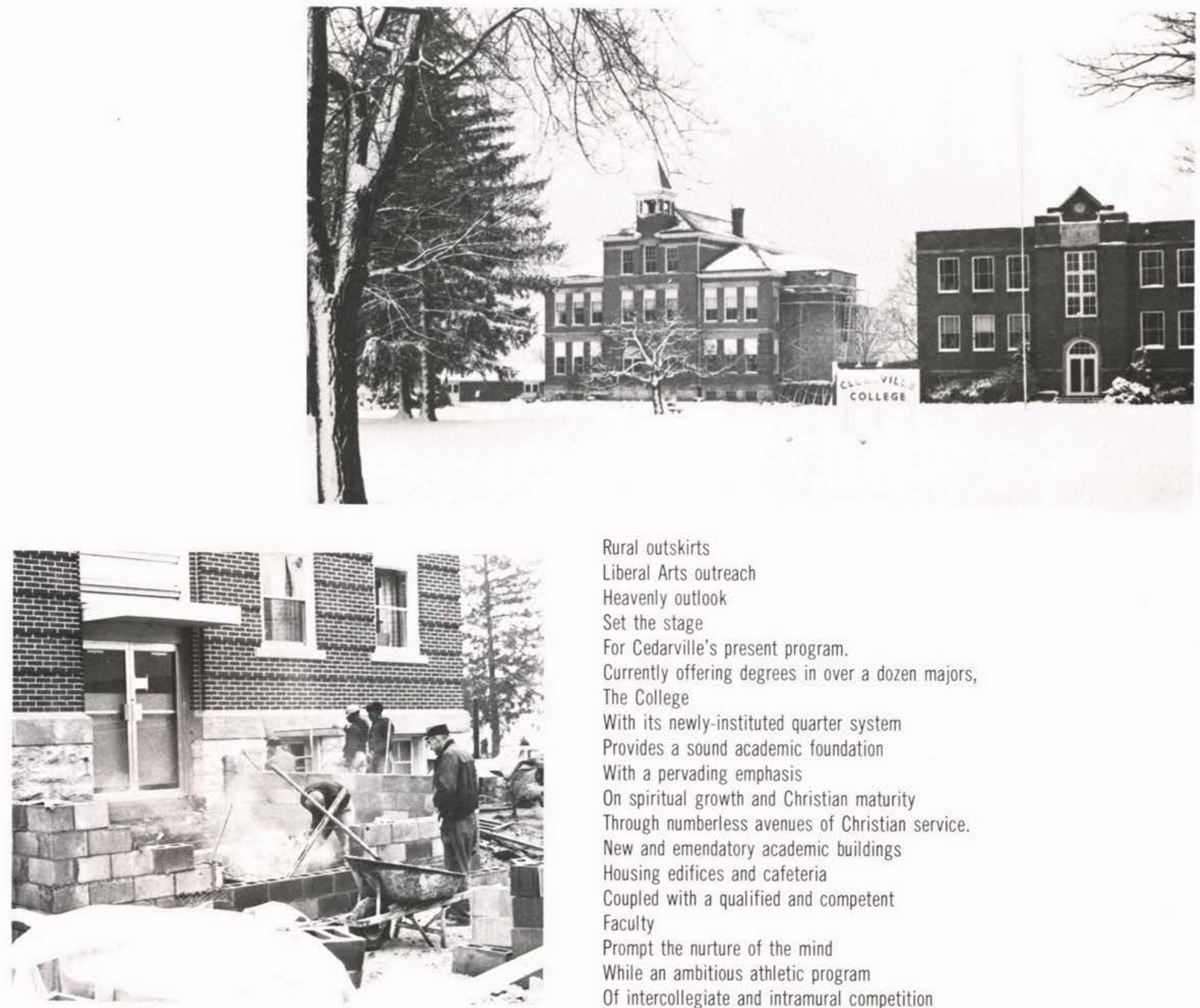

Rural outskirts

Liberal Arts outreach

Heavenly outlook

Set the stage

For Cedarville's present program.

Currently offering degrees in over a dozen majors,

The College

With its newly-instituted quarter system

Provides a sound academic foundation

With a pervading emphasis

On spiritual growth and Christian maturity

Through numberless avenues of Christian service.

New and emendatory academic buildings

Housing edifices and cafeteria

Coupled with a qualified and competent

Faculty

Prompt the nurture of the mind

While an ambitious athletic program

of intercollegiate and intramural competition

Provide for the body.

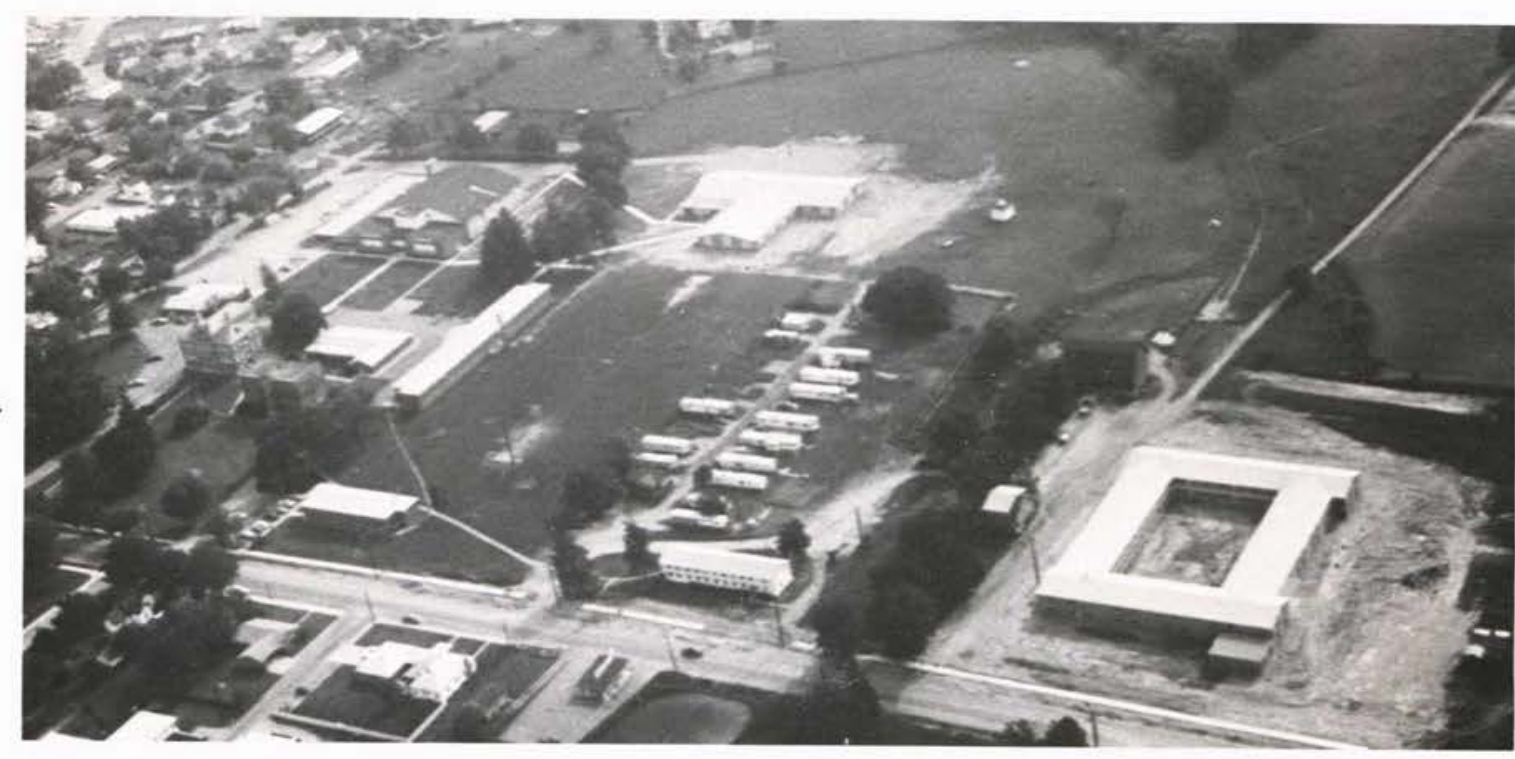


PRESENT ...

\section{0th ANNIVERSARY}
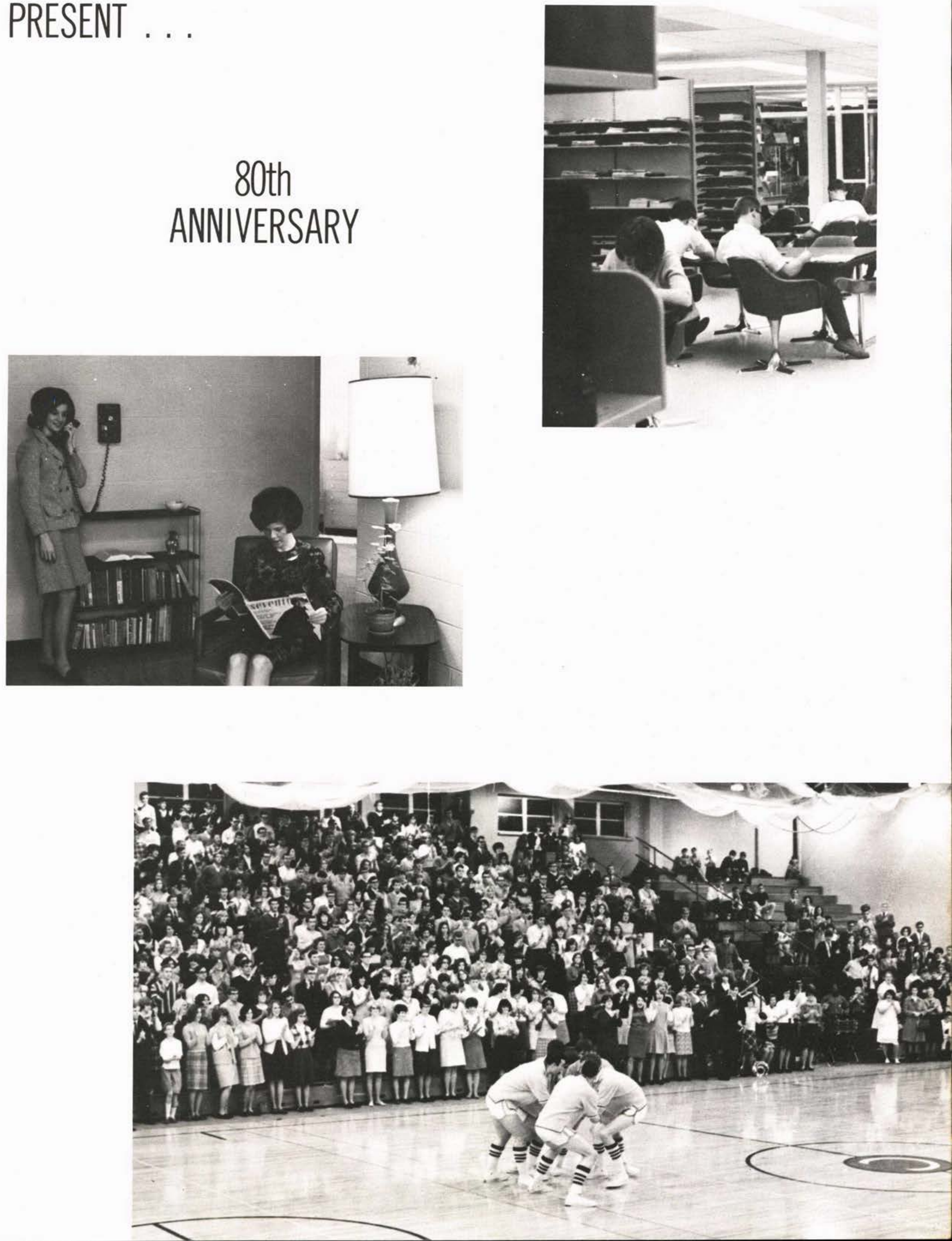
Cedarville is responding

To the challenge of the future

With a zealous

Development plan

That awaits anxiously the funds

Which will remove it

From the architect's desk

And project it into fulfillment.

A new dorm, chapel, and science building Are proposed

And will increase

Cedarville's capacity in the future

For mature instruction,

While contending

For the Word of God

And the testimony of Jesus Christ.

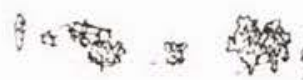

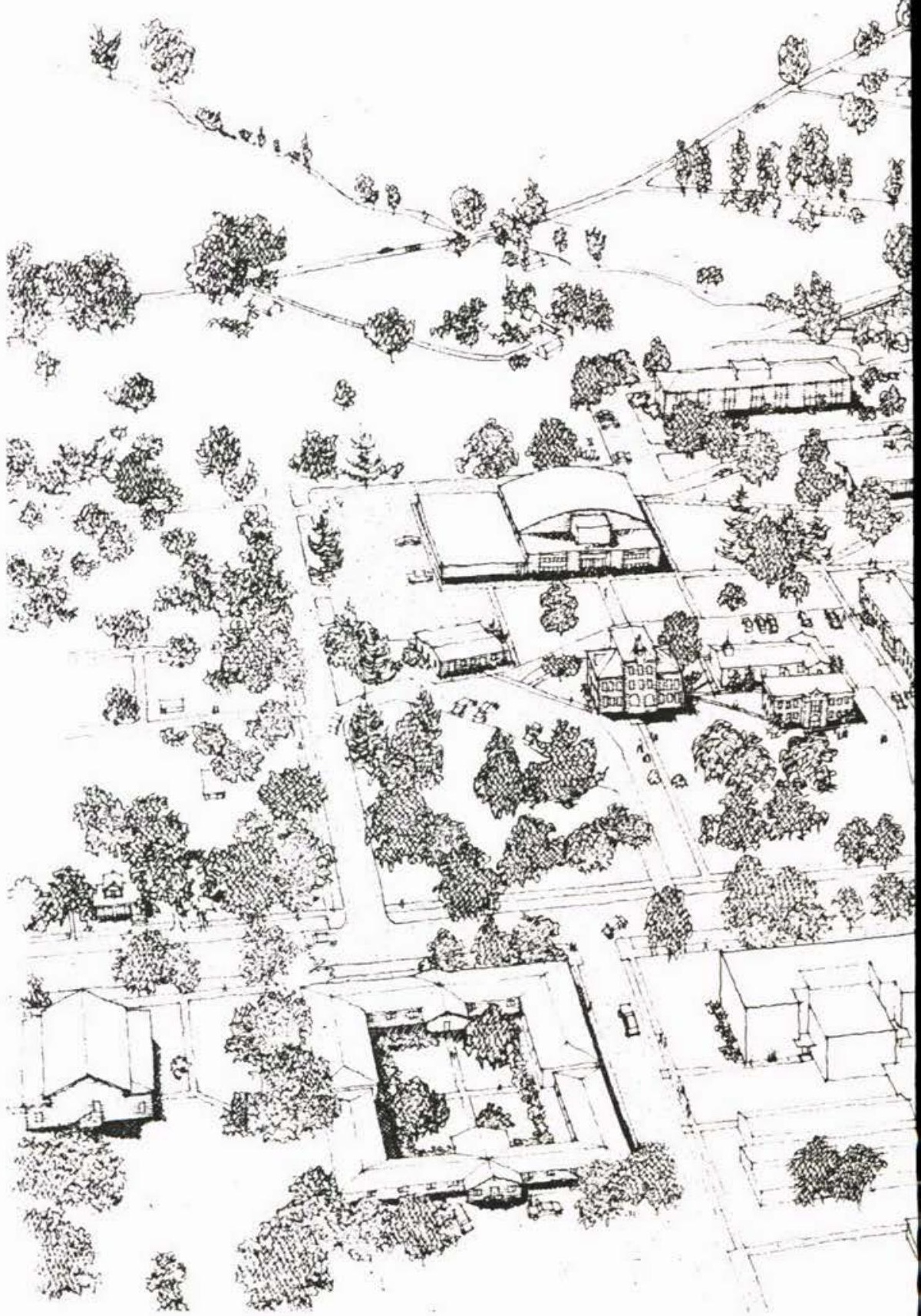




\section{THE FUTURE . . .}

${ }_{4}^{3}-\frac{3}{4}$

is

3. 需

然

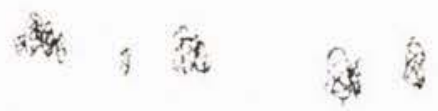

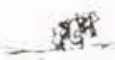

整

4

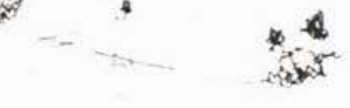

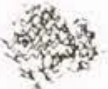

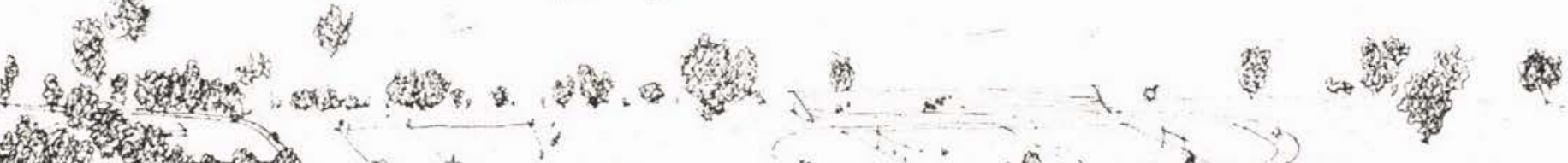
A then

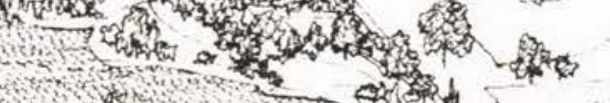

\%

S.

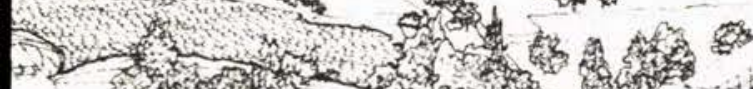

201

管

A. Ad

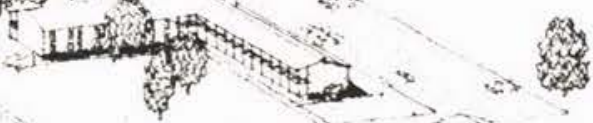

繁㱍

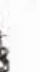

寚的

4 (1)

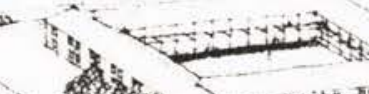

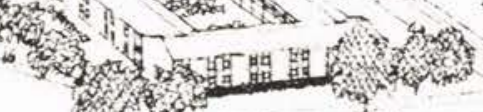

A

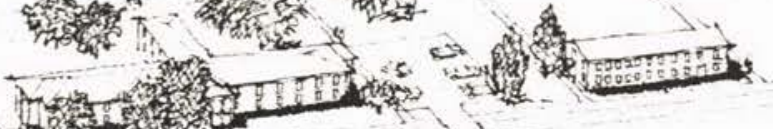

$$
\text { tas. }
$$

a c c t

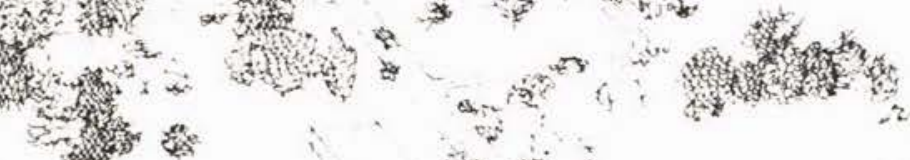

a.

i A w

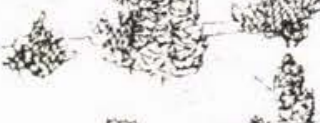

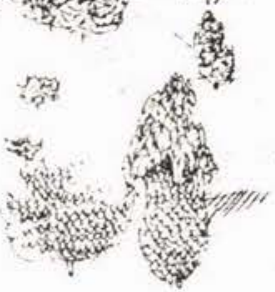




\section{Groups and Organizations}

As you think, you travel; and as you love you attract. You are today where your thoughts have brought you; you will be tomorrow where your thoughts take you. You cannot escape the results of your thoughts, but you can endure and learn, can accept and be glad. You will realize the vision (not the idle wish) of your heart, be it base or beautiful, or a mixture of both, for you will always gravitate toward that which you, secretly, most love. Into your hand will be placed the exact result of your thoughts; you will receive that which you earn ... no more, no less. Whatever your present environment may be, you will fall, remain, or rise with your thoughts, your vision, your ideal. You will then become as small as your controlling desire or as great as your dominant aspiration. 


\section{STUDENT COUNCIL}

A responsible student government

Employing the democratic processes

of representation and voiced opinion

Provide an open forum

For free debate and discussion

And encourages

The exchange of ideas and suggestions

For the improvement and advancement

of Cedarville College.

The Student Council

Officiates as an operative liaison

Between Administration and Student Body.

From the activities of new student week

Early in the fall

To Homecoming

To recommendations for

Student Handbook revisions

To the formation of a Student Court

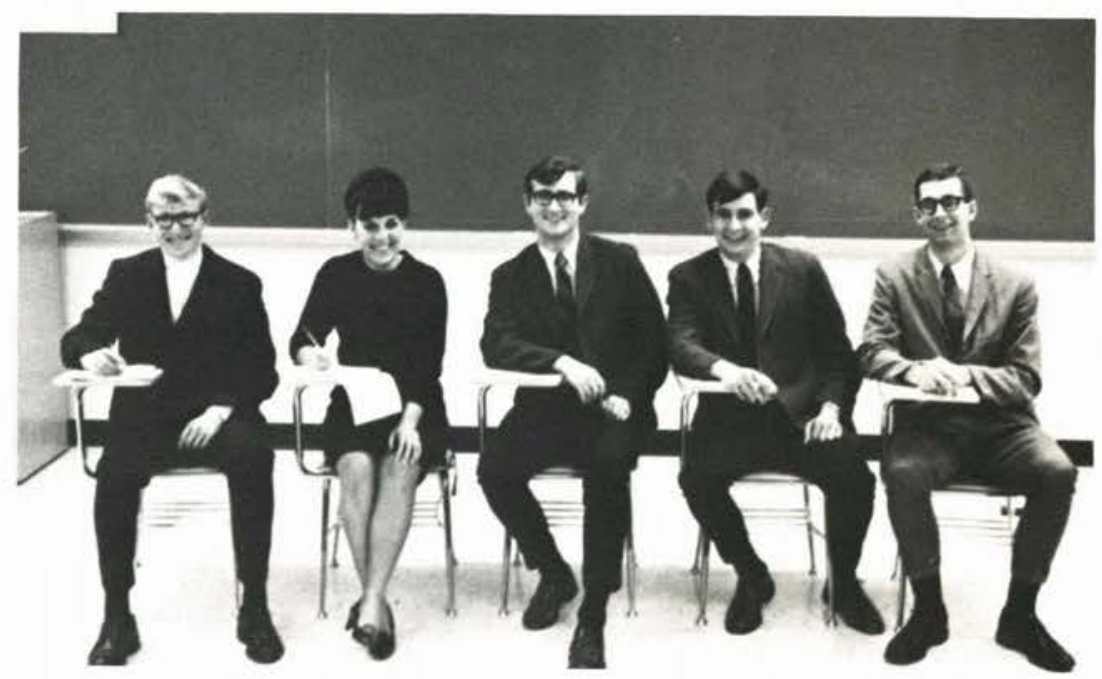

The Student Council

Promotes and offers

Service to the school.

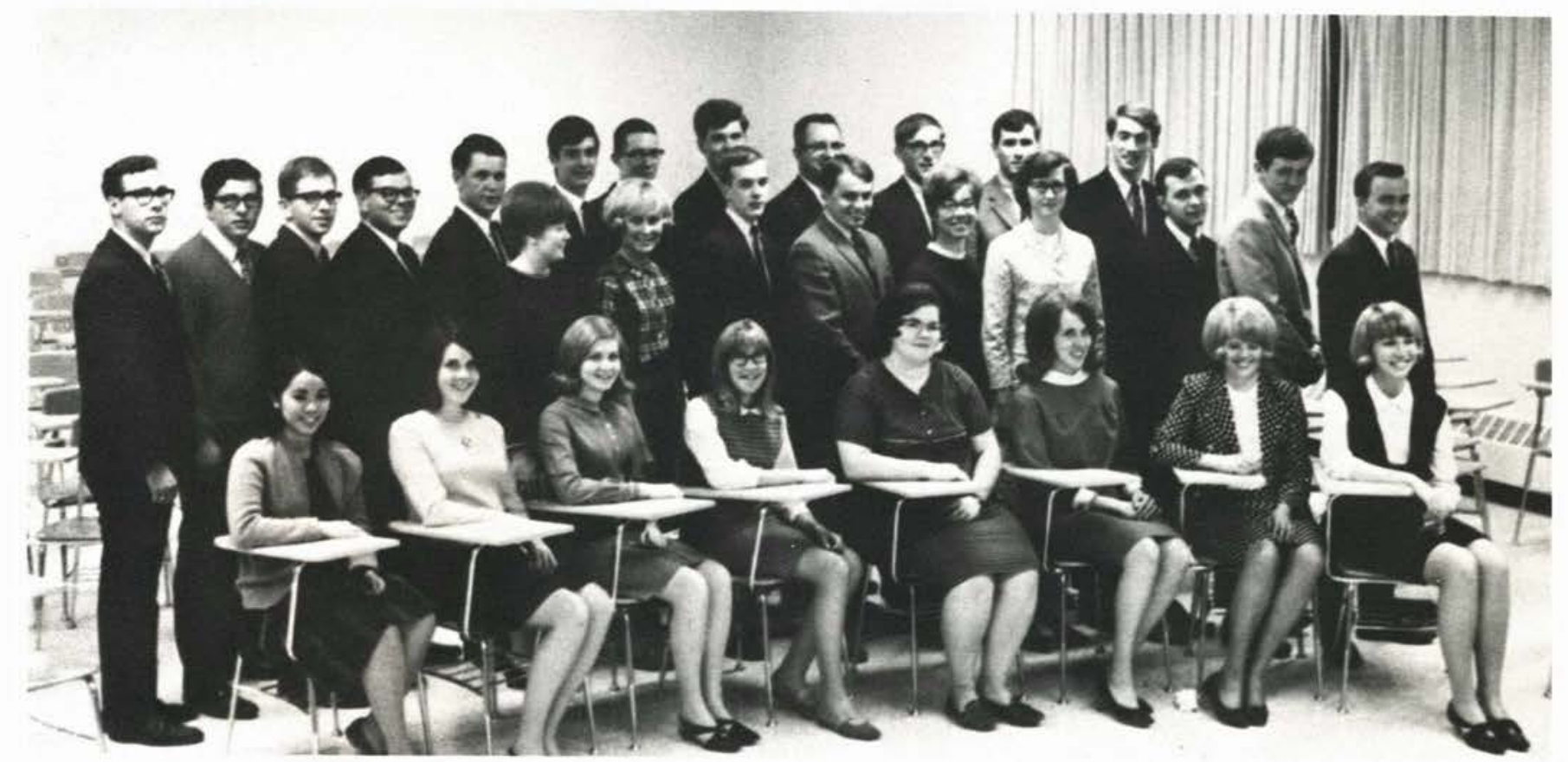

First Row: A. Kusumoto, N. Norton, D. Hamilton, D. Chaffe, D. Bratka, J. Hoag, M. Stanbery, D. Bush. Second Row: J. Austin, P. France, K. Cole, L. Walker, M. Beitler, S. Eury, D. Wall, J. Jeremiah, R. Allerton. Third Row: K. Muck, R. Hamilton, R. Roderick, S. Snyder, R. Edwards, D. Durham, K. Heldreth, S. Olsen, Dean Mclntosh, Advisor; P. Entner, L.
Reno, D. Yoder.
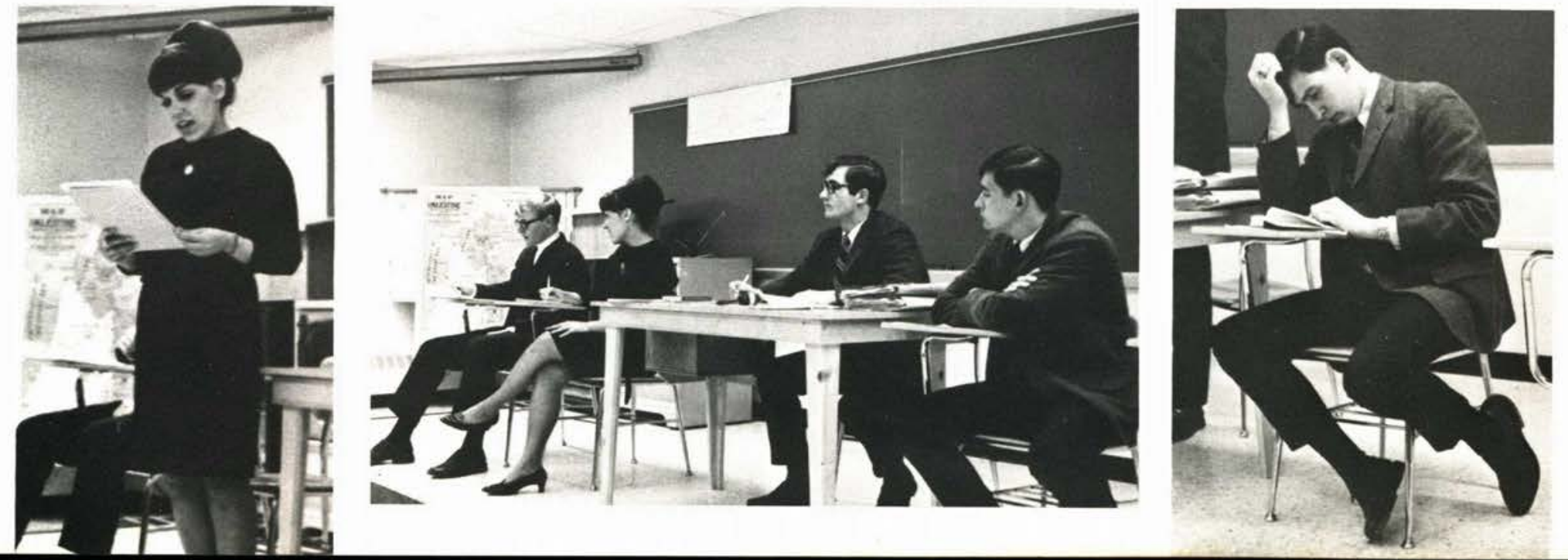


\section{PUBLICATIONS CO.}

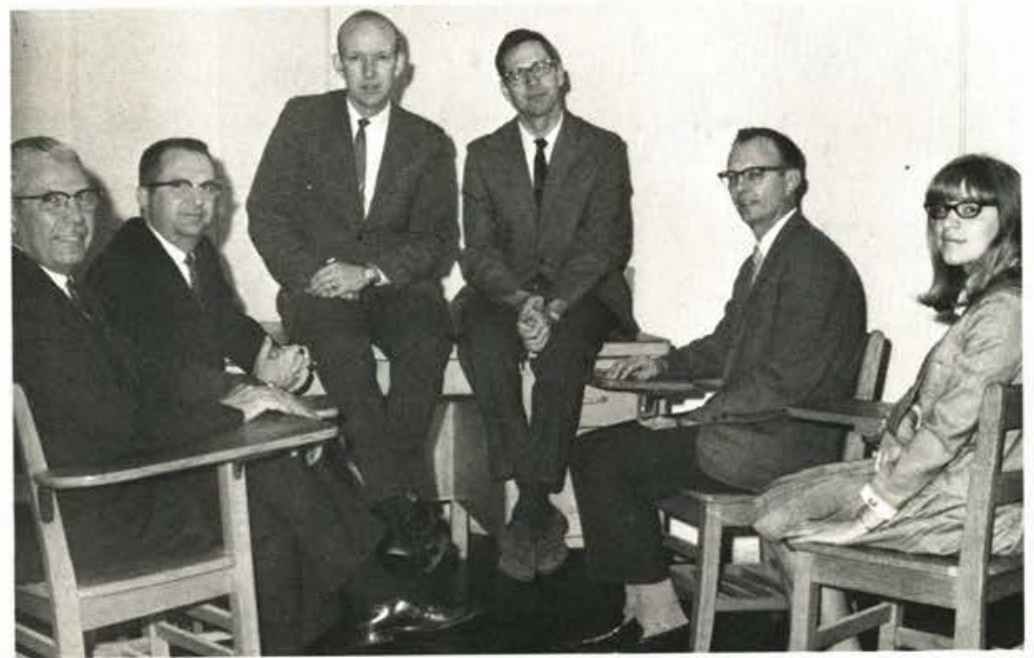

Mr. Hurst, Mr. Mclntosh, Dr. Baumann, Mr. Vanderkoy, Dr. Johnson, Dianne Chaffe. Not Pictured: Debbi Bush.

\section{DISCIPLINE CO.}

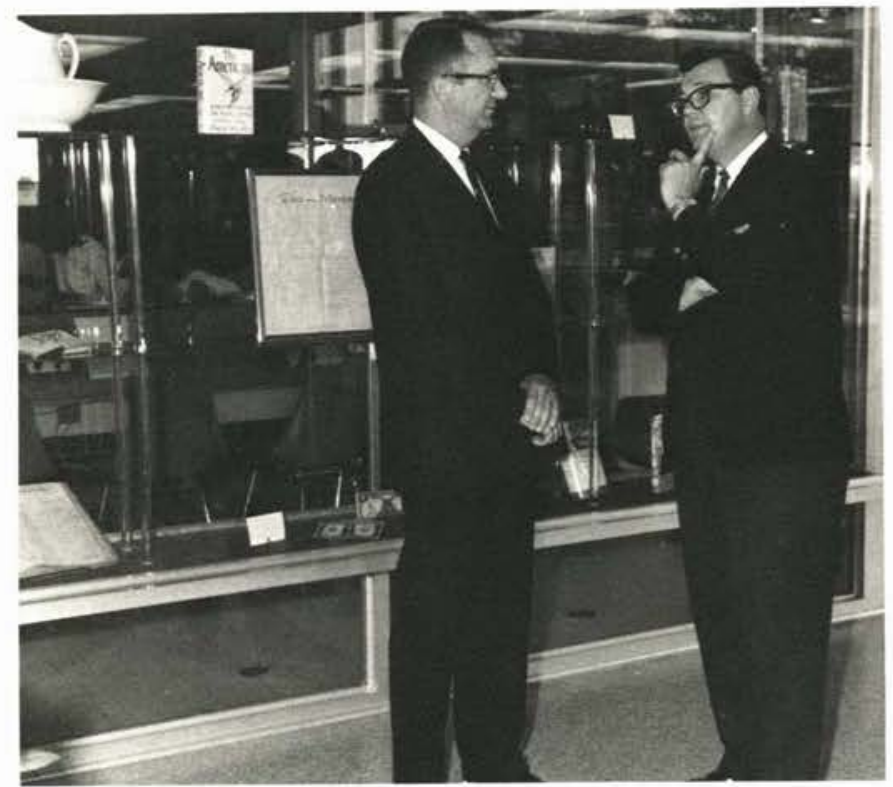

Mr. McIntosh, Mr. Monroe. Not Pictured: Mr. Frye, Deane Allen, Debbi Bush, Larry Waite.

\section{"WHO'S WHO"}

\section{DORM COUNSELORS}

In personal and spiritual problems,

To lead in devotions

And dorm prayer meetings,

The willingness to be a friend,

To set an example

To understand -

This requires a dorm counselor
"Quiet!"

"Where's your date permit?"

"Did you take an L.P.?"

Any good policeman

Can maintain discipline.

But the desire to help

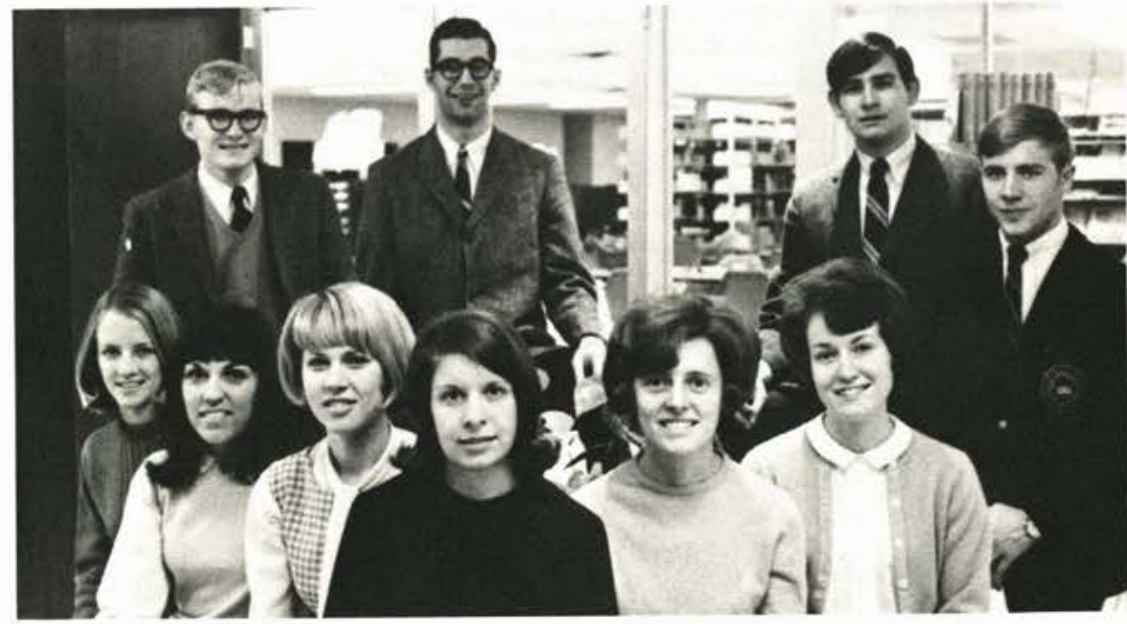

First Row: L. Riggs, D. Daab, D. Bush, K. Hubbard, M. Schonscheck, J. Field. Second Row: F. Jenista, L. Waite, D. Haffey, J. Phipps.

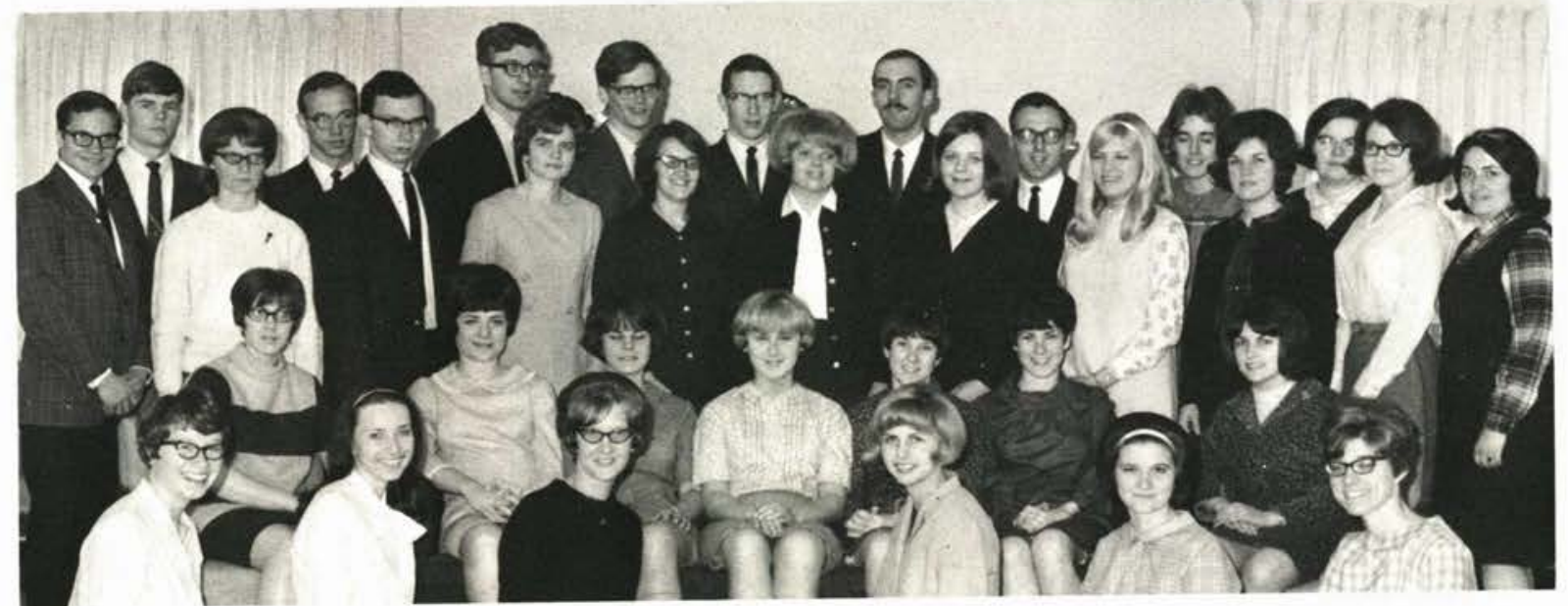

First Row: S. Pader E Knott. B. Miller, L. Taylor, M. Muirhead, M. Beitler. Second Row: B. O'Keefe, M. Grable, K. Guthrie, P. France, C. O'Shell, S. O'Shell, P. Fow Work. I. Clater, E. Smelser, S. Brown, K. Heldreth, J. Berry, D. Staples, A. Moorman, D. Bratka. 


\section{MIRACLE}

"School days, school days

Good ole Golden Rule days,"

Their memories and events

Are Captured

Forever

In a yearbook.

Amusing candids, poignant copy

Pen a diary

That records for posterity

Reminiscences

Of a school year that is somehow always

Bigger and better than last year.

Dedication, imagination, and pride went into

The Miracle's memorabilia.

It beautifully reflects

The accomplishments and the personalities

of Cedarville College, 1967-68.

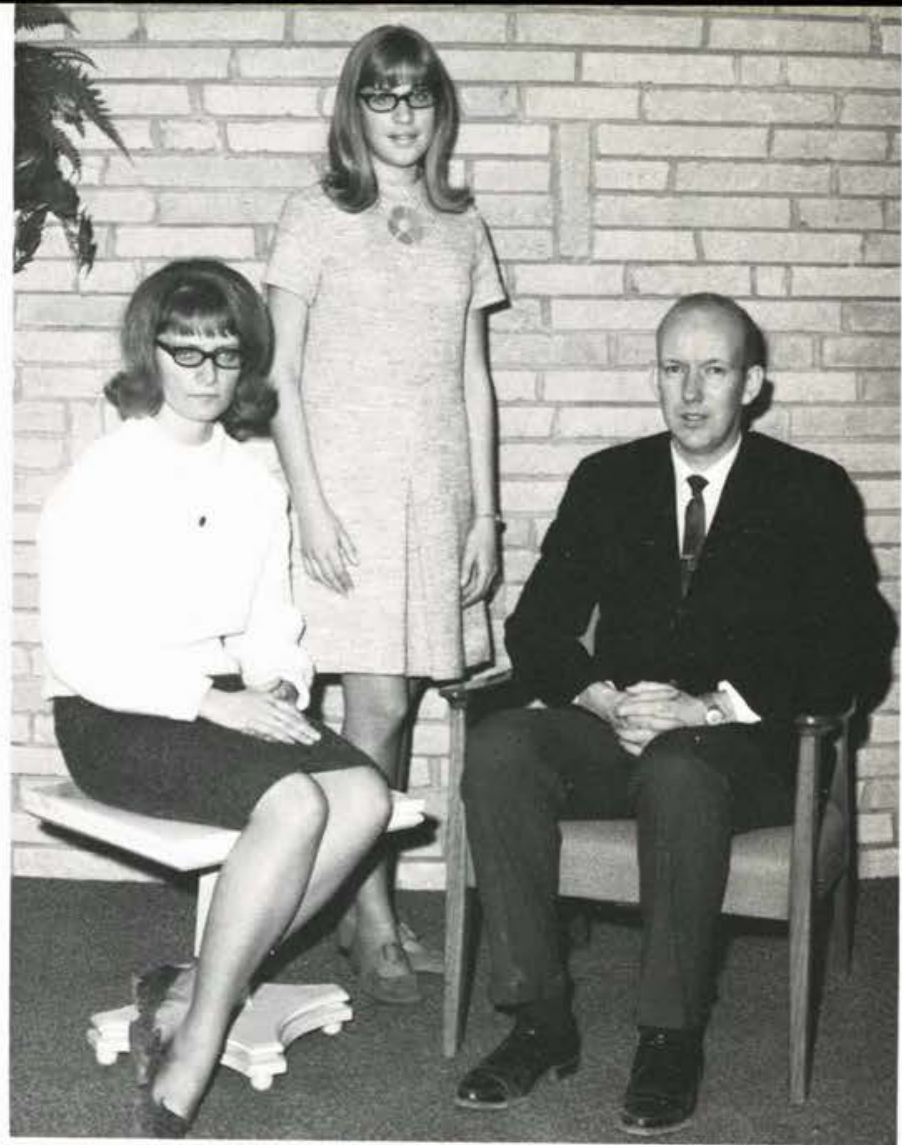

Dianna Hutchison, Ass't Editor; Dianne Chaffe, Editor: Dr. Baumann, Advisor.
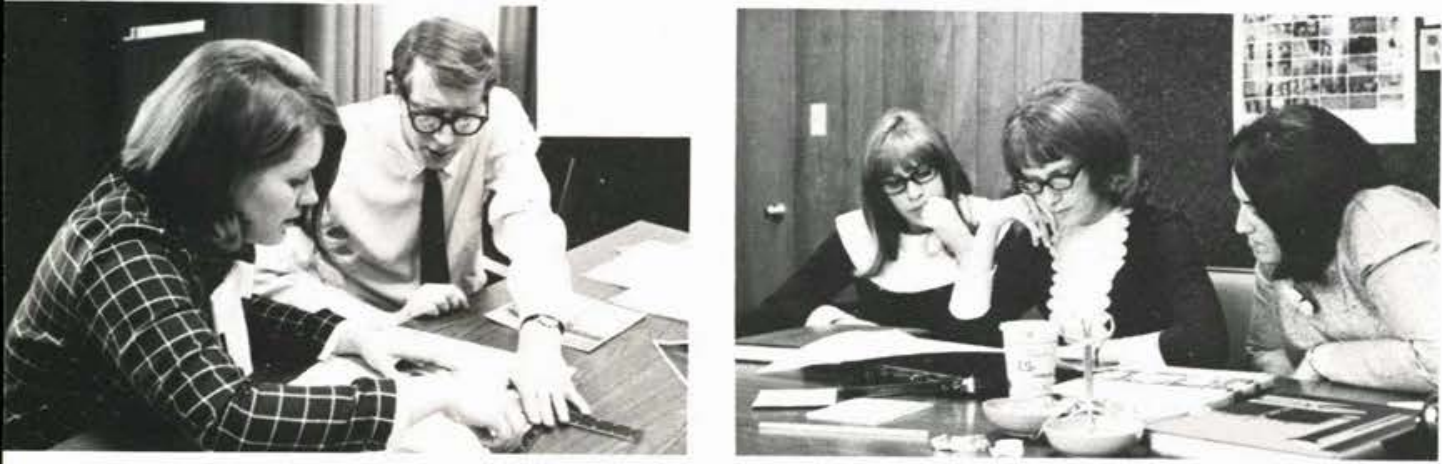

EDITORS

Layout: Jan Phenix

Copy: Pat France

Photography: Jon Brown

Men's Sports: John Watson

Women's Sports: Kathy Spencer

Advertising: David Chumbler

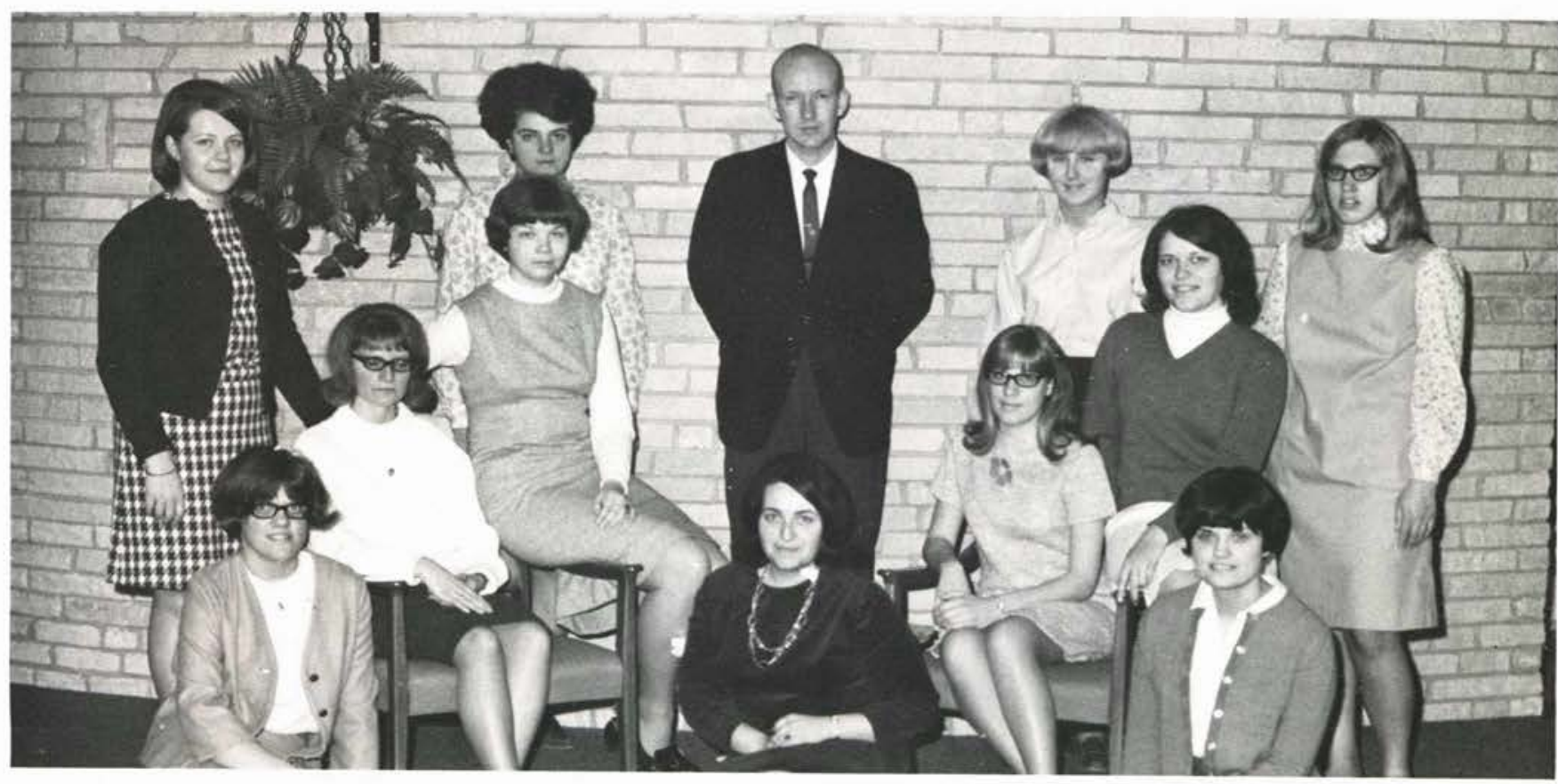

Seated: Pat Richardson, Dianna Hutchison, Becky Rudig, Jo Ann Waltz, Dianne Chaffe, Diane Thompson, Carol Stewart. Standing: Janet Phenix, Tina Lautt, Dr. Baumann, Patti France, Becky Harman. 


\section{WHISPERING CEDARS}

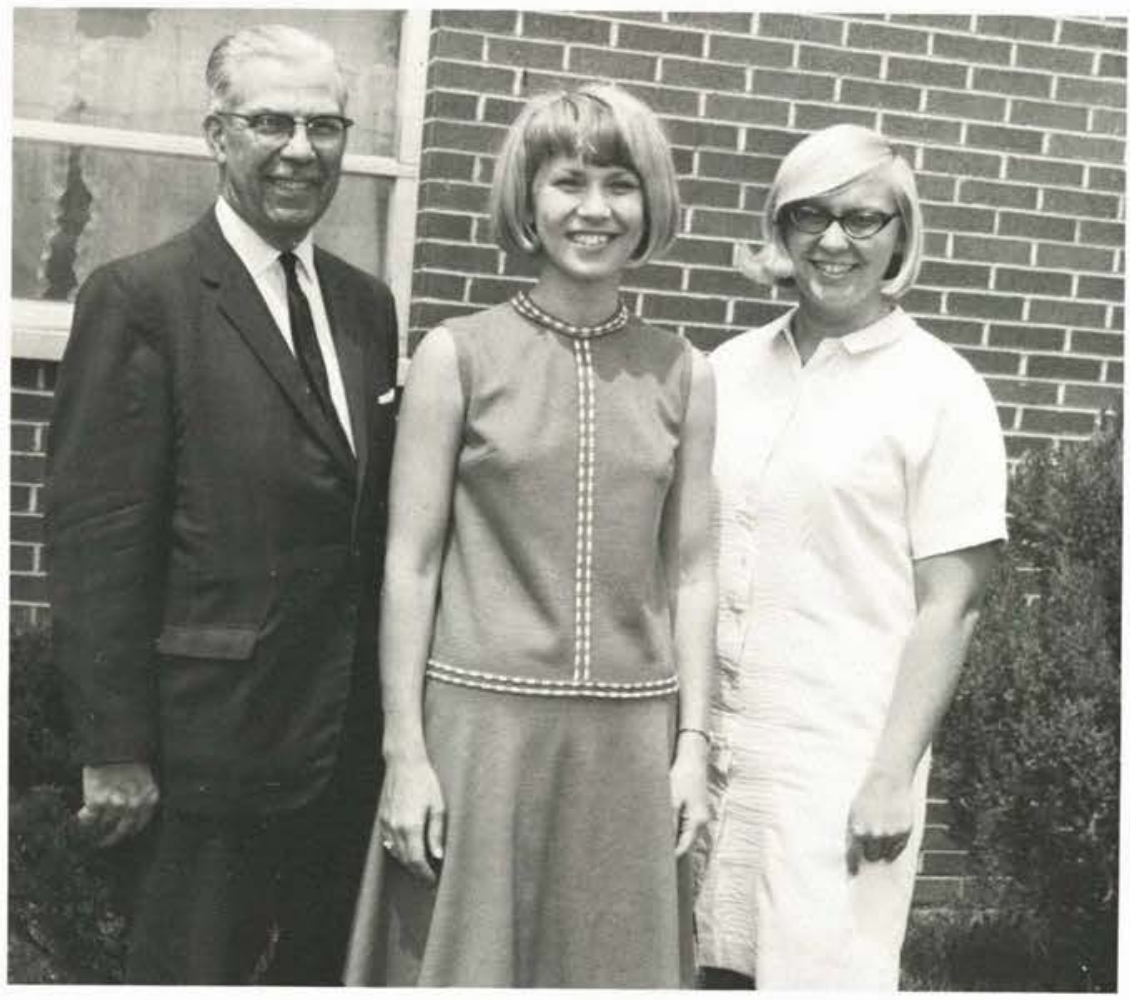

A free press,

So essential to a democracy,

Finds its counterpart

In the campus newspaper.

Whispering Cedars

Operates as an agent

Of student opinion,

A medium of praise and dissent.

Its journalism

Is the mouthpiece of news and views.

Integrity, veracity

Are its desired characteristics.

With a disdain for sensationalism

A love for truth

And a thirst for facts,

The paper

Serves the college family

As the manifestation

Of campus spirit and feeling.

Mr. C.B. Hurst, advisor; Debbi Bush, Editor; Bev Carlson, Circulation Manager.

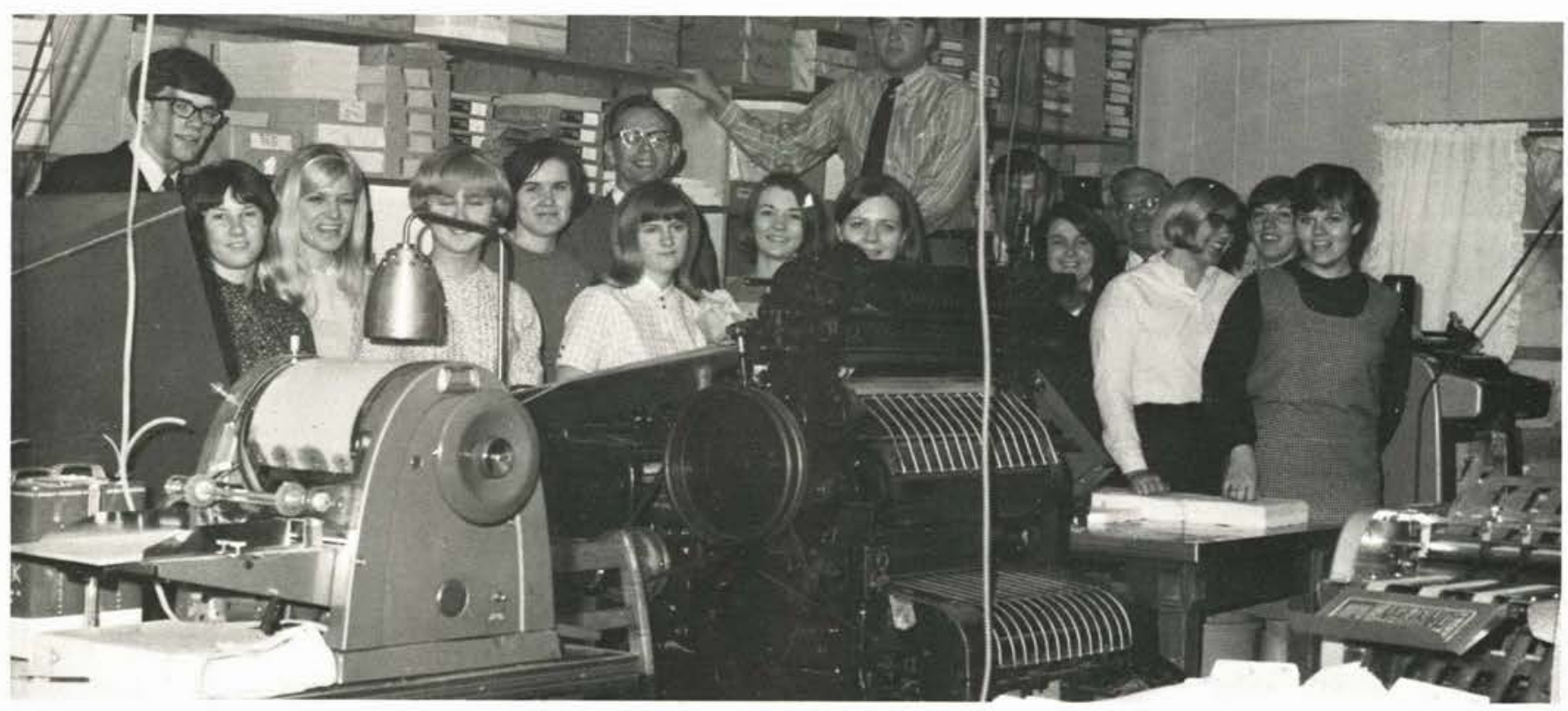

R. Mitchell, C. O'Shell, G. Sears, P. France, J. Winston, C. Averitt, Dr. C. Johnson, K. Griswold, J. Phenix, M. Woodend, D. Connelly, K. Spencer, Mr. C. Hurst, Advisor, B. Carlson, J. Kleymeer, M. Brewer.

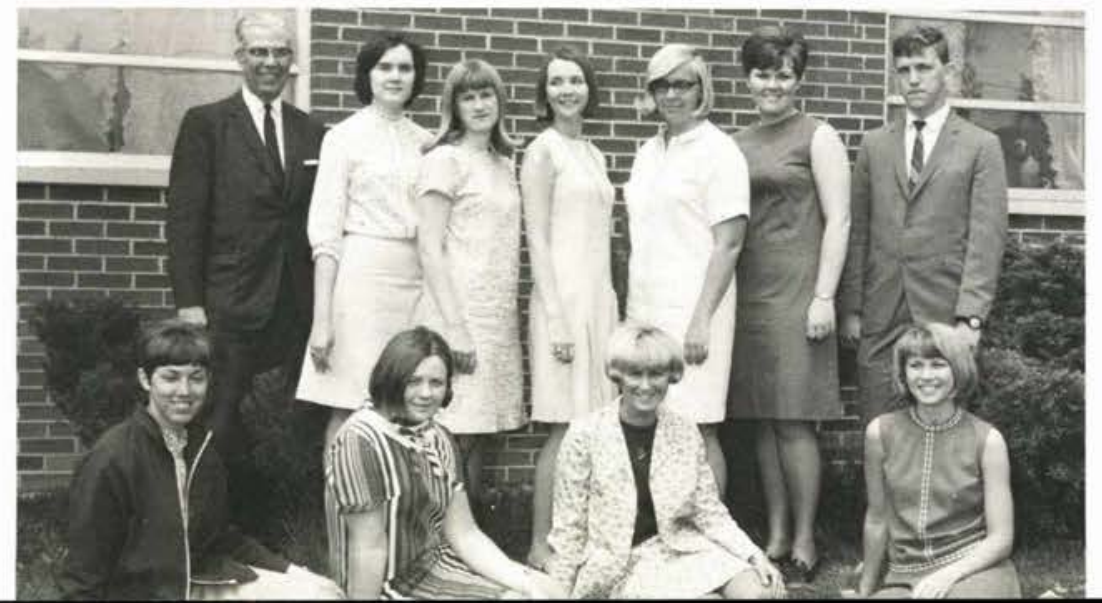


FBA

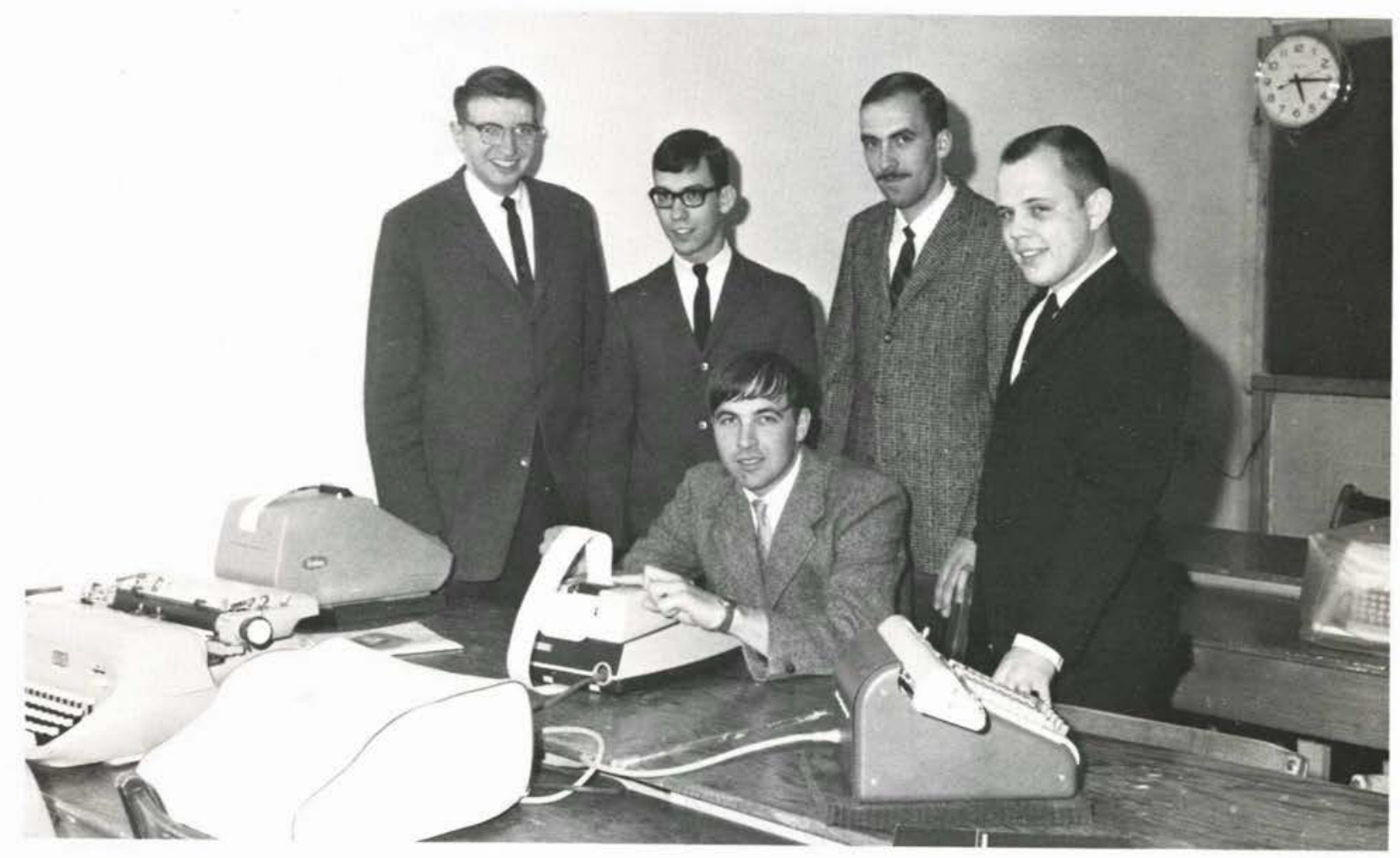

Seated: D. Kinniburgh. Standing: Mr. Wilcoxin, advisor; P. Spencer, J. Berry, K. Wagner.

E. Smelser, B. Schill, A. Shuter, B. Reed.

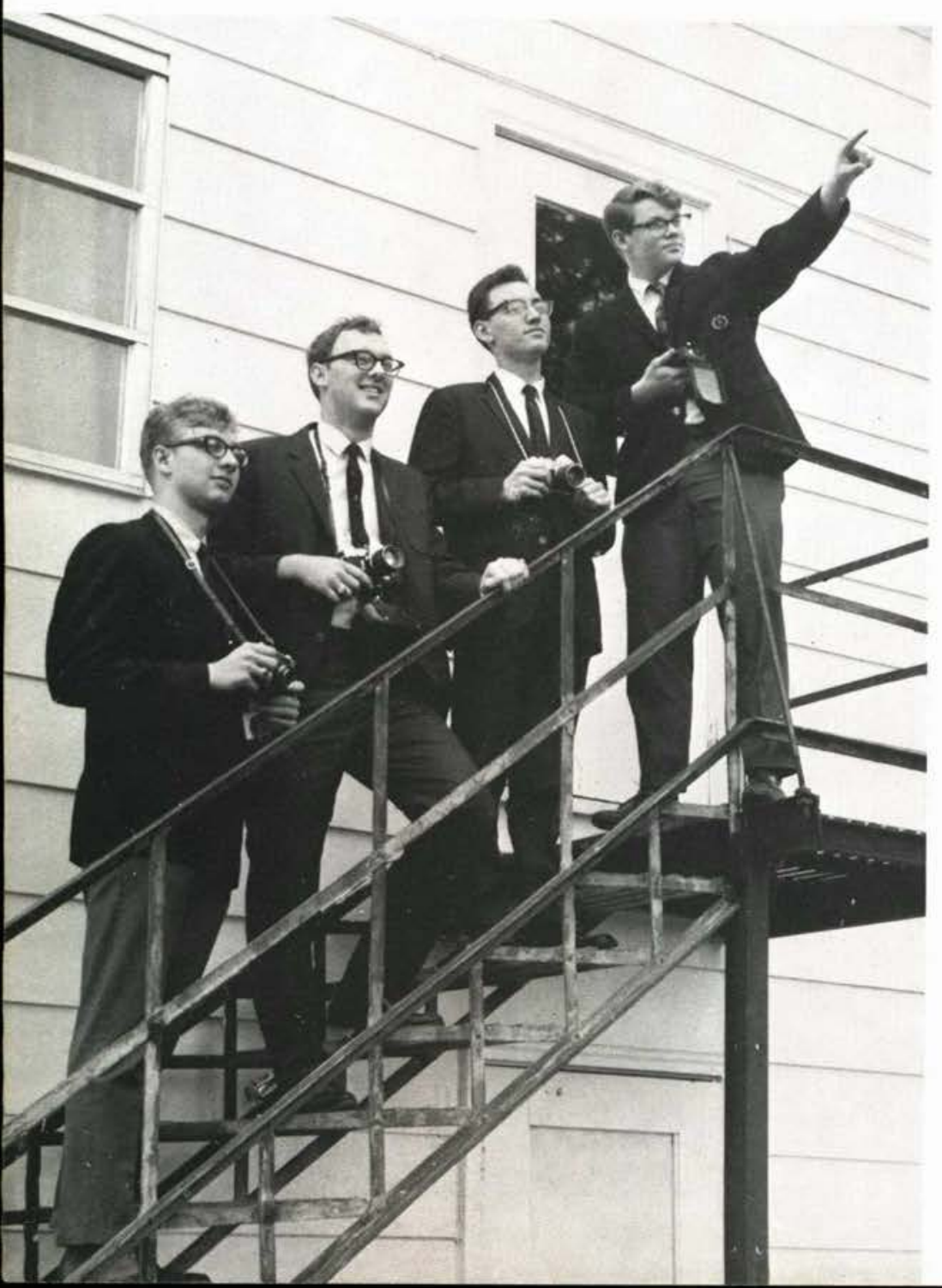

PHOTO CLUB 


\section{COLLEGE FIRE DEPARTMENT}

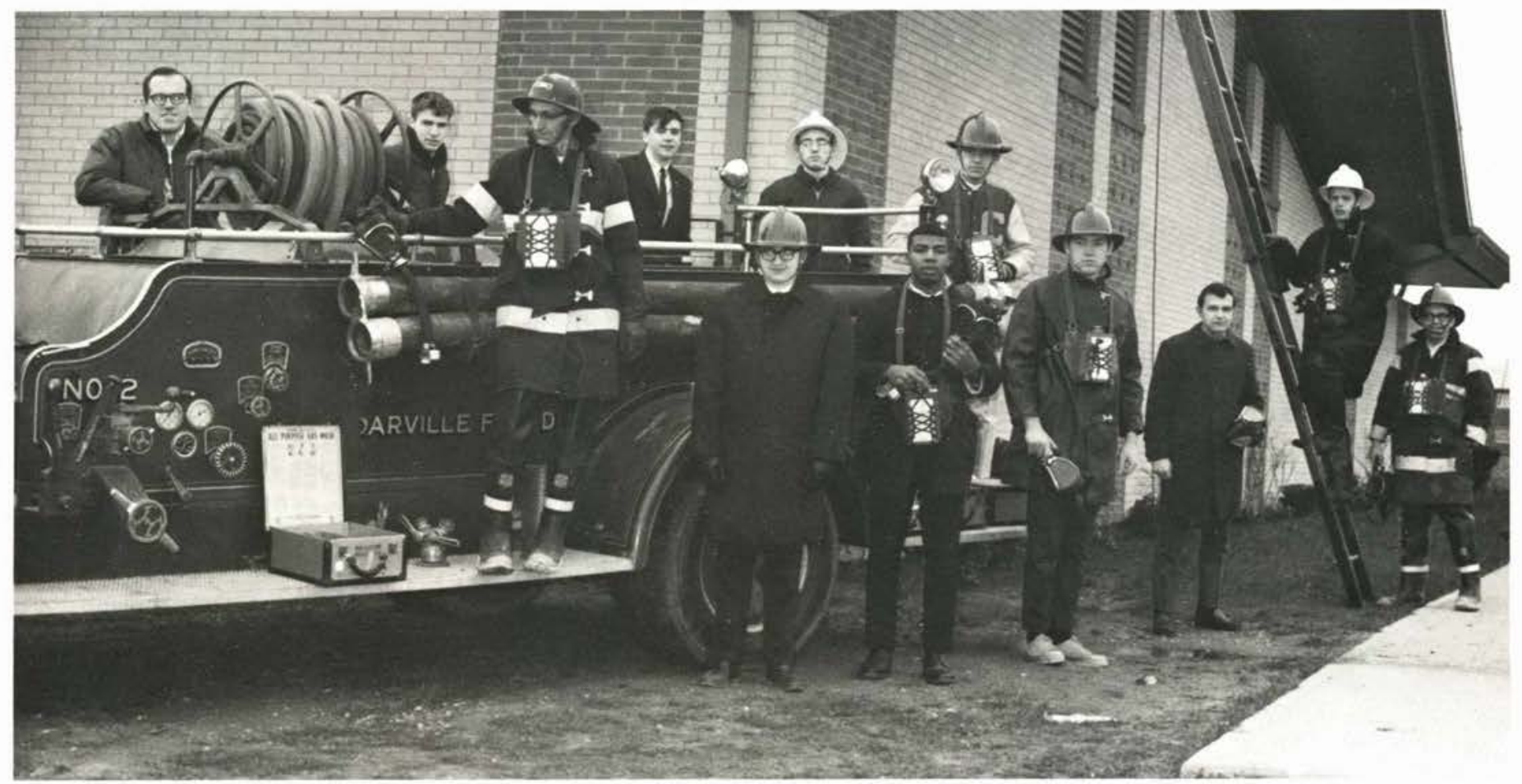

First Row: G. Marshall, R. Coombs, B. Hunter, J. Motter, D. Wall, B. Sweeney, R. Hamilton. Second Row: R. Leach, B. Lapp, R. Meyers, A. Shuter, R. Coriell.

\section{RADIO CLUB}

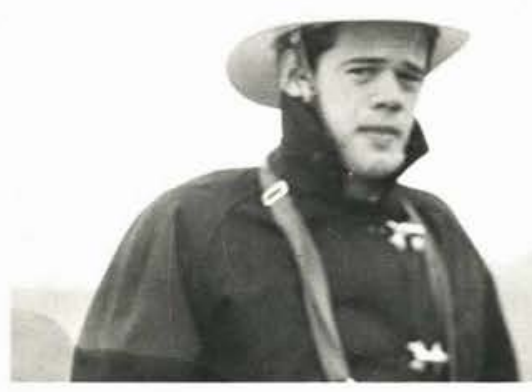

Seated: G. Richardson. Standing: Mr. Gathany, L. Welch, R. Patten, R. Hamilton, A. Shuter, L. Eichelber ger, C. Eckart.

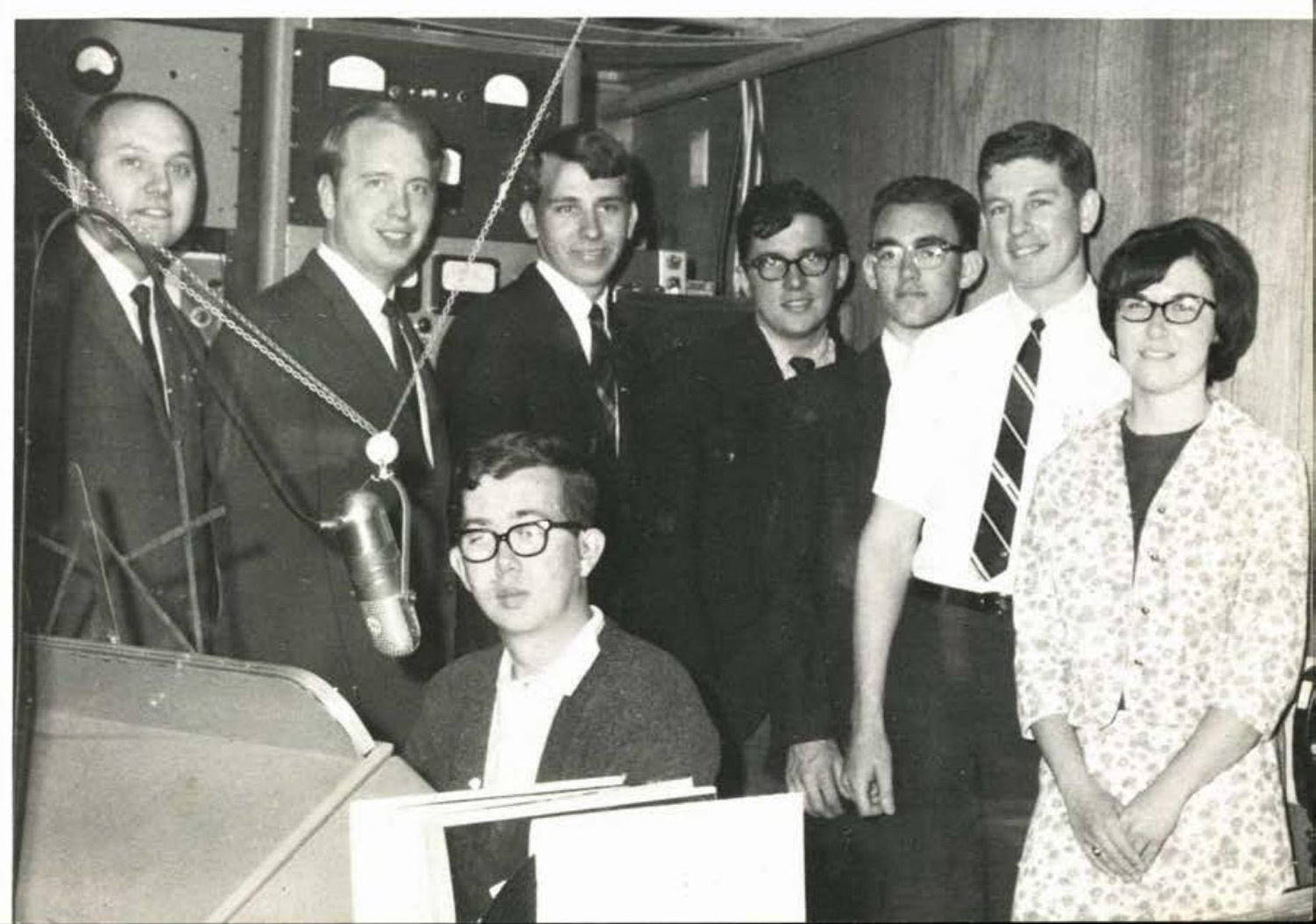




\section{CHRISTIAN SERVICE}

OLD FOLKS HOME

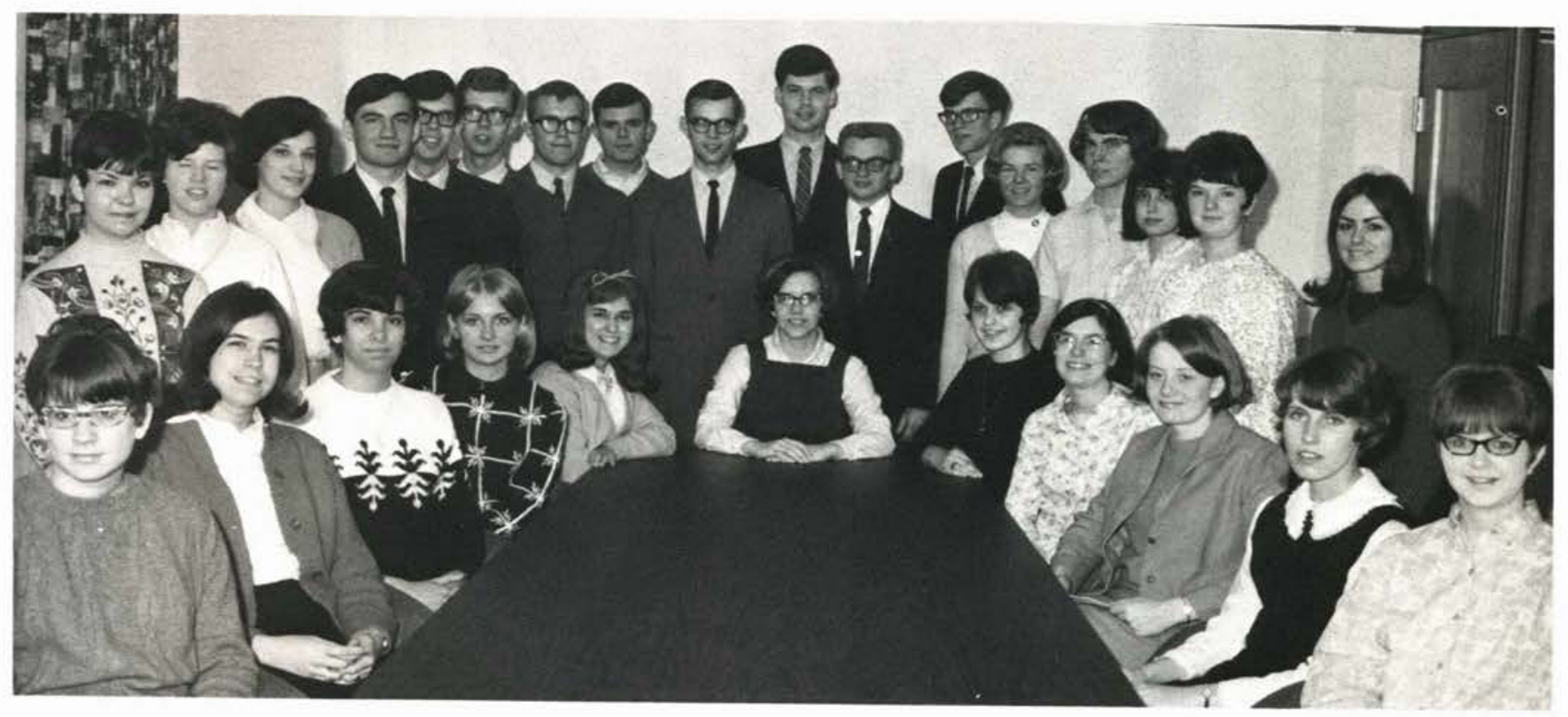

First Row: J. Boyd, C. Nieboer, N. Haffey, B. Bennett, B. Decker, J. Strychalski, K. Donaldson, E. Phillips, J. Austin, M. Bair, J. Grover. Second Row: A. Russell, C. Endicott, K. Borger, C. Rowe, B. Bixel, T. Greenwood, P. Oehmcke, F. Stickle, D. Acker, J. Price, S. Gordey, D. Mann, J. Eveland, N. Leapline. Third Row: R. Mitchell, B. Brown, S. Olsen.

\section{JAIL GROUP}

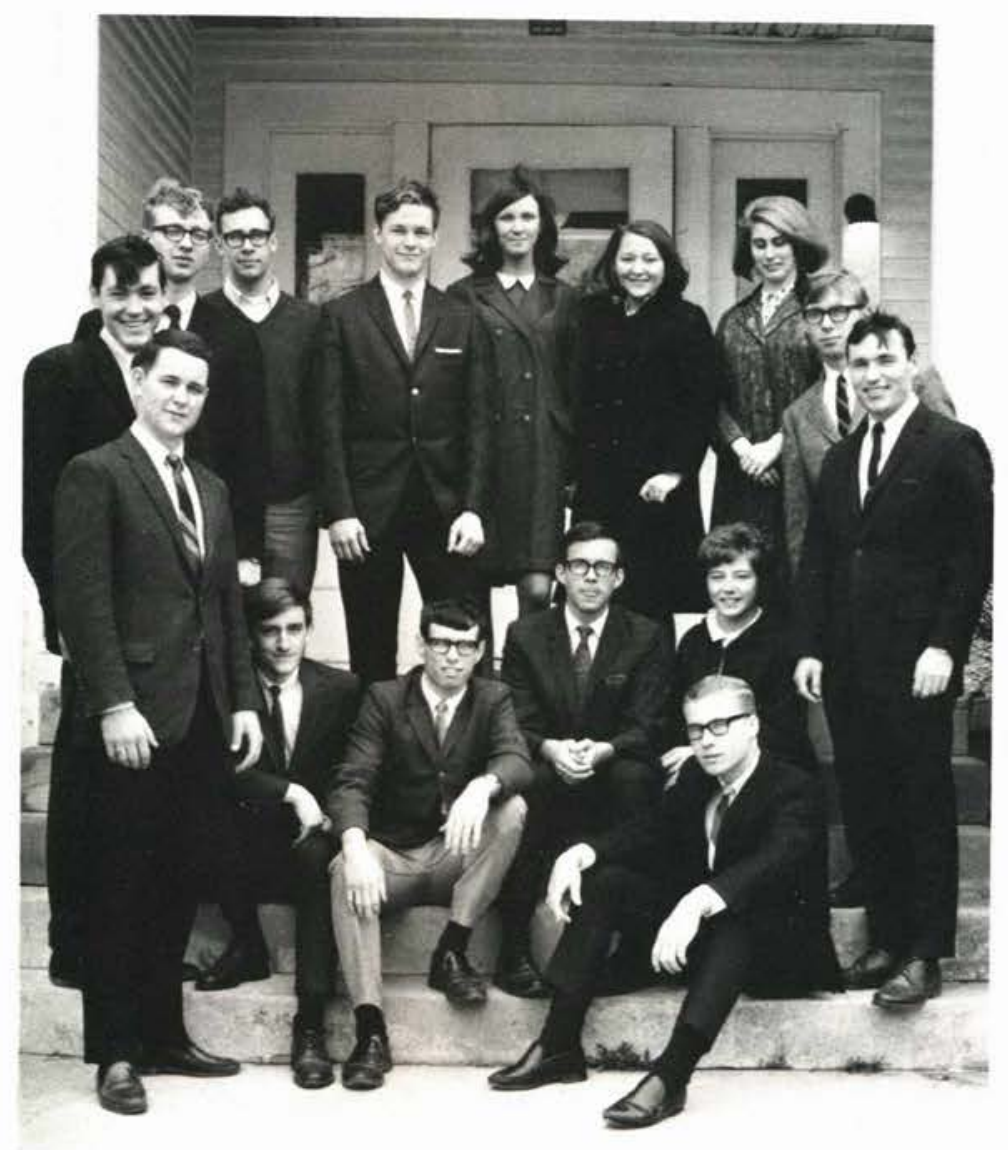

Sitting: B. Rehkopf, D. Mays, D. Deiringer, D. Kearney, L. Davis. Standing: R. Edwards, J. Motter, E. Smelser, K. Muck, M. Satler, V. Palmer, C. Jenista, R. Kauffold, D. Rohm, D.

\section{VIET NAM PRAYER}

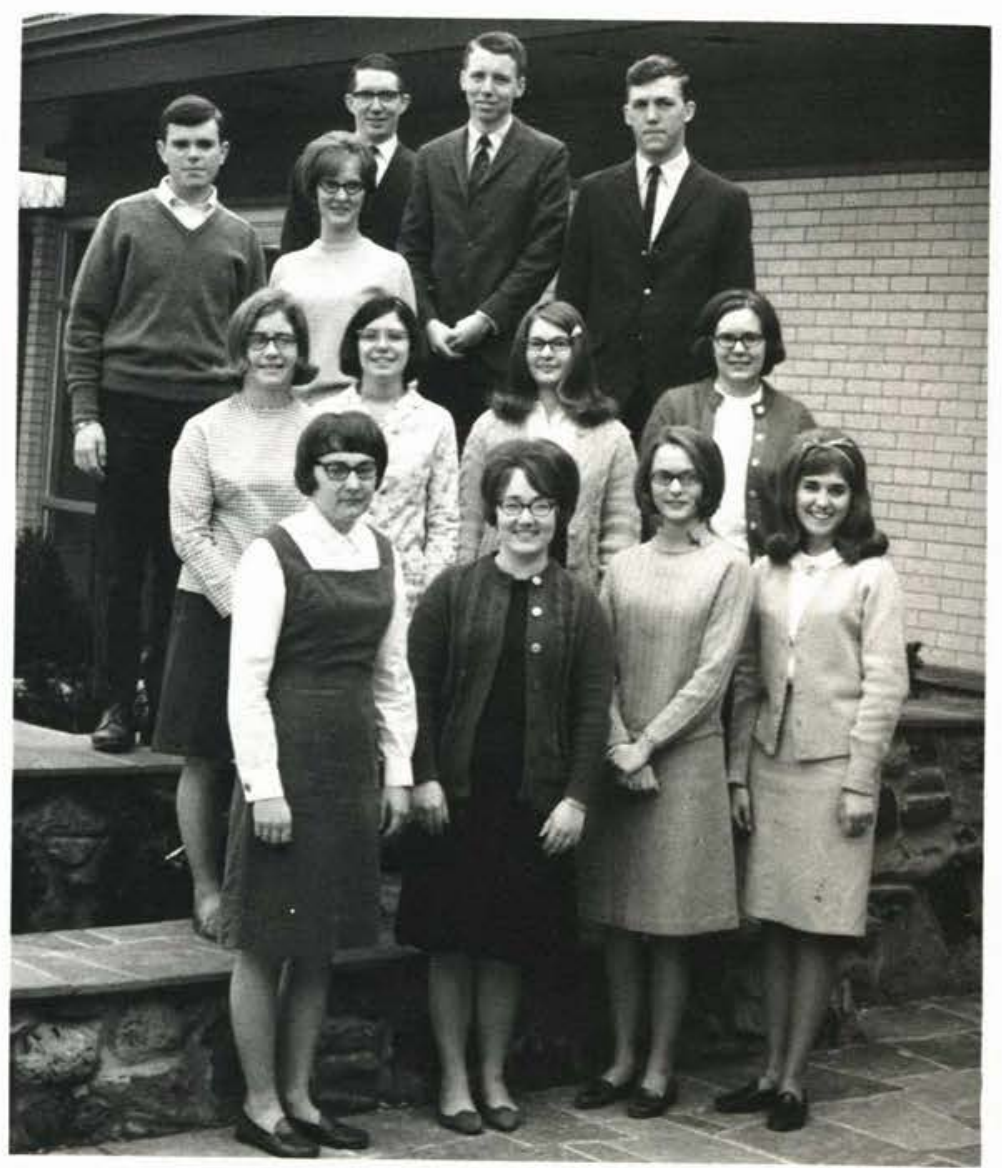

First Row: D. Baker, S. Trotter, D. Radcliffe, B. Decker. Second Row: M. Beitler, E. Phillips, S. Olsen, E. Price. Third Row: B. Brown, B. Miller. Fourth Row: K. Hildreth, L. Radcliff,
D. Connelly. 


\section{SWORDBEARERS}

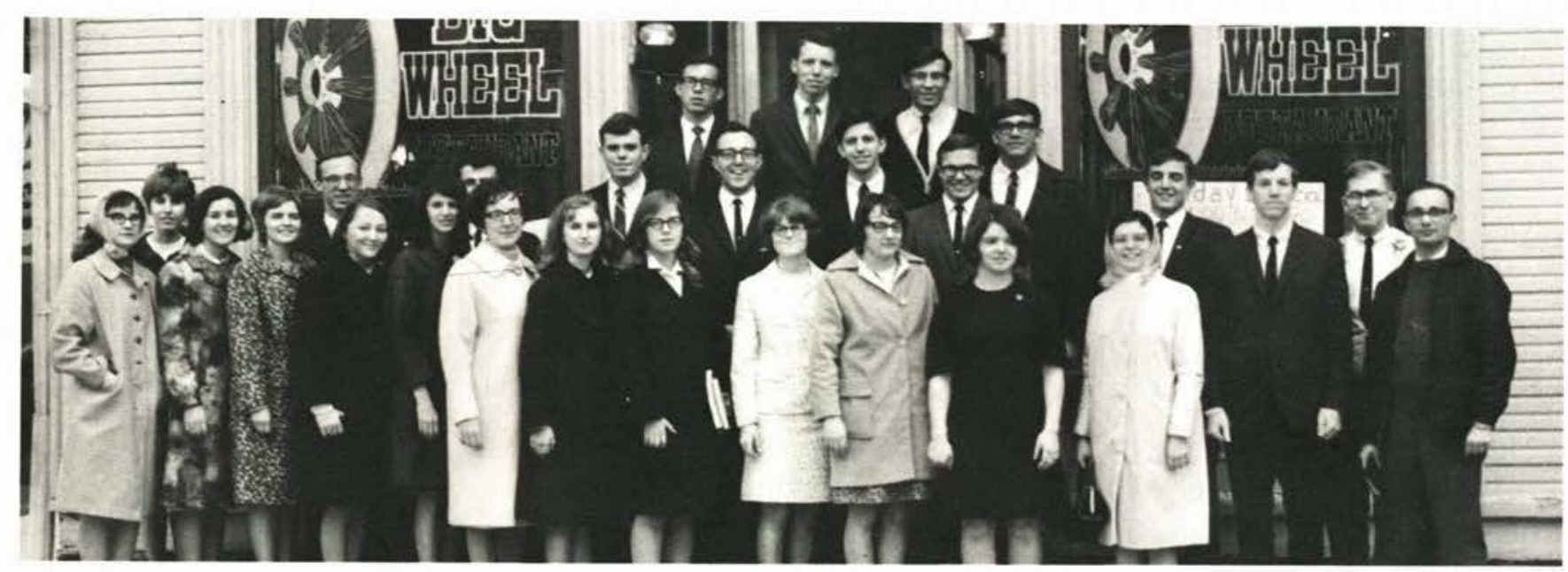

First Row: P. Prindle, E. Thomas, S. Dufford, C. Jenista, L. Myers, D. Myers, N. Paugh, M. Bartlett, R. Holtzhouse, D. Baker, R. Duell, E. Phillips, D. Gilbert, D. Fidler. Second Row: M. Bair, T. Clater, D. Shreve, B. Brown, D. Staples, P. Bolton, B. Smith, B. Sine, T. Northey, B. Reeder. Third Row: D. Dieringer, L. Radcliffe, B. Kilko.

\section{DETENTION HOME}

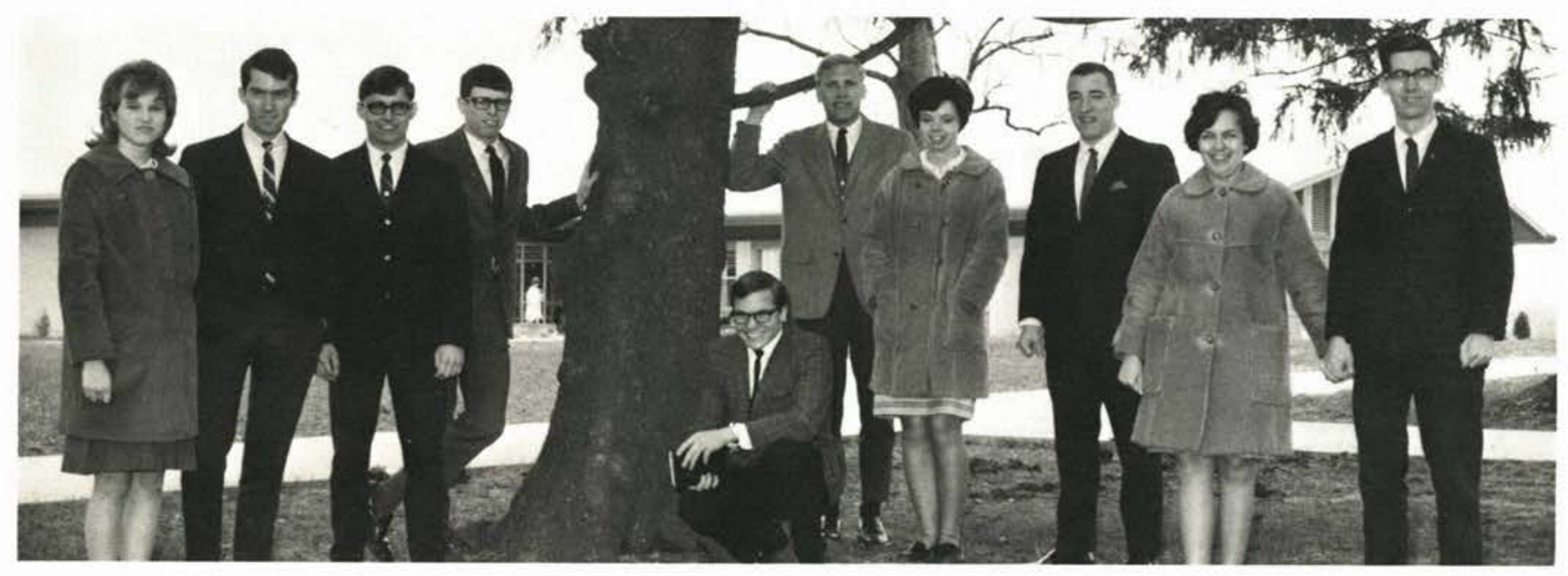

C. Willener, A. Call, G. Creviston, D. Mays, B. Smith, D. Mills, J. Miller, C. Packard, K. Albright, C. Hull.

\section{CAMPUS LIFE}

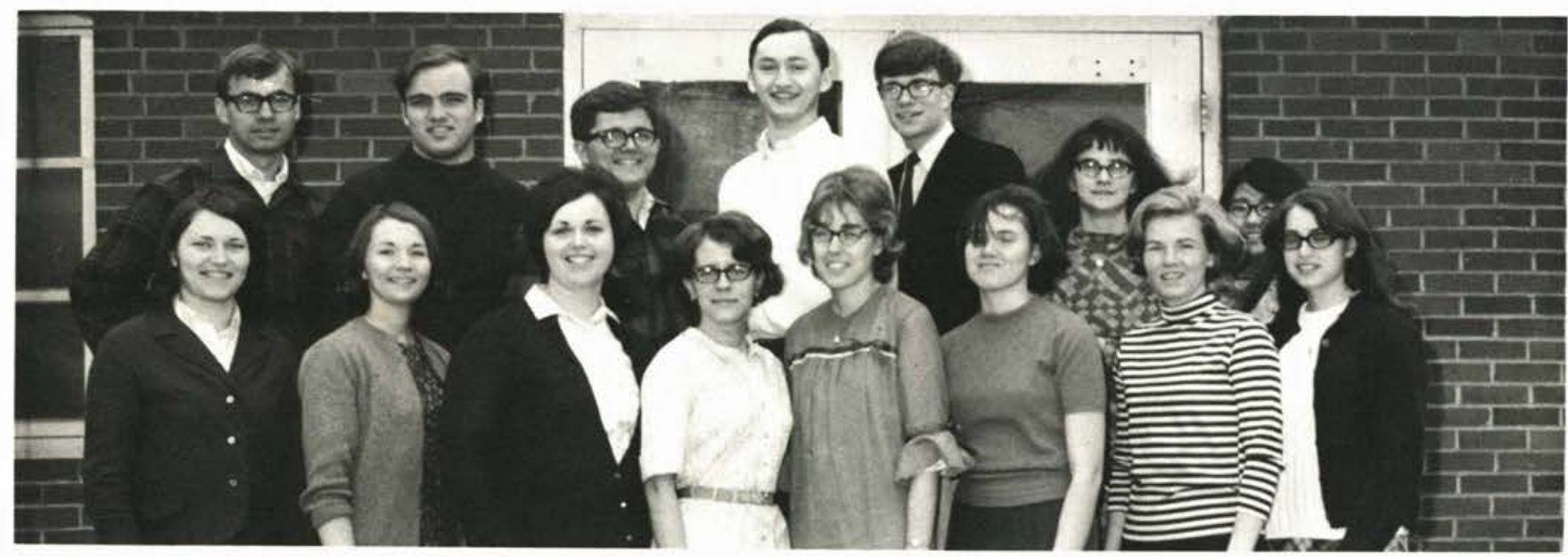

First Row: D. Thompson, K. Griswold, M. Crunk, M. Storey, A. Moorman, J. Winston, J. Price, J. Kever. Second Row: B. Schultz, D. Acton, W. Amstutz, T. Yoder, R. Mitchell, P. Prindle, M. Ishuin. 


\section{SUNDAY SCHOOL \& JUNIOR CHURCH}

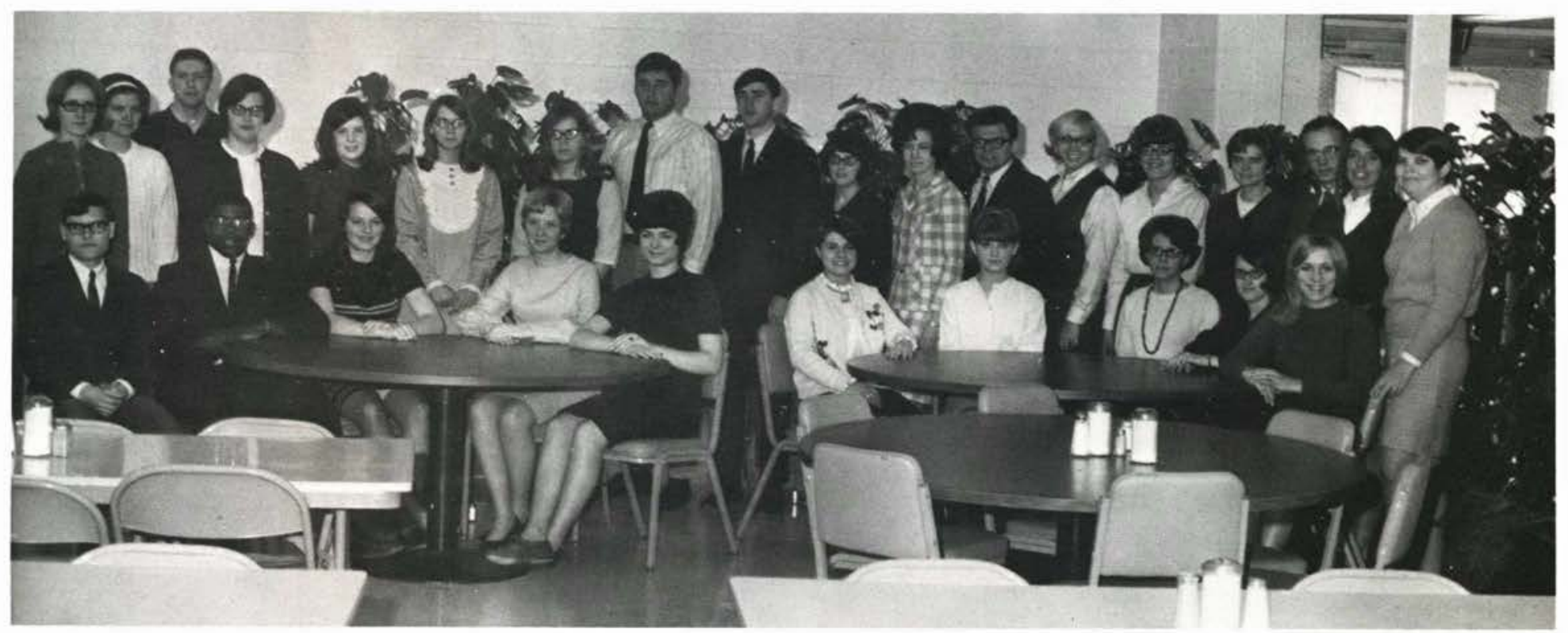

Seated: R. Jezowski, K. Hammonds, J. Hoerath, B. Meyers, M. Grable, B. Thomas, N. Schwarm, M. Storey, R. Mclnnes, C. Collins. Standing: B. Cleckner, M. Muirhead, D. Handyside, C. Zickefoose, V. Tallman, D. Lewis, M. Bartlett, M. Woodend, R. Meyers, J. Motts, N. Wright, W. Connell, B. Carlson, S. Prather, S. Dufford, T. Clater, V. Conant, L. Lescure.

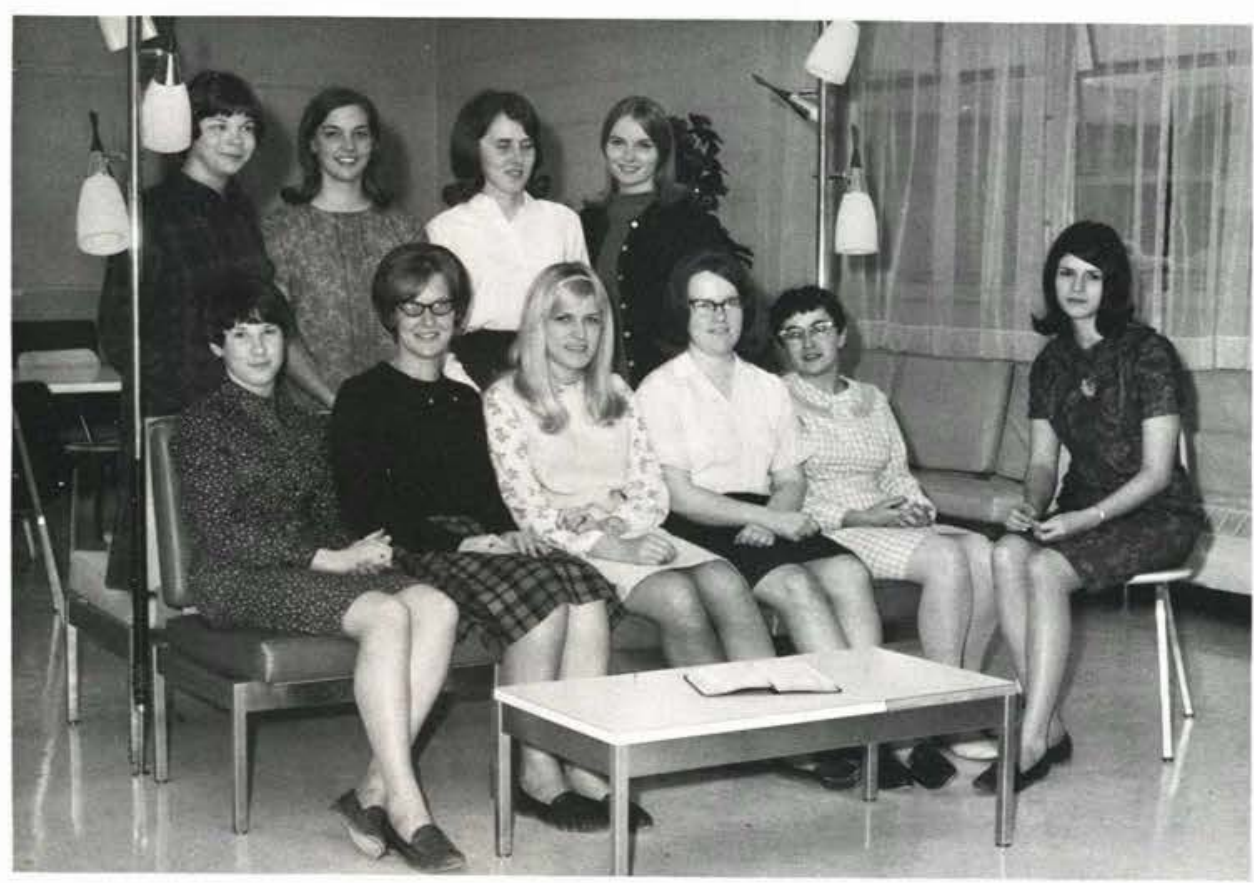

\section{BIBLE \\ CLUB}

First Row: C. O'Shell, B. Miller, G. Sears, N. Towle, J. Mossman, L. Myers. Second Row: B. Rudig, L. Madsen, J. Hoag, C. Beerer.

\section{CHRISTIAN EDUCATION

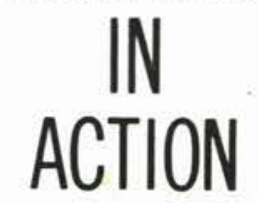

D. Nevitt, S. Dufford, B. Meyers, Miss Jean Fisher, M. Kapp, M. Stanbery, J. Boyd.

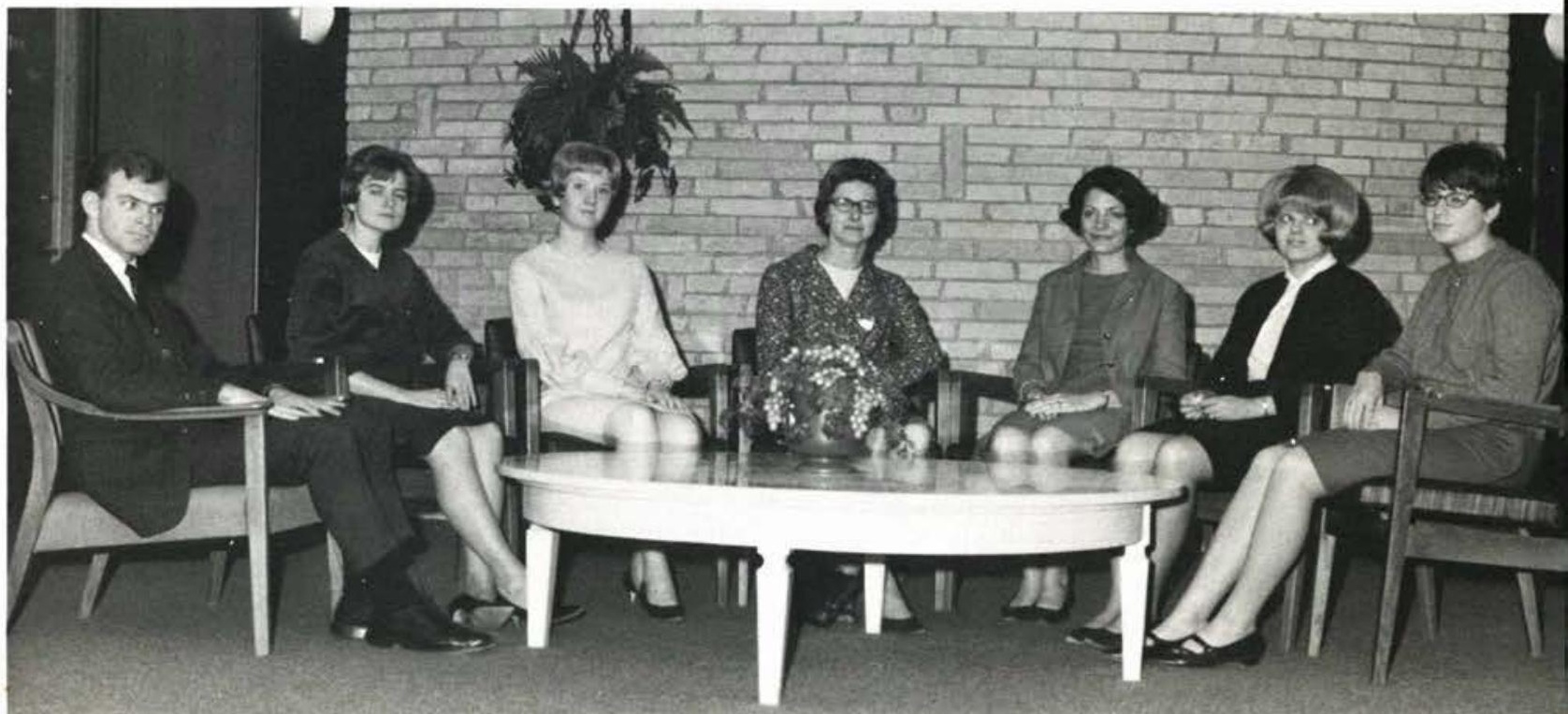




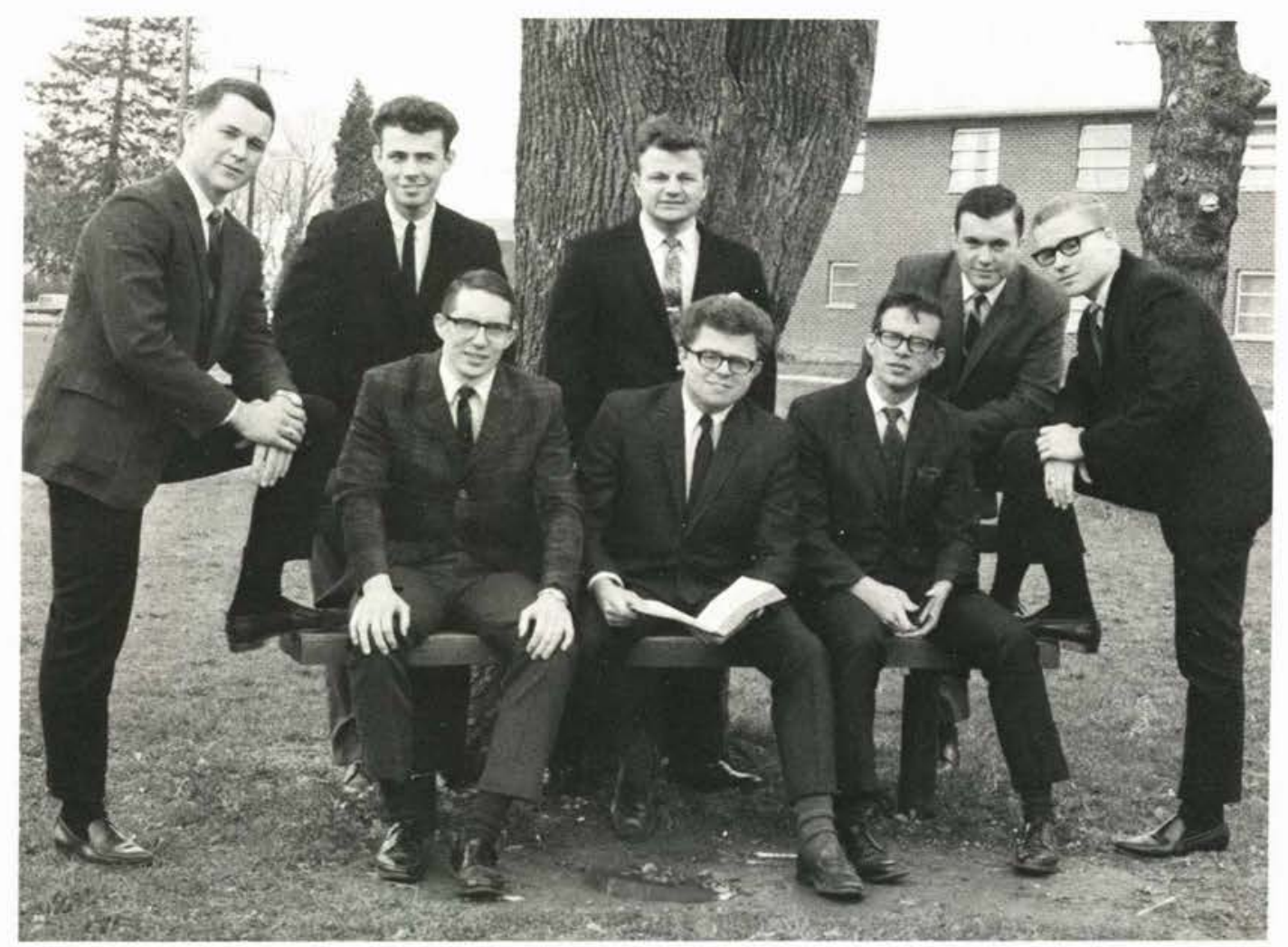

Seated: K. Heldreth, W. Connell, D. Dieringer. Standing: R. Edwards, D. Shreve, P. Friedrich, S. Hesler, D. Kearney.

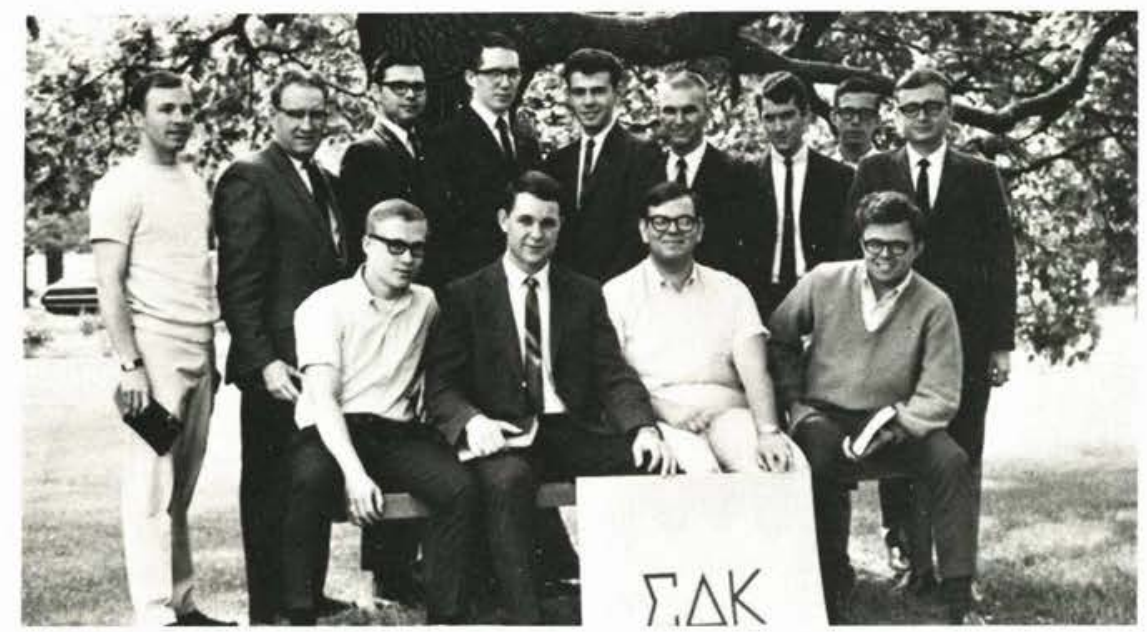

\section{SIGMA DELTA KAPPA}

Seated: D. Kearney, R. Edwards, S. Snyder, W. Connell. Standing: D. Rockwell, Mr. Armstrong, Advisor; P. Jones, K. Heldreth, D. Shreve, M. McCullough, A. Call, D. Dieringer, B. McVey.

\section{PEN PALS}

Dianna Hutchison, Diana Radcliffe, Barb Miller, Bob Pereira.

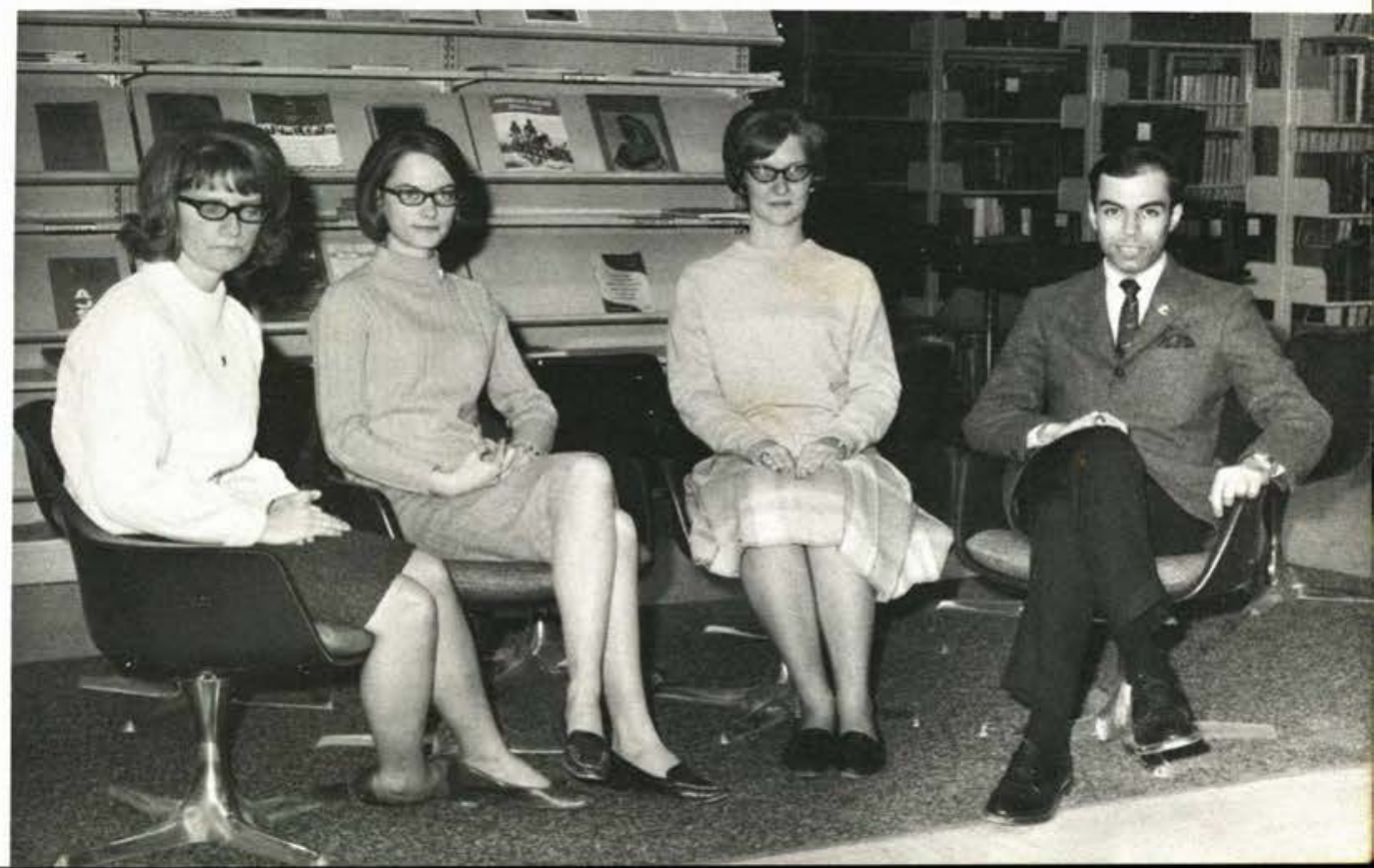




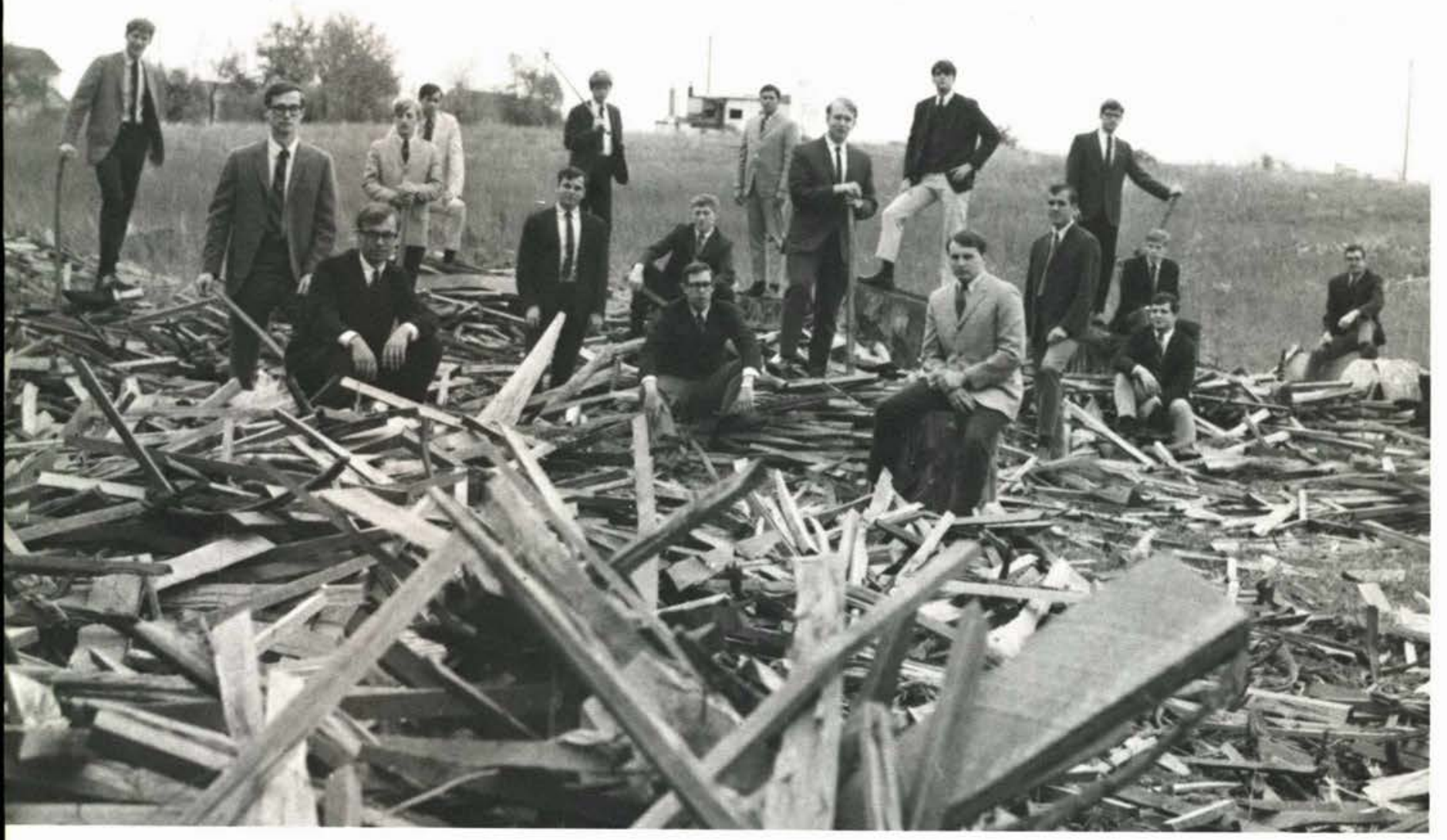

Pres........ Walker V.Pres..... K. Muck Sec...... J. Rooke Treas.... R. Daubert

G. Darrow, R. Allen, R. Schultz, R. Adams, J. Lee, A. Keim, J. Rooke, K. Muck, D. Southwell, M. Woodend, L. Weich, T. Warren, L. Walker, R. Cook, A. Gathany, D. Marshall, R. Barnhart, D. Lauener.

PI SIGMA NU

\section{ALPHA CHI}

First Row: D. Haseltine, D. Mills, D. Rohm, K. Evans, R. Hamilton, R. Coriell, K. Hammonds, R. Allerton, R Coombs. Second Row: B. Schill, R. Patten, M. Sattler, B. Hunter, S. Walker, M. McCullough, C. Rowe, B. Bixel, R. Jezowski. Third Row: C. Dutton, D. Dieringer, K. Cole, B. Grunert, L. Eichelberger, A. Knott, M. Norris, D. Bodden, P. Large, T. Gelo. Fourth Row: D. Hull, J. Phipps, D. Mays, D. Wall, D. Gish, J Collier D. Seigneur, J. Motter, D. Mohler, P. Senseney, R. Leach, A. Call. Fifth Row: G. Crevistons, I Seely, A Sweeney, D. Gaffner, I. Bosworth, R. Myers, S. Olsen, B. Lapp, R. Mitchell, J. Frye. Sixth Row: D. Manross, J. Rumley, D. Braun.
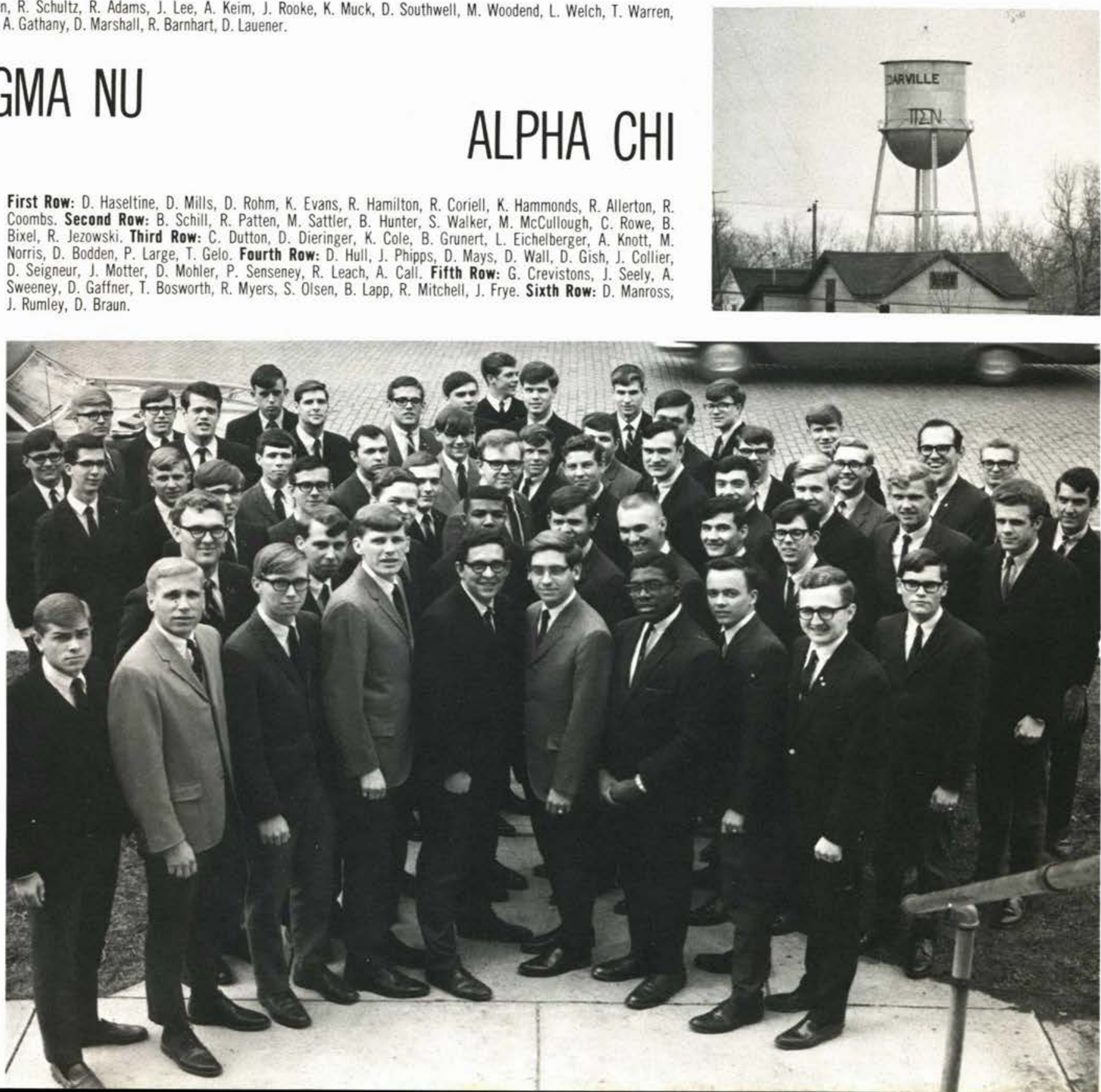


\section{GAMMA CHI}

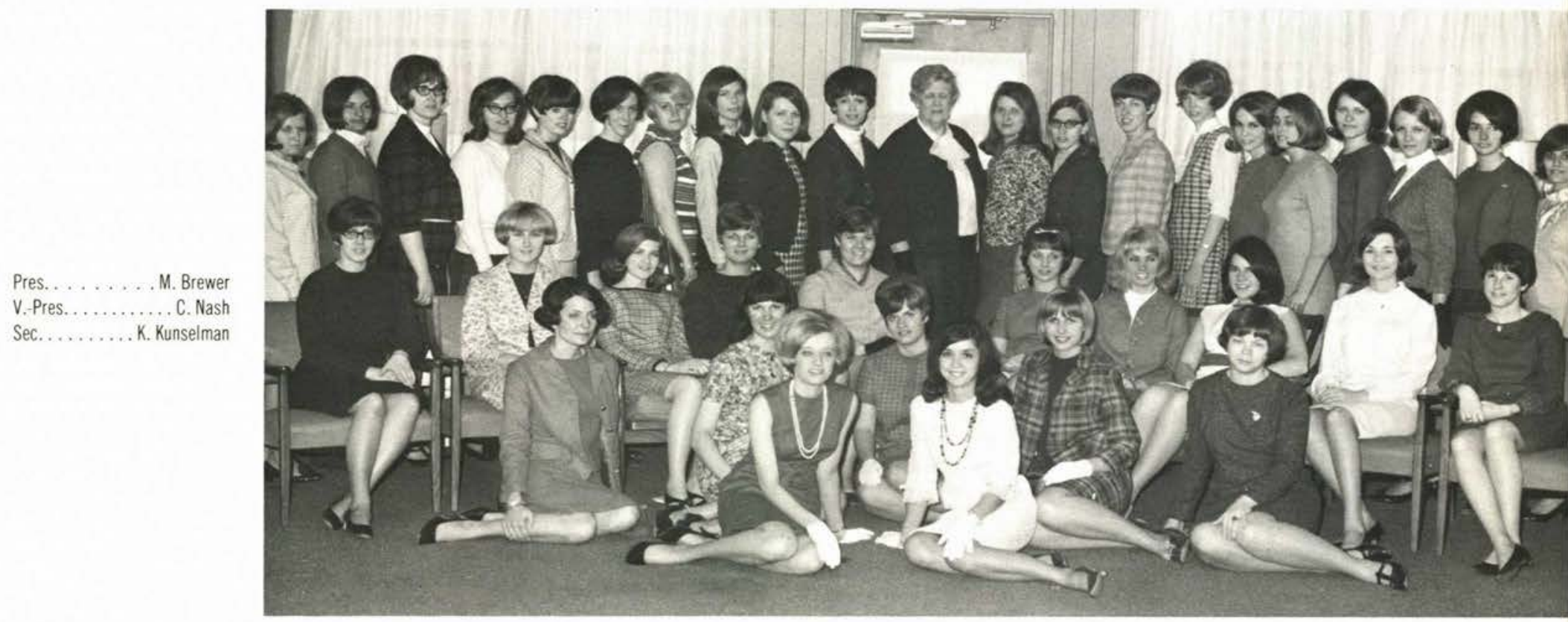

First Row: M. Reed, N. Price. Second Row: M. Kapp, D. Fitch, K. Johnson, L. Taylor, B. Rudig. Third Row: B. O'Keefe, P. France, D. Hamilton, E. Johnson, M. Brewer, K. Kunselman, C. Nash, N. Norton, K. Griswold, C. O'Shell. Fourth Row: L. Browning, A. Plate, S. Lucas, G. Foster, G. Gatliff, B. Maidment, V. Meyer, H. Stowell, J. Phenix, M. Bales, Mrs. M. Maddox, K. Gildan, B. Harman, J. Kleymeer, R. Silverthorn, J. Saemenes, R. Kauffold, D. Thompson, L. Riggs, D. Wilkes, B. Grosh.

\section{KAPPA DELTA CHI}

First Row: Mrs. N. McPheeters, Advisor, J. Corbin, J. Hoag, R. Butler, D. Simpson, J. Kever. Second Row: M. Rieken, R. Hartikainen, C. Beerer, C. Stevenson, B. Meyers, B. Vincent, C. McGee, D. Morgan.

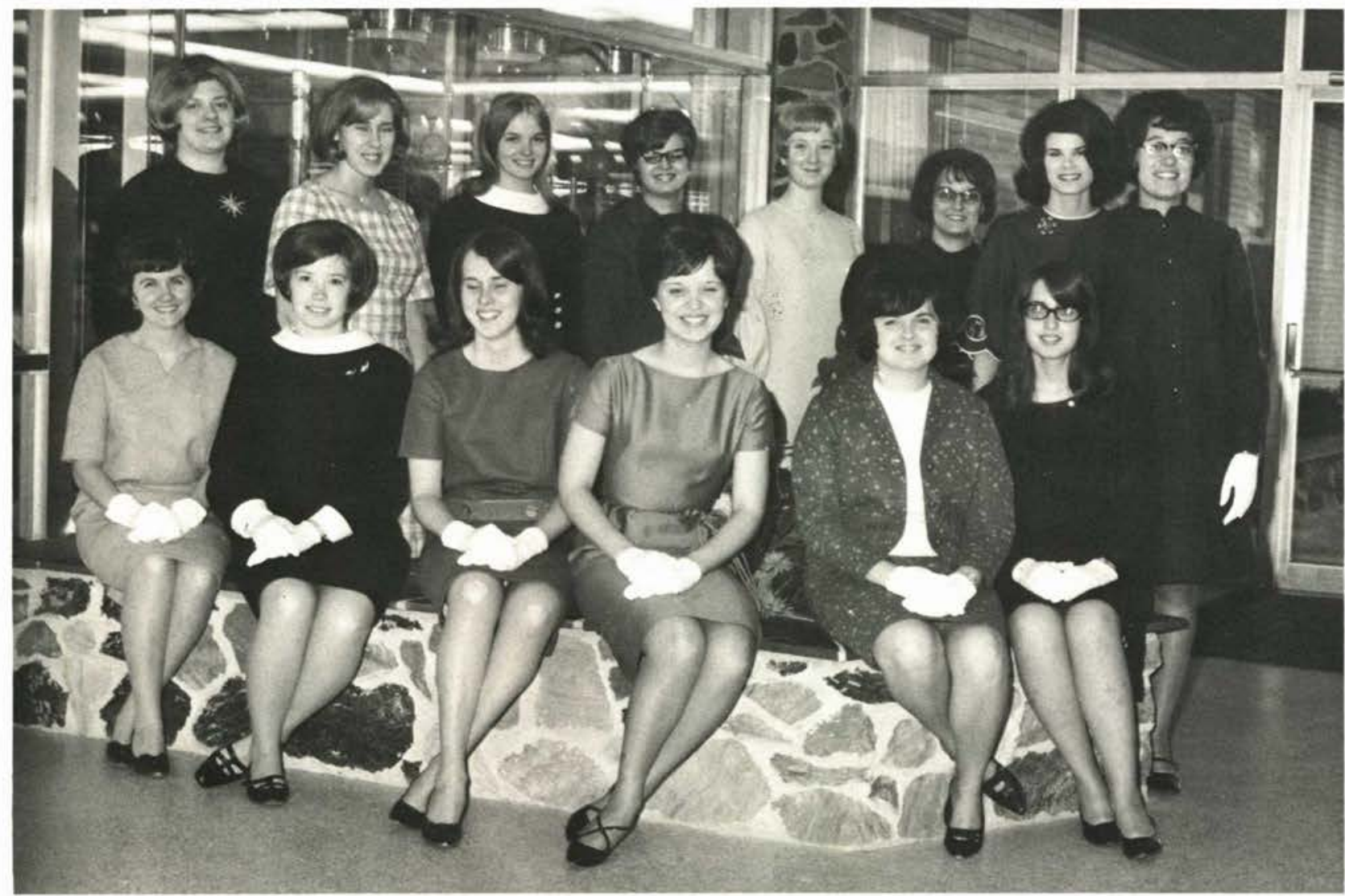

Pres. ..... C. Stevenson V.-Pres. ... R. Hartikainen Sec..........M. Rieken 


\section{AMBASSADORS}

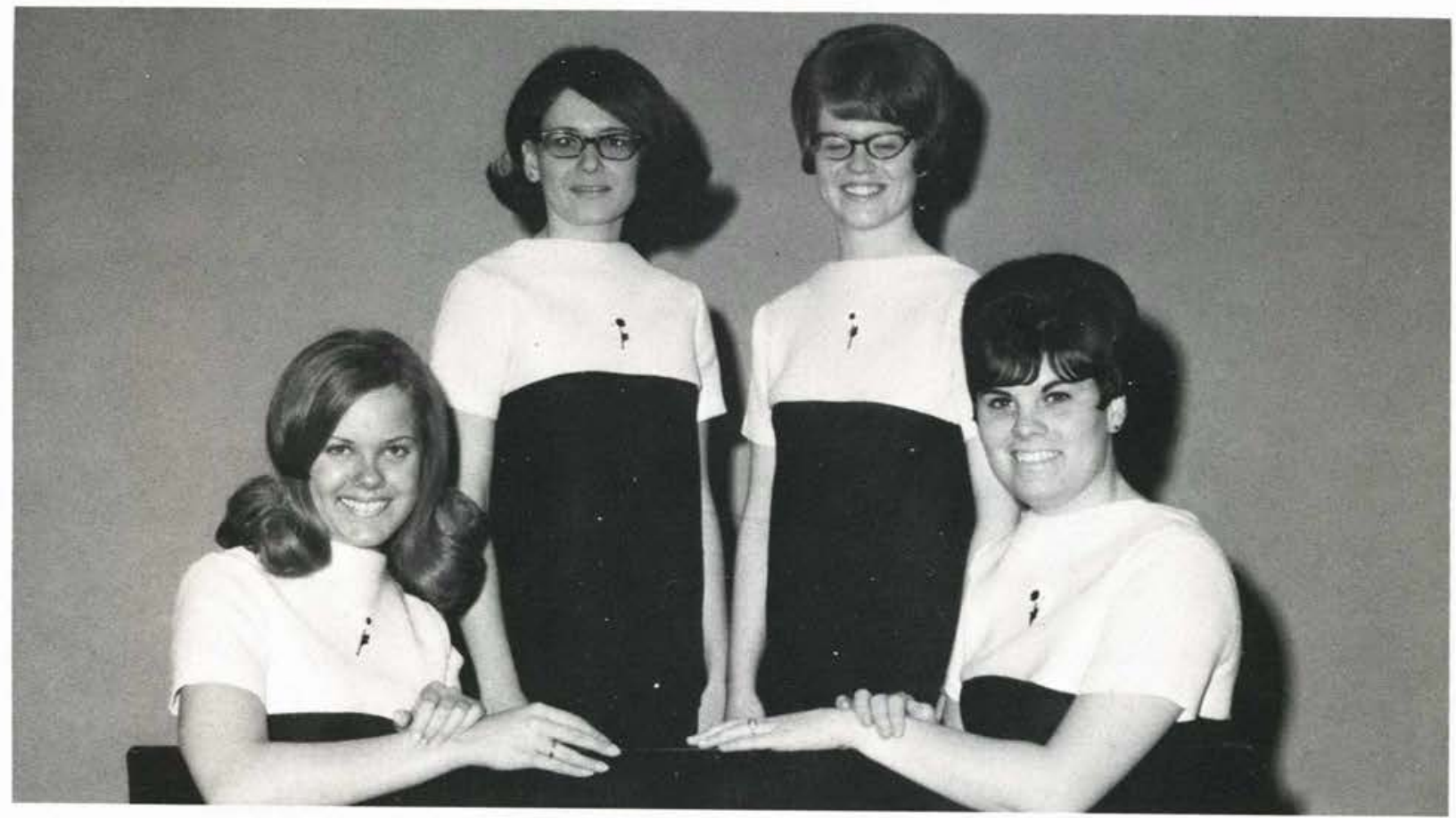

Janet Beesley, Cindy Cartner, Janice Brock, Cheryl Steenburg.

\section{FOLLOWERS}

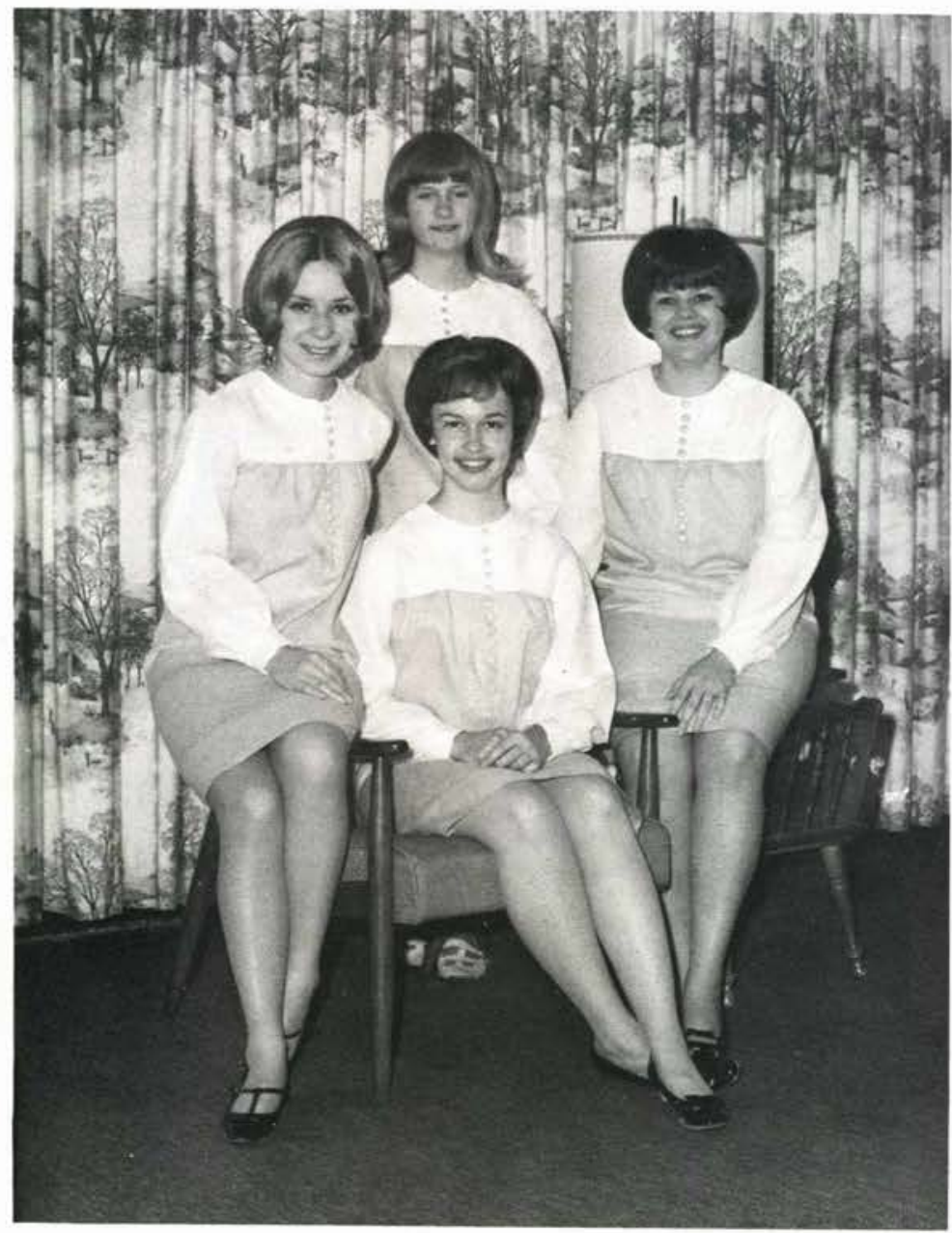

Sitting: Laura Payne, Jan Gabriel, Cathy Cartner. Standing: Connie Averitt.
LIFE LINERS

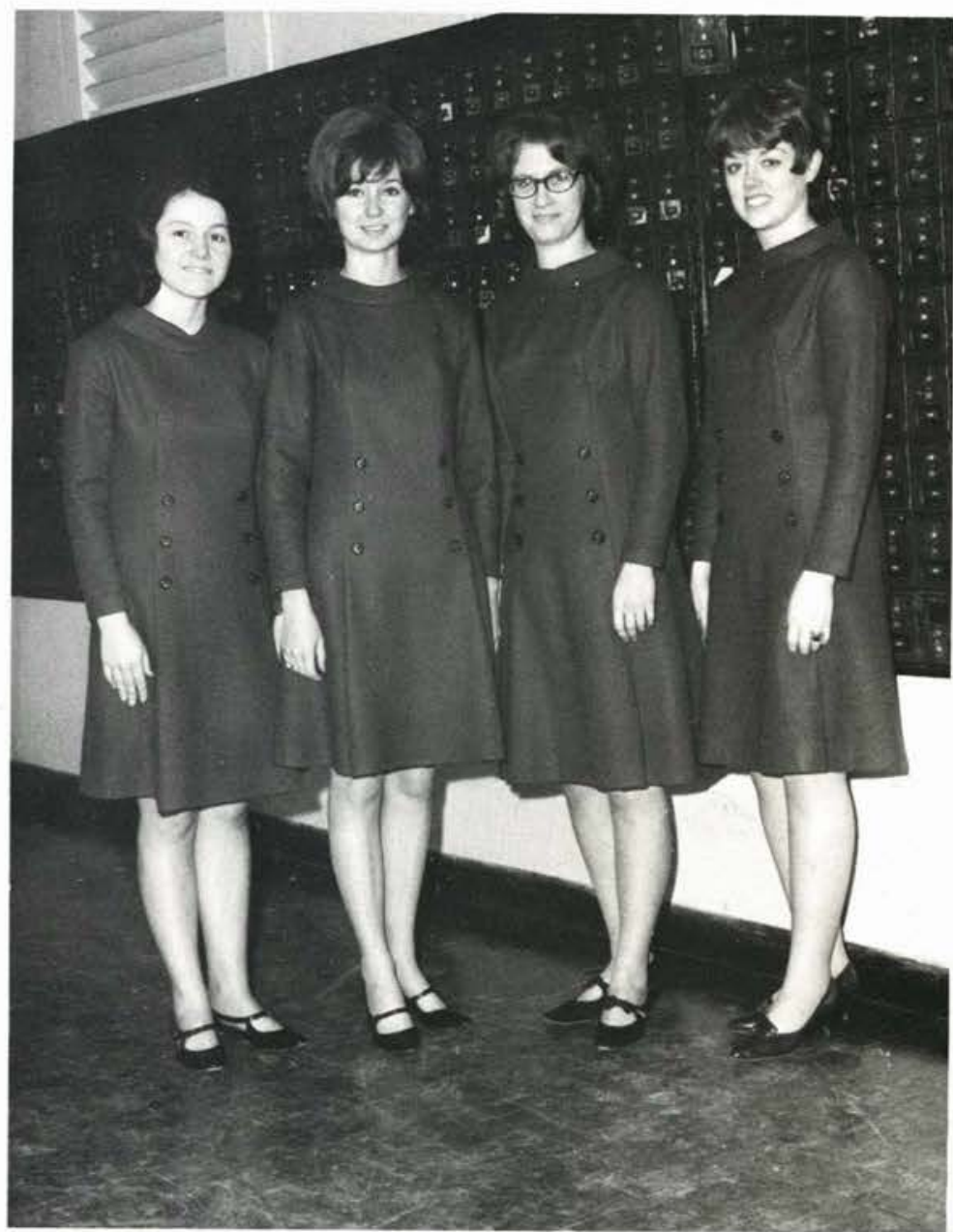

Ruth Hardy, Betsy Bodenmiller, Jane Hess, Gail Gatliff. 


\section{VICTORS}
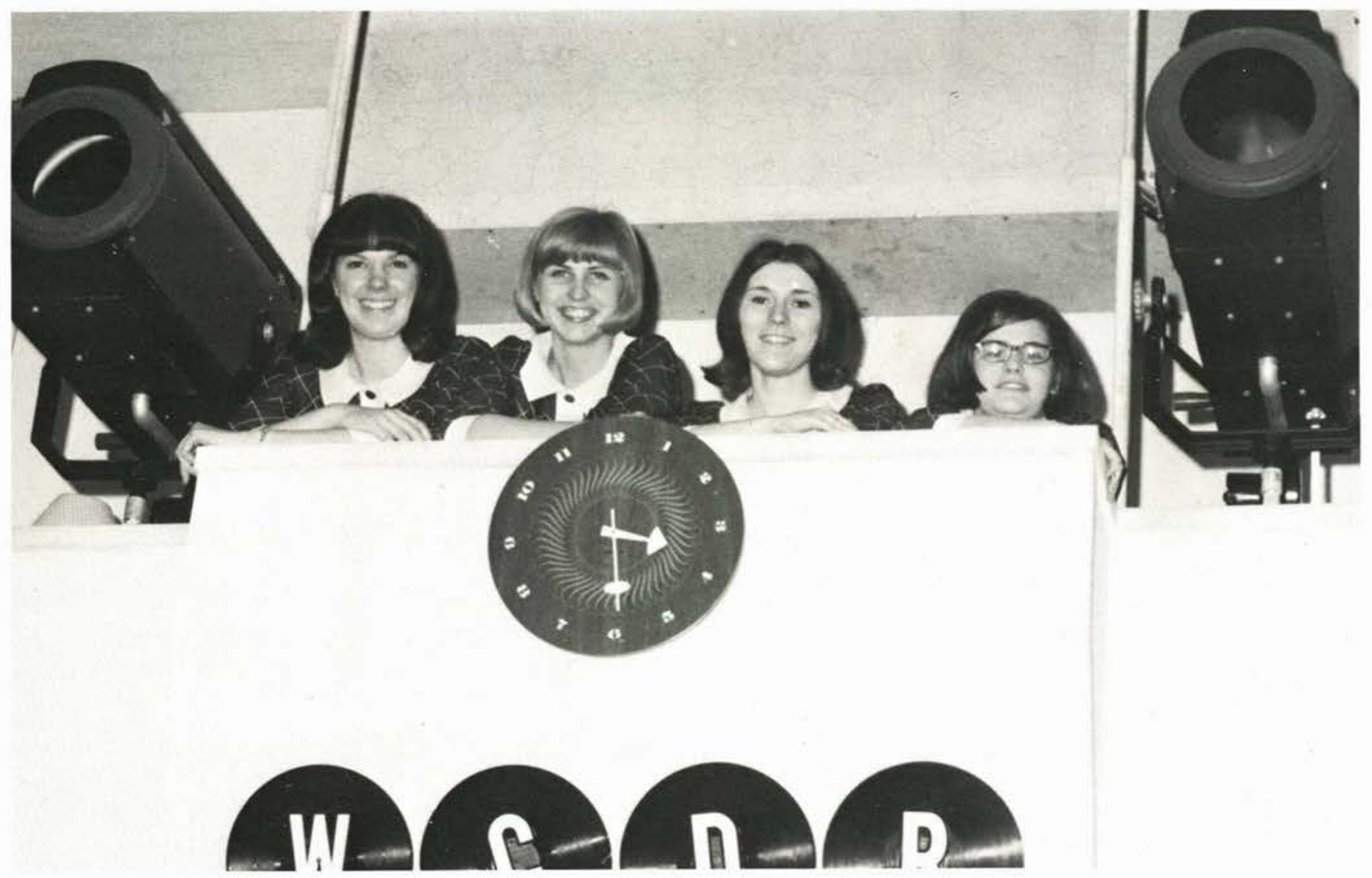

Darlene Fitch, Laura Taylor, Esther Greenwood, Margaret Anderson.

\section{GUARDSMEN}

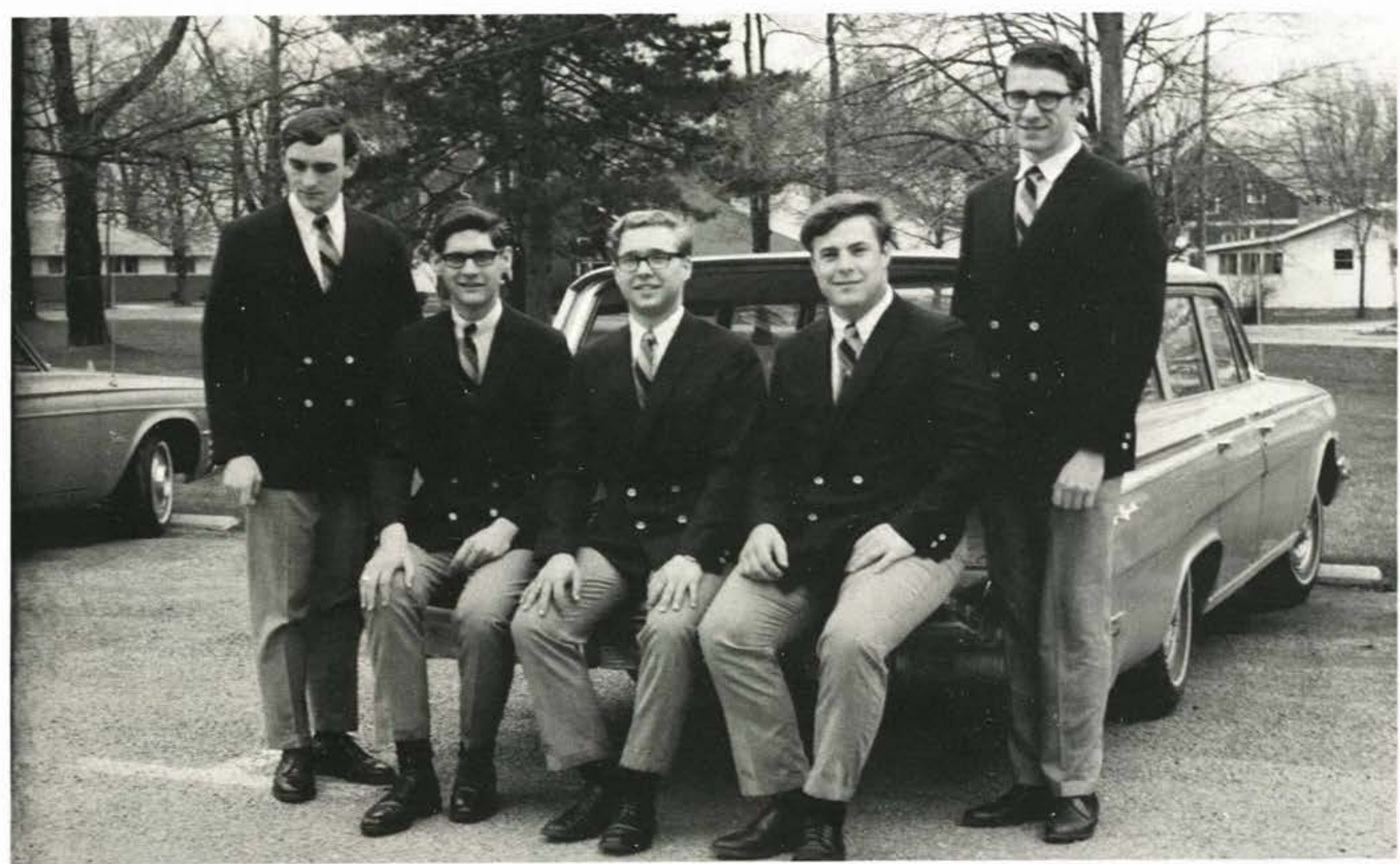

Lyle Anderson, Ron Spieth, Phil Senseney, Dennis Bunting, Philip Mohler. 


\section{SAILORS}

\section{TRUMPET TRIO}

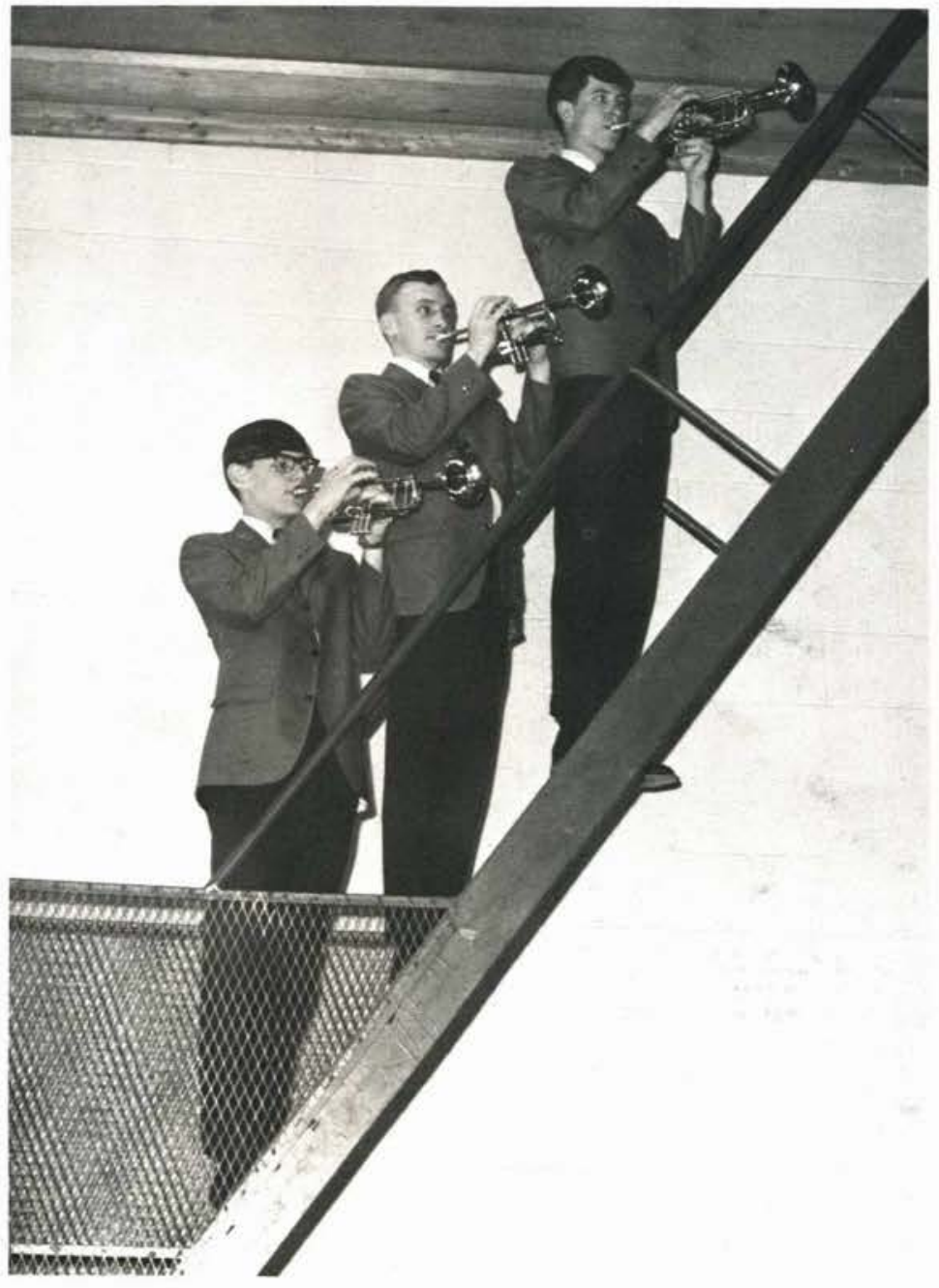

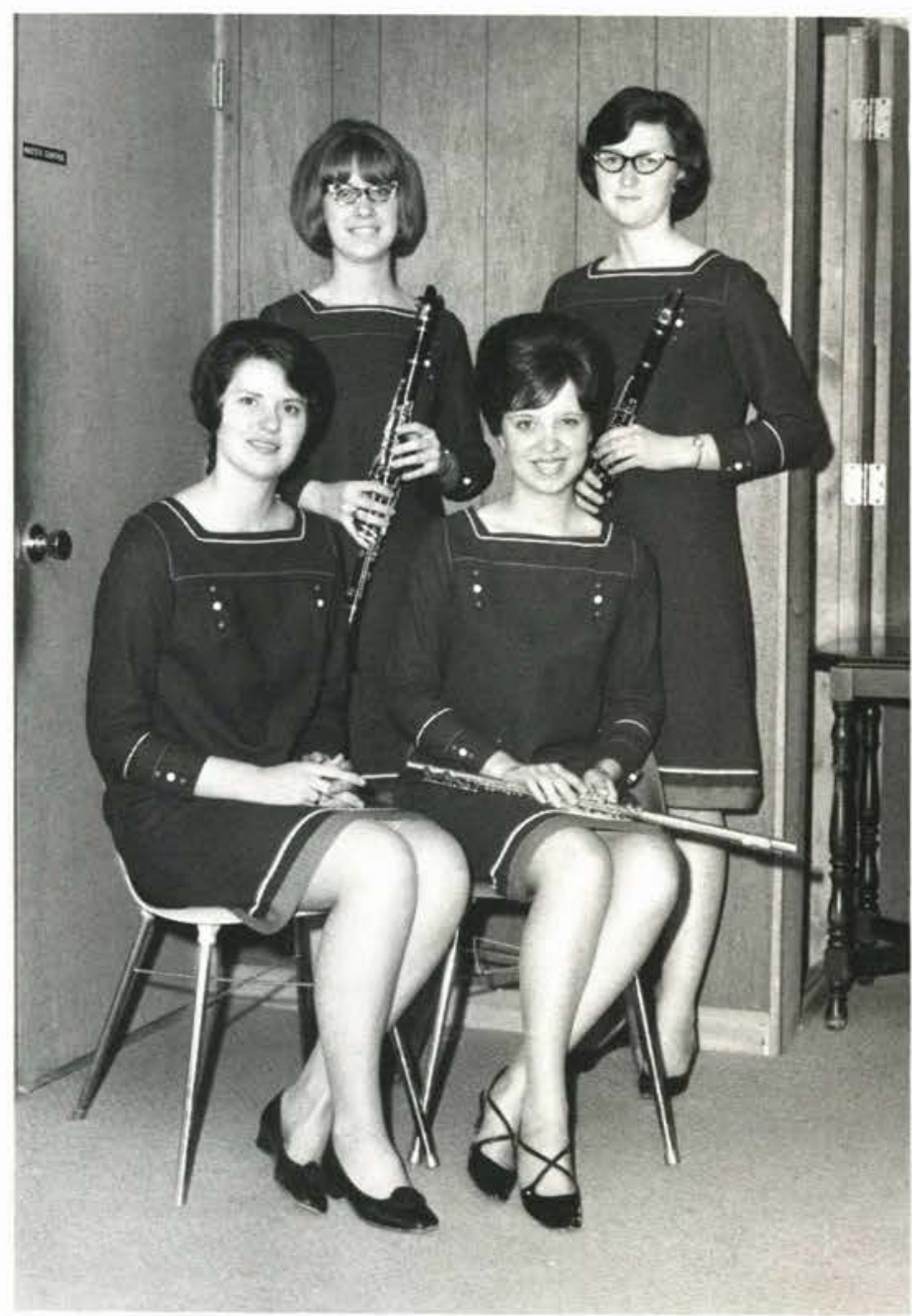

Sitting: M. Brown, R. Butler. Standing: C. Busho, S. Eury.

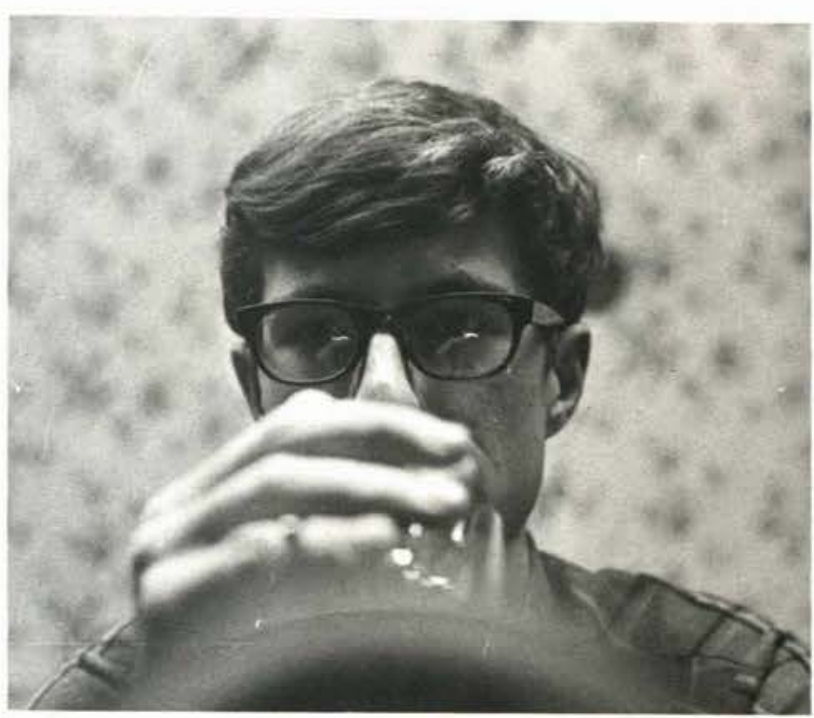

D. Gish, L. Pugh, D. Smith. 


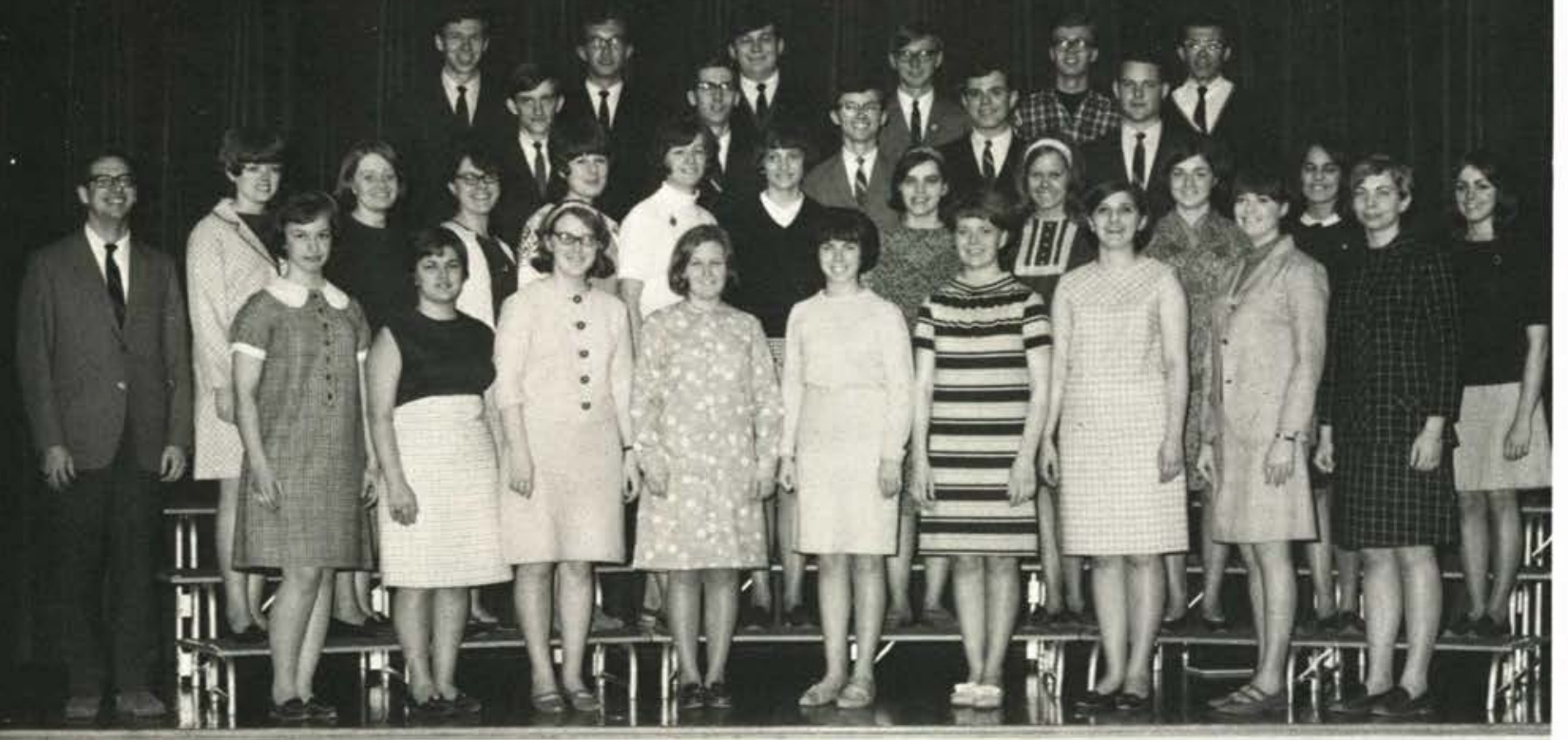

First Row: J. Gabriel, J. King, L. Hirschy, L. Browning, C. Morse, N. Queen, J. Risko, K. Newberry, H. Boothroyd. Second Row: Mr. P. Vanderkoy, G. Gatliff, J. Austin, D. Abrams, J. Eichelberger, S. Gift, S. Witt, N. Watson, J. Beesley, J. Lewis, M. Lima, N. Leapline. Third Row: B. Rehkopt, D. Hull, S. McMillen, B. Brown, R. Edwards. Fourth Row: J. Davis, L. Davis, D. Liechty, D. Rohm, T. Greenwood, R. Kilko.

Practice

Prayer

Polish

Produced precision

In sacred concert.

An extensive tour of the East Coast

Combined with numerous weekend engagements

Provided the choir

With invaluable opportunities

For praise, testimony, service

Through song

In order to win

The Master's smile

"When He Shall Come."

\section{CHORALAIRES AND CHOIR}

First Row: M. Anderson, R. Comps, P. France D. Kearney, S. Pape H. Moore. R. Hardy Second Row: J. Hirschy, B. O'Keefe, R. Hamilton, J. Brock, D. Daab, L. Anderson, E. Greenwood, J. Hess. Third Row: K. Cartner, T. Clater, M. Bales, J. Schneider, K. Borger, D. Bunting, J. Saemenes, J. Herwig, V. Butler, J. Jeremiah Fourth Row: L. Welch, E. Johnson, P. Mohler, D. Fitch, S. Olsen, L. Taylor, D. Yoder, M. Brewer, M. Crampton, E. Knott, G. Taylor, Mr. D. Matson, director.

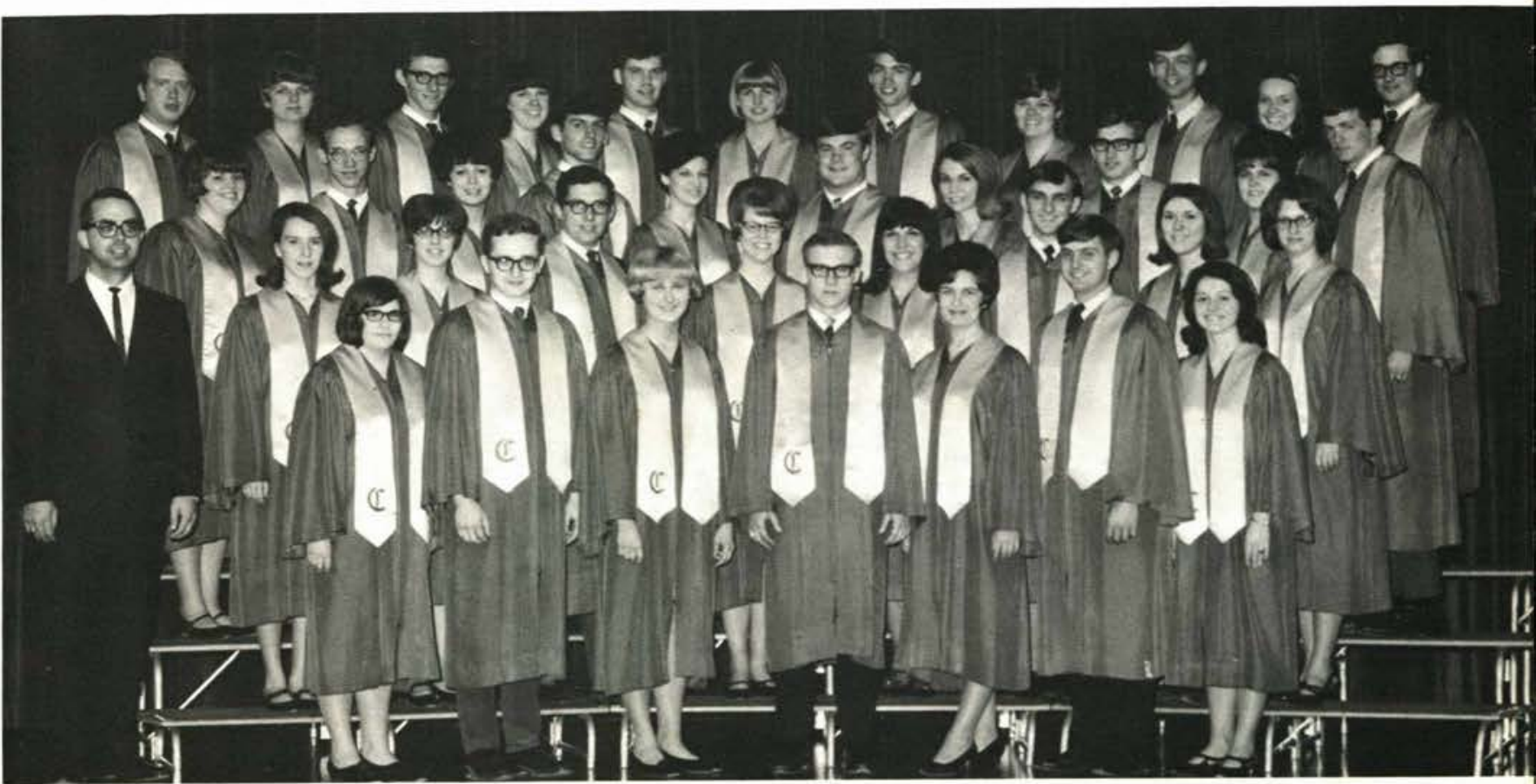




\section{BAND}

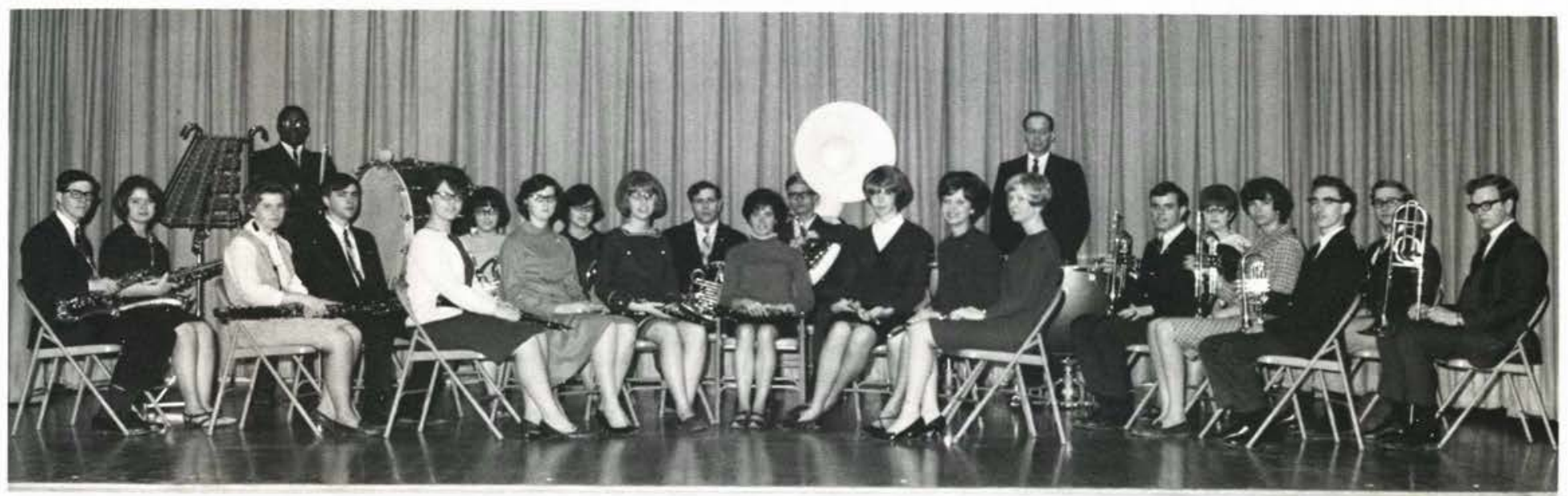

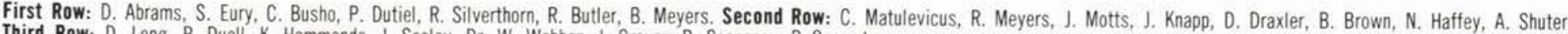
Third Row: D. Long, R. Duell, K. Hammonds, J. Seeley, Dr. W. Webber, J. Grover, P. Senseney, B. Grunert.

\section{MEN'S GLEE CLUB}

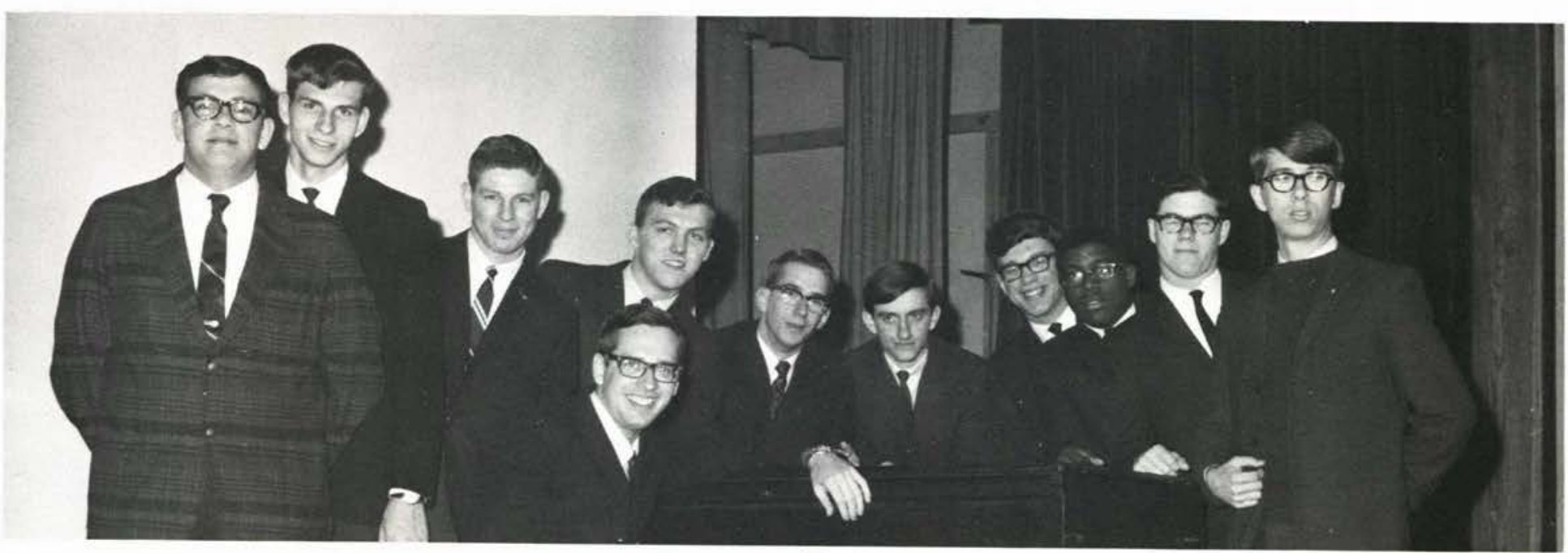

Mr. P. Vanderkoy (at piano), R. Sine, B. Lapp, L. Eichelberger, D. Connelly, B. Powley, B. Rehkopf, G. Stutzman, K. Hammonds, D. Long, C. Hull.

MADRIGALS

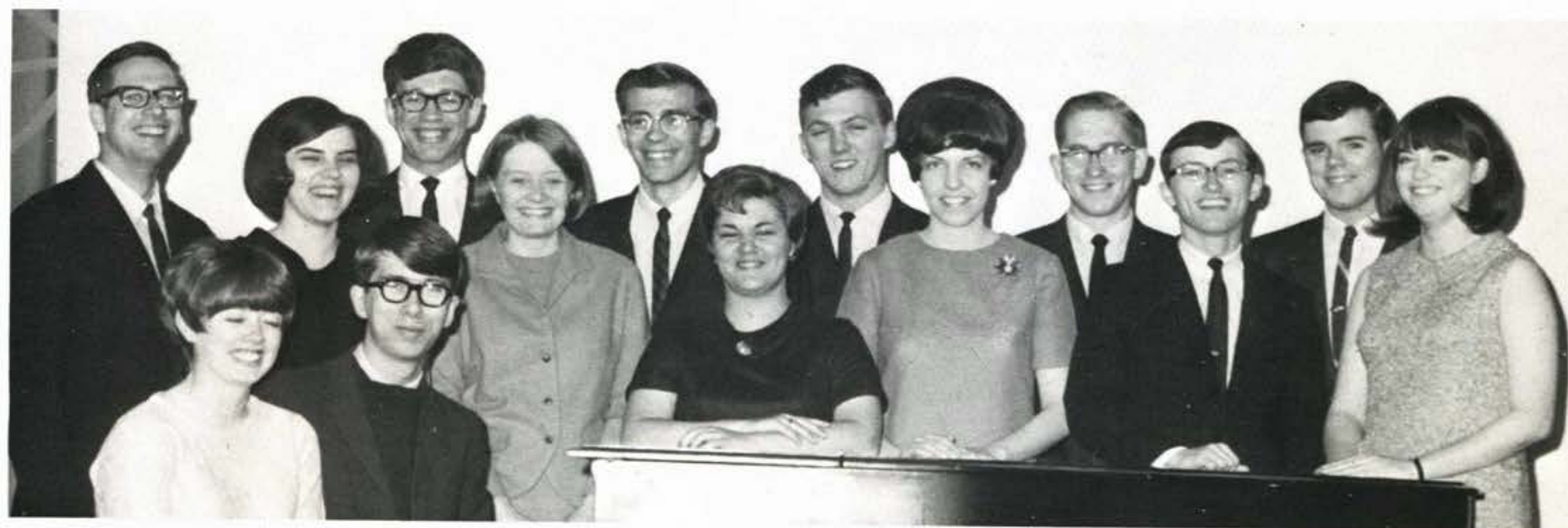

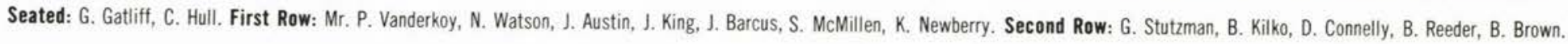




\section{MODERN MUSIC MASTERS}

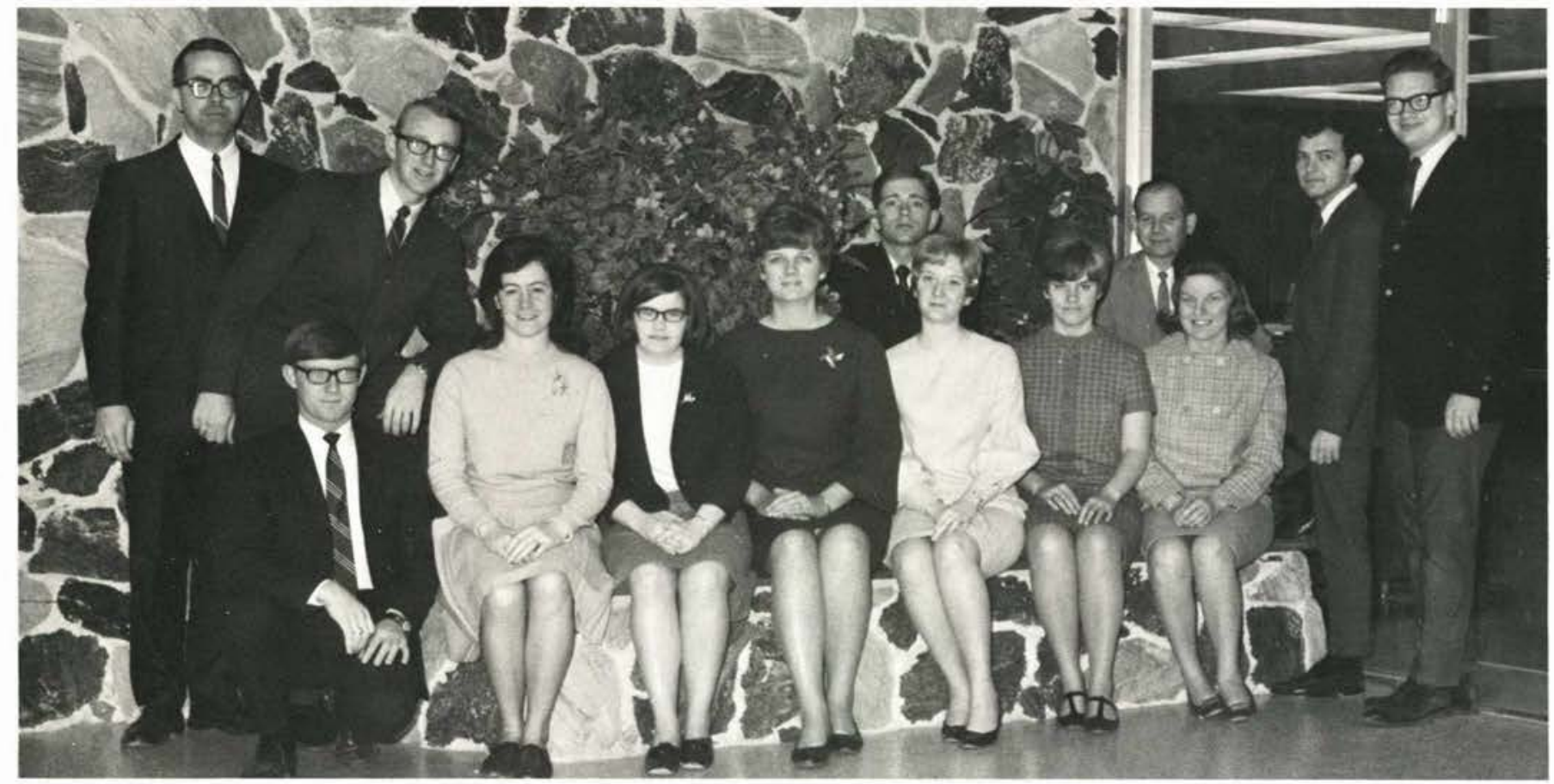

First Row: C. Layner, P. Boyd, M. Anderson, E. Johnson, B. Meyers, K. Johnson, S. Byrd. Second Row: Mr. D. Matson, D. Boyd, R. Roderick, Dr. W. Webber, D. Wall, B. King. Not Pictured: H. Moore, D. Daab, T. Clater.

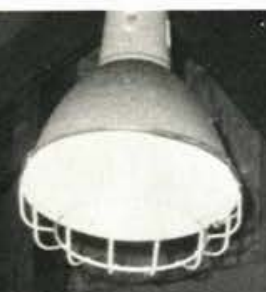

\section{SOCK 'N BUSKIN}

First Row: S. Gift, Mrs. M. Maddox, Advisor, L. Field, M. Brewer, J. Phenix. Second Row: D. Liechty, D. Hamilton, R. Meyers, S. Lathrop. Third Row: B. Lapp, R. Leach, L. Browning, L. Anderson.

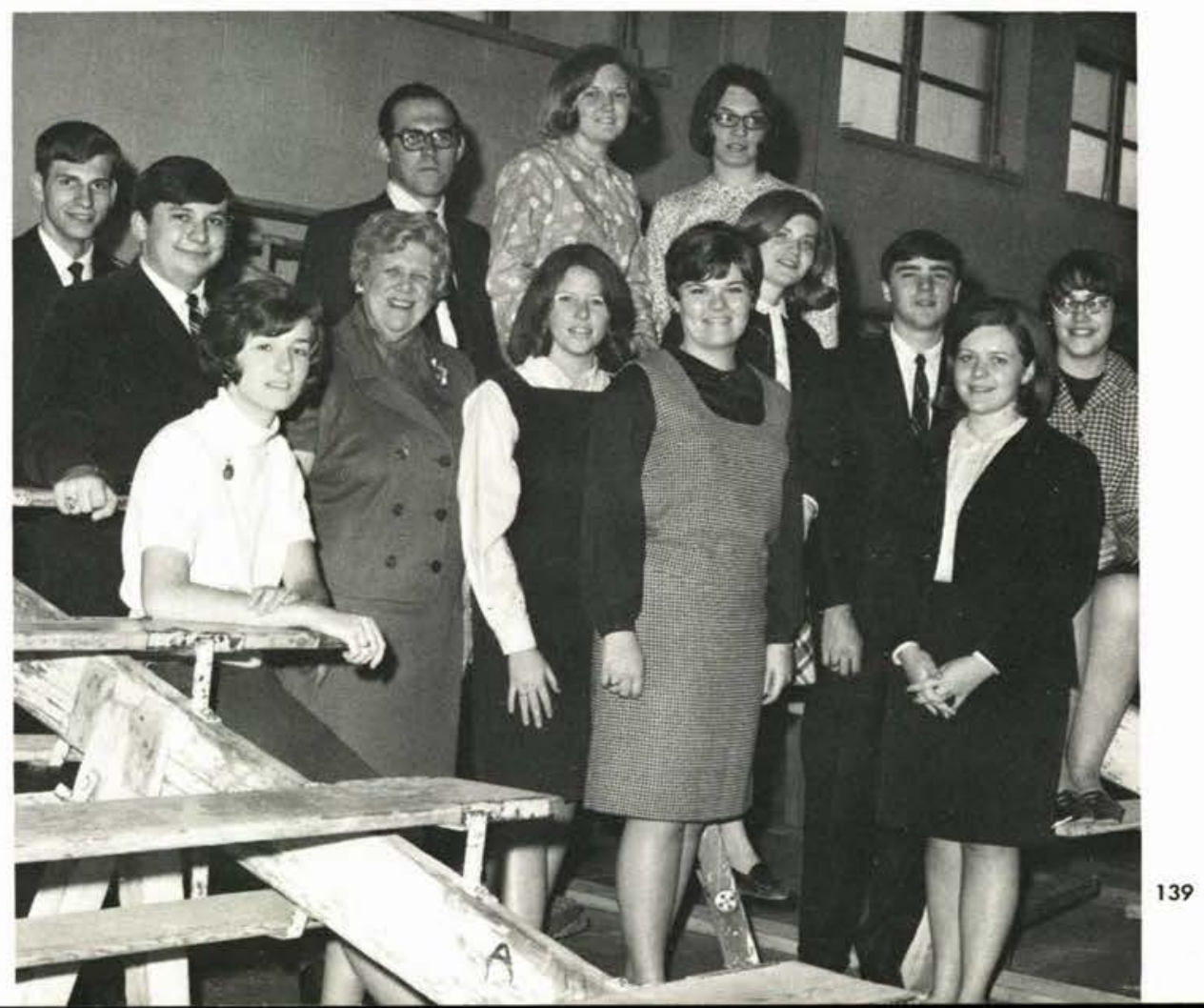


WRA

Intramural programs

Picnics, outings, athletics

For the women of the recreation association

Mean gratification and fulfillment.

Honoring its members with pins, letters,

Trophies and plaques.

The most esteemed laureation being

The "athlete of the year" award.

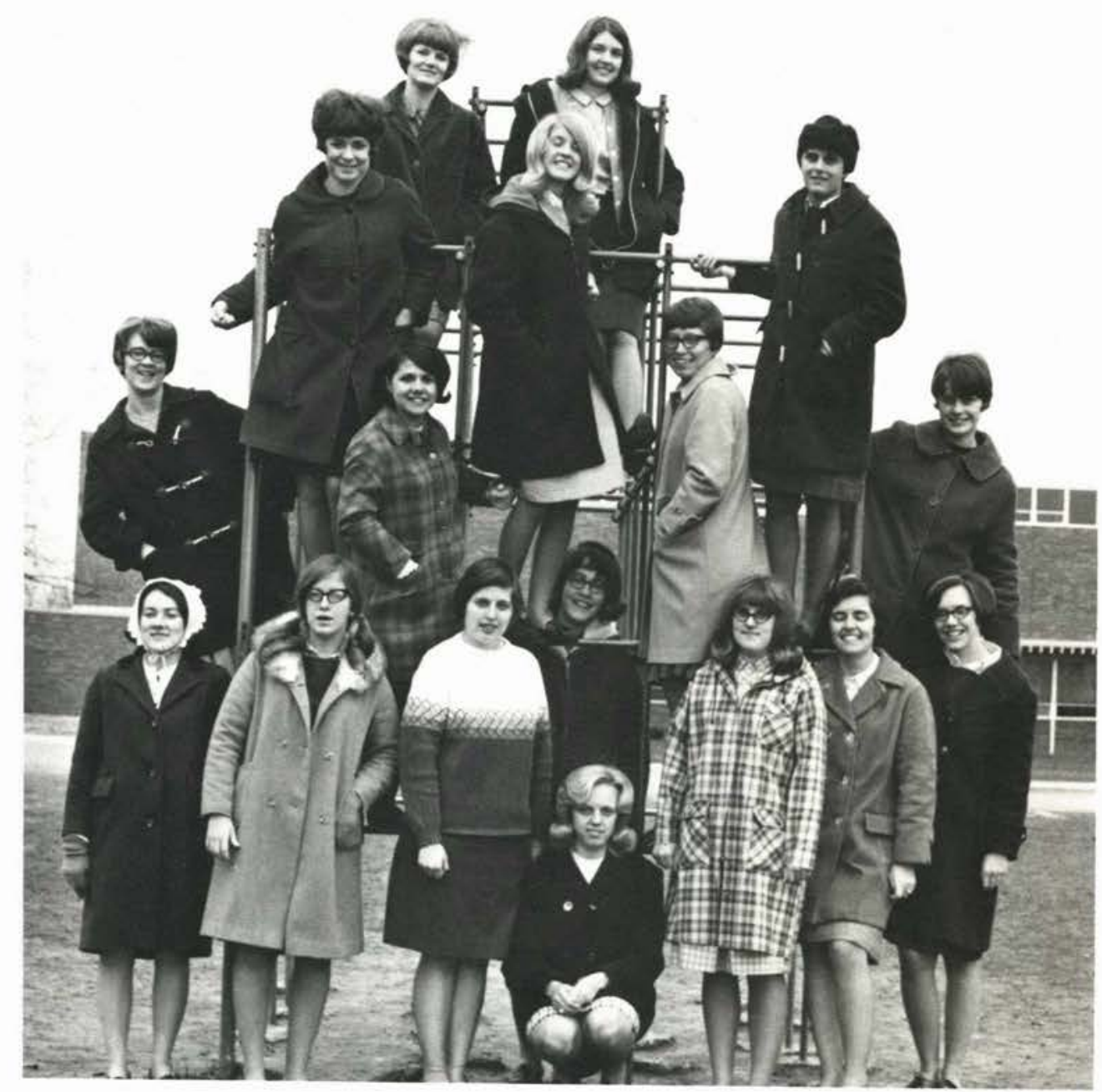

First Row: R. Bigley, M. Bartlett, R. Miller, C. Cunningham, L. Tanner, Miss Kearney, advisor, J. Strychalski, Second Row: S. Prather, B. Thomas, S. Lathrop, S. Rader, K. Donaldson. Third Row: M. Grable, P. Wissinger, J.' Stamatis. Fourth Row: D. Blake, L. Hollaway.

\section{VARSITY C}

R. Adams, T. Duff, M. Causey, L. Reno, D. Cooper, D. Yoder, M. Zazvrskey, D. Curcio, J. Rooke, B. McDonald, G. Darrow, D. Handyside.

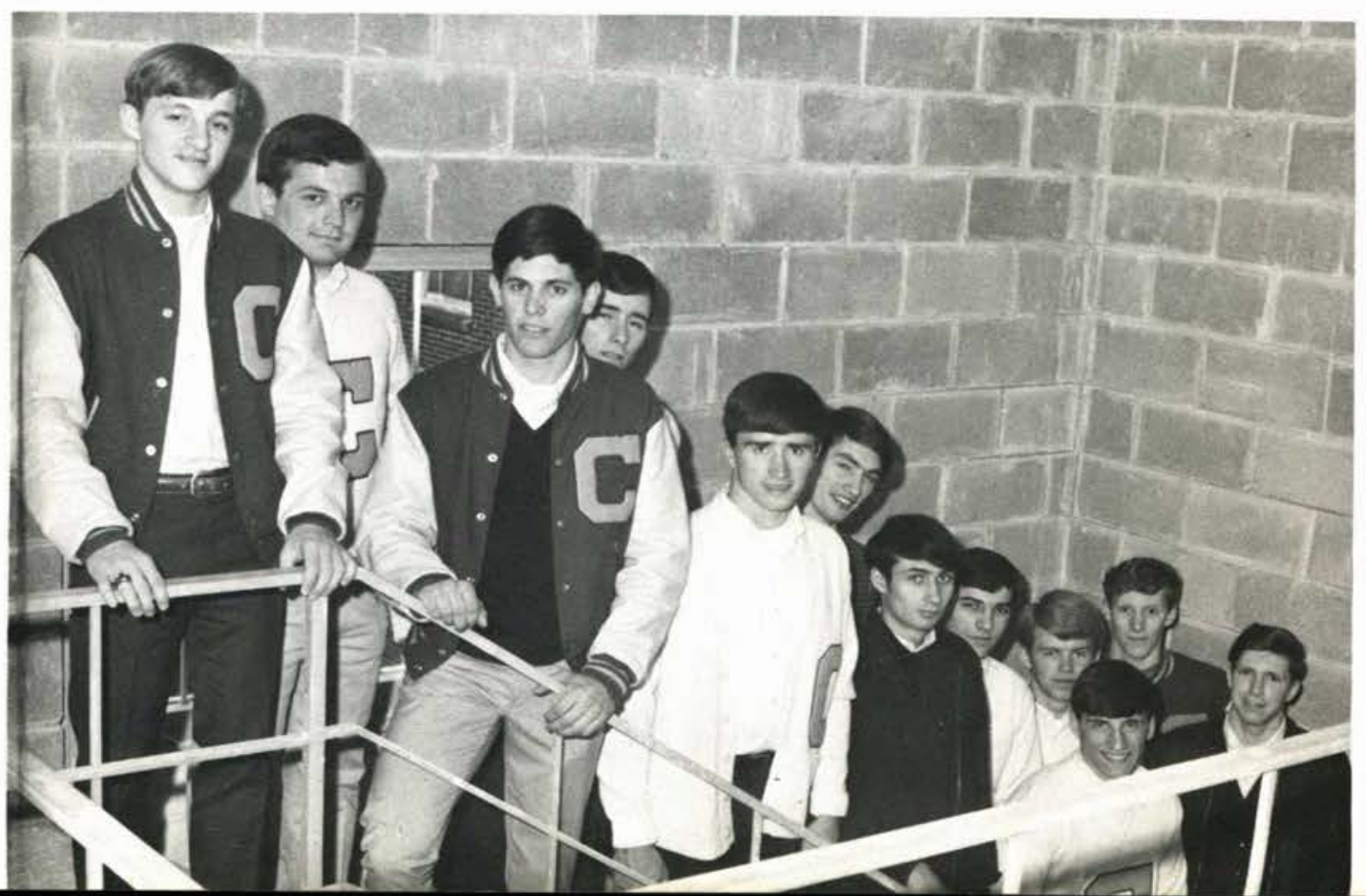




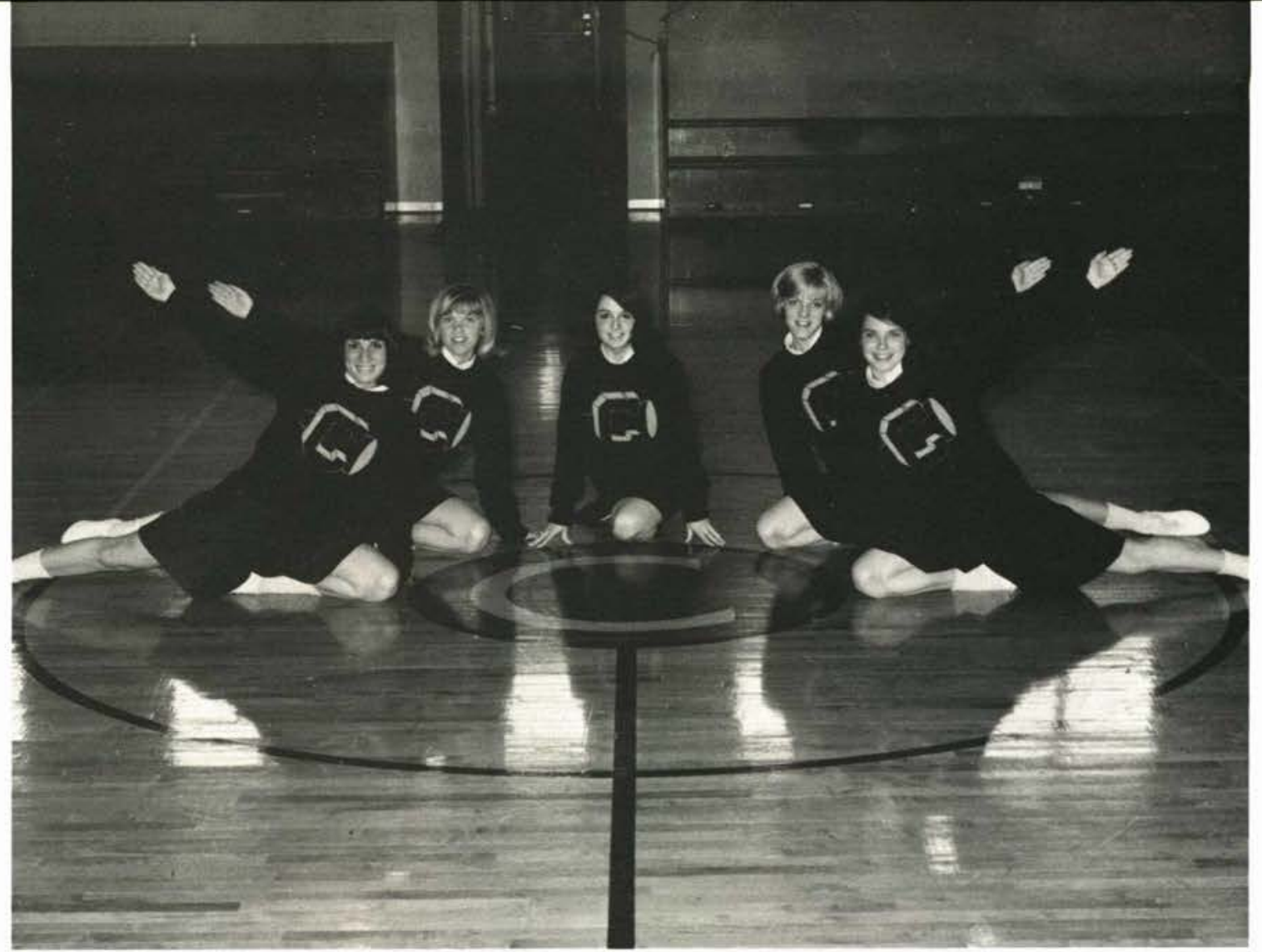

J. McCann, D. Koehn, P. Jenkin, D. Koehn, B. Mason.
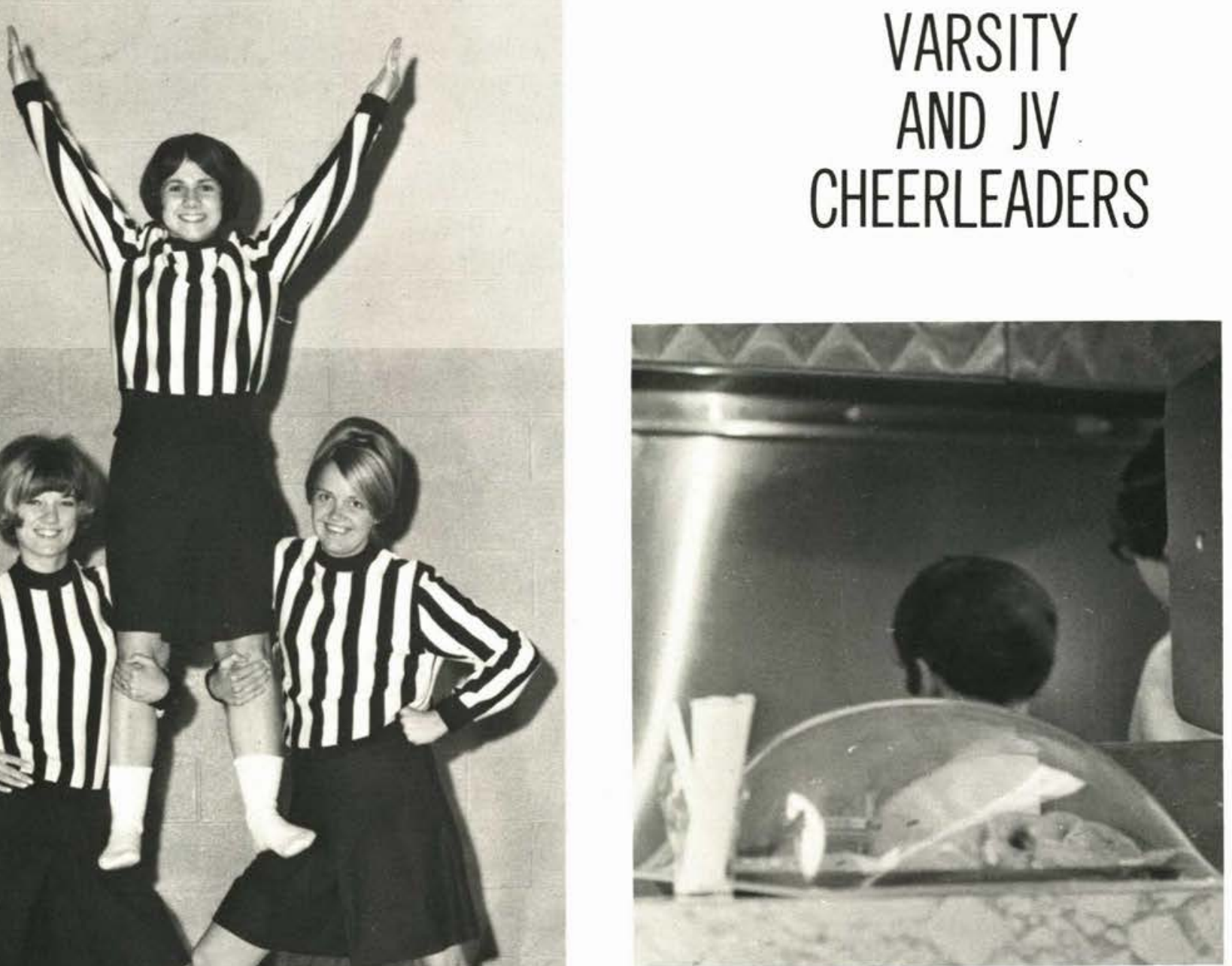


\section{SOCCER}

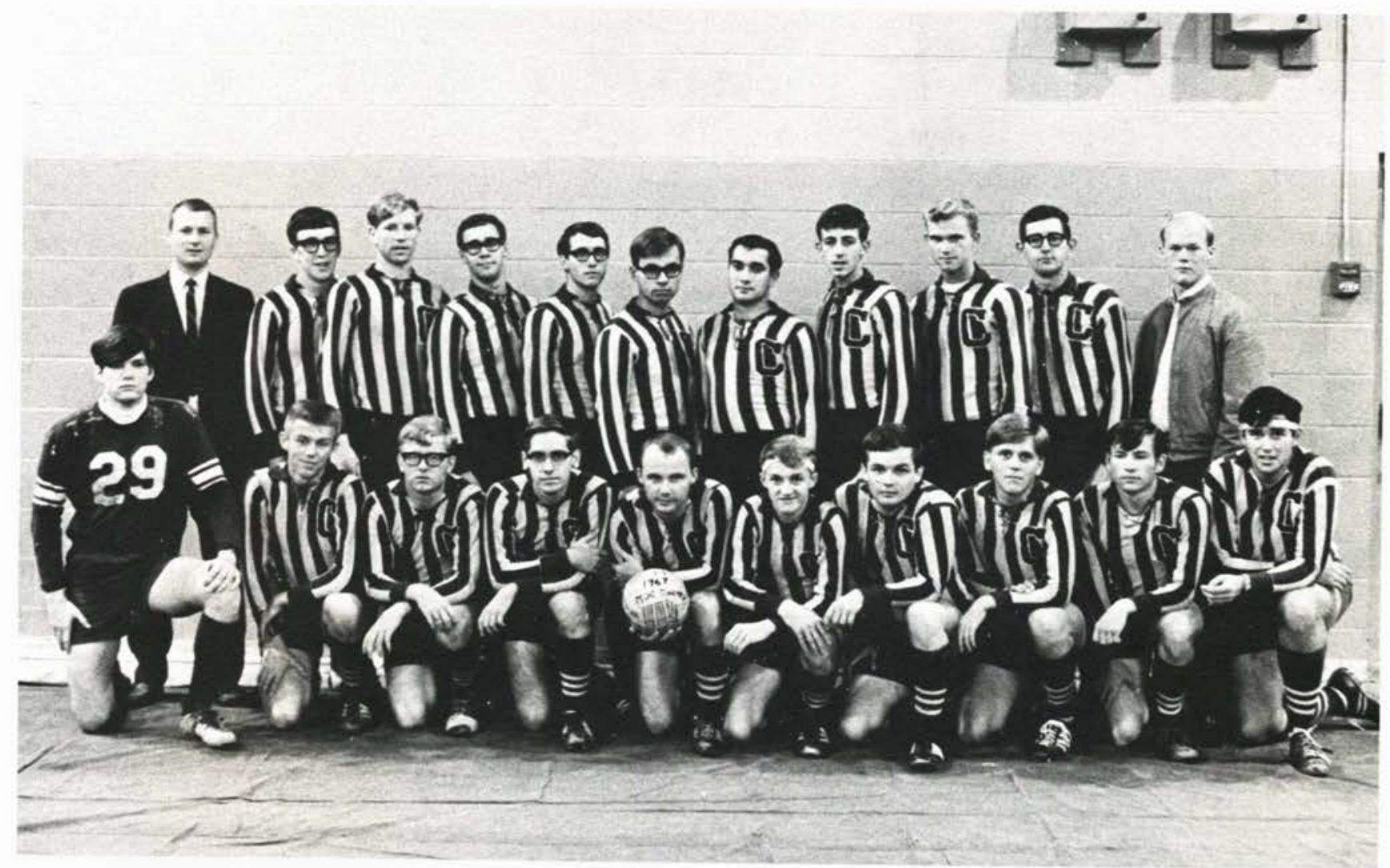

First Row: T. Warren, goalie; J. Rooke, G. French, R. Coriel, J. Rueck, R. Adams, T. Duff, B. Rooke, M. McCullough, M. Woodend. Second Row: Coach Olson, J. Brown, R.
Daubert, K. Muck, L. Reno, R. Schultz, T. Young, D. Price, P. Large, D. Lauener, N. Heyer, manager.
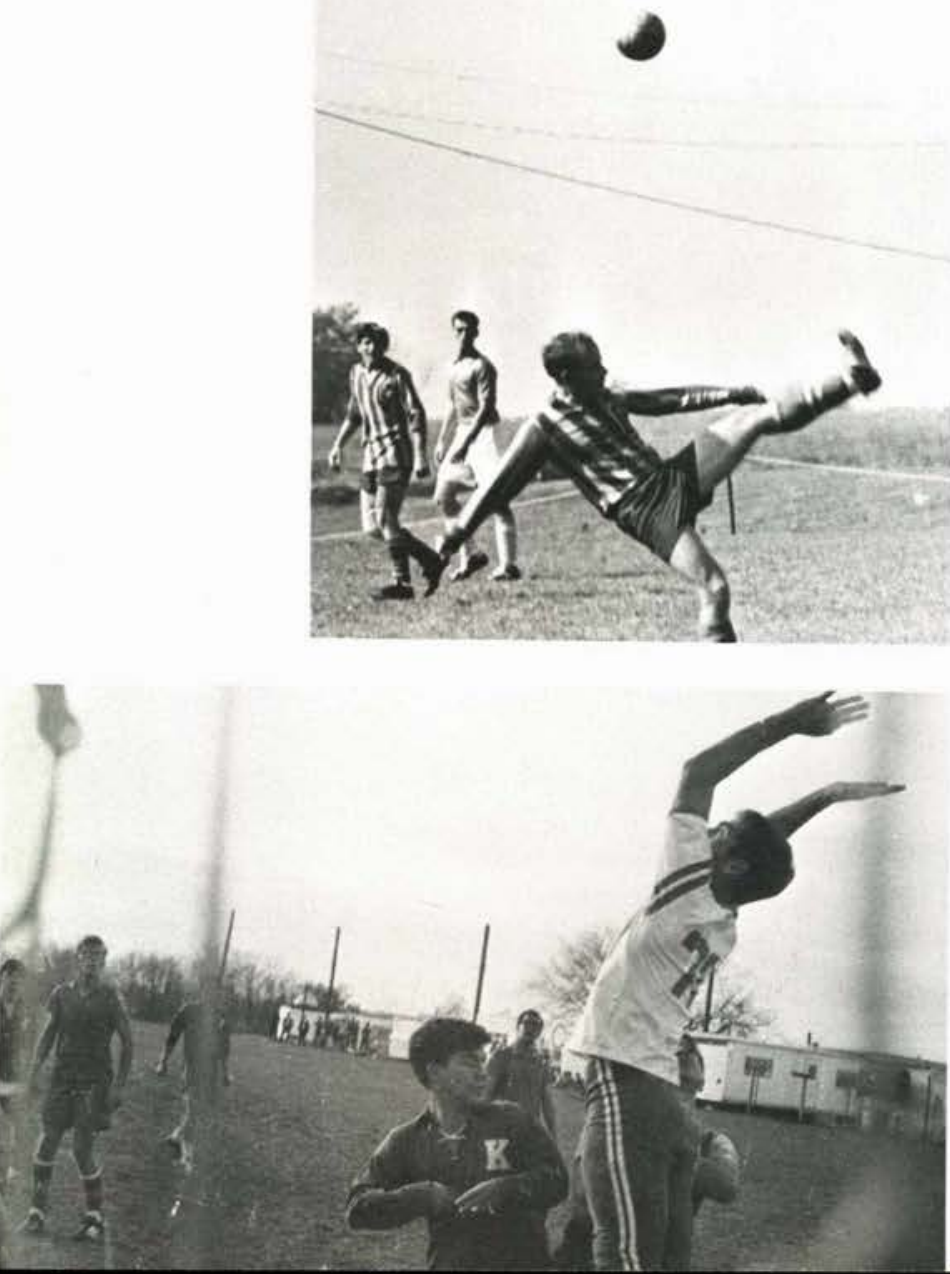

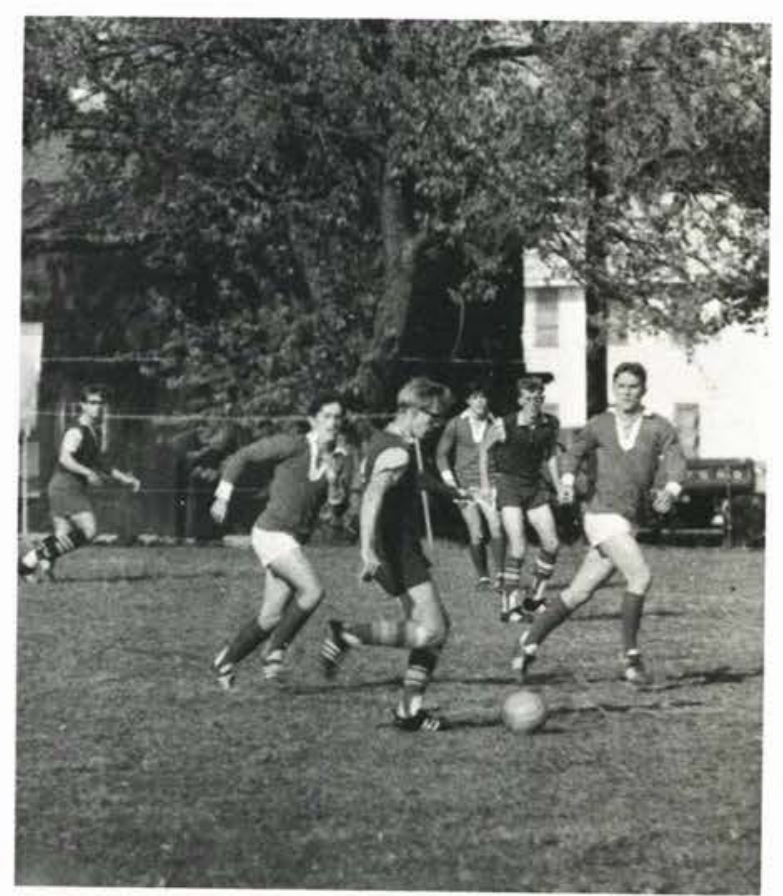

JV game 


\section{CC'S BASKETBALL TEAM}

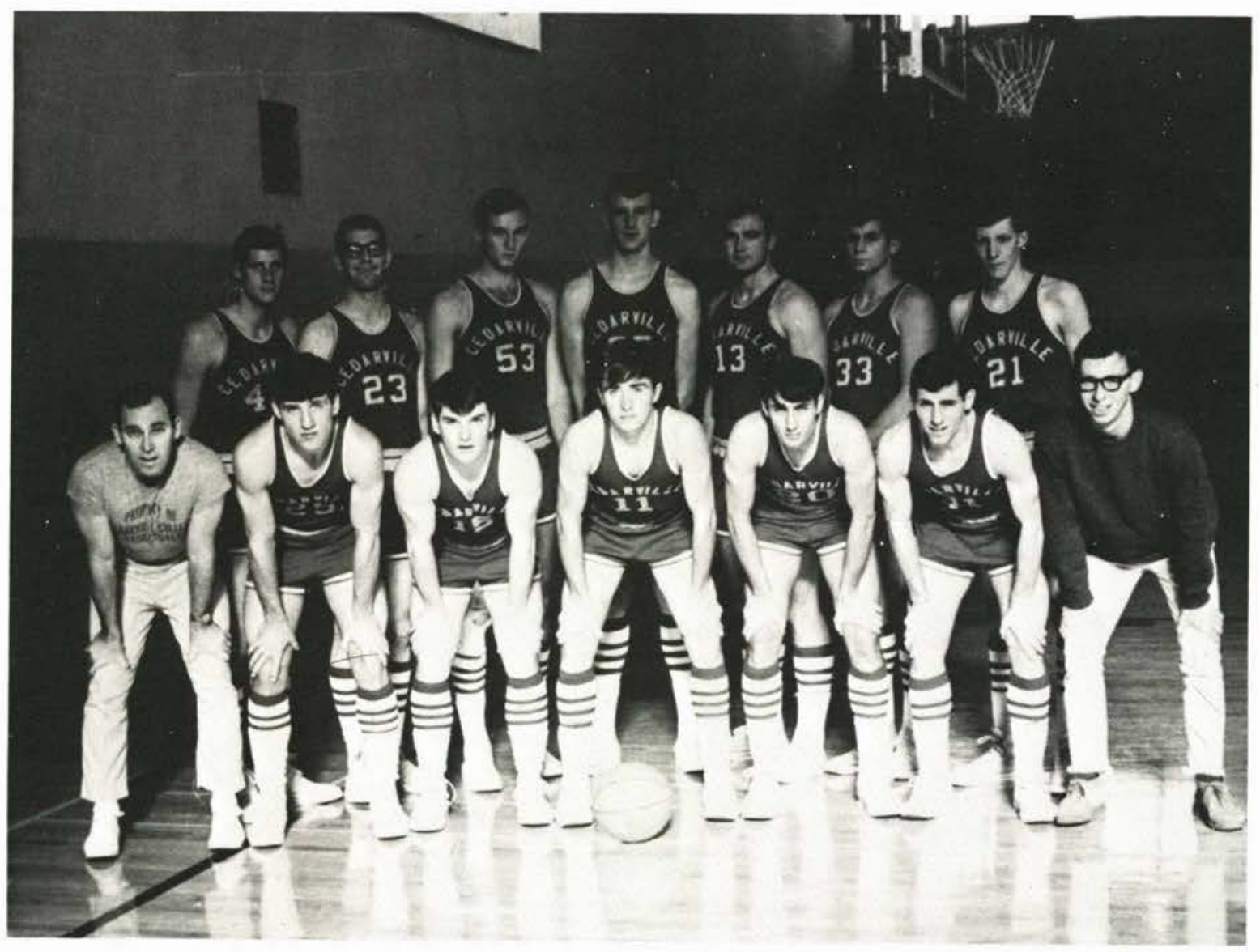

First Row: Coach Don Callan, B. McDonald, J. Watson, D. Cooper, M. Zazvrskey, R. Fisher, J. May. Second Row: D. Gaffner, L. Waite, H. Hurst, D. Atherton, A. Knott, W. Trefzger, G. Darrow.

\section{AND JUNIOR VARSITY}

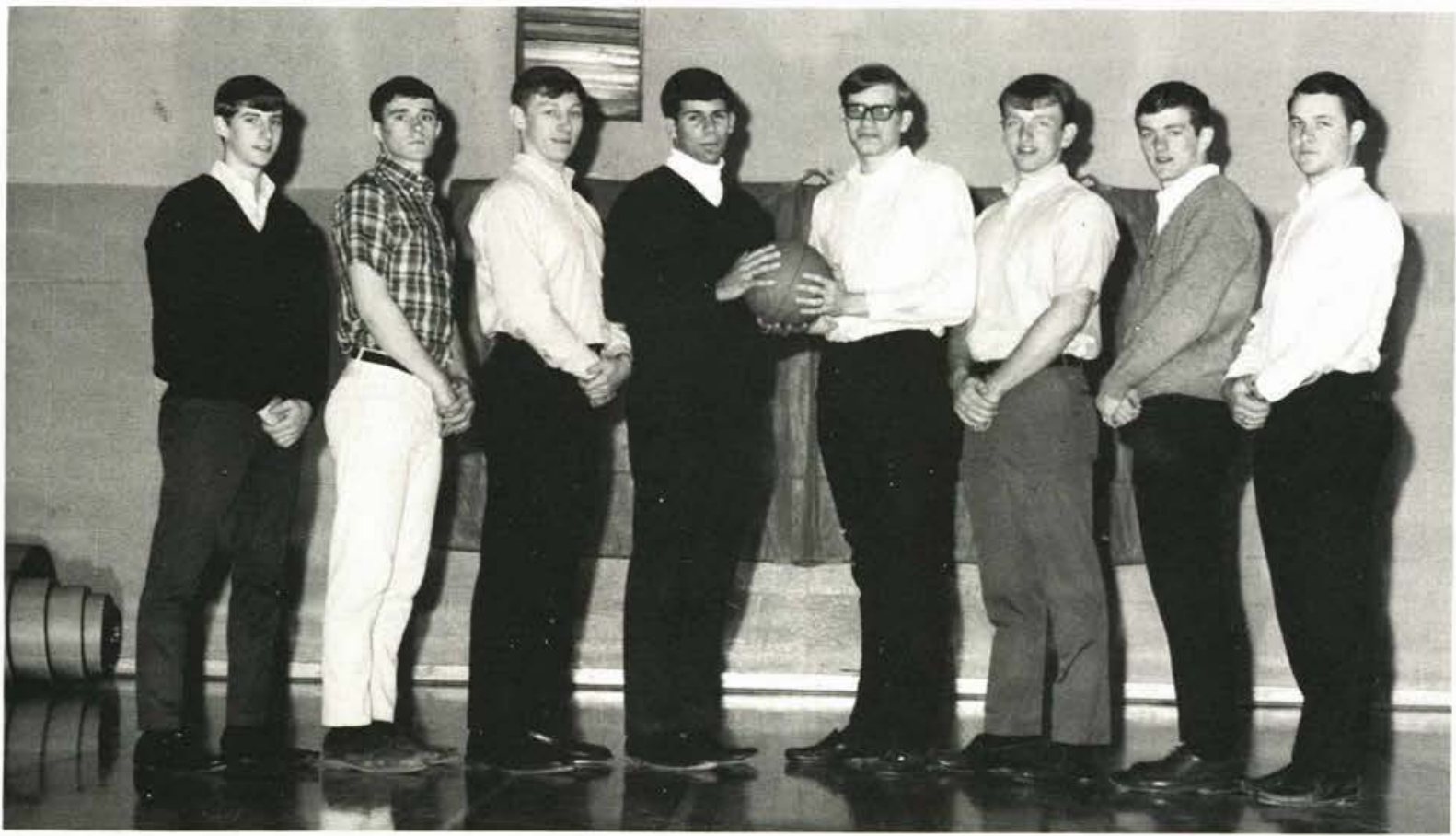

D. Carroll, D. Butler, D. Southwell, B. Trefzger, G. McDowell, B. Lunney, J. Jeremiah, R. Edwards.

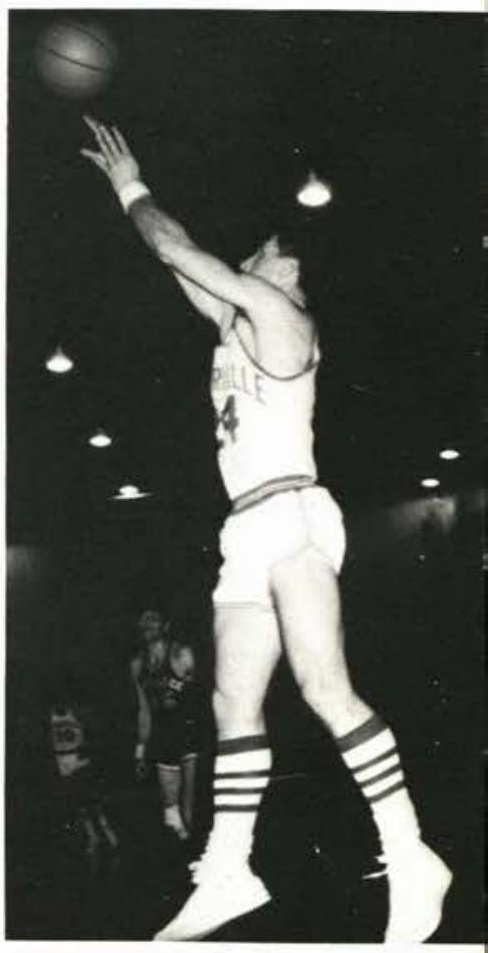




\section{OUR TENNIS TEAM}
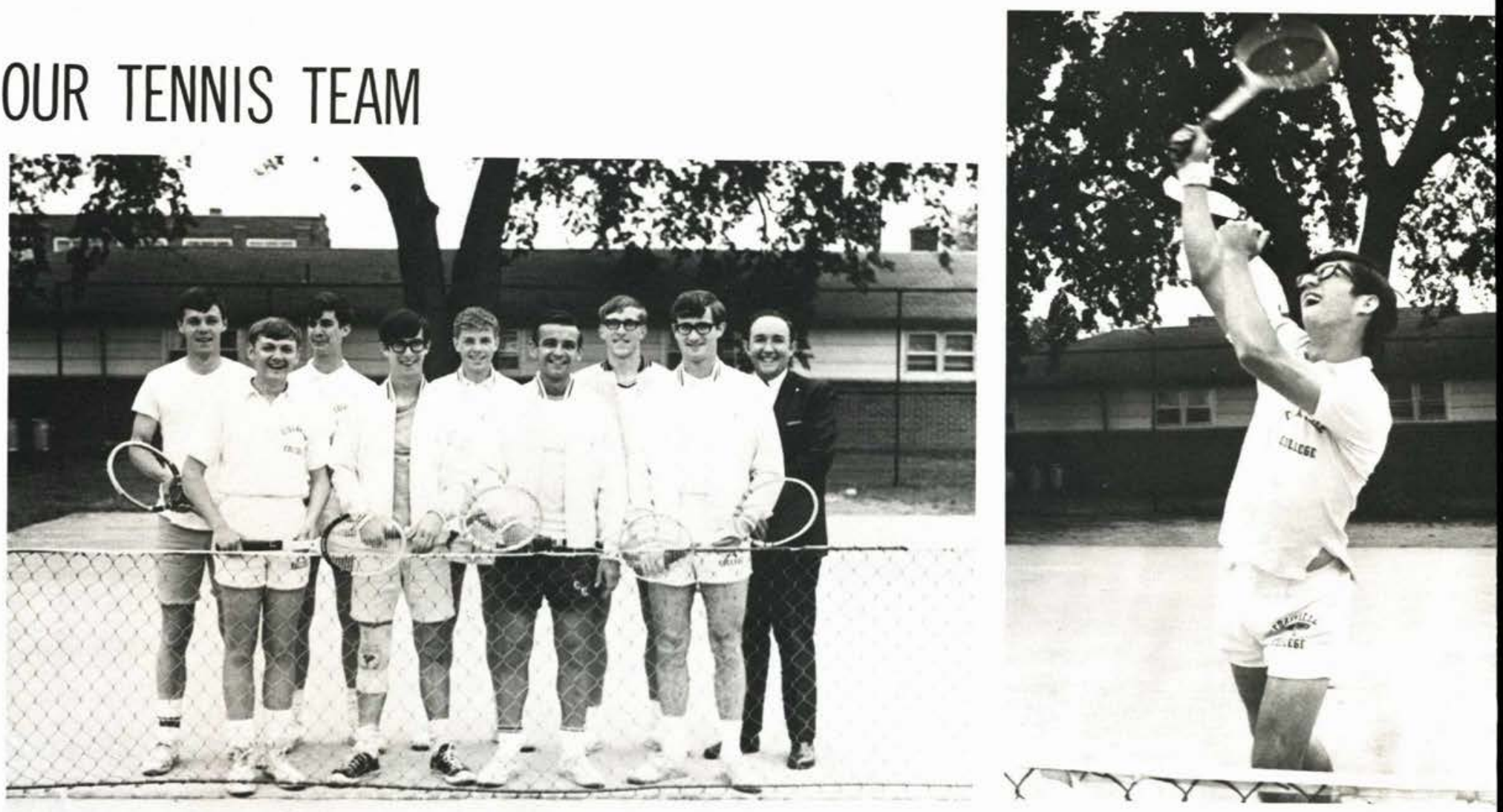

First Row: M. Boyce, W. Pettitt, R. Baker, D. Allen. Second Row: D. Ruffin, D. Durham, T. Carpenter, P. Entner, Mr. Murdoch,
Coach.

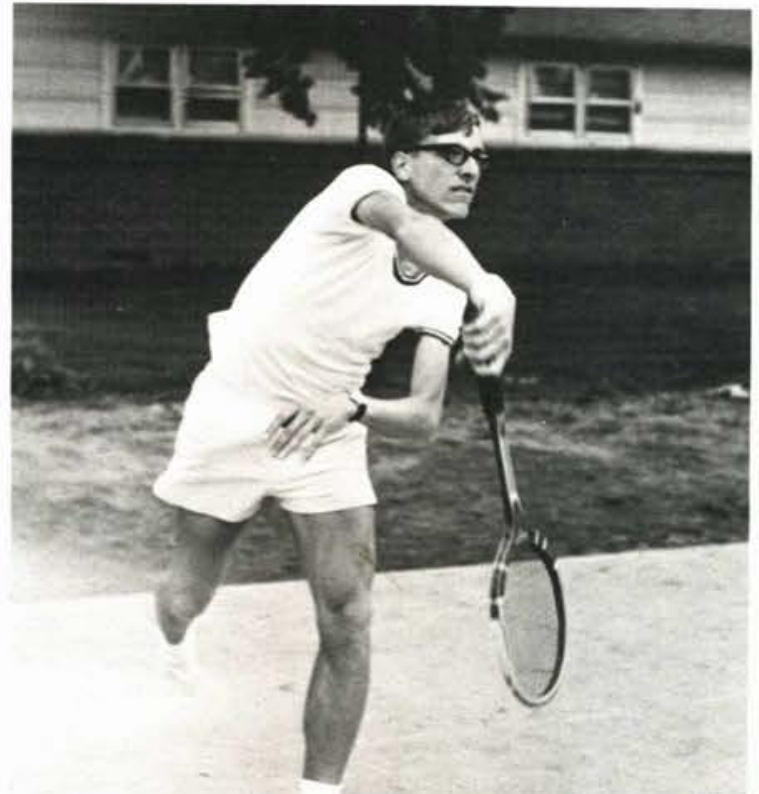

\section{CHAMPIONS}

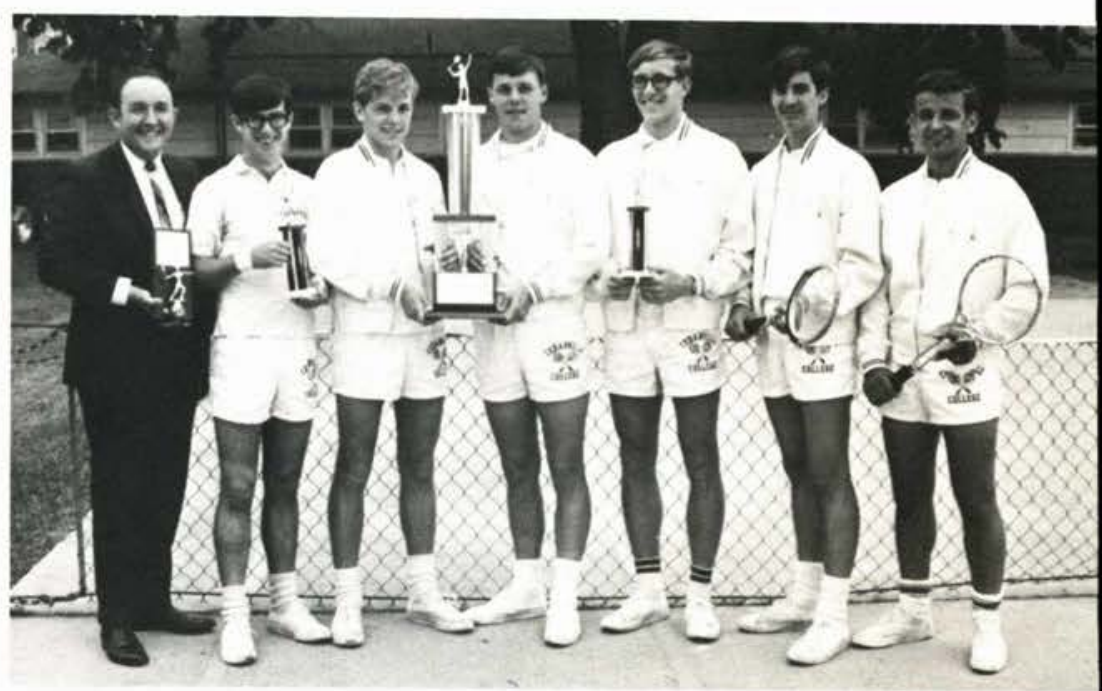

Coach Murdoch, Pettitt \#3, Carpenter \#6, Ruffin \#2, Entner \#1, Durham \#4, Baker \#5.
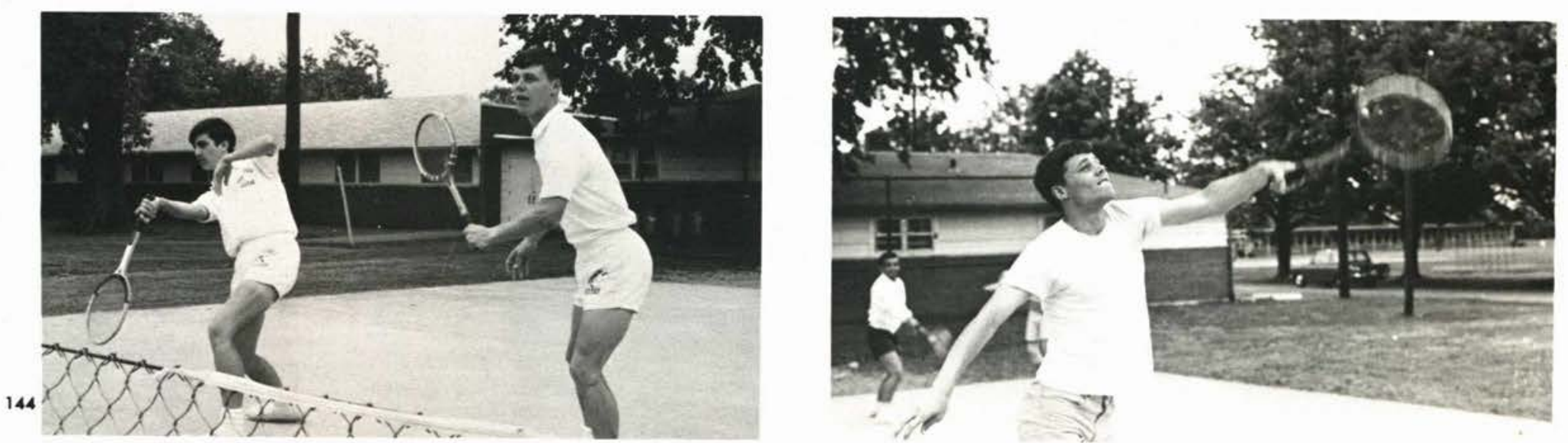


\section{BASEBALL TEAM}

Coach: Dennis Olson

Infield:
J. Colyer
D. Cooper
R. Edwards
J. Frink
R. Inghram
D. Mays
B. McDonald

Outfield:
D. Gregory
D. Haseltine

M. Kintz

J. Simons

Catcher:

T. Duff

Pitchers:
D. Atherton
D. Carrol
M. Causey

B. Lunney

B. Trefzger

M. Wolford

Manager:

Steve Taylor

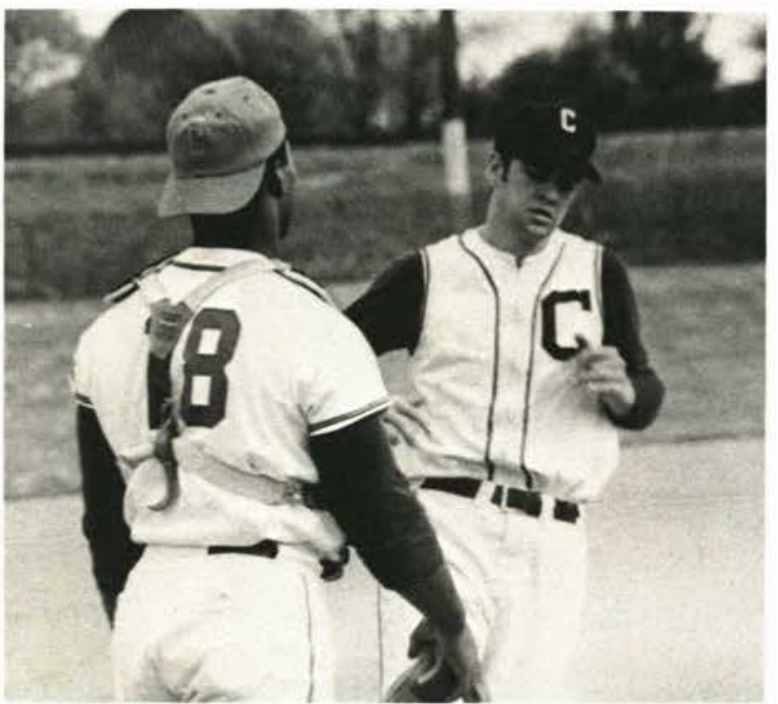

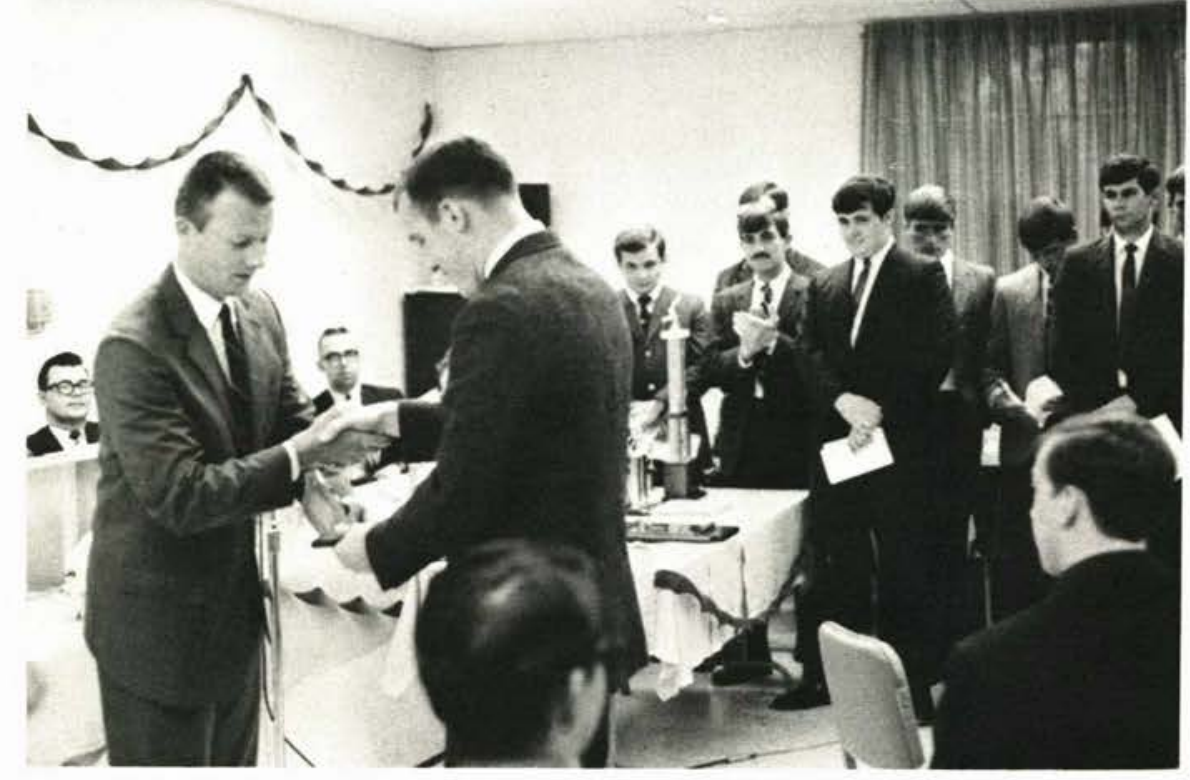

TENNIS AND SOCCER TROPHIES

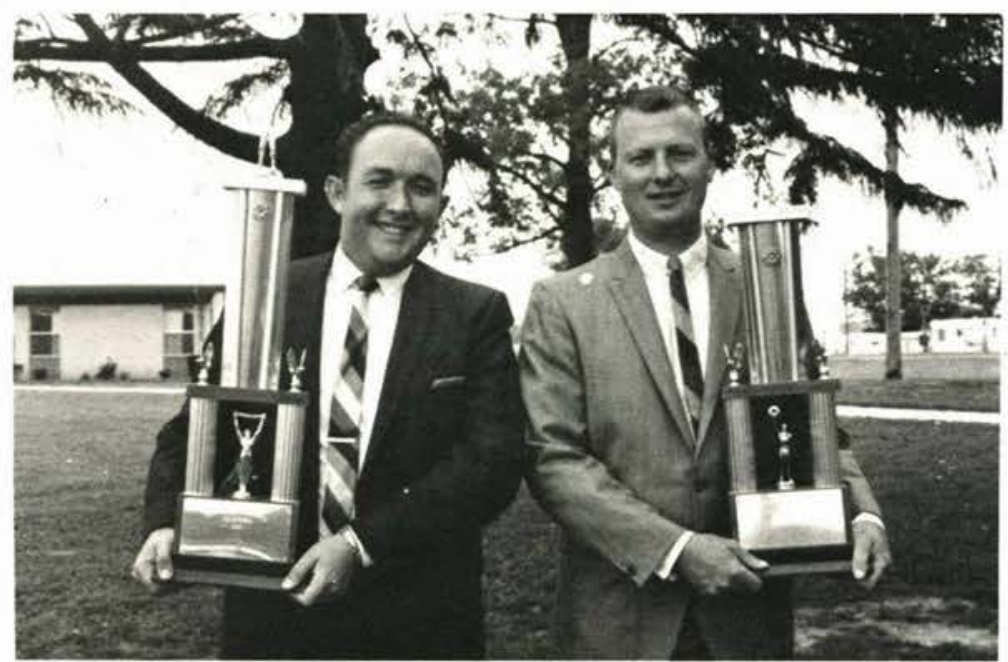




\section{CROSS COUNTRY \& TRACK}

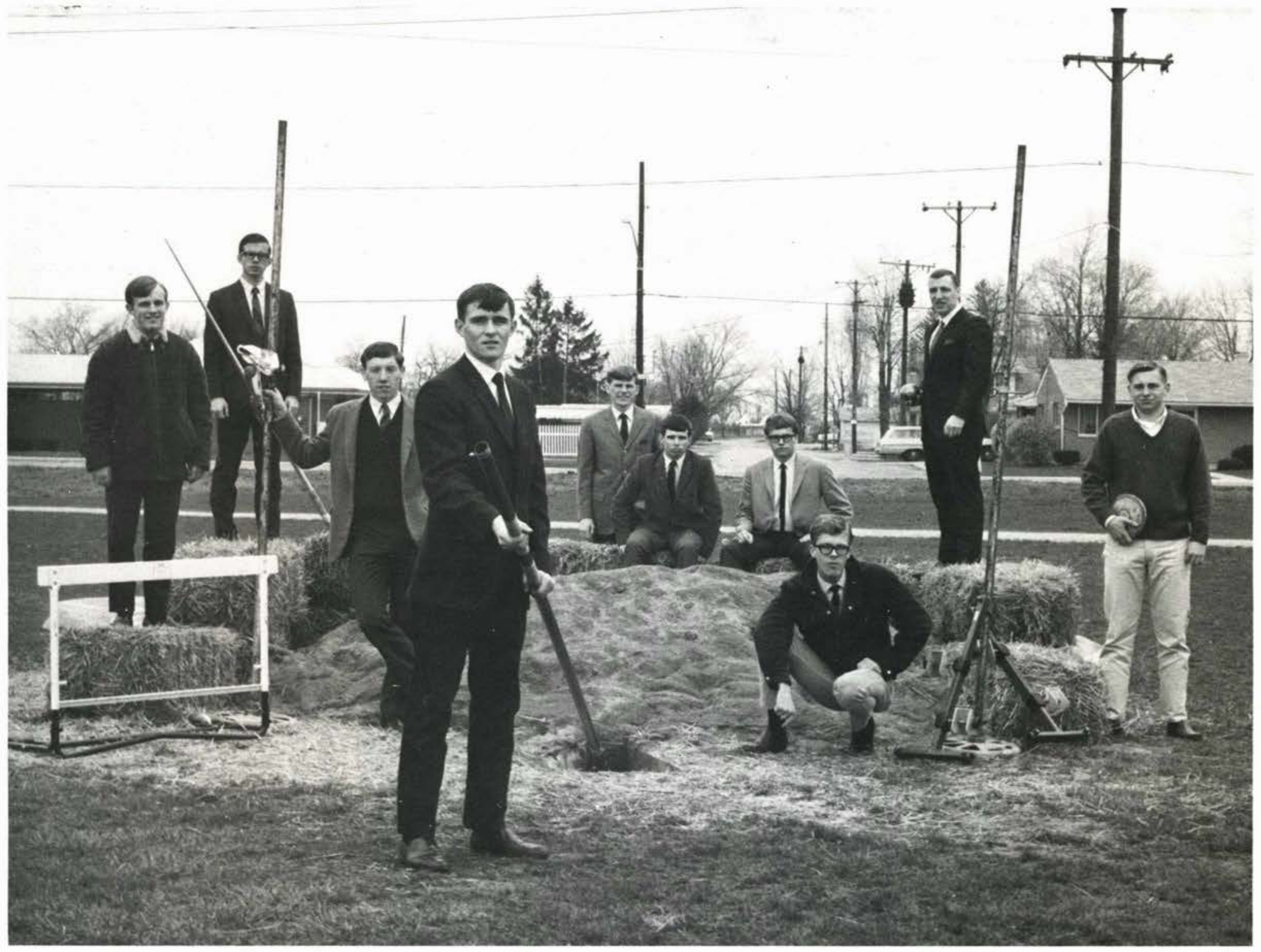

Steve Fowler, Dennis Dieringer, Mr. Dunstan, coach; Dick Butler, Ken Evans, Dee Mays, Doug Ambroza, Tom Greenwood, Cal Packard, Dennis Huddleston. 

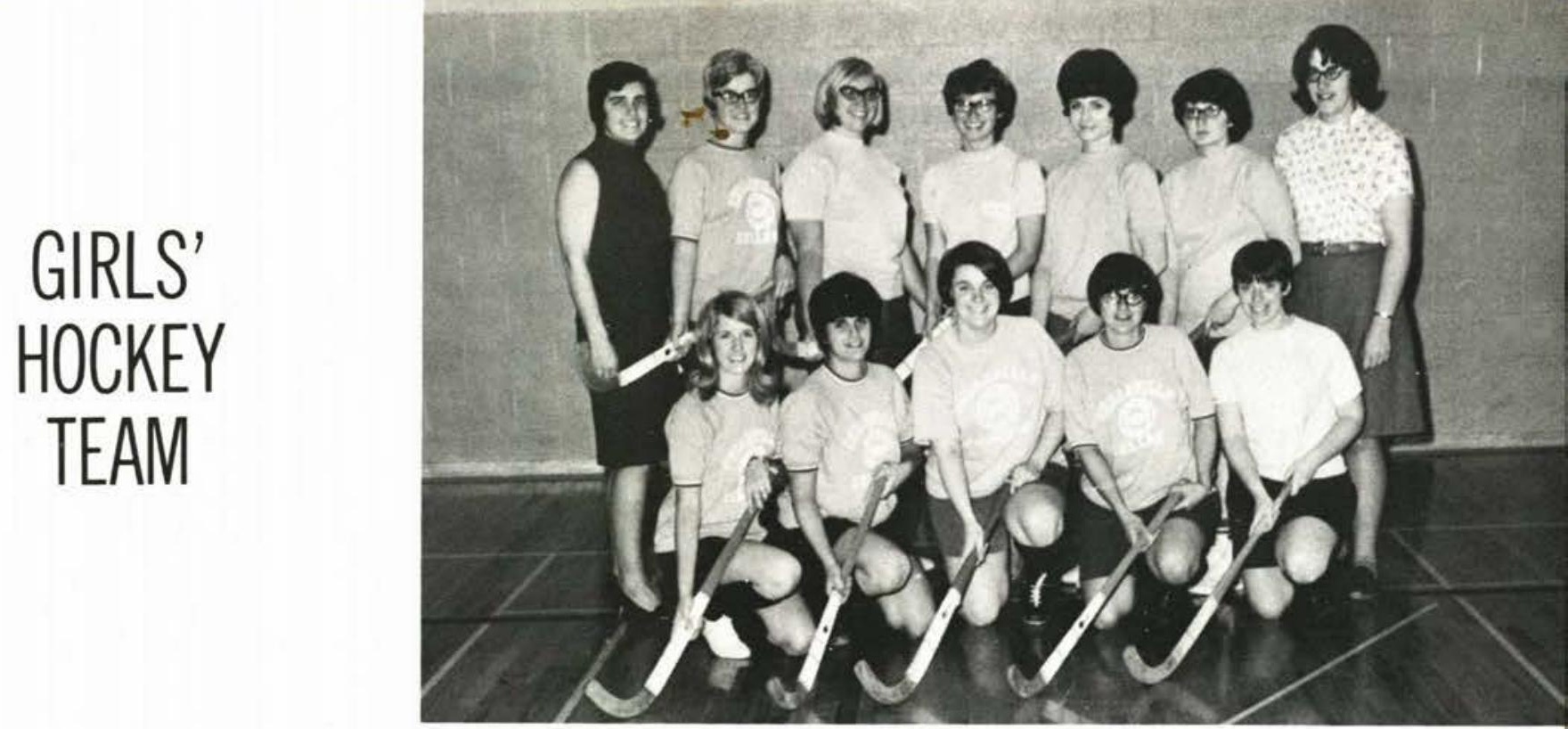

First Row: Miss Kearney, Becky Rader, Bev Carlson, Suzy Rader, Mary Lynn Grable, Barb Kensil, Sandy Lathrop. Second Row: Pat Wissinger, Judy Stamatis, Kathy Spencer, Karen Rau, Nancy Brown.

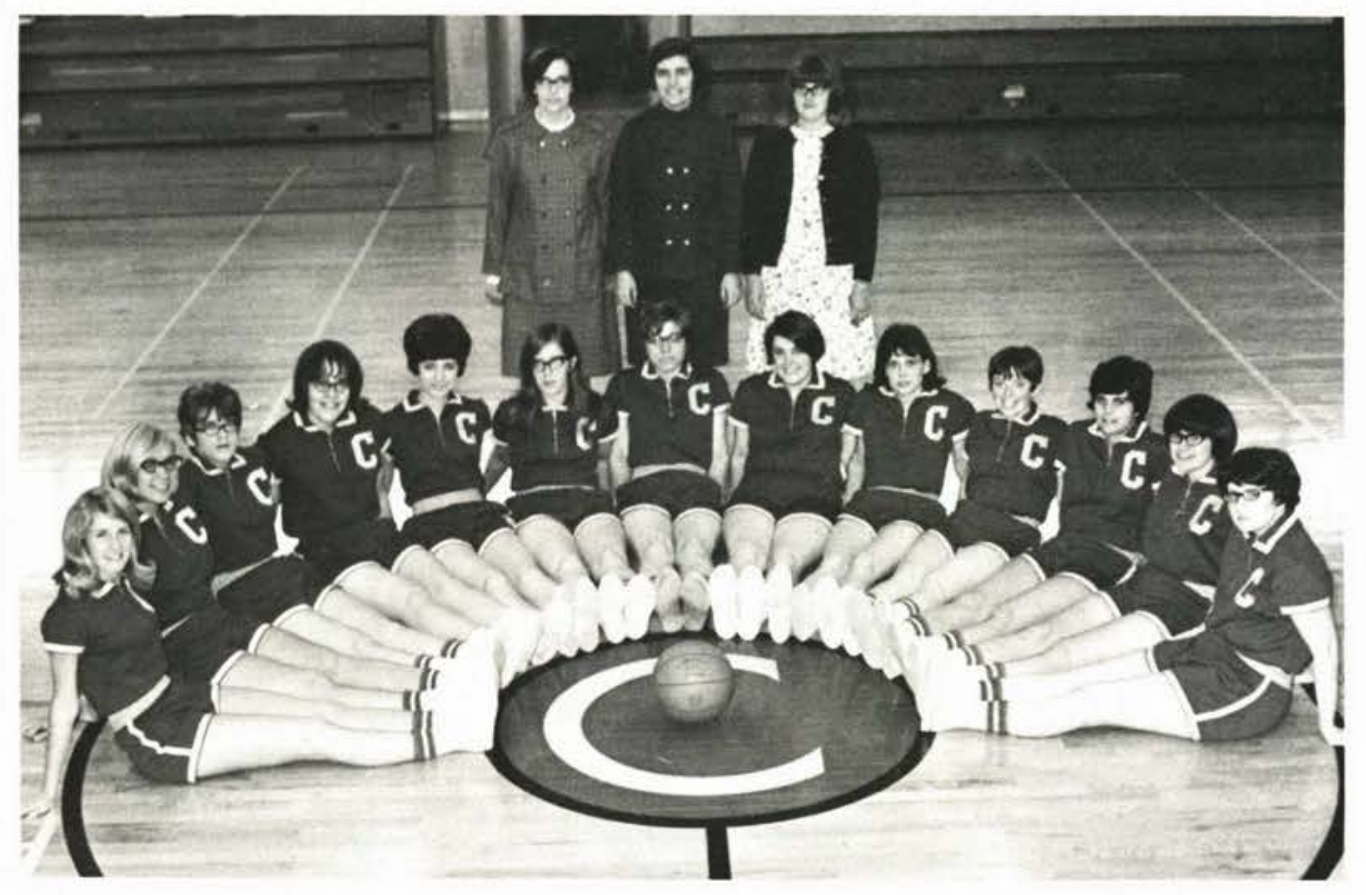

BASKETBALL TEAM

Standing: J. Strychalski, Miss Kearney, L. Tanner. Seated: P. Wissinger, B. Carlson, S. Prather, S. Lathrop, M. Grable, M. Bartlett, M. Beitler, K. Spencer, D. Mann, N. Brown, J. Stamatis, K. Rau, B. Kensil.

First Row: Bev Carlson, Mary Lynn Grable, Miss Kearney, Suzy Rader, Nancy Leapline. Second Row: Kathy Spencer, Barb Kensil, Pat Wissinger, Karen Rau, Judy Stamatis.

VOLLEYBALL TEAM 


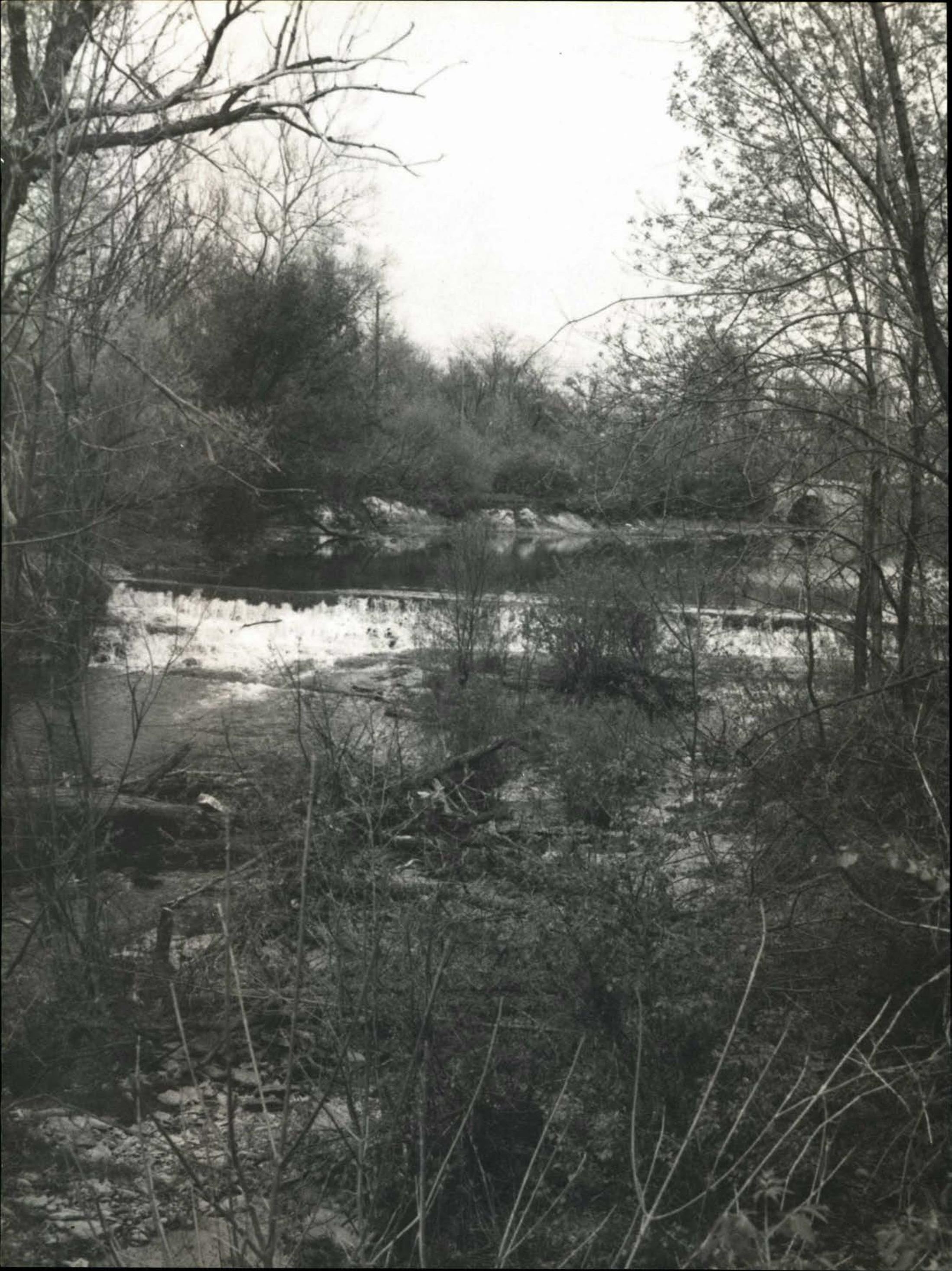




\section{Activities and Special Events}

Look upward to the beginning of the day. This simple act will elevate your mind and impel you to go forth with new confidence and power. A day is well-lived in which you have put a fine resolution into practice, achieved a definite purpose, done some worthy act of kindness or rendered a noble service to others. True service is not limited to time or place. Today is the day to do something fine and noble.

This day brings to you its share of duties, opportunities and responsibilities. The spirit in which you approach the work and activities of this day will vitally affect the results. Go forward with large confidence and high expectation. Be alert to the fresh opportunities of this day and do everything possible to advance your highest and best interests.

IDEALS 


\section{AUTUMN QUARTER}

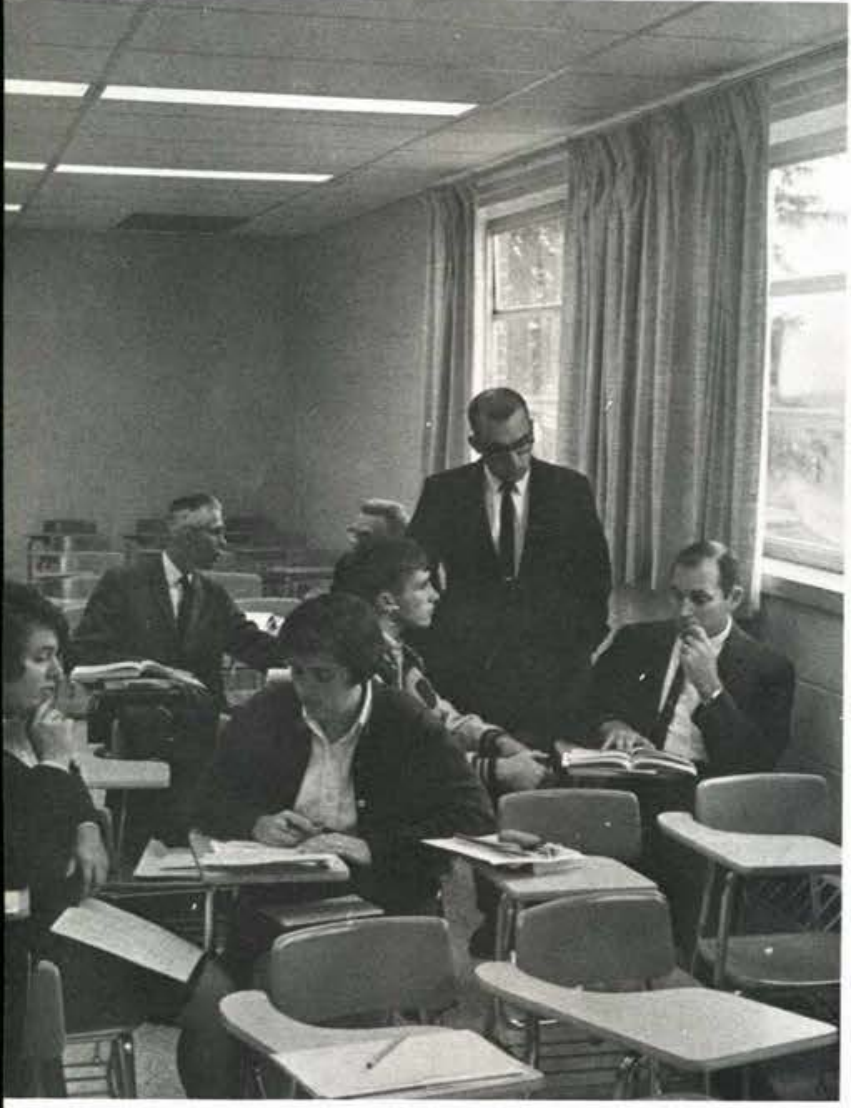

REGISTRATION

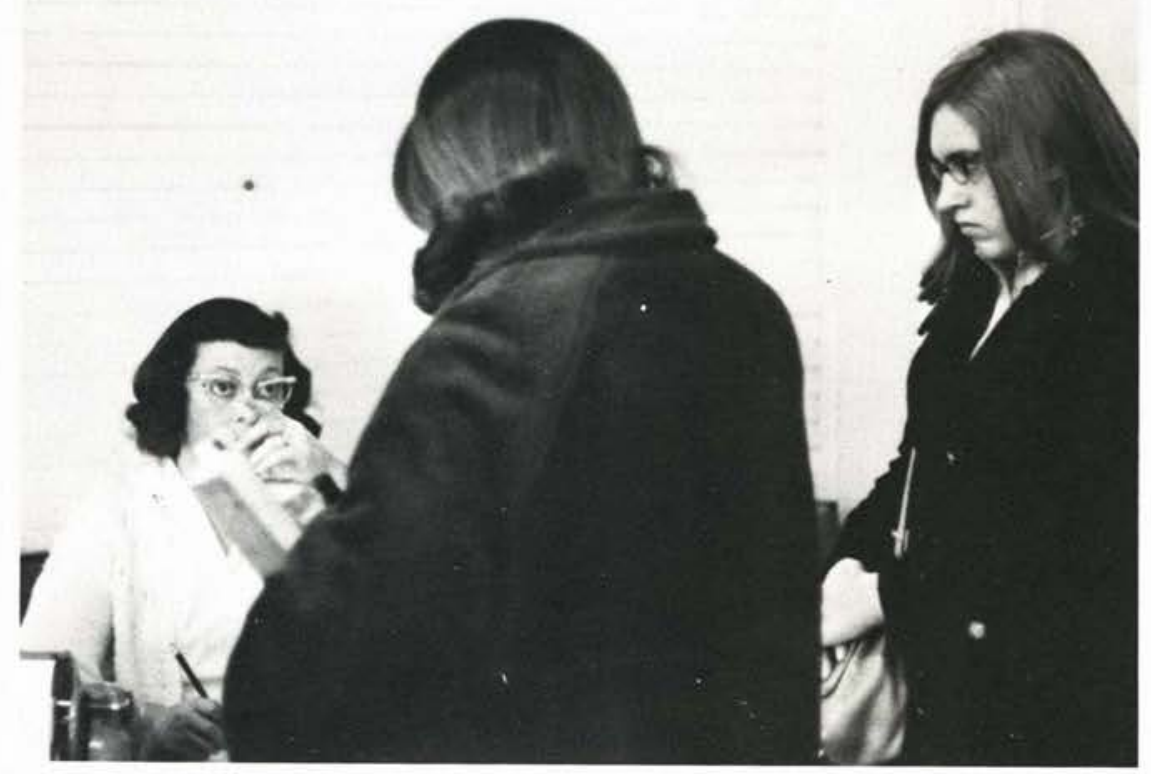

INITIATION
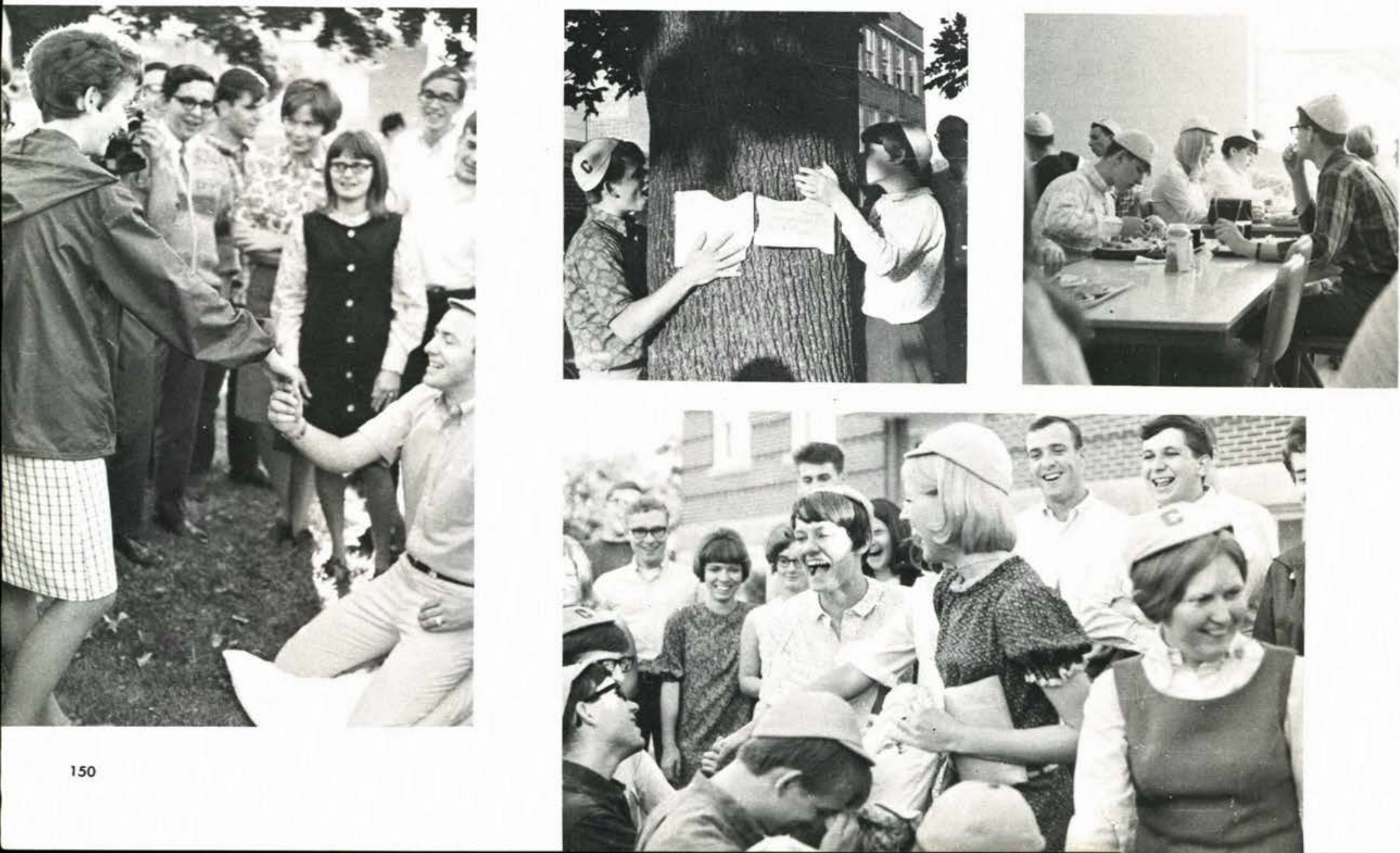
The beginning of a new school year as seen by freshmen and returning students.

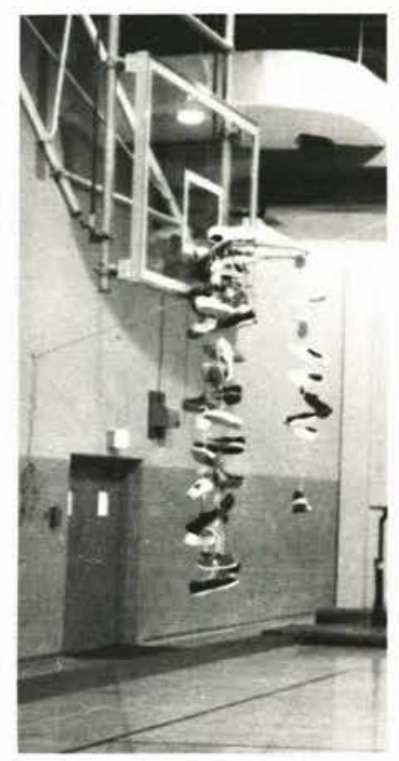

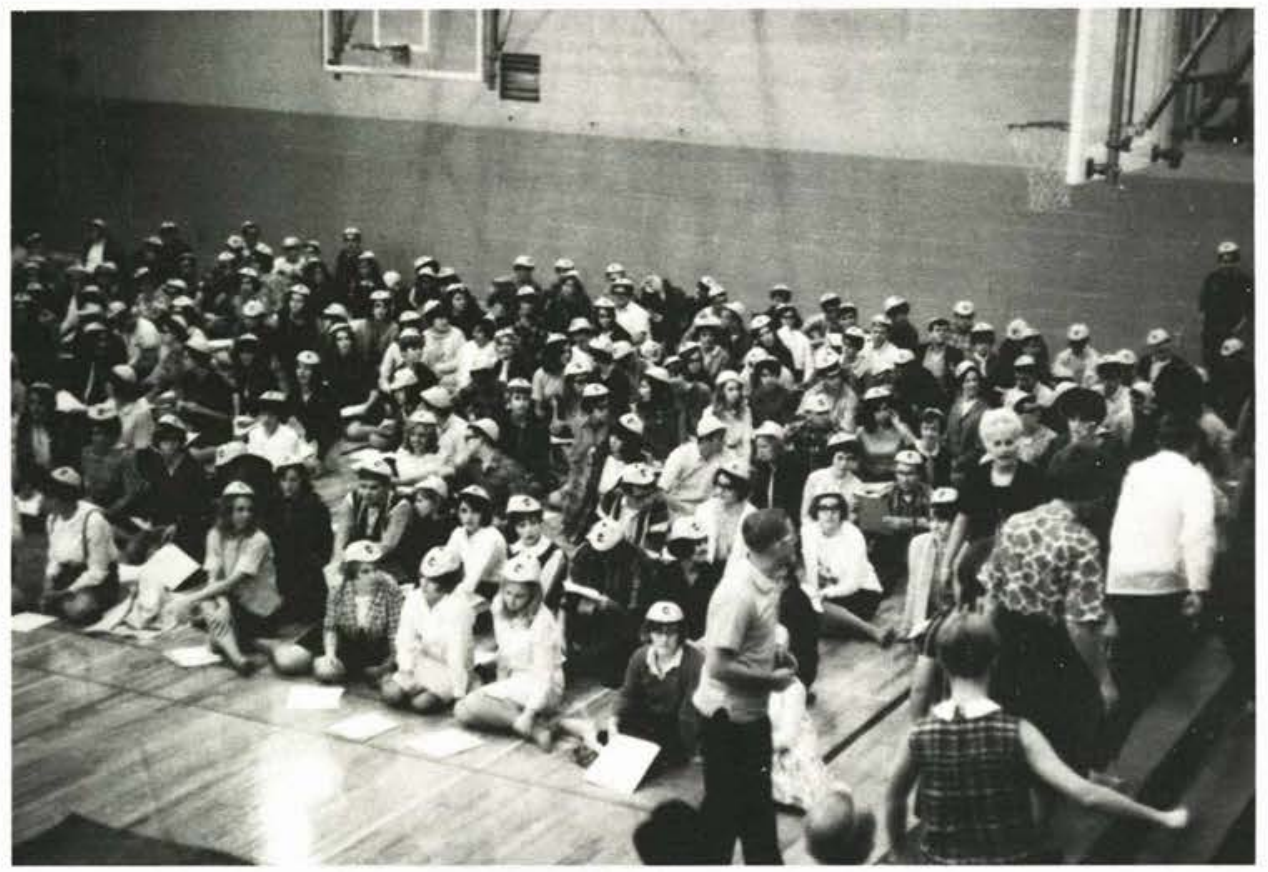

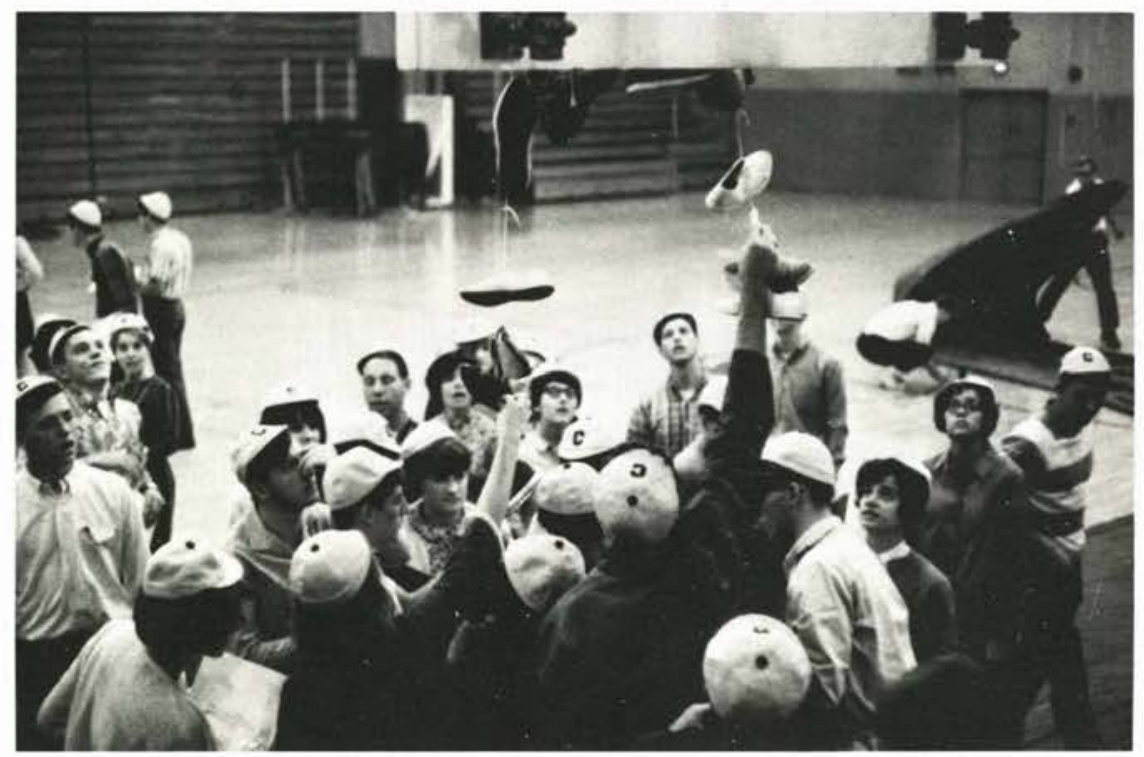

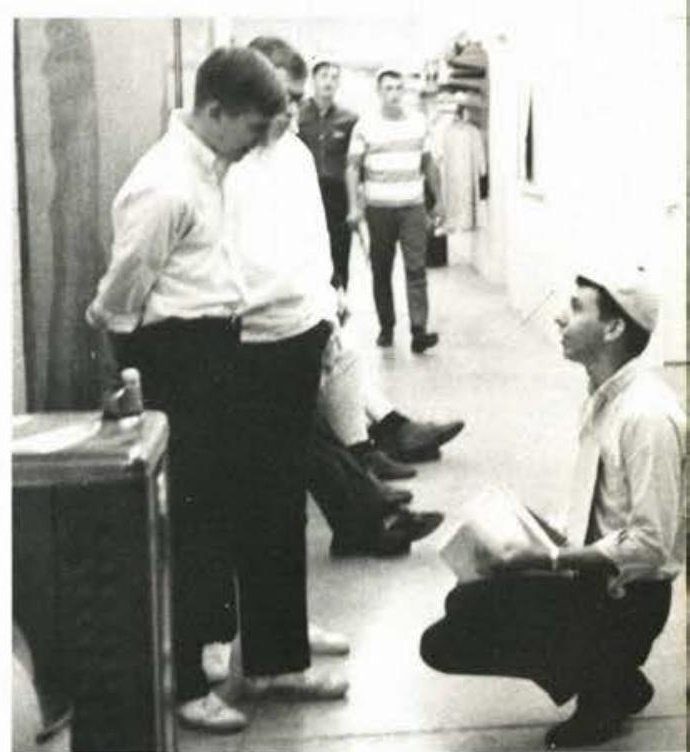
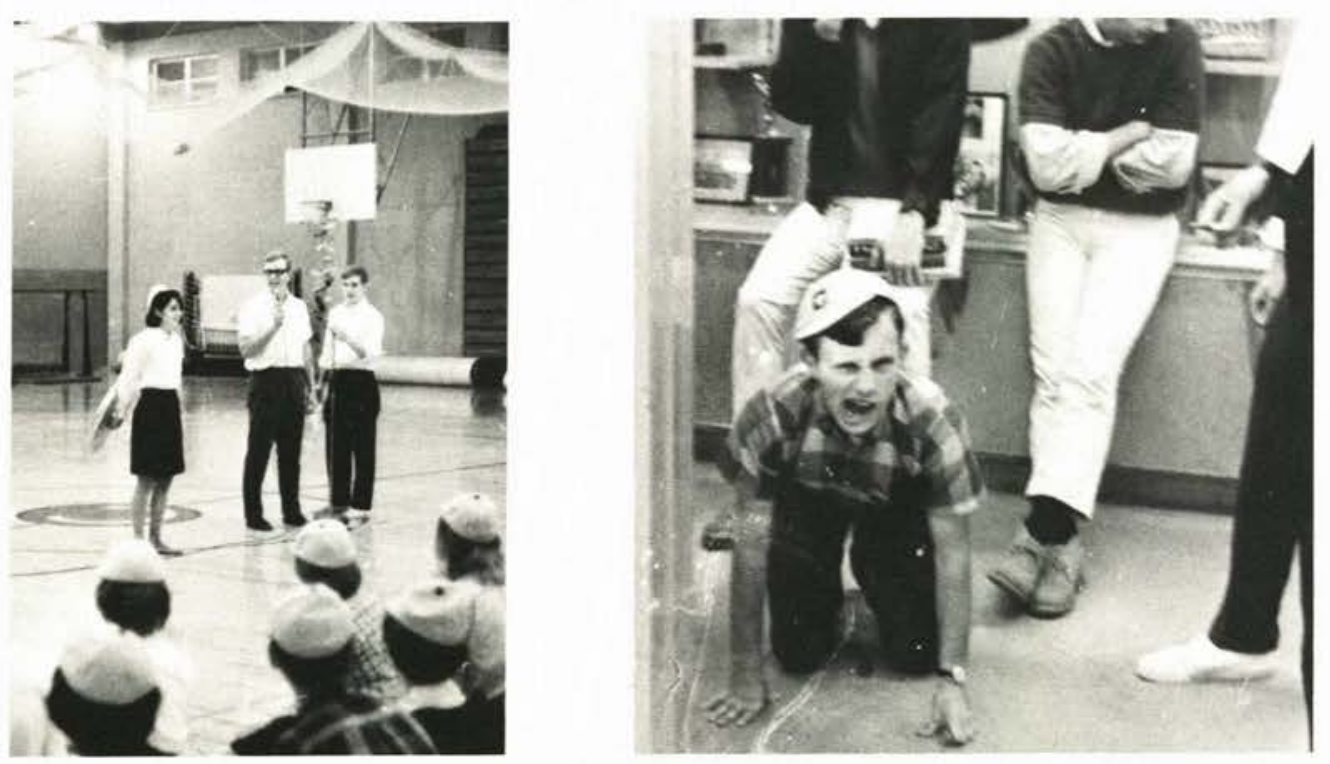


\section{HOMECOMING}

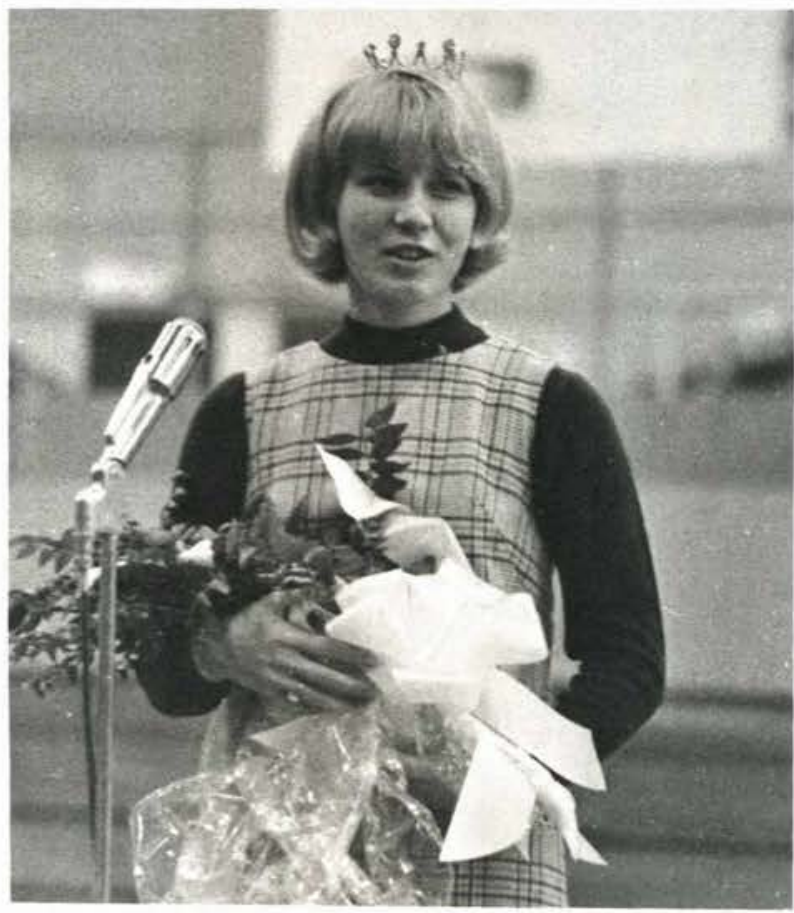

As I reflect on my years at Cedarville, I see years full of every emotion, every experience. There have been times of laughter and times of tears; times of honor, and times of shame. But each experience-academic, religious, social - has forced me to look at myself - many times hating what I saw - and work at making those all-important changes that must be made in order to mature. It is here at Cedarville that I have learned the importance of having a thirst for self-improvement. As I look around the campus, I see memory after memory returning, reminding me that in the future, I will spend many a wistful evening reflecting on the learning experiences I was involved in here at Cedarville. It is here that I came to place a premium on my faith. It was living with students who had what I was looking for that made me see the importance of a constant communion with God. I shall always thank the students and faculty at Cedarville for forcing me to find myself, and then to try to improve all those imperfections which seem never to diminish. Truly the Lord was thinking of me when he had Paul write the words, "Not as though I had already attained, either were already perfect, but I follow after, if that I may apprehend that for which I am already apprehended of Christ Jesus." I am so thankful that God doesn't give up on us, but rather keeps prodding us along to newer fields.

Debbi Bush
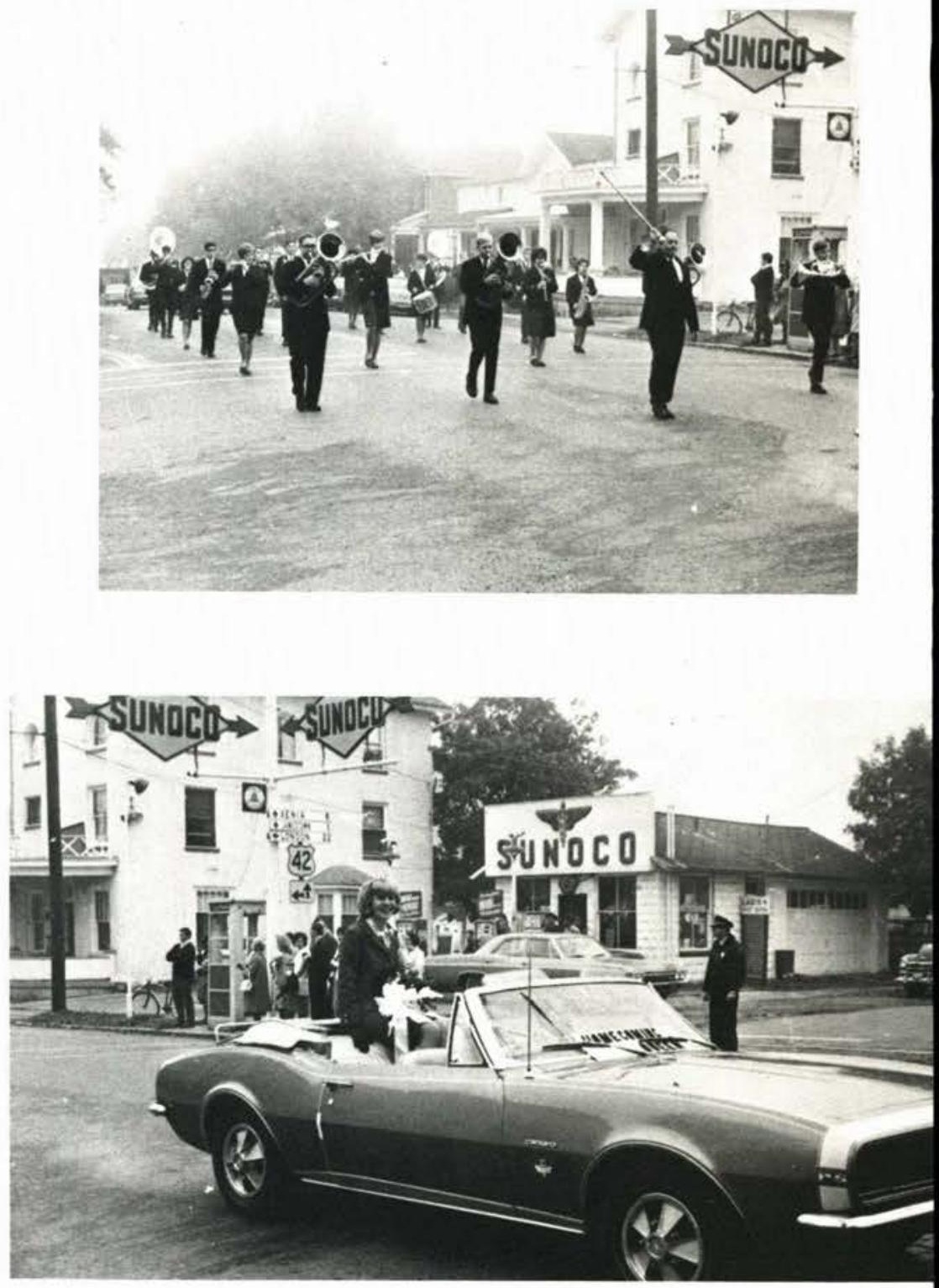

Homecoming Queen - Debbi Bush

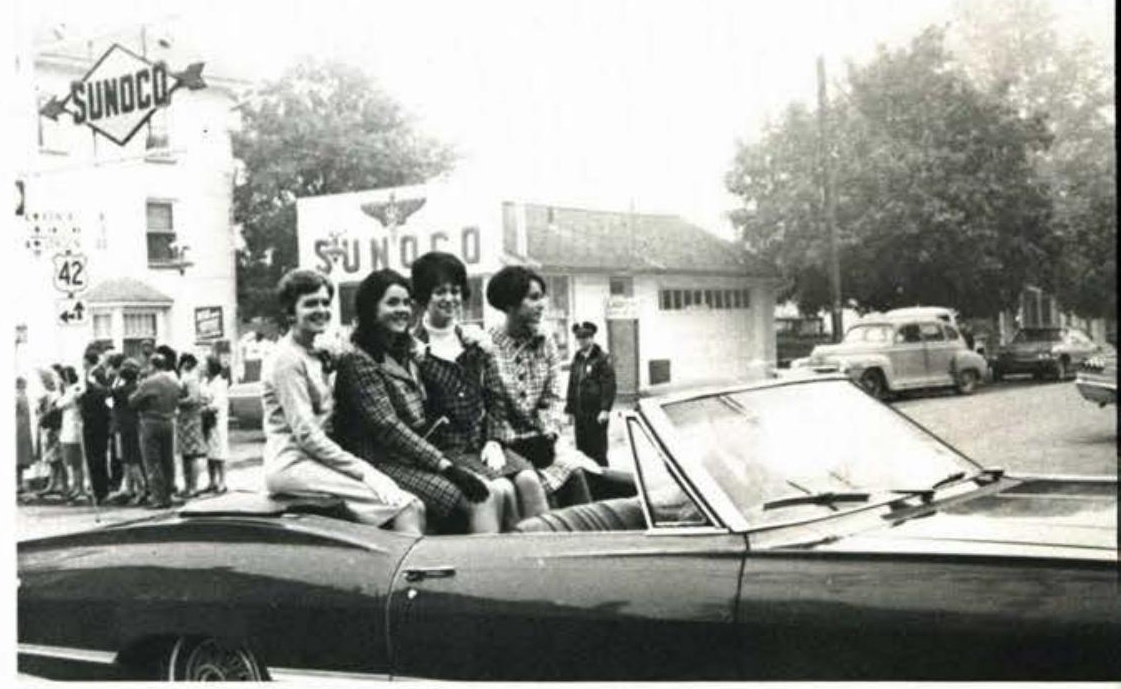

Queen's Court - Shirley Dufford, Nancy Norton, Marilyn Bales, Iris Lima. 
OCT. 14, 1967

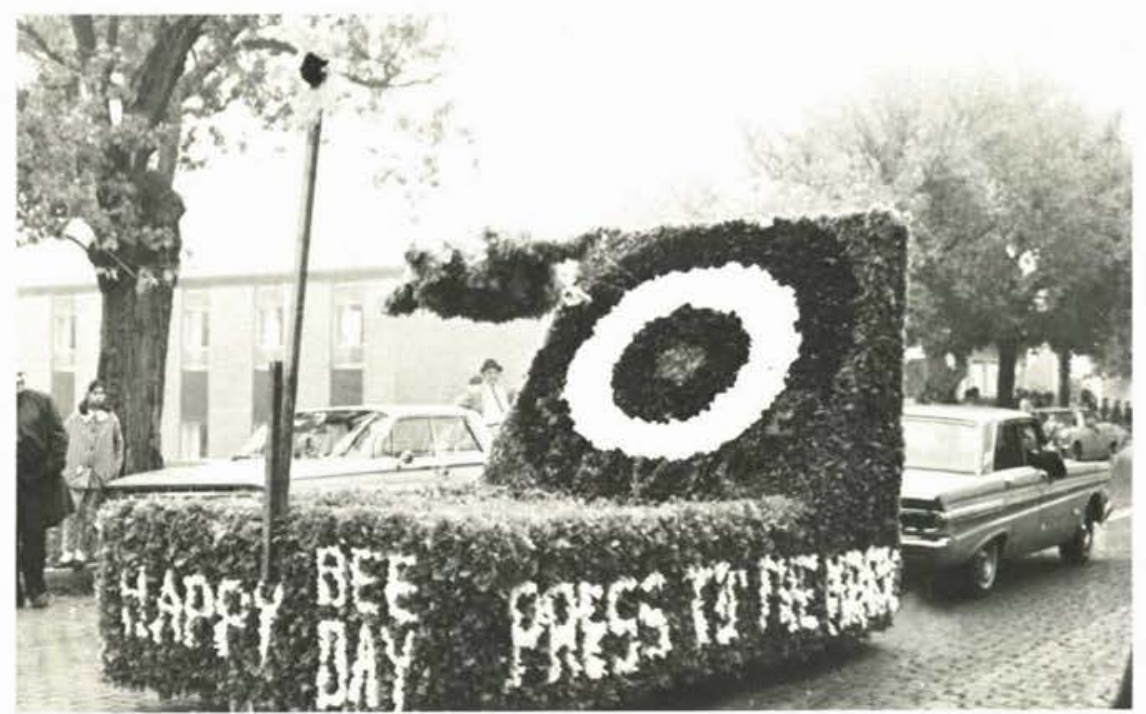

FRESHMAN FLOAT

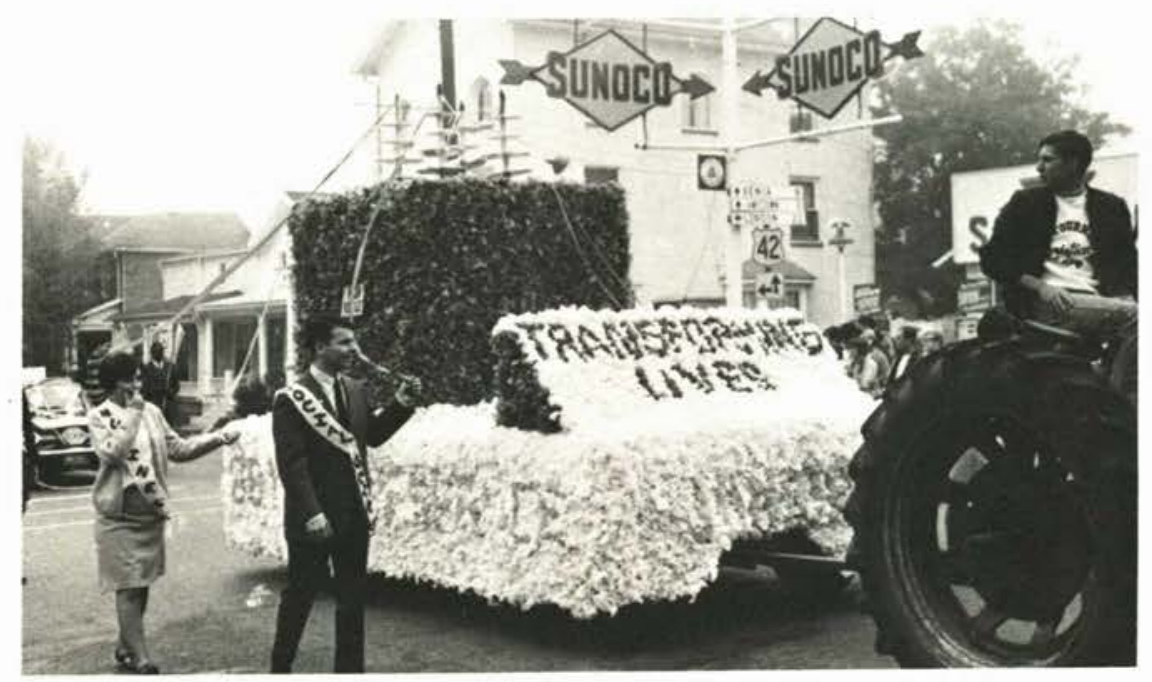

\section{CLASS FLOATS}

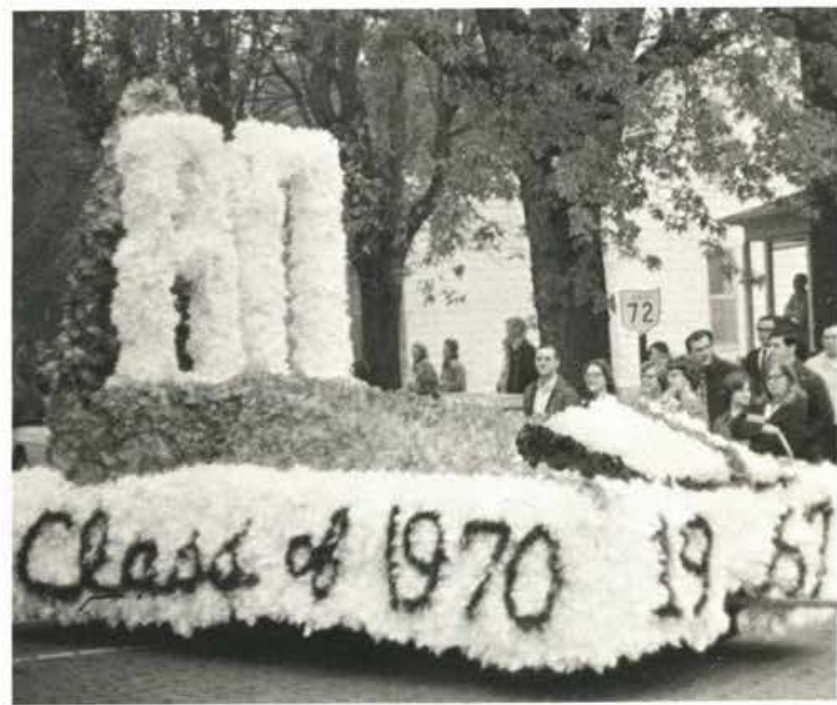

SOPHOMORE FLOAT

JUNIOR FLOAT
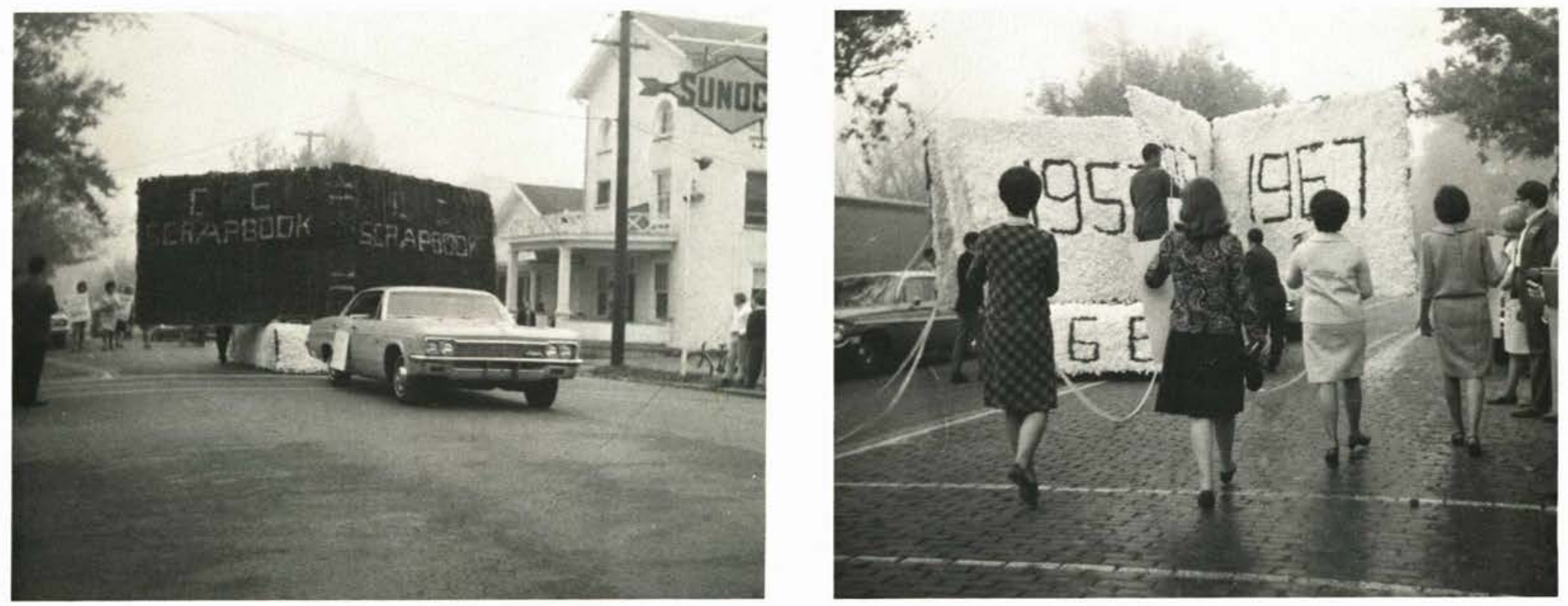

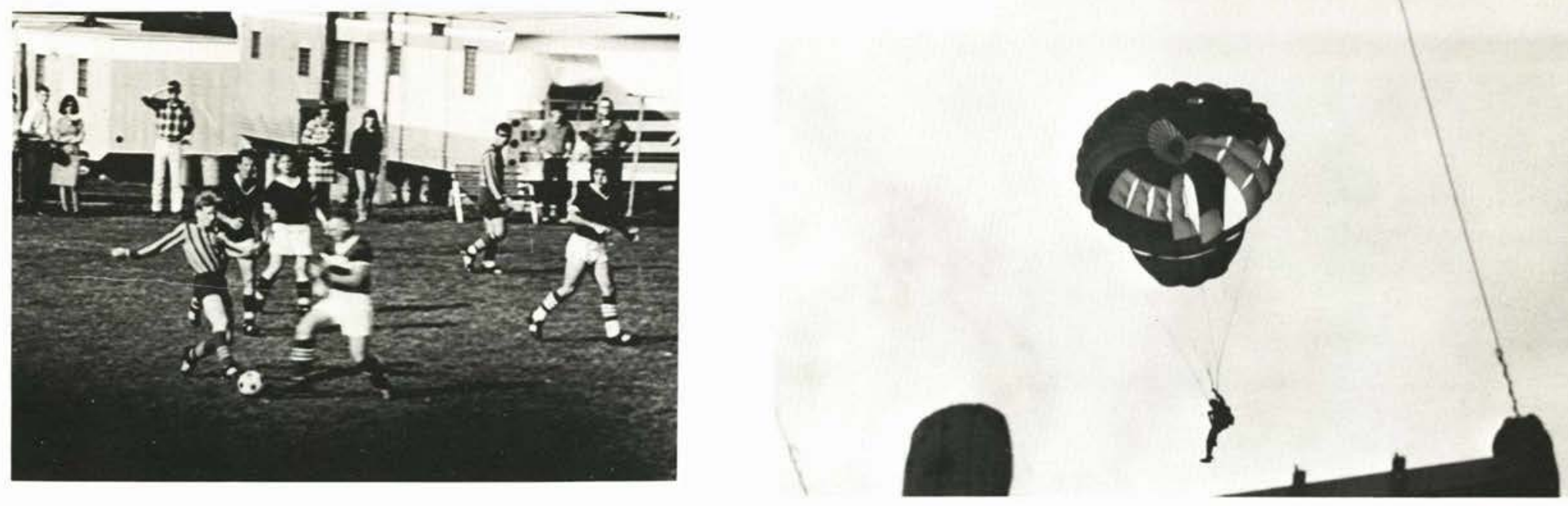

$\frac{1}{3}+\frac{1}{20}$

A. $x^{2}$.

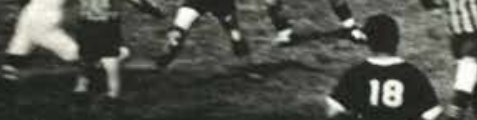

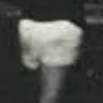

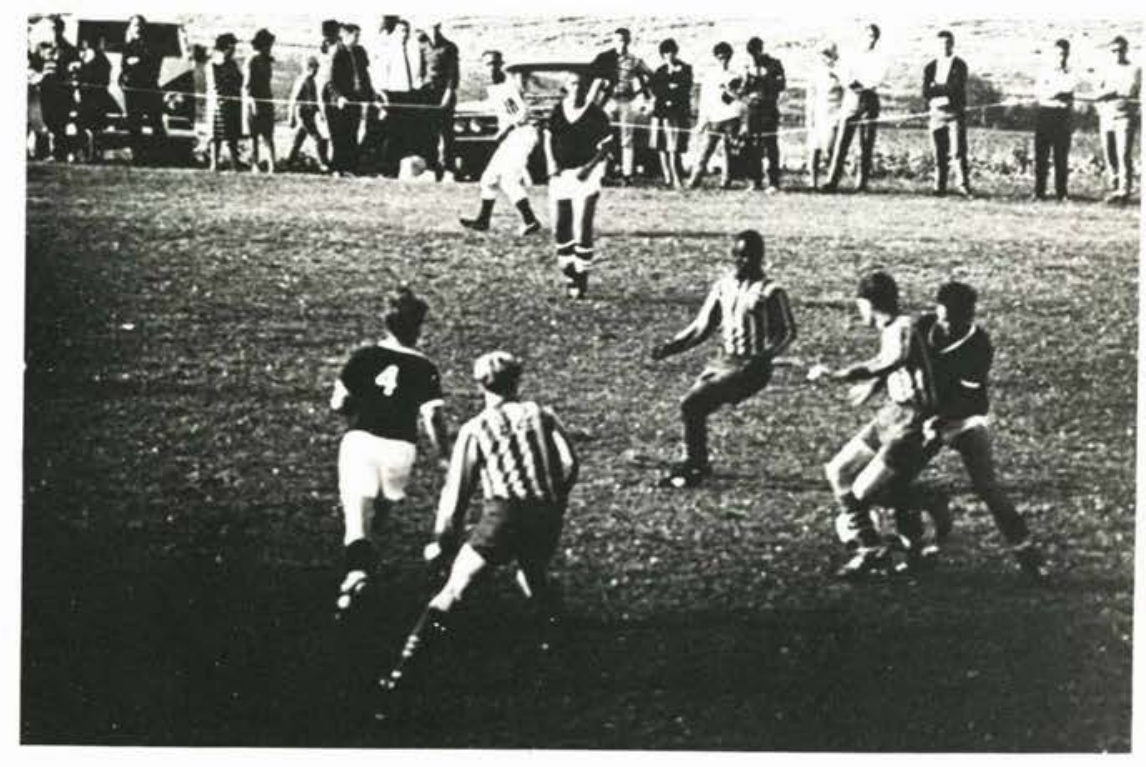
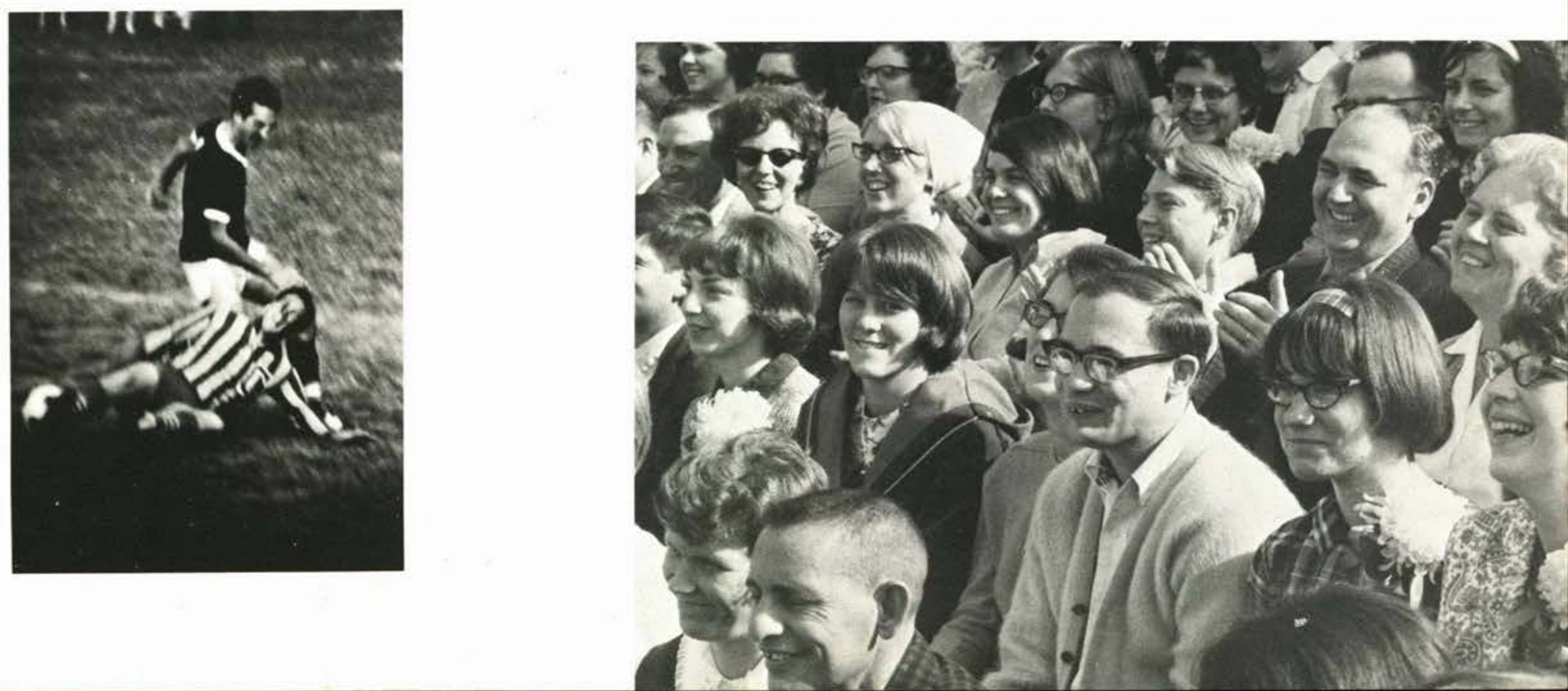


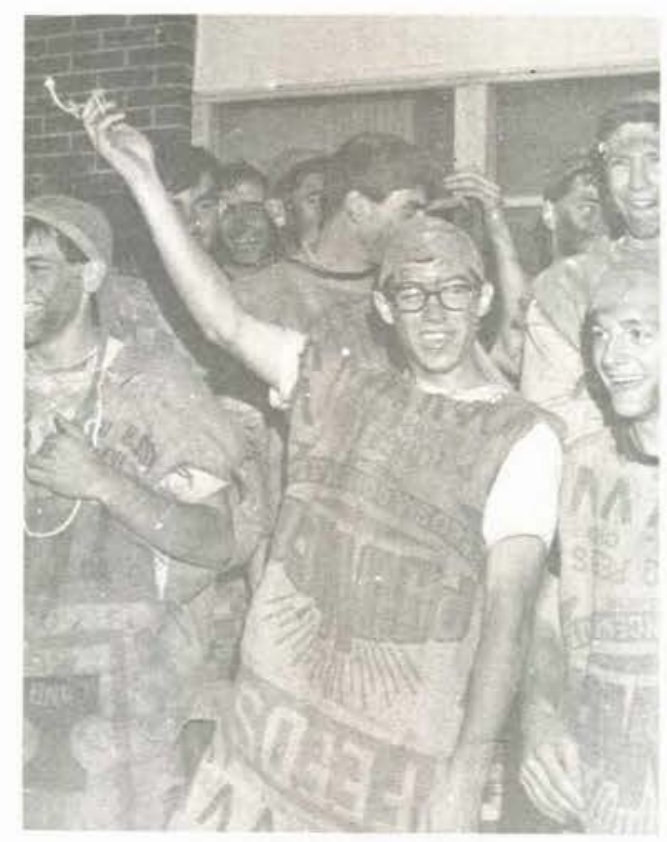

NELSON \& NEAL

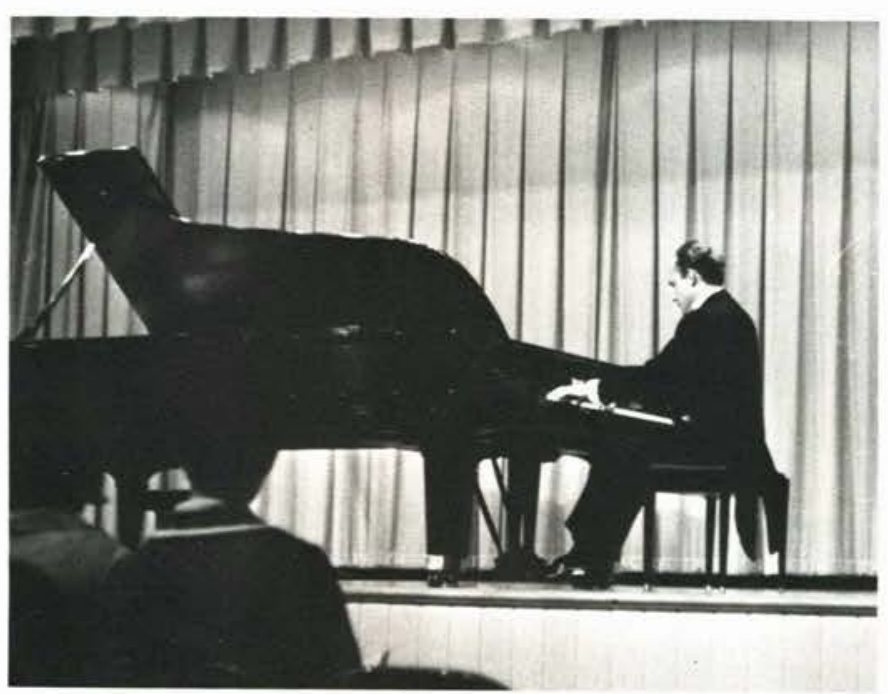

DUO-PIANO

CONCERT

OCT. 31
VARSITY "C" INITIATION OCT. 20
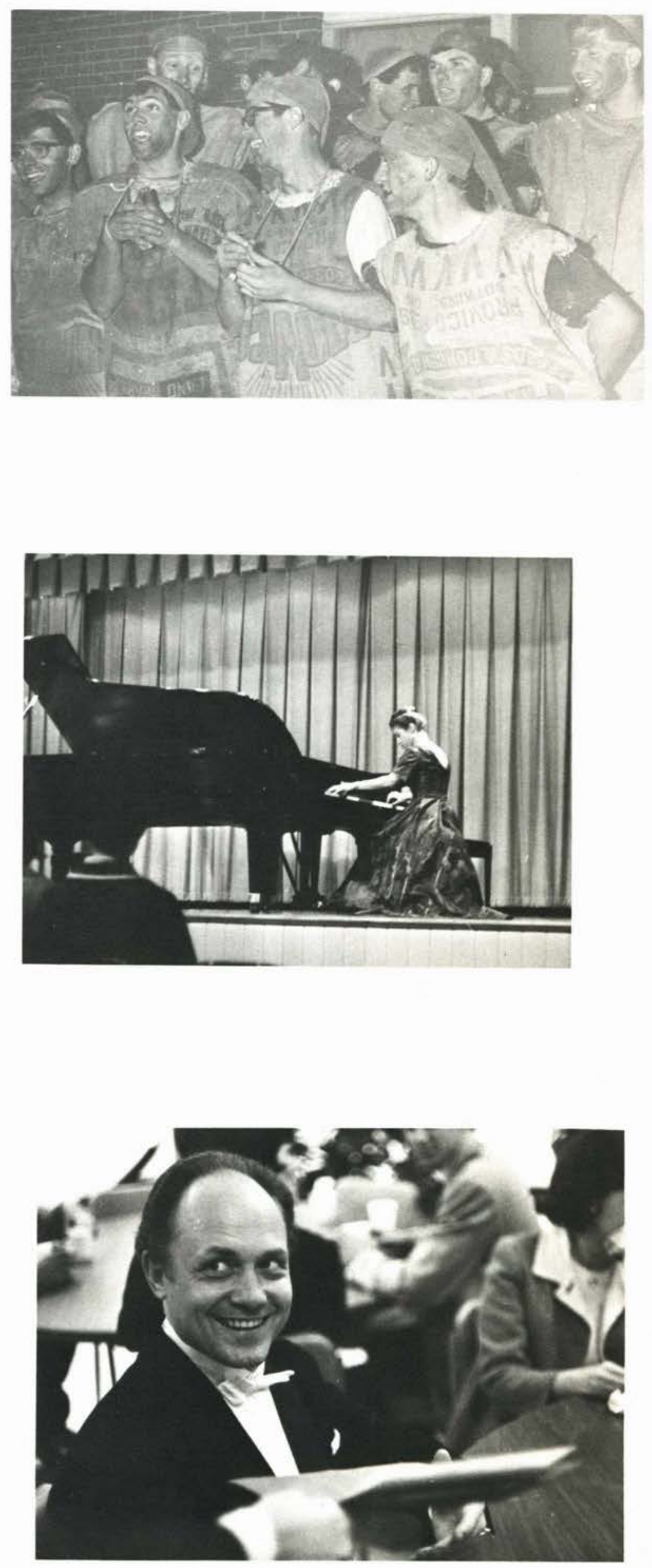
SOCCER
GAMES
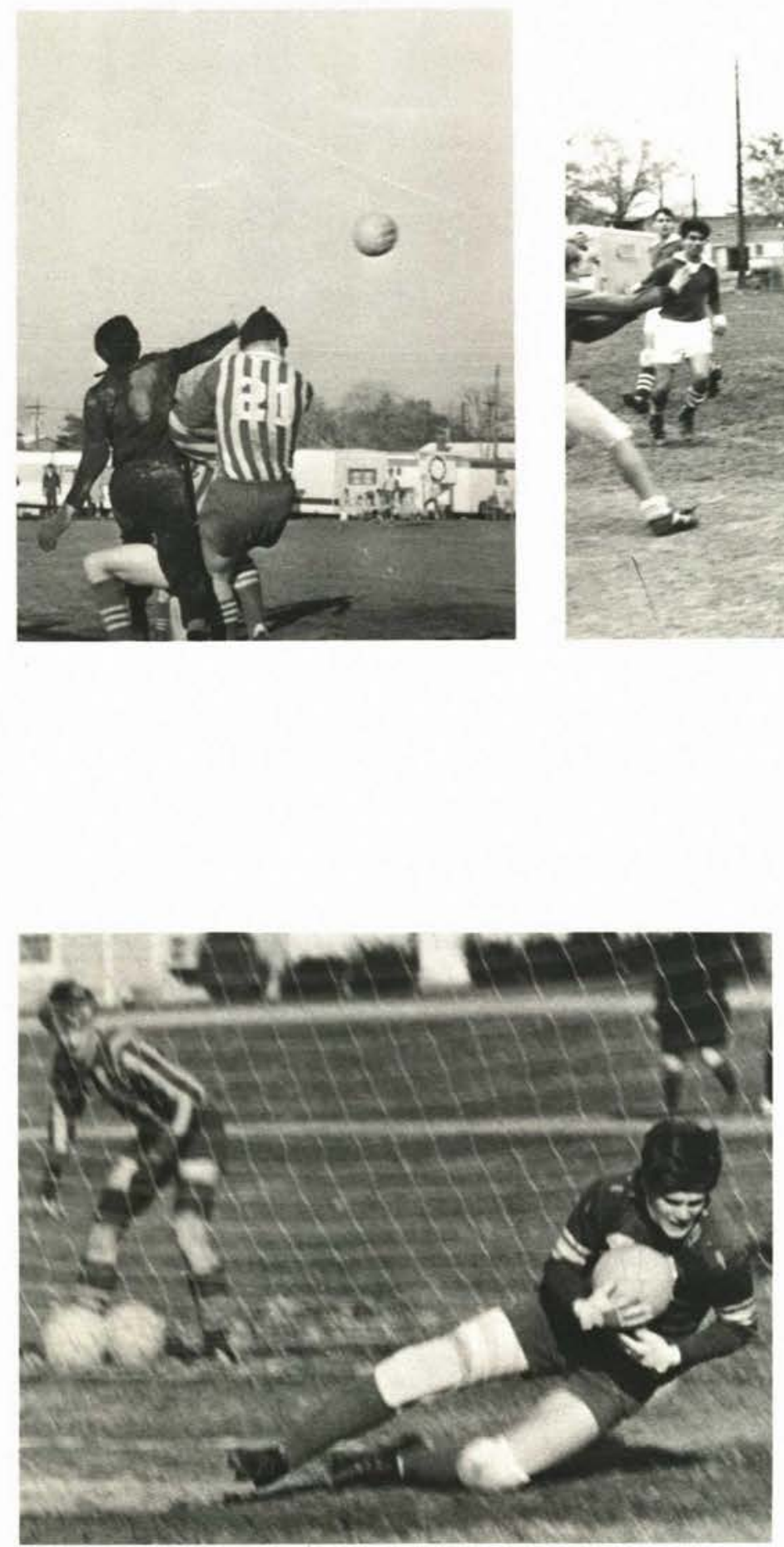
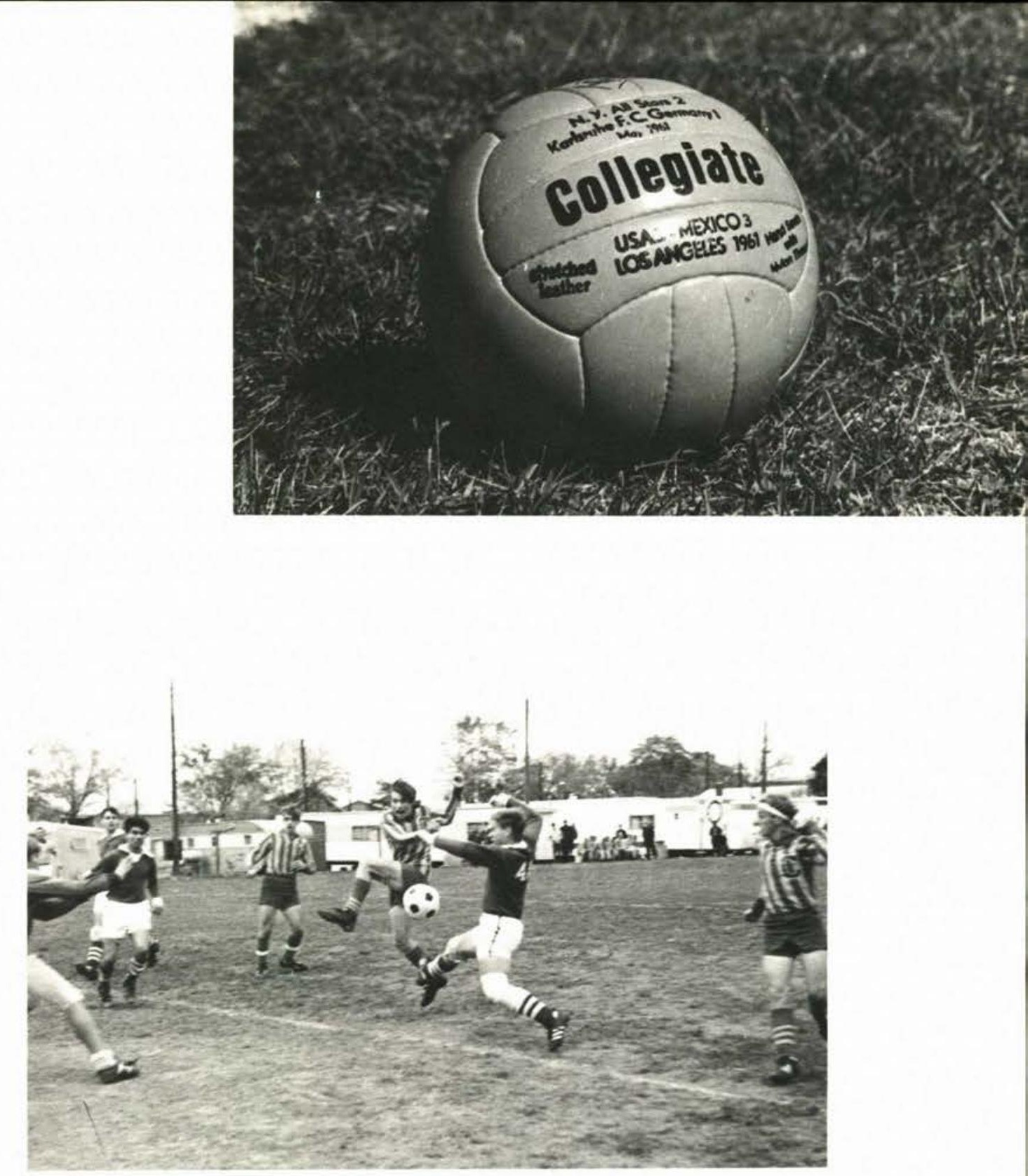


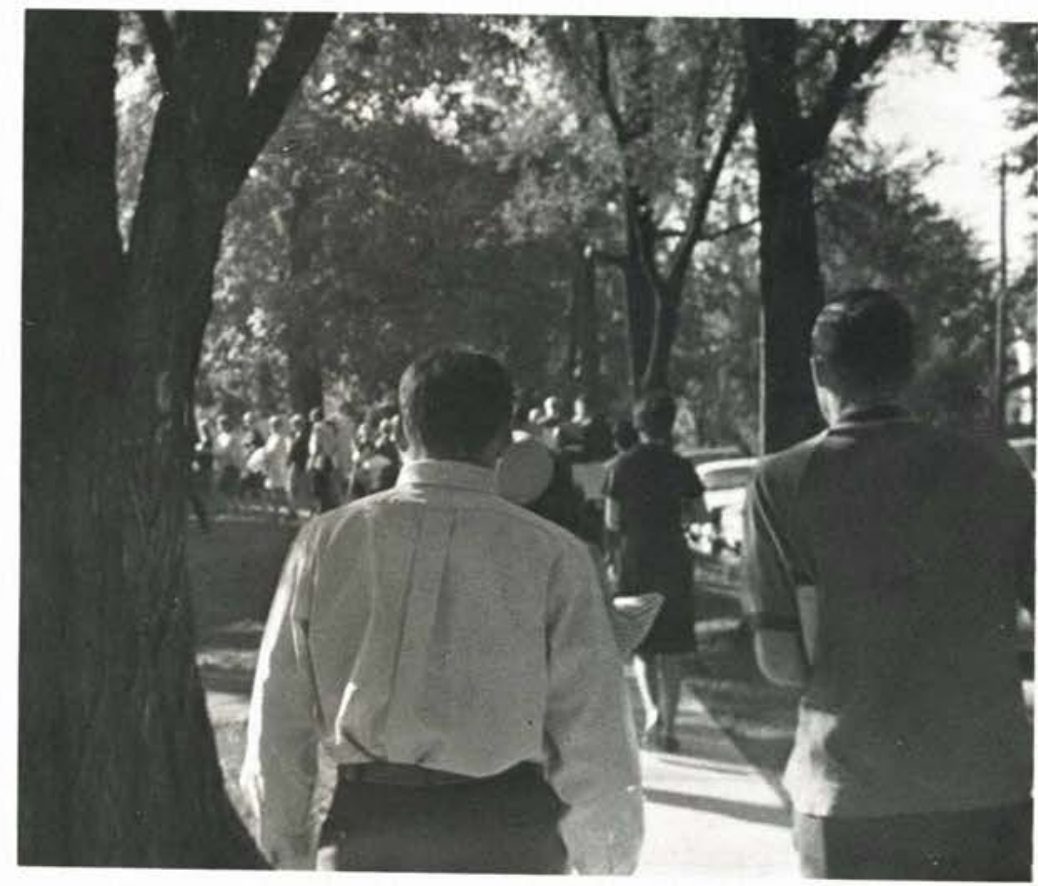

CHAPEL

\section{Ten o'clock every day}

The favorite seat, friends

Sweet Hour of Prayer.

An inspiring forty-five minutes

At the start of a new day.
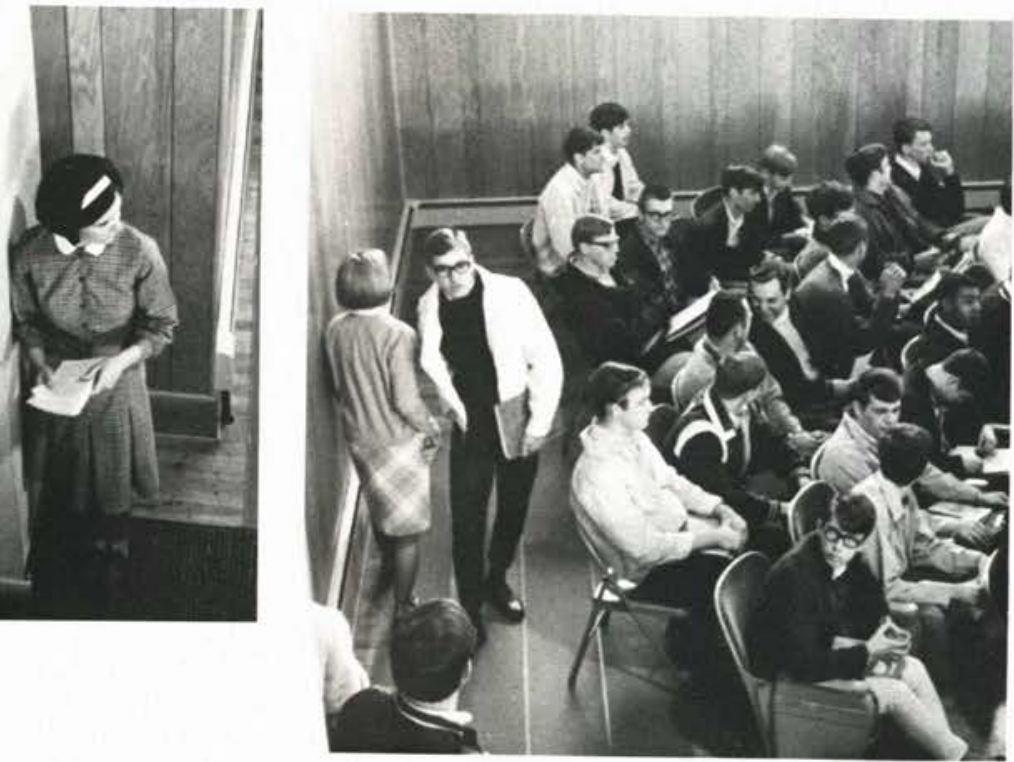

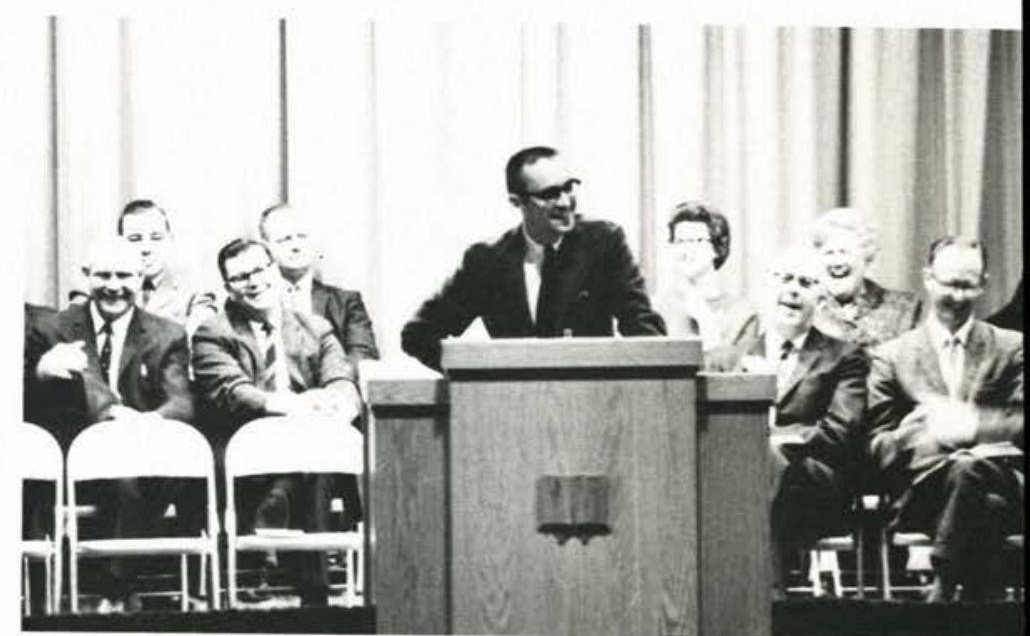

Rev. Sam Canine

Dr. John G. Balyo
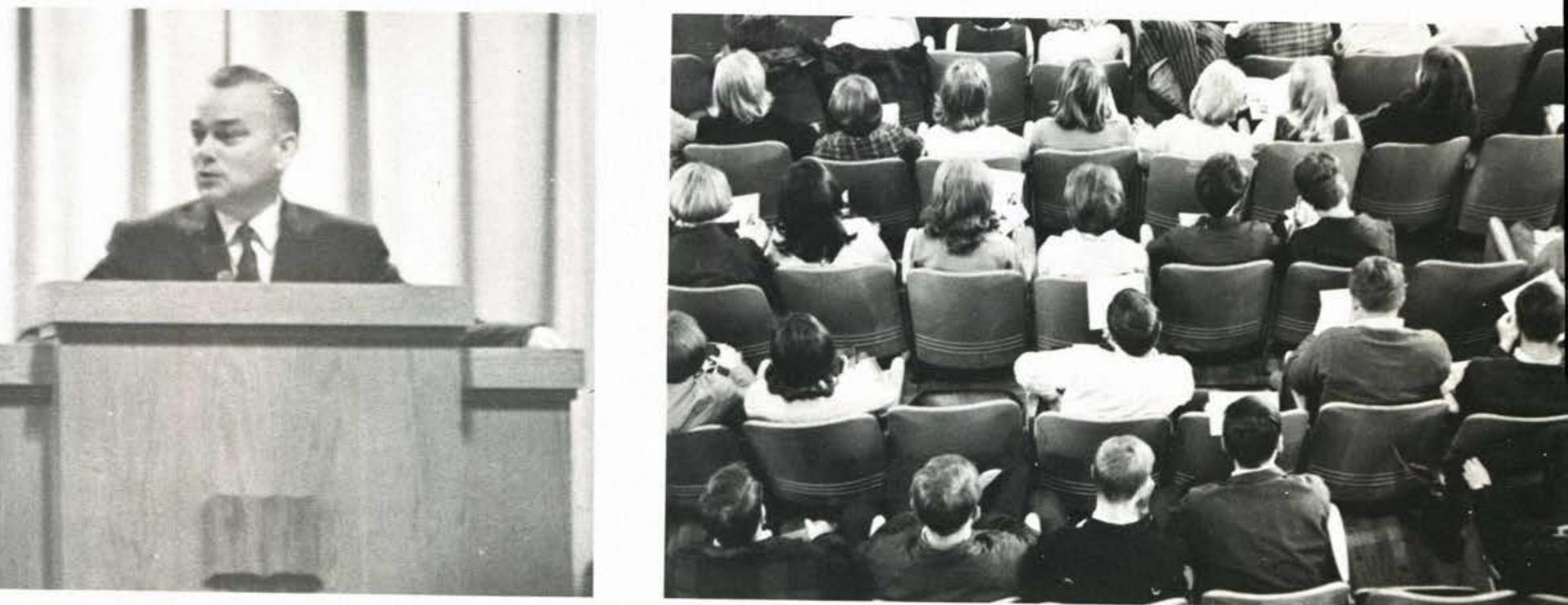
NOV. 13

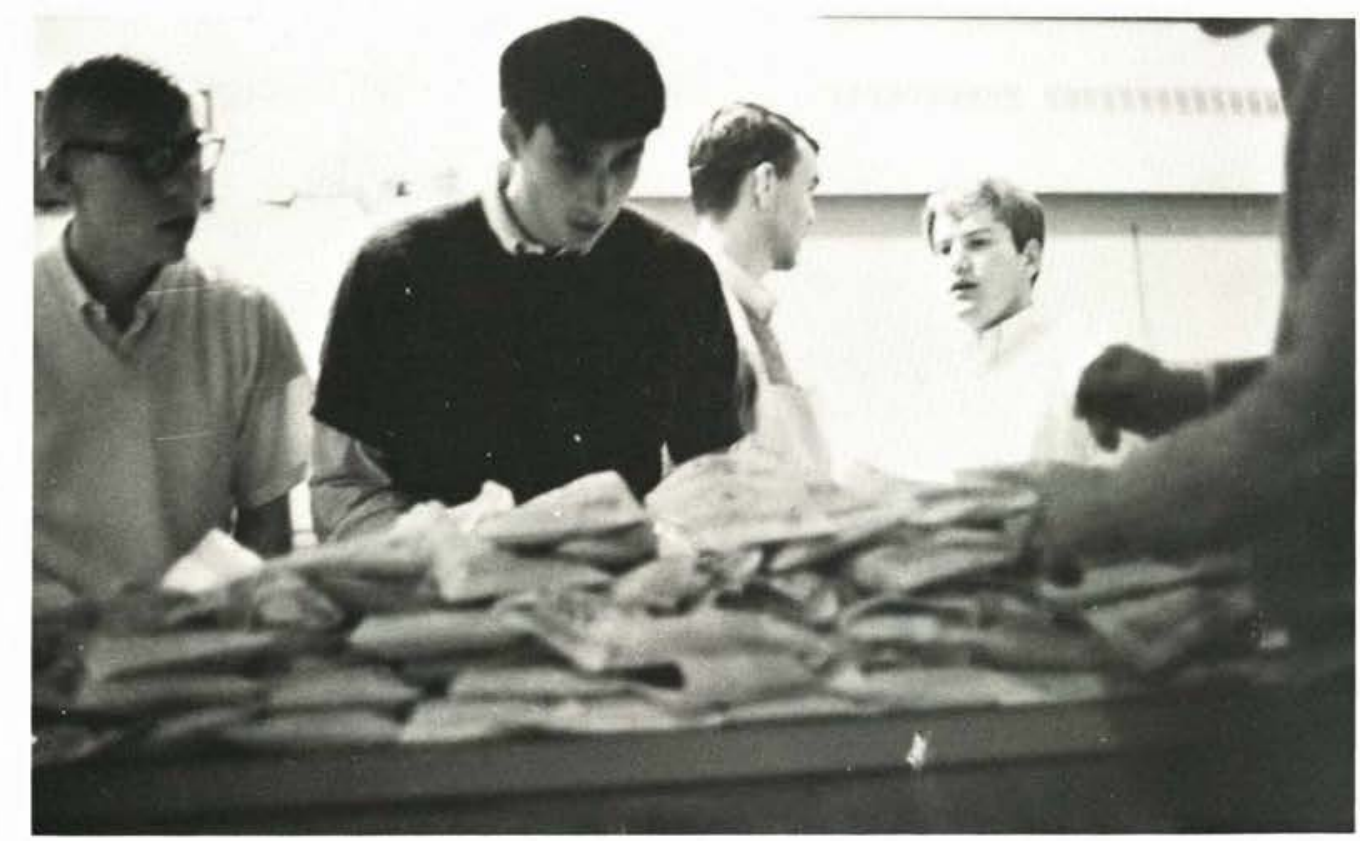

Pi Sigma Nu members make sandwiches for the National Guard during the riots at Central State University,

FALL AT C.C. MEANS ...

\section{"CARE PACKAGES" ...}

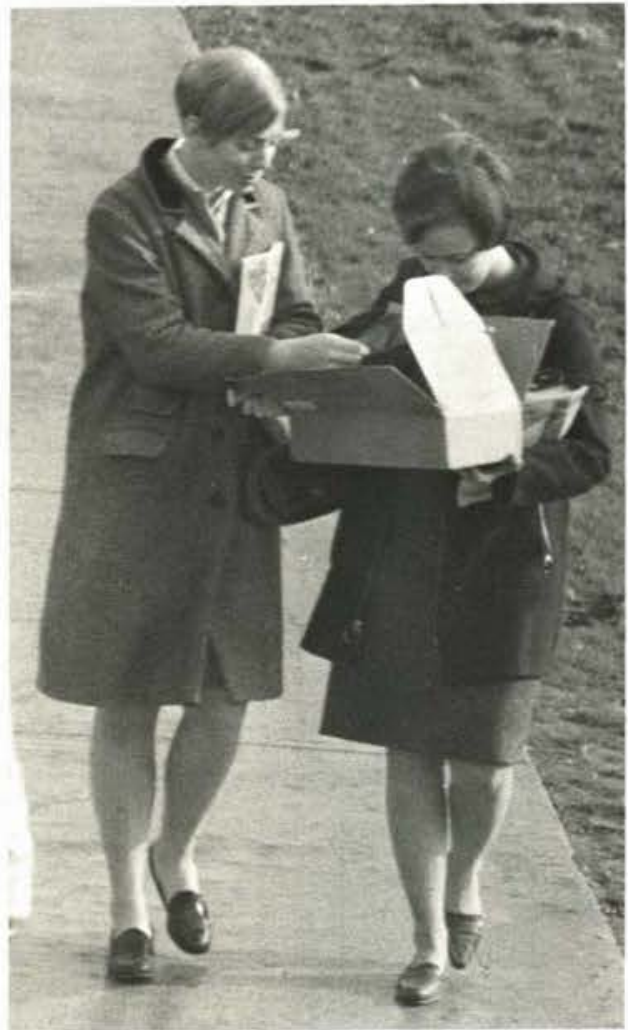

VARIETY SHOWS ...

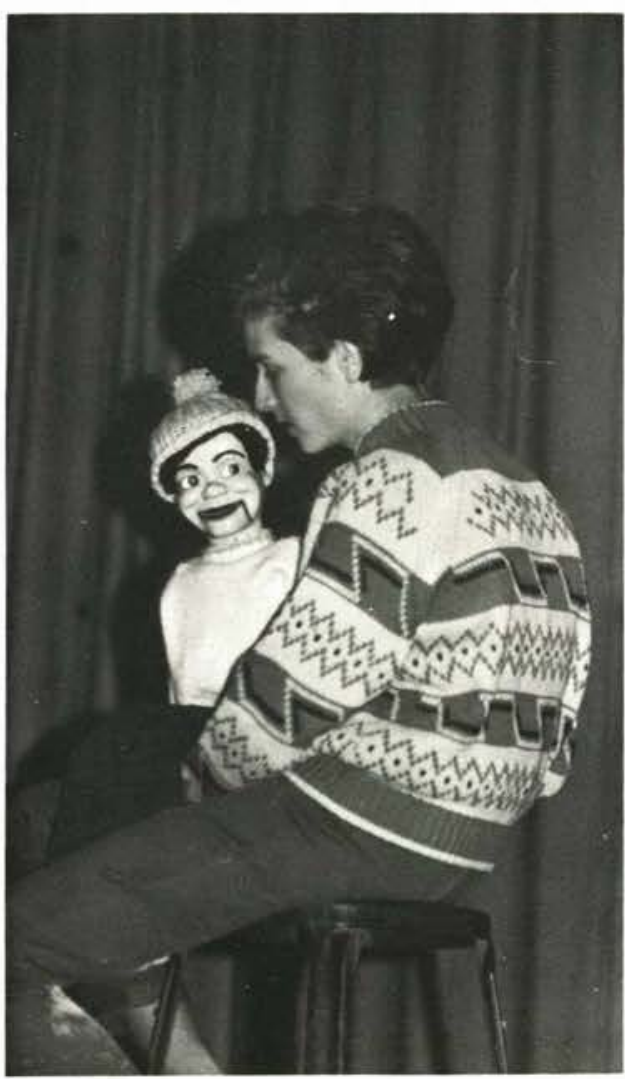

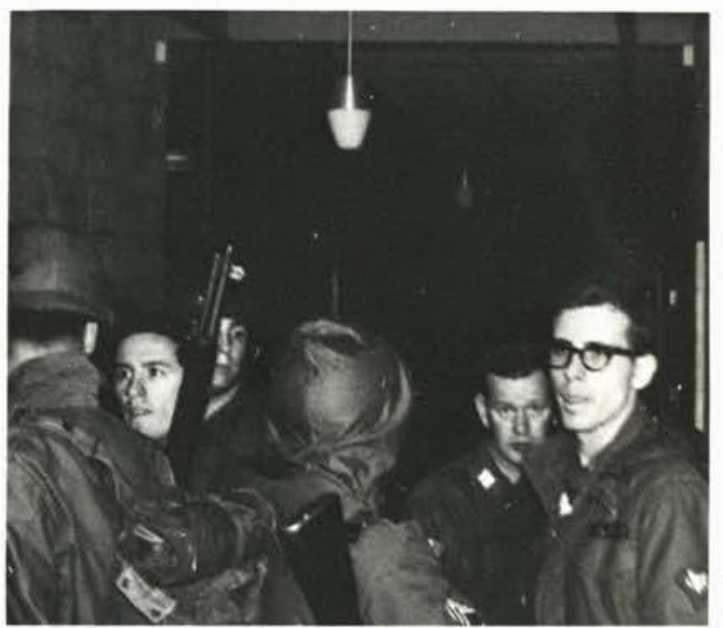

RAIN

AND -

MUD.

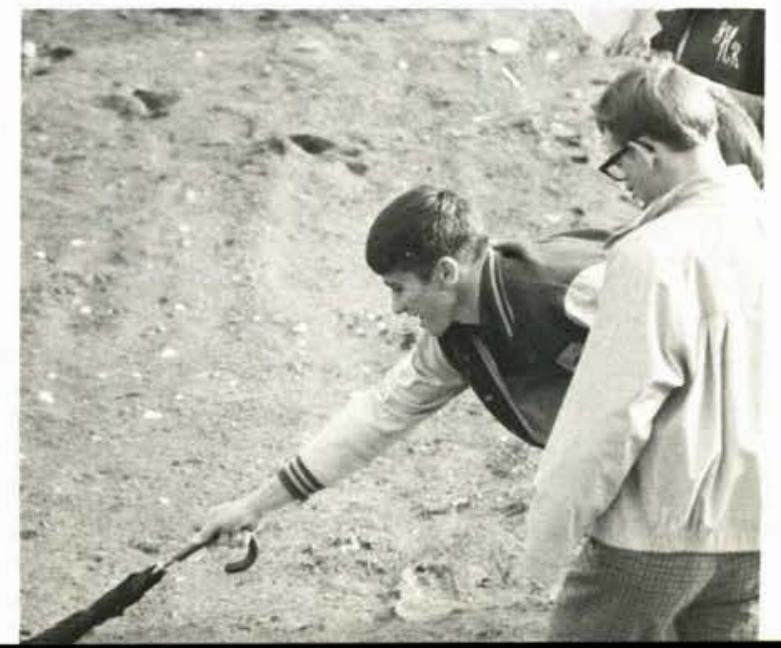


WINTER
QUARTER

1968

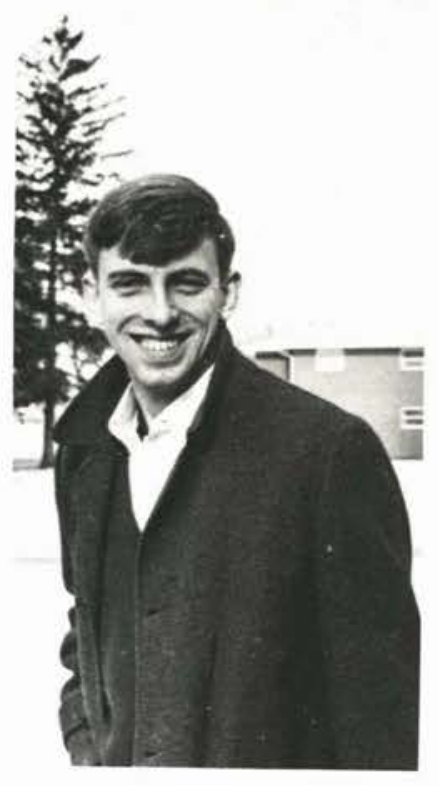

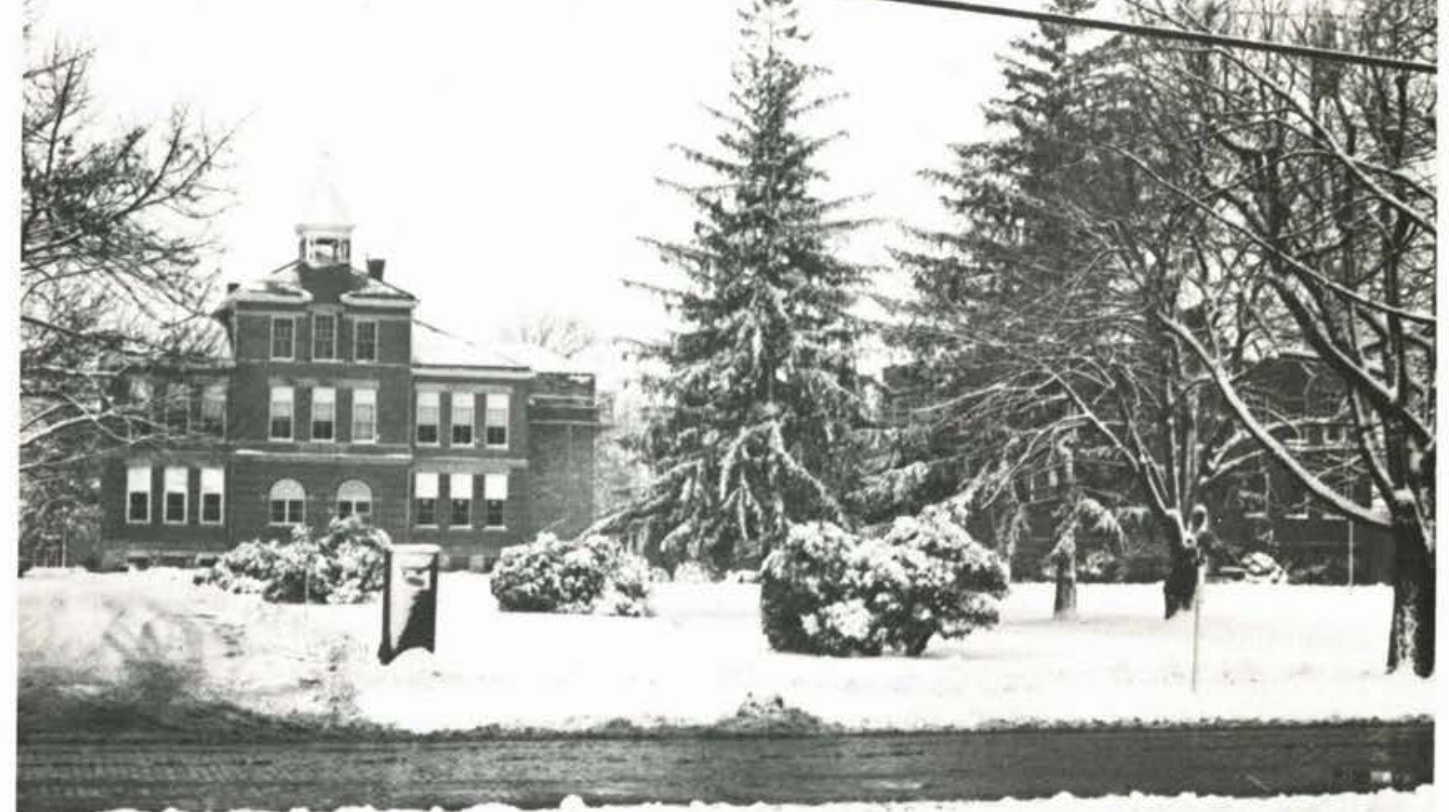
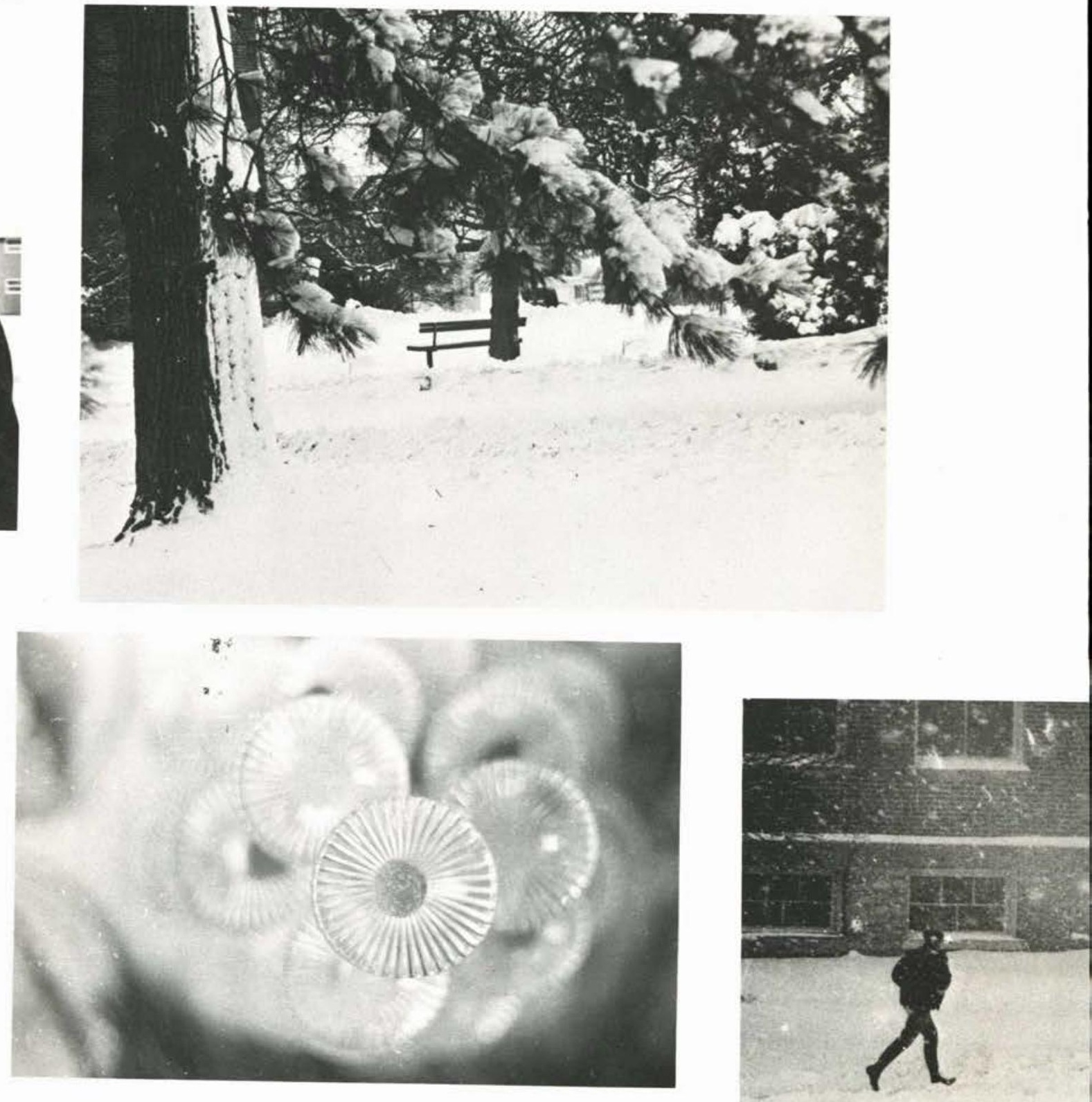


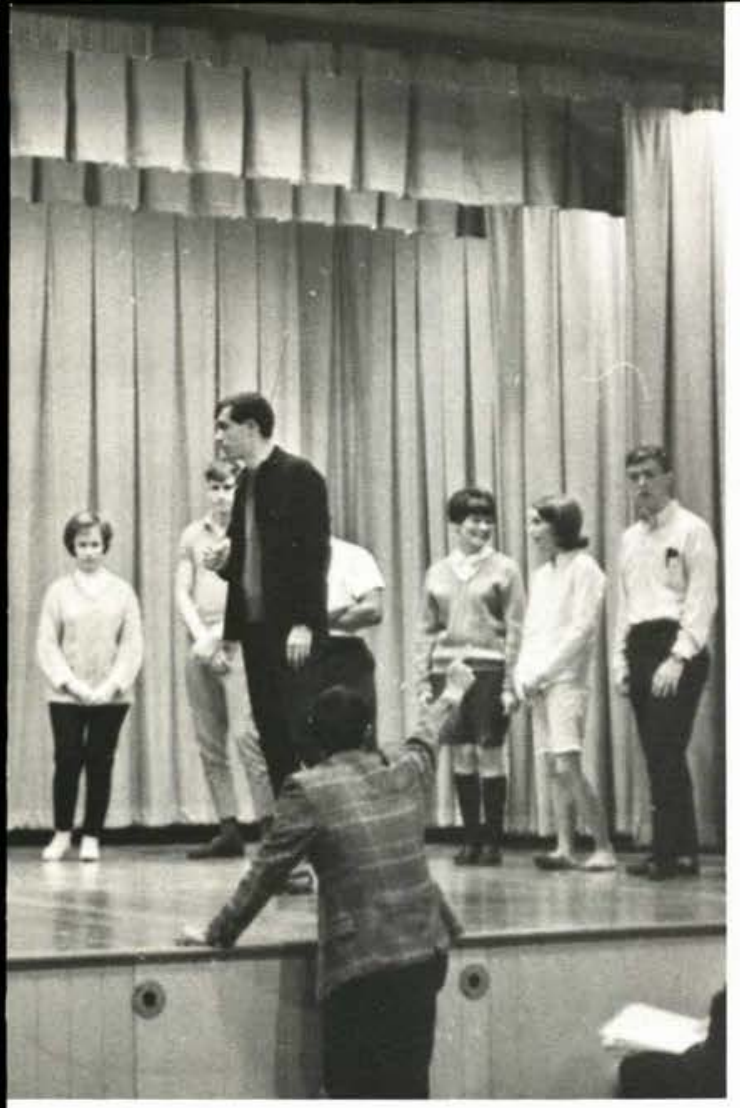

"PIRATES of PENZANCE"

FEB. 8, 10
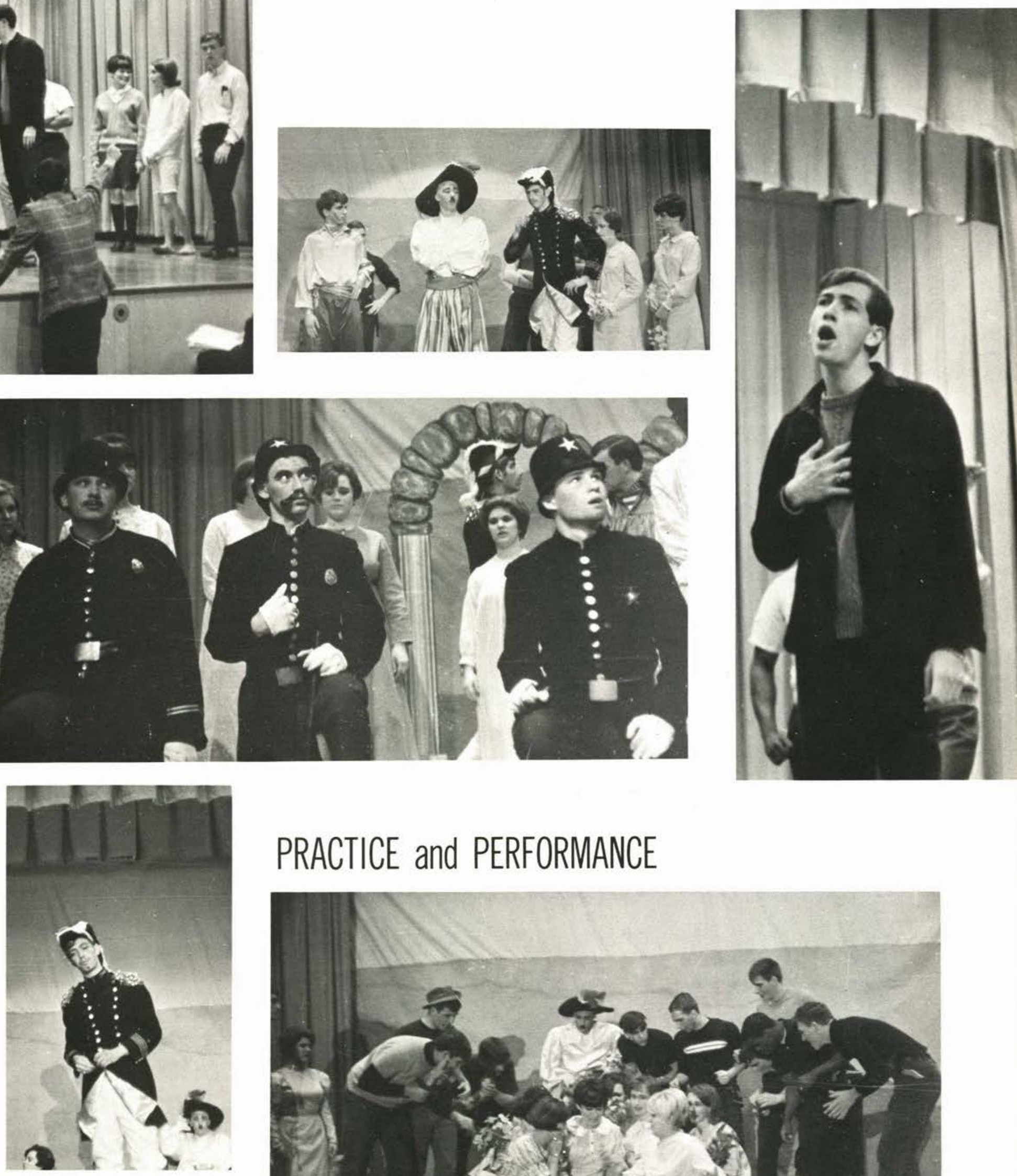

PRACTICE and PERFORMANCE

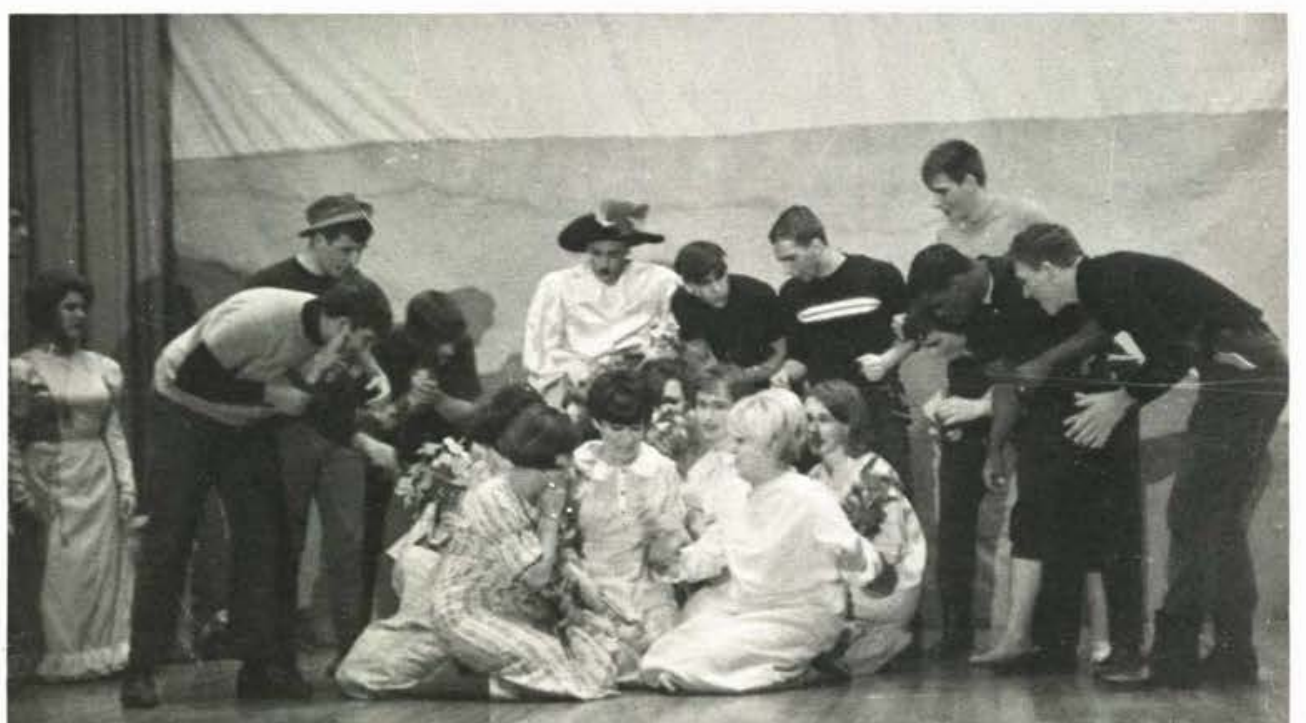




\section{ALPHA CHI QUUEEN}
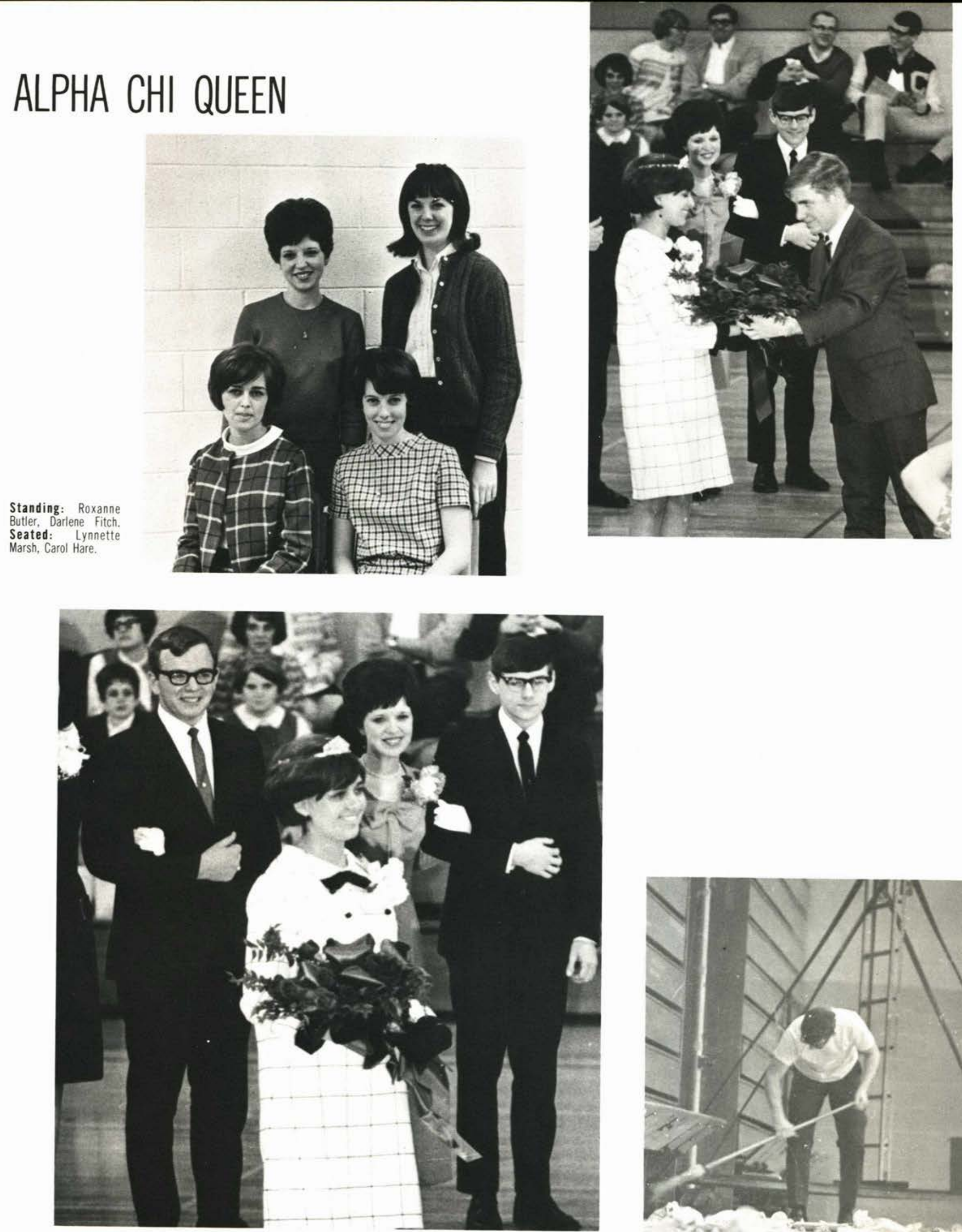

QUEEN LYNNETTE MARSH

The duties of the Queen of Alpha Chi include being hostess to the school functions sponsored by Alpha Chi. One of her activities this year was to preside over the Cedar Day festivities.

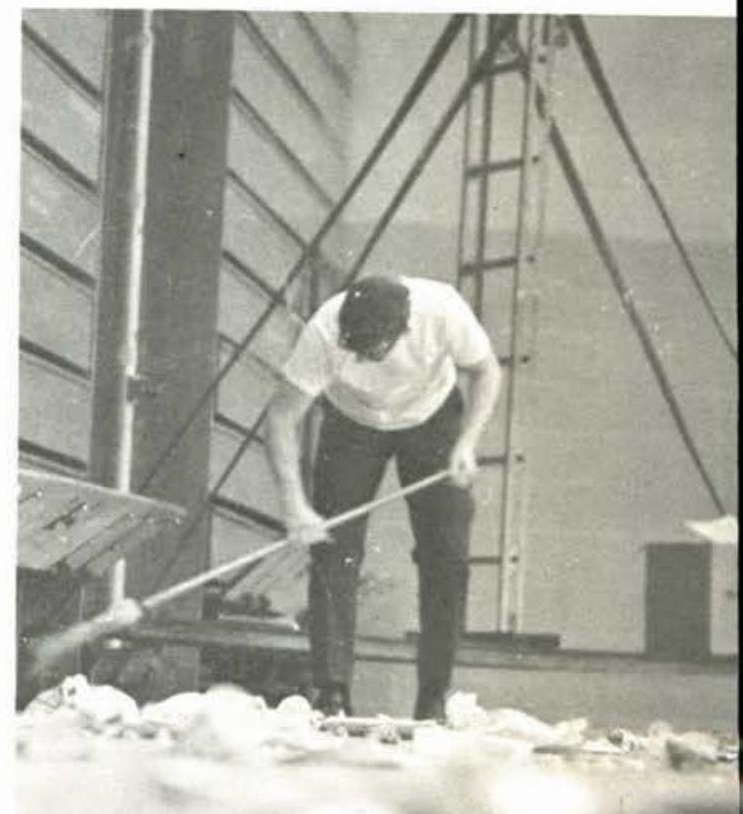


TOM BILLER SENIOR RECITAL

FEB. 17
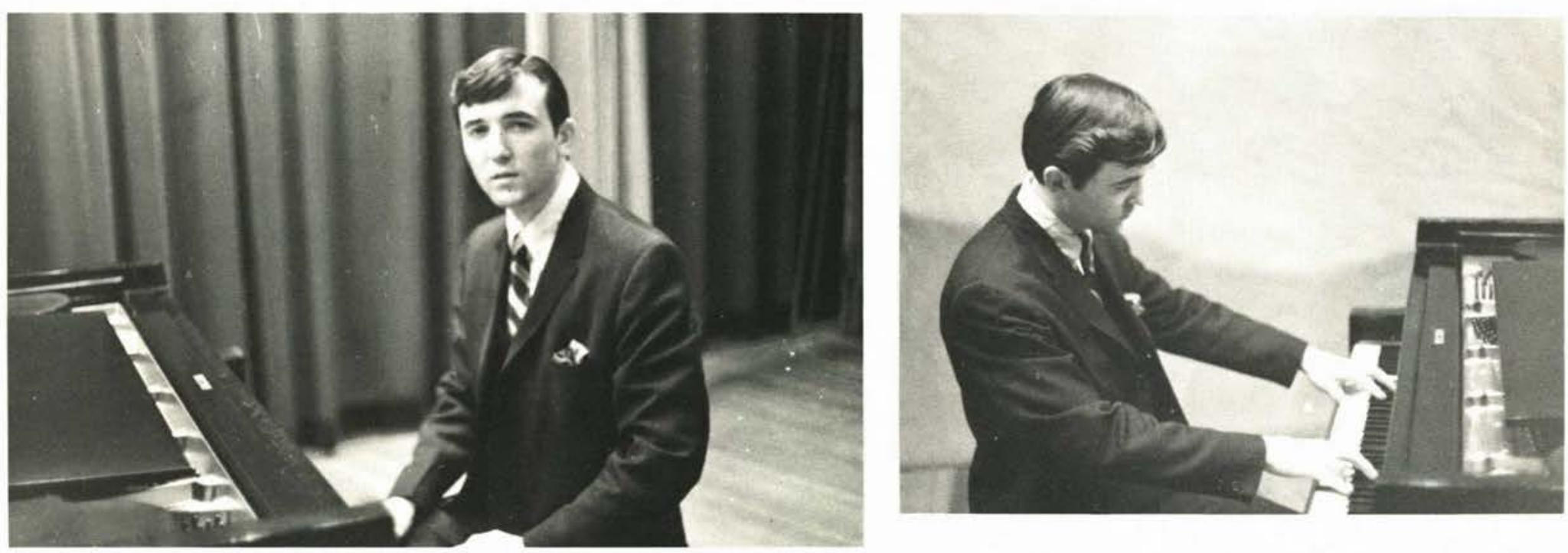

A favorite meeting place on campus, especially during the cold winter season, is the college snack shop. In January, a part of it was converted on weekends into an "atmosphere room" using an Old English

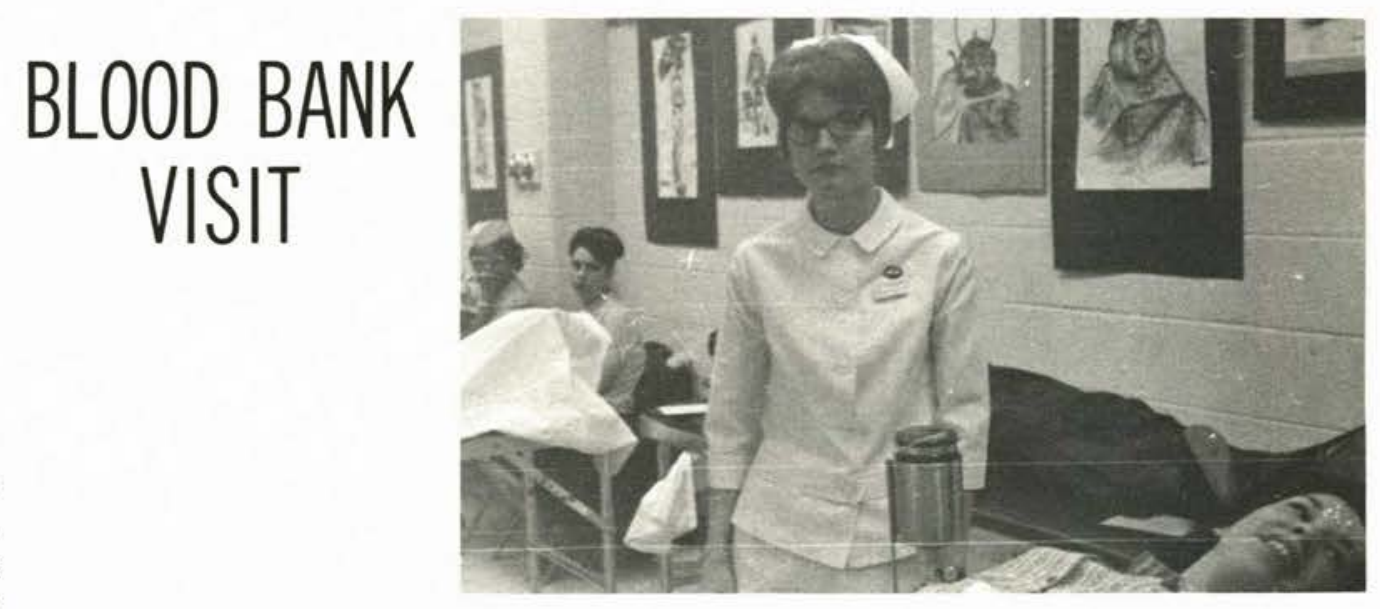
theme.

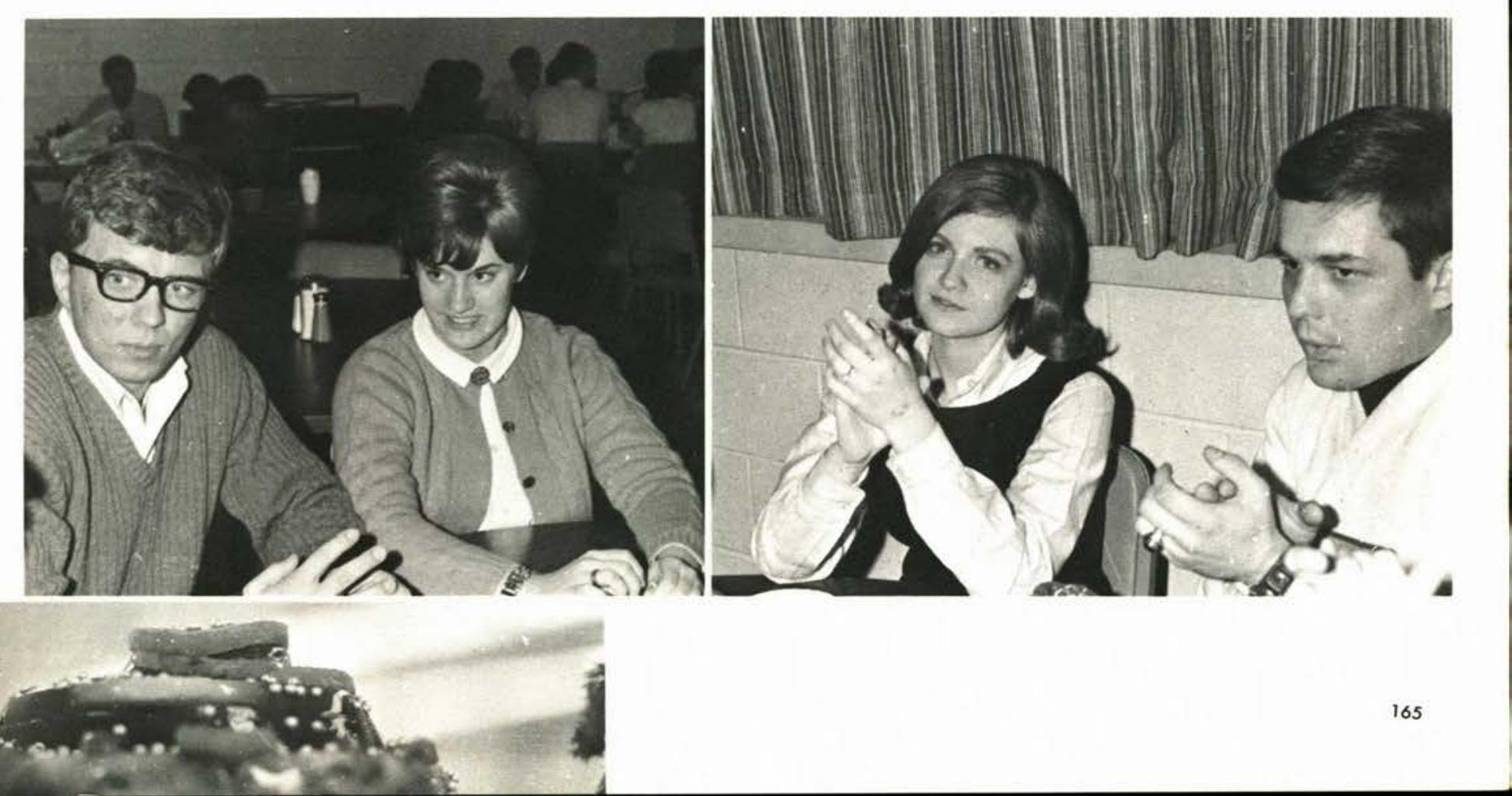




\section{BASKETBALL SEASON}

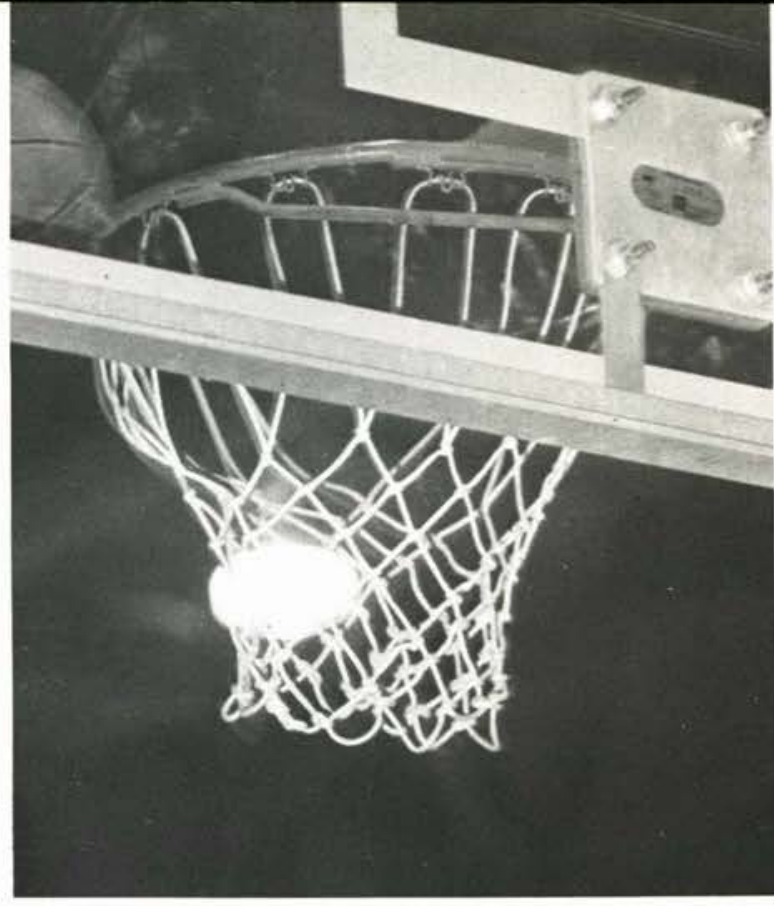

23 in

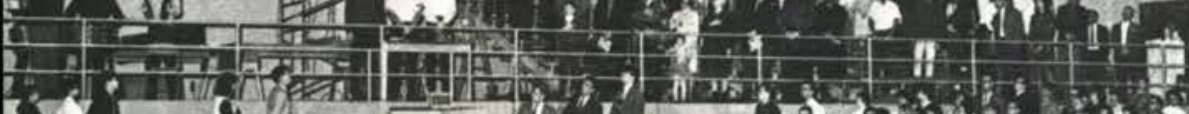

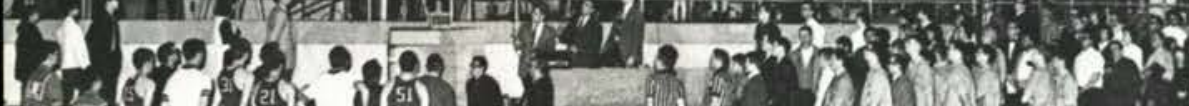
$30407=0.9$ G) Put

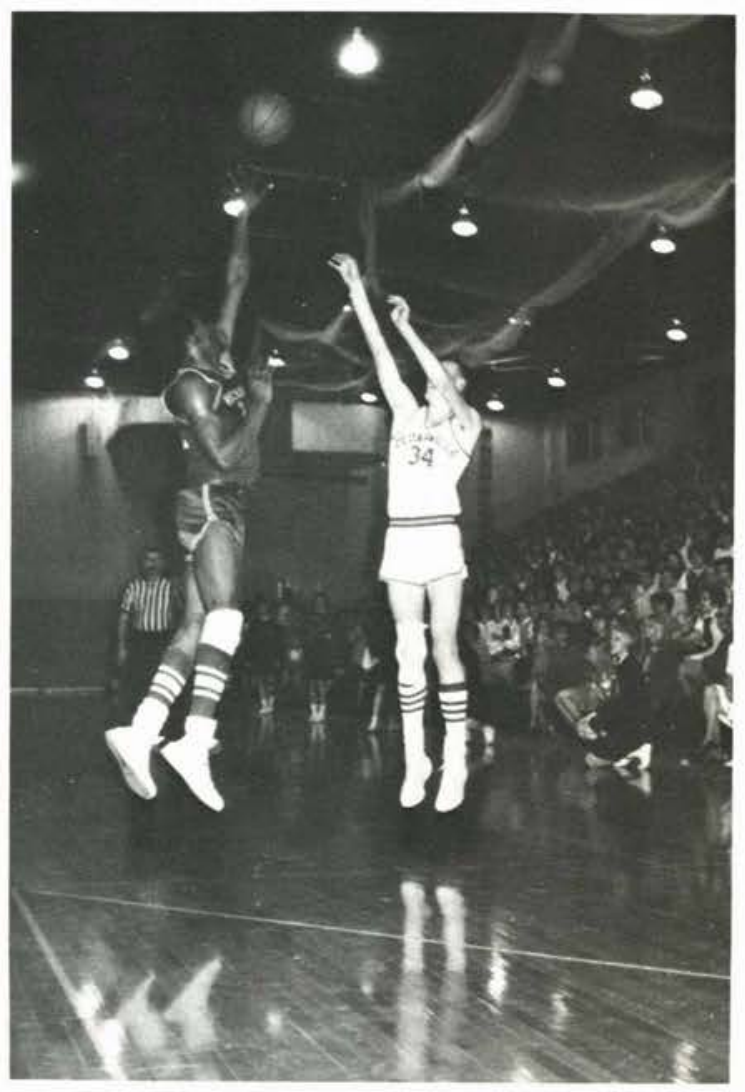

Bruce McDonald

Defiance - Feb. 3.
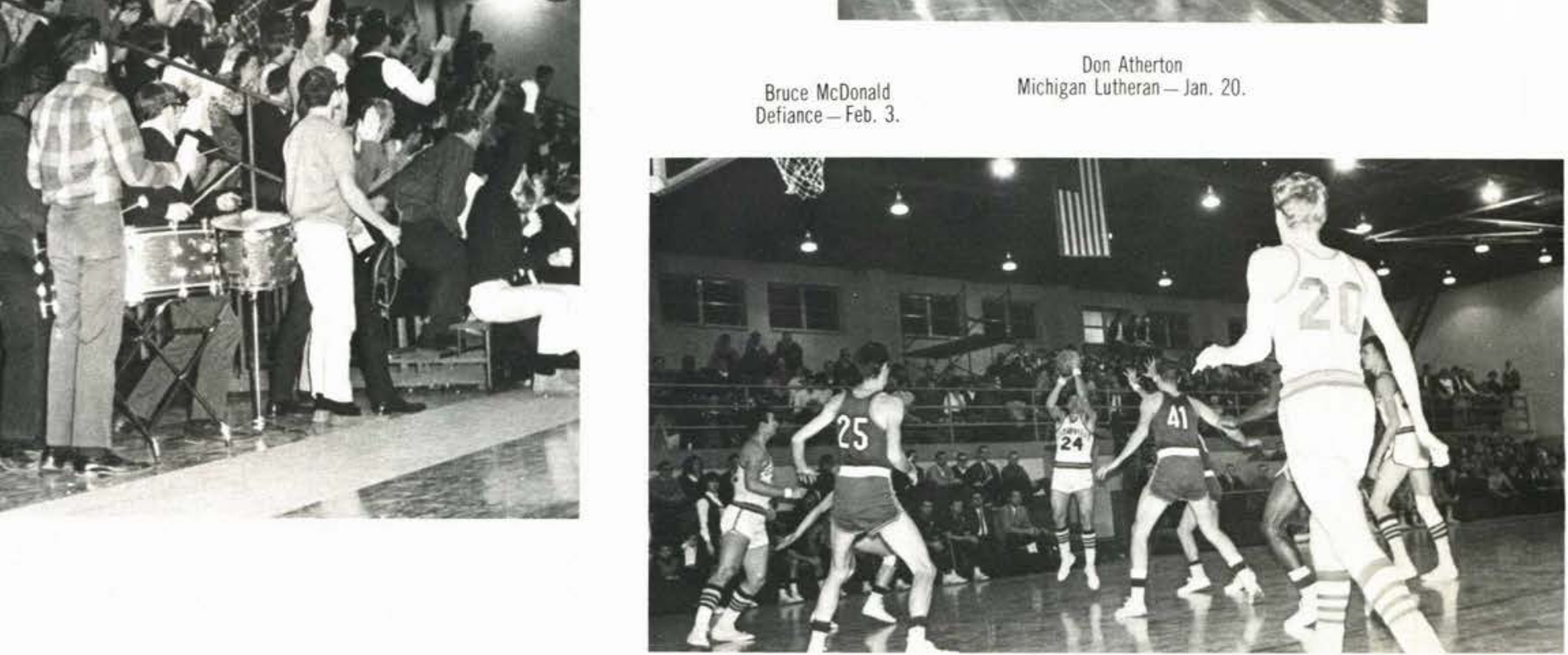


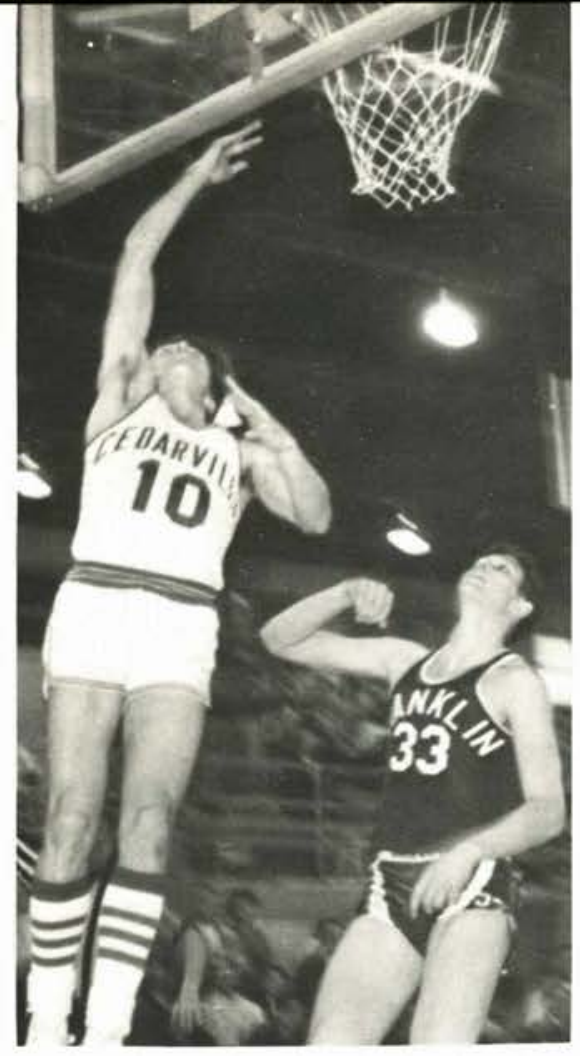

Don Cooper Franklin-Jan. 27.

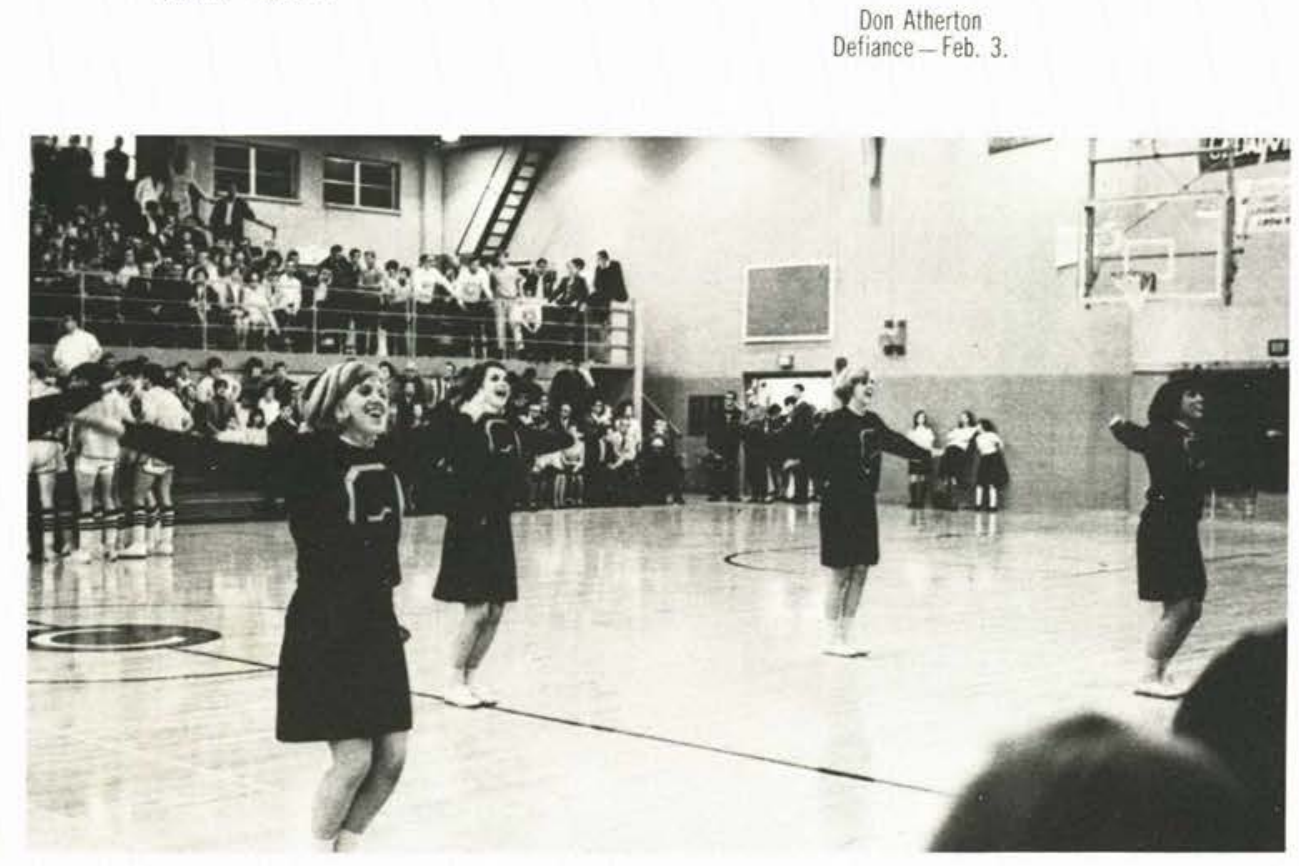

Gary Darrow

Wilmington-Feb. 13.

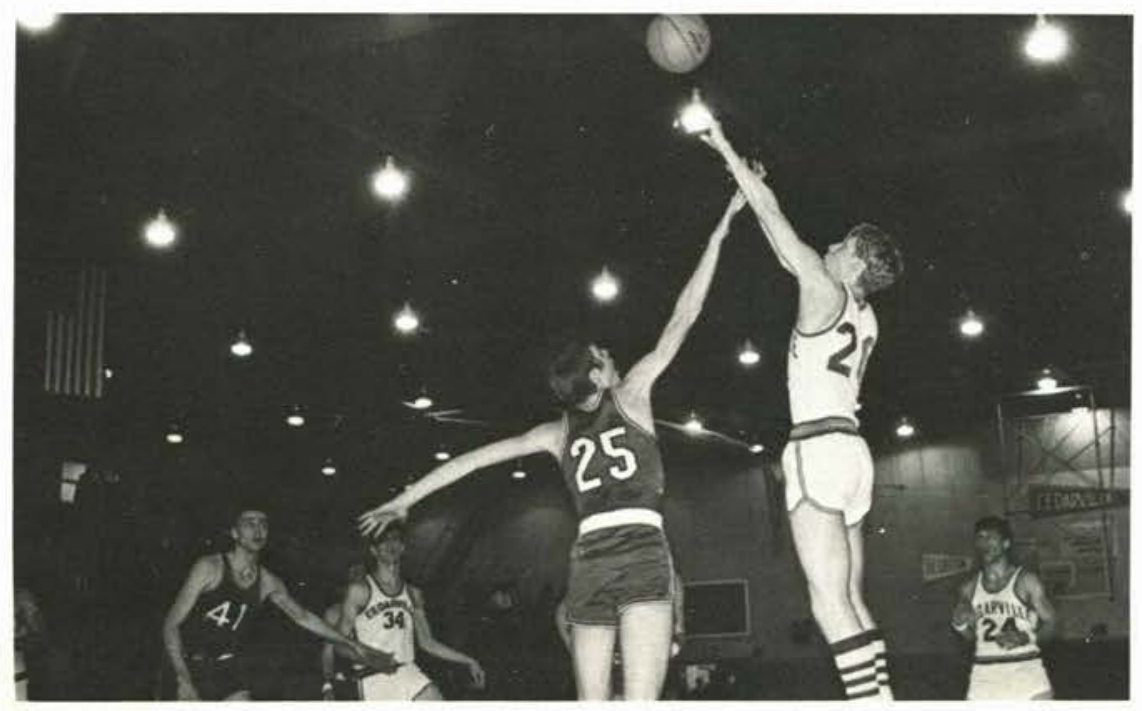

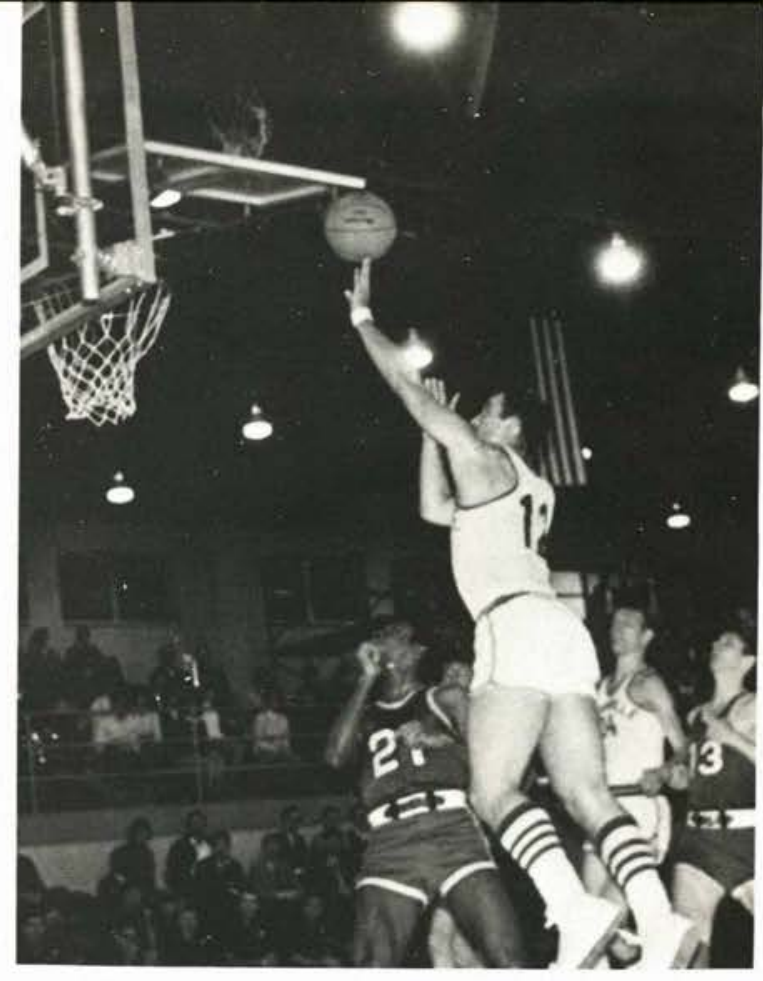

Al Knott
Defiance-Feb. 3

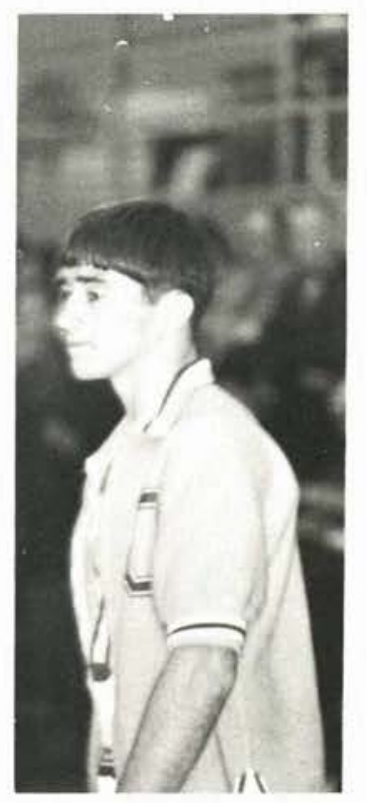

Don Cooper

Defiance-Feb. 3.

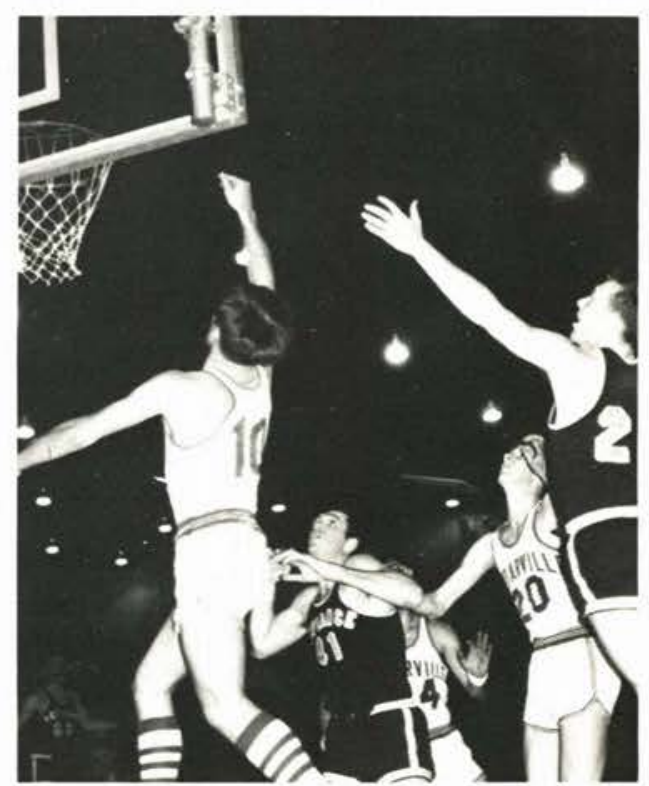



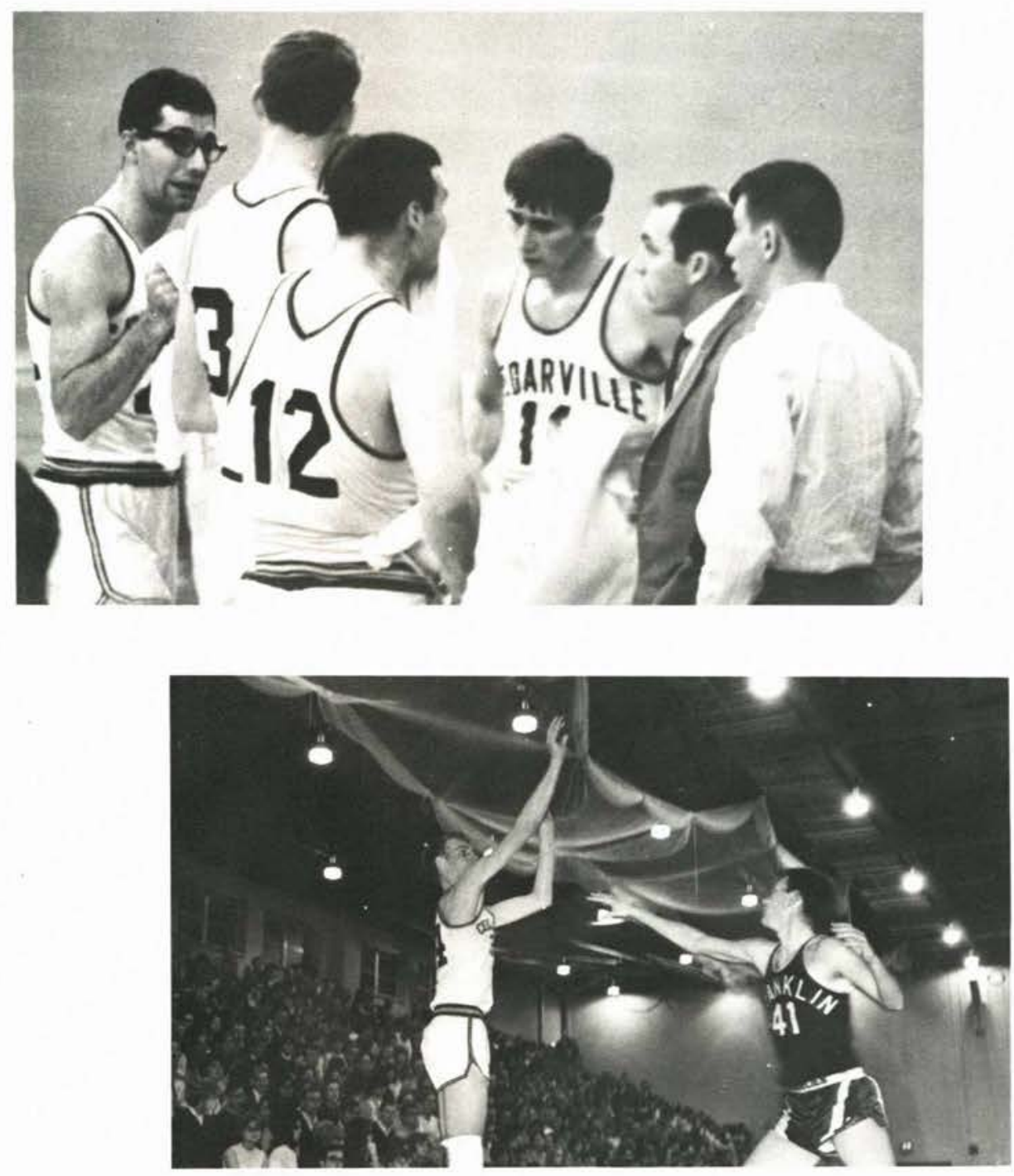

Mich. Lutheran - Jan. 20
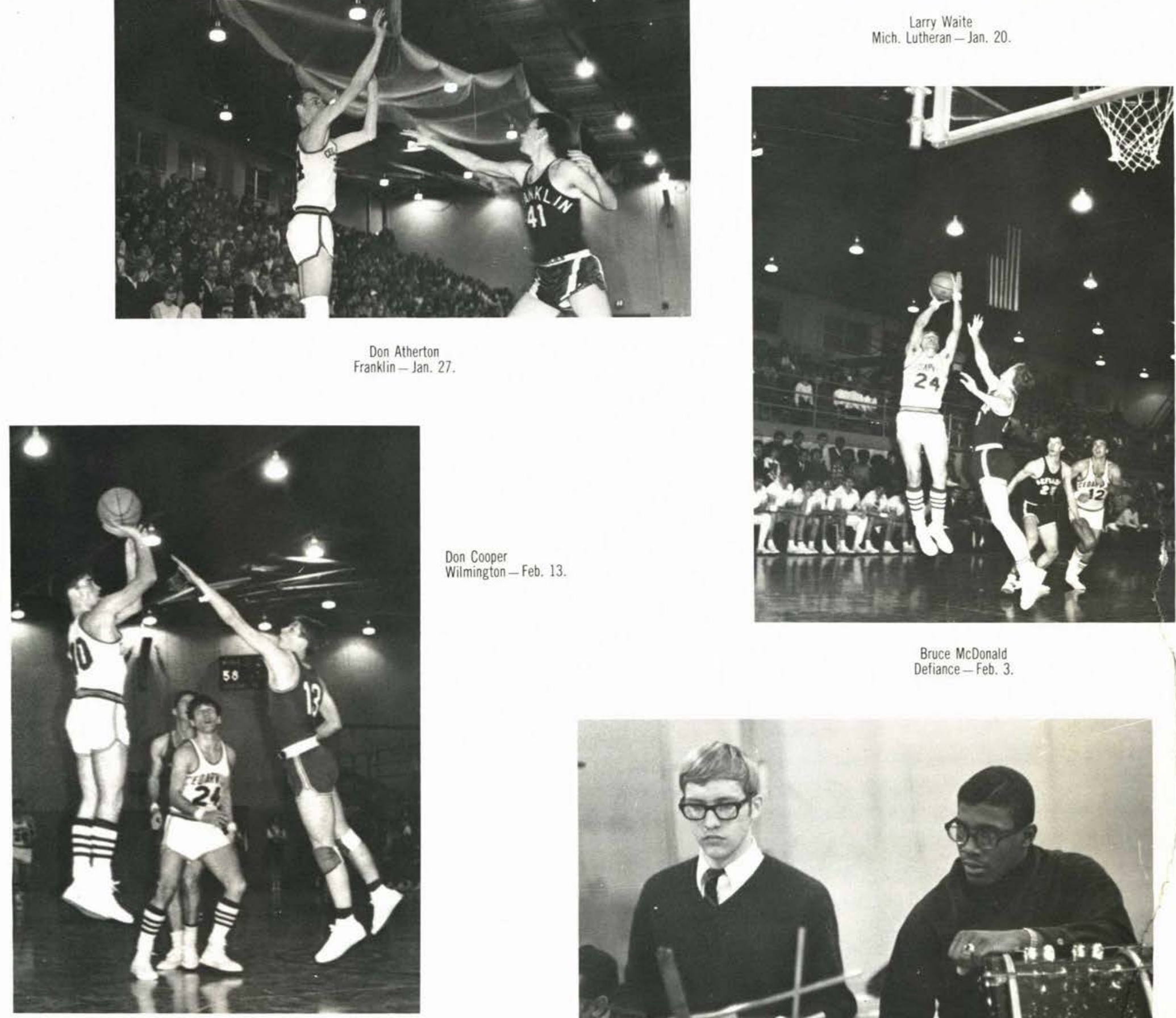

Don Cooper

Wilmington-Feb. 13.

Bruce McDonald Defiance-Feb. 3.

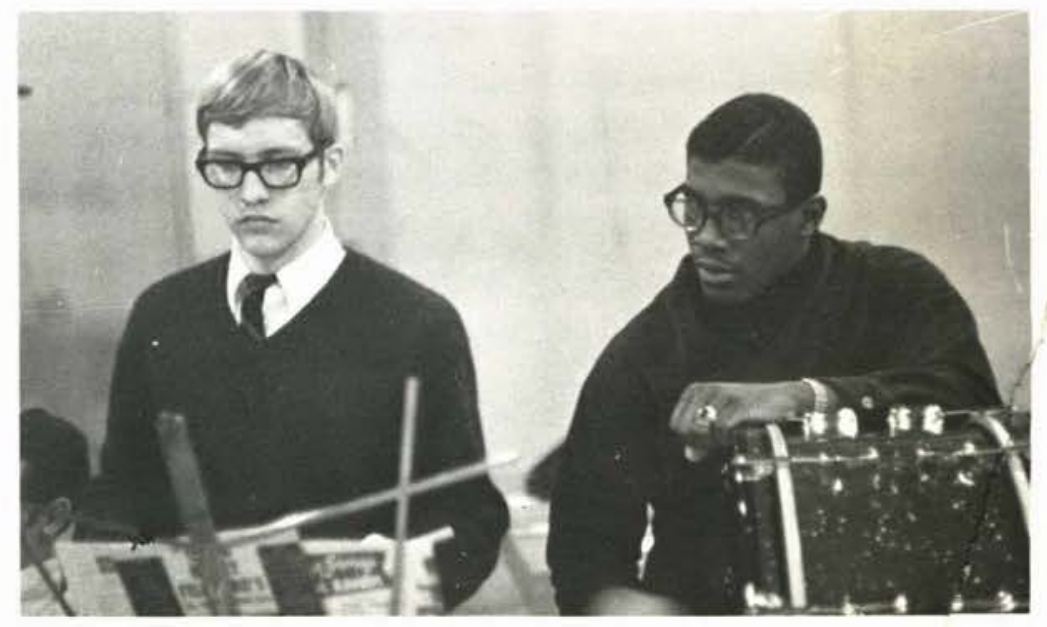


"REMEMBER WHEN" - FEB. 23rd
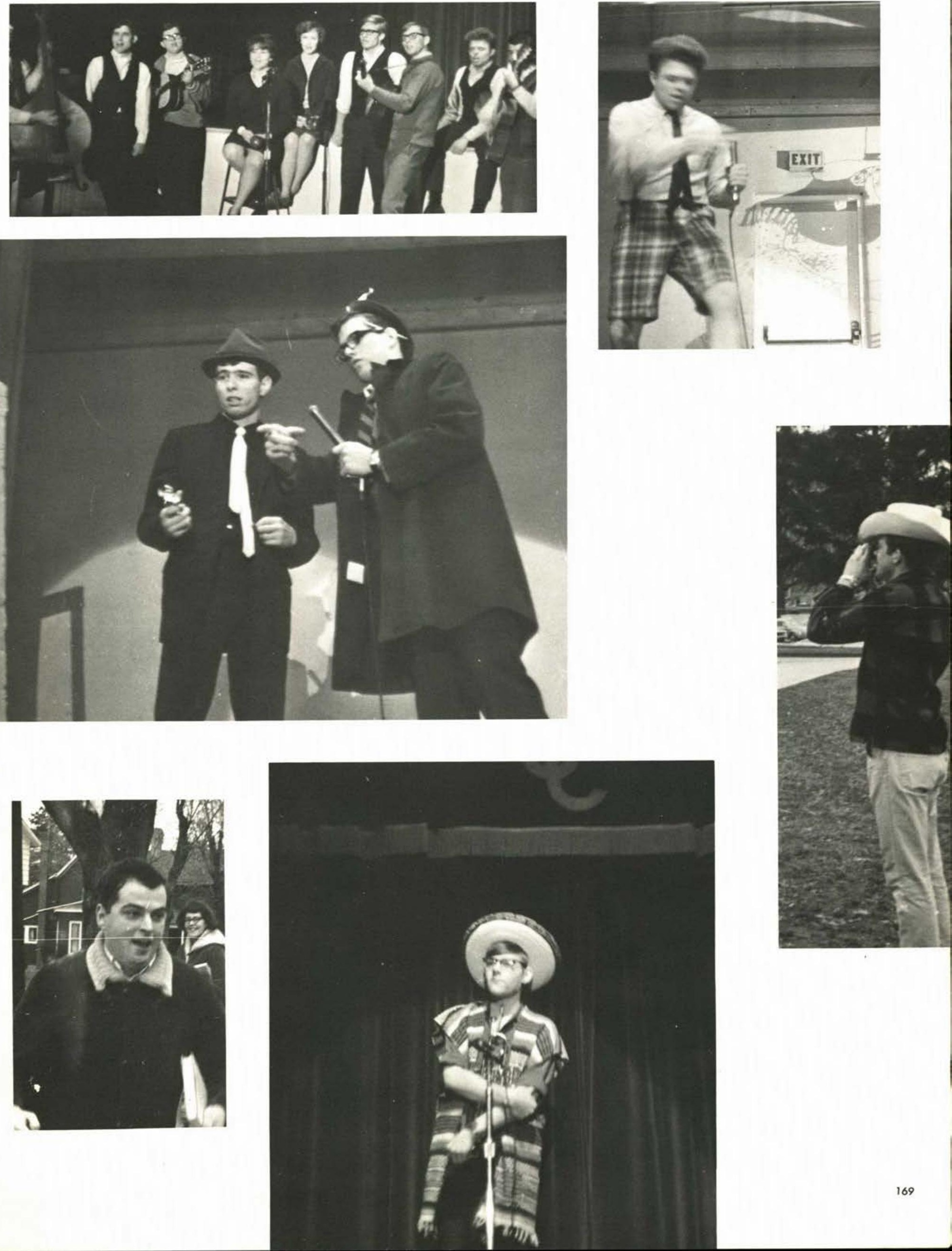
SPRING

\section{QUARTER}
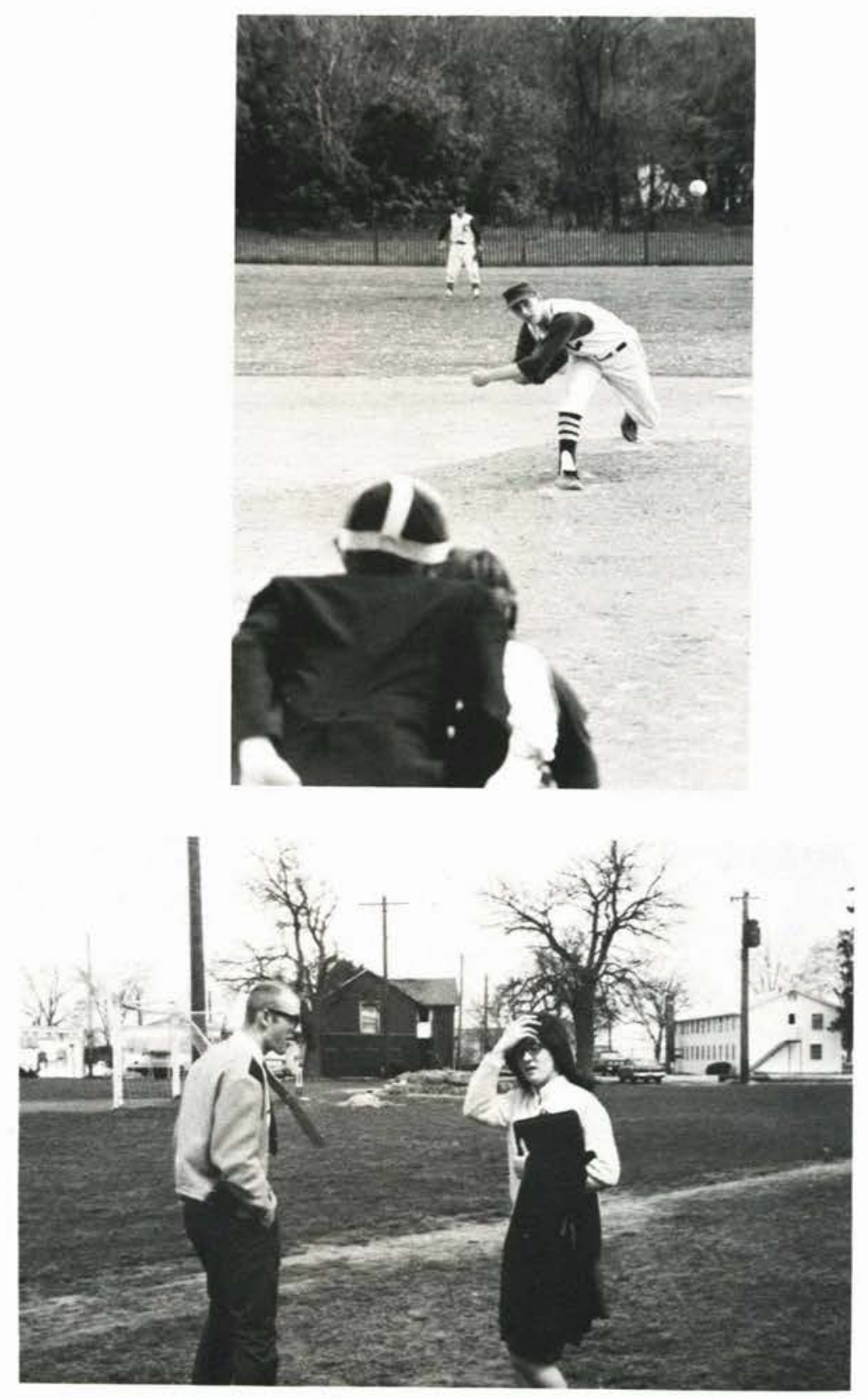

\%

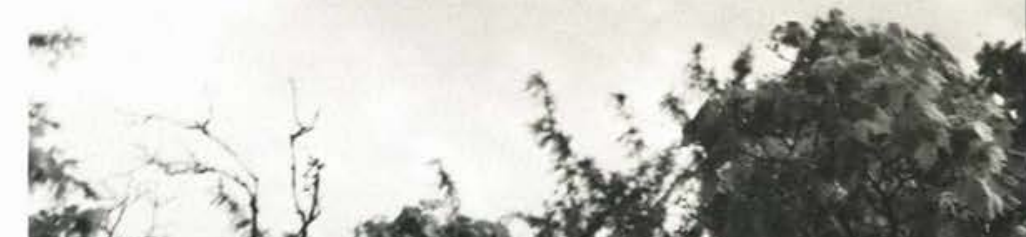

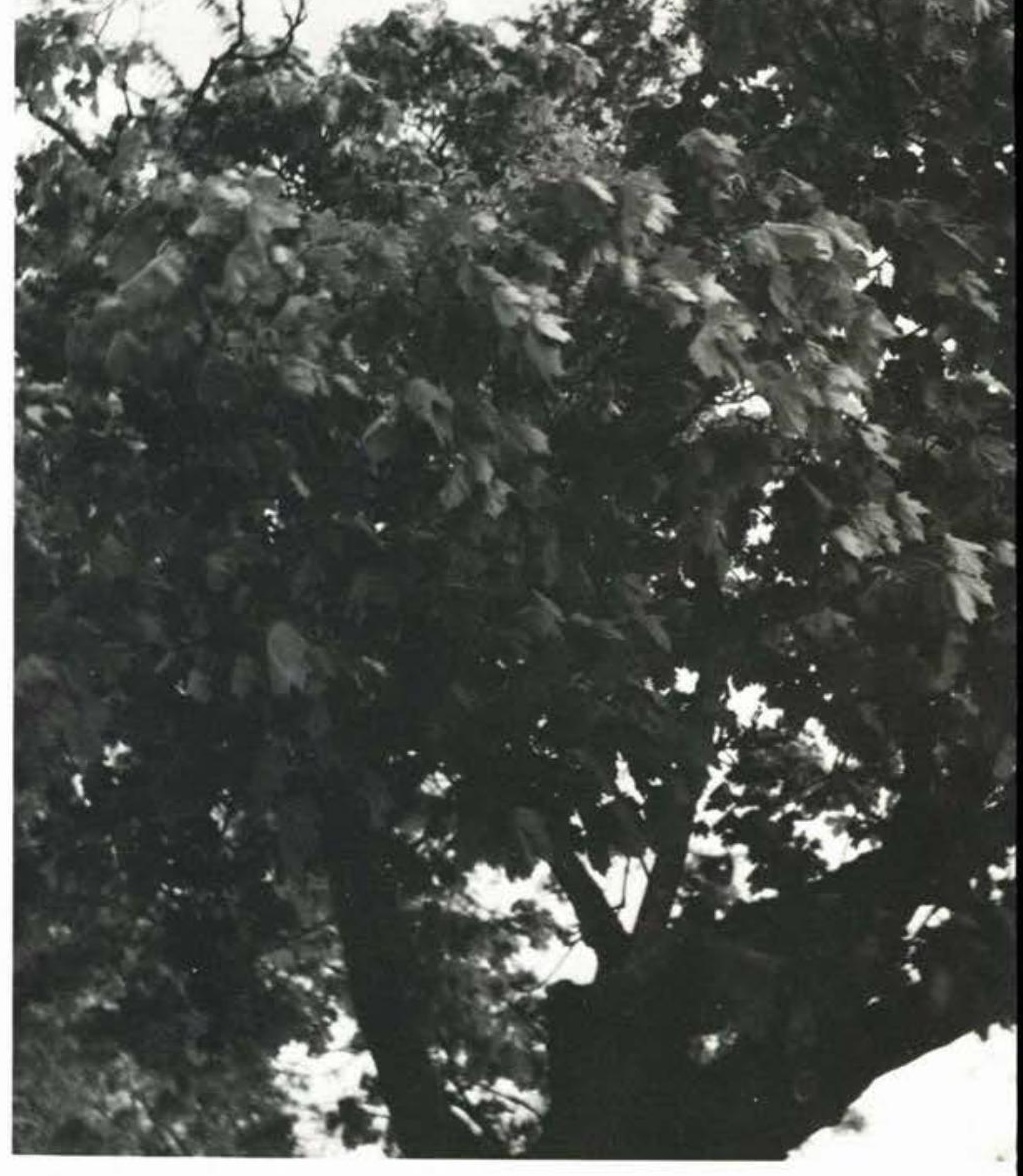

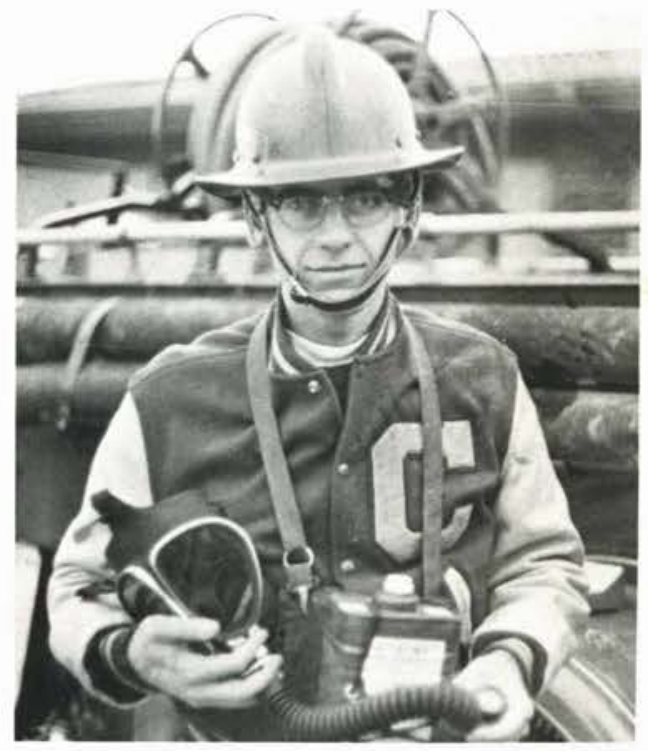

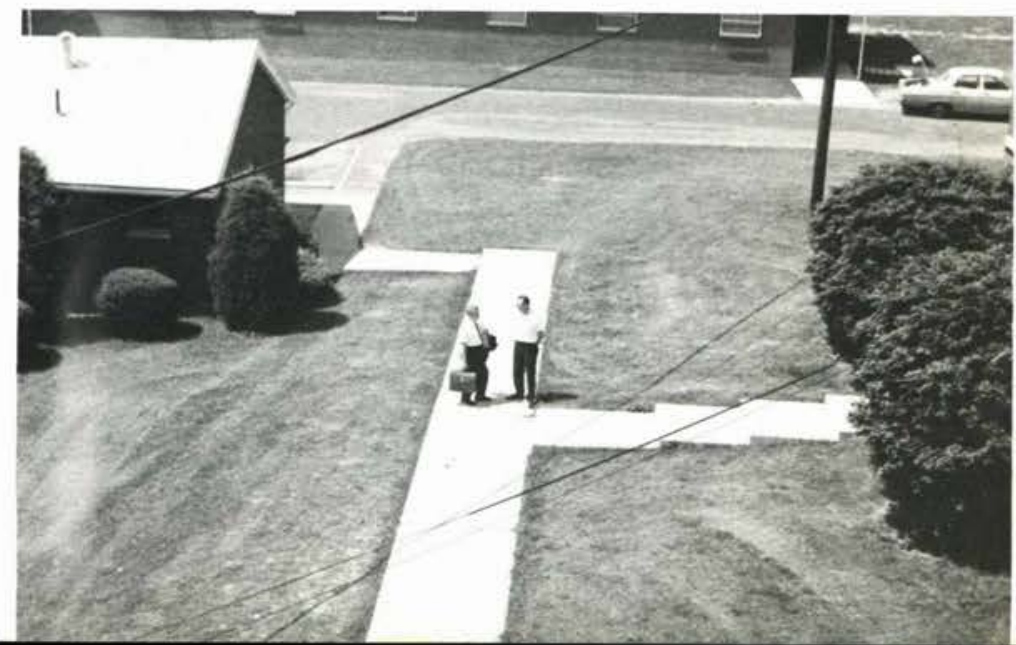




\section{"BEN FRANKLIN" ARTIST LECTURE - MAR. 23}
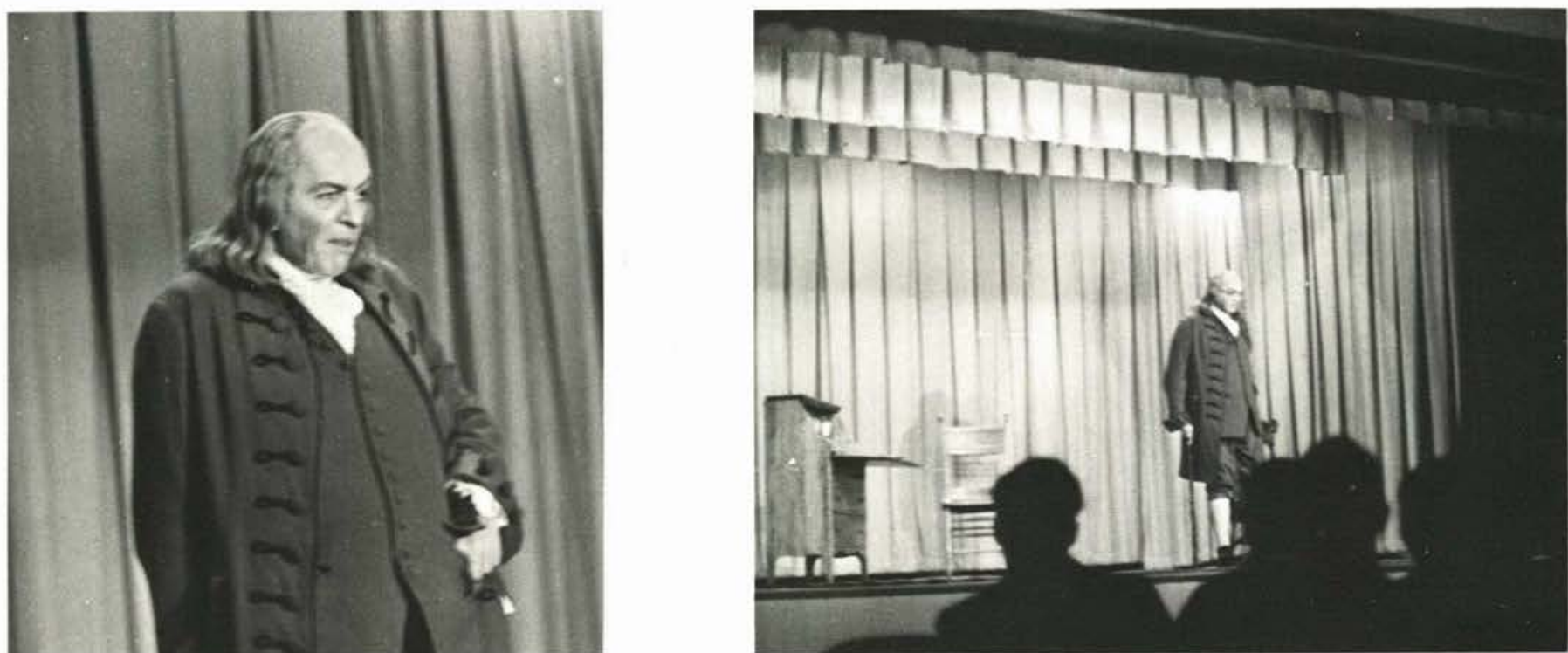

\section{"PENNY MILE" MARCH - MAR. 30}
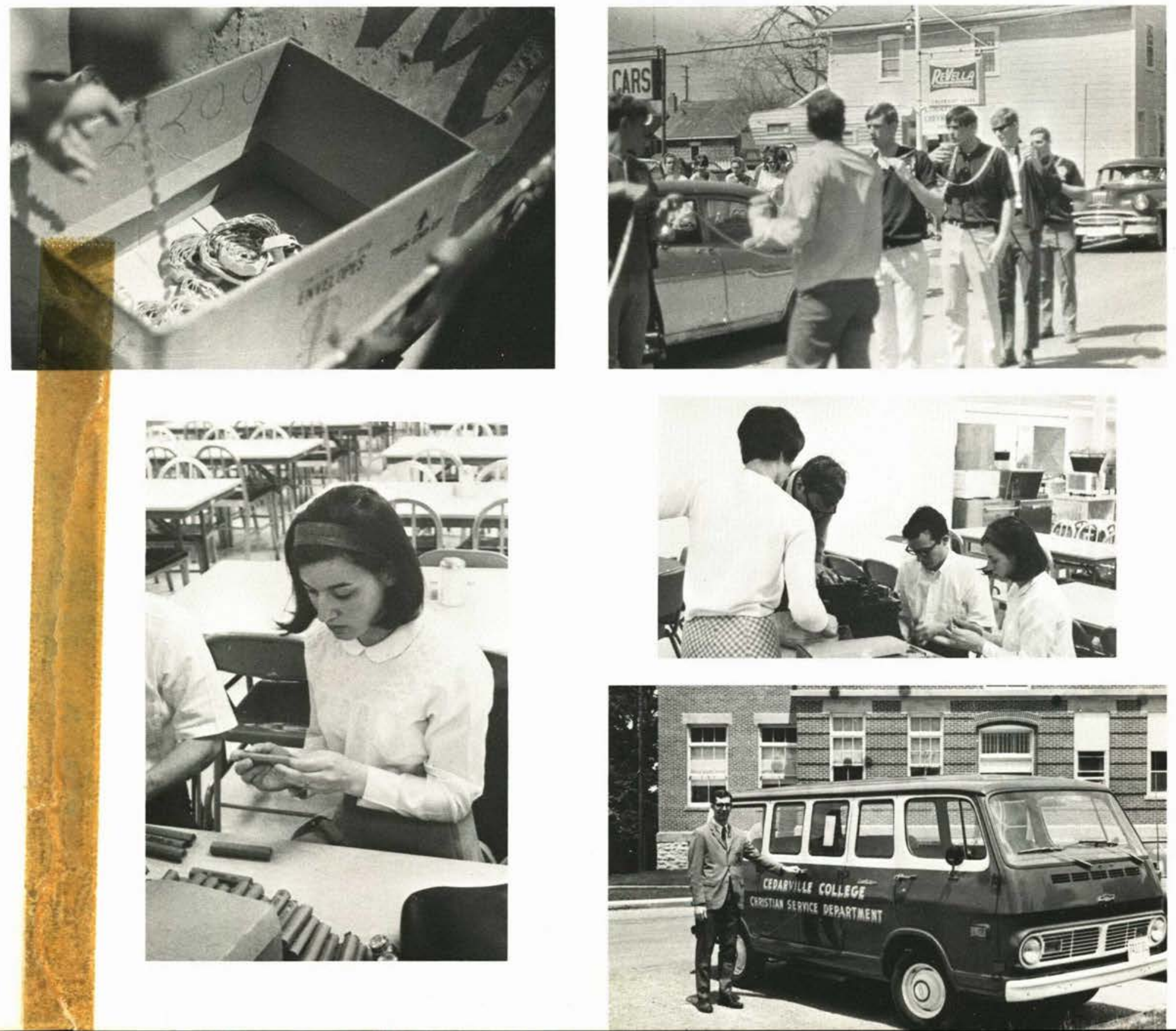
BASEBALL

AT C.C.
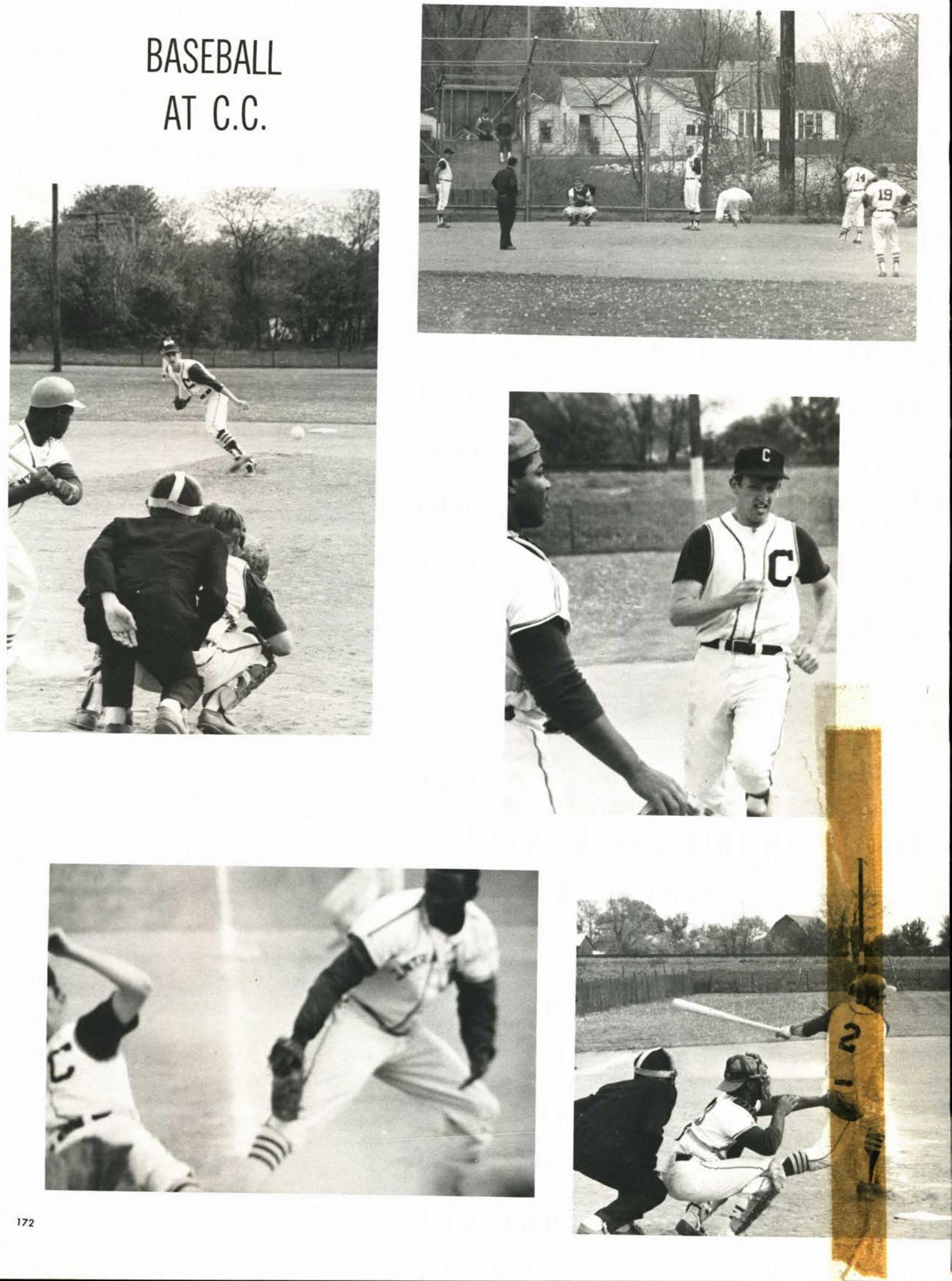

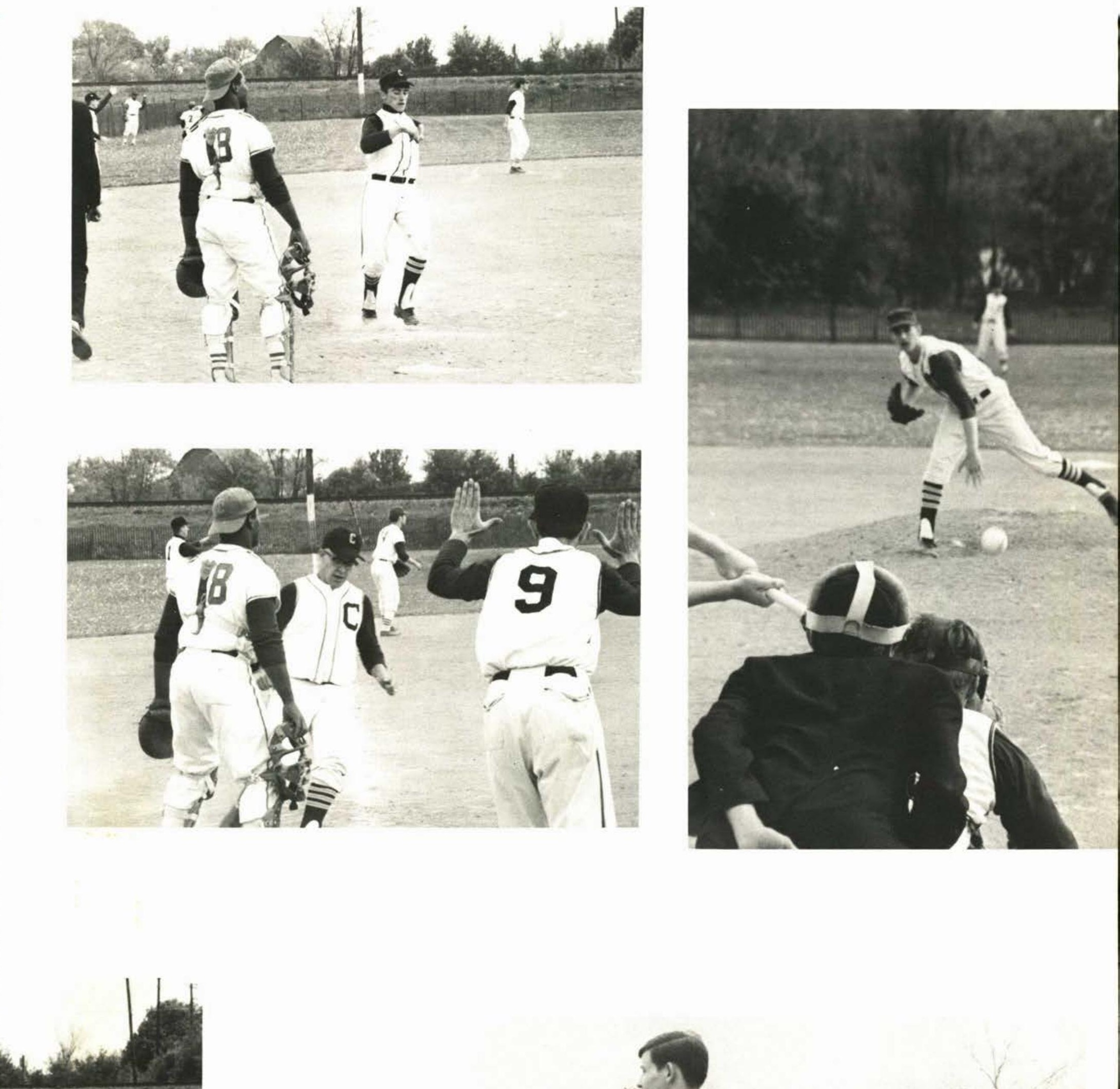

a

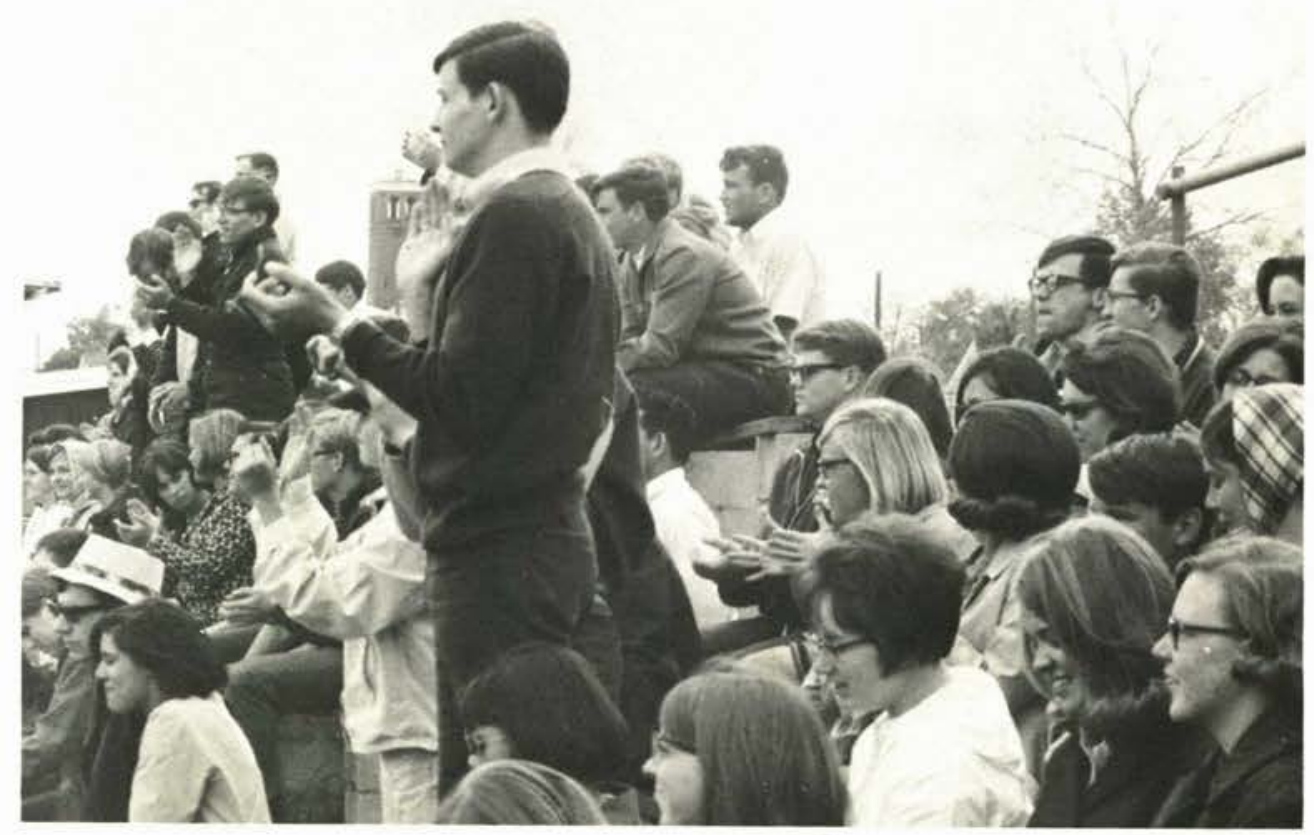




\section{MISSIONARY CONFERENCE - APRIL 1-5}
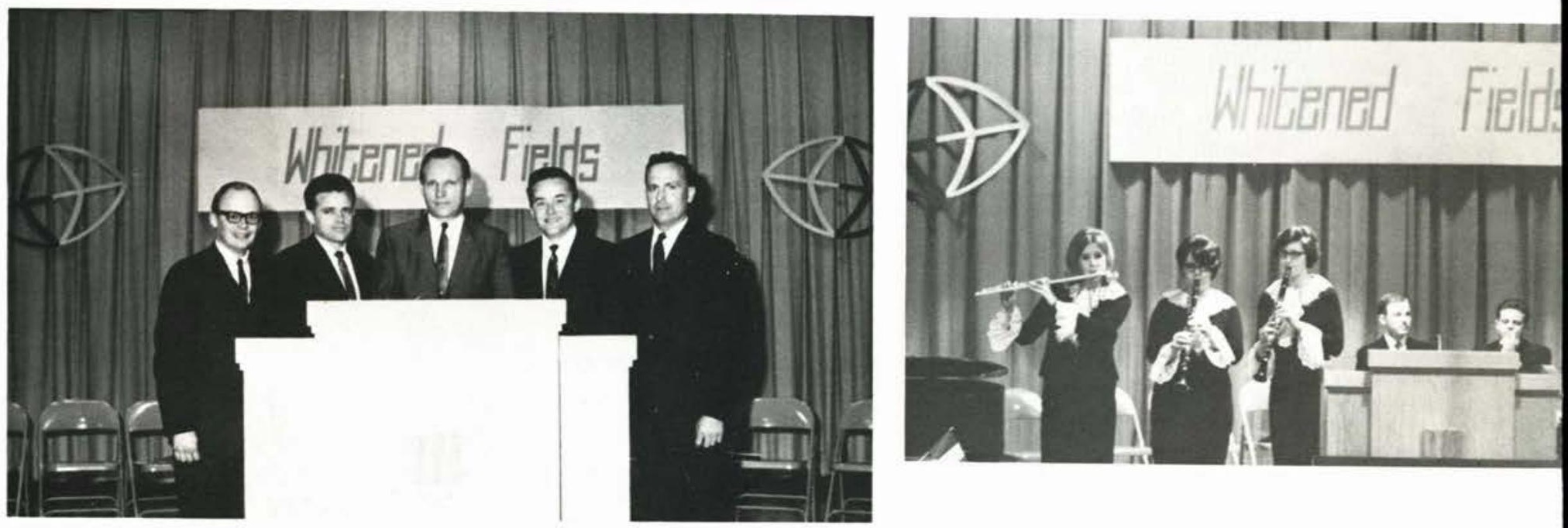

John B. Mitchell, Russell E. Ebersole, Jr., Karl W. Luyben, William Stoner, Donald Curtis.

\section{GAMMA CHI BANQUET}

APR. 6
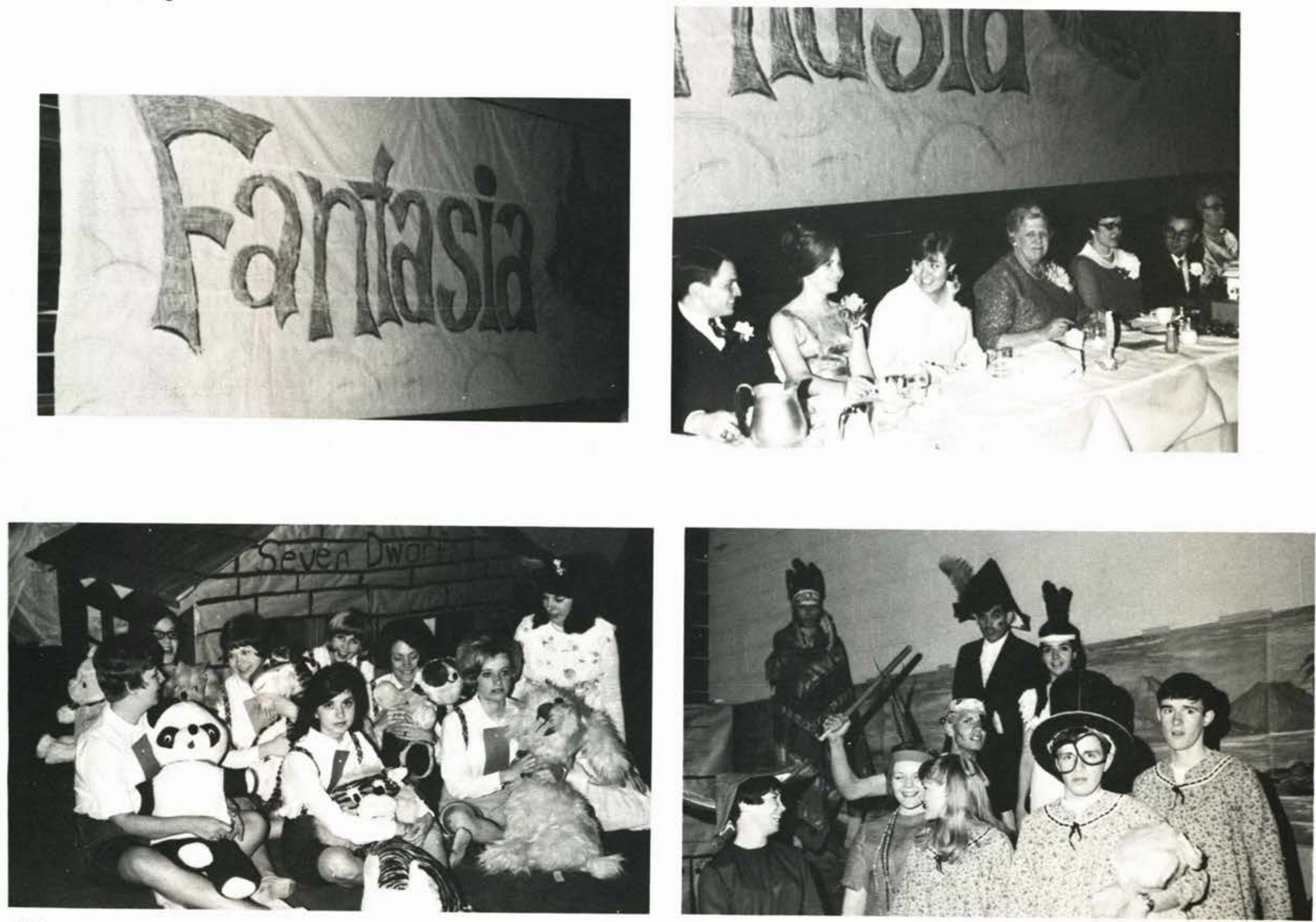


\section{TRAIN WRECK in CEDARVILLE - APR. 6}
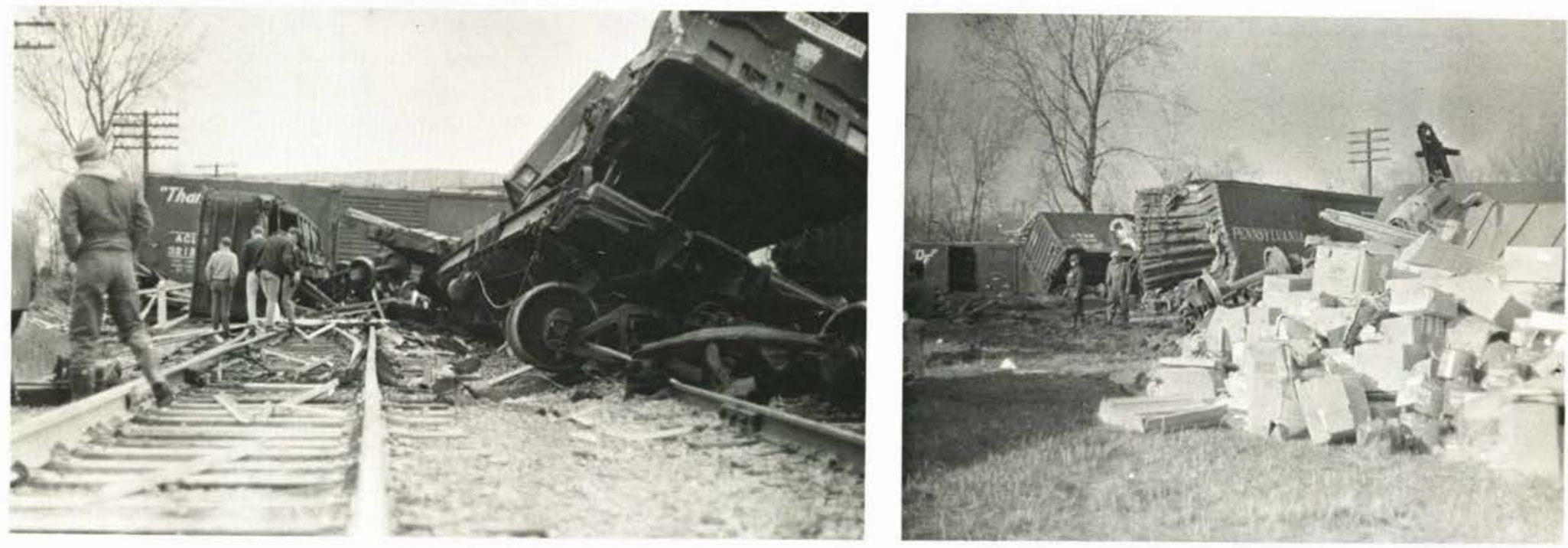

EASTER SUNRISE BREAKFAST

APR. 14
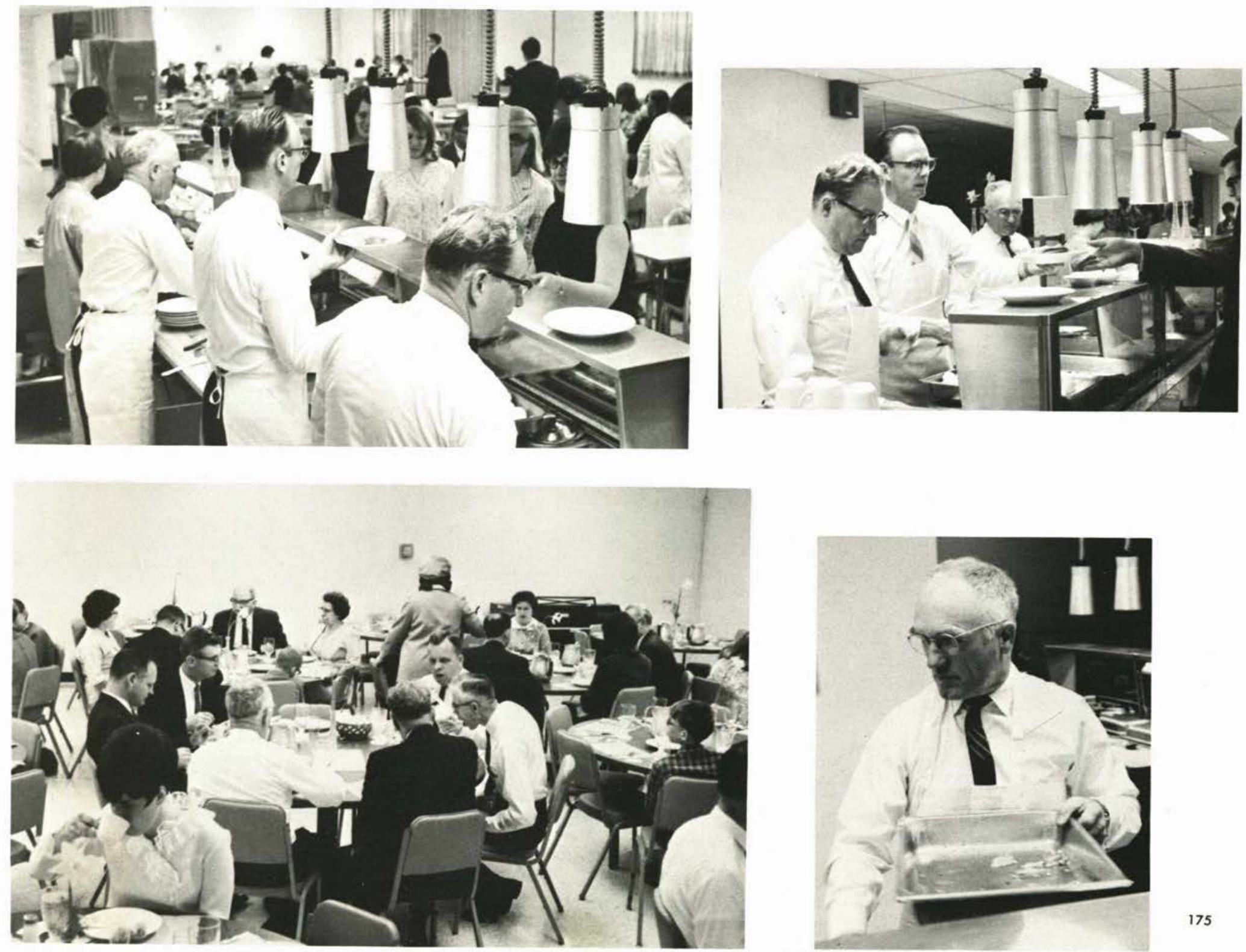


\section{HONORS DAY - MAY 10}
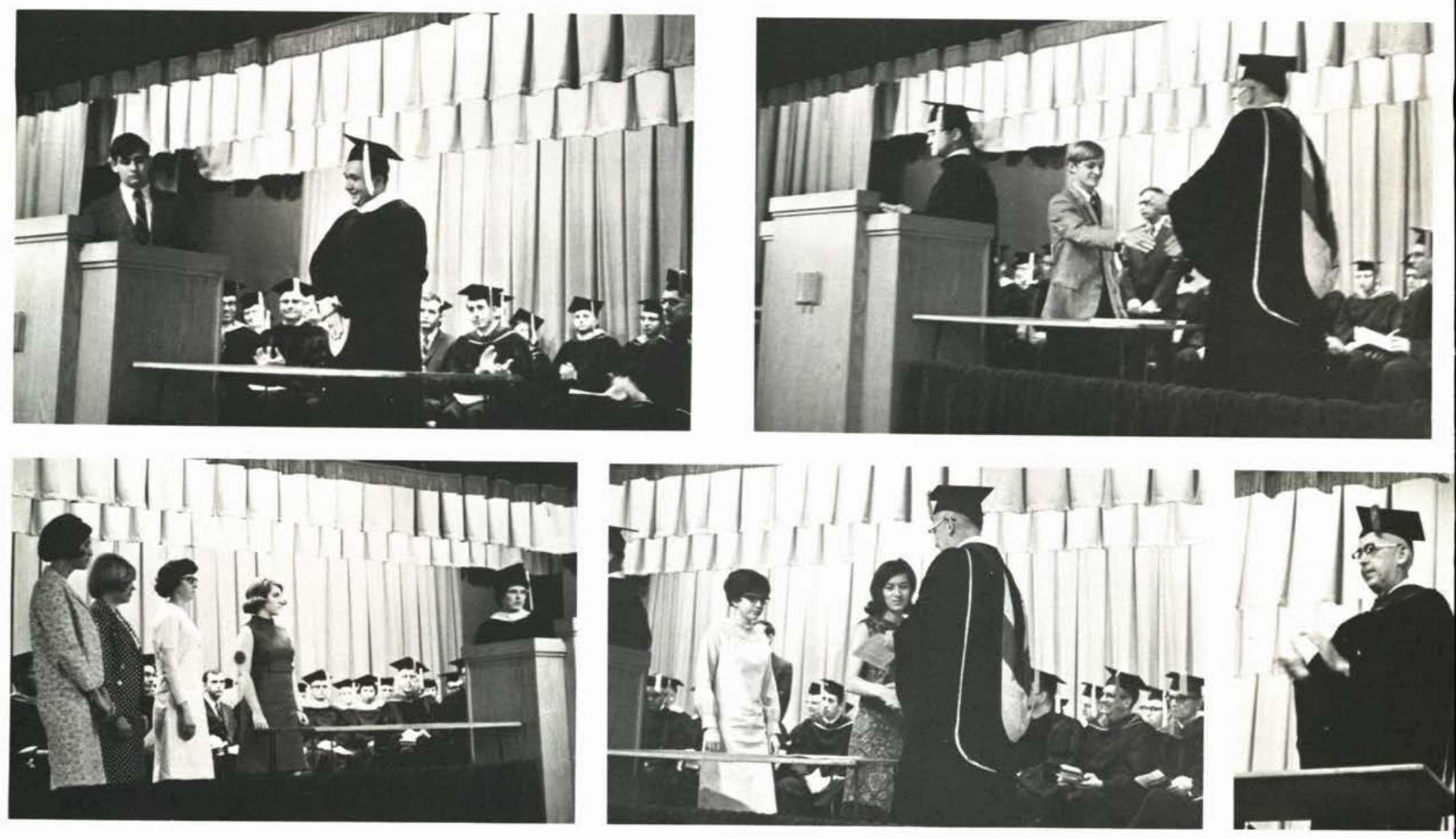

"THE IMPORTANCE OF BEING EARNEST" - MAY 10, 11
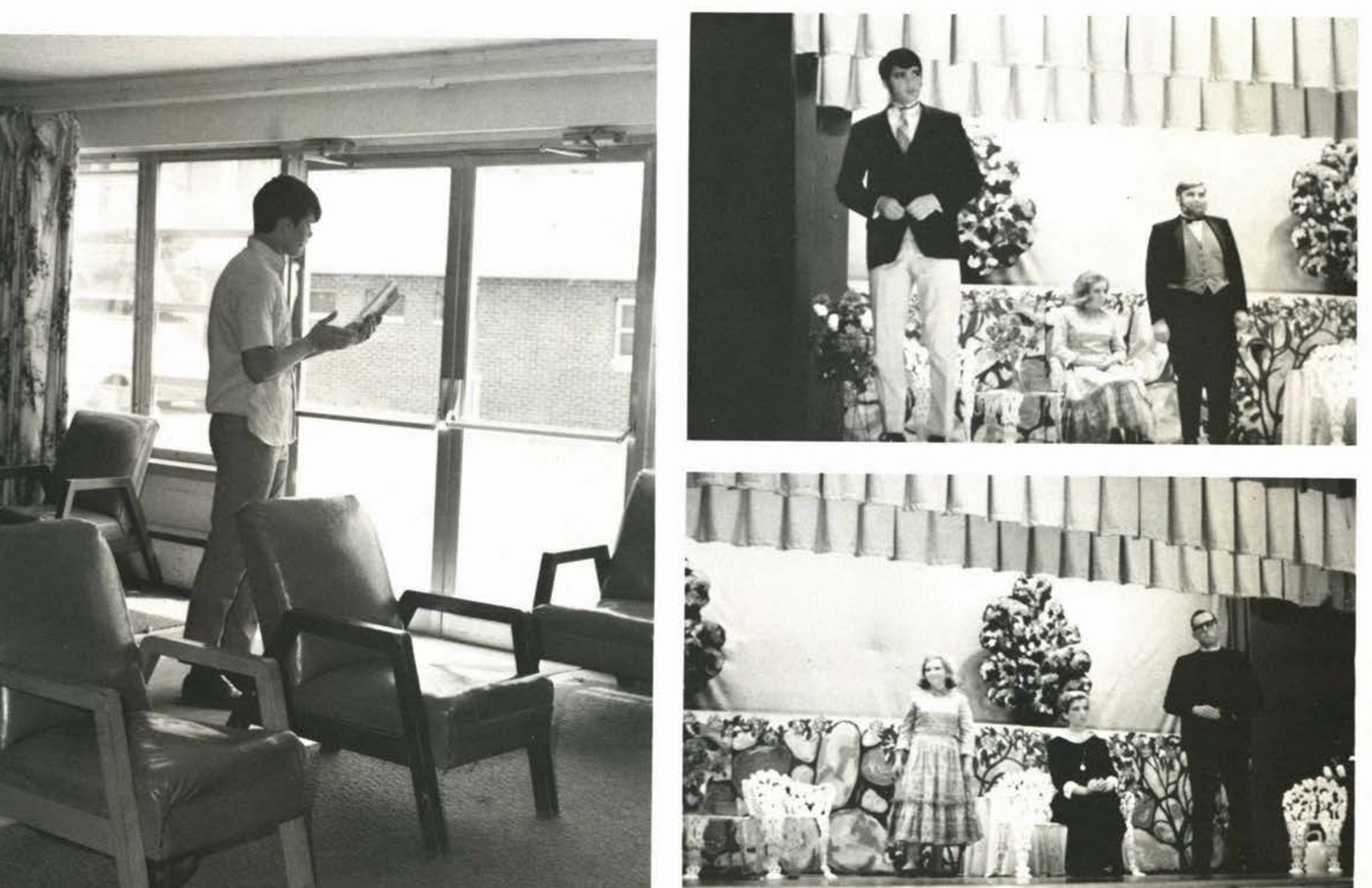

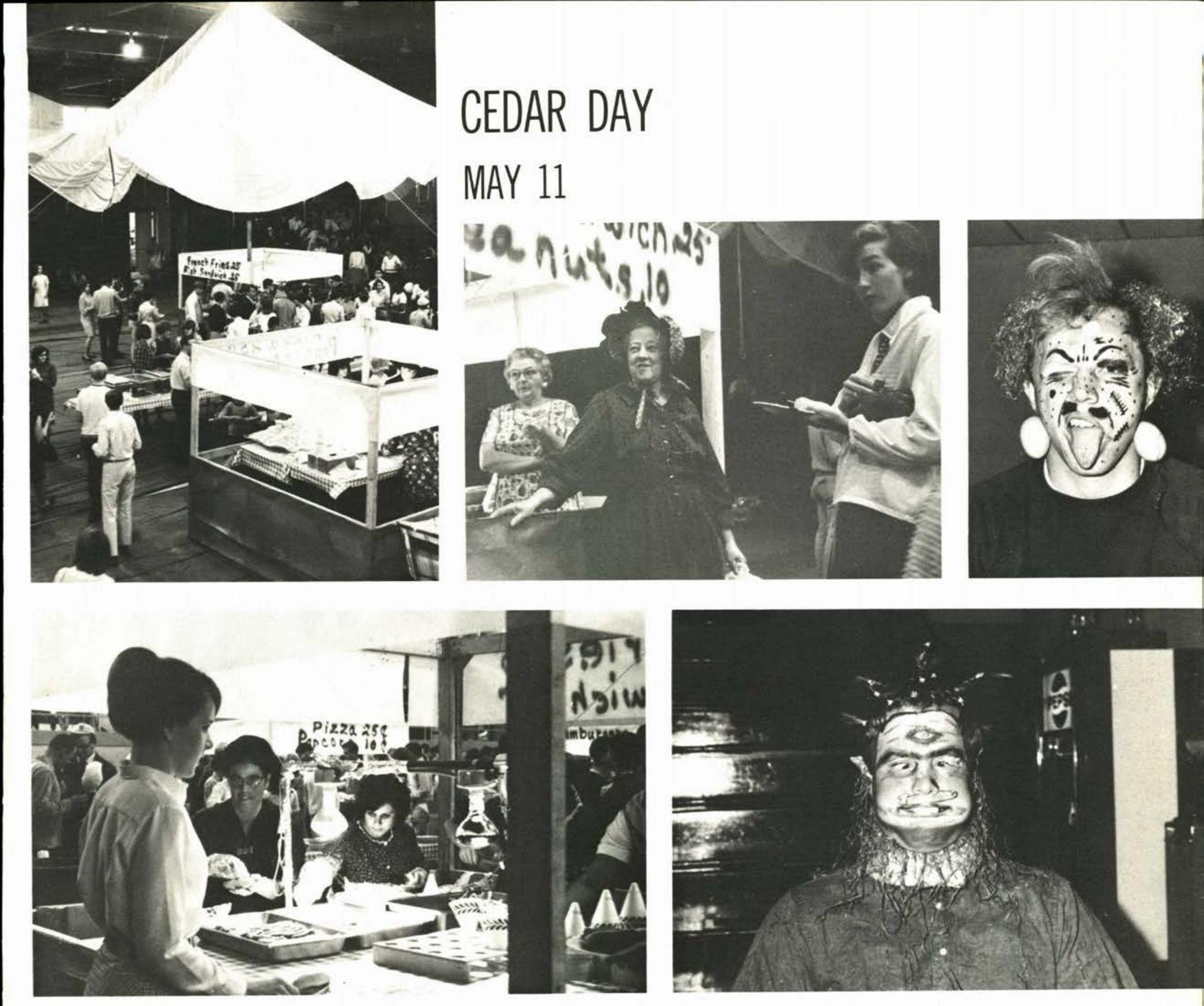

Food Booth Country Fair Style.

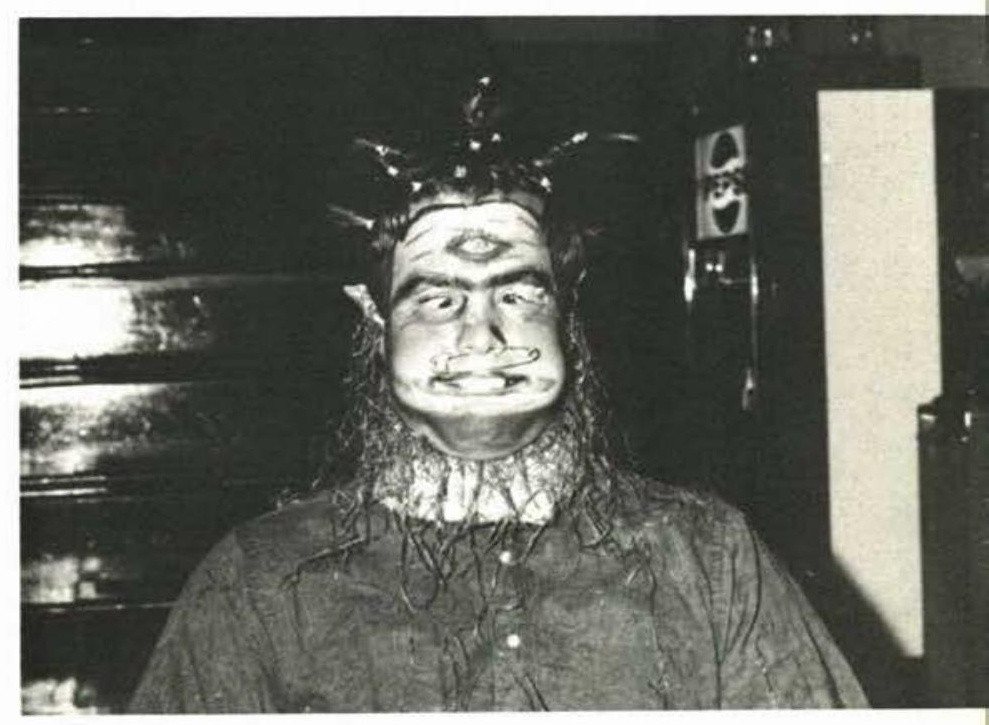

Ugly Man Contest Winner - Dennis Bunting.

\section{JUNIOR-SENIOR BANQUET - MAY 17}
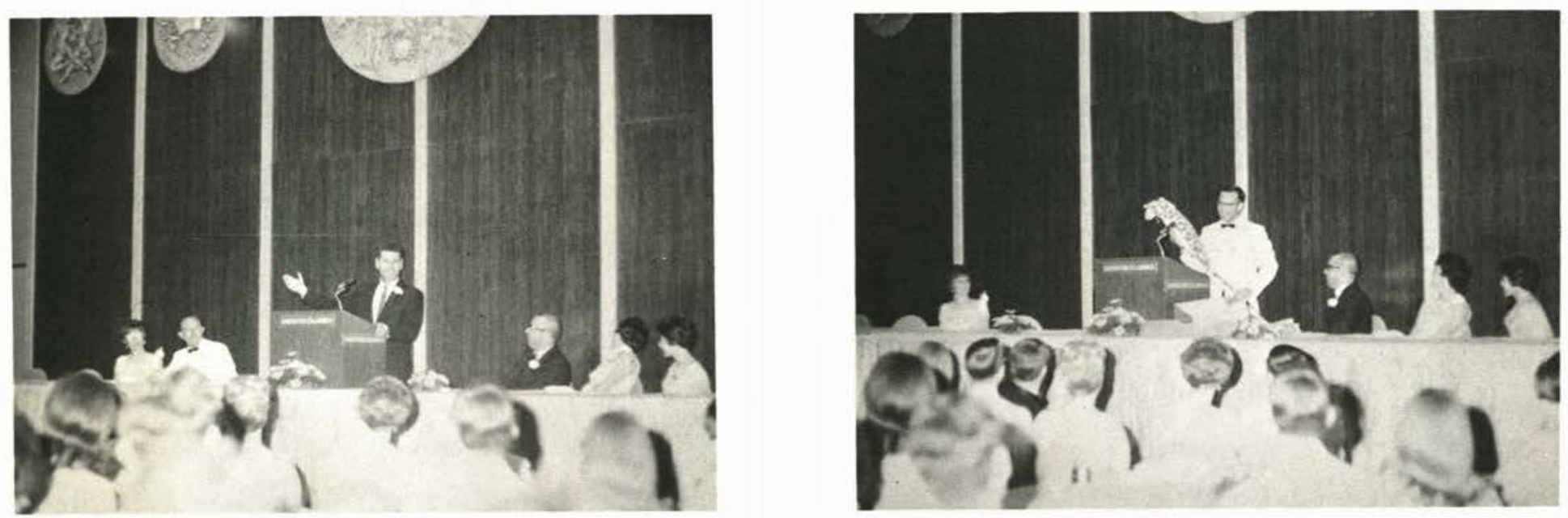

Special Speaker Dr. Hugh Horner.

Gift Presentation to Mr. Riter from the Senior Class. 


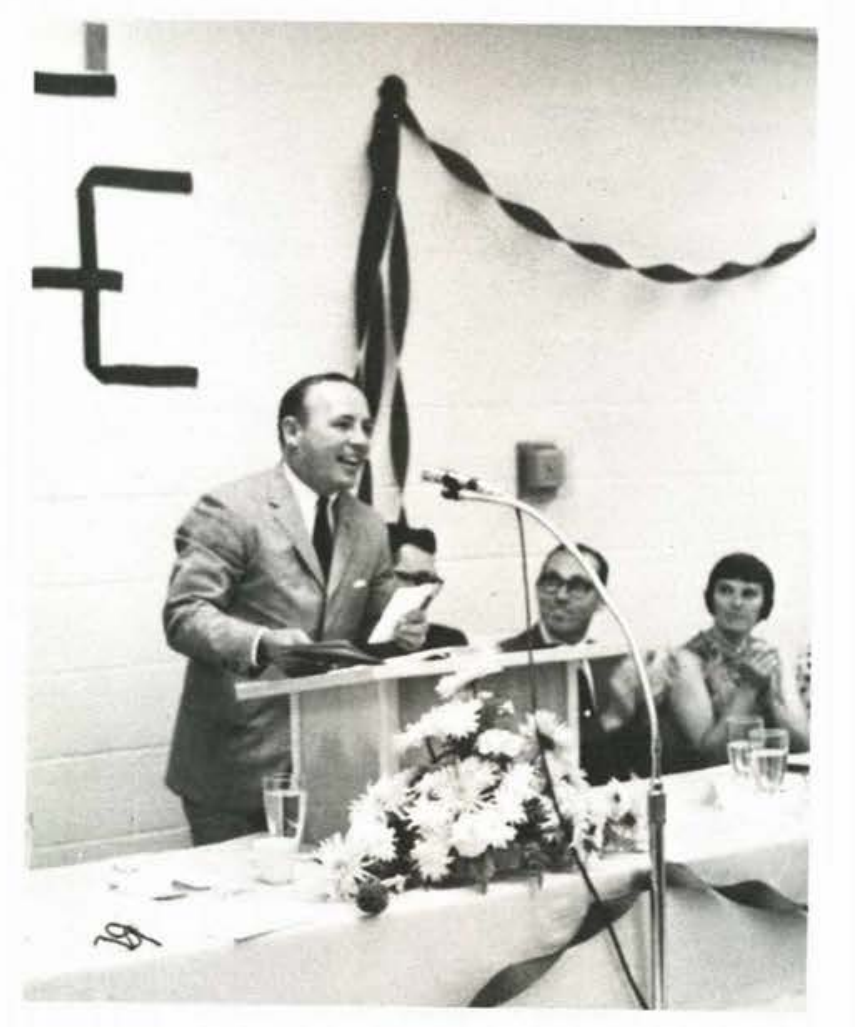

\section{ATHLETIC BANQUUET}

MAY 27
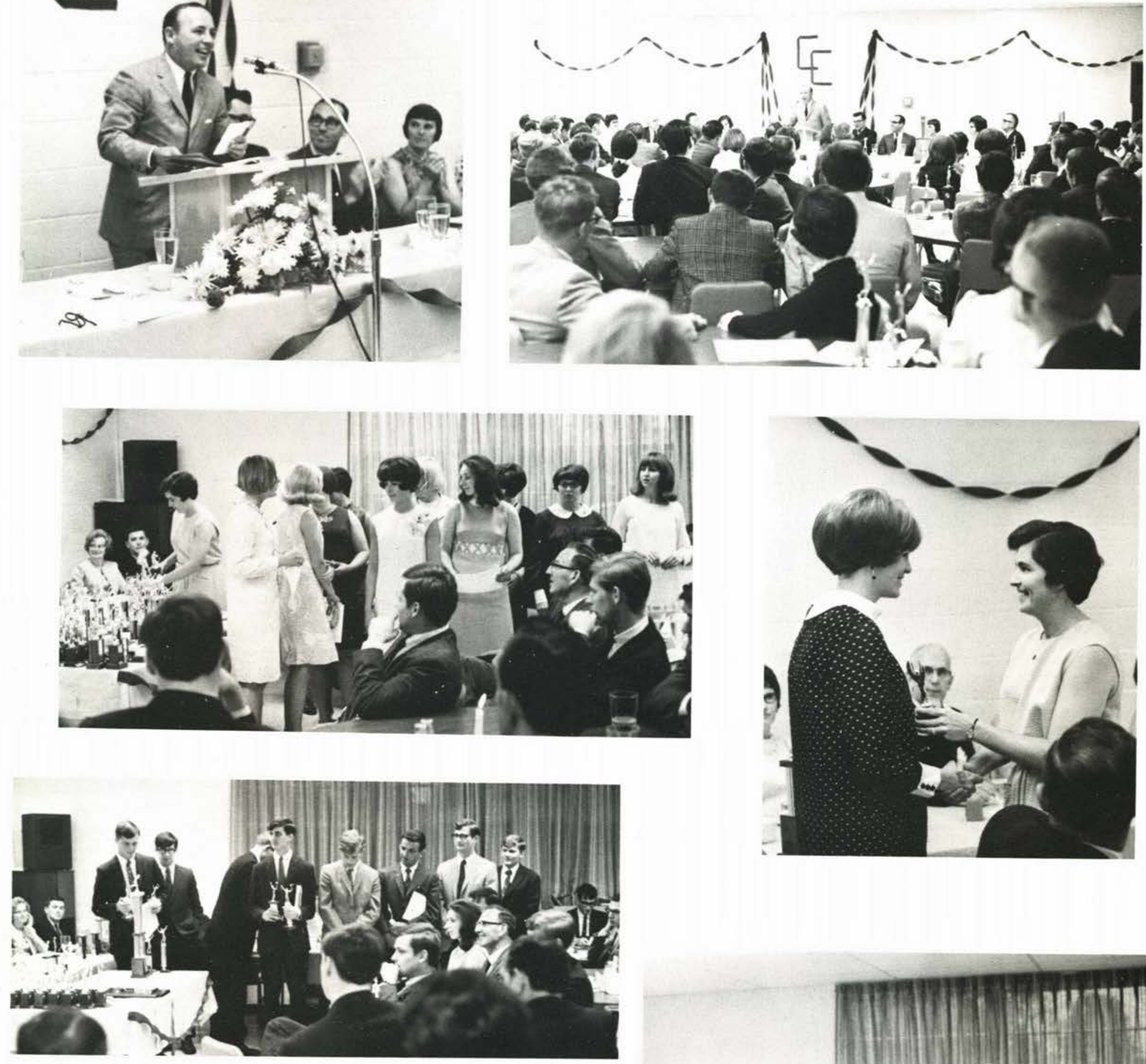

1

8

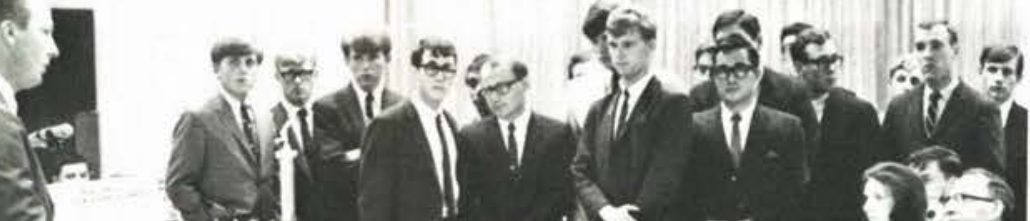

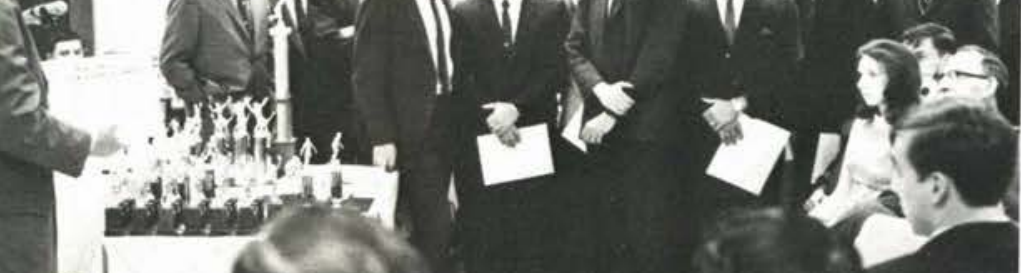

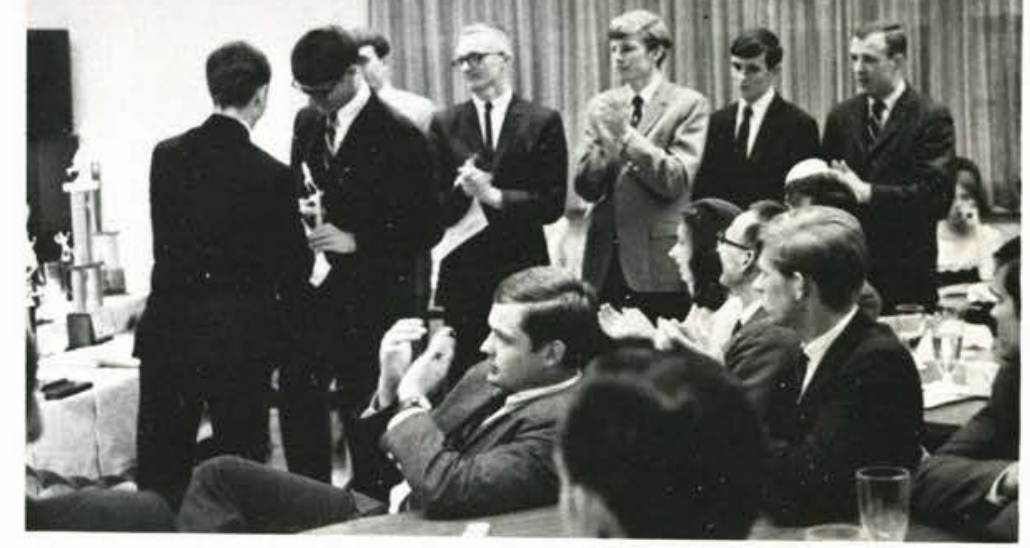




\section{In Memoriam}

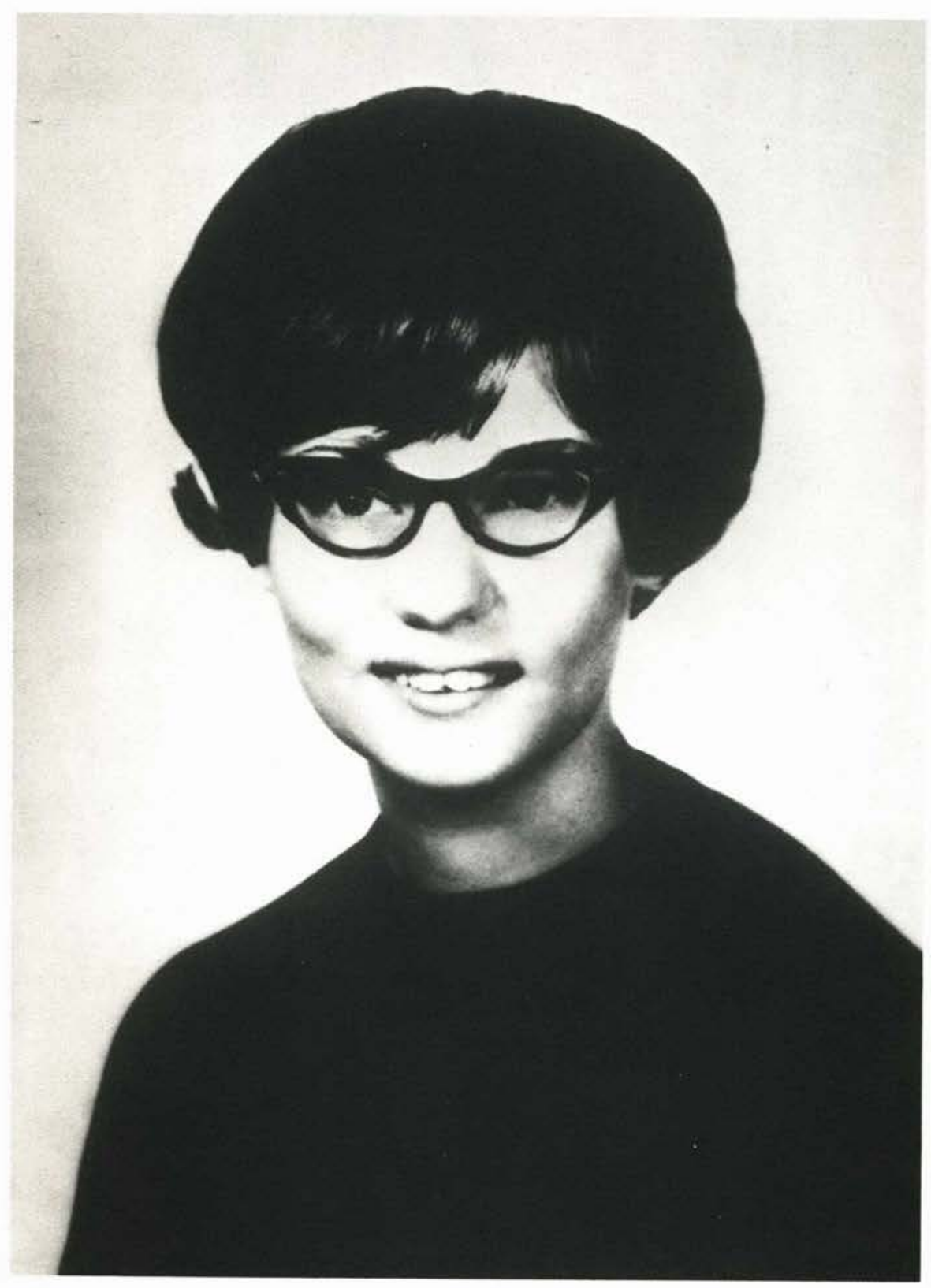

Martie Brown, 1946-1967

Death is strange at times. Martie and I were both acquainted with its results. Often were the times we talked about what heaven was like. Although we were very close on earth it is an added joy to know we will meet again in Heaven. I may sorrow here because we are separated, but really we still share many things. She knows my joys; I just miss telling her. She knows my sorrows; I miss her sympathy. People thought Martie quiet. She was in her own way, but not really. In the quietness of her death, she has spoken to many hearts. A wedding dress, satin shoes, a promise and a secret remain to remind us that the door of Heaven may soon open to us. 
Across the hills from yesterday

And just beyond today,

Tomorrow waits so patiently

To greet you on your way;

Elusive though it seems to be

Beyond the setting sun,

Although today is in the past

Tomorrow cannot come.

A treasured thought is in our minds

Of yesterdays gone by,

And sweet remembrance fills our hearts

To light a weary eye;

Today, a treasure to behold,

A shining glowing dream,

Tomorrow, ever waiting there,

Beyond our reach would seem.

Tomorrow, what a precious word!

A promise rich and real,

A chance to realize a hope,

A glowing dream ideal;

Another dawn that's yet to be

A day that's ever fair,

Beyond the realm of yesterday ...

A new tomorrow there.

Garnett Ann Schultz 


\section{STAFF}

LAY-OUT

Diane Thompson

Becky Rudig

Pat Richardson

JoAnn Waltz

Carol Stewart

Viann Meyer

Alberta Plate

Karolyn Wagner

Tina Lautt
COPY

Ann Kusumoto

Carol Stewart

Viann Meyer

Pat Richardson

Becky Harmon
PHOTOGRAPHY

TYPISTS

Bill Reed

Art Shuter
Barbara Jordan

Becky McDonald

Olivia Anderson

Sheryl Zerbe
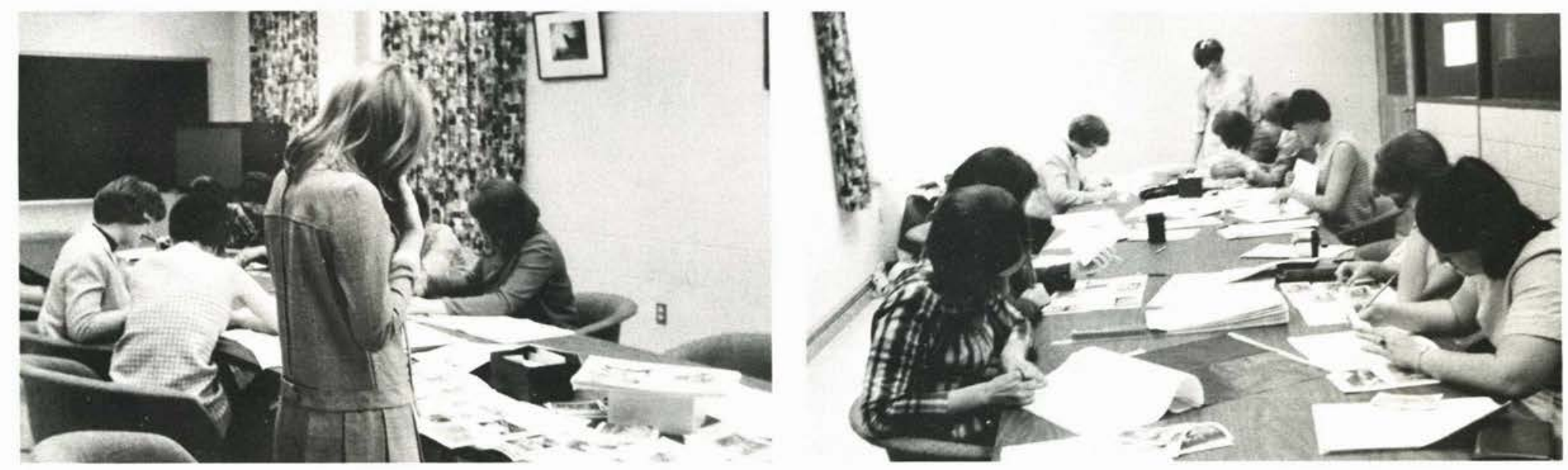

\section{REFLECTIONS OF THE EDITOR}

Although this has been the busiest year of my life, it has been the happiest. I have enjoyed working on the yearbook very much and the things I have learned through this experience have been invaluable. Most of all I have learned to trust Christ more completely. Knowing that He would help me through this responsibility, I found strength at the right times instead of panic. I will always remember this year as the year in which I had the opportunity to serve the school by attempting to preserve the events, thoughts, people and the school as it was in 1967-68.

I would like to thank my staff for the fine work they have done this year and for being patient as I learned. I would also like to express my appreciation to Mr. Turner and Mr. Yarnell for helping me locate pictures, to Dale Braun and Art Shuter for taking many of the pictures we needed, to my parents for their encouragement and help and to those who took time out to help in the last minute rush.

Dianne Chaffe 
INDEX 
Abernathy, Betty ... 81

Abrams, Dorothy ...81, 137, 138

Abuhl, Barbara ... 71

Acker, Donald ... 71, 128

Acton, Daniel ... 95, 129

Adams, Richard ...71, 86, 132, 140, 142

Adams, Sandra ...95

Adams, Susan ...95

Afolabi, John ... 95

Albright, Kathryn ...81, 125, 129

Allen, Deane ... 47, 120, 144

Allen, Robert ... 81, 132

Allerton, Ronald ... 46, 120, 132

Ambroza, Douglas ... 81, 146

Amstutz, William ... 95, 129

Anderson, Lyle ... 73, 81, 135, 137, 139

Anderson, Margaret ... 71, 135, 137, 139

Anderson, Olivia ... 71

Andrews, Thomas

Anthony, Caroline

Ashley, Janet ...81

Atherton, Donald ... 95, 143

Augsburger, Maxine ... 71, 125

Augspurger, Ruth ... 71

Austin, Bonfiles ...8 81

Austin, Jane ...81, 120, 125, 128, 137, 138

Averitt, Constance ... 95, 123, 134

Bailey, Francis ...81

Bailey, Nancy ... 81

Bair, Marsha ... 95, 125, 128, 129

Baker, Donita ... 81, 128, 129

Baker, Rebecca ... 47

Baker, Ronald ... 47, 144

Bales, Marilyn ...8 81, 124, 133, 137, 93

Barcus, Joanna ...71, 138

Barlow, Jacqueline ...95

Barnhart, Roy ... 71, 132

Bartlett, Mary ...95, 125, 129, 130, 140, 147

Beatty, Rita ... 110

Beck, Linda ... 81

Beerer, Carol ...71, 125, 130, 133

Beesley, Janet ... 95, 134, 137

Beitler, Mareanne...95, 120, 125, 147

Beitler, Marilyn ... 71, 121, 125, 128

Bell, Terry ... 71

Bellew, Larry ... 47

Bellintani, Loris ... 47

Beltle, Allen ... 81

Bennett, Barbara ...95, 128

Bennett, Beth ... 81

Benton, Cheri ... 71

Bernheisel, Lois ... 95

Berry, James ... 71, 121, 128

Bertschinger, Betty

Biddison, Barbara ...99, 125

Biddle, Calvin ... 47, 121

Bigley, Regina ...95, 125, 140

Biller, Tom ... 47

Bird, Bonita ... 47

Bird, John ... 71

Bixel, Robert ... 71, 128, 132

Blake, Diane ... 47, 140

Blaylock, James ...47

Boblitt, Janice ... 48

Bodden, David ... 81, 132

Bodenmiller, Betsy ...71, 134

Boertje, Darwin ...81 81

Bogert, Margaret ...69, 110

Bolen, Paula ... 71

Bolton, Paul ... 95, 129

Bonzo, Patricia ... 81, 121

Booghier, Stephen ... 71

Boothroyd, Hilda . . 73, 95, 137

Borger, Karen ... 81, 128, 137

Bosworth, Timothy ... 95, 124, 132

Bowersox, Richard ... 48
Boyce, Michael ... 95, 144

Boyd, Dan ... 48, 139

Boyd, Joan ...995, 128, 130

Boyd, Patricia ... 111, 139

Bramblet, Joanne ... 95

Braswell, Sylvia ...81

Bratka, Donna ... 81, 120, 121, 125

Braun, Dale ... 81, 132

Brewer, Margaret ... 48, 123, 133, 137, 139

Brock, Janice ... 81, 134, 137

Brock, Lynn ... 48

Brookhart, Joyce ...95, 125

Brower, Daniel ... 48, 128

Brower, Steve ... 95

Brown, Carolyn ...7 71

Brown, Jon ... 48, 142

Brown, Julia ... 96

Brown, Kirby ...996

Brown, Martha ... 71, 136

Brown, Nancy ... 96, 147

Brown, Robert ...996, 128, 129, 137, 138

Brown, Stanley ... 71, 121

Browning, Linda ...996, 133, 137, 139

Buchanan, Karen ...996

Bunting, Dennis ... 135, 137, 121

Bush, Deborah ... 48, 75, 120, 123

Busho, Connie ...99, 136, 138

Butcher, Preston ... 82

Butler, Richard ... 96, 143, 146

Butler, Roxanne ... 71, 128, 133, 136, 138

Butler, Valerie ... 82, 137

Buzzard, James ...996

Byers, Ronald

Cady, Audrey ...48 48

Cagwin, Daniel

Cagwin, Rebecca ... 48

Call, Alan ...8 82, 129, 131, 132

Campbell, Hugh

Campbell, Jerry ... 82

Caraway, Daniel ...63, 71, 100

Carity, Marcia ... 96

Carlson, Beverly ...49, 123, 130, 147

Carmean, Robert ... 82

Carpenter, Timothy ...72, 144

Carroll, Donald ... 96, 143

Carter, Kathy ... 72

Carter, Verna

Cartner, Cynthia ... 82, 121, 134

Cartner, Kathleen ... 72, 134, 137

Causey, Mark ...82, 140

Chaffe, Dianne ...72, 91, 120, 121, 122

Chandler, Herman ... 111

Chapin, Janet ... 82

Chapman, Dennis

Chumbler, David ... 72

Cianfrani, Carmen ... 72

Clark, Carolyn ...82 82

Clark, Cathy ... 96

Clark, Susan ... 96

Clater, Theodore ... 41, 49, 121, 129, 130, 137

Claypool, Kenneth ... 82

Cleckner, Barbara ... 72, 130, 125

Cochenour, Donald ...996

Cockerill, Diane ... 96

Cole, Ken ... 41, 82, 120, 132

Collins, Cherrill ... 72, 130

Collogan, James ...996

Colyer, James ... 97, 132

Combs, Gay ... 72

Comstock, Faith ...82

Conant, Joy

Conant, Virginia ... 72, 130

Connell, William ... 72, 79, 130, 131

Connelly, Don ...97, 123, 128, 138

Cook, Roger ... 82, 132

Cooley, Mark ... 97 
Coombs, Ronald ... 97, 127, 132, 137

Cooper, Donald ... 49, 108, 140, 143

Cooper, Gwytha ... 49

Cooper, Wendy ...997

Corbin, Joan ... 82, 133

Coriell, Ronald ... 72, 127, 132, 142

Cornett, Laurence

Cosgray, Robert ... 49

Cossins, Martin ... 97

Courtot, Kathryn ... 82

Courtot, Max ...73

Cox, Martha ....82

Crampton, Michael ... 41, 49, 137

Crampton, Roger

Creviston, Gary ... 97, 129, 132

Crowel, Robert

Crunk, Maureen ... 97, 124, 129

Culberson, Lyle

Cunningham, Cynthia ...99, 140

Curcio, Charles ... 83, 140

Curtis, Mary ... 49, 125

Cypher, Richard

Daab, Donna ... 73, 89, 120, 137, 121

Darrow, Gary ... 73, 132, 140, 143

Daubert, Russell ... 49, 142

Davis, James ... 49, 137

Davis, Larry

Davis, Lynn ... 128

Davis, Marilyn ...997

Decker, Rebekah ... 83, 128

DeDario, James

Denner, David ...997

Denner, Dennis

Dennis, George ... 97

Dennis, Steven

Dennis, William

DeOliveira, Luiz ... 49, 78

Devor, Elizabeth ... 97

Dewar, Barbara ... 73, 125

Dieringer, Dennis ...97, 128, 129, 131, 132, 146

Dietz, Edward ... 97

Dillon, Berdine

Dillon, Jeri

Dohogne, Diane ... 98

Donaldson, Karen ... 73, 128, 140

Donley, John

Douglass, Sandra ...98 98

Draxler, Dave ... 41, 93, 138

Duell, Regina ... 98, 125, 129, 138

Duff, Thomas ... 50, 63, 140, 142

Dufford, Shirley ... 50, 121, 129, 130

Durham, David ... 98, 120, 144

Dutiel, Pamela .... 138

Dutton, Charles ... 68, 98, 132

Eberhart, Michael ... 73

Eckart, Cynthia ... 83, 124, 125, 127

Edlund, Barbara ...998

Edwards, Brian ... 98

Etwards, Luther

Edwards, Ronald ... 83, 120, 128, 131, 137, 143

Eichelberger, Janet ... 98, 137

Eichelberger, Leland ... 41, 83, 127, 132, 138

Eifert, Wilbur ... 50

Eldridge, Elaine ... 83

Elliott, Harry ...73

Empet, Sara ... 73, 124

Endicott, Connie ... 83, 128

Entner, Paul ... 73, 120, 144

Epps, Janet ...998

Erickson, Randall ...98

Estep, Gary ...98 98

Eury, Sylvia ... 83, 120, 136, 138

Evans, Gladie ...8 83

Evans, Kenneth ... 98, 132, 146

Evans, Sylvia ...73

Evans, Thomas
Eveland, Jean ... 99, 128

Falci, John ....50

Farrar, Robert ... 99

Farrell, Kathleen ...83

Fawley, Jean

Felter, Charlene ... 83

Fidler, David ... 110, 129

Field, Jennifer ... 121

Field, Laura ... 83, 139

Fisher, James ...8 83

Fisher, Ronald ... 83, 143

Fitch, Darlene .. 41, 73, 111, 133, 135, 137

Fletcher, John

Flint, Mark ...99

Foster, Dale

Foster, Glenda ... 74, 133

Foulkrod, Beverly

Foulkrod, Tom ... 110

Fourman, Nancy ... 50

Fowler, Gary ... 50

Fowler, Steven ... 99, 107, 146

Fraley, Tomecia ...99

France, Patricia ... 83, 120, 121, 122, 123, 124, 125, 133, 137

Frawley, Jean

Freed, Patricia ... 99

Freehauf, Heidi ...999

French, Gary ... 99

French, Gerald ... 50, 142

Friedrich, Paul ... 83, 131

Frields, Mark ... 99

Frink, James ... 99

Frye, Jonathan ...99, 99, 132

Furushima, Gayle ... 99, 124

Gabriel, Janet ... 99, 134, 137

Gaffner, David ... 74, 132, 143

Ganschow, Ann ... 50

Gariss, David ... 89, 99

Gariss, Ronald ... 84

Garlock, Linda ... 74

Gathany, Andrew ... 84, 132

Gatliff, Gail ... 74, 125, 133, 134, 137, 138

Gauwitz, Donald

Gavitt, Leroy E. ... 50

Gelo, Thomas ... 84, 132

Gerber, Lonnie ... 99

Getts, Jeannie ... 99

Getz, Cathy ... 99

Gibbs, George

Gidley, David

Gift, Susan ... 68, 84, 97, 137, 139

Gilbert, Dan ... 99

Gilbert, David ... 99, 129

Gildan, Kathleen ... 110, 133

Gillenwater, Glenda ... 84

Ginaven, Sheryl

Gingrich, Susan ... 99

Gish, Duane ... 99, 132, 136

Gladding, Linda ... 99

Goodman, John ... 74

Goodwin, Joyce ... 50

Gordey, Sonya ... 74, 128

Gottwals, Douglas ...64 64

Gowman, Sandra ... 99

Grable, Mary ... 74, 121, 125, 130, 140, 147

Graham, John ... 84

Graham, Sharon ... 74, 84

Graves, Richard

Greene, Preston

Greenwood, Esther ... 99, 135, 137

Greenwood, Thomas ... 74, 128, 137, 146

Gregory, David ... 74, 89

Gregory, Joseph

Griffis, Darry

Griswold, Karen .... 100, 123, 129, 133

Gromacki, Gloria

Grosh, Barbara ... 51, 133 
Grover, Judy ... 100, 128, 138

Grunert, Robert ... 84, 132, 138

Gunlock, James ... 51

Guthrie, Konnie ... 84, 121

Gutow, Martha ... 74

Guy, Thomas ... 100

Haffey, David ... 74, 120, 121

Haffey, Nancy ... 100, 128, 138

Hales, Timothy ... 100

Hall, Floyd

Hamill, Loren ... 68

Hamilton, Carol ... 51

Hamilton, Donna ...51, 120, 125, 133, 139

Hamilton, Karen ...51, 125

Hamilton, Louis 84

Hamilton, Michael ... 51

Hamilton, Randall ... 41, 120, 127, 132, 137

Hamilton, William

Hammer, Arnold

Hammond, Michael ... 74

Hammonds, Kenneth ...84, 130, 132, 138

Handyside, David ... 74, 125, 130, 140

Hansmann, Paul ... 84

Hanthorn, Rebecca ... 100

Hardin, Joy ... 100

Hardy, Ruth ... 74, 134, 137

Hare, Carol . . . 74

Hare, Evelyn ... 84, 89

Harman, Rebecca ... 84, 122, 125, 133

Harris, Jane ... 51

Hart, Barb ... 100

Hart, Ruth ... 52, 133

Hartman, Tyler ...84 84

Haseltine, Dan ... 84, 132

Hayes, Bruce ... 100

Heldreth, Kirk ...85, 120, 121, 131

Hemphill, Barbara ... 100

Henderson, Thomas

Hendrickson, Rose ... 74

Herwig, John ... 137

Hesler, Sherrill ... 85, 131

Hess, Jane ... 75, 134, 137

Hetrick, Sally ... 75, 121

Heyer, Kent ... 100

Heyer, Neal ...75, 142

Higdon, Donald ... 52

Hildreth, Kirk ... 128

Hirschy, Judith ...75, 137

Hirschy, Linda ... 100, 137

Hoag, Jennifer ...75, 120,130, 133

Hockenberry, David

Hoerath, June ... 100, 130

Hoffman, Robert F. ... 75

Hoffman, Robert J.

Hogan, Donna ... 75

Holderby, Perry

Hollaway, Joseph ... 52

Hollaway, Linda ... 75, 140

Holliday, Jack ... 85, 110

Holtzhouse, Ruth ... 100, 129

Hook, John

Hopkins, Donna ...8 85

Horner, Carol .... 100

Hostetler, James ... 100

Howell, Francis

Hoyer, Barbara . . . 85

Hubbard, Cathy ... 52, 121

Hubbard, Judy ... 101

Huddleston, Dennis ... 101, 146

Hughes, William ...75

Hull, Charles ... 52, 129, 138

Hull, David ... 85, 132, 137

Hulto, Eunice ... 111

Hume, James ... 52

Hume, Linda

Humphrey, Ronald
Hunter, Robert ... 75, 128, 132

Hurst, Harold ... 101, 143

Hurtt, Sharon ...85

Hutchison, Dianna ... 85, 92, 121, 122, 131

Inghram, Richard ...68, 101

Irwin, Nora ... 101, 125

Irwin, Patti ... 50, 101

Ishuin, Geraldine ... 101, 129

Ison, Robie ... 85

Jacoby, Elaine ... 101

Jefferies, Linda . . . 85

Jefferies, William ... 75

Jenista, Carol ... 101, 128, 129

Jenista, Frank ... 52, 120, 121

Jenista, Ruth ... 52

Jenkin, Patricia ...93, 101, 141

Jensen, Val ...75

Jeremiah, James ... 85, 120, 137, 143

Jezowski, Ronald ... 75, 130, 132

Johnson, Dorena ... 101

Johnson, Elaine ... 52, 133, 137, 139

Johnson, Faye ... 101

Johnson, Kathleen ... 52, 125, 133, 139

Johnson, Richard

Johnson, Stephen ... 85

Johnson, Thomas ... 53

Jones, David ... 53, 67

Jones, Frances ....53

Jones, Kenneth

Jones, Paul ... 85, 131

Jones, Verna ...85

Jordan, Barbara ... 75

Kahler, Carolyn ... 85

Kanengieter, Rosalie

Kapp, Marian ... 53, 130, 133

Karschner, Wayne ...886

Kauffold, Ruth ... 53, 125, 128, 133

Kaufman, Joan ... 68, 97, 101

Kearney, David ... 86, 128, 131, 137

Keever, Jan ... 125

Keib, Donna ...53

Keim, Alan ... 53, 124, 125, 132

Kelley, Samuel

Kensil, Barbara ... 75, 147

Kever, Janet ... 75, 129, 133

Keys, Jocele ...886

Kilko, Robert ... 129, 137, 138

Kincannon, Donna .... 76

King, Donald

King, Jean ... 101, 137, 138

King, Maureen ... 101

King, Robert ... 76, 139

Kinniburgh, Daniel ... 76, 124, 125, 126

Kintz, Michael ... 76

Kirchner, Dennis

Kiser, James

Kishpaugh, Deanna ... 86, 125

Kishpaugh, JoAnne ...8 86

Klein, George ... 86

Kleymeer, Joy ... 76, 123, 133

Klomparens, Ruth ... 86

Knapp, Jaris ... 41, 101, 138

Knott, Allen ... 53, 132, 143

Knott, Eileen ... 86, 121, 137

Koehn, Darlene ... 102, 141

Koehn, Deborah ... 102, 141

Koogler, Arthur

Krikke, Florence ... 102

Kukasky, Thomas ... 102

Kunselman, Karen ... 76, 125, 133

Kusumoto, Ann ... 102, 120, 125

Lacey, Daniel

Lake, Harry . . . 76

Lake, Sharon ... 11

Lapp, Robert ... 102, 127, 132, 138, 139

Large, Donald ... 102, 124, 125, 132, 142 
Larsen, Bryan ... 102

Lathrop, Sandra ...88, 139, 140, 147

Lavener, Dale . . 53, 132, 142

Launer, Linda ... 53

Lautt, Tina ... 54, 93, 122

Lavender, Larry ... 76

Layner, Clifford . . . 76, 139

Leach, Don ... 54

Leach, Judith ... 102

Leach, Roger ... 127, 132, 139

Leapline, Nancy ... 128, 137, 147

Lear, Patricia ... 102

Lee, John ... 87, 132

Leiby, Stephen

Lepline, Barbara ...88 87

Lescure, Lynn ... 54, 130

Lewis, Carol . . . 76

Lewis, Darlene ... 102, 130

Lewis, Judy ... 102, 137

Lewis, Nancy ... 54

Lewis, Ron ... 54

Lewright, Barbara ... 102

Liechty, Daniel ...87, 137, 139

Lillback, Larry ... 102

Lima, Iris . . 102, 125

Lima, Margarita ... 102, 137

Liming, Rebecca ... 102

Little, Steven ... 102

Lloyd, Kathleen

Long, Donald ... 102, 138

Lucas, Sandra ... 54, 125, 133

Lucius, Bruce ... 54

Lunney, Robert ... 102, 143

Lyon, Warren . . .87

Madsen, Linda ... 102, 130

Maidment, Beverly ... 102, 133

Maidment, Lee

Malmstrom, Janet . . .87

Mann, Darlene ... 102, 128, 147

Manross, Daniel ... 102, 132

Mansker, Pamela ...87

Margraff, Linda

Margraff, Paul ... 103

Marsh, Lynnette ... 76

Marshall, Dennis ... 132

Martin, Janis ... 87, 124, 125

Mason, Beverlee .... 103, 104, 141

Matkins, Diane ... 103

Matulevicus, Carol ... 103, 138

May, John ... 54, 143

Maynard, Janice ... 103

Mays, Dee... 128, 129, 132, 146

Mays, Loren ... 103

McCann, Judy ... 87, 141

McClain, Richard ... 103

McComber, Rodney ... 103

McCulloch, James ... 103

McCullough, Max ...87

McCullough, Michael ... 131, 132, 142

McDaniel, Tanis ... 103

McDonald, Bruce ... 76, 140, 143

McDonald, Rebecca ...87 87

McDowell, Gary ... 87, 143

McGee, Katherine ... 54, 133

McGillivray, John ... 76

McGinnis, Thomas ... 76

McGreevy, Walter

Mclinnes, Roberta ... 54, 121, 130

Mckenney, Roger ... 55

Mcknight, Rick

McMillen, Samuel ... 103, 137, 138

McNiece, Melinda ... 103

McVey, William ... 55, 131

Mellish, Ron ... 76

Merriman, Douglas ... 103

Metzger, Norine
Meyer, Viann ... 87, 133

Meyers, Betty ... 55, 130, 131, 133, 138, 139

Meyers, Ronald ... 103, 124, 127, 130, 132, 138, 139

Millard, Linda ... 87

Miller, Alice ... 87

Miller, Barbara ... 76, 121, 125, 128, 130

Miller, Darryl ... 65, 103

Miller, Donald ... 103

Miller, James ... 55

Miller, Janice ... 77, 125

Miller, Joan ... 103, 125

Miller, Joyce . . 87, 129

Miller, Priscilla ... 77

Miller, Roberta ...55, 125, 140

Mills, Dale ... 103, 129, 132

Mills, Roger ...993

Mitchell, Beverly ... 103

Mitchell, Charles ... 55

Mitchell, Marilyn ...55 55

Mitchell, Richard ...87, 123, 124, 128, 129, 132

Mix, Beverly ... 103

Modrall, Larry ... 103

Mogle, Claire

Mohler, David ... 103, 132

Mohler, Philip ... 103, 135, 137

Moore, Harold ... 77, 137

Moore, Joyce

Moore, Terva ... 103

Moorman, Alice ... 87, 121, 129

Moose, Helen ... 55

Moots, J. ... 130

Morgan, Donna ... 55, 125, 133

Morrell, Thomas ... 77

Morrow, Coletta ... 104

Morse, Carole ... 104, 137

Moseley, Beverly ... 55

Mossman, Janice ... 111, 130

Mott, Shirley ... 87

Motter, Jerome ... 87, 127, 128, 132

Motts, Joyce ... 104, 138

Moulder, Steven ... 104

Mozingo, Edgar ... 104

Muck, Kenneth ... 77, 120, 128. 132, 142

Muirhead, Margaret ...88, 121, 125, 130

Mulford, Arlan ... 77

Murdoch, Ruth

Murphy, Coleen ... 87

Murphy, James ...5 56

Murphy, Phyllis

Music, Ernest . . . 77

Myers, Dawn ... 104, 129

Myers, James ... 56

Myers, Linda ... 129, 130

Nash, Charlotte ... 77, 133

Nelson, Dennis

Nelson, Karen ...87

Nelson, Linda ... 104

Nelson, Wayne ...87

Nevitt, Dan ... 56, 124, 130

Newberry, Kathryn ... 104, 137, 138

Nichols, Kenneth ... 111

Nichols, Pamela ... 104

Nichols, Penny ... 88

Nieboer, Constance ... 104, 128

Norman, Lee

Norris, Maynard ... 56, 132

Northey, Timothy ... 104, 129

Norton, Daniel ... 104

Norton, Nancy ... 77, 120, 133

Oehmcke, Peter ... 88, 128, 125

O'Keefe, Carolyn ... 104

O'Keefe, Elizabeth ...88, 121, 133, 137

O'Keefe, James ... 88

Olsen, Samuel ... 88, 120, 128, 132, 137

Olsen, Susan ... 104, 128

O'Shell, Constance ... 77, 123, 130, 133, 121 
O'Shell, Suzanne ... 46, 121, 125

Osinuga, Emmanuel ... 88

Osman, Marsha ... 104

Ostella, Richard ... 56

Ostrander, Albert ... 56

Overholt, Connie

Overholt, Kenneth

Packard, Calvin ... 77, 129, 146

Paige, Lynn ... 100, 104

Palmer, Milton ... 41, 105

Palmer, Vicki ... 105, 128

Pape, Sue ... 56, 137

Parcher, George ... 105

Parry, Jeffery

Parshley, Florence ... 105

Patten, Randall ... 41, 105, 132

Paugh, Nancy ... 105, 129

Pauling, Susan

Payne, Laura ... 105, 134

Peek, Jeanette ... 105

Penrod, David ... 105

Pereira, Robert ... 77, 124, 125, 131

Perrine, Cheryl ... 105

Perrin, Bonita

Pestel, Gary ... 56

Pettitt, Warren ... 88, 144

Petty, Tony .... 105

Phelps, James ... 77

Phelps, Ronald ...88 88

Phenix, Janet ...88, 121, 122, 123, 124, 133, 139

Phillips, Edith ...88, 128, 129

Phipps, James ... 41, 56, 93, 121, 132

Plate, Alberta ... 88, 124, 133

Podhaski, Lynn ...89

Powell, Gregory

Powley, Robert ... 89, 138

Poyner, Lyman ... 105

Prather, Sandra .... 105, 130, 140, 147

Prentice, Larry ... 56

Pressnell, Walter

Preston, Donna ... 77

Price, Connie ... 105

Price, Daniel ... 56, 142

Price, Elaine ... 105, 128

Price, Judith ... 105, 128, 129

Price, Nedra ... 56, 133

Price, Wilbur ...89

Prindle, Priscilla ... 89, 129

Pritchett, Jennifer ...5 57

Pugh, Lawrence ... 89, 136

Radcliff, Larry ... 89, 128, 129

Radcliffe, Diana ... 89, 125, 128, 131

Rader, Rebecca ... 57, 147

Rader, Susan ... 77, 121, 140, 147

Rakes, David ... 77

Ramsey, Roy

Rau, Karen ... 77, 147

Ray, Susan ...88

Reagan, Wilborn ... 111

Reed, Barbara ... 106, 69

Reed, Gary

Reed, Mary Jean ... 57, 133

Reed, William ... 89, 126

Reeder, Bill ... 129, 138

Reeder, John ... 89

Reese, Betty ... 89

Rehkopf, Robert ... 128, 137, 138

Rehn, Judith ...89

Rejiester, James ... 90

Reno, Lee ... 41, 106

Reno, Loren ... 41, 90, 120, 140, 142

Richardson, Gerald ... 77, 127

Richardson, Pat ... 106, 122

Rieken, Marlene ... 57, 133

Riggs, Linda ... 57, 121, 133

Risko, Jeanne ... 77, 137
Roberts, Craig ... 106

Rockwell, David ... 77, 125, 131

Rockwell, George ... 90

Roderick, Richard ...57, 120, 139

Rodriguez, Ruth ... 990

Rogers, Ruth ...990

Rohm, Dane ... 106, 124, 128, 132, 137

Rohm, Lynn ... 57

Rohm, Robert ... 57

Rooke, Ben ... 106, 142

Rooke, John ... 77, 93, 132, 140, 142

Rose, Bonnie ... 57

Rose, Linda ... 90

Rose, Russell

Ross, Elizabeth

Rothwell, Susan ... 57

Rouch, Deborah .... 77

Rowe, Charles ... 106, 128, 132

Ruder, Esther

Rudig, Rebecca ... 90, 122, 125, 130, 133

Rueck, Faye

Rueck, John ... 46, 142

Ruffin, Danny ... 90, 144

Rumage, Lois ... 90

Rumley, John ... 77, 132

Russell, Adrienne ...990, 128

Russell, Saundra ... 90

Saemenes, Joyce ...99, 125, 133, 137

Sattler, Melbourne ... 106, 128, 132

Schill, William ... 77, 126, 132

Schilling, Dale ... 106

Schisler, Roy ... 58

Schlechty, Sandra ... 58, 125

Schneider, John ...99, 137

Schneider, Marikay ... 106

Schonscheck, Marlene ... 58, 121

Schrader, Tonya

Schroeder, Peter ... 64, 90

Schulte, Elaine ... 58

Schultz, Robert ... 78, 124, 129, 132, 142

Schwarm, Norma ... 106, 125, 130

Scott, Stephen

Seaman, James ... 111

Sears, Gail ... 90, 121, 123, 130

Seeley, Jeffrey ... 106, 132, 138

Seigneur, Dean ... 58, 125, 132

Senseney, Philip ... 90, 132, 135, 138

Sewell, Gerald ... 106

Sexton, Mary ... 106

Shafer, Chery ... 106, 124, 125

Shamblin, Rebecca ... 78

Sharp, Rebecca ... 90

Shearer, Byron ... 58, 91

Shelley, Melanie ... 106

Shepherd, Bruce ... 106

Sherman, Margaret ... 106

Sherrill, Patricia ... 106

Shreve, Arthur ... 106, 129, 131

Shuter, Arthur ... 41, 90, 126, 127, 138

Silverthorn, Renee ... 106, 133, 138

Simmons, James ... 106

Simpson, Donna ... 58, 125, 133

Sine, Robert ... 106, 129, 138

Slates, Linda ... 107

Sleischter, Mary ... 58

Smallwood, Paul

Smelser, Edward ... 78, 121, 128, 126

Smith, Dan ... 68, 90, 136

Smith, Melba ... 107

Smith, Michael

Smith, William ... 90, 121, 129

Snider, Nancy ... 107

Snyder, Stephen ... 78, 131, 120

Southwell, David ... 90, 132, 143

Sparks, Marvin

Spaulding, James ... 107 
Spencer, Kathryn ... 58, 147, 123

Spencer, Philip ... 58, 126

Spieth, Ronald ... 107, 135

Spinks, Linda .... 125

Sprigg, Kathy

Sprunger, Marcia ... 78

Stamatis, Judith ... 90, 140, 147

Stanbery, Maxine ... 46, 120, 130, 121

Staples, David ... 110, 121, 129

Steenburg, Cheryl ... 90, 134

Stephens, Linda ... 75, 107, 141

Stevens, Daniel ... 78

Stevenson, Connie ... 58, 125, 133

Stewart, Carol ... 91, 122

Stewart, Gwendolyn ... 78

Stickle, Fred ... 78, 128

Stillwell, Dennis ... 91

Stockwell, James ... 111

Stora, Dennis ... 91

Storey, Mary ... 107, 129, 130

Stout, Roger ... 78

Stowell, Hannah ... 107, 133

Strychalski, JoAnn ... 78, 128, 140, 147

Stryker, Timothy ... 91

Stumbo, Gaye ... 78

Sturdevant, Patricia ...991

Stutzman, Gary ... 107, 138

Sumner, Rita ... 107

Suter, Terry ... 107

Swan, Susan ... 58

Sweeney, Adrian ... 46, 127, 132

Tallman, Sharon ... 59

Tallmon, Verna ... 107, 130

Tanner, Lela ... 78, 125, 140, 147

Taylor, Gary ... 41, 78, 137

Taylor, Laura ... 78, 111, 121, 133, 135, 137

Taylor, Linda ... 78

Taylor, Steve

Taylor, Richard ....91

Tennant, Mercy ... 107

Thomas, Billie ... 107, 130, 140, 141

Thomas, Elaine ... 107, 129

Thomas, George ... 78

Thomas, John

Thomas, Neil ... 107

Thompson, Dale ... 59

Thompson, Diane .... 108, 122, 124, 129, 133

Thompson, Jerry ... 108

Thorne, Susan ... 79

Tompkins, Gail ... 59

Towle, Nancy ... 79, 125, 130

Treat, Kenneth ... 91

Trefzger, William ... 108, 143

Tripp, William

Trotter, Sharon ... 91, 128

Trumbull, Cheryl ... 79

Trumphour, Mary ...91

Turner, Virginia

Underwood, Norman

Urch, Suzanne ... 108

Vincent, Beverly ... 133

Vlug, Terrance ....59

Vos, Janice ... 108, 74

Wagner, Karolyn ... 91, 63

Wagner, Keith . .. 59, 128

Waite, Larry ... 59, 79, 120, 121, 124, 143
Walker, Leland ... 60, 120, 132

Waiker, Lois ... 60, 79

Walker, Thomas ... 108, 72, 132

Wall, David ... 46, 120, 127, 132, 139

Wallis, Nelson ... 79

Walstrum, Ann ... 91

Walthall, Gary ...60 60

Waltz, JoAnn ...91, 121, 122

Ward, Diana ... 60

Warnken, Estelle ...91

Warren, Carol ... 79

Warren, Timothy ... 79, 104, 132, 142

Watson, John ... 79, 143

Watson, Nola ... 91, 137, 138

Weaver, Judith .... 108

Webb, Susan .... 108

Webster, Daniel ...91

Welch, Glenda ... 68, 108

Welch, Loren ... 60, 127, 132, 137

Wessel, Lynda

Whaley, Janis ... 108

Whatoff, Robert ... 79

Whipple, Ellen

Whitaker, Patricia .... 108

White, Connie ... 108

White, Martha ...92 92

Whitmire, James ... 60

Whitney, Leah ... 60

Wilhite, Mark ... 109, 124

Wilkes, Deborah ... 109, 133

Willenee, Carol ... 109, 129

Williams, Dwight

Williams, Joseph

Williams, James

Williams, Linda ... 109

Willis, Pamela ... 41, 79

Wilson, Mike

Winston, Judith ... 92, 123, 124, 129

Winters, Thomas ... 109

Wintrow, Linda ... 60, 125

Wissinger, Patricia ... 92, 140, 147

Wita, Ronald ... 41, 109

Withers, Lawrence ... 109

Witt, Shirley ... 92, 137

Wolford, Michael

Woodend, Michael ... 46, 123, 130, 132, 142

Worden, Ken ... 109

Work, Dale ... 79, 121

Wright, David

Wright, Edith ... 109

Wright, Homer ....92

Wright, Nancy ... 79, 130

Wuest, Darlyne ... 109

Yaple, David ... 109

Yeater, Sharon ... 79

Yoder, Douglas ... 79, 120, 137, 140

Yoder, Terence ... 125, 129

Young, Jeff ... 79

Young, Joyce ... 92, 141

Young, T. ... 142

Zazurskey, Michael ... 140, 143

Zerbe, Sheryl ....111

Zickefoose, Cynthia ...92, 130

Zimmerman, Barry ... 92

Zwiesler, Catherine ... 109 


\title{
FIRST BAPTIST CHURCH
}

\author{
Main \& Lawrence \\ Mishawaka, Indiana \\ 46544
}

"Proclaiming the Bible as it is for men as they are."

Sunday School

Morning Worship

Youth Groups

Evening Service

Victory Hour

Wednesday
9:25 AM

10:30 AM

6:00 PM

7:00 PM

7:00 PM

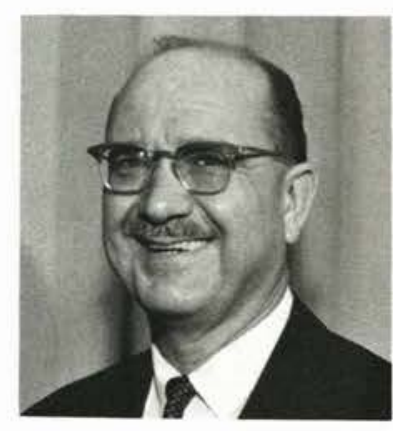

Dr. Roy G. Hamman Pastor
THE OHIO

INDEPENDENT BAPTIST

CONGRATULATIONS

TO

THE SENIORS OF '68

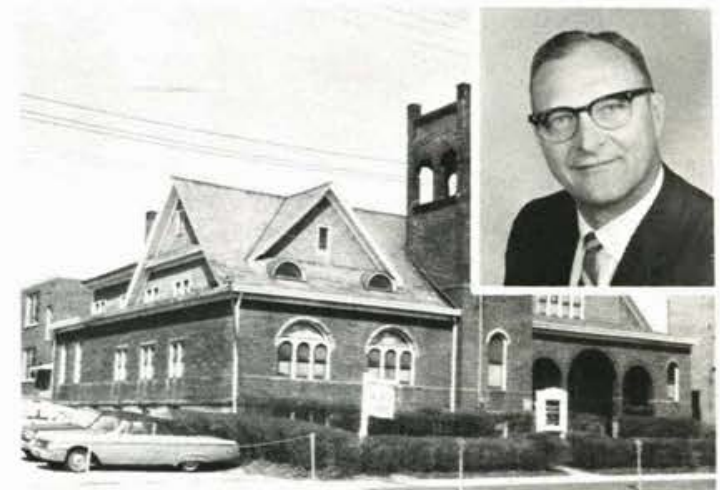

"GREETINGS"

FIRST BAPTIST CHURCH MEDINA, OHIO

"In the heart of Medina with Medina on its heart." 


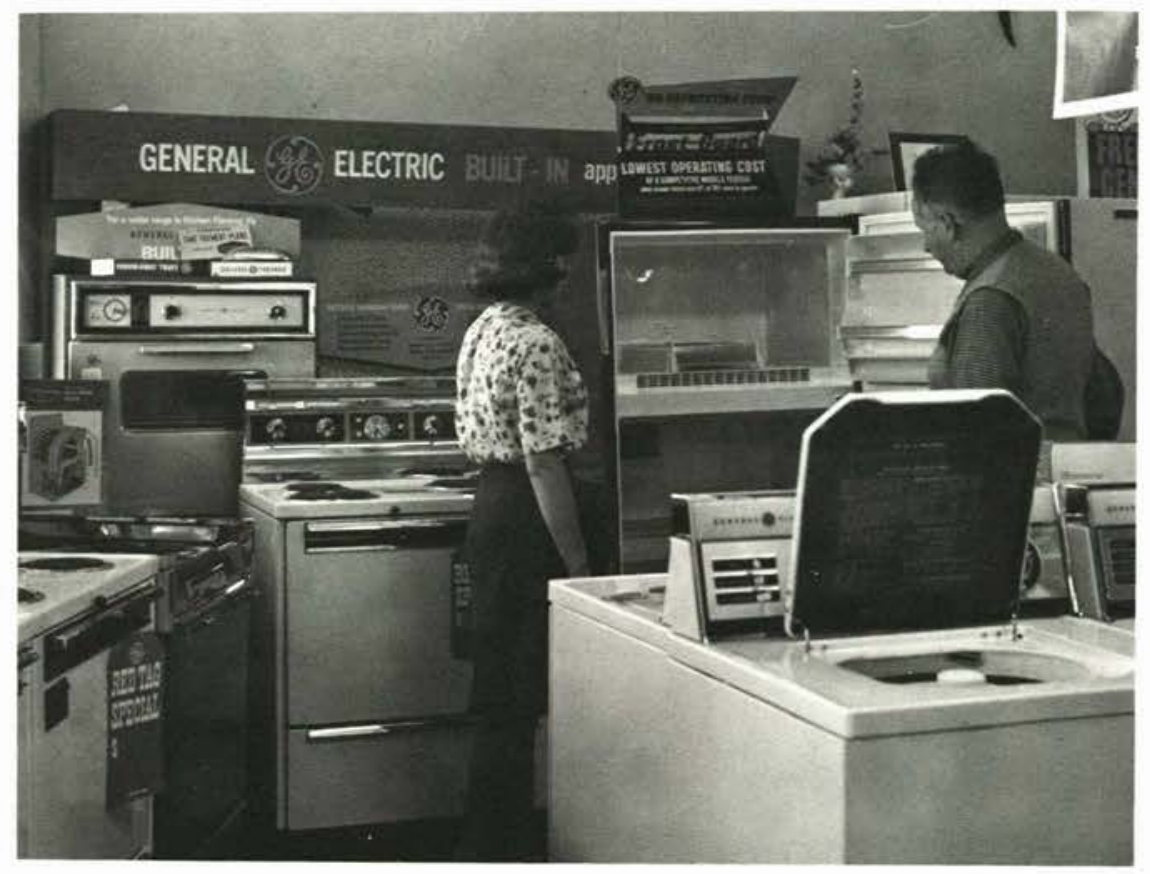

\section{CEDARVILLE HARDWARE}

"PROGRESSIVE HARDWARE SERVICE" 766-1941

FOR YOUR BEST DEAL IN ELECTRICAL APPLIANCES

\section{FIRST BAPTIST CHURCH}

\section{"DEPEND on the CAMERA SHOP" for PERSONALIZED SERVICE}

“. . THAT WE MAY PRESENT EVERY MAN PERFECT (MATURE) IN JESUS CHRIST."

Francis A. Crown Pastor
George D. Muzzey D.C.E.

CONGRATULATIONS

THE MEMBERS AND FRIENDS OF THE FIRST BAPTIST Church of Elyria,

Ohio congratulate the graduating

class of 1968, and wish you God's

best as you seek to do His perfect will in your life.

FIRST BAPTIST CHURCH OF ELYRIA, OHIO 276 Washington Avenue

Woodrow W. McCaleb, Pastor David F. Truit, Associate

\section{FIRST BAPTIST CHURCH}

Galion, Ohio

Rev. Wilfred Booth, pastor 


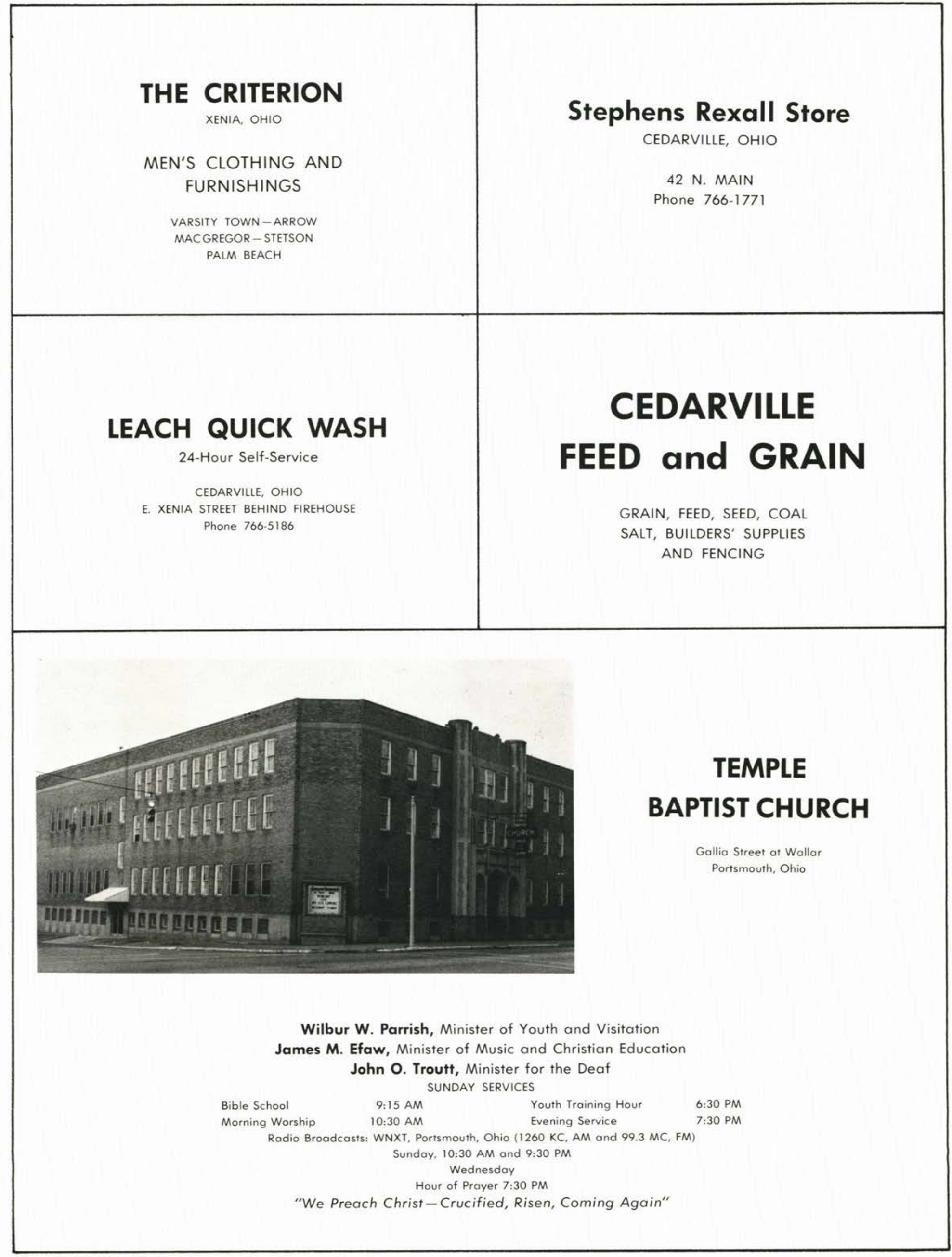




\section{Congratulations to the Class of ' 68}

Sherwin Williams

47 W. Main

Xenia, Ohio

Rogers Jewelers

37 East Main Street

Xenia, Ohio - 372-7681

Nilson-Bone Florists

68 West Main Street

Xenia, Ohio - 372-4211

Carriage House Restaurant

37 West Main Street

Xenia, Ohio

McDorman's Men Clothing

24 South Detroit

Xenia, Ohio

The Xenia Daily Gazette

"Growing with all Greene County"
Berean Church and Book Supplies 53 South Detroit Street

Xenia, Ohio

Daum's

114 South Detroit Street

Xenia, Ohio

Hitchcock's Jewelry Store 68 South Detroit Street

Xenia, Ohio

Tiffany Jewelry Store

20 South Detroit Street

Xenia, Ohio-372-3161

Famous Auto Supply

Sporting Goods

64 E. Main St.

Xenia, Ohio-372-4071

Adair's

Xenia, Ohio

"Worth a Trip to Xenia"
Hurley's Florist

544 W. Main St.

Xenia, Ohio

Calvary Baptist Independent Church

Cresaptown, Maryland

Compliments of Norton Baptist Church 4239 Cleveland-Massillon Rd.

Norton, Ohio 44203

"Standing on the Truth of God's Word"

Modern Shoe Repair Shop

53 West Main

Xenia, Ohio

Home Furniture

84 East Main St.

Xenia, Ohio

Litt Brothers

25 East Main Street

Xenia, Ohio 


\section{Cedarville Super Valu}

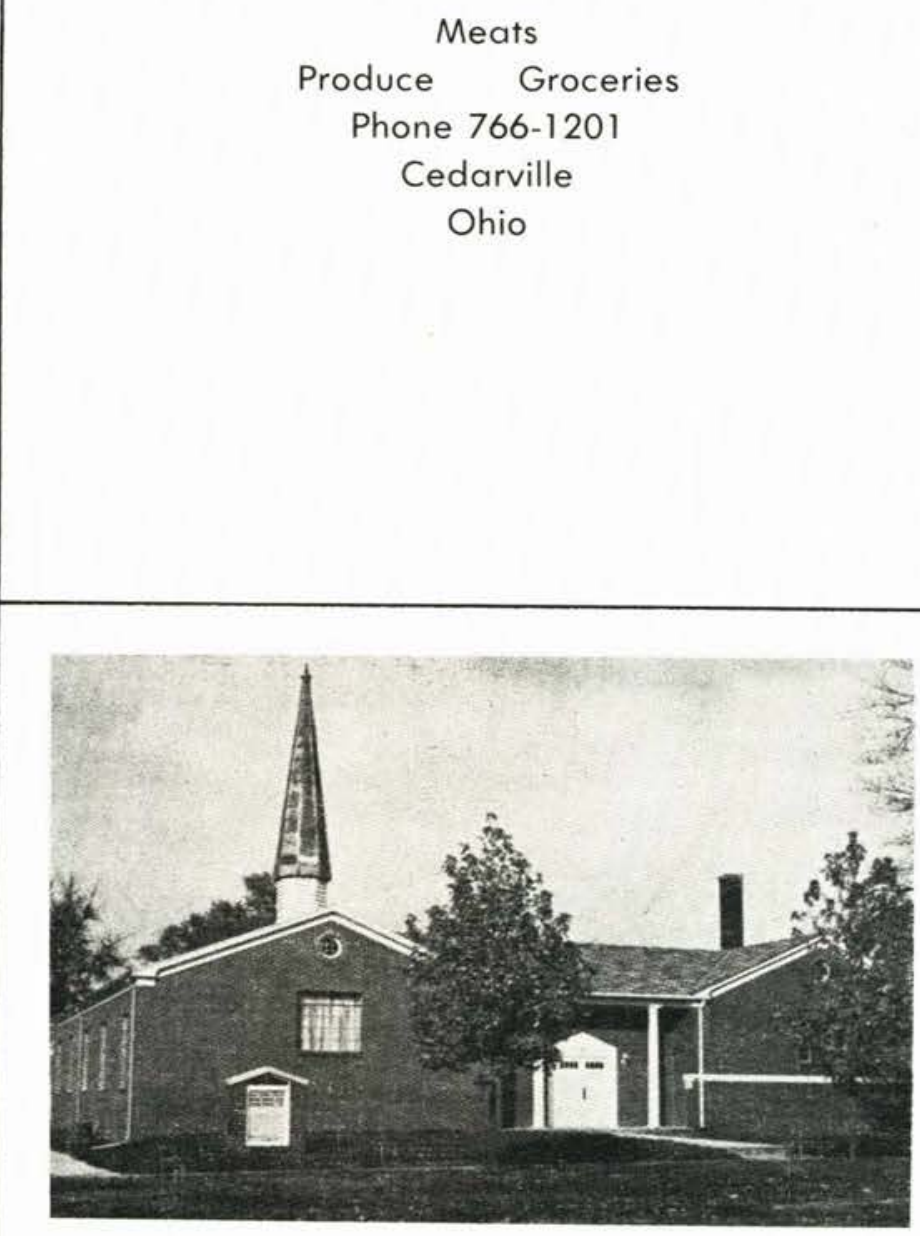

Faith Baptist Church

Western Ave. and 23rd St.

Mattoon, Illinois

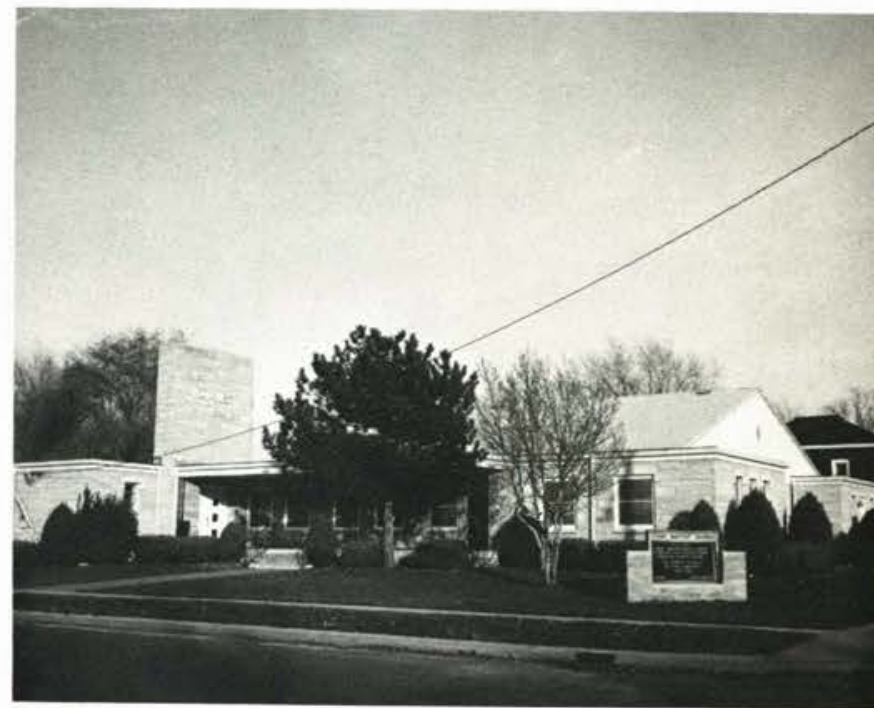

First Baptist Church

Pana, III.

Albert Rust, Pastor

Sunday School

Morning Worship

Study and Youth Groups

Evening Service

Mid-Week Prayer Service - Wed.

"Holding Forth the Word of Life"

Phil. 2:16

\section{HADDON HEIGHTS BAPTIST CHURCH}

Third and Station Avenues

Haddon Heights, New Jersey 08035

Dr. K.W. Mastellor, Pastor

Rev. F.D. Henzler, Ass't Pastor

Rev. D.P. Jeremiah, Minister of

Christian Education
9:00 AM

10:00 AM

$6: 30$ PM

7:30 PM

7:30 PM 


\title{
DINNEN'S SOHIO SERVICE
}

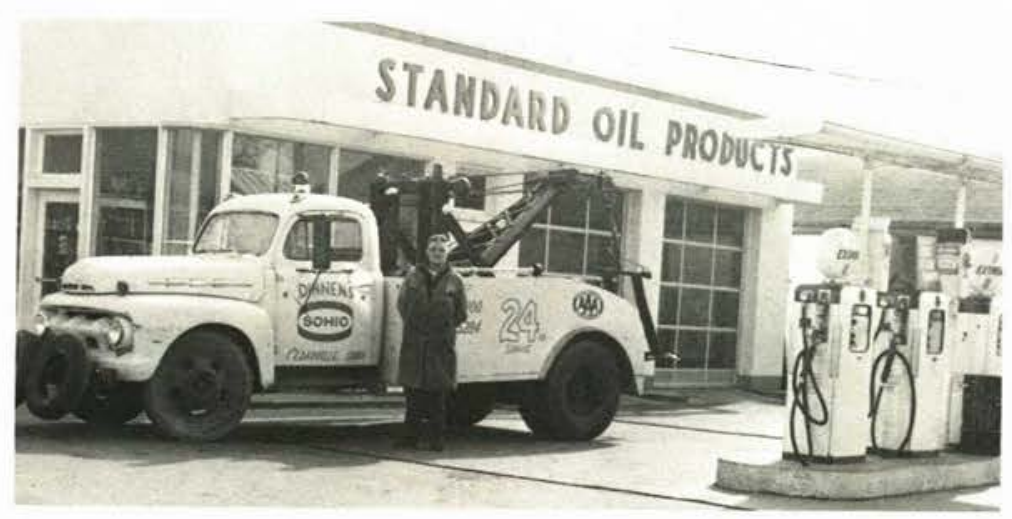

\author{
Batteries \\ Lubrication \\ Wrecker Service
}

Myron Dinnen,

Proprietor

\section{First Baptist Church}

Ninth Avenue and Eleventh Street

Silvis, Illinois 61282

Harland L. Sauser, pastor

\section{Congratulations}

\author{
to the \\ Class of '68
}

\author{
From \\ the \\ Miracle \\ Staff
}
Sunday Services
9:30 AM Sunday Bible School
10:45 AM Morning Worship
6:00 PM Youth Fellowship
Thursday
7:30 PM Prayer Meeting











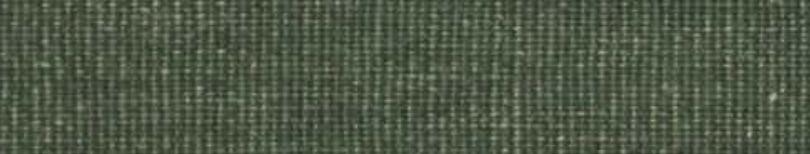

(P)

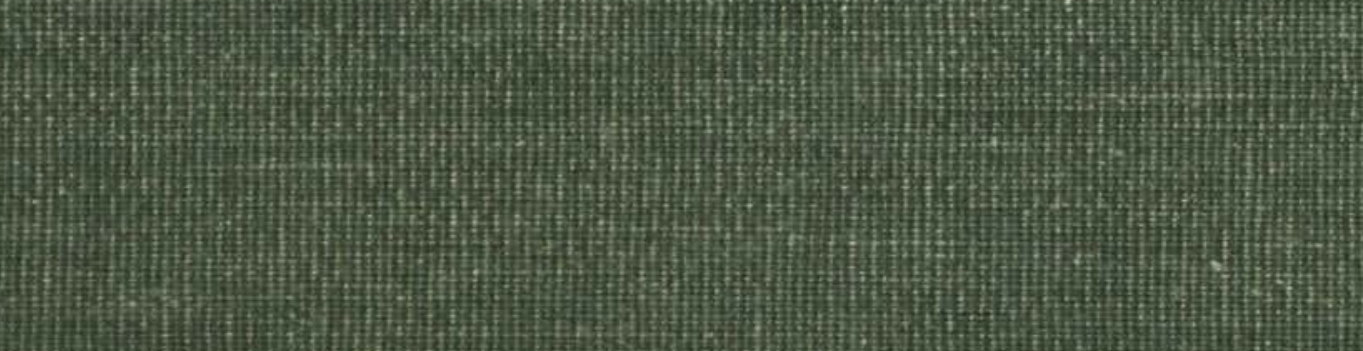

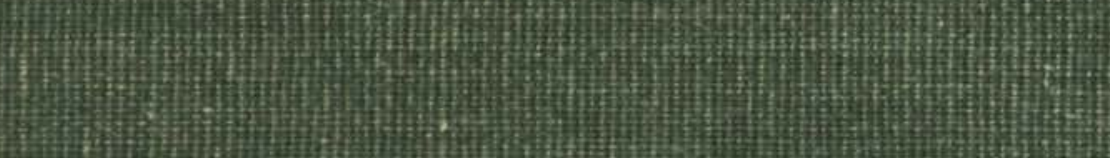

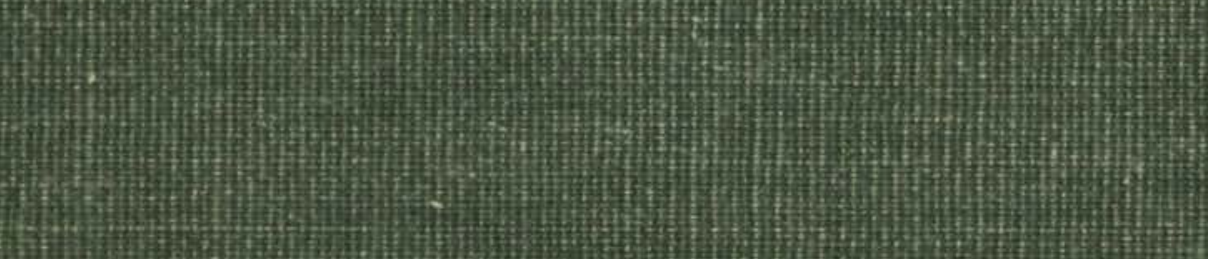

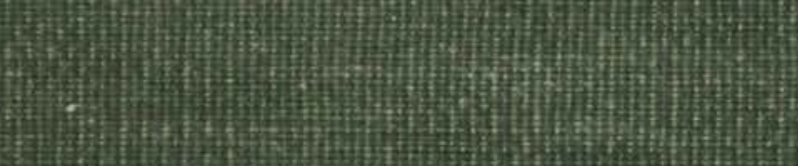

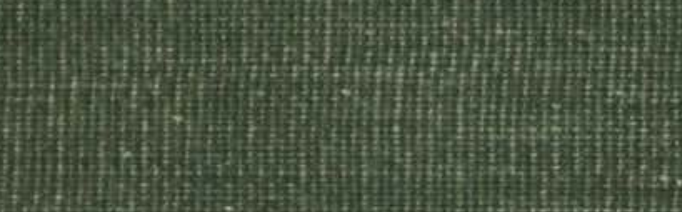

(1)

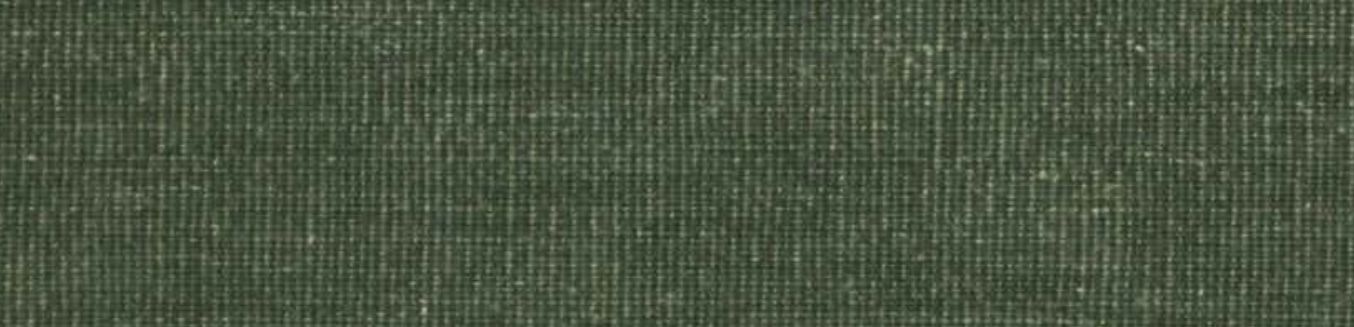

4)

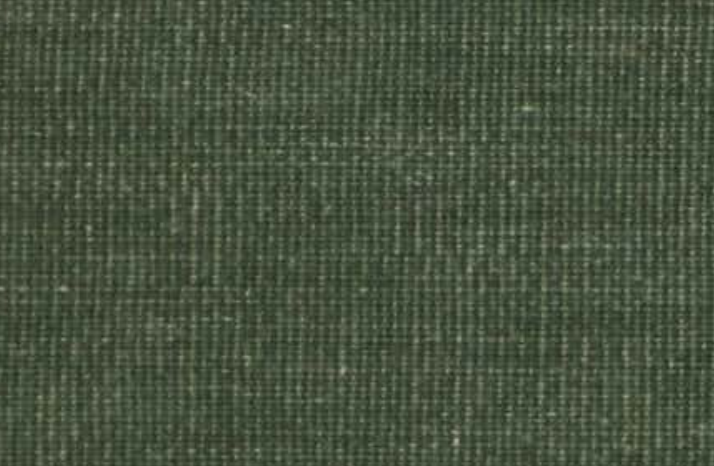

P(1)

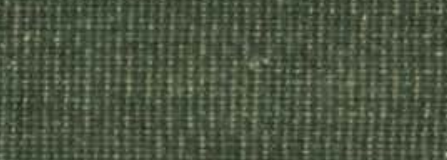

